LIBRARY OF CONGRESS

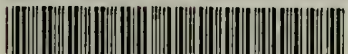

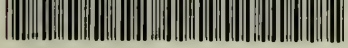

000039442680 
D.

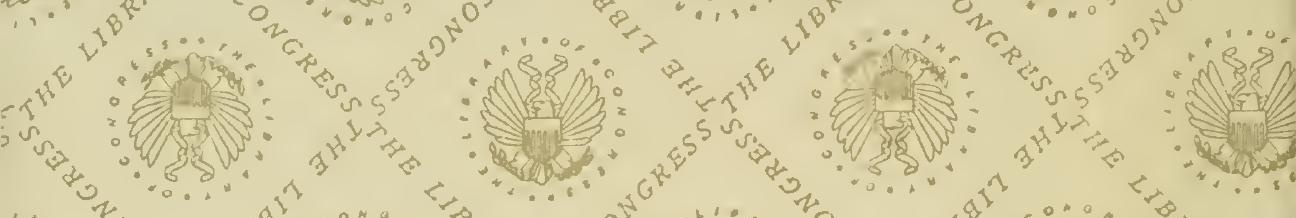
0
0

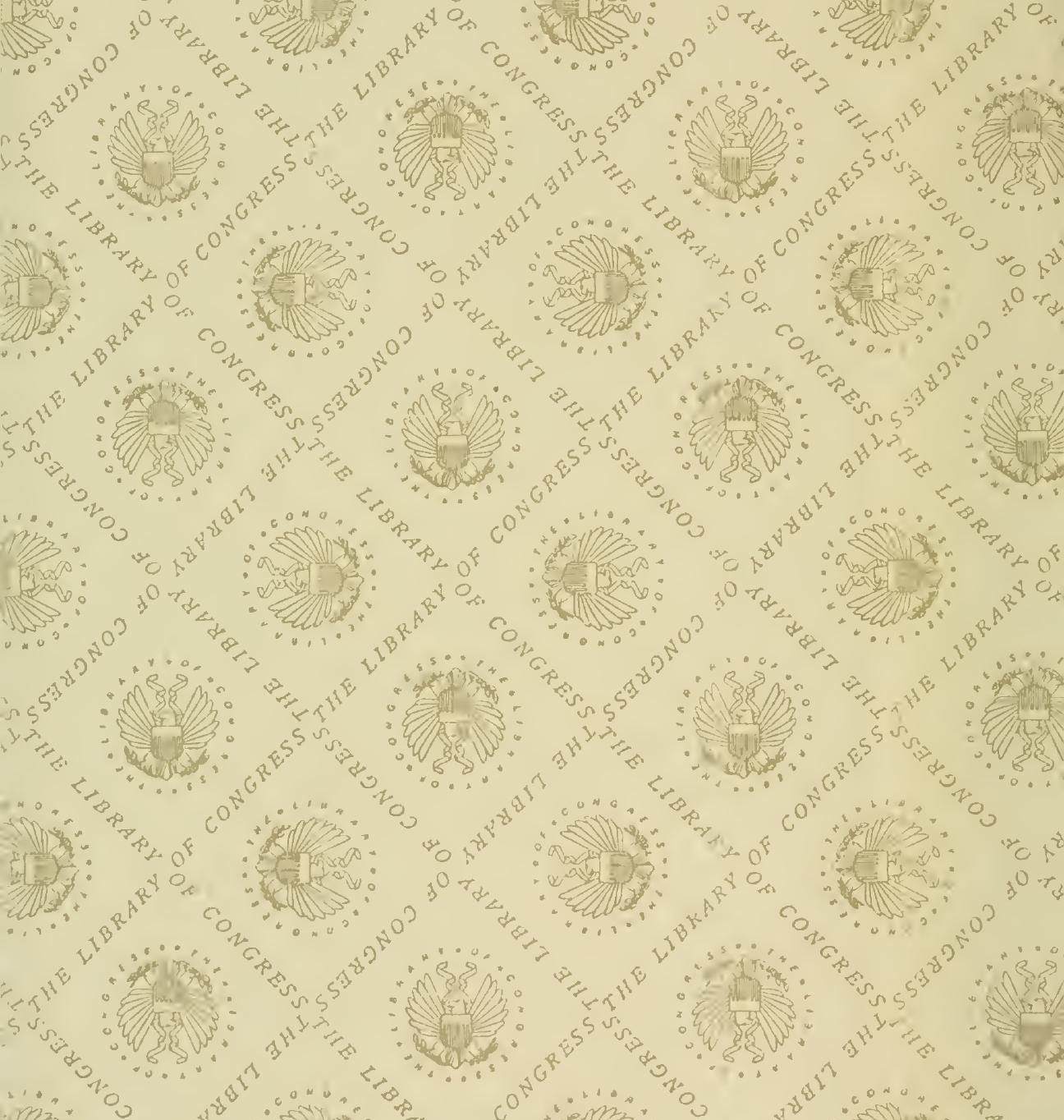

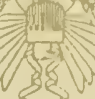


(a) 年

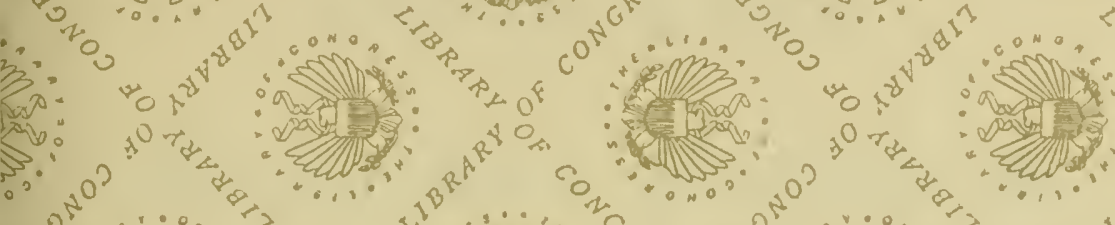
16. 14.
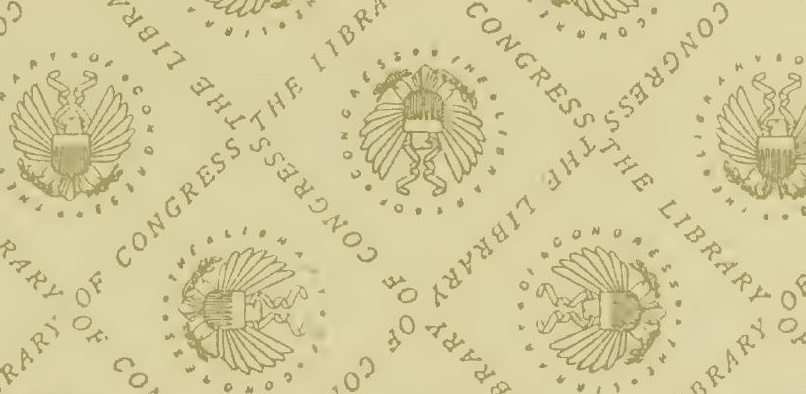

70
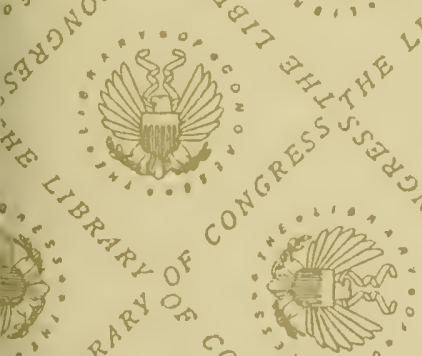

\section{清

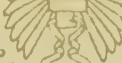

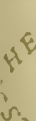
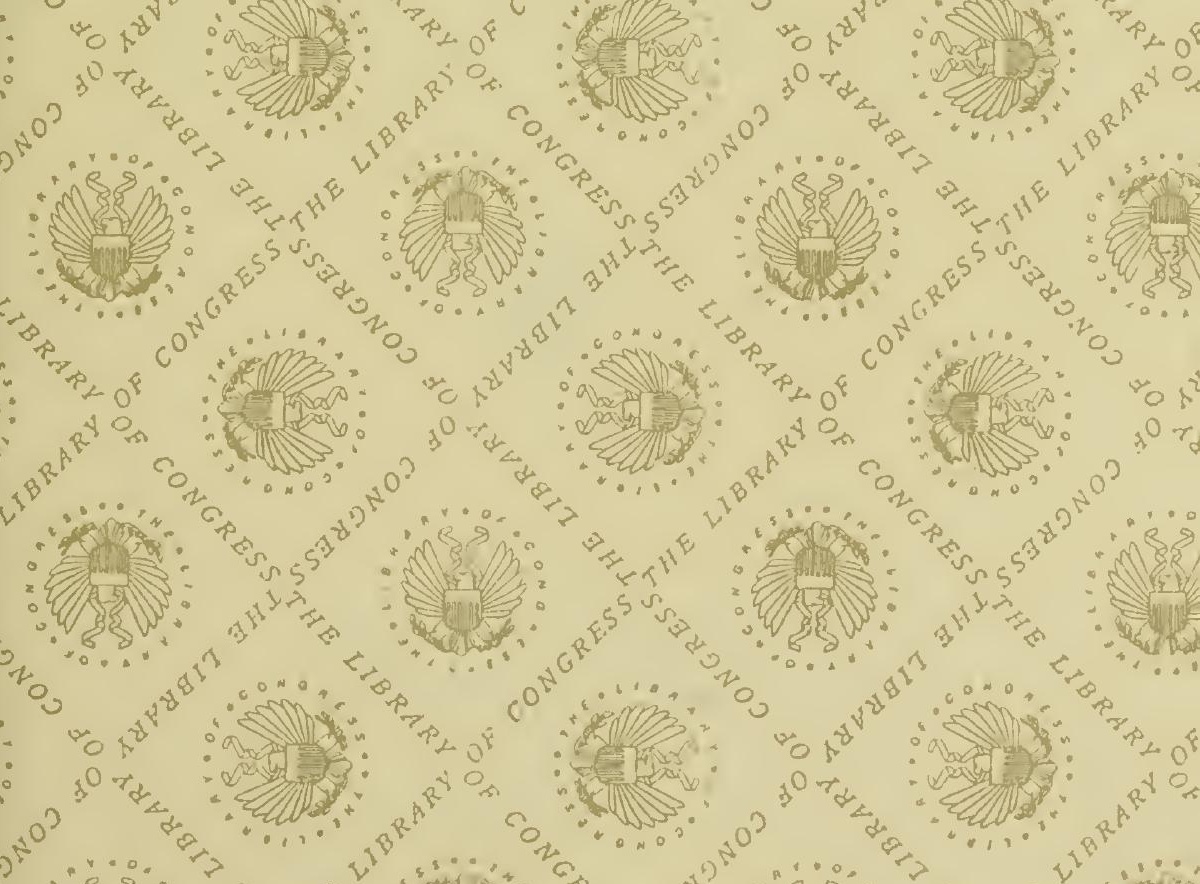



\section{VETERINARY}

\section{Matereta Medica}

A ND

\section{THERAPEUTICS}

\section{$\mathrm{BI}$}

Kenelm Winslow, M.D.; M.D.V.; B.A.S. (Harv.)

FORMERLY INSTRUCTOR IN ZOOLOGY, BUSSEY (AGRICULTURAL) INSTITUTE, AND ASSISTANT PROFESSOR OF THERAPEUTICS, VETERINARY

SCHOOL (BOTH) OF HARVARD UNIVERSITY

TIIRD EDITION, REVISED

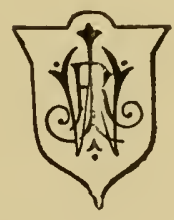

New York

WILLIAM P. JENKINS

851-853 Sixth Avenue

1905 

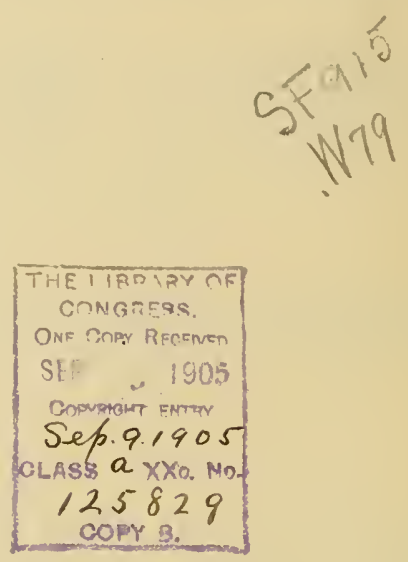

Copyrigit, 1901, 1905

By William R. Jenkins

[Registered at Stationers' Hall, London]

Printed in the United States of America

PRINIED BY THE

Press of Wildiam R. Jenkins

NEW YorK 


\section{PREFACE TO THIRD EDITION.}

In preparing this third edition of "Veterinary Materia Medica and Therapentics" the work has been thoroughly revised and re-written, much new matter having been added to bring the book up-to-date.

Among wholly new subjects included may be mentioned the following: Adrenaliu; Soluable Silver Compounds, including Crede's Ointment and Collargol; Gelatin and Calcium Chloride, in their new rôles as hemostatics; Orthoform; Tallianine; The Oxygen Treatment for milk fever in cows; an entirely new section on Serum Therapy, embracing Antitoxins and Vaccines and also some of the Toxins; another new section on the use of the stomach tube in view of the recent interest which has been aroused in the employment of this aid to stomach-therapy.

Every endeavor has been made to keep the book up to that high standard attained as a text book in the Veterinary Colleges and to meet the needs of veterinarians and agriculturists at home and abroad.

KENELM WIN̦SLOW.

August, 1905. 



\section{PREFACE TO FIRST EDITION.}

The writer wishes to acknowledge his indebtedness to the works of Brunton, Wood, Hare, Edes, Ringer, Bartholow, White and Mann, in human medicine; and to those of the following veterinary writers: Finlay Dun, Friedberger and Fröhner, Ellenberger, Koch, Cagny, Müller; and to the leading veterinary periodicals.

The matter on "properties," "description," and, in many cases, "derivation," is according to the U. S. Pharmacopœia, while the inportant preparations of both the U.S. and British Pharmacopoias are included. The classification and arrangement of drugs employed in this book are modifications of those adopted by W. Hale White in his excellent treatise on Materia Medica, Pharmacology and Therapeutics.

Kenelm WinsLow. 



\section{CON'TEN'TS.}

PAGE

Preliminary Considerations....................... 1

Definitions.................................... 1

Mode of Action of Drugs ....................... 2

Absorption of Drugs............................ 3

Elimination of Drugs......................... 4

Circumstances Modifying the Action of Drugs ........... 6

Mode of Administration......................... 6

Dosage.................................... 10

Anatomy and Physiology ....................... 11

Time of Administration $\ldots \ldots \ldots \ldots \ldots \ldots \ldots \ldots \ldots \ldots \ldots, 13$

Habit................................... 14

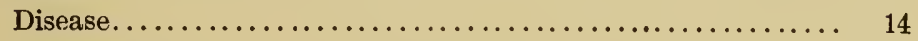

Idiosyncrasy ............................... 14

General Actions of Drugs....................... 15

Drugs Acting on the Digestive Organs.............. 15

" " " " Circulation................... 32

" " " " $"$ " Nervous System................ 38

" " " " " $"$ " $"$ "

" " " " $"$ " $"$ " $"$ "Sinary Organs................. 52

" " " $"$ "Sexual Organs................... 56

" Influencing Metabolism ..................... 59

" " Bodily Heat...................... 60

"Acting on the Skin...................... 62

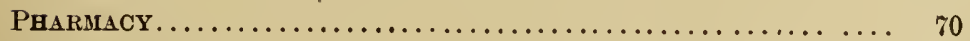

INCOMPATIBILITT $\ldots \ldots \ldots \ldots \ldots \ldots \ldots \ldots \ldots \ldots \ldots \ldots \ldots \ldots \ldots \ldots$

Prescription Writing............................ 86

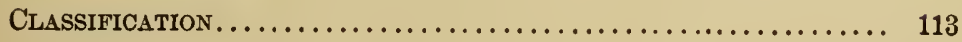




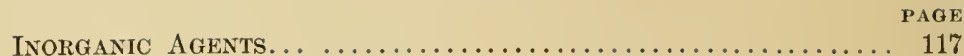

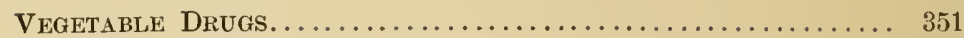

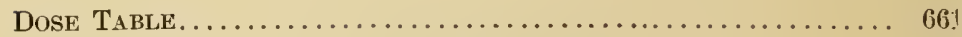

General Therapeutic Measures................... 679

Food and Feeding......................... 679

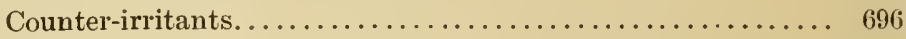

Cold and Heat............................. $r 04$

Disinfectants, Antiseptics and Deodorants............. 718

Venesection.................................. 728

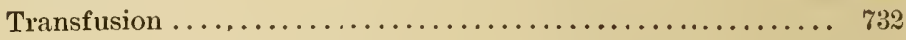

Intravenous Saline Infusions. . . . . . . . . . . . . . . 732

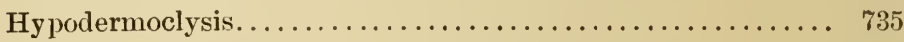

Enteroclysis.............................. 736

Kunsel's Treatment for Milk Fever in Cows............. 737

Lavage ................................. 739

Serum Therapy.......................... $₫ 41$

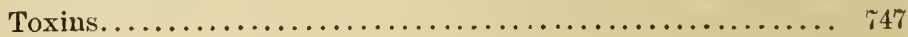

Vaccines.............................. 


\section{.}





\section{PRELIMINARY CONSIDERATIONS.}

\section{Definitions.}

Pharmacology is derived frum the Greek, Pharmakos, a drug, and is the sum of all exact knowledge pertaining to drugs, and therefore embraces Materia Medica, Therapeutics, and Pharmacy.

Materia Medica, derived from two Latin words signifying medical materials, treats of the derivation, natural history, physical and chemical properties, physiological actions, doses, and tests of purity of drugs. A special term sometimes used to describe the physical and chemical properties of drugs is Pharmacognosy, while Pharmacodynamics refers to the action of drugs on healthy animals.

Therapeutics, derived from the Greek, Ther(ipevo, meaning to serve or attend the sick, is that brancl of knowledge which treais of the application of all means-medicinal or otherwise-to the cure of disease or relief of pain. The term has been further subdivided as follows: Rational Therapeutics, which treats of the application of drugs as. fonnded on their physiological actions; Empirical Therapeutics, the use of drugs as based on clinical evidence; and General Therapentics, the nse of remedial agents other than drugs, e.g., Heat, Cold, Flectricity, Food, etc.

Pharmacy is the art of preparing, componnding, dispensing and preserving drugs.

Toxicutory, derived from the Greek, Toxition, a poison, is that branch of knowledge which treats of the nature, actions, detection and tereatment of poisons.

Amedicine is an angent of animal, vegetable or mineral origin nsed for the cure of disease or relief of pain. The word cure, signifies literally to care for, from the Latin 
which tends to delay absorption and lessen the action of medicines given by the mouth.

If drugs are irritating they should be given to animals. on the food, or after feeding, in order that they be sufficiently diluted. Some remedies require hydrochloric acid for their solution-as iron-and they should therefore be administered at or after the time of feeding, because hydrochloric acid secretion is then active.

\section{Elimination of Drugs.}

A drug is as much outside the body when within the digestive tube-so far as any action it may have on the body (unless an irritant) - as if it were on the skin. When absorbed, a medicine passes into the blood vessels or lymphatics and thence into the general circulation. That portion which enters the portal circulation reaches the liver and may be destroyed in part (some alkaloids) by this organ. After entering the blood the drug is thought to form unknown combinations with the tissues for which it has an affiuity-thereby exerting its remedial effect-and is decomposed or rarely accumulates in the body, but usually is eliminated either unchanged or as decomposition-products in the breath, or by the excretions or secretions of the kidneys, bowels, liver, sudoriparous, salivary and mammary glands, and mncous membranes. The urine is the most frequeut channel of elimination for soluble drugs. The bowels constitute the next more common pathway of elimination. Volatile drugs (chloroform, ether) are eliminated very rapidly, usually in the breath. If a drug is eliminated slowly the duration of its action is correspondingly long, and vice versa. This fact will guide us in the frequency of administration of medicines, since if a drug which is tardily eliminated be given at frequent intervals it may be absorbed faster than it is excreted and so accumulate in the body and canse poisoning. The so-called Cumulative Action of a drug refers to the occurrence of a sudden and violent effect during its mediciual administration. This may be due, 
(1) to delayed followed by rapid absorption from the alimentary canal; or (2) to slow-or sudden arrest of-elimination. The salts of lead, mercury and arsenical preparations are eliminated slowly. Digitalis and strychnine are said to be especially prone to produce a cumulative action. Strychnine may, however, be given subcutaneously in gradually increasing doses without the likelihood of poisoning. Digitalis may canse a cumulative effect in being slowly oxidized in the body or in leading to contraction of the renal vessels and suppression of urine-elimination. The drugs likely to cause a cumulative action must be administered infrequently, once, twice, or thrice daily; whereas medicines which are rapidly decomposed and eliminated (alcohol, nitrites, etc.) may be given at very frequent intervals if desirable. The term excretion is often used synonymously with elimination, but, strictly speaking, a drug is not eliminated unless it has been first absorbed. On the other hand, an insoluble drug passing unabsorbed through the alimentary canal is said properly to be excreted in the frees. 


\section{CIRCUMSTANCES MODIFYING THE ACTION OF DRUGS.}

\section{Mode of Administration.}

The following table gives the various methods of administering drugs in order of their rapidity of absorption, beginning with the method by which absorption is most rapid, and following with those by which absorption is less and finally least rapid:

1. Intravenous, by injection into the reins.

2. By inhalation (volatile drugs).

3. Subcutaneous, by injection into subcutaneous tissue.

4. Intratracheal, by injection into the trachea.

5. Oral, by the mouth.

6. Rectal, by the rectum.

7. Inunction, by the skin.

1. Injection into the veins (usually into the jugular) is not so commonly practiced as the subcutaneous method, as there is a certain minute danger of inducing phlebitis, embolism and thrombosis. The danger is more theoretical than real, however, as we have frequently thrown from $5 \bar{\xi}(150 \mathrm{cc}$. $)$ to $6 \frac{1}{2} \overline{3}(200 \mathrm{cc}$. $)$ of fluid extract of cannabis indica into the jugular, and even chloral hydrate, a most irritating and caustic drug, in the dose of $1 \bar{\xi}$ (30.) dissolved in $8 \overline{3}(240$ cc.) of water, without producing any untoward symptoms. No method of administration can secure more rapid absorption, since intravenous injection is absorption. This has constituted one of the theoretical objections to the method, that the sudden entrance of a drug might create shock. Injection into the jugular is useful when very rapid and effective action is imperative, as in causing immediate catharsis in colic and intestinal 
obstruction of horses. In such cases barium chloride and eserine sulphate are employed intravenously. The jugular is occluded with the hand and the injection is made with the same care described below in reference to the subcutaneous method. The intravenous use of hot normal salt solution is frequently valuable in hemorrhage, shock and poisoning (see p. 733).

2. Volatile drugs are absorbed with great rapidity and effect owing to the enormous vascular surface of the lungs in contact with the inhaled vapor. Ether, chloroform, ammonia and amyl-nitrite are given by this method. Inhalation of medicated steam and sprays, used mainly for their local action on the respiratory tract, are also absorbed to some extent by the bronchial mucous membrane and lungs. This is a convenient and effective mode of applying local medication to horses in inflammatory troubles of the upper air passages, including the bronchial tubes, and in many cases may effect a cure without the use of internal remedies.

3. Subcutaneous or hypodermatic injection is suitable for soluble, non-irritating drugs of small bulk, when a sure and rapid action is desired. The medicinal solution shonld be free from solid particles and microörganisms. If the solution is not clean, or is irritating, abscess may occur. The syringe and needle must also be absolutely clean. Solutions made by dropping tablets in pure drinking water will rarely cause abscess, and the syringe may be male aseptic by filling it with alcohol, and wiping the needle with the same, previous to their employment. Solutions may be preserved for hypodermatic use with boric acid ( 1 per cent.), but solnble tablets are more convenient.

In practising this method the hair should be remover from the seat of injection-preferably the thin skin underlaid by connective tissue behind the elbow or on the abdimen-and the part washed; then a loose fold of skin is picked up and held firmly between the thumb and forefinger of the left hand, while the needle is thrust under the skin, 
but not into a vein. The syringe is slowly emptied and the needle withdrawn, keeping slight pressure over the point of injection with the thumb for a few seconds. The use of irritating drugs-permissible in emergencies-as fluid extract of ergot, tincture of digitalis, ether and ammonia, is less apt to be followed by abscess if injected deeply into the muscular substance, but this method causes more pain than ordinary injections. To avoid getting air in the veins, all the air is lemoved from the syringe before n-ing, by holding it, needle upwards, and pushing in the p) lunger till a few drops of the solution are forced ont of the needle. The danger of introducing air into the blood stream is greatly exaggerated, however, as the writer has proved by forcing vast quantities of air into the jugnlar vein of a horse without producing any untoward symptoms. The proper quantity of a solution for subcutaneous use is 5-30 minims for dogs: 1-2 drachms for horses, although large amounts of salt solution may be injected into the subcutaneous tissue or muscles (hypodermoclysis) with great benefit in hæmorrhage, etc. See 1. 735.)

The minimum doses of drugs should be employed by the subcutaneous method.

\section{INDICATIONS FOR SUBCUTANEOUS INJECTION.}

(a) To secure a rapid action, as in relieving intense pain or motor excitement; and to support a failing heart, respiration and vascular tone in severe operations, a:ıesthesia, or other poisoning.

(b) When arministration of drugs by the mouth is inadvisable or impossible, as in unconsciuusuess, dysphagia, convulsions or vomiting.

(c) When a local as well as general action is beneficial, e.g., the use of strychnin in roaring and otler local paralyses; atropin in local muscular spasms ; veratrin in muscular rheumatism.

4. Intratracheal injection is a strictly veterinary procedure. The skin is incised aseptically with a sharp scalpel midway in the neck, and a stout needle (attached to a syringe) is thrust $b$ tween the rings into the trachea. Larger quantities [H. ( 3 i.-ii.) (30.-60.)] and more irritating drugs are given in this way than by the subcutaneous method, and absorption is about as rapid; the dose is the same. There 
is undoubtedly danger; however, in giving irritant drugs by this method, especially chloral, and several cases of foreignbody pneumonia have come under our notice as the result of this mode of administration. It is the best method of benumbing or killing the parasites (S. filaria and micrurus) infesting the trachea and bronchi, and has been employed to influence the mucous membrane of the lárynx and trachea in certain inflammatory conditions.

5. Drugs are usually given by the mouth and are absorbed from the stomach and intestines. Many non-irritating and not umpleasant drugs are taken voluntarily in the food, gruel, milk or drinking water by animals. Cats and dogs will often swallow medicine enclosed in a piece of meat. Absorption is more tardy than by the subcutaneous method, more rapid when given in solntion into an empty stomach; slower when administered in powder, pill or ball, and on a full stomach. Some drugs are probably absorbed from the stomach, only to be destroyed in part by the liver, or eliminated by the bile, and do not enter the general circulation at all.

When drugs are administered for their local action on the stomach, in citarrh or ulcer, they should be given half hour to an hour before feeding; if given for their action in or on the intestines, they should be administered two or three hours after meals.

6. Rectul injections of medicines (enemata or clysters) are practised when the use of drugs by the mouth is inadvisable or impossible, as in uncousciousness, dysphagia, convulsions; also to destroy parasites (oxyurides) in the rectum, to influence an inflamed or ulcerated rectal mucous membrane, and to remove intestinai contents (oil and glycerin).

The dose of drugs by this method is generally twice that by the mouth, and absorption is slower and more imperfect. The drug should be non-irritating, soluble, and not too bulky, since a small amount is necessary ( 3 i. $-\overline{3}$ i. dogs; $\overline{3}$ ii. $-\overline{3}$ viii. horses); to avoid tenesmus and expulsion. Warm starch solution (made by boiling) or linseed tea with a little laudanum is a good vehicle for medicinal enemata, and re- 
tention of enemata is facilitated by pressure on the auus with a towel for some minutes after the injection is given.

Solids are sometimes employed by rectum in suppositories. For general uses of enemata, see p. 30.

7. Drugs are absorbed very slightly by the skin, and then only when rubbed very vigoronsly into the epidermis (inunction) with lanolin, fat or oil of some kind. Mercury, silver and iodine are most commonly employed for absorption, but drugs are usually applied extemally for their local action only and not to influence the general system through the blood.

\section{Dosage.}

The study of dosage is known as Posology. The action of drugs is altered both in degree and in kind by the dose. Thus, increasing the dose would naturally lead to an increase in the intensity of a drug's action, but it frequently changes the entire character of the action as well.

Drugs, as opium and alcohol, acting especially on the nervous system, often excite in therapeutic doses, but depress and paralyze in toxic doses. Drugs, as digitalis, stimulating the heart in medicinal doses, usually depress and paralyze the organ in poisonous doses. Many drugs promoting urinary secretion, in ordinary closes, cause inflammation and minary suppression in large doses. The best way to determine the dose of a drug is to estimate the amount required for each pound of live weight. This only applies to the same species and to animals of ordinary build. Fat is a comparatively inert tissne as far as the action of drugs is concerned, so that a very fat horse, weighing, for example, 1,200 pounds, would be affected in a more prononnced manner by a dose of medicine than would a lean horse of the same weight and taking the same dose. In the case of young animals, and of those either above or under the ordinary size of the adult of any species, the dose shonld be proportioned-according to weight-to the average dose for the adult animal of that species. Thus, if the average weight of a horse is 1,090 ponuls, the dose of any drug for a colt weighing 
500 pounds would be half the usual dose for adult horses. In a general way the dose for all animals from birth to a few weeks old, is one-twentieth of that suitable for the mature animal of the same species; for yearlings, about onethird of the adult dose. The dose recommended for dogs is commonly the same as that given to man, but this rule does not apply in the case of some powerful drugs (strychnine), where the dose should be adjusted to the weight, i.e., so much per pound, live weight.

It is impossible to calculate the dose for all domestic animals as based on that for animals of one species, because the differences in anatomy and physiology modify the actions of drugs in degree and kind, but the dose for sheep is about one-fourth of that for the larger ruminants.

The repetition of a dose is determined to a considerable extent by the duration and rapidity of a drug's action. Agents used for their immediate effect, as those relieving pain and stimulating the circulation and respiration, are repeated frequently till the desired effect is attained. Medicines improving the condition of the digestion, blood and nutrition, as tonics of various kinds, require time for the aceomplishment of their mission, and are usually given two or three times daily for a period of some weeks.

\section{Anatomy and Physiology.}

Certain differences in the action of medicines may be observed as occurring in the various species of animals, and in animals as contrasted in this respect with man.

ACTION OF DRUGS ON ANIMALS AS COMPARED WITH THAT ON MAN.

From a comparative standpoint the action of drugs on the nervous system of animals differs from that on man. This follows according to the "law of dissolution," which teaches that the more highly developed a part of the nervous system is in the evolntionary scale, the more sensitive is it to the influence of drugs. Since the cerebrum of man is relatively larger and more highly developed, in propor- 
tion to his weight, than is the case in animals, and since the spinal cord is larger and more highly developed in proportion to the brain in animals, it happens that drugs impressing the nervous system exert less effect on the brain, and more on the spinal cord, of animals than they do in man.

Thus opium is more powerful in its inflnence on the brain of man, and strychnin is more potent in its action on the spinal cord of animals. Drugs are not absorbed so rapidly or perfectly in the enormous digestive apparatns of ruminants as in man; neither do emetics act in these animals, nor in horses; while in none of the lower animals are agents cansing sweating so efficient as in man.

ACTION OF DRUGS ON HORSES AS COMPARED WITH THAT ON OTHER

\section{ANIMALS.}

Differences exist relative to the action of drugs on the horse, as compared with other animals, chiefly in respect to the digestive apparatus. Emetics do not act on the horse, as this animal does not vomit unless the stomach is greatly distended with gas, which canses dilatation of the cardiac ontlet. Otherwise the stomach is too small to be successfully compressed by the abdominal walls, and the great length of the osophagus between the stomach and diaphragm, together with the horseshoe-like band of fibres at its cardiac extremity, prevent the regurgitation of food. The intestines of the horse, on the other hand, are as voluminous as the stomach is small, and therefore are powerfully influenced by irritants (as purgatives), although the action of cathartics is slow. The bowels of horses excrete vastly more of the fluid ingested than is the case in man or dogs -whose kidneys chiefly assume this function-and these latter organs are said to eliminate about 15 per cent. of the fluid ingesta in the former animals, as against 50 per cent. in man and dogs.

ACTION OF DRUGS ON RUMINANTS AS COMPARED WITH THAT ON OTHER ANIMALS.

The capacious four-fold stomach of ruminants always 
contains large amounts of food in the rumen and abomasum, while the impervious, poorly vascular and skin-like gastric mucous membrane renders absorption feeble and imperfect and enforces a comparatively larger dosage than is proper for horses of greater weight. Ruminants are also generally insusceptible to emetics. The skin and kidneys of ruminants are still less active than is the case in horses.

ACTION OF DRUGS ON DOGS AND PIGS AS COMPARED WITH THAT ON OTHER ANIMALS.

The action of medicine on dogs and pigs is similar in kind to that observed in man, but the former animals are less sensitive to drugs as a rule, since the dose suitable for a man weighing 150 pounds is appropriate for a dog of 40 ponuds weight. As exceptions to this rnle, we find that dogs will not bear the human dose of calomel, oil of turpentine, or strychnine. In fact, the ordinary tonic dose of strychnine (gr. $\frac{1}{30}$ ) for man will throw a medium-sized dog into convulsions, and may kill a small animal, notwithstanding that this amount is recommended as a suitable canine dose in veterinary text books.

Contrariwise, the dog is comparatively insensitive to many drugs powerfully influencing man,--notably aloes, colocyuth and rhubarb. Most cathartics act more quickly on dogs than is the case with the other domestic animals, but saline purgatives are less appropriate in often cansing vomiting, and because of their bulk.

\section{Time of Administration.}

This matter has been alluded to in speaking of the absorption of drugs. Medicines readily undergoing decomposition in the presence of other substances, as iodine and hydriodic acid, should be given on an empty stomach ; and likewise all drugs, when a speedy action is desired. Irritants should be administered on a full stomach; while agents requiring hydrochloric acid for their solution-as iron- 
must be exhibited on the food or immediately after the time of feeding.

Habit.-This circumstance does not have the same importance in veterinary medicine which it possesses in human practice, since we control drug habits in animals. Animals usually become less susceptible to the action of drugs on their repetition, e.g., opium and cathartics. This rule does not hold in the case of drugs having a cumulative action, nor in the repeated use of irritants on the skin, for then their action is strongly intensified.

Disease.-The action of drugs is profoundly influenced by disease. It is only possible to enumerate a few examples. Pain is almost an antidote to opium, and large repeated doses of the drug, previously innocuous, may, on the sudden cessation of pain, induce poisoning. Opium is also borne in enormous doses in peritonitis. Inflammation and congestion of the digestive organs hinders the absorption of all medicines. A congested condition of the alimentary canal, and even of the respiratory tract in lorses, contraindicate the use of strong purgatives in these animals, since superpurgation may occur. A high temperature alters the action of many drugs.

Opium is not so efficient as an analgesic in fevers, while antipyretics will not lower the temperature in health. Stimulants are not nearly so potent in depressed bodily conditions, and counter-irritants will not produce their characteristic actions on the skin when the circulatory functions are at a low ebb.

Idiosyncrasy.-Individual susceptibility to drugs is infrequent, but unfortunately cannot be anticipated. The writer has seen simple zinc oxide (free from adulteration or impurities) cause a frenzy of irritation when rubbed on a dog's skiu, and a small dose of tartar emetic cause violent vomiting in a cow. Some animals are very susceptible to counter-irritants. Well-bred animals are commonly more responsive to drugs than others. 


\section{GENERAL ACTIONS OF DRUGS.}

\section{Drugs Acting on the Digestive Organs.}

Siclagogues are agents iucreasing the secretion of saliva. Antisialagogues are agents diminishing salivary secretion. Amoug the sources of saliva-the parotid, sublingual and submaxillary glands-the latter have received most study. The chorda tympani, with its centre in the medulla, is one of the two nerves supplying the submaxillary glind. It contains two sets of fibres, the secretory and vasodilator. Hence stimulation of this nerve, or its centre, whether immediately or reflexly, leads, by meaus of its vasodilator fibres, to dilatation of the blood vessels and enhanced vascularity of the gland, and so indirectly to greater secretion; while, through excitation of the secretory fibres, the protoplasm of the glaudular cells is influenced and secretion directly increased.

Reflexly the gland is stimulated by drugs exciting the peripheral terminations of the gustatory (lingual branch of the fifth nerve) and glossopharangeal nerves in the mouth; the vagus endings in the stomach; by agencies sending pleasurable impressions to the brain through the medium of the eyes or nose; or by stimulation of other sensory nerves. The submaxillary gland is also supplied by a branch of the cervical sympathetic accompanying the submaxillary arteries. Stimulation of this nerve, or its centre, causes vascular constriction in the gland and inhibition of secretion.

Sialagogues are often classed under three heads. 1st, Specific sialagogues, acting directly on the mechanism concerned with secretion, i.e., the gland cells, or nervous apparatus. Pilocarpine is the best example of the specific class. It stimulates the gland cell or peripheral nerve endings. 2nd, Reflex sialagogues, exciting sensory nerve terminations 
and indirectly or reflexly stimulating the nerrous mechanism controlling secretion. As examples of this class, may be mentioned alkalies, acids, emetics, and other agents stimulating the mucous membrane of the mouth and stomach. 3rd, Mixed sialagogues, acting both specifically and reflexly. Physostigmine, nicotine or tobacco and mercury preparations may be included in this category.

Antisialagoyues. - These drugs may act in various ways to lessen salivary secretion, but atropiue is most notable in this regard. It acts by depressing the peripheral endings of the secretory nerves.

Uses.-Sialagogues are not of much therapentic value. Some are added to the drinking water given to animals suffering from fever, to relieve dryness of the mouth and thirst. They are then called refrigerants; as, for example, potassinm nitrate, diluted phosphoric and other acids. The reflex sialigogues are sometimes employed to stimulate the mucons membrane of the pharynx in sore throat and relaxed conditions; as, for example, potassium chlorate in electuary for horses.

Excessive salivation produced by mercury salts or pilocarpine is relieved by an antisialagogne, i.e., atropine.

Stomachics are drugs which, in therapentic doses, mildly stimulate the mucons membrane of the mouth and stomach, thereby increasing the secretions and vascularity of these parts, the appetite, and, in a less degree, gastric peristalsis. These agents also influence the intestines, but this effect will be considered muler carminatives.

Stomachics may be divided into bitters, aromatics (drugs containing a rolatile oil and often very pungent), and aromatic bitters (drugs containing a volatile oil and a bitter principle). While both the bitters and aromatics enliance the appetite, the action of the latter is more porverful and fleeting. Very large doses of stomachics are distinctly irritating, and cause anorexil, nausea, and vomiting in animals capable of the act. 


\section{STOMACHICS.}

Gentian

BITTERS

Calumba

Quassia

Hrdrastis

Taraxacum

AROMATIC BITTERS

Cascarilla

Chamomile

Serpentaria
AROMATICS

Coriander

Capsicum

Pepper

Ginger

Cardamon

Fennel

Fenugreek

Anise

Calamus

Mustard

Spearmint

Peppermint
Alcohol

Ether

Chloroform

Alkalies (see Antacids)

Uses.-Stomachics-particularly bitters-are serviceable in improving the appetite and gastric digestion in atonic indigestion, and in enfeebled states of the digestive organs occurring in the course of chronic diseases or during convalescence from acute disorders. The aromatics are more frequently employed for their action on the intestines, when they are called carminatives. Bitters are contra-indicated in irritable or inflamed conditions of the alimentary tract.

Antacids are drugs which are nsed to counteract acidity in the stomach and bowels resulting from indigestion and fermentation, or from excessive secretion of gastric juice. Some (not ammonia compounds) are also occasionally employed to alkalize the blood and urine.

It has been cornmonly tanght that if antacids are given immediately before or at the time of eating, they increase the flow of acid gastric juice, althongh diminishing the secretion of alkaline saliva. They are thus said to improve gastric digestion, especially when combined with bitters.

This fact lias been substantiated by analyses of the stomach-contents in experiments on man.

If administered several hours alter eating, antacils counteract acidity due to fermentation and relieve pain caused by this condition. Since fermentation is frequently the cause of tympanites, the antacids are conjoined to advantage with carminatives (sodium bicarbonate and ginger). 
The alkaline carbonates allay pain by means of the carbonic dioxide set free in their decomposition in the digestive tract, and the antacids are also beneficial in dissolving an excessive secretion of mucus in catarrhal conditions of the alimentary canal.

The antacids are synonymous with alkalies, with the exception of the neutral vegetable salts--acetates, citrates and tartrates-of potassium and sodium, which are sometimes classed under this hear. These do not alkalize the contents of the stomach, but nevertheless are broken up in the body and transformed into carbonates and thus render the urine more alkaline during their elimination. Among those included in the following list the sodium compounds are much less active in alkalizing the urine than the potassium salts. Sodium bicarbonate is in most frequent use in digestive disorders, but ammonium carbonate is particularly appropriate in flatulence, because it possesses the added power of stimulating peristaltic action and expelling flatus.

\section{ANTACIDS.}

Sodium carbonate

Sodium bicarbonate

Potassium carbonate

Potassium bicarbonate

Solution of potash

Ammonia
Ammonium carbonate

Magnesia

Magnesium carbonate

Calcium carbonate (chalk)

Solution of lime (lime water)

Acicls.-The mineral acids-in contradistinction to the alkalies-lessen the secretion of gastric juice and increase the secretion of saliva. Diminution of the gastric secretion is undesirable, and to avoid it acids should be griven two hours after meals when gastric secretion is complete. Acids appear to excite movements of the stomach as the normal motility increases with the secretion of gastric juice. Hydrochloric acid seems to be an exception and not to lessen gastric secretion, but even to excite the normal production of this acid in the stomach. It should not, lowever, be given until an hour or two after feeding animals, and stimulates the formation of pepsin from pepsinogen, as well as the amount of acid in the gastric juice. 
Carminatives include the same drugs which were mentioned as stomachics, including valerian, asafetida and volatile oils generally, but the term as generally employed refers to their effect in exciting peristaltic action, and so expelling gas from the stomach and bowels. The aromatics are considerably more valuable for this purpose than the bitters. Carminatives also prevent griping caused by many cathartics, aid digestion, and disguise the taste of disagree- । able drugs. Capsicum and ginger are most frequently prescribed in Veterinary practice.

Digestives.-Pepsin is occasionally of benefit in the treatment of dogs and young animals in cases of enfeebled gastric digestion resulting from acute diseases or other general causes. It should be administered directly after eating, and is prescribed to advantage with hydrochloric acid. As a general proposition it is inadvisable to give agents which merely substitute an artificial for the natural digestion, except as a temporary expedient. A wiser course consists in removing the cause of indigestion by proper feeding or by enforcing abstinence from food, and in the use of remedies calculated to strengthen the natural digestive functions.

Pancreatin may be given during, or immediately after, eating, and will assist gastric digestion for some time before sufficient acid is secreted to destroy it. In fact, some authors (Hare) insist that this substance is more valuable in any case than pepsin in aiding stomach digestion, although pancreatin is more commonly given, several hours after eating, to promote intestinal digestion. Papain is another agent which is employed as an artificial digestive of vegetable origin. Its value is not yet definitely determined.

Antiseptics.-These agents are sometimes used to prevent or arrest fermentation of food in the stomach and bowels. Since fermentation is primarily due to indigestion, it is essential to remove the cause by diet and other rational means rather than to combat the effects of indigestion. Large doses of antiseptics hinder the digestive processes and may endanger the life of the patient, so that it is difficult to attain perfect antisepsis in the alimentary canal. 
Among the drugs more commonly employed for their antiseptic action on the contents of the digestive tract may be mentioned :

\section{Carbolic acid \\ Creosote \\ Creolin \\ Naphtol \\ Naphtalin}

Bismuth subnitrate

\author{
Bismuth salicylate \\ Bismuth subgallate \\ Sodium sulphite, bisulphite and \\ hyposulphite \\ Hydrogen dioxide
}

Emetics are drugs which cause vomiting. The act of vomiting proceeds from irritation of the romiting centre in the medulla, which is in close prosimity to the respiratory centre. This centre is either acted npon directly by drugs circulating in the blood, or refiexly by agents stimulating sensory nerves in various parts of the body. Thus, irritation of the sensory nerve-endings of the mouth, throat, gullet, lungs, heart, stomach, bowels, biliary passiges, peritoneum, uterus and kidneys, may produce vomition. Vomiting is occasioned by simultaneous contraction of the abdominal walls and the diaphragm. In this process the stomach is squeezed between the abdominal walls and diaphragm, and contraction of the longitudinal fibres, radiating from the lower end of the gullet, draws the stomach towards the diaphragm and so pulls open the cardiac orifice, while the pylorus is firmly contracted and closed. Some peculiarities must be noted in reference to vomition in the domestic animals. Dogs, pigs and eats vomit readily and may be placed in the same category as man in this respect. Horses rarely vomit and are not easily nauseated by emetics. Vomiting. is prevented in these animals by : 1 . The small size of the stomach, which is not readily compressed between the abdominal walls and diaphragm. 2. The length of the gullet. between the stomach and diaphragm, which forms a valvelike obstruction when the tube is shortened by contraction of the longitudinal fibres at its lower extremity in attempts. at vomition. 3. A horse-shoe-like band of fibres at the cardiac orifice, which hinders dilatation of this opening. Rumi- 
nants are likewise comparatively insusceptible to emetics because of the large size of their digestive apparatus, which is not easily compressed between the parietes and diaphragm. Therefore the vomiting centre remains probably in a state of non-development in the horse and ruminant, by reason of non-use.

Cattle and horses do, however, occasionally vomit. Cattle at sea frequently suffer from mal de mer, and the writer has observed actual romition in them following the use of tartar emetic. Horses may romit when the stomach is greatly distended with gas.

Emetics may be classed as: 1. Specific, acting on the vomiting centre through the blood. 2. Local, by stimulation of the sensory nerve-endings in the mouth, throat, gullet and stomach. 3. Mlixed, those acting in both ways.

It is impossible, in our present state of knowledge, to apply this classification accurately to individual drugs, but the following statements may be made: If an emetic is injected into the carotid and vomiting instantly occurs, the drug has probably acted upon the vomiting centre; if some time elapses before the occurrence of vomition, it is probable that the drug has acted upon the stomach during its elimination by that organ. Contrariwise, if, after the ingestion of an emetic, a considerable period intervenes before vomiting comes on, it is probable that the agent has acted on the vomiting centre.

Again, if a larger quantity of a drug is required when injected into the blood than when swallowed, to cause emesis, it is fair to suppose that the agent acts on the stomach directly or during its elimination. Finally, if an emetic is thrown into the blood after the removal of the stomach and substitution of a bladder in its place, and vomiting does not occur (Majendie's experiment with tartar emetic), it shows that the agent only acts on the stomach; but if vomiting does occur, it indicates that the agent acts on the vomiting centre and causes emesis by contraction of the parietes and diaphragm, with this reservation, that the 
drug may have been eliminated by the oesophagus and intestines and have reflexly stimulated the vomiting centre through the medium of these parts. These remarks demonstrate the complexity of the subject.

EMETICS.

Specific

Apomorphine

Senega

Squills

Lukewarm water
Local

Tepid water

Mustard

Salt

Alum

Ammonium Carbonate
Mixed

Tartar Emetic

Ipecac:

Zinc Sulphate

Copper Sulphate

Apomorphine is the only emetic given under the skin. It also acts well by the month, but causes more nausea and allied effects than drugs acting locally.

Mustard and salt, 1 teaspoonful each, in a cup of lukewarm water, form a convenient emetic for dogs. Ipecac is nseful in respiratory diseases as an expectorant as well as emetic, and zinc sulphate is a prompt emetic in poisoning. The other emetics are practically unimportant.

Emetics cause, beside vomition, several other phenomena which are sometimes ntilized therapeutically. Among these may be mentioned,-nansea, salivation, violent respiratory efforts, compression of the abdominal glands and ducts and extrusion of their contents, passive congestion of the head, chest and peripheral parts by reason of compression of the abdominal veins. Increased secretion of the mucous membranes of the nose, eyes, stomach, gullet and bronchial tubes follow passive congestion. Muscular relaxation always accompanies nausea, and sweating ensues from relaxation of the skin and leaking out of the secretion. The flow of bile is increased on account of pressure on the liver and gall-bladder, while the secretion is also augmented.

The pulse and respiration are more frequent during emesis, but are diminished in force and frequency afterwards. All these phenomena are more apparent after the use of specific emetics.

Uses.-These apply particularly to dogs.

1. To empty the stomach in case of poisoning, over- 
loading of the organ, and indigestion with convulsions in young animals :-Mustard, salt or zine sulphate.

2. To expel foreign bodies from the fauces and gullet (apomorphine subcutaneously); or, by the forcible expiration attending vomition, to expel excessive secretion or exudation from the air passages in laryngitis or bronchitis :Ipecac.

3. To empty the gall-bladder in catarrhal jaundice and biliousness and to expel bile from the stomach.

4. To lower blood pressure and increase secretion in the first stage of bronchitis :-Ipecac.

5. To stop vomiting:-Ipecac in minute doses.

Contra-indications.-Pregnancy; hernia; inflammation of the stomach, brain or abdominal viscera; bleeding from the stomach, bowels or lungs; aneurism and asthenia.

Gastric sedatives and anti-emetics are agents used to relieve pain in the stomach and vomiting. These include:

\section{Ice}

Hot water

Bismuth subcarbonate

Bismuth subnitrate

Carbon dioxide

Hydrocyanic acid

Morphine

Menthol

Carbolic acid

Creasote

Aconite

Belladonna

Hyoscyamus
Cocaine

Cerium oxalate

Lime water

Minute doses of arsenic

" ". " "ipecac

" " " " alcohol

" " " iodine

" " " silver nitrate

Chloroform

Chloral

Bromides

Nitrites

Most of these agents act locally, but opium and morphine, chloral, the bromides, prussic acid and the nitrites act centrally.

USES OF GASTRIC SEDATIVES AND ANTI-EMETICS IN CANINE PRACTICE.

It must be recognized that vomiting is merely a symptom. It is, therefore, essential to remove the cause. This may sometimes be accomplished by starving, the use of an 
emetic, or tepid water. If vomiting is due to acute irritation of the stomach, as is frequently the case in dogs, ice and bismuth subnitrate (gr. x.-xx.), with tincture of aconite (M.i.-ii.), form suitable remedies. When vomiting arises from indigestion and fermentation, carbolic acid with bismuth often act farorably. The vomiting following anæsthesia is probably of central origin. Here enemata of laudanum (M.x.-xxx.) and sodim bromide (gr. xx.-xxx.) are beneficial. Ipecac, iodine, silver nitrate and the like are nseful in vomiting dependent upon an atonic or depressed state of the stomach. Then vomiting is continuous, small quantities of milk and lime water, equal parts, or peptonized milk ( $\mathrm{ij}$ ij.-iv.), or a drachm of cracked ice with a few drops of brandy, should be given at half-hour intervals. It may be rarely necessary to resort to recta! feeding.

Purgatives or rathartics are agents which empty the bowels. They act: (1) By stimulating peristaltic action. (2) By increasing the secretions (succus entericus) of the intestinal glands and, perhaps, transudation of fluid from the blool vessels in the walls of the intestines. (3) By hindering absorption of secretions alid fluids which normally occurs in the lower bowels. (4) By a combination of two or more of these methods. Purgatives may be divided into:

1. Laxalives. -These include such agents as:

\section{Olive oil}

Cottonseed oil

Magnesia

Sulphur

These drugs slightly increase intestinal action, chiefly by stimulation of peristalsis.

2. Simple Purgatives. - These stimulate secretion and peristaltic action. Among then may be mentioned:

Aloes

Calomel

Linseed oil

Castor oil

Rhubarb

Senna

Cascara sagrada

Frangula

3. Drastic Purgatives.-Drastics are essentially gastrointestinal irritants, and in large doses canse mucous and 
bloody diarrhoa, congestion of the mucous membrane of the alimentary canal and severe colic. They may produce death in poisonous doses with collapse by reason of gastroenteritis. Drastics greatly increase both peristaltic action and secretion, and are contra-indicated in irritable and inflamed conditions of the digestive tract. They are, however, indicated for their revulsant or derivative effect (i.e., to dilate the blood vessels in the alimentary canal and to cause an ontpouring of serum from the blood, thus relieving congestion in other parts) in some acute inflammations, as in brain diseases. Their medicinal action is often attended with considerable apd irregular peristaltic contractions, so that griping occurs. The latter is prevented by suitable combination with other purgatives; with hyoscyamus and belladonna; or with carminatives, as ginger. The drastics include :

Croton oil

Colocynth

Gamboge

Scammony

Jalap

Elaterium

4. Hydragogue Purgatives.-Hydragogues are agents which chiefly increase the fluidity of the intestinal contents. They include:

(a) SALINE PURGatives

Magnesium sulphate

Sodium sulphate

(b) DRASTICS

Jalap

Elaterium
Sodium phosphate

Potassium bitartrate

The salines stimulate secretion by reason of their bitterness, and by their irritant and specific properties. They, moreover, hold on to the fluid thus secreted and hinder its absorption because of their slow diffusibility. Purgation follows, owing to the mechanical effect of the increased fluidity in the bowels, and since the angmented bulk of the intestinal contents excites peristaltic action. When it is desirable to remove fluid from the blood the salt should be given in concentrated solution, but when a speedy purgative 
action only is required the saline should be administered in considerable dilution. This happens because salines continue to cause an outpouring of fluid (succus entericus) into the intestines until a 5 to 6 per cent. solution of the salt is reached. The nearer to this degree of dilution (5-6 per cent.), therefore, the dose is given, the more quickly will it purge.

The drastics inclnded in this class of purgatives have the power of markedly increasing intestinal secretion as well as peristiltic action.

5. Cholagogue Purgatives. - Cholagogues are agents which assist in removing bile from the body. They do this in two ways. 1. By directly stimulating the secretion of bile. These are cilleıl Direct Cholagogues, or Hepatic Stimulants. 2. By increasing peristalsis in the upper portion of the small intestines, and thus hastening the expulsion of bile from the bowels. These are called Indirect Cholagogues. Some cholagogues are not generally considered purgatives, but it is proper to classify all of them thus, since bile stimulates peristilsis.

The functions of the liver which cholagogues influence -more or less-are as follows:

1. Destructive Action.-The liver not only destroys the toxicity of peptones and other poisonous fermentative and putrefactive prolucts of digestion, but antagonizes as well the effect of toxins and alkaloids derived from various sources, including those absorbed from the alimentary tract.

2. Constructive Action.-The liver completes the assimilation of peptones by converting them into albumin and globulin for immediate use. An important hepatic function is the formation of glycogen from peptones and sugar occurring as digestive products. Finally urea is produced in the liver from prodncts of oxilation in the body, as lencin.

3. Excretory Action.-The liver produces bile which is both an excretion and secretion. In bile are eliminated, as toxic matters, biliary acids (the product of liver cells), bile pigment (the result of decomposition of red blood cells in 
the spleen), and lecithin and cholesterin (the waste products of nerve tissue and cellular activity). The bile pigment and acids are rendered inert in the bowel by precipitation in the acid chyme.

4. Secretory Action.-Bile, as a secretion, is utilized in the intestines, where it is instrumental in the emulsification and absorption of food-fat. Experiments on fasting, curarized dogs-from which all the bile was removed through glass tubes connected with the common bile duct-have shown that the following drugs particularly stimulate biliary secretion:

\title{
DIRECT CHOLAGOGUES.
}

*Podophyllum

Aloes

*Rhubarb

Colchicum

Sodium Sulphate

* Sodium Phosphate
Ipecac

Euonymus

*Nitro-hydrochloric Acid

Corrosive Sublimate

Sodium Salicylate

These experiments may not apply to all animals owing to the differences in food requirements and anatomy. The drugs marked with an asterisk have been found by clinical evidence most valuable. The purgatives above-mentioned act most successfully as cholagogues when given in small doses.

\author{
Calomel \\ Mercury \\ Most purgatives in a less degree.
}

INDIRECT CHOLAGOGUES.

The bile occurring at any time within the bowels is in part absorbed and then re-secreted. This process may be repeated inclefinitely, but is prevented by purgatives, especially those increasing peristalsis in the duodenum and upper part of the jejunum (calomel), because they hurry along and expel the bile in the gut before it has time to be absorbed.

In this way calomel and purgatives are indirect cholagogues in removing bile from the body; not by stimulating its secretion, but by hastening its excretion from the bowels. 
Cholagogues are serviceable in indigestion and constipation with disordered hepatic functions, as shown by icterus, lightcolored frecs, etc. A more complete method of ridding the blood of bile consists in the use of both an indirect cholagogne (to sweep it ont of the bowels) and a direct cholagogue (to increase biliary secretion). The urea-forming and glycogenic functions of the liver are not influenced to advantage by drugs, with the exception of opium, morphive and codeine, which lessen the amount of sugar in the urine in Diabetes Mellitus.

\section{GENERAL USES OF PURGATIVES.}

1. To empty the bowels. - In this way are removed freal accumulations and poisonons matters resulting from bacterial infection, and from fermentative and putrefactive changes in the intestinal contents in indigestion. Foreign bodies, bile, pathological discharges and intestiual parasites are also expelled.

Peristaltic action is quickened in chronic constipation, while spasmodic and painful conditions (colic) are relieved by ridding the bowels of the source of irritation causing the trouble.

2. To remove fluid from the body.-This effect is more marked after the use of concentrated solutions of saline purgatives and other hydragogues. Concentration of the blood and resulting absorption of dropsies of renal and cardiac origin, or inflammatory effusions, can be accomplished by these agents.

3. To revulse. - That is, to canse dilatation of the blood ressels in the intestinal walls and so withdraw blood from remotely congested areas, as in cerebritis. The drastics are appropriate for this service. Pain and nervous phenomena in other regions are sometimes benefited by the counterirritant action of drastic cathartics.

4. To deplete. - Cathartics, particularly concentrated saline solutions, deplete the body both locally and generally by withdrawal of serum from the blood ressels. Purgatives 
tend to combat inflammation (antiphlogistic action) in this way by lowering blood tension while they also favor reduction of a febrile temperature. Local depletion by salines is especially indicated in diarrhoa and dysentery, and in the first stages of acute inflammation of the digestive tract. Plethora and obesity are of ten treated by a depletive method with cathartics.

5. To eliminate-Deleterions material in the blood resulting from renal insufficiency, and probably from infecion in acute diseases, may be eliminated to a considerable extent by purgatives. So also may be bæmic sources of rheumamatism, lymphangitis and hæmoglobinæmia.

Contra-indications.-These refer rather to the special agent than to any disorder, for there is scarcely a condition in which some cathartic is not permissible.

Drastics are inadmissible under the following circumstances: in catarrhal conditions of the respiratory and digestive tracts, intestinal hæmorrhage, collapse, anæmia, hernia, prolapse of rectum, metritis, nephritis, pregnancy, general debility, and in wounds of and operations upon the pelvic or abdominal viscera.

In well-defined enteritis and peritonitis cathartics are to be avoided. In mechanical obstruction of the intestines surgical interference is indicated when practicable, but where this is impossible enemata and possibly purgatives may be employed. The intestines, developed to an extent disproportionate to the size of the stomach in the horse, are powerfully influenced by cathartics, so that in catarrh of the respiratory organs and influenza, metastasis, or change in the site of the inflammation may occur, and the intestines may become involved with the occurrence of excessive purging (super-purgation) after the ingestion of any but the mildest cathartics, as linseed oil. Aloes is the purgative given horses for ordinary purposes, while epsom and glauber salts are suitable for ruminants and pigs, and calomel and castor oil for dogs. Water assists the action of purgatives, and its ingestion should be encouraged by supplying a liberal 
quantity of common salt either with the purgative or on the food, and also by sweetening the drinking water with molasses in the case of cattle. If the action of cathartics is delayed, it is usually advisable to give enemata.

Enemata, or Clysters. - These are fluid injections into the rectum and are used for the following purposes:

1. To empty the lower bowels when purgatives are inadmissible, as in intestinal obstruction, ulceration and inflammation, frecal accumulations, debilitated conditions, obstiate romiting, unconscionsness, and in inability to swallow (sore throat and tetanus).

2. To relieve pain, spasm (of intestines and bladder), and shock, when deep, hot enemata $\left(105^{\circ}-115^{\circ}\right.$ F.) are used.

3. To save life. After severe hæmorhage, deep injections of hot normal salt solutions, $110 \mathrm{~F}^{\circ}$. (Enteroclysis, see p. 703.)

4. To accelerate the action of purgatives, and as a preparation for abdominal and pelvic operations.

5. To supply food. (See artificial feeding, p. 663.)

6. For their local effect upon inflammation of the mucous membrane of the rectum and colon. Opium and boiled starch solution; silver nitrate and tannic acid-in diarrhœea, dysentery, colitis and proctitis.

7. To kill intestinal parasites (oxyurides), - solutions of quassia and cornmon salt.

8. To administer medicines in dysphagia due to pharyngitis, tetanus, unconsciousness (apoplexy, coma and convulsions); to obstinate romiting and other causes.

9. To reduce temperature,-cold enemata in ferer.

10. To produce diuresis,-deep injections for retention and absorption into the blood.

11. To improve muscular tone and intestinal peristalsis in chronic constipation, - cold enemata $\left(55^{\circ}-60^{\circ} \mathrm{F}\right.$.).

12. To overcome twist and intussusception.

13. To stimulate peristalsis, relieve congestion, and increase the flow of bile in catarrhal jaundice,-cold, deep irrigations $\left(55^{\circ}-60^{\circ} \mathrm{F}\right.$.) are liere indicated. 
En smata are best given by allowing water to gravitate into the bowel from a height of 2 to 4 feet. The ordinary fountain syringe of human practice is suitable for the smaller animals, while for deep injections or irrigatious a human rectal tube should be slipped over the hard unber tip. In the case of horses or cattle enemata may be siploned through a rubber tube or piece of small hose. This is accomplished by filling the tube with water and compressing it at either end to prevent the escape of water, while one end is submerged in a pail or tub raised 2-4 feet above the patient, and the other end is then introduced directly within the bowel; or affixed to a rectal tube six feet long, when deep injections or irrigations are in order. A continuous flow is thus obtained. A still simpler method consists in pouring water into a funnel which has been fitted to one end of a rubber tube while the other end is passed into the rectum. That portion of the tube which is to be placed within the gut should always be lubricated with vaseline, oil or soap. Manual removal of hardened frees (scybala) must be practised in all animals before the use of enemata. The finger or blunt curette may be utilized for this purpose in small patients. The injection of linseed or cottonseed oil (H.oi.; D. 3 ii.) an hour before the use of larger enemata assists in softening the intestinal contents.

When deep injections are indicated, the hind quarters of the animal should be raised-small animals may be partially inverted-and the fluid allowed to flow in slowly, pushing in the rectal tube as the gutdistends. Such enemata are more effective whether the object be to simply unload the bowels, to cause retention and absorption of the fluid, or to wash out the intestines. One to several gallons of warm water form a suitable quantity for unloading the bowels of large animals; one-half pint to a quart, in the case of small patients. The injections should be repeateri until a good evacuation is secured. To increase the purgative effect of enemata a cup each of soft soap, salt and molasses are added to a gallon of water; or a tablespoonful 
each to a pint. Linseed oil or cottonseed oil are also mixed with water. Epsom salts are still more efficacions (H.円ii. to gallon of water. (D. $\overline{3}$ i.-iv. to pint); while oil of turpentine (H. $\overline{3}$ ii.-iv.; D. 3 i.-iv.) is very active and especially useful in colic and flatulence, mixed with the enema. When clysters are given to be absorbed they should always be deeply injected in quantities of one-half to one gallon for horses; or one-half to one pint for dogs. In chronic constipation and torpidity of the bowels plain cold water $\left(55^{\circ}-60^{\circ} \mathrm{F}\right.$.) injections are indicated.

Medicated irrigations are most serviceable in catarrhal disorders of the bowels (dysentery, etc.), i.e., the fluid is allowed to flow in and out again till the solution returns clear.

\section{Drugs Acting on the Circulation.}

\section{I.-Activg Upon the Blood.}

(a) Blood Plasma.-The alkalinity of the blood plasma can be increased by the use of the salts of the alkaline and earth metals; i.e., potassium, sodium, lithium, ammonium, ragnesium and calcium compounds. This effect is of value therapeutically in rheumatism, hæmoglobinæmia and uricacidæmia. In the former two disorders, increasing the alkalinity of the blood plasma appears to assist in the elimination of toxic material, while in the latter condition the excretion of uric acid-existing in the blood as uratesis thought to be favored by potassium and lithium salts. These salts also alkalize the urine and increase its secretion. Drugs which remove considerable fluid from the body, as purgatives, diaphoretics and diuretics, necessarily alter the composition of the blood plasma. By removing fluid from the plasma, these agents are useful in aiding absorption of inflammatory exudations, dropsies and oedemas, since the mass of fluid removed is soon replaced from that contained in the food and tissues. In the virions infectious and constitutional diseases treatment is largely directed to exciting the secretions and excretions with the purpose of eliminating products of tissue wasie anul hacterial action from the blood, 
which prove detrimental to the system. This line of treatment is pursued in uræmia, hæmoglobiuæmia and lymphangitis. Venesection, saline infusions, hypodermoclysis and enteroclysis alter the character of the plasma and often have a life-saving value. (See p. 728-736.)

(b) The Red Corpuscles. - The so-called blood tonics, or homatinics influence the red corpuscles, increasing their number and content of hæunoglobin when there is a deficiency of either. The effect upon the augmentation of hæmoglobin is more marked.

\section{HAMATINICS.}

Iron and its salts

Arsenic

Corrosive sublimate

Copper saits

Potassium permanganate

Manganese dioxide

The first two are immensely superior to the others in blood-making properties. Iron especially favors the formation of hrmoglobin; arsenic increases the number of red corpuscles. Certain agents possess toxicological significance by destroying the composition of hromoglooin. Large doses of the coal tar products, as acetanilid, antipyrin and phenacetin, nitrites and potassinm chlorate, convert hæmoglobin into methæmoglobin, a mixture, probably, of hematin and soluble albumin; while carbonic oxide, phosphorus, sulphur, arsenic, iodine, liydrogen sulphicle and turpentine, in large doses, reduce oxyhæmoglobin and prevent its combination with oxygen. Acetanilid, potassium chlorate and amyl-nitrite destroy the red blood cells, if absorbed in considerable amount.

(c) Wlite Corpuscles.-It is possible experimentally to arrest purulent exudations caused by irritation and inflammation when quinine is introduced into the blood or applied locally to blood vessels. This happens because quinine and all cinchona salts, berberine sulphate and acetanilidlike other poisons to amobae - prevent the amoboid movement or migration (diapedesis) of lencocytes throngh the vessel walls. Unfortunately it is impossible to give large enough doses iu practice to realize such favorable results in inflammatory disorders. An enormous increase 
of leucocytes (leucocytosis) occurs in acute diseases accompanied by a local exudutive process, and also in leukæmia, etc. Recent experience with nucleic acid and tallianine (see p. 545) proves that they induce leucocytes and are valuable in bacterial infections. Arsenic, and in some cases quinine, appear to reduce the leucocytosis, and in lenkæmia seem to thus aid recovery. Drugs altering the consistency of the blood are: Calcium chloride and (to a less extent) other calcium salts, gelatin and potassium iodide, which increase the rate and degree of coagulation; cod-liver oil, which angments the solids in the blood; and toxic doses of mercury, which lessen the solids and coagulation and increase the fluidity of the blood.

\section{II.-Drugs Acting on the Heart.}

The mechanism controlling the heart, which is influenced by drugs, is as follows :

1. Heart-muscle and ganglia.

2. Iuhibitory apparatus, including the vagus nerve roots originating from the medullary centre, and its fibres terminating in the heart.

3. The accelerator apparatus, consisting of the accelerator nerve-with centres in the cerebrum and modullaand its fibres passing down the spinal cord to the dorsal nerves; from thence through the first thoracic ganglion to the sympathetic, and so on to the cardiac plexus, with peripheral terminations in the heart. The ganglia in the heart are sitnated about the auriculo-ventricular groove and at the entrance of the superior and inferior venæ cavæ, and at the orifices of the pulmonary veins.

The ganglia have been divided into the inhibitory, connected with the vagus nerve; the motor; and the accelerator gauglia; and they are supposed to be influenced by drugs as well as the rest of the mechanism detailed above. Our knowledge of the functions of these ganglia is imperfect and therefore of the action of drugs on them.

The action of drugs on the heart-muscle has been determined by estimating their influence on the lower two-thircls 
of the apex, which is comparatively free from nerve supply. The action of drugs on the inhibitory apparatus is of more importance than that exerted on the accelerator nerve, and we know more about it. Drugs usually affect the roots of the vagus nerve in the centre, or its peripheral terminations in the heart, rather than its trunk. The heart is influenced by drugs as follows :

1. Stimulation of the inhibitory apparatus leads to slowing or weakening of the heart-beats, or to both.

2. Depression of the inhibitory apparatus results in quickening or strengthening the heart-beats, or both.

3. Stimulation of the accelerator apparatus causes an increase in the rate or force of the heart-beats, or both.

4. Depression of the accelerator apparatus induces decrease in the rate or force of the heart-beats, or both.

5. Stimulation of the heart-muscle produces increase in the rate or force of the heart-beats, or both.

6. Depression of the heart-muscle lowers the rate or force of the heart-beats, or both.

The vagus centre is stimulated by agencies increasing blood-pressure, or cansing aspliyxia. On the other hand, agencies reducing blood-pressure depress the vagus, or stimulate the accelerator nerve, or both. Thus, the nitrites, as amyl nitrite, nitro-glycerin and spirit of nitrous ether, quicken the heart by lowering vascular tension. Exterual stimuli to sensory nerves reflexly stimulate the heart, as also do many locally irritating agents taken internally; e.g., strong alcoholic or ammoniacal preparations.

Since drugs commonly influence more than one part of the mechanism controlling the heart, and since it is difficult to determine the exact physiological details in such complex actions, we shall content ourselves with tabulating the actions of drugs ordinarily employed for their influence on the heart, remembering that while moderate doses produce the effects enumerated below, poisonous doses often give rise to diametrically opposite actions.

(a) Drugs increasing the force of the heart-beat.

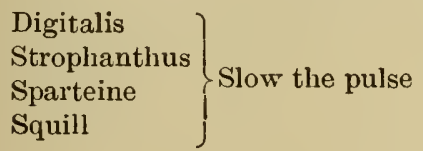

$\left.\begin{array}{l}\text { Barium salts } \\ \text { Camphor } \\ \text { Physostigmine }\end{array}\right\} \begin{aligned} & \text { Do not alter rate } \\ & \text { particularly }\end{aligned}$ 
(b) Drugs increasing the rate of heart-beats.

$\begin{array}{ll}\text { Belladonna } & \text { Stramonium } \\ \text { Atropine } & \text { Cocaine }\end{array}$

Hyoscyamus

(c) Drugs increasing the force and rate of heart-beats.

$\begin{array}{ll}\text { Alcohol } & \text { Strychnine } \\ \text { Chloroform } & \text { Caffeine } \\ \text { Ether } & \text { Quinine } \\ \text { Ammonia } & \text { Arsenic }\end{array}$

Ammonium carbonate

(d) Drugs decreasing the force and rate of the heart-beats.

Aconite

Veratrum viride

Prussic acid

Ergot

Antimony salts

The drugs most frequently given to animals for their action on the heart are alcohol, ether, digitalis, strophanthus, ammonia, ammonium carbonate, camphor, caffeine, strychnine, atropine, aconite and veratrum viride. The reader is referred to special articles on these drugs for therapentical indications and other details.

\section{III.-Drugs Acting on the Blood Vessels.}

The following table includes the mechanism regulating vascular tension :

1. In the walls of the vessels. $\left\{\begin{array}{l}\text { Smooth muscular fibres } \\ \text { Terminations of vasodilators and vasu- } \\ \text { constrictors }\end{array}\right.$

2. Nerve supply of vessels.... $\left\{\begin{array}{l}\text { Vasodilators } \\ \text { Vasoconstrictors }\end{array}\right.$

3. Centres.............. $\left\{\begin{array}{l}\text { Vasomotor centres in the medulla and } \\ \text { subsidiary centres in the spinal } \\ \text { cord and sympathetic system, con- } \\ \text { trolling the vasodilating and con- } \\ \text { stricting nerves }\end{array}\right.$

Each vessel is governed by two sets of fibres,-the constricting and dilating,-but we cannot discriminate between the action of a drug on the muscular fibres and the peripheral nerve endings in the vessel walls; nor can we 
always tell whether a drug acts to stimulate one set of peripheral fibres or depress the other.

Vascular tension is increased not only by contraction of vessels, but also by drugs which cause the heart to beat more quickly, and by those making its pulsations more forcible and complete, so that all the blood is squeezed ont of the ventricle at each contraction. Contrariwise, blood pressure is diminished, not only by those drugs inducing vascular dilatation, but by those reducing the rate or force of the heart, or both.

We shall simply classify drugs influencing the vessels according as to whether they act after absorption into the blood, or only when applied locally to the vessel walls.

(a) Drugs acting systemically to contract vessels.

\section{Adrenalin}

Cocaine

Ergot

Digitalis
Strophanthus

Squill

Sparteine

Strychnine
Hamamelis

Hydrastis

Physostigmine

(b) Dirugs acting systemically to dilate vessels.

\section{Amyl nitrite}

Nitroglycerin

Spirit of nitrous ether

Alcokol

Ether

Chloroform

\section{Chloral}

Aconite

Opium

Belladonna (secondary action)

Hyoscyamus

Stramonium

6

(c) Agents acting locally to contract vessels. Cold

Astringents

(d) Agents acting locally to dilate vessels.

Heat

Counter-irritants

Uses.-Drugs or agencies causing general dilatation of vessels are useful in overcoming internal congestions and colds by equalizing the circulation; that is, by causing the blood to be distributed more equably about the body. They are also of benefit in morbic conditions attended with a high, vascular tension; and are serviceable in dilating peripheral vessels and in causing perspiration and loss of heat in fevers (spirit of nitrous ether and alcohol). Drugs inducing general contraction of vessels are employed in 
disorders characterized by loss of tone, as in shock and collapse; and in heart weakness or disease (digitalis and strychnine); also in internal hæmorrhage and inflammations (ergot); and to aid the absorption of dropsies and cedemas. The uses of drugs locally contracting vessels will be described under Astringents (p. 63) and of ageuts locally dilating vessels under ccunter-irritants (p. 62).

\section{Drugs Acting on the Nervous System.}

\section{I.-Drugs Influencing the Brain.}

It is impossible to classify drugs according to their action on the various centres of the brain, because our knowledge is insufficient. In a general way, dritgs affecting the nervous system fall into two gromps,- the excitant and depressant. But another difficulty arises in regard to classification from the fact that the same drug usually both excites and depresses. Probably all drugs influencing the nervous apparatus possess some exciting action, and inost drugs which excite in small doses cause depression and paralysis after poisonous quantities.

For example, alcohol, ether and chloroform are cerebral excitants in small doses, but in large doses are depressants (hypnotics) and paralyzants (anæsthetics). Belladonna and its congeners, on the other hand, only excite the brain in large amounts; while opium and cannabis Indica may excite the brain in small doses, but are used for their more common depressant action. The condition of the patient has some bearing on the action of a drug influencing the brain. Thus moderate doses of alcohol depress and stupefy healthy animals while stimulating the enfeebled and ill-nourished.

The brain of the lower animals is undeveloped compared to that of man, and, in accordance with the general fact that the more highly a portion of the nervous mechanism is organized the more powerfully is it influenced by drugs, it follows that drugs acting on the brain and cord are more 
prone to affect the cord in veterinary patients, while impressing the brain more potently in man. For this reason we notice in the horse that the primary period of excitability (sometimes seen in man) following the administration of morphine is much prolonged and not infrequently completely obliterates the somnifacient action of the drug in this animal.

We shall be content to classify drugs acting on the nervous system according to their most pronounced action in moderate doses.

(a) Cerebral Excitants.

$\begin{array}{ll}\text { Alcohol } & \text { Caffeine } \\ \text { Anæsthetics } & \text { Quinine } \\ \text { Camphor } & \text { Cocaine }\end{array}$

Uses.-These drugs are rarely used simply to excite the brain, but formther purposes. Camphor, caffeine and quinine are employed to generally excite the nervous system in depressed conditions. Caffeine is a valuable antidote to the depressing cerebral action of opinm in poisoning.

(b) Cerebral Depressants. - It is fortunate that drugs progressively paralyzing the functions of the brain follow the so-called law of dissolution-i.e., paralyze the various functions of the brain in the inverse order of their evolutionary development. The centres last to be acquired are the first to be paralyzed (cerebral centres); while those of earliest origin (the respiratory, vagus and vasomotor centres) are last to succumb to the action of cerebral depressants. The cerebral depressants are used mostly to relieve pain, when they are called anodynes or analgesics. Pain is due to irritation of any sensory nerve, or the sensory tract in the spinal cord, or of the sensory centres in the brain. Pain may be relieved by paralyzing any portion of this path and destroying connection with the perceptive centres in the brain.

(1) Anodynes, by reason of their action on the brain.

Opium

Morphine

Codeine
Alcohol

Anæsthetics

Chloral
Cannabis Indica

Gelsemium Bromides 
(2) Narcotics.-This term is a broad and somewhat inclusire one. Narcotics embrace drugs which depress the brain and cause sleep (hypnotics or soporifics) and stupor (some anodyues and anrsthetics), and finally paralyze the respiratory and circulatory functions. The following may be included in this group :

\begin{tabular}{|c|c|c|}
\hline Opium & $\begin{array}{l}\text { Anresthetics } \\
\text { Chloral }\end{array}$ & $\begin{array}{l}\text { Cannabis Indica } \\
\text { Belladonna }\end{array}$ \\
\hline
\end{tabular}

(a) Hypnotics or Strporifics (drugs cansing sleep):

Opium Rromides Paraldeñde Sulphonal Morphine Cannabis Indica Urethrane Trional Chloral (Of little importance in veterinary

Uses.-Hypnotics are not of much value in Veterinary medicine by simply promoting sleep. Their general sedative and anodyne actions are utilized in relieving motor excitement (spasms) or sensory excitement (pain).

(b) General Ancesthetics.

Fther

Nitrons nxirle Ethylene dichloride

Methylene bichloride

Chloroform (Of slight value in veterinary medicine)

Anæsthetics are agents which abolish sensation generally or locally. It is thought that the general anæsthetics act directly on the nerve cells. Anæstletics-like uarcotics generally-first stimulate and then depress the nerve centres, but depression is by far their most salient and useful effect.

Anæsthetics destroy the functions of nerve centres in the cerebrum and spinal cord, and so abolish pain, sensation and reflex action. The law of dissolution is exemplified in their action. Anæsthesia is commonly described in three stages. (1) The first or stimulant stage is exhibited by excitement and struggling, owing in part to fright and in part to stimulation, first of the higher cerebral centres, and then of the lower cortical centres. There are also conghing and choking in this stage, following the local irritation of the vapor on the respiratory tract. There may be vomiting, and the circulation and respiration are temporarily stimulated. Stimulation now ceases and depression of the cerebrum, together with the motor, sensory and reflex spinal 
centres, appears, and ushers in the (2) ancesthetic stage, characterized by muscular relaxation and complete abolition of consciousness, sensation and motion. Between these two stages-the stimulant and anæsthetic-there sometimes occurs a transient state in which sensation is lost before conscionsness. This has been styled the anodyne stage.

Finally, the (3) paralytic stage ensues, accompanied by depression and then paralysis of the three great rital medullary centres controlling the circulation and respiration, together with that of the lowest reflex centres, so that involuntary micturition and defacation occurs. The animal dies of a combination of vasomotor, heart and respiratory fililure. If recovery should follow the paralytic stage, the bodily functions return in the reverse order to that in which they were lost; i.e., the lower vital fuuctions first appear, followed finally by the higher cerebral functions.

Uses.-Anæsthetics are employed in surgical operations to prevent pain and struggling; in obstetrical operations and in the reduction of fractures, dislocations aud hernia, to secure complete muscular relaxation; to overcome spasms and convulsions resulting from disease or poisons ; to arrest severe pain in colic; and finally to destroy aged or sick and useless animals.

For fuller details see Auæsthesia (p. 299).

(c) Drugs acting on the cortical motor centres of the brain.

(1) DRUgis stimulating the yotor centres.

Strychnine

Atropine

(2) DRUGS DEPRESSiNG THE MOTOR CENTRES.

\section{The Bromides}

Chloral
Physostigmine

Alcohol

Anæsthetics

The action of drugs on the cerebral cortical centres has been found by comparing the local effect of electrical stimulation before and after the internal use of drugs.

Uses.-The drugs depressing the cortical motor area of the brain are valuable in convulsions and spasmodic disorders 
and in motor excitement, particularly in epileptiform convulsions of dogs.

\section{II.-Drugs Acting on the Spinal Cord.}

The functions of the cord consist in the conduction of sensory impulses forward to the brain and of motor impulses backward to the muscles; in the origination of nervous force in centres controlling certain functions (sexual, sweating, etc.); and in reflex action by which the cord transmits impulses from sensory to motor tract of the same side of the body, or laterally, from sensory to motor columns on opposite sides.

While drugs probably influence the various centres in the cord, our knowledge of their action is chiefly limited to that exerted on the motor cells of the inferior cornua.

If a drug stimulating the motor cells of the cord is given experimentally, slight peripheral irritation will reflexly cause convulsions, and, if the cord is severed from the brain, the same phenomena appear.

(A) Drugs stimulating the motor cells of the inferior cornua.

Strychnine

Brucine

Thebaine
Ammonia

Anresthetics $\left.\begin{array}{l}\text { Opium } \\ \text { Ergot }\end{array}\right\}$ Primary action

Uses.-Strychnine is employed in paraplegia resulting from diseases of the spinal cord after irritation caused by the lesion has passed away.

(B) Drugs depressing the motor cells of thie inferior cornua.

(1) WITHOUT PRIMARY STIMULATION

Physostigmine
Bromides
Ergot
Nitrites
Gelsemium

Chloral

Morphine

A pomorphine

Alcohol
Emetine

Turpentine

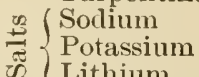

(2) With PRIMARY STIMULATION

\section{Ether}

Chloroform

Camphor

Carbolic acid

$$
\stackrel{\text { 尔 }}{=}\left\{\begin{array}{l}
\text { Antimony } \\
\text { Silver } \\
\text { Zinc } \\
\text { Saponin }
\end{array}\right.
$$

Nicotine

Veratrine

Mercury

Arsenic 
Uses.-Drugs depressing the motor cells of the cord are serviceable as antidotes in the treatment of poisoning by those exciting the same (chloral and bromides in strychnine poisoning), and in convulsive and spasmodic disorders, as chorea and tetanus.

\section{III.-Drugs Acting on the Nerves.}

The nerve terminations, rather than their trunks, are influenced by drugs.

(A) Drugs influencing peripheral sensory nerve-endings.

(1) Stimulating sensory nerve-terminations. - Counterirritants. (See p. 62.)

General Uses.-They are applied externally (mustard and heat) to stimulate the heart and respiration in heart failure, shock and collapse.

(2) Depressing sensory nerve-terminations.-These include local sedatives or anodynes, which lessen sensation; and local anæsthetics, which abolish sensation.

LOCAL ANODYNES

Aconite

Menthol

Carbolic acid

Atropine

Cocaine

Eucaine

$\begin{array}{ll}\text { Morphine } & \text { Veratrine } \\ \text { Chloral } & \text { Heat } \\ \text { Prussic acid } & \text { Cold }\end{array}$

Sodium bicarbonate

LOCAL ANESTHETICS

Uses.-The local anodynes are employed to relieve pain of an inflammatory, rheumatic or neuralgic character, and itching. The local anxesthetics are employed to prevent pain in surgical operations.

(B) Drugs influencing peripheral motor nerve-endings.

(1) STIMULATING HOTOR NERVE-TERMinAtIONS

Strychnine

Pilocarpine

Conium

Amyl-nitrite
Aconite

Nicotine

(2) DEPRESSING MOTOR NERVE-TERMINATIONS

Atropine

Cocaine

Camphor
Prussic acid

Nicutine and many others 
Uses.-Drugs influencing the peripheral motor nerveendings are not of any practical therapeutical value.

\section{IV.-Drugas Acting on the Nerves of Special Sense.}

\section{(A) Dirugs acting on the eye.}

(1) Drugs infuencing the pupil.-The mechanism controlling the pupil cousists of the centres for the contraction of the pupil (in corpora quadrigemina?), the centres for the dilatation of the pupil (in the medulla and aqueduct of Sylvius?), the third nerve, the cervical sympathetic and the circular and radiating (latter sometimes absent) muscular fibres of the iris. Drugs may act either centrally or locally on these structures. The pupil is dilated by drugs (1) depressing the contracting (oculomotor) centre, (2) the terminations of the third nerve or (3) the circular fibres of the iris ; and contrariwise, by (4) stimulating the dilating centre, (5) the terminations of the sympathetic or (6) the radial fibres of the iris; and, finally, by a combination of these actions.

Again, the pupil is contracted by drugs stimulating (1) the oculomotor centre, (2) the terminations of the third nerve or (3) circular fibres of the iris; and by depressing (4) the dilating centre, (5) the terminations of the sympathetic or (6) the radial fibres of the iris ; and also by a combination of these actions. Drugs may act locally on the pupil throngh the medium of the circulation as well as when dropped into the eye. Furthermore, absorption and central action may occur when drugs are dropped into the eye as well as when entering the blood through the more orfinary channels.

The drugs used in the treatment of the diseases of the eye are only those acting locally. Drugs influencing the pupil are divided into two classes: (1) those that contract the pupil (myotics) and (2) those that dilate the pupil (mydriatics). 


\section{(1) Mydriatics.}

(a) ACTING LOCALLY.

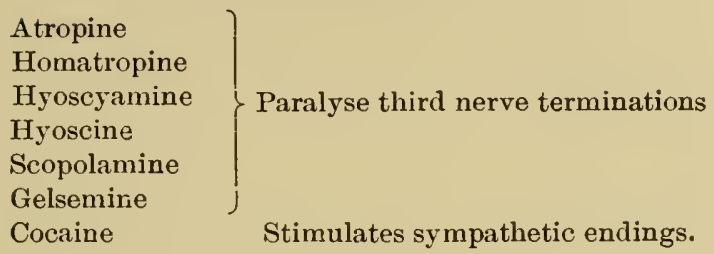

(b) ACTing Centrally.

Anæsthetics (late in their action)

The dilating centre is stimulated by carbonic dioxide in the blood, and therefore dilatation of the pupil occurs in asphyxia; also after irritation of sensory nerves, the sexual organs and digestive apparatus.

Uses.-Mydriatics are useful in dilating the pupil for examination of the eye, and to prevent adhesions of the iris in central corneal ulcers; in keratitis, to overcome photophobia and blepharospasm; and in iritis, to secure rest of the iris and ciliary muscles.

(2) Myotics.

(a) ACTING LOCALLY.

Physostigmine
Pilocarpine Stinulate third nerve-endings

(b) acting centrally.

Anæsthetics Opiun

Uses.-Myotics are employed to prevent prolapse of the iris in wounds and ulcers of the cornea; to antagonize the effect of atropine; to prevent the entrance of light in painful disorders of the eye; to lessen intra-ocular tension in glaucoma; and, in alternation with mydriatics, to break up adhesions to the iris. All the local mydriatics and myotics mentioned above act on the ciliary muscle to destroy the power of accomodation. Intra-ocular tension in glaucoma is usually increased by atropine and other mydriatics, but is liminished by eserine. 


\section{V.-Drugs Acting on the Ear.}

Strychnine makes the hearing (and sight) more acute; while salicylic acid, salicylates and quinine cause, in man, subjective symptoms, including fulness, roaring and buzzing noises in the ears.

\section{Drugs Acting on the Respiratory Organs.}

It is impossible to describe under this head all the drugs influencing the respiratory tract. Thus, agents affecting the circulation exert a powerful action on the blood supply and functions of the respiratory organs. The application of counter-irritants and heat and cold externally, reflexly prod nce notable alterations in pulmonary conditions. Emetics are indirectly serviceable in assisting the expulsion of exudations from the upper air passages in dogs. Furthermore, medicines having a depressing action on the nervous system are of importance in respiratory disorders in relieving cough and spasm. We shall consider here those agents acting on the respiratory apparatus itself.

\section{Drugs Acting on the Respiratory Mucous Membrane.}

(A) Drugs Acting Locally.

1. Stimulating the mucous membranes and causing rascular dilatation, increased secretion and muscular contraction of the walls.

2. Producing a sedative action.

3. Exerting an antiseptic influence.

4. Relixing spasm.

5. Causing a local astringent action.

6. Thinning exudations.

Errhines, or sternuatories, are drugs which are introduced into the nostrils to cause irritation, coughing and sneezing and expulsion of secretions, parasites and foreign bodies from the nasal chambers and upper air passages. They are rarely of any value, and include tobacco, ipecac, euphorbium, ammonia, chlorine and sulphurous anhydride. 
By inhalation (in pint of water at temp. of $140^{\circ} \mathrm{F}$. or over, unless otherwise stated).

(1) DRUGS EXERTING STIMULANT ACTION.

Carbolic acid ....................... $x \mathbf{x x .}$

Creosote.........................

Oil of cubebs......................

Tincture of benzoin.............. $z_{\text {ss. }}$

Tincture of ipecac .................

Oil of turpentine.................

Oil of pine...................

(2) DRugs exerting a SEDative iNfluence.

Diluted hydrocyanic acid............ $(\pi \mathrm{x},-\mathrm{xv}$. in $5 \mathrm{i}$. cold water).

(3) DRUGS PRODUCING AN ANTISEPTIC ACTION.

Thymol........................... vii.-xii.

Carbolic acid....................

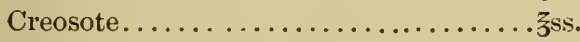

Compound tincture of bellzoin........ 3 ss.

Sulphurous anhydride gas.

Formaldehyde vapor.

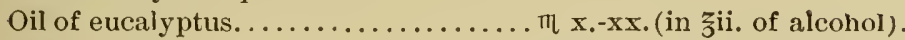

Oil of cubebs. . . . . . . . . .

Oil of juniper...................

Benzoic acid.................. (in z siii. of alcohol).

Tar water, undiluted.

Potassium permanganate................ $\mathrm{xv},-3 \mathrm{i}$.

Quininehydrochlorate..............

(4) DRUGS RELAXING SPASM.

Amyl nitrite..................... (5ss.-i.) D. (mii.-v.) undiluted. Extract of belladonna............. gr. ii -iv.

" " "hyoscyamus..........gr. viii. $-\mathbf{x v}$.

" " conium............. gr. viii.

(Burning stramonium leaves).

(5) DRUGS CAUSING A LOCAL ASTRINGENT ACtion.

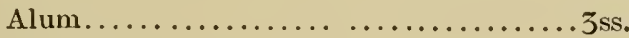

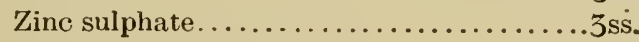

Solution of ferric chloride............ 3 i.

Silver nitrate......................

(6) DRUGS THINNING SECRETION.

Sodium bicarbonate.................

Solution of lime, undiluted.

Ammonium chloride................ 3ss.

Vinegar............................

Lactic acid...................... $\mathbf{j}^{\mathrm{i}}$. 
Uses.-Inhalations are often beneficial in the treatment of coryza, pharyngitis, laryngitis, tracheitis and bronchitis. In the first stage of catarrhal inflammation of the upper air passages, autiseptics may cut short the attack. Simple steaming with vinegar or sodinm bicarbonate moistens and soothes the dry, irritable mucous membrane and relieves congestion by promoting secretion. In the exudative stage, agents stimulating the respiratory mucous membrane and making the secretions less viscid are in order. If the mucous or purulent discharges are excessive, astringent sprays or inhalations are useful; and if they are foul-smelling, drugs combining stimulant and antiseptic actions may be employed. Inhalations may be given by means of a bronchitis kettle, or by atomization, to small animals. Care must be exercised that too large a quantity of the solution be not used lest absorption and poisoning ensue. Dogs may be placed urer the perforated seat of a cane-bottomed chair with the steaming apparatus underneath.

A hot brick is sometimes employed to give inhalations to horses by dropping it into a pail containing the proper solution. The head should not usually be covered during inhalation if the breathing is embarrassed or the respiratory tract obstructed, siuce fresh air is imperative. Simple steaning may be conductel for an hour. Inhalations containing special drugs may be given for fifteen minutes.

\section{(B) Drugs Acting Systemically.}

Expectorants are agents which influence the bronchial mucous membrane and its secretion. They aid or hinder expectoration in man, but are much less efficient in this respect in Veterinary medicine, because the act of expectoration is performed with difficulty by the lower animals. Nevertheless, expectorauts are useful in altering the character of the secretion and lessening the irritation caused by diry, tenacions discharges, and in stimulating the mucous memhrames and improving their circulation and nutrition. Mirenver, some drugs (volatile oils) exert an antiseptic: 
action on the bronchial mucous membrane during their elimination.

\section{Expectorants}

(1) INCREASING SECRETION

Depressing the heart and lowering vascular tension ("depressing expectorants")

$\begin{array}{lll}\text { Apomorphine } & \text { Ipecac } & \text { Antimony and potas- } \\ \text { Potassium iodide } & \text { Pilocarpine } & \text { sium tartrate }\end{array}$

Stimulating the heart and increasing vascular tension ("stimulating expectorants")

$\begin{array}{lll}\text { Ammonium chloride } & \text { Sulphur } & \text { Terpin hydrate } \\ \text { Squill } & \text { Tar } & \text { Terebene } \\ \text { Camphor } & \text { Turpentine } & \text { Volatile oils }\end{array}$

Balsams

(2) DIMINISHING SECRETION

$\begin{array}{lll}\text { Belladonna } & \text { Stramonium } & \text { Opium } \\ \text { Hyoscyamus } & \text { Acids } & \end{array}$

Volatile oils, and drugs containing them, first increase and then decrease bronchial secretion as a secondary effect.

(3) ALTERING THE NUTRITION OF BRONCHIAL MUCOUS MEMBRANE

Potassium iodide Cod-liver oil Sulphur

Ammonium chloride

(4) EXERTING AN ANTISEPtic ACtion

Turpentine

Terebene

Terpin hydrate

$\begin{array}{ll}\text { Tar } & \text { Balsam of Tolu } \\ \text { Ammoniacum } & \text { Cubebs } \\ \text { Balsam of Peru } & \text { Copaiba }\end{array}$

Uses.-Expectorants are chiefly prescribed in bronchitis. In the early or dry stage drugs increasing secretion and at the same time depressing the circulation are often employed in sthenic cases. These drugs possess less value in the treatment of the horse, on account of comparative insusceptibility to them, than in the case of dogs.

If exudation is excessive, then drugs lessening secretion are indicated. When the disorder is persistent, agents altering and improving the nutrition of the brouchial mucous membrane are beneficial. Bronchitis accompanier? 
copious foul secretion is treated with volatile oils, which exert an antiseptic action on the air passages. Expectorants are usually administered with other agents influenciug the respiratory tract; e.g., drugs relieving cough and spasm and those stimulating the respiratory movements or circulation.

\section{Drugs Stimulating the Respiratory Centres.}

\section{Strychnine \\ Atropine \\ Belladonna \\ Hyoscyamus}

External counter-irritation and heat.

Drugs Depressing the Respiratony Centres.

Many drugs depress and paralyze the respiratory centres in large doses, but they are seldom used medicinally for this purpose.

Uses.-Drugs stimulating the respiratory centres and movements are of great value in diseases of the chestespecially bronchitis-attended with obstruction in the air passages aud cyanosis. They promote coughing and efforts at expulsion of secretion and facilitate the entrance of oxygen into the blood. Some, possibly ammonia, stimulate the movements of the ciliæ lining the tracheal mucous membrane.

Strychuine is, perhaps, the most powerful respiratory stimulant; atropine is indicated where exudation is abundant, while ammoniun carbonate is prescribed to increase secretion. Certain drugs sometimes cause in large doses CheyneStokes breathing in animals, e.g., opium, chloral, bromides, digitalis, ammonium carbonate and strychnine, owing to disturbance of the respiratory centre.

Drugs Relaxing Spasm of the Bronchial Muscular Tunic and Relieving Cough.

LOCALLY

White of egg

Mucilage
Linseed tea

Syrups

External counter-irritation and heat 
SYSTEMICALLY.

\section{Opium}

Codeine

Hyoscyamus

Strainonium

Cannabis Indica
Nitrites

Chloral

Bromides

Chloroform

Phenacetin

\section{Uses of Drdgs allaying Spasm and Codgh.}

Coughing is a reflex act following irritation of sensory , nerve endings in any part of the respiratorv tract (usually of afferent vagal brauches), in the pharynx, pleura, ears, teeth, stomach and liver. Sensory impulses conreyed to the reflex centre for conghing, - near the respiratory centre in the medulla,-are there transformed into motor impulses and result in coughing. Drugs may stop coughing by acting locally to relieve congestion and irritation (demulcents) or they may exert a topical sedative action on the nerve endings. They also act systemically by quieting the reflex centre for coughing, or the sensory or motor nerve endings; also by abating. congestion in promoting secretion (expectorants), or in influencing the circulation.

Congh may be beneficial when it assists the expulsion of exudation, but is not so when it is constant and ineffective, as in congestion of the trachea, bronchial mucons membranes, lungs or pleura; in pulmonary consolidation; and in conghs originating outside of the respiratory tract. We should try to arrest coughing by agents removing the cause (congestion or irritation), such as counter-irritants, expectorants, local applications (sprays, inhalations) and heart stimulants; but if these are inefficient and coughing is immoderate, we may resort to the use of sedative agents. Some preparation of opium is most frequently employed to stop coughing, but should be avoided if cyanosis exists, since inspiratory and expulsive efforts are weakened by the drug. Belladonna, on the other hand, stimulates the respiratory centres and arrests cough by depressing both the afferent and efferent vagal terminations in the lungs, whilelike opium-lessening secretion. These drugs are often 
combined. When spasm of the bronchioles exists, as in asthma, and sometimes in bronchitis, the nitrites are particularly valuable.

\section{Drugs Acting on the Urinary Organs.}

Diuretics are drugs increasing the secretion of urine. Three factors are concerned with the urinary flow. (1) The composition of the blood. (2) The state of local (renal). and general blood pressure. (3) The activity of the renal cells. The renal mechanism influenced by drugs comprises :

(1) The Malpighian glomerules, which excrete water, salts and some excrementitious matters from the blood by osmosis. Their activity depends upon their blood supply, which is increased by agents cansing dilatation of their afferent vessels, or contraction of the efferent vessels; and by drugs increasing general blood tension. Drugs augmenting the mass of blood and tension in the Malpighian bodies enlarge the surface of cubical epithelium covering the capillary loops and promote osmosis of fluid into the cavity of the Malpighian capsule.

(2) The nucleated polyhedral cells lining the convoluted tubes. - These secrete the solid products resulting from the retrograde metamorphosis of nitrogenous bodies circulating in the blood, as urea, together with water.

(3) The constricted tubes.-These regulate the urinary secretion by either impeding its passage by constriction of their walls, thus aiding absorption, or by their active peristalsis facilitating the flow of urine.

(4) Nervous mechanism.-This governs the secretory activity of the cells of the convoluted tubes, regulates the tension in the vessels of the malpighian bodies, and possibly controls the unstriped muscle of the constricted tubes. It comprises : 
(a) Four medullary centres.

Vasomotor Vasodilator centres

(controlling renal vessels) $\}$ Vasoconstrictor centres

Secretory (Stimulating cells of convoluted tutes)

Inhibitory (Depressing activity of renal cells)

(b) Subsidiary centres in the spinal cord, solar and renal plexus.

(c) Secretory nerve-endings in kidneys.

(d) Muscular fibre in renal ressels.

(e) Renal cells.

These structures may be either stimulated or depressed, or the same drug may cause diuresis in various ways by a combination of actions.

Diuretics more commonly act in two ways. (1) By influencing renal circulation; (2) by stimulating the secretory nerve-endings in the renal cells or the cells themselves.

(1) Diuretics increasing general or local (renal) blood tension.

These drugs increase cardiac action in some cases, and in other's stimulate or depress the rasomotor centres, or the terminations of the vasoconstrictors or dilators in the renal vessels-to canse dilatation of the branches of the renal artery carrying blood to the capillary loops in the Malpighian bodies, or to produce contraction of the artcrial branches conducting blood away from the Malpighian tufts-in either way increasing local blood tension and secretion of urine.

Local blood tension is thought to be augmented by the nitrites and alcohol acting on the rasomotor centre or renal vessels to dilate the afferent vessels of the Malpighian glomerules, in addition to stimulating the heart. Buchu, turpentine, juniper and cantharides are said to act locally on the kidneys to contract the efferent vessels of the Malpighian bodies. Digitalis, strophanthus, squill, caffeine and strychnine stimulate the heart and contract the ressels generally, while locally they are beliered to cause contraction of the efferent vessels of the Malpighian bodies by action on the vasomotor centres. The entrance of water into the circulation increases the mass of blood, vascular tension, and thus the flow of urine. 
(2) Diuretics stimulating the secretory nerve-endings or renal cells.

$$
\begin{array}{cl}
\text { Potassium nitrate } \\
\text { “ } & \text { acetate } \\
\text { “ } & \text { citrate } \\
\text { bitartrate }
\end{array}
$$

Lithium salts

Salines generally
Colchicum

Calomel

Urea

Theobromine sodio-salicylate

Caffeine

(Diuretin)
Uses.-Diuretics are useful in removing deleterious and waste solid matters in the blood resulting from disease or the imperfect oxidation of albuminoid substances. In fevers the potassium nitrate and other potassium salts are employed with spirit of nitrous ether, alcohol and digitalis. They are antipyretics by eliminating pyrogenic material. Tissue waste is increased by diuretics, and they are serviceable in plethora, rheumatism and obesity. In acute diseases of the kidneys, salines and digitalis are indicated; in chronic renal disorder's more stimulating agents are often used, as juniper, buchu, etc. Diuretics remove water from the system. They are, therefore, employed in odema and dropsy of renal or cardiac origin, and in chronic effusions, as in pleuritis and pericarditis. Blood tension is lowered by withdrawal of water from the blood, and congestion may be relieved in various parts of the body. Diuretics lessen irritation of the kidneys by diluting the urine when the secretion is concentrated or contains toxins or other irritants (uric acid, calcium oxalate, etc.). Finally, stimulating diuretics (buchu, turpentine, etc.), are indicated in chronic inflammatory diseases of the kidneys and bladder, and in relaxed and paretic disorders of the bladder (incontinence of urine) to excite the reflex and motor functions of the sphincter and detrusor muscles.

\section{Drugs Influencing the Reaction of Urine.}

In man and animals secreting an acid urine, the basic phosphates of sodium and potassium in the blood are decomposed by the renal cells, and acid phosphate of sodium or potassinm-being more diffusible-are eliminated, giving the urine its characteristic reaction, while the bases remain 
behind. In the case of the herbivora the urine is alkaline, because there are larger quantities of magnesium and calcium salts in the food, which precipitate phosphoric acid in the stomach, and becanse there is an excess of alkaline sodium and potassium salts in the blood. The urire may best be made acid by benzoic acid, which is converted into hippuric acid during its passage through the kidneys. Salicylic acid, urotropin, the mineral acids (except nitric acid), aud large quantities of the regetable acils and boric acid tend to acidify the urine in a less degree. An acid urine may be made alkaline by alkalies, as salts of potassium, lithium, sodium and calcium, together with the vegetable salts, tartrates, citrates and acetates, which circulate as carbonates in the blood. Drugs promoting diuresis make the urine less acid because the basic sodium phosphate in the blood is not so readily broken up in the kidney when it diffuses through the cells in great dilution. Nitric acid renders the mine slightly alkaline by being converted into and eliminated as ammonia, while ammonia fails to make the urine alkaline because it is transformed iuto urea, uric and nitric acids (?).

Uses.-Benzoic acid is sometimes of benefit in acidifying and disinfecting an alkaline decomposing urine of pyelitis or cystitis. Recently urotropin has been used more successfully for these purposes. The alkalies are thought to be useful in alkalizing the blood in certain disorders (rhenmatism), hæmoglobinæmia, etc.), and the urine of carnivora, to prevent the precipitation of uric acid in the urine or to aid its solntion when already precipitated.

\section{Drugs Influencing the Composition of Urine.}

Drugs contracting efferent ressels of the Malpighian bodies diminish the flow of blood and urea-excretion aud increase the elimination of water; while those clilating the afferent vessels canse more blood to pass through the kidney and promote the secretion of solids and water. Drugs stimulating the cells of the convoluted tubes augment especially the urinary solids. The composition of the urine 
is also altered by most drugs eliminated in it, leading to changes in color, odor, reaction and the appearance of blood pigment, etc.*

\section{Urinary Antiseptics.}

Certain drugs are sometimes given with the purpose of preventing decomposition of the urine in purulent pyelitis and cystitis. Among these are:

$\begin{array}{lll}\text { Urotropin } & \text { Salicylic acid } & \text { Copaiba } \\ \text { Benzoic acid } & \text { Silol } & \text { Cubebs } \\ \text { Boric acid } & \text { Buchu } & \text { Volatile oils }\end{array}$

\section{Urinary Sedatives.}

The foregoing list, in preventing decomposition, and :

$\begin{array}{ll}\text { Hyoscyamus } & \text { Opium } \\ \text { Belladonna } & \text { Alkalies (with an acid urine) }\end{array}$

\section{Drugs Acting on the Sexual Organs.}

\section{(A) Influencing Chiefly the Male Generative Organs.}

The mechanism concerned with the sexual functions is presided over by cerebral and spinal lumbar centres. Agents may immediately excite the spinal centres or cause local irritation of sensory nerves in varions parts of the bodymore particnlarly in the neighborhood of the genital organs -and thus reflexly stimulate the lumbar centres.

* Thus blood appears in the urine after toxic doses of turpentine, cantharides and salicylic acid; and bluod pigment, in poisoning by potastium chlorate, acetrnilid, nitrites, glycerin and mushrooms (mus(arin); and occasionally by over(oses of mineral acids, naphtol, naphtalin and arsenic. Rhubarb and senna impart their coloring matter (chrysarobin) to urine, which makes acid urine brown, but alkaline urine a deep blood or purplish red. Carbolic acid, creosote, naphtalin and other tar-products, together with g:ultheria and uva ursi (due to contained arbutin), stain the urine a greemsh-brown or blackish hue. Sintonin dyes an alkaline urine cherry or purple-red, while an acid urine is turned yellow or greenish. Logwood gives its color to acid urine, while an alkaline urine is rendered red or violet. Poisonous d ses of sulphonal and trional give rise to a claret-colored urine, owing to hematoporphyrin. Gamboge and carrots bestow their colors on the urine. Turpentine is said to give urine the odor of violets, but large doses impart the preculiar odor of the oil itself. Cubebs, copaiba, encalyptus, valerian, musk, asafetida, sandal wood oil, asparagus and turpentine (large doses) communicate their special odor to the urine. 
The cerebral centres are mainly affected by visual, nasal or oral impressions, and also reflexly by irritation of sensory nerve-endings, more especially those situated in the sexual organs.

(1) Aphrodisiacs are drugs exciting sexual desire (and increasing sexual power in the male). They include:

\section{DIRECT APHRODISIACS}

Strychnine, Phosphorus, Alcohol (act on centres)

Cantharides (local irritant)

INDIRECT APHRODISIACS

In debility : Iron, Strychnine, Arsenic, (full diet)

(2) Anaphrodisiacs are drugs lessening sexual desire. They are :

Opium, Bromides, Purgatives, Nauseants, (bleeding), (spare diet)

Uses.-Drugs directly exciting sexual appetite are of no therapeutic valne, and the local irritants, as cantharides, are, moreover, likely to work harm by producing inflammation of the urinary tract. Loss of sexnal desire and power should usually be treated by improving the general nutrition with tonics and good feeding and by regulating the use of the sexual organs, unless the trouble is due to organic disease. Drugs diminishing sexual appetite may be useful in quieting the centres and rendering them less sensitive to sources of local irritation. It is, however, more sensible to remove the canse of irritation, as smegma preputii, acid urine, urinary calculi, intestinal parasites, scybala, fissure of the rectum, hæmorrhoids, etc. Anaphrodisiacs may be employed to subdue excessive sexnal excitement and nervousness (hysteria) sometimes accompanying "heat" in the fermale.

(B) Influencing the female sexual organs.

(1) Einmencegogues are drugs which favor the occurrence of "heat" (ovulation) in the female when it is irregular or abnormally absent. We are at present ignorant of their exact mode of action. Some act directly, perhaps, by stimulating the centres or nterns. 
DIRECT EMMENAGOGUES

Savin, Rue, Cantharides (irritants)

INDIRECT EMMENAGOGUES

Purgatives (Aloes)

In debility : Iron, Arsenic, Strychnine (full diet)

Uses.-The irritant emmenagogues are usually ineffectual in medicinal doses, while they may cause inflammation of the urinary tract and abortion (in pregnant animals) in large doses. The use of the indirect emmenagogues is more rational and effective. Aloes is thought to stimulate the uterus reflexly by irritation of the large intestines, and may also act locally on the uterus after absorption. Absence of œestrum, ordinarily a symptom resulting from a general or local condition, should be treated if possible by removing the cause (lebility, plethora, deformity).

(2) Ecbollics, or oxytocics, are drugs stimulating uterine contraction during or directly after parturition. The exact physiological details concerned in this action are unknown. They are :

$\begin{array}{ll}\text { Ergot } & \text { Quinine } \\ \text { Cotton root bark } & \text { Hydrastis } \\ \text { Corn smut } & \text { Savin }\end{array}$

(3) Drugs restraining uterine contractions.

$\begin{array}{ll}\text { Anrestheties } & \text { Chloral } \\ \text { Opium } & \text { Bromides } \\ \text { Cannabis Indica } & \end{array}$

Cannabis Indica

Uses.-Ecbollics (preëminently ergot) are used to contract the nterus and arrest hæmorrhage after parturition; or to stimulate the womb during parturition in inertia. In poisonons doses they may lead to abortion duriug preguancy. Drugs restraining uterine contraction (especially opium) are sometimes given to prevent threatened abortion.

(4) Drugs influencing mill-secretion.

(a) Galactagogues are drugs increasing the flow of milk. They include :

Pilocarpine

Alcohol

(Full diet)
Leaves of castor oil plant (internally or locally on udder as poultice). 
Drug treatment is ineffective in increasing the secretion of milk; rich feeding is the chief desideratum. Many drugs are eliminated in milk and may produce their characteristic effects in animals or man drinking it. Among these are:

Opium
All volatile oils
Purgative salts
Rhubarb
Senna
Castor oil
Scammony
Jalop
Iodine
Potassium iodide
Antimony

Arsenic
Mercury
Lead
Zinc
Iron
Bismuth
Neutral salts
Ammonia
Acids
Sulphur
Atropin

Copper

Carbolic acid

Colchicum

Euphorbium

Ergot

Salicylic acid

Veratrin

Strychnine

Croton oil

Aloes

Turpentine

(b) Antigalactagogues.-Belladonna is the ouly efficient drug lessening the secretion of milk, applied locally or given internally. It paralyzes the peripheral secretory nerveendings and is useful in mammitis by diminishing the circulatory activity in the mammary gland.

\section{Drugs Influencing Metabolism.}

(1) Alteratives.-The term "alterative" is a vague, indefinable word used to describe the action of certain drugs, modifying tissue change and improving nutrition in some disorders, which cannot easily be classified uuder other heads. It is impossible here to recount the actions of alteratives, because they probably act in various ways, and because we are generally ignorant of their actions. The value of alteratives has been discovered by clinical experience. The following are of ten classed as alteratives :

Arsenic and its preparations

Mercury and its salts

Iodine and its salts

Cod liver oil
Phosphoric acid

Colchicum

Sarsaparilla

Sulphur

Uses.-Alteratives are employed in those diseases in which experience has proved them to be beneficial. 
Tonics.-The word "tonic" is another term even more vague and all-embracing than "alterative," and, therefore; more difficult to accurately define. Tonics improve the general mutrition and health, and, as ordinarily understood; refer to drigs promoting appetite and digestion (bitter tonics, as gentian); the state of the blood (hrmatinics, as iron and arsenic); or the condition of certain organs (heart tonics, as digitalis ; nerve tonics, as strychnine).

Tonics are indicated in the treatment of debility (general or special) and anæmia.

\section{Drugs Influencing Bodily Heat.}

Antipyretics are drugs lowering the temperature of the body in fever. The mechanism concerned with temperature changes is as follows :

(1) Heat production.-There is a centre in the corpus striatum for lieat production. Probably this controls muscular activity, which is chiefly instrumental in the production of bodily heat.

(2) Heat loss.-There is a centre for heat dissipation situated in the medulla. This controls the vasomotors and state of vascular tension; the activity of the sweat glands and respiration; and, therefore, the amount of heat lost by radiation from the blood vessels of the skin, by evaporation of sweat, and by the act of respiration. Heat is also lost by the passage of fieces and urine.

(3) Heat regulation.- There are heat-regulating centres in the cortex cerebri (?) which coördinate or adjust the relations existing between the heat-producing and heatdissipating centres.

Finally, the bodily temperature is reflexly influenced by sensory impulses originating in various parts of the body, and conveyed by afferent nerves to the three brain-centres controlling temperature. Agents lowering bodily temperature in fever may act to depress the lieat-producing centre; to stimulate the heat-dissipating centre; to dilate vessels; 
to increase the secretions (particularly of sweat) ; to influence the circulation by drugs or venesection; to physically abstract heat, and to remove sources of fever in the digestive tract.

TABLE SHOWING THE PROBABLE MODE OF ACTION OF ANTIPYRETICS.

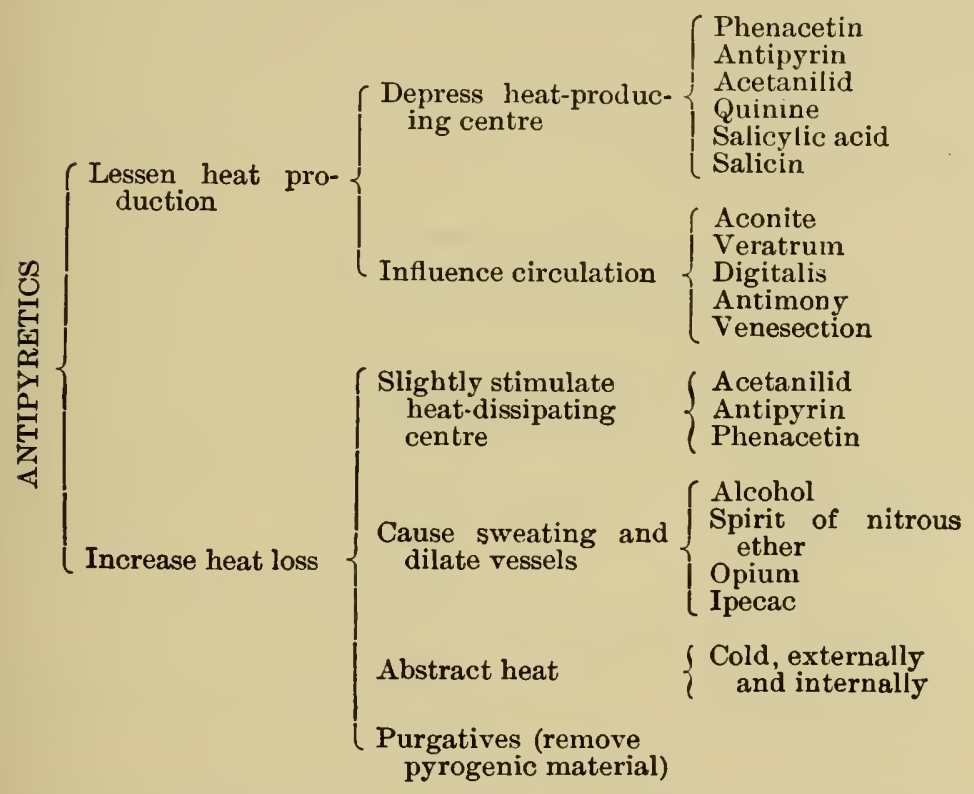

Antipyretics do not lower temperature in health when given in medicinal doses.

Uses.-Antipyretics are employed to diminish fever. It is wiser to lessen heat production than to increase heat loss (by diarphoretics, diuretics, circulatory depressants, bleeding, etc.), because heat production is stimulated by the latter process. The modern antipyretics (acetanilid, antipyrin and phenacetin) act chiefly to lessen heat production, and are, therefore, most serviceable, but even these drugs should usually be avoided unless fever is exceedingly high or longcontinued. This follows because a high bodily temperature is ofteu inimical to bacterial life and growth, and because 
fever is a natural protective agency, being the result of increased oxidation, and destroys toxins and pyrogenic substances. Acetanilid, antipyrin and phenacetin, moreover, depress the circulation in large doses and alter the red corpuscles so as to interfere with their oxygen-carrying functions. Cold is the best antipyretic agency when it can be employed, in not only reducing temperature and increasing the elimination of toxins in the urine, but in powerfully stimulating the nervous system and vital functions. (See p. 704.)

\section{Drugs Acting on the Skin.}

(A) Drugs Influencing the Blood Vessels of the Skin.

(1) Locally dilating superficial vessels. IRRITANTY OR COUNTER-IRRITANTS.

Cantharides

Iodine

Mustard

Capsicum

Croton oil

Oil of turpentine and other volatile oils

Ammonia water

Camphor

Red mercuric iodide
Corrosive mercuric chloride

Arsenous acid

Silver nitrate

Zine chloride

Carbolic acid

Mineral acids

Caustic alkalies

Anæsthetics and alcohol(when evaporation is prevented)

(Heat)

Drugs are classified as follows, according to the degree of irritation they produce:

Rubefacients are drugs which cause vascular dilatation and redness of the skin when locally applied, such as mustard and iodine (and heat).

Vesicants are drugs producing inflammation of the skin and exudation of serum nnder the epidermis (vesicles), when locally applied, such as cantharides.

Pustulants are drugs inducing a still higher grade of inflammation when locally applied, accompanied by migration of leucocytes from the vessels into the vesicles, forming pustules. 
Caustics, or Escharotics, are agents which, when locally applied, lead to so great a degree of irritation that the vitality of tissnes is destroyed, e.g., nitric acid, caustic potash and the white-hot iron.

Uses.-Irritants are often called comnter-irritants when they are used against (counter) existing irritation or inflammation by reflexly causing contraction of vessels in congested or inflimed underlying parts. Thus a blister on the chest leads to contraction of the vessels in the inflamed pleura and relieves pleuritis. Counter-irritants are, therefore, employed locally to overcome internal congestion and inflammation. Rubefacients are often applied over the whole surface of the body (mustarl and turpentine) to dilate superficial vessels and equalize the circulation in colds, chills and internal congestions. Vesicants are used to alter the circulation and nutrition of adjacent parts and to secure resolution and absorption of inflammatory products in joint and periosteal disorders. (For details see Counter-irritants, p. 665.)

\section{(2) Locally contracting superficial vessels.}

Astringents are dings which, when locally applied, make the tissues drier and denser and lessen secretions. Their action is probably dependent on various factors: partial coagulation of the albuminous fluids of the tissues; coagulation of morbid secretions; removal of water; and contraction of the muscular coat of the blood vessels. They are local irritants with the exception of lead acetate and bismuth salts. The astringents are:

Salts $\left\{\begin{array}{l}\text { Lead } \\ \text { Aluminum } \\ \text { Zinc } \\ \text { Silver } \\ \text { Copper } \\ \text { Ferric: } \\ \text { Bismuth } \\ \text { Hamamelis } \\ \text { Ergot }\end{array}\right.$

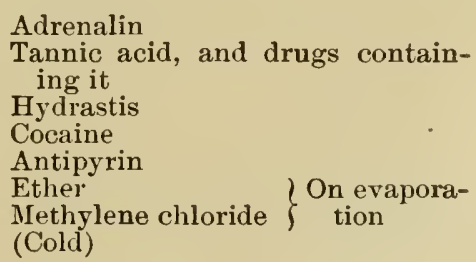

Styptics, or Hcemostatics, are drugs which arrest hæmor- 
rhage, when locally applied, both by coagulation of albumin of the blood and by direct contraction of the vessels and tissues surrounding them. Adrenalin and solutions of ferric alum, ferric chloride and subsulphate are the most powerful styptics, although all astringents possess an hæmostatic action.

Uses.-Astringents are employed in local loss of tone and relaxation of tissues accompanied by serons, mucous or purulent exudation from mucous membranes or raw surfaces. The irritating astringents are usually contra-indicated in acute inflammatory conditions, but are thought to prevent the out-wandering of lencocytes through the blood vessels, which results in purulent exudation.

Styptics are employed in the treatment of hæmorrhage from mucous membranes, or in bleeding from other parts which cannot be stopped by surgical means, heat or cold. The coagulated blood is prone to sepsis, speedy decomposition and infection.

(B) Drugs Locally Softening, Soothing and Protecting THE Skin, oR EMOLLIENTS.

They include :

Lard

Petrolatum

Cacao butter
Olive oil

Cottonseed oil

Lanolin

(Fomentations-Poultices)

Demulcents are drugs exerting a soothing, protecting and softening influence on the mucous membrane of the alimentarý canal, when given internally. They are mostly gums, syrups and albuminous fluids, as:

Acacia
Linseed infusion or tea
Liquorice
Syrup
Molasses
Honey

Glycerin

White of egg

Milk

Starch

Sweet oil

Uses.-Emollients are serviceable in softening the skin when it has a tendency to be dry and fissured; also in 
chafing and superficial inflammation when emollients protect the skin from the natural irritation of the air.

Demulcents are of value in inflammation of the mucous membrane of the digestive tract, and again in catarrh of the mucous membrane of the upper respiratory tract, because they not only exert a direct sellative inflnence on the throat, but, either reflexly or in some measure directly, act on the bronchial tubes. Demulcents are often $€$ mployed to relieve irritation in the urinary tract, but it is difficult to understand how they can there exert a demulcent influence after their decomposition in the alimentary canal and absorption into the blood, since they have never been found in that fluid or in the secretions.

\section{(C) Drugs Influencing the Secretion of Sweat.}

The mechanism controlling the sudoriparons glands and secretion of sweat, comprises sweat centres in the spinal cord ; secretory nerves proceeding from them with terminations in the glands; the gland cells, and blood vessels of the skin. Any portion of this mechanisu may be affected. by drugs to increase or diminish sweat secretion.

(1) Drugs increasing the secretion of sweat, or diaphoretics: They may :

(1) Stimulate the sweat centres.

(2) Excite the secretory nerve-endings in the glands.

(3) Increase the activity of the gland cells.

(4) Stimulate the vasodilators and increase the vascularity of the skin. Stimulation of the secretory and vasodilator nerves usually go hand in hand, since they accompany each other in their course to the sweat glands.

Pilocarpine

Alcohol

Spirit of nitrous ether

Heart stimulants

External heat

(Warm drinks)
DIAPHORETICS

Pilocarpine stimulates the gland cells, or secretory nerve-endings. The othrrs act indirectly by promoting the vascularity of the skin and thus the activity of the sweat glands. 
Antimony salts

Solution of ammonium acetate

Opium

Camphor

Ipecac

Potassium acetate

Potassium citrate

Aconite

Stimulate sweat centres

(2) Drugs diminishing the secretion of sweat, or anhidrotics. They may depress the varions parts of the mechanism which are stimulated by diarphoretics. They are :

Atropine

Belladonna

Hyoscyamus

Stramonium

(Cold externally)
Acids

Zinc salts

Nux vomica

Quinine

Salicylic acid
Action undetermined

Uses.-There are two indications for the use of diaphoretics. First, to bring blood to the surface and to cause sweating, thus equalizing the circulation in "colds," chills and congestions and reducing temperature in fever by evaporation and radiation of heat from the skin. Ammonium acetate, alcohol and spirit of nitrous ether are commonly used in the treatment of the disorders first noted, but acetanilid and phenacetin are more powerful antipyretics. Second, to eliminate morbid material from the blood in failure of the kidneys, as urinary suppression, or uræmia. These conditions are comparatively rare in veterinary practice. The skin of the lower animals generally is much less responsive to diaphoretics than that of man, while horses and cattle are more susceptible to these agents than dogs, cats or pigs.

A warm covering and atmospliere assist the dilation of the peripheral vessels and activity of the gland cells and should always be secured to aid diaphoresis. Anhidrotics are of little service in veterinary medicine. Excessive sweating is usually a sign of debility and is remedied by rest, tonics and good feeding. 


\section{Drugs Which Destroy Microörganisms and Parasites.}

(1) Disinfectants or Germicides are agents which destroy the microörganisms causing infectious and contagious diseases, fermentation and putrefaction. Examples:

$\begin{array}{ll}\begin{array}{l}\text { Corrosive mercuric chloride } \\ \text { Carbolic acid }\end{array} & \begin{array}{l}\text { Sulphurous acid } \\ \text { Chlorine } \\ \text { Lime }\end{array} \\ \text { Heat }\end{array}$ development of the microörganisms occasioning fermentation, putrefaction and disease; more especially the micrococci producing suppuration. Those used externally for surgical purposes are :

Corrosive sublimate

Carbolic acid

Creolin

Lysol

Hydrogen dioxide

Potassium permanganate

\section{Zinc chloride \\ Zinc sulphate \\ Formalin \\ Iodoform \\ Salicylic acid \\ Aristol}

Iodol

Boric acid

Benzoin

Thymiol

Balsam of Peru

Alcohol

Antiseptics which are given internally:

Naphtol
Salol
Creolin
Carbolic acid

Deodorants, or Deodorizers, are agents which destroy or counteract a foul odor. Those possessing any real value are also disinfectants and antiseptics, and remove the source of the odor.

(For further details see special article on disinfectants, antiseptics and deodorants, p. 718.)

Anthelmintics or Vermicides are drugs which kill intestinal worms, as the various species of tænia (tape worm); of ascaris (round worm), and oxyuris (thread, seat or whip worm), inhabiting the alimentary canal of the domestic animals. 
Anthelmintics inimical to trenia are:

Aspidium (horse and dog)

Oil of turpentine

Kousso

Areca nut (sheep and lambs)

Pumpkin seed
Calomel

Arsenic

$\left.\begin{array}{l}\text { Aloes } \\ \text { Linseed, cotton- } \\ \text { seed or castor } \\ \text { oil }\end{array}\right\} \begin{aligned} & \text { Aid in expulsion } \\ & \text { of dead para- } \\ & \text { sites }\end{aligned}$

Anthelmintics destroying ascarides:

Creolin

HORSES.

Aloes

Oil of turpentine

Arsenic

Calomel

Areca nut

Santonin

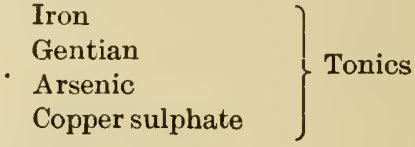

DOGS.

Spigelia

Anthelmintics killing oxyurides.

Rectal injections containing salt, solution of lime, quassia, iron salts, alum and oil of turpentine are used to. destroy these parasites infesting the lower bowel.

Anthelmintics destroying cestri equi ("Bots").

Carbon disulphide

Chloroform

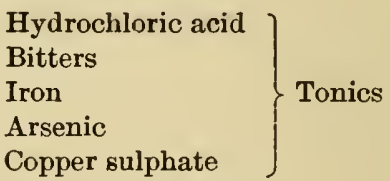

Vermifuges are purgatives (as aloes and oil) used to expel dead parasites from the bowels after the administration of anthelmintics.

Parasiticides, or antiparasitics, are drugs which destroy parasites, more especially those inhabiting the skin. We may classify them as those used :

(1) Against Tinea (Ringworm and Favus).
Mercurial ointments

Tincture of iodine

Glycerite of carbolic acid

Creolin

Creosote
Chrysarobin ointment Salicylic acid

Cantharides

Boric acid

Thymol

Sulphurous acid
Croton oil

Formalin 
(2) Against Acari. (Scab, itch and mange.)

$\begin{array}{ll}\text { Sulphur } & \text { Carbolic acid } \\ \text { Tar } & \text { Corrosive sublimate } \\ \text { Peruvian balsam } & \text { Salicylic acid } \\ \text { Styrax } & \text { Cantharides }\end{array}$

(3) Agains Paticuti. (Lice.)

$\begin{array}{ll}\text { Staphisagria } & \text { Carbolic acid } \\ \text { Oil of tar } & \text { Creolin } \\ \text { Perurian balsam } & \text { Tobacco } \\ \text { Styrax } & \text { Pyrethrum }\end{array}$

Oil of anise

(4) Against Pulex irritans. (Flea.)

Pyrethrum

Oil of anise

Carbolic soap

(5) Against Actinomycosis.

Tincture of iodine

Iodoform

Potassium iodide

Copper sulphate

Glycerite of carbolic acid

Corrosive sublimate

(6) Against Oideum albicans. (Thrush, aphtha, aphthous stomatitis.)

\section{Boric acid}

Potassium chlorate

Potassium Permanganate
Alum

Salicylic acid

Hydrochloric acid

(7) Against Strongylus micrurus and filaria. (Intratracheal injections of carbolic acid and turpentine.)

(For details concerning the use of parasiticides, see special articles on the drugs enumerated above.) 


\section{PHARMACY.}

\section{The More Important Medicinal Bodies and Principles Contained in Drugs.}

Alkaloideum, pl. Alkaloidea,-Alkaloids. Characteristics :

1. Alkaloids are nitrogenous bodies, being the active principles of many vegetable drugs.

2. They resemble mineral bases in that they have an alkaline reaction and unite with acids to form soluble crystalline salts. Hence their name, alkaloids.

3. Chemically they are ammonia compounds. One or more atoms of $\mathrm{H}$ (in $\mathrm{NH}_{3}$ ) are replaced by various radicals.

4. They are mostly insoluble in water, but very soluble in alcohol. Solutions possess a bitter taste.

5. They are similar to animal alkaloids and probably have a like origin,-i.e., from the decomposition of albuminoid material.

6. Alkaloids are precipitated in solutions by tannin, forming insoluble tannates.

7. They are usually solids and their salts are soluble and convenient for hypodermic use.

8. Their Latin ending is ina ; English, ine; viz. : Morphina, morphine.

Examples: An alkaloid of cinchona is quinine; of belladouna, atropine.

Glucosideum, pl. Glucosidea.-Glocosides are neutral, non-nitrogenous organic bodies, representing the active principles of many vegetable drugs. They yield glucose and other substances on decomposition. Hence their name. Their Latin ending is inum; English, in ; viz., salicinum, salicin.

Examples: A glucoside of digitalis is digitalin; of santoninum is santonin. 
Oleum, pl. Olea.-Fixed oils are combinations of glycerin with fatty acids; usually oleic, stearic, margaric or palmitic acids. They are liquid at ordinary temperatures and soluble in benzin, chloroform and ether. Exposed to the air they undergo acid fermentation, resulting in "rancidity." Fixed oils are expressed from fruits and seeds of plants and animal tissue. They are "fixed" because they cannot be distilled. They leave a greasy mark on paper.

Examples : Cod liver oil, castor oil, olive oil, linseed oil and croton oil.

Oleum, pl. Olea._Fats are solid, fixed oils.

Examples: Lard, cacao butter.

Oleum destillatum, pl. Olea destillata.-Distilled oils are obtained by the distillation of flowers, fruits, leaves or seeds of plants; by maceration, infusion, expression; or by extraction with solvents. They are either liquid or solid and possess an aromatic odor and taste. They are lighter than water, and soluble in alcohol, ether, and very slightly soluble in water. Distilled oils are miscible with fatty substances and mineral oils. They do not leare a greasy mark on paper.

Synonyms: Essential oils, volatile oils and ethereal oils. Alcoholic solutions of these oils are known as essences.

Examples: Oil of peppermint, oil of cloves, oil of wintergreen.

Oleo-resina, pl. Oleo-resince.-Oleo-resins are semi-solid mixtures of resins and volatile oils. Many are natural products, exuding from trees, as crude turpentine, which contains the volatile oil of turpentine and a resin, or what is commonly termed "rosin." They are soluble in ether.

Examples: Oleo-resin of capsicum, oleo-resin of aspidium.

Resina, pl. Resince.-Resins are brittle, amorphous solicls, solnble in alcohol and alkalies. They are obtained from oleo-resins by simple distillation, as in the case of turpentine; or may be extracted from plants by means of heat or alcohol. 
Examples: The resins of Burgundy pitch and podophyllum.

Balsamum, pl. Balsama.-Balsams are oleo-resins with the aldition of either cinnamic or benzoic acids, or both. They are soiids or liquids.

Examples: Balsam of Peru, balsam of Tolu.

Gummus, pl.Gummi.*-Gums are solid exulations from plants. They are geverally soluble in water, and their solutions are precipitated by alcohol.

Example: Gum acacia.

Gum Resina, p1. Gum-Resince, are solid exudations from plants consisting of a mixture of a gum and a resin.

\section{Pharmaceutical Processes.}

Many of the technical methods are those employed in chemistry, but of those more especially used in pharmacy are the following:

PROCESSES OF MECHANICAL DIVISION.

Slicing.-This prepares the drug for further reduction, and is the first of all pharmacentical processes.

Braising or Contusion.-Consists in breaking the drug by force, and is nsually performed with an iron mortar and pestle.

Rasping or Fiting.-For those drugs not easily reduced by the former processes, as guaiac wood.

Triturction.-Performed with mortar and pestle. The effect produced where there is a circular motion, accompanied by pressure.

Grinding and Sifting.-In order to exhanst drugs some must be giound and sifted to a finer powder than others. To accompiisin this, after grinding, we use sieves of different degrees of fineness, designated by numbers $20,40,60$, 80,100 . These have reference to the number of meshes contained to the square inch. Therefore, when a 60 powder is directed to be used, it is that which will pass through a sieve containing 60 meshes to the square inch.

* Gummi (indeclinable noun), often used for gum or gums. 
Levigation.-Somewhat similar to trituration, but performed with a slab and muller. These should be made of glass, or some non-absorbent material.

Elutriation.-This consists in mixing the powder, obtained by some of the former comminuting processes, with water; agitating it; allowing the coarser particles to settle, and pouring off the supernatant liquid which holds the finer particles in suspension. The powder settles from the latter and is dried.

\section{MECHANICAL PROCESSES.}

Processes by which liquids are separated from solids, and by which active principles and soluble constituents are separated from the inert portion of the drug.

Decantation.-The process by which solids are allowed to subside in a mixture and the supernatant liquid is slowly poured off or decanted. Liquids which will not mix, or that are of different Sp. Gr., may be decanted one from the other.

Filtration.-This is the process of separating a solid insoluble substance from a liquid by passing the liquid containing it through a porous substance called a filter. The filter commonly used consists of unsized or bibulous paper, although cotton, musin, felt, earthenware, and other substances are employed.

Percolation consists in the following process: A pulverized vegetable drug (continining both soluble and insoluble constituents) is placed in a conical vessel, or percolator, and subjected to the action of a liquid called a menstrum, when the soluble portion, or percolate, flows from the lower opening. The menstruum as it descends becomes more and more saturated with the soluble constituents of the drug. The first that escapes is the strongest, ancl each successive portion of the percolate becomes weaker until the drug is exhausted. The percolate should not drop faster than one or two drops a second. Percolation is employed in making tinctures, fluid and solid extracts, syrups and some other fluid pharmaceutical preparations. 
Clarification.-Is the process whereby cloudy substances are made clear by the addition of some coagulable substances, as albumin or ichthyocolla. It is the adding to any fluid, containing a sediment, a substance which will carry down all undissolved particles, then filtering or decanting the liquid.

\section{CHEMICAL PROCESSES USED IN PHARMACY.}

Solution.-The process by which soluble substances assume the fluid state through the action of a liquid.

Lixiviation.-Employerl to separate a soluble constituent from an insoluble prorous body. The substance to be lixiviated is mixed with water and placed in a conical vessel, the bottom of which is covered with straw or coarse sand, and, after maceration lias contiunerl sufficiently, the satnrated portion, caller the lye, is drawn off from an opening in the lower part of the vessel.

Crystallization. - Is the concentration of a liquid containing a soluble solid by means of heat, when on allowing it to stand until cool, crystals form. Stiring dnring cooling will produce granulation. Solution, filtration and crystallization are the three best ways of obtaining pure salts.

PROCESSES REQUIRING THE APPLICATION OF HEAT.

Liquefaction.--Is the melting of substances which at ordinary temperature are hard, and when cool returu to the sane condition as before heating, as resin, wax, lard, tallow.

Exuporation. - Is the conversion of a liquid into steam or vapor. Licpnids which evaporate at ordinary temperature are called volutile, as alcohol. To keep the heat below the boiling point of water, we use the water bath, which cannot reach a higher temperature than $100^{\circ} \mathrm{C}$. To gradially increase the heat, use the sand bath. 
Distillation.-Is the vaporization of a liquid in a retort or a still, by heat, aud conduction of the vapor through a cooled tube, where it is condensed and passes into a receiver and is called the distillate. Distillation is used to purify liquids or recover a volatile liquid from a solid, solution or mixture. Where two liquids ars mixed that have different points of vaporization and are separated in this way, the process is called rectification.

Sublimation.-Distillation of a volatile solid. When the product is in a solid form, it is called a sublimate, as iodine, camphor, etc.; when in flakes, it is called flowers, as sulphur.

Maceration.-A term used to denote the action of liquids upon drugs at ordinary temperature.

Digestion. - Same process, with heat raised to $40^{\circ} \mathrm{C}$.

Menstruum.-Any fluid substance used to dissolve a solid body or extract its medicinal principles.

Excipient.-Any substance used to give a pill mass proper consistency.

Exsiccate.-The process of removing all moisture, even the water of crystallization, from a crystal, by the use of heat.

Desiccate.-To remove all excess of moisture. Heat not usually employed in this process.

Incineration.-The combustion of a substance for its ashes.

Reduction is employed to recover a metal in its purity, when in a combined state.

\section{Pharmaceutical Preparations.}

The "United States Pharmacopœia" is an authoritative book, including the drugs of most value, with a description of their properties, tests for their purity, and methods for making their preparations. It is revised each decade by a convention of representative delegates from medical schools 
and societies, schools of pharmacy and pharmaceutical associations, with the collaboration of medical officers from the army, navy and marine hospital service. The last edition dates from September 1, 1905. All matter occurring in the "Pharmacopoia" is said to be according to the U. S. P., or official. That occurring in the "British Pharmacopœia" is marked B. P.

\section{Official Preparations.}

\section{I.-Preparations Whose Solvent is Water.}

Decoctum, pl. Decocta.-Decoctions are solutions of crude drugs in water, obtained 'by boiling. Unless specified otherwise, their strength is 5 per cent., and the boiling is conducted fifteen minutes. Decoctions tend to undergo rapid decomposition, and are only snitable in case of those drugs whose active principle is soluble in water. They are nnsuitable when the active principle of a drug is volatile, decomposed by heat, or when it contains much starch, which would form an easily decomposable, thick mass.

Drugs containing hard, woody substances, especially albumin, which coagulates in boiling and remains in the crude drug, are those especially adapted for this method.

Infusum, pl. Infusa.-Infusions are aqueous solutions of drugs made by maceration in boiling water without the aid of ebullition. Maceration is done for half an hour, and the strength is 5 per cent., unless otherwise ordered. The same disadvantages apply in the case of infusions as with decoctions, in addition to the longer time required for their preparation. Some drugs, as digitalis or ergot, which yield their active principles to water and are more powerful when freshly made, are suitable for this process.

Liquor, pl. Liquores.-Solntions are preparations holding active non-volatile principles in solution in water. They have no uniform strength. 
Aqua, pl. Aqua.-Waters are aqueous solutions of volatile principles. They have no uniform strength and are mostly solutions of volatile oils or gases.

Mistura, pl. Misturce.-Mixtures are compounds consisting of a combination of fluid preparations or compounds in which solid substances are dissolved or held in suspension by an appropriate vehicle. The term is thins very comprehensive, including most prescriptions for fluids used in practice, but nore narrowly it applies in pharmacy to insoluble materials suspeuded by suitable menstrua in water, the whole to be shaken before used. They bave no definite strength.

Emulsum, pl. Emulsa. - Emulsions are similar to mixtures in that an oily substance in a state of fine division is held in suspension in a gummy or albuminous vehicle. They have no uniform strength.

Syrupus, pl. Syrupi.-Syrups are saccharine solutions. The vehicle is usually water, although rinegar and alcohol may be used, and they are all medicated except the simple syrup. They are not of uniform strength.

\section{II.-Preparations Whose Solvent is Alcohol.}

Tinctura, pl. Tincturce.-Tinctures are alcoholic solutions of non-volatile principles, made usually by maceration and percolation of the crude drug. They vary in strength, usually from 5 to 20 per cent., but even exceed these limits. The alcohol in tinctures is often an important factor in the action of the preparation. Strong or diluted alcohol is used according to the solubility of the active principle.

Spiritus, pl. Spiritus.-Spirits are alcoholic solutions of volatile substances, either gases, liquids, or solids. They have no uniform strength.

Elixir, pl. Elixirs.-Elixirs are alcoholic solutions of drugs containing sugar and aromatic substances.

III.-Preparations Having Wrne as a Solvent.

Vinum, pl. Vina.-Wines are weak tinctures containing 
a small amount of alcohol, the remainder of the solvent being white wine. They have no definite strength.

\section{IV.-Preparations Having as a Solvent Diluted ACETIC ACID.}

Acetum, pl. Aceta.-Vinegars are solutions of the active principles of drugs in acetic acid prepared by maceration and percolation. They are not of miform strength.

\section{V.-Preparations Made by Solution and Evaporation.}

Extractum, pl. Extracta. - Extracts are concentrated preparations of the crude drug. Ordinarily alcoholic solutions of the crude drug are obtained by maceration and percolation, and then evaporated to a pasty mass. They are usually stronger, weight for weight, than the crude drug, but are not of uniform strength.

* Extractum Fluidum, pl. Extracta Fluida.-Fluid extracts are permanent, concentrated medicinal solutions of uniform strength. $1 \mathrm{cc}$. of the fluid extract is equivalent to $1 \mathrm{gm}$. of the crude drug. This result is obtained by percolation (usually with alcohol) and partial evaporation.

\section{VI.-Preparations Made by Distillation on Occurring}

Naturally, as Exudations from Trees.

Oleo-Resina, pl. Oleo-Resince-Oleo-Resins are officially extracts obtained by percolation of the crude drug with ether, and evaporation of the solvent. They have no uniform strength.

Pulvis, pl. Pulveres.-Powders are preparations of finely pulverized drugs. Sugar of milk is frequently added, on account of its hardness, to aid in pulverization and as a diluent. Comparatively tasteless, non-irritating and often insoluble drugs are given in this form. Drugs that are volatile, deliquescent or irritating are otherwise administered. Powders are used to advantage in veterinary practice, since they are often taken voluntarily on food.

Trituratio, pl. Triturationes.-Triturates are combina-

\footnotetext{
* Now written, according to the U. S. P., 1905, Fluidextractum, Fuidextracta.
} 
tions of lrugs and sugar of milk, prepared by trituration. They occur in powder or are pressed into tablets.

Oleum, pl. Olea._Fixed oils are usually obtained by mechanical expression from the product of plants or the tissue of animals.

Pilula, pl. Pilula.-Pills are preparations of drugs made into globular form by the addition of a suitable excipient, and should be of a convenient size for swallowing.

Mrassa, pl. Massce.-Masses are pasty mixtures suitable for making pills.

Suppositorium, pl. Suppositoria. - Suppositories are medicinal substances incorporated with cacao butter and moulded into solid, usually conical, bodies intended for introduction into the rectum or vagina, where they melt at the temperature of the body.

Confectio, pl. Confectiones.-Confections are pasty masses, consisting of powder incorporated with syrup.

\section{ViII.-Preparations Intended for External Use.}

Linimentum, pl. Linimenta.-Liniments are liquid preparations with an oily, alcoholic or soapy basis.

Oleatum, pl. Oleata.-Oleates are medicinal solutions in oleic acid.

Unguentum, pl. Unguenta.-Ointments are preparations having a fatty basis ( 80 per cent. of lard).

Ceratum, pl. Cerata.-Cerates are similar to ointments but harder, owing to the addition of 10 per cent. more wax.

Glyceritum, pl. Glycerita.-Glycerites are preparations whose solvent is glycerin.

Emplastrum, pl. Emplastra.-Plasters are solid, sticky, supple preparations intended for application to the skin, where they become adhesive at the temperature of the boily. In veterinary parlance they are often known as "charges."

Charta, pl. Chartce.-Papers are fragments of medicated paper.

Collodium, pl. Collodia.-Collodions are solutions of gun 
cotton in ether and alcohol, leaving a thin, dry, adhesive coating when applied externally.

\section{IX.-Preparations Having Honey as an Excipient.} Mel, pl. Melita.-Honeys.

X.-Non-Official Preparations Peculiar to Veterinary Practice.

Botus, pl. Buti.-Balls, are substitutes for pills. They are of elongated, cylindrical shape, about two and one-half inches long, and should weigh about two ounces when intended for horses. Various excipients are used to make a mass of the proper consistency. For immediate use, molasses and licorice root may be employed, and brown tissue paper is used as a covering for the balls. Gelatine capsules may take the place of balls, and should be covered with paper to prevent slipping through the fingers when wet with saliva. Linseed meal is a good excipient on account of its gum. Soap is often used, and glycerin makes a good preservative and keeps the mass moist.

Heat is often necessary in preparing a ball mass when the materials are resinous (as aloes) or waxy. A ball is given to a horse by holding it in the right hand, the tips of the fingers and thumb surrounding it in the form of a cone. The tongue of the animal being drawn to the operator's left with his left hand, the right is then quickly passed along the roof of the patient's mouth (avoiding the edges of the back teeth) until the back of the tongue is reached, when the ball is dropped, the right hand rapidly removed and the tongue released.

If the mouth is narrow or the animal unmanageable, a balling iron or speculum is used to keep the mouth open. The horse may be backed into a narrow stall and the head. steadied by an attendant with the assistance of a "twitch" on the nose. Substances of an irritating nature may begiven in this form, and balls are also used when the disposition of the patient does not almit the giving of a drench. 
Haustus, pl. Haustus.-A drench is an extemporaneous fluid mixture, intended for immediate use as a single dose.

Soluble substances are best given in solution to obtain the most rapid results, muless irritating. Even then they may be prefarabie when sufficiently diluted with water and demulcents. Insoluble drugs may at times be given to advantage in a mixture rither than in the form of a ball or powder. Most official fluid preparations require dilution before administration, but for convenience small doses of tinctures and fluid extracts are dropped npon the tongne of horses unless the preparations are exceptionally acric. Drenches are particularly applicable $f(r$ cattle and sheep, as solids are not quickly absorbed in their capacions digestive apparatus, and drenches are given them with ease.

The amount of liquid conveniently administered to horses is from one to two pints; to dngs, from two to funr ounces; to sheep, six to eight onnces. Cattle take readily unlimited quantities. Care should be ohserved that drenches: are so diluted as to be harmless to the mucous nembrane, and, if containing insoluble drugs, that these be held in suspension by a suitable vehicle or thoronghly shaken before: using. Drenches are best given to horses by making a loop on the end of a rope, passing the upper jaw through this, the other end of the rope being passed throngh a pulley in the ceiling and held by the operator or assistant. The horse shonld be first backed into a narrow stall. The neck of the bottle (which should properly be made of horn or tin) containing the drench, being introduced and held in the right hand of the operator between the ontside of the back teeth and the inside of the cheek of the patient, the left hand is used to steady the nose of the animal, but the nostrils should not be obstructed.

In giving drenches to cattle the operator stands on their left and passes his right arm between the horns over the poll and down in front of the face, grasping the nasal septum between the thumb and forefinger. The neck of the bottle is then thrust with the left hand into the animal's mouth. 
Dogs are given drenches with the aid of an assistant, who holds the mouth closed with one hand, while he makes a cup by pulling the corner of the lip away from the teeth with the other hand, into which the medicine may be slowly poured, the animal easily swallowing it. Small dogs may be placed sitting upon a table. A large dog may be put upon his hind quarters in a corner, and his head held between the knees of the operator. Cats are given drenches by rolling them in a heavy blanket with only the head out and the jaws held apart by means of two loops of tape about either jaw behind the incisor teeth. The jaws are then pulled apart by drawing on either loop from above and below the animal's head.

Drenches should never be poured into animals if in an unconscious condition, for then they are unable to swallow, and the fluid may gravitate into the trachea. If coughing ensues during the administration of a drench, the procedure shonld be immediately stopped.

Electuarium, pl. Electuaria.-Electuaries are medicinal pastes intended to be smeared on the teeth of animals, where they melt at the temperature of the body and become absorbed. Molasses, honey, glycerin, syrup or mucilare are used as excipients. Electuaries are used for their local action on the month and throat, and for convenience in administration, if so crude a method may be thus described. A certain specified quantity of the electuary may be weighed by the dispenser and serve as a sample, or a domestic utensil may be employed to measure the dose, which is smeared with a thin, flat stick on the back teeth or tongue of the patient. 


\section{INCOMPATIBILITY.}

Before entering upon the study of prescription writing, it is essential to consider the results of improper combination of drugs, i.e., incompatibility.

While a knowledge of chemistry, pharmacy and the physiological actions of drugs is necessary to avoid incompatibility, it is yet possible to formulate certain rules which will assist us in escaping unfortunate combinations.

Incompatibility is conveniently divided into three classes: I. Chemical. II. Physical. III. Physiological.

I. Chemical incompatibility occurs when drugs are so mixed that an unsuitable alteration in their chemical composition takes place. Certain substances should usually be prescribed alone because of the frequency with which chemical changes arise when they are combined with other medicines. These are :

Lead, silver and zinc salts

Iodine and iodides

Tannic and gallic acids

Liquid iron preparations

Corrosive sublimate

\author{
Mineral acids \\ Solution of potassa and lime \\ Quinine sulphate \\ Hydrocyanic acid
}

The possibilities of the following combinations must be kept in mind to avoid incompatibility :

1. Solutions of alkaloids are incompatible with tannic acid, alkalies, alkaline salts, and iodides and bromides, i because precipitation occurs.

2. Glucosides are decomposed by acids and are, therefore, incompatible with them.

3. Acids may not be added to alkalies, alkaline salts or vegetable acid salts, because decomposition and chemical change will ensue. 
4. A mixture of salts in solution will decompose if either an insoluble compound or double salt can be formed; otherwise no change will take place.

5. Chloral is incompatible with alkaline solutions, because chloroform is generated.

6. Chloroform and potassium cyanide form prussic acid.

7. Potassium chlorate, nitrate, or permanganate liberate oxygen and should not be mixed with readily oxidizable substances, such as charcoal, sugar, sulphur, glycerin, carbolic acid, iodine, turpentine and organic materials, lest explosive compounds be formed.

8. Lime water precipitates mercury salts.

9. Both calomel and antipyrin are incompatible with sweet spirit of nitre.

10. Calomel may not be combined with nitrohydrochloric acid lest corrosive sublimate result.

11. Calomel and prussic acid form the poisonous mercuric cyanide.

12. Liquid iron compounds are incompatible with fluid preparations of the vegetable bitters (except those of calumba and quassia), because the tannic acid in them throws down a precipitate.

13. Considerable quantities of acid are incompatible with tinctures, since ethers are produced.

14. Water precipitates resinous tinctures.

15. Gum arabic is incompatible with lead and iron salts, and mineral acids.

16. Strychnine is precipitated in solution by potassium. bromide.

17. Pepsin and pancreatin are mutually destructive in fluid combination.

18. Solutions of potassium chlorate and iodide unite to. form a poisonous compound.

It is beyond our scope to attempt the enumeration of all possible drug-incompatibilities. The special incompatibilities of each drug may be found under the proper heading in the detailed description of them. Furthermore, we may 
avoid incompatibility by (above all) simplicity in prescription writing, i.e., the use of few drugs in combination. Water or alcohol are generally the best solvents.

II. Physical incompatibility consists in the production of unsightly-looking mixtures, but withont necessarily any chemical alteration of their ingredients; for example, the addition of water to insoluble powders, oils and chloroform. While such combinations are pharmacentically improper, they may sometimes be used to advantage in practice.

III. Physiological incompatibility consists in the union of drugs possessing antagonistic physiological actions. For instance, the combination of purgatives and astringents; of morphine and atropine; of digitalis and nitroglycerin. Such prescriptions may be valuable therapeutically when the antagonism is not complete. This follows because, while the deleterious action of one drug may be offset by another, its beneficial effect may at the same time exist or be accentuated. Thus the anodyne influence of morphine is increased by combination with atropine, but both the depressing action of morphine on the respiration and its constipating tendencies are lessened by atropine. 


\section{PRESCRIPTION WRITING.}

Words and Phrases Commonly Used in Prescription Writing, With their Abbreviations.*

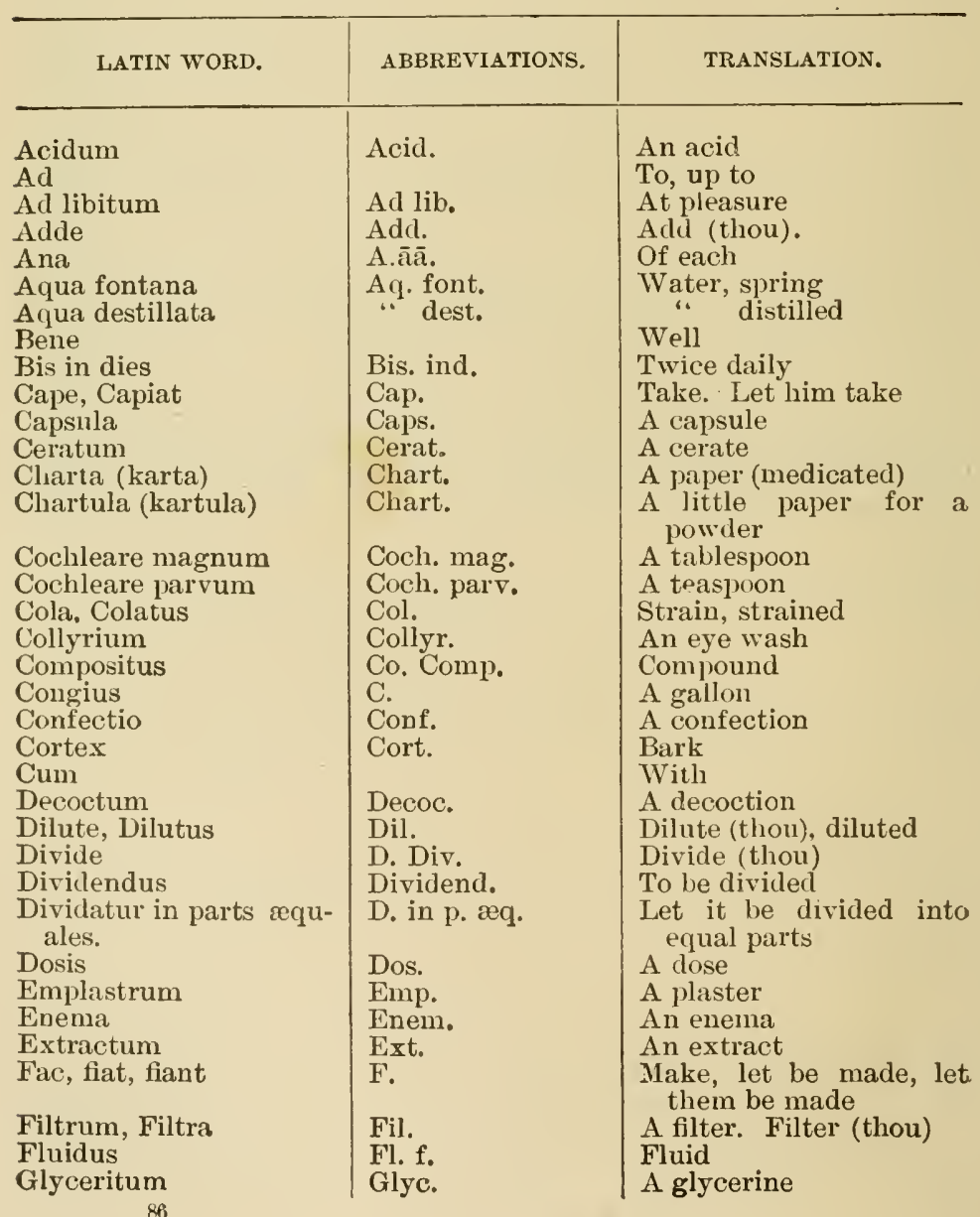




\begin{tabular}{|c|c|c|}
\hline LATIN WORD. & ABBREVIATIONS. & TRANSLATION. \\
\hline $\begin{array}{l}\text { Gutta, Guttæ } \\
\text { Guttatim } \\
\text { Haustus } \\
\text { Hora } \\
\text { In dies } \\
\text { Infusum } \\
\text { Injectio } \\
\text { Lac } \\
\text { Libra } \\
\text { Liquor, or Liq'uor } \\
\text { Lotio (losheo) } \\
\text { Magnus } \\
\text { Massa } \\
\text { Misce } \\
\text { Mistura } \\
\text { Mucilago } \\
\text { Nox, Nocte Maneque }\end{array}$ & $\begin{array}{l}\text { No. } \\
\text { O. } \\
\text { P. æ. } \\
\text { Parv. } \\
\text { Pil. } \\
\text { P. r. n. } \\
\text { Pulv. } \\
\text { Q. S. (followed by } \\
\text { genitive) } \\
\text { Q. h. } \\
\text { Sat. } \\
\text { Ss. } \\
\text { Semidr: } \\
\text { Sesunc. } \\
\text { S. Sig. } \\
\text { Solv. } \\
\text { Sol. } \\
\text { Spr. } \\
\text { Suppos. } \\
\text { Syr. } \\
\text { Tal. } \\
\text { Tra. Tr. } \\
\text { T. i. d. } \\
\text { Ungt. } \\
\text { Vin. } \\
\text { Vehic. }\end{array}$ & $\begin{array}{l}\text { A drop, drops } \\
\text { Drop by drop } \\
\text { A draught } \\
\text { An hour } \\
\text { Daily } \\
\text { An infusion } \\
\text { An injection } \\
\text { Milk } \\
\text { A pound, a Troy pound } \\
\text { A solution } \\
\text { A lotion } \\
\text { Large } \\
\text { A pill-mass } \\
\text { Mix } \\
\text { A mixture } \\
\text { A mucilage } \\
\text { Night, at night and in } \\
\text { the morning } \\
\text { A number, in number } \\
\text { A pint } \\
\text { A part (governs genitive) } \\
\text { Equal parts } \\
\text { Small } \\
\text { A pill } \\
\text { According to circum- } \\
\text { stances; occasionally } \\
\text { A powder } \\
\text { As much as is necessary } \\
\text { Every hour } \\
\text { Saturated } \\
\text { A half } \\
\text { A lialf drachm } \\
\text { An ounce and a half } \\
\text { Sign } \\
\text { Dissolve, dissolved } \\
\text { A solution } \\
\text { A spirit } \\
\text { A suppository } \\
\text { A syrup } \\
\text { Such, or, like } \\
\text { A tincture } \\
\text { Three times a day } \\
\text { An ointment } \\
\text { A wine } \\
\text { A menstrum } \\
\text { a }\end{array}$ \\
\hline
\end{tabular}

* Abstracted from Mann's " Prescription Writing." 
A prescription, derived from the Latin Prae, before, and Scriptum, written, comes to us from the early custom of physicians in writing down their advice beforehand for their patients' guidance. As now used it is the written formula of the practitioner describing to the pharmacist the manner of compounding and dispensing medicines, and to the attendint the mole of administering them.

Formulis are official when simply taken from the "United States Pharmacopœia," and extemporaneous when concocted off-hand by the practitioner. Extemporaneous formule are simple when composed of one ingredient; a compound prescription is composed of several parts, which may be considered as follows:

I. Heading.

II. Names and quantities of drugs.

III. Direction to compounder.

IV. Direction to attendant.

V. Siguature of writer.

The heading, "Recipe," is derived from the Latin, the imperative of the verb meaning to take, and is ordinarily represented by the sign $D_{x}$, a corruption of 2 , the sign of the Zodiac for Jupiter. After the Christian era the sign of the Cross was used, or N. D., for Nomine Deo, in God's name; J. D. for Juvane Deo, meaning God helping, etc. We have now reverted to the old sign, which is all that remains of an appeal to Jupiter. This symbol seems to put the practitioner, even if involuutarily, into a position of reverence in thus offering a prayer in embryo (the old physicians always wrote one) whenever one writes a prescription. The custom also suggests that we are not yet sufficiently sure of our Materia Medica after all these centuries, to sacrifice the efficacy of prayer.

In regard to the names and quantities of drugs, we find in the text books that one should always strive after a classical arrangement, whereby four ingredients are essential to accomplish any result. These include : 
I. The basis, or active medicinal substance.

Ir. The adjuvant, or assistant.

III. The corrigent, or corrective.

IV. The excipient, vehicle, or menstruum.

But we shall find that while such a classical arrangement may exist in the text-books, we are usually content in practice with the basis, together with a vehicle. The classical arrangement is essential in order that the old Latin motto be fulfilled: "Curare cito, tuto et jucunde." Curareto cure (the basis) ; cito-quickly (the adjurant); tuto-safely (the corrigent); jucunde-pleasantly (the excipient).

In a physic ball for horses we may employ aloes as a basis; calomel as an adjurant; ginger as a corrective; molasses as an excipient. More commonly in fluid preparations we prescribe several bases, or ingredients for curative purposes, neglecting any adjuvant or corrigent and simply using water as a velicle. It is often of distinct advantage to write for a combination of several drugs whose action looks towards a common encl. Iet one should always lean to simplicity rather than complexity in the number of ingredients. While it is difficult to avoid chemical antagonism, how much harder is it to prevent untoward physiological combinations in the body, which we can in nowise foretell. In olden times ignorance led practitioners to try the effect of an enormous number of drugs, with the hope that out of the charge one at least of the pellets in these shot-gun prescriptions might strike the desired spot, if the others failed to do so. But we now believe that the damage done by all the shot which miss far surpasses the good accomplished by the successful missile. Four hundred different remedies are included in one of these old formulæ, whereas now it is rare to find four in a prescription.

In relation to the third part of the prescription (the directions to the compounder), we find that a few regulation Latin phrases or words express these directions. If one is unfamiliar with Latin, one can easily memorize these words 
and phrases understandingly. The directions to the attendant are heralded by the Latin Signa, or Signetur, meaning label, or let it be labelled ; abbreviated, "Sig.;" or merely "S.," and being for the use of the attendant of the patient, are in English. The directions should be very precise. One should not write : "Use as directed," or "Give in water," but indicate exactly the quantity of medicine to be administered, the precise amount of water with which it is to be diluted, and the time at which it is to be given. For instance: "Give one tablespoonful in half a pint of water three times daily after feeding."

Poisons should be marked as such. It is well sometimes to indicate that the prescription is "for a horse," in order to avoid mistakes and to quell the qualms of the conscientious druggist.

Preparations which are not to be used internally should be labelled "external use." Under "Signature" the name of the writer and date is included. If desirable, one may inscribe "Do not repeat." Quantities used in prescription writing are indicated by the signs of the apothecaries or Troy system of weights for solids. For liquids, sigus representing units of the wine measure are employed. The Troy grain and ounce are used by apothecaries as units of weights in dispensing prescriptions. In ordering large quantities (as pounds) the avoirdupois pound of 16 ounces is employed, and in buying ounces of drugs withont a prescription the avoirdupois onnce is also utilizer. The avoirtupois omnce contains 437 grains; the Troy onnce contains 480 grains. The grain is of similar value in both systems.

TROY, OR APOTHECARIES' WEIGHT.

$\begin{array}{llcll}\text { Weight } & & \text { Sign } & & \text { Latin name } \\ \text { Pound } & \ldots & \text { tb } & \ldots & \text { Libra } \\ \text { Ounce } & \ldots & \xi & \ldots & \text { Uncia } \\ \text { Drachm } & \ldots & 3 & \ldots & \text { Drachma } \\ \text { Scruple } & \ldots & \text {. } & \ldots & \text { Scrupulum } \\ \text { Grain } & \ldots & \text { gr. } & \ldots . & \text { Granum. }\end{array}$


WINE MEASURE.

\section{Measure}

Gallon

Pint

Fluid Ounce $\ldots$...

Fluid Drachm ....

Minim

\section{Sign}

C

o

Fl.

Fl. 3

$\pi$

\section{Latin Name}

Congius

Octarius

Fluida Uncia

Fluida Drachma

Minimum

A drop is often used synonymously with minim, which is correct if the substance spoken of is water, or a liquid of nearly similar density. If the liquid is not of similar density, then a minim, or the sixtieth part of a drachm, is far from being a drop as measured by dropping a liquid from any ordinary utensil. Any amount from 45 drops to 276 drops, measured in this way, may be obtained from a drachm of fluid, according to its density, mode of clropping, and kind of vessel from which it is dropped.

A gutta (gtt.), then, is of no fixed value, but means a drop as dropped from a vessel; while a minim is always the sirtieth part of a drachm.

RELATIVE VALUE OF UNITS IN THE WINE MEASURE.

$$
\begin{aligned}
& \begin{array}{lllll}
\mathrm{C} & \mathrm{O} & \overline{3} & 3 & \mathrm{~m}
\end{array}
\end{aligned}
$$

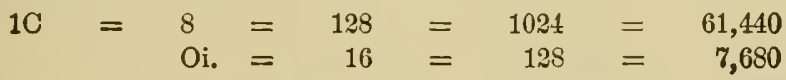

$$
\begin{aligned}
& \text { zi. }=8=480 \\
& \text { ji. }=60
\end{aligned}
$$

RELATIVE VALUE OF UNITS IN TROY SYSTEM.

$$
\begin{aligned}
& \text { to } 3 \text { j } 3 \text { Gr. } \\
& 1=12=96=288=5,760 \\
& 3 \text { i. }=8=24=480 \\
& \text { 3i. }=3=60 \\
& \text { Эi. } \doteq 20
\end{aligned}
$$

The abbreviation, Fl., is usually omitted in prescription writing, as referring to fluids, the character of the preparation being sufficiently apparent. The Roman numerals are used to express the quantities employed. The Roman numerals are written under a horizontal line, the i's or j's are dotted (they are identical in Latin) and the dot serves to enforce and check the numbers used. Fractions are 
usually expressed in ordinary Arabic characters, except $\frac{1}{2}$, which is often indicated by a double s (ss), standing for semis, the Latin for one-half.

APPROXIMATE EQUIVALENTS OF WINE UNITS IN DOMESTIC MEASURES.

\begin{tabular}{|c|c|c|c|c|}
\hline Teaspoon & $=$ & 3 i. -ii. & $=$ & 5 Cc. \\
\hline Desert spoon & $=$ & $3 \mathrm{ii.}$ & $=$ & $10 \mathrm{Cc}$. \\
\hline Table spoon & $=$ & इss. & $=$ & $15 \mathrm{Cc}$. \\
\hline Cup & $=$ & jiv. & $=$ & $120 \mathrm{Cc}$ \\
\hline Tumbler & $=$ & viii. & $=$ & $250 \mathrm{C}$ \\
\hline
\end{tabular}

There are usually about six teaspoonfuls to the fluid ounce. It is a good plan to have some regard for the size of vials generally kept by druggists, and to write for a quantity to fill the bottle. The bottles commonly in use in human and canine practice are the 2 and 4 drachm; the 1 , $2,3,4,5,6,8,12$, and 16 ounce.

The 2 drachm bottles are useful for measuring the dose of fluid extracts for horses; the 3 ounce bottle is convenient in writing prescriptions in the metric system for dogs, as it holds approximately $100 \mathrm{Cc}$. The 4 ounce bottle is the common size, employed in canine practice, containing 24 doses of one teaspoonful each. The $\frac{1}{2}$ pt. and pt. bottles are more appropriate for larger animals.

THE METRIC SYSTEM.

The metric system will be described, because it is the universal system employed in scientific writings, and is now official. It is based on the fact that a uniform, unchangeable standard is employed as the unit of all measures, whether of weight, capacity or area. This standard is the ten-millionth part of the distance from either pole to the equator, and is denominated a meter (39.371 inches), and is the standard of length. The cube of 0.1 of a meter is taken as the unit of capacity and called a litre (2.1135 pints). The weight of water at its greatest density, $4^{\circ}$ C. $\left(39.2^{\circ}\right.$ F.), which this cube will contain, is termed a kilogram (2.2046 lbs. Avoirdupois), and is the unit of the measure of. weight. 
METRIC DIAGRAM.

The weight of water that the small cube will contain is one gramme. This is the unit of weight of the metric system.

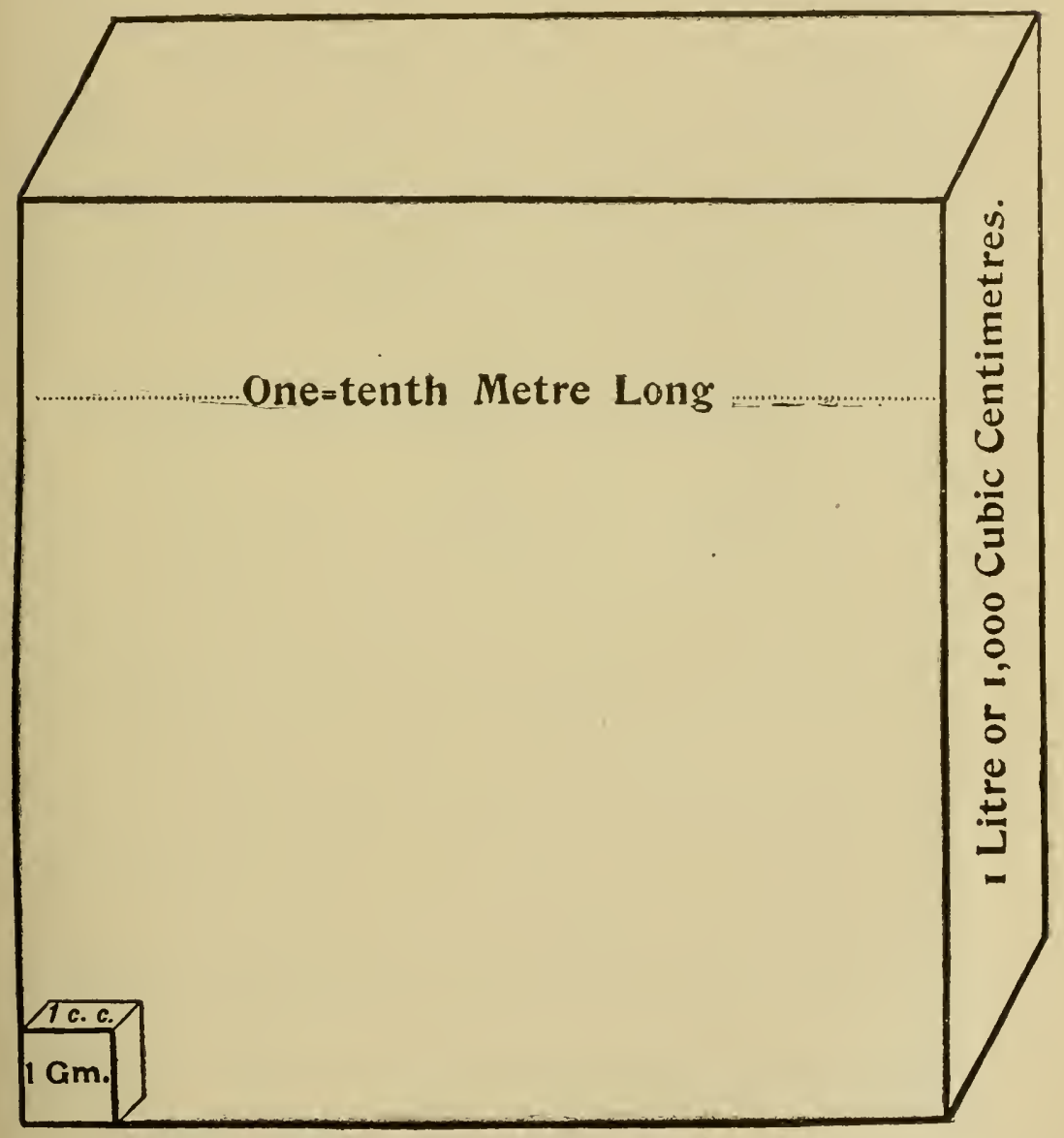


But for prescriptions and other small weighings lesser mits than the kilo and liter are required and therefore the cube of one-hundredth of a meter is taken and the weight of water which this eube holds is recognized as the unit of weiglit and called a gram $(15,432$ grains). The quantity of water contained in the cube of one-hundredth of a meter is used as a nuit in measuring capacity in chemical and pharmacentical practice and termed a cubic centimeter. The multiples of these measures, proceeding in decimal progression, are distinguished by Greek numerals as prefixes, i.e., Deca-10, Hecto-100, Kilo-1000. The subdivi-ions of the unit are representerl hy the Latin prefixes, as Deci-0.1, Centi-0.01, Milli-0.001. Hence, using the gram as the nuit, we can arrange a table as follows:

\begin{tabular}{|c|c|c|c|c|}
\hline Kilogram & $=$ & 1.000 grams & $=$ & 1.000 .0 \\
\hline Hectogram & $=$ & 100 grams & $=$ & 100.0 \\
\hline Decagram & $=$ & 10 grams & $=$ & 10.0 \\
\hline Gram & $=$ & 1 gram & $=$ & 1.0 \\
\hline Decigram & $=$ & iti gram & $=$ & 0.1 \\
\hline Centigram & $=$ & ton & $=$ & 0.01 \\
\hline Milligram & & 100 & & 0.001 \\
\hline
\end{tabular}

The metric system has the advantage of being arranged decimally, which makes the computation of percentages easy, and the transference of a quantity of one denomination to that of another, by merely shifting a decimal point. There are other advantages which make it of value to practitioners. Our present system is not uniform with that of any other country. The English, while nsing the sane nomenclature for weights and measures, put a different value upon them. The system has another value, at least theoretically, in having one unit for weights and measures. The unit of the fluid measure is 1 cubic centimeter of water, which at $4^{\circ} \mathrm{C}$. weighs one gram. As a matter of fact, fluids are dispensed in the metric system by measuring them in Cc., and if liquids were all of the same density as water, they would be equivalent to grams of water when measured in Cc. Unfortunately, this is not the case. Theoretically, medicine should be dispensed by 
weight in the metric system, but as medicines, when given to patients, are usually measured by bulk, they cannot be lispensed by weight without producing a complication. For example, suppose that we order chloroform in a prescription in the metric system,-

R.

Chloroformi 30 |

S. Two teaspoonfuls in water.

Јону SмIтн.

thinking we are dispensing 10 grams, $^{*}$ for a teaspoonful holds 5 grams of water. But as chloroform weighs $\frac{1}{2}$ more than water, we really have ordered $10+\frac{1}{2}=15 \mathrm{Gm}$. of chloroform. Therefore, in writing a prescription for chloroform with other ingredients, weighed in Gm., we would have to add $\frac{1}{2}$ of the number of $\mathrm{Gm}$. of chloroform in the prescription to the number previously estimated in order to make the chloroform of the same bulk as other liquids of the density of water.

In order to avoid reducing substances of density differing from that of water, to terms of equivalency with that of water, it is the custom, and now official, to weigh solids in Gm. and measure liquids in Cc. This is an exact method if the doses of drugs are learned in the same way: i.e., if the doses of solids are learned in $\mathrm{Gm}$. and prescribed in G.m., and the doses of liquids are learned in Cc. and prescribed in Cc.

In writing prescriptions in the metric system a line is drawn perpendicularly across the right-hand side of the blank to indicate the decimal point; multiples of the unit being placerl to the left of the line, while fractions are written to the right of the decimal line. In using this system we are spared the annoyance of special signs and different tables for weights and measures. As matters now stand we must be cognizant of both systems, and be able to convert the old into the new, or vice versa. One drachm is equivalent to four grams, $3 \mathrm{i} .=\mathrm{Gm} .4$. Therefore, Gm. $1=3 \frac{1}{4}$ or Gr. 15. Then, Gr. i. $=\frac{1}{15}$ of Gm. i.; or

15) $1.000(.066$

$\frac{90}{100}$

90

* For eaclı dose. 
The equivalent of $\mathrm{Gr} .1$ is $\mathrm{Gm}$. .06. In order to determine the equivalent of fractions of a grain in grams, we divide .06 by the denominator and multiply the result by the numerator of the fraction of a grain. For example:

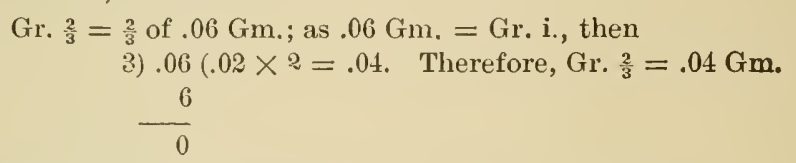

Again : to find the equivalent of $\mathrm{Gr} \cdot \frac{1}{8}$ in $\mathrm{Gm}$.

$$
\text { 8) } .066(.008 \times 1=.008
$$

Therefore, Gr. ${ }_{8}^{\frac{1}{8}}=.008 \mathrm{Gm}$.

We stated that $3 \mathrm{i} .=4 \mathrm{Gm}$. It follows that $\xi \mathrm{i}$. would equal $32 \mathrm{Gm}$. As a fact, 3 i. apothecaries' weight, is equivalent to a trifle less than Gm. 4.; and an ounce, apothecaries' weight, is usually considered equal to $30 \mathrm{Gm}$. (exactly 31.10 Gm.) for the sake of convenience. A fluid ounce in wine measure is precisely equivalent to $29.57 \mathrm{Cc}$. We have here another reason why both solid and fluid ounces should be valued at $30 \mathrm{Gm}$. or Cc. Although it is the custom to regard the minim of liquid as the equivalent of one grain, it is inexact. An apothecaries' ounce weighs $480 \mathrm{Gr}$; ; fluid ounce of wine measure weighs $457 \mathrm{Gr}$. Multiples of grams or cubic centimeters may be designated as such, instead of using the technical terms. It is perfectly proper to speak of $100 \mathrm{Gm}$. as one hundred grams, although technically equal to a hectogram. .1 Gm. may be called one-tenth gram, although technically a decigram. Similar remarks apply to Cc., yet 1,000 Cc. (approximately 1 quart) equal one liter, and this term is in common use. 
TABLE FOR CONVERTING APOTHECARIES' WEIGHTS AND MEASURES INTO GRAMS AND CUBIC CENTIMETERS.*

\begin{tabular}{|c|c|c|c|}
\hline \multicolumn{2}{|c|}{ SOLIDS. } & \multicolumn{2}{|c|}{ FLUIDS. } \\
\hline Troy Weight. & Metric. & $\begin{array}{c}\text { Apothecaries' } \\
\text { Measure. }\end{array}$ & Metric. \\
\hline Grains. & Grams. & Minims. & Cc. \\
\hline 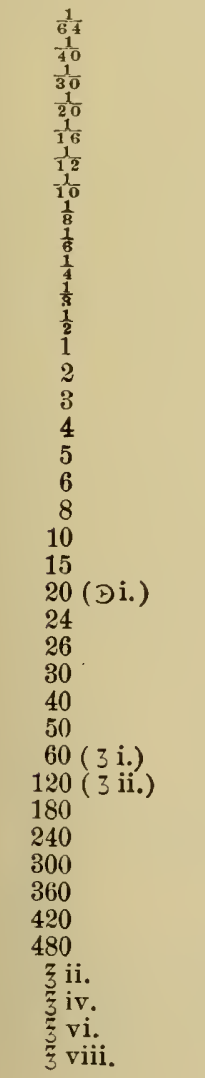 & $\begin{array}{r}.001 \\
.0015 \\
.002 \\
.003 \\
.004 \\
.005 \\
.006 \\
.008 \\
.010 \\
.016 \\
.02 \\
.03 \\
.065 \\
.13 \\
.20 \\
.26 \\
.32 \\
.39 \\
.52 \\
.65 \\
1.00 \\
1.30 \\
1.50 \\
1.62 \\
1.95 \\
2.60 \\
3.20 \\
3.90 \\
7.80 \\
1165 \\
15.50 \\
19.40 \\
23.30 \\
27.20 \\
31.10 \\
62.20 \\
124.40 \\
186.60 \\
248.80\end{array}$ & 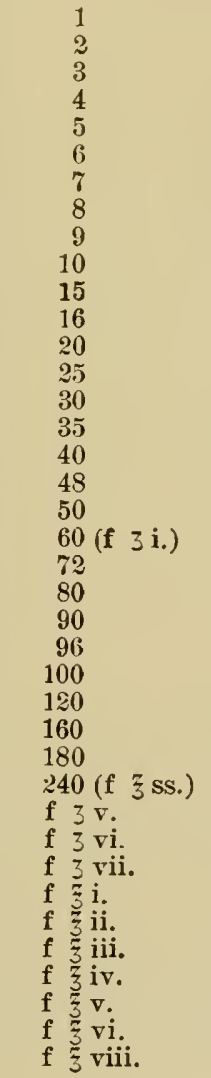 & $\begin{array}{r}.06 \\
.12 \\
.18 \\
.24 \\
.30 \\
.36 \\
.42 \\
.50 \\
.55 \\
.60 \\
.72 \\
1.00 \\
1.25 \\
1.55 \\
1.90 \\
2.20 \\
2.50 \\
3.00 \\
3.12 \\
375 \\
4.50 \\
5.00 \\
5.60 \\
6.00 \\
6.25 \\
7.55 \\
1000 \\
11.25 \\
15.00 \\
18.75 \\
2250 \\
26.25 \\
3000 \\
60.00 \\
90.00 \\
120.00 \\
15000 \\
180.00 \\
240.00\end{array}$ \\
\hline
\end{tabular}




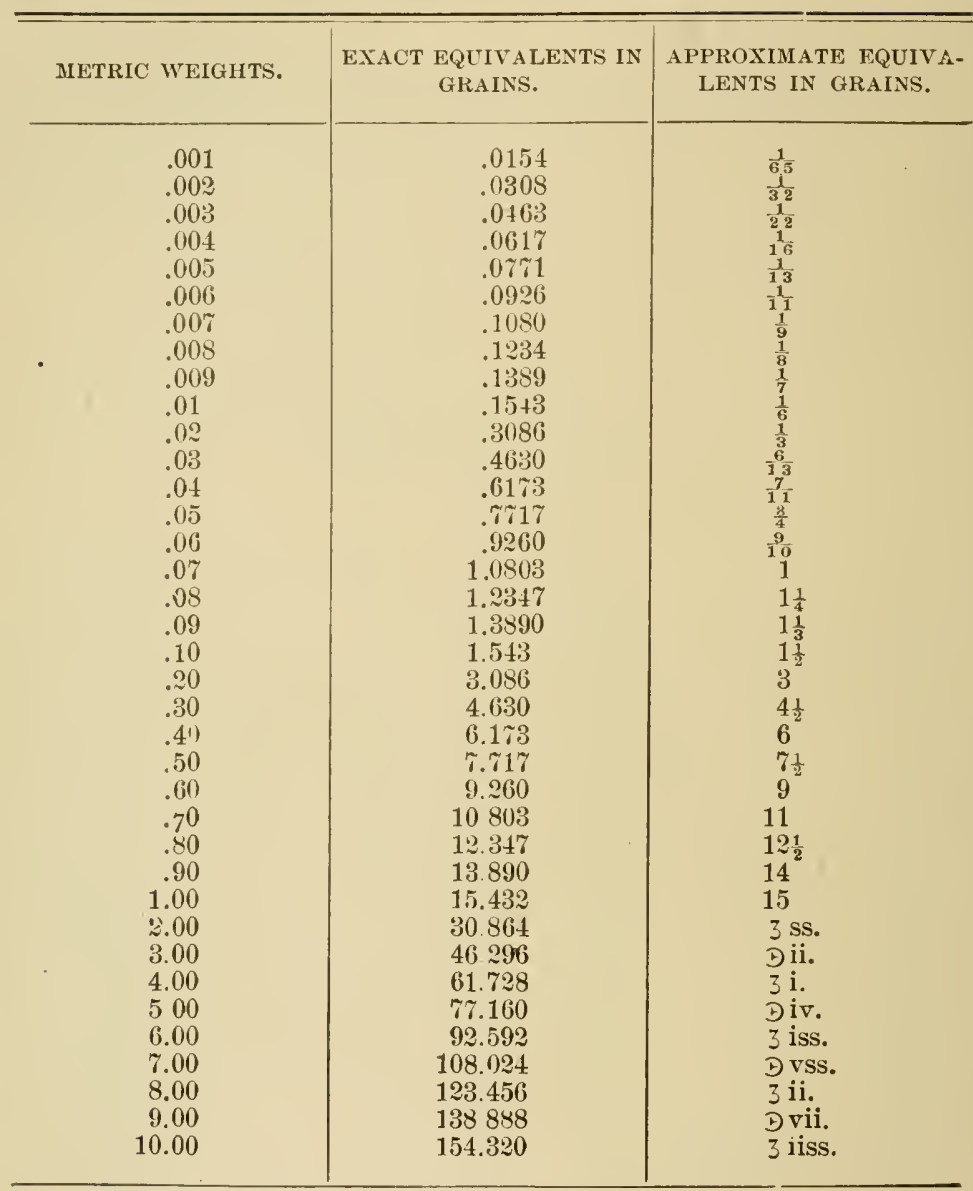

*Tables from Mann's Prescription Writing.

\section{Rules for Forming the Genitive Case in Prescription Writing.}

The Latin names of drugs, as we learn them, when consisting of a single word, are in the nominative case. For example: oleum, an oil. The genitive case of a Latin word means of (the word), and is equivalent to the English pos- 
sessive. Thus the Latin name Oleum Lini, consists of two words. The first, oleum, is the Latin nominative for oil, and the second word, lini, is the Latin genitive of the word meaning linseed. The name signifies, then, oleum (oil) and lini (of linseed). In writing prescriptions it is usually essential to put the Latin name of the drug in the genitive case, following the use of the heading $P_{x}$, standing for the Latin imperative Recipe, meaning in English, Take.

For example:

$\mathrm{I} x$ Potassii nitratis.................

Literally translated this means :

Take

Of potassium nitrate, ounce 1.

There is only one other case which is nsed in writing prescriptions (the accusative), but this can be avoided by using abbreviations in the few instances in which it shonld be employed. The following rules can be memorized (with their exceptions) in order to form the genitive case (singular):

(1) Latin names of drugs ending in " $a$ " form their genitive in ae. The only exceptions are three: Physostigma takes the genitive in tis, physostigmatis. Folic (leaves), pl. genitive, foliorum. Theobroma, genitive theobromatis. In most instances the genitive of Latin nouns ending in $a$, can be made in practice by dropping the nominative ending $(a)$ and adding $a e$ in its place, viz. : Tinctur- $a$ (a tincture), genitive tinctur-cle (of a tincture).

(2) All pharmacopoial nouns ending in us, um (os and on), form their genitive in $i$. This genitive case can be formed in practice by dropping the nominative ending ( $u s$ or $u m$ ) and adding $i$ in its place, viz.: bol-us, a ball; genitive bol-i, of 1 a ball. Extract-um, an extract; genitive extract- $i$, of an extract.

The nouns ending in os and on are very few. Exceptions : Rhus, genitive rhois. Flos, genitive floris. Fructus, quercus and spiritus do not change in the genitive, as it is the same as the nominative case.

L. OF C. 
(3) All other Latin names of drugs, of whatever termination (except those ending as described under rules 1 and 2) have their genitive in $s$ and $i s$.

The genitive case can be formed in practice, in some instances, by adding is to the nominative, as, for example, chloral, genitive chloral-is; xther, genitive ather-is. To many nouns ending in the nominative in as or is, we not only add $i s$ to the nominative, but also change the latter letter of the nominative case. For instance, to sulphas (sulphiite) not only do we add $i s$, sulphas-is, but we change the latter letter, $s$, of sulphas into a $t$, so that the proper genitive of sulphas is sulphat-is.

The same remark applies to all the other Latin names of salts, as sulphis, genitive sulphit-is ; nitras, genitive nitrat-is ; hydrochloras, genitive hydrochlorat-is; citras, genitive citrat-is; phosphas, genitive phosphat-is; acetas, genitive acetat-is. Cortex is not cortex-is in the genitive, but corticis. Mas, genitive not mas-is, but maris. Adeps, genitive not adeps-is, but adipis. Mucilago, pepo and pulvis lengthen and change in the genitive to mucilagin-is, pepon-is and pulver-is. Aloe, genitive not aloeis, but aloës, adding $s$ and not is.

(4) Some Latin names of drugs do not change their ending in the genitive because indeclinable and not latinized, or else they belong to the fourth declension, where the genitive case is the same as the nominative. Examples: Spiritus, quercus and fructus, already mentioned, as exceptions to rule 2. Cannabis, digitalis, sinapis and hydrastis. The genitive of these nouns is the same as the nominative.

The following are indeclinable: Amyl, buchn, catechu, coca, curare, jaborandi, kino, phenol, salol, naphtol, thymol, menthol, cusso, gummi, etc.

If the Latin names for quantities and amounts thereof are written out in full (instead of using signs for quantities, and numbers for the amounts), the quantities and amounts in Latin must be put into the accusative case, as they are. the objects of the verb, recipe. 
For example :

R

Sodii Sulphatis, uncias duas.

Translated :

Take

Of sodium sulphate, ounces two.

The Latin noun uncia (ounce) is in the accusative case, and the adjective durs is also in the accusative, agreeing with uncias. But to write ont prescriptions in full, as above, is not customary and would be considered pedantic.

Again: The Latin names of the ingredients should be written in the accusative case when no noun for weight or measure is employed. For example:

$\mathrm{R}$

Pilulas catharticas compositas duas.

Translated literally :

Take

Pills cathartic compound, two. Or, take two compound cathartic pills.

Pilulas (pills) is in the accusative, object of the verb recipe. Catharticas, compositas, and duas are adjectives, agreeing with pilulas. We can only write this prescription correctly, without using the accusative case, by abbreviating it as follows :

B

Pil. Cath. Co. ii.

\section{Examples of Prescriptions for Different Preparations.}

TO WRITE A PRESCRIPTION FOR A PILL.

1. We calculate the number of pills we wish to prescribe and then multiply the dose of each ingredient in the pill by that number. We will suppose that we desire to prescribe 30 pills to a dog, containing reduced iron, socotrine aloes and sulphate of strychnine. The dose of reduced 
iron is gr.ii.; of socotrine aloes gr.ss.; of strychnine sulphate gr. $\frac{1}{120}$. Mrultiply each dose by 30 :

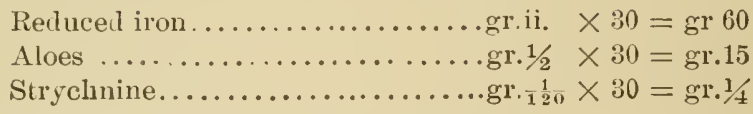

The Latin of aloes socotrine is aloe, genitive aloe-s, of aloes; socotrina, genitive socotrin-re, of socotrine.

The Latin of strychnine sulphate is strychnina, genitive strychnin-ce of strychnine; sulphas, genitive sulphat-is, of sulphate.

The Latin of reduced iron is ferrum, genitive ferr-i, of iron : reductum, genitive reduct- $i$, of reduced. Hence :

$\mathrm{B}$

Ferri reducti................ $3 \mathrm{i}$.

Aloës socotrinæ..................

Strychnina sulphatis ..........gr. $1 / 1$

Misce et divide in pilulas.........xxx.

(Abbreviated) M. et div. in pil. $\mathrm{xxx}$.

Signa. Give one pill three times daily.

JOHN SMITH.

The Latin names of the drugs being put in the genitive, and the signs and numbers for the proper quantities and amounts added, we come to the Latin directions to the pharmacist. (Misce) mix (et) and (divide) divide (in pilulas, accusative plural) in pills $x x x$. This is a regulation phrase and can be employed whenever we write a prescription for pills, so that it should be memorized. It can be abbreviated correctly as follows :

M. et div. in pil. $\mathrm{xxx}$.

Instead of writing the prescription as just described, we can calculate the dose needed of each ingredient in the pill, and then write a prescription for one pill and direct the pharmacist to make 30 pills like it.

P Ferri reducti......................ii.

Aloës socotrinæ................gr.ss.

Strychninæ sulphatis............gr. $\frac{1}{1 \frac{1}{20}}$

Misce et fiat pilula 1.; dispense pilulas tales numero $\mathrm{xxx}$. 
(Abbreviated) M. et f. pil. 1. Dispense pil. tales No. xxx.

Signa or S. (as before.)

Translated : (Misce) mix (et) and (fiat) let there be made (pilula) pill 1 ; (dispense) dispense (pilulas) pills, (tales) such, (numero) in number, xxx. Abbreviated as above, (Signa) S. = Label.

The same prescription may be written in the metric system: Gr.1 = .06 gm. Fractions of a grain are converted into grams, therefore, by dividing .06 by the denominator of the fraction and multiplying the result by the numerator. The dose of aloes (gr. $\frac{1}{2}$ ) is transformed into grams then, as follows :

$$
\begin{aligned}
& \text { 2) } .06(.03 \times 1=.03 \\
& -\frac{.0}{00}
\end{aligned}
$$

$\mathrm{Gr} \cdot \frac{1}{120}$ is converted into grams thus :

$$
\text { 120) } .0600(.0005 \times 1=.0005 \mathrm{gm} \text {. }
$$

$\mathrm{B}$

Solids in Gm. Liquids in Cc.

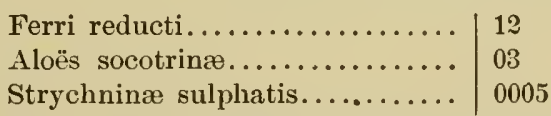

M. et f. pil. 1; dispense pil. tales No. xxx.

Sig. (as before).

This prescription may be abbreviated in this manner:

$\mathrm{P}$

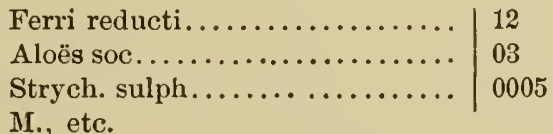

Prescriptions for balls are calculated and written in every respect like those for pills. We may write the above prescription in another form, in case we prescribe a pill or ball mass to be made, or an official mass to be divided into pills. Suppose we write a prescription for a physic mass, suitable for horses. We conclude to write for a quantity of the mass sufficient to make eight balls. Each ball con- 
tains a single dose of aloes and sufficient excipient to make the mass of the proper consistency. The dose of aloes is one ounce, and we know by experience that it will take an equal amount of molasses and one drachm of powdered ginger to make a proper ball mass. Multiplying each of the ingredients, then, by 8 , we find we need 8 onuces each of aloes and molasses, and 1 ounce of pulverized ginger, to make a mass which shall be divided into 8 balls.

The Latin names and genitives of socotrine aloes we have already described. Molasses is syrupus fuscus in Latin, or brown syrup. Syrupus, genitive syrup-i, of syrup. Fuscus, genitive fusc-i, of brown. The Latin for powdered ginger is pulvis, powder, genitive pulver-is, of powder. Zingiber, ginger, genitive zingiber-is, of ginger.

We will proceed to write the prescription thus:

$\mathrm{IR}$

Aloës socotrinæ

Syrupi fusci............ āā $\bar{z}$ viii.

Pulveris zingiberis......... $\bar{j} \mathrm{i}$.

Misce et fiat massa, in bolos viii., dividenda.

(Abbreviated) M. et f. mass., in bolos viii., dividend.

Sig. Give one ball at once.

JoHN SMITH.

The Latin directions to the pharmacist are translated: (Misce) mix (et) and (fiat) let there be made (massa) a mass (in bolos, accusative pl.), in balls viii. (dividenda) to be divided.

This is also a stock phrase and should be memorized as applying to pills or balls made from a mass.

The prescription is abbreviated :

R

Aloës soc.

Syr. fusci .āā ż viii.

Pulv. zingiber.............. $\overline{3}$ i.

M. et $\mathrm{f}$. mass., in bolos viii., dividend. (as above)

Or : Misce et divide in bolos viii.

(Abbrev.) M. et div. in bolos viii.

Translated: Mix and divide into balls 8 . 
Or : M. et fac bolos viii. (abbrev.) Mr. et f. bolos viii.

Translated: Mix and make balls S.

Mixtures are compounds in which fluids are mixed or solids dissolved or held in suspension by a suitable vehicle, We unst first decide upon the number of doses which we wish to prescribe, and then the quantity of the mixture to be given at each dose.

Suppose we wish to give sweet spirit of nitre and quinine to a horse. We conclude to give the mixture three times daily for several days. The dose of the nitrous ether will be an ounce; the quinine will be dissolved in it. Bottles are in use containing 12 to 16 ounces, or 1 pint. We will decide upon the pint bottle. This, then, will hold 16 ounces, or 16 doses of sweet spirit of nitre. In each dose of the nitre we want dissolved gr.20 of quinine sulphate. $16 \times$ gr. $20=$ gr.320 $=3$ v. . i. Now, 5 drachms of quinine sulphate will not dissolve in 16 ounces of sweet spirit of nitre, so that we will add enough diluted sulphuric acid to dissolve the quinine. We do not know how much sulphuric acid will be required, so we write after acid sulphuric, Q. S., for quantum sufficiat, i.e., as much as suffices (to dissolve, understood).

Again, we do not know exactly how much bulk the quinine will take up when dissolved in the nitre; nor what amount of acid will be required. Yet, on the other hand, we want to fill our bottle. To get over these difficulties we will write after sweet spirit of nitre ad, underlined (to); in nther words, we order the druggist to take of sweet spirit of nitre enough to (make, understood) a pint.

The Latin for quinine is quinina, genitive quinin-ce, of quinine. The Latin for sulphate is sulphas, genative sulphat-is, of sulphate. The Latin for spirit of nitrous ether is spiritus, genitive spiritus, of spirit; nitrosus, genitive nitros-i, of nitrous; ather, genitive ather-is, of ether. The Latin for sulphuric acid diluted is acidum, genitive acid-i, of acid; sulphuricus, genitive sulphuric-i, of sulphuric; dilutus, genitive dilut-i, of diluted. 
We may now write our prescription as follows:

$\mathrm{I}$ i

Quininæ sulphatis............ $\mathrm{j}$ v. Đi.

Acidi sulphurici diluti....... Q. S.

Misce.

Spiritus atheris nitrosi ad ..... Oi.

(Furnish $¥$ i. bottle for measure).

Signa. Small bottleful three times daily in half a pint of water. Abbreviated : JoHN SMITH.

$\mathrm{P}$

Quin. sulph............... jv. Эi.

Acid. sulphurici dil............. S.

Spts. æther. nitrosi ad .......... Oi.

II.

S. (as above).

We will write a prescription for a mixture containing 12 doses of chloral and potassium bromide for a dog. The quautity of the mixture given at each dose will be a teaspoonful. Now, there are six teaspoonfuls in one ounce. We will order a 2-ounce bottle, which will, therefore, hold 12 doses of a teaspoonful each. The dose of chloral is gr.v. $5 \times 12=$ gr. 60 , or $3 \mathrm{i}$. The dose of potassium bromide is gr.x. $10 \times 12=$ gr.120, or 3 ii. Then we will order enough water to fill the bottle. The Latin for chloral is chloral, genitive chloral-is, of chloral ; Latin for potassium bromide is potassium, genitive potassi-i, of potassium; bromidum, genitive bromicl- $i$, of bromide; Latin for water is aqua, genitive aqu-ce, of water.

B

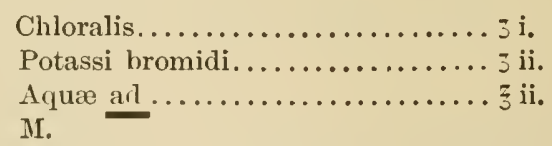

Signa. Teas, in 1 tablespoonful of water every 3 hours.

JoHN SMith.

A drench is a mixture which is given the horse in one. dose. We will write a prescription for a horse, containing ether, chloroform and laudanum, to be administered as a drench. The Latin for ether is cether, genitive cether-is, of ether; 
dose, $\underset{j}{ } \mathbf{i}$. The Latin for chloroform is chloroformum, genitive chloroform- $i$, of chloroform; dose, $j$ ii. The Latin for laudanum is tincturce opii; tinclura, genitive linctur-ce, of tincture; opium, genitive opi-i, of opium; dose, $\overline{3}$ ii. The prescription reads:

P

Etheris.....................

Chloroformi.................. $\lesssim$ ii.

Tincturæ opii................ $\overline{3} \mathrm{ii}$.

Nisce et fiat haustus.

Translated: (Misce) mix, (et) and (fiat) let there be made (haustus) a clrench.

(Abbreviated) M. et f. haust.

Sig. Give at once in one dose in pint of water.

JoHN SMITH.

In writing a prescription for powders, we may either write for one powder and direct the druggist to dispense several more like it, or write for the whole amount of the ingredients and order them divided into the required number of doses or papers. In the first case we will write for a powder containing one dose of each of the drugs.

For example, we may write a prescription for calomel and santonin, with sugar of milk as an excipient, since the dose of the drug is inconveniently small. This powder is suitable for a medium-sized dog.

The Latin for calomel, or the lower chloride of mercury, is hydrargyrum, genitive hydrargyr-i, of mercury; chloridum, genitive chlorid-i, of chloride; mite, genitive mit-is, of lower; dose, gr.ii. The Latin for santonin is santoninum, genitive santonin- $i$, of santonin; dose, gr.i. The Latin for sugar of milk is saccharum, genitive sacchar-i, of sugar; lac, genitive lac-tis, of milk; amount, gr.s. The prescription will read :

$\mathrm{B}$

Hydrargyri chloridi mitis.........gr.ii.

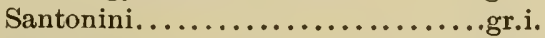

Sacchari lactis...................

Misce et fiat pulvis 1 ; dispense pulveres tales vi. 
Translated: Mix, and let there be made powder 1 ; dispense powders such vi.

(Abbreviated) M. et f. pulv. 1; dispense pulv. tales vi.

Sig. Give one powder every two hours.

JoHN SMITH.

In the second case, if we write a prescription for six powders, we multiply the dose of the ingredients in each powder by 6 , and then order the prescription to be dispensed in six papers.

$\mathrm{P}$

Hydrarg. chlorid, mitis............gr.xii.

Santonin. ......................

Sacch. lactis................. $\mathrm{i}$.

Misce et divide in chartulas numero..vi.

Translated: Mix and divide into papers in number vi. (Abbreviated) M. et div. in ch't. No. vi.

Sig. (as before).

To write the above in the metric system. The dose of calomel is gr.ii. $=.12 \mathrm{gm} . .12 \times 6=.72$, or gr.xii. The dose of santonin is gr.i. $=.06 \mathrm{gm} . .06 \times 6=.36 \mathrm{gm}$., or gr.vi. The amount of sugar of milk used as an excipient in each powder is gr.x. $.06 \times 10=.6$ gm., the amount prescribed in each powder. The amount necessary for six powders is $.6 \times 6=3.6 \mathrm{gm}$., approximately $4 \mathrm{gm}$.

$\mathrm{P}$

Solids in Gm. Liquids in Cc.

Hydrarg. chlorid. mitis.... $\mid 72$

Santonin.............. 36

Sach. lactis............ 4

M. et div. ch't. in No.......vi.

Sig. (as before).

We will write a prescription for a horse, in the form of a powder, containing dried iron sulphate, nux vomica and sodium bicarbonate. The Latin for iron sulphate (dried) is ferrum, genitive ferr-i, of iron ; sulphas, genitive sulphat-is, of sulphate; exsiccatus, genitive exsiccat-i, of dried; dose, $3 i$. The Latin for nux vomica is nux, genitive nuc-is, of nut; vomica, genitive vomic-a, of vomica; dose, $3 \mathrm{i}$. The 
Latin of sodium bicarbonate is soctium, genitive sodi-i, of sodium; bicarbonas, genitive bicarbonat-is, of bicarbonate. We will order a sufficient quantity of the ingredients to make thirty powclers. The close of iron and nux vomica is 3 i. $\times 30=5$ iii., 3 vi. The dose of sodium bicarbonate is 3 ii. $\times 30=3$ vii.ss.

$\mathrm{P}_{\mathrm{x}}$

Ferri sulphatis exsiccati

Pulveris nucis vomicæ........āa $\bar{\zeta}$ iii., 3 vi.

Sodii bicarbonatis........... $\overline{3}$ vii.ss.

Misce et diviale in chartulas $\mathbf{x x x}$.

Translated: Mix and divicle into papers $\mathrm{xxx}$.

(Abbreviated) M. et div. in ch't. xxx.

Sig. Give one powder three times daily on the food.

ЈонN SмIтH.

In order to avoid the expense of having powders divided into papers, we may frequently direct one dose to be weighed by the druggist, and a measure to be furnished holding the quantity.

B

Ferri sulph. exsicc.

Pulv, nucis vom........... āā ž iii., 3 vi.

Sod. bicarb............... 3 vii.ss.

Misce et fiat pulvis.

Translated : Mix and let there be made a powder.

(Abbreviated) M. et f. pulv. (Furnish measure holding $\xi \mathrm{ss}$. )

Sig. Give measureful on food three times daily.

JоHN SMITH.

To transform this prescription into terms of the metric system: $\overline{3}$ i. $=$ Gm.30.; 3 i. $=$ Gm.4. Hence 3 iii., 3 vi. = Gm.114; $\overline{3}$ vii.ss. $=\mathrm{Gm} .225$.

$\mathrm{B}$

Solids in Gm. Liquids in Cc.

Ferri sulph. exsicc.

Pulv. nucis vom....... āā 114

Sodii bicarb............225

M. et f. pulv., etc.

S. (as before). 
Electuaries are not suitable preparations in which to prescribe powerful drugs, as we cannot secure any degree of accuracy in the dosage. This happens because we do not usually know the exact amount of excipient which will be required to make the paste of the proper consistency. We will write for an electuary containing potassium chlorate, licorice and molasses. The Latin for potassium chlorate is polassium, genitive potassi- $i$, of potassium; chloras, genitive chlorat-is, of chlorate; dose, 3 ii. The Latin for powder of licorice root is (powder lras been given before) glycyrrliza, genitive glycyrrtiz-ce, of licorice; radix, genitive radic-is, of root. The Latin for molasses is syrupus, genitive syrup-i, of syrup; fuscus, genitive fusc-i, of brown; dose of licorice root and molasses immaterial. They are used as excipients.

B

Potassi chloratis.

Pulveris glycyrrhizæ radicis ..āā.. ž iv.

Syrupi fusci....................S.

Misce et fiat electuarium.

Translated: Mix and let there be made an electuary.

(Abbreviated) M. et $\mathrm{f}$. electuarium.

(Weigh 3 vi. as sample).

S. Give amount equal to sample every 2 hours smeared on teeth. JoHN SMITH.

We cannot tell precisely what quantity of potassium chlorate will be administered in the 3 vi. ordered in this prescription, but we can be assured that it will not be larger than 3 drachms, which is a small dose for the horse.

Suppositories are occasionally prescribed to dogs. The excipient is cacao butter, of which about 15 grains is required. We will write a prescription containing iodoform and extract of Belladonna root, to be dispensed in suppositories for a medium-sized dog. The Latin for iodoform is iodoformum, genitive iodoform-i, of iodoform; dose, gr. $\frac{1}{2}$. The Latin for extract of belladonna root is belladonna, genitive belladonn-ce, of belladonna; extractum, genitive extract- $i$, of extract; radix, genitive radic-is, of root; dose, gr.1. The Latin for cacao butter is oleum theobroma; 
olenm, annitive ole-i, of oil ; thentroma, genitive theobromalix, of theobroma. The quantity of cacan lutter may be safely left to the discretion of the pharmacist. We will multiply the dose by ten, to make ten suppositories.

$\mathrm{P}$

Iodoformi....................

Extracti bellactonne radicis .....gr ii.ss.

Olei theobromatis. .............S.

Misce et fiant suppositoria $\mathbf{x}$.

Translated: Mix and let there be made suppositories $\mathrm{x}$. (Abbreviated) M. et f. suppos. $x$.

Sig. Introduce one into the bowel every 4 hours.

\section{JoHN SMITR.}

In writing prescriptions for ointments the degree of dilution of the medicinal substance, or substances, must be determined. In case the dilution is done in percentage, the metric system is particularly useful. A five-per-cent. ointment of the yellow oxide of mercury is of value in some cases of conjunctivitis. We will write for $5 \mathrm{Gm}$.

The Latin for yellow oxide of mercury is hydrargyrum oxidum flavum; hydrargyrum, genitive hydrargyr-i, of mercury; oxidum, genitive oxid- $i$, of oxide; flavum, genitive flavi, of yellow.

The excipient will be simple ointment. Latin for simple ointment is unguentum, genitive unguent- $i$, of ointment. If we order $5 \mathrm{gm}$. of simple ointment we can determine the amount of mercury necessary to form a 5-per-cent. preparation with it by simply moving the decimal line forward two places, .05 , which will give a 1 -per-cent. ointment of mercury; and then, by multiplying by $5, .05 \times 5=.25$, we secure a 5 -per-cent. ointment.

$\mathrm{B}$

Solids in Gm. Liquids in Cc.

Hydrargyri oxidi flavi..... $\mid \mathbf{2 5}$

Unguenti............. 5

Misce et fiat unguentum.

Translated: Mix and let there be made an ointment.

(Abbreviated) M. et f. ung.

Sig. Use externally.

JоHN Sмтн. 
There is nothing of special note to consider in regard to writing prescriptions for liniments. We will write a prescription for Carron oil as an example.

Carron oil is composed of equal volumes of solution of lime and cottonseed oil. The Latin for oil of cottonseed is oleum gossipii seminis; oleum, genitive ole-i, of oil ; gossypium, genitive gossypi-i, of cotton; semen, genitive semin-is, of seed. The Latin for solution of lime is liquor calcis; liquor, genitive liquor-is, of liquor ; calx, genitive calc-is, of lime.

$\mathrm{B}$

Liquoris calcis.

Olei gossypii seminis......... āā $\tilde{z}_{3}$ iii.

Misce et fiat lınimentum.

Translated: Mix and let there be made a liniment.

(Abbreviated) M. et $\mathrm{f}$. liniment.

Sig. Apply externally.

Jонм Sмттн. 


\section{CLASSIFICATION.}

PART I.

Inorganic Agents.

Section I.-Water, and Solution of Hydrogen Dioxide.

Section II.-Alkaline Metals; Potassium, Sodium, Anmonium and Lithium.

Section III.-Alkaline Earth Metals; Calcium, Barium, Magnesium, Aluminum and Cerium.

Section IV.-Plumbum, Argentum, Zincum, Cuprum, and Bismuthum.

Section V.-Ferrum, Manganum.

Section VI.-Hydrargyrum.

Section VII.-Arsenic, Antimony.

Section VIII.-Phosphorus.

Section IX.-Chlorine, Indine, Bromine, Orthoform.

Section X.-Sulphur.

Section XI.-Acids.

Section XII.-Carbon Compounds.

Class 1. Carbon.

Class 2. Alcohol, Ether, Chloroform.

Class 3. Nitrites.

Class 4. Chloral.

Class 5. Antipyretics, Analgesics.

Class 6. Antiseptics.

Class 7. Miscellaneous Carbon Compounds.

\section{PART II.}

\section{Vegetable Drugs.}

Section I.-Drugs acting on the brain.

Class 1. Depressing the brain; Opium.

Class 2. Stimulating the brain; Belladonna.

Section II.-Drugs acting on the spinal cord.

Class 1. Stimulating the inferior cornua; Nux Vomica, Strychnine.

Class 2. Depressing the inferior cornua ; Physostigma, Gelsemium. 
Section III.-Drugs acting on the spinal cord and motor nerves.

Class 1. Depressing the inferior cornua and motor nerves; Tobacco and Conium.

Section IV. -D Drugs acting on the sensory nerves.

Class 1. Depressing the sensory nerves ; Cocaine, Eucain, Holocain.

Section V.-Drugs acting on the secretory nerves.

Class 1. Stimulating the secretory nerves; Pilocarpus.

Section VI.-Drugs acting on the heart.

Class 1. Increasing the force and decreasing the frequency of the heart ; Digitalis, Strophanthus, Convallaria and Squill.

Class 2. Decreasing the force and frequency of the heart; Aconite, Veratrum Viride and Album, Veratrine.

Section VIJ.-Drugs acting on the respiration.

Ipecac.

Section VIII.-Vegetable Antipyretics and Antiseptics.

Class 1. Cinchona and its Alkaloids.

Class 2. Salicylic: Acid, Salicin, Salol, Oil of Gaultheria and Methyl Salicylate.

Section IX. - Volatile Oils or 1)rugs containing them.

Class 1. Used mainly for their action on the skin : Turpentine, Oil of Turpentine, Terebene, Terpiu Hydrate, Burgundy Pitch, Canada Turpentine, Resin, Tar, Pitch, Oil of Cade, Balsam of Peru, Balsam of Tolu, Benzoin, Benzoic Acid, Black and White Mustarl, Eucalyptus, Arnica, Myrrh.

Class 2. Used mainly for their stomachic and carminative action upon the digestive tract: Capsicum, Ginger, Peppermint, Menthol, Spearmint, Anise, Cardamon, Coriander, Fennel, Fenugreek.

Class 3. Used mainly for their antispasmodic action in stimulating the nervous system: Valerian, Ammonium, Ferric and Zinc Valerianates, Asafoetida and Ammoniacum.

Class 4. Used mainly for their stimulant and diuretic action on the genito-urinary tract: Buchu and Oil of Juniper.

Class 5. Used mainly for its emmenagogue action on the female generative organs; Savin. Tallianine.

Section X.-Vegetable Bitters.-Gentian, Quassia, Cascarilla, Calumba,

Taraxacum, Hydrastis, Calamus.

Section XI. - Vegetable Cathartics.

Class 1. Simple Purgatives.-Aloes, Linseed Oil, Castor Oil, Rhamnus Purshiana, Frangula, Rhamnus Catharticus, Rhubarb (Chrysarobin), Senna. 
Class 2. Drastic Purgatives.-Croton Oil, Scammony, Jalap, Gamboge, Elaterin, Colocynth.

Class 3. Cholagogue Purgatives.-Podophyllum, Podophyllin.

Section XII.-Tannic Acid and Drugs containing it.

Nutgall, Tannic Acid, Gallic Acid, Pyrogallol, White Oak-

Catechu, Kino, Krameria, Hæmatoxylon, Hamamelis.

Section XIII.-Vegetable Demulcents.

Olive Oil, Cottonseed Oil, Soap, Soft Soap, Glycerin, Linseed, Acacia, Tragacanth, Althæa, Sugar.

Section XIV.-Vegetable Drugs killing Parasites.

Class 1. Used to destroy tape worms: Aspidium, Areca Nut, Kamala, Kouso, Granatum.

Class 2. Used to destroy round worms : Santonica.

Class 3. Used to destroy oxyurides: Quassia.

Class 4. Used to destroy lice: Stavesacre.

Class 5. Used to destroy fleas: Pyrethrum.

Section $\mathrm{XV}^{\top}$.-Vegetable Drugs stimulating unstriated muscle, pa ticularly of the uterus.

Ergot, Cotton Root Bark.

Section IVI.-Colchicum.

Section XVII.-Vegetable Drugs acting mechanically.

Starch, Oil of Theobroma, Purified Cotton, Pyroxylin, Collodion, Euphorbium, Adrenalin.

Section XVIII.-Medicinal Agents of Animal Origin.

Cantharides, Lard, Suet, Hydrous Wool Fat, Yellow and White

Wax, Spermaceti, Honey, Milk, Sugar, Pepsin, Pancreatin,

Ox Gall, Papain, Cod Liver Oil, Ichthyol, Thiol.

NOTE.

ABBREVIATIONS USED IN REFERENCE TO THE SYNONYMS IN THE DESCRIPTIONS OF DRUGS.

B. P...British Pharmacopœia.

E .....English.

P. G...German Pharmacopœia.

Fr.... French.

G.....German.

Three doses of each medicine are usually given; one for horses and cattle; one for sheep and swine, and one for cats and dogs, unless otherwise specified. The quantities are expressed in units of the Apothecaries' Weight or Wine Measure and also in the metric system. The 
solids in the latter to be dispensed in grams, the liquids in cubic centimeters. Only those official drugs and preparations of the Uniter States. and British Pharmacopcia's will be mentioned, which are considered to be of value to practitioners of Veterinary Medicine. In connection with doses the following abbreviations are used :

$$
\begin{aligned}
& \text { H......... Horse. } \\
& \text { C......... Cattle. } \\
& \text { Sh. \& Sw. Sheep and Swine. } \\
& \text { D......... Dogs. }
\end{aligned}
$$

The same dose may be given to either dogs or cats of equal weight. 
PART I.

INORGANIC AGENTS.

\section{SECTION I.}

Water.

Aqua, Water. Aqua Destrliata, Distilled Water. $\mathrm{H}_{2} \mathrm{O}$.

(The latter used in filling many prescriptions.)

Action external.-The reader is referred to special articles on "Cold and Heat" (p. 704), "Food and Feeding" (p. 679), and "Counter Irritants" (p. 696), for details concerning the action and uses of water, respectively, as a medium of heat and cold, as an article of diet in health and disease, and as a connter irritant. Cold water, externally, at first stimulates reflexly beat production, with slight rise of temperature, increased carbonic acid elimination and contraction of the vessels and muscles of the skin. If the cold water application is continued, the bodily heat falls, owing to physical abstraction of heat. "Reaction" follows the removal of cold, if properly applied, with dilatation of the superficial vessels (and sensation of warmth and exhilaration in man). Moderate warm water $\left(105^{\circ} \mathrm{F}\right.$.) applications stimulate cutaneous vascularity, favor diarphoresis, and diminish urinary secretion. Hot water $\left(110^{\circ}-120^{\circ}\right.$ F.) applications act as counter irritants in dilating the peripheral vessels, contracting those in more remote parts, and relieving pain, spasm, congestion and inflammation.

Action internal.-Water is quickly absorbed and thus swells the secretion of urine, and, to a less extent, that of bile, saliva and pancreatic juice. Intestinal peristalsis is facilitated by a considerable amount of water. Water also increases tissue change, and elimination of carbonic dioxide and urea; promotes the appetite and washes out the tissues and urinary tract, thus removing waste matters from the body. 
The elimination of uric acid is lessened by water. Large quantities of water, if not taken at meal time-when they dilute the digestive juices and disorder digestion--favor the formation of fat.

Uses external.-See "Heat and Cold," p. 704.

Uses internal.-Healthy animals may be given as much water as they ciesire, with certain restrictions in relation to work and feeding. It is unwise to allow horses much water, either immediately before or after severe work, or after feeding. If water is given before severe work it increases the bulk of intestinal contents, is apt to canse digestive disturbance, and interferes with the movements of the diapliragm. For these same reasons water should only be permitted in small amount (at a time) in "heaves" of horses.

If a quantity of water is allowed horses after hard work, colic is very likely to occur. Working horses should, therefore, be watered, in reasonable amount, while at work; and, if this is impracticable, may be allowed but a few mouthfuls of water, or a gallon of oatmeal gruel after severe work, with whole hay but no grain until after an hour's rest. When horses at rest drink much water after eating, the contents of the stomach (which is unusually small in this animal) are washed into the intestines and are not so thoroughly digested. This accomplishes two bad results: it deprives the animal of some nourishment and engenders digestive trouble and diarrhœa.

The best plan is to give resting horses water before eating, or to keep it at their command at all times. Cold water is desirable, frequently and in limited quantities, in fever, although there is a popular fear of it. Hot water assists the action of diaphoretics; cold water that of dinreties. Lukewarm water is an emetic, but hot water, in small and repeated doses, allays nansea and vomiting. Water is restricted in ordinary diarrhoeas, obesity, and to assist the absorption of exudations. The drinking of water should be encouraged by a liberal allowance of salt (which in itself aids digestion), in animals in a poor condition, to increase their appetite and flesh. Water is valuable in diluting a con- 
centrated urine from which calculi are liable to be deposited. High rectal injections of water are absorbed, and consequently flush out the kidneys.

\section{Solution of Hydrogen Dioxide.}

Aqua Hydrogenr Droxmi. Solution of Hydrogen Dioxide.

Synonym.-Hydrogen Peroxide. A slightly acid aqneous solution of hydrogen dioxide $\left(\mathrm{H}_{2} \mathrm{O}_{2}\right)$ containing, when freshly prepared, about 3 jer cent., by weight, of the pure dioxide, colresponding to abont 10 volumes of available oxygen. U.S.P.

Derivation.-Add barium dioxide, 300 , to cold, distilled water, 500 ; agritate and keep at a temperature of $100^{\circ} \mathrm{C}$. $\left(50^{\circ} \mathrm{F}.\right)$. To this mixture (of barium hydrate) add a solntion of phosphoric acid, 96, in cold distilled water, 320 , anci shake them together thoroughly. Filter, and wash the precipitate (barinm phosphate) with distilled water until the filtrate measures 1000. Add dilnted sulphuric acid to the filtrate (until cloudiness disappears in a small filtered portion of it; absence of barium), and starch 10. Agitate frequently. Filter and refilter till the solution becomes clear.

Properties. - A colorless liquid, without odor, acidulous to the taste and producing a peculiar sensation and soapy froth in the mouth; liable to deteriorate by age, exposure to heat, or protracted agitation. Spec. gr.1,006 to 1,012.

Reaction slightly acid. When exposed to the air at the ordinary temperature, or when heated in a water bath at a temperature not exceeding $60^{\circ}$ C. $\left(140^{\circ} \mathrm{F}\right.$.) the solution loses chiefly water. When rapidly heated it is liable to decompose suddenly. (The value of a given sample of hydrogen dioxide may be roughly ascertained by adding a few drachms to a few crystals of potassinm permanganate in a test tube. The greater the amount of effervescence the better the hydrogen dioxide.-Wallian.)

Dose.-H. \& C., 亏̈i.-ii. (30.-60.); D., з i.-ii. (4.-8.)

Action and Uses.-Hydrogen dioxide is probably the most powerful surgical antiseptic and disinfectant in use. 
It is not poisonous to higher animals, and liberates oxygen immediately in the presence of oxidizable matter, thus destroying all bacteria and organized ferments.

The microbicidal action is transient and not persistent; only water remains. Therefore hydrogen dioxide is useless for the production of continnons antiseptic action. The drug is an antiseptic in the digestive tract, and some oxygen may be absorbed by the blood, but this is extremely doubtful. The official solution contains 10 volumes of oxygen; that is, it yields up 10 times its bulk of oxygen gas. Most proprietary preparations are stronger, and conitain 12 volumes of oxygen, and are more powerfully disinfectant.

Hydrogen peroxide is particularly valuable as an antiseptic on suppurating and septic wounds, uecrosed tissue, abscess cavities, sinus', nlcers, moribid growths and suppurating mucous membranes. In fistulæ of the withers and poll, hyclrogen dioxide acts as an efficient cleansing and decomposing agent (through its oxidizing powers), and it should be injected prior to the use of other antisepties, stimulants and canstics, as curbolic acid in glycerin (see p. 329). Hydrogen dioxide is probably the best remedly we possess in the treatment of acute caturrh of the pharynx and tonsils in dogs and cats when applied directly to the throat, dilnted with two parts of lime witer, with an atomizer or brush, or on absorbent cotton on in applicator. It is commonly employed in full strength and only in glass, porcelain, or hard rubber vessels or instruments. The drug shonld not be nsed in cavities where an ontlet for the free escape of gas is wanting. Peroxile of hydrogen decomposes pus with effervescence, and thus is a guicle to its presence or absence; it also destroys the pus cocci.

Hydrogen dioxide is a safe and efficient agent in disinfecting drinking water, and is of some value in gastric fermentative indigestion of dogs where the vomitus and frees show evidence of gas formation or frothiness, and is absolutely safe. Recently good results have been reported from the use of hydrogen dioxide, diluted with three parts 
of lime water, in dysentery when given as high enemata twice daily.

\section{SECTION II.}

Alkaline Metals-Potassium, Sodium, Ammoniom, Lithium.

\section{Potassium.}

Potassinm is not used in medicine in the metallic state. Its compounds may be considered in three groups: 1 , Potassa; 2, the Carbonates (acetate and citrate); 3 , the Mineral Salts.

Potassium compounds were formerly obtained from wood ashes by lixiviation; from sea water by evaporation, and from argol, a substance deposited in wine casks. Now they are obtained from potassium muriate, mined in Stassfurt, Saxony, which is thought to result from the boiling away of sea water in past ages.

GENERAL ACTION OF POTASSIUM SALTS.

In lethal doses the action of all the potassinm compousds is very similar.

Stomach and Intestines.-The potassium salts, with the exception of the vegetable compounds, are irritants to the gastro-intestinal tract, if ingested in concentrated form.

Heart.-Marked depression of the heart is one of the most characteristic symptoms of poisoning by the potassium salts. Potassium has a direct, paralyzing action on the heart muscle, and in lethal doses there is cardiac arrest in diastole. Much the same action is, moreover, observed on all the higher forms of tissue. The functional activity of the nerves and muscles is depressed and abolished, more especially that of the brain and cord, so that paralysis of central origin occurs. 
Blood.-It has been taught that the potassium salts give up their oxygen to the blood. In the case of the nitrate and chlorate we know that this is untrue, since they are eliminated unchanged. When given for any considerable period, the potassium, like the sodinm salts, impoverish the system and produce a more fluid state of the blood. Large doses of the potassium salts are likely to occasion purging, , while small closes are apt to cause diuresis. The carbonates and regetable salts resemble each other in action, but that of the mineral salts is peculiar to the individual compound.

\section{Potassa. Potassa. KOH. (U. S. \& B. P.)}

Synonyms. - Potash, potassium hydrate, potassium hydroxide, canstic potish, lappis cinsticus chirurgorum, E.; potasse caustique, Fr:; aetz killi, G.; kali causticum fusum, P. G.

Derivation. - A solution of potassinum hydrate is evaporated; the residue is fused and rum into moulds.

Properties.-Dry, white, translucent pencils or fused masses, hard and brittle, showing a crystalline fracture; odorless, or having a faint odor of lye, and of a very acid and caustic taste. Exposed to the air, it very rapidly absorbs carbon dioxide* and moisture, and deliquesces. Soluble at $15^{\circ}$ C. ( $59^{\circ}$ F.) in abont 0.5 part of water, and in 2 parts of alcolıol; very soluble in boiling water and in boiling alcohol; slightly soluble in ether.

\section{Liquor Potass.:. Solution of Potassa. (U. S. \& B. P.)}

An iqueous solution of potassinm hydrate $(\mathrm{KOH})$, containing abont 5 per cent. of the hydrate.

Synomym.-Solution of potassium hydrate, kali hydricum solutum, lixivium causticum, solution of potash, E.; potasse caustiqne liquide, lessive caustique, Fr.; aetzkalilange, G.; liqnor kali caustici, P. G.

Derivution.-Boiling a solution of potassium carbonate

* When solubility is mentioned hereafter, reference will be had to solubility at the abore temperature. 
with calcium hydrate leaves potassium hydrate in solution, while calcium carbonate is precipitated.

$$
\mathrm{K}_{2} \mathrm{CO}_{3}+\mathrm{Ca}(\mathrm{OH})_{2}=2 \mathrm{KOH}+\mathrm{Ca} \mathrm{CO}_{3} \text {. }
$$

Properties.-A clear, colorless liquid, odorless, having a very acrid and caustic taste and a strongly alkaline reaction. It has a soapy feel and taste.

Dose.-H. \& C., ₹ss.-i. (15.-30.); Sh. \& Sw., 3 ss.-i. (2.-4.); D., १v.-xx. (.3-1.3).

\section{Potassa Cum Calce. Potassa with Lime. (U. S. P.) (Equal parts of potassa and lime.)}

Synonym.-Vienna paste.

Properties.-A grayish white powder, deliquescent, having a strongly alkaline reaction; should be soluble in diluted hydrochloric acid without leaving more than a small residue.

Actions and Uses.-Potassa, liquor potassæ and potassa cum calce are mainly of value as escharotics. Liquor potassæ is unfit for internal use unless greatly diluted with water. It resembles potassium carbonate in its effects. Caustic potash is very destructive of tissue by combining with water and fatty matters. It is most diffusible and, therefore, difficult to limit its action. This we may do, however, by applying a plaster to a part, with a hole in it, through which the caustic stick is applied. Before using the caustic, the outside of the plaster should be covered with oil or grease, but not the part under the aperture in it. After removing the plaster the operation of the caustic may be arrested by vinegar. Cauterization by this means is very painful ander ordinary circumstances, but may be made comparatively painless by incorporating one part of morphine muriate with three parts of potassa cum calce, and adding enough chloroform to make a paste.

Caustic potash is indicated where extensive destruction of tissue is desirable, as in the treatment of the bites of rabid dogs and of snakes. It is less commonly used for the 
removal of warts and small growths, and as a caustic on indolent or exuberant granulations.

Potash has been employed to form an issue, or artificial nlcer for the production of connter irritation. Potash may be prescribed in bronchitis, for its action, common to the alkalies, in thinning and increasing the bronchial secretions. An excellent mild stimulating liniment consists of :

$\mathrm{R}$

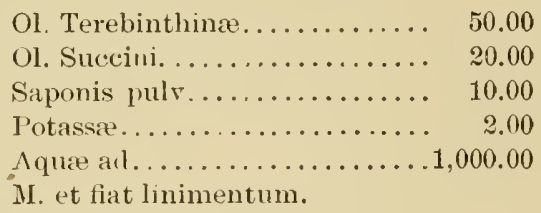

The potash siponifies the oils and exerts a stimulating action on the skin. Potassinm bicarbonate is less irritating and mor suitable for the latter indication. Potash is sometimes recommendied as an antacir and sedative in gastric disnrders, but is inferior to sodium bicarbonate for this purpose.

Potassir Carbonas. Potassium Carbonate. $\mathrm{K}_{2} \mathrm{Co}_{3}$ (U. S. \& B. P.)

Synonym.-Salts of tartar.

Derivution.-The solution resulting from the lixiviation of wood ashes is boiled to dryness, and the resultant mass is the "potash" of commerce. This is purified to some extent by burning in ovens, forming "pearlash," a mixture of the hydrate and carbonate. Water dissolves mainly the carbonate which is obtained by evaporation of the aqneous solution.

Properties.-A white, grannIar powder, odorless, and having a strongly alkaline taste; very deliqnescent. Soluble in 1.1 parts of water ; insoluble in alcohol.

Dose.-H. \& C., 3 ss.-i. (15.-30.); Sh. \& Sw., 3 ss.-i. (2.-4.); D., gr.v.-xx. (.3-1.3). 
Potassin Bicarbonas. Potassium Bicarbonate. KHCo

$$
\text { (U. S. \& B. P.) }
$$

Synonym.-Doppolkohleusaures kali, G.

Derivation. - Obtained by passing a stream of $\mathrm{Co}_{2}$ through a solution. of the carbonate.

$$
\mathrm{K}_{2} \mathrm{CO}_{3} \cdot+\mathrm{CO}_{2}+\mathrm{H}_{2} \mathrm{O}=2 \mathrm{KHCO}_{3} \text {. }
$$

Properties.-Colorless, transparent, monoclinic prisms, odorless and having a saline and slightly alkaline taste. Permanent in the air. Soluble in 3.2 parts of water. Almost insoluble in alcohol. Becomes converted into the carbonate by boiling.

Dose.-H. \& C., 亏 ss.-i. (15.-30.); Sh. \& Sw., 3 ss.-i. (2.-4.); D., gr.v.-xx. (.3-1.3).

Preferable to carbonate for internal use, as it is less irritating.

Potassit Acetas. Potassium Acetate. $\mathrm{KC}_{2} \mathrm{H}_{3} \mathrm{O}_{2}$ (U. S. \& B. P.)

Synonym.-Terra foliata tartari, E.; Essigsaures kali, G.

Derivation.-Add acetic acid in excess to potassium carbonate. Evaporate to dryness and fuse residue.

$\mathrm{K}_{2} \mathrm{CO}_{3}+2 \mathrm{HC}_{2} \mathrm{H}_{3} \mathrm{O}_{2}=2 \mathrm{KC}_{2} \mathrm{H}_{3} \mathrm{O}_{2}+\mathrm{H}_{2} \mathrm{O}+\mathrm{CO}_{2}$.

Properties. - A white powder, or crystalline masses of a satiny lustre; odorless and having a warming, saline taste. Very deliquescent on exposure to the air. Soluble in 0.36 part of water and in 1.9 parts of alcohol.

Dose.-Same as bicarbonate.

Potassi Citras. Potassium Citrate. $\mathrm{K}_{3} \mathrm{C}_{6} \mathrm{H}_{5} \mathrm{O}_{7}$ (U. S. \& B. P.)

Synonym.-Citrate de potasse, Fr.; Citronsaures kali, G.

Derivation.-Neutralize potassinm carbonate with a solution of citric acid, and evaporate to dryness.

$3 \mathrm{~K}_{2} \mathrm{CO}_{3}+2 \mathrm{H}_{3} \mathrm{C}_{6} \mathrm{H}_{5} \mathrm{O}_{7}=2 \mathrm{~K}_{3} \mathrm{C}_{6} \mathrm{H}_{5} \mathrm{O}_{7}+3 \mathrm{H}_{2} \mathrm{O}+3 \mathrm{CO}_{2}$.

Properties.-Transparent, prismatic crystals, or a white, granular powder; odorless and having a cooling, saline 
taste. Deliquescent on exposure to air. Soluble in 0.6 part of water; sparingly soluble in alcohol.

Dose.-Same as bicarbonate.

ACTION OF THE CARBONATES AND VEGETABLE SALTS.

The carbonate is too irritating for internal use, while the bicarbonate is more so than the similar sodium salt. Therefore the latter is in more common use as an antacid. Antacids nentralize abnormal acidity, due to fermentation, if given some time after eating in large doses.

The vegetable salts (the acetate and citrate) are converted into the carbonate in the blood and are non-irritating. The bicarbonate is also absorbed into the blood as the carhonate. These salts, together with the hydrate, alkalize the blood and urine. It is thought by many authorities that they increase oxidation within the body, as it is known that they do so ontside the body in contact with organic matter. Not only water, but also the solids (urea and uric acid) in the mine are increased by their administration. The chlorate and nitrate, not undergoing decomposition in the luxly, do not give up their oxygen or alkalize the blood or nrine. The carbonates are mainly useful as antacids; the citrate and acetate as diuretics.

Uses. - Potassium carbonate forms a useful addition to mixtures for application to the skin, as, through its strong alkalinity, it removes grease and sebaceous matter, permitting medicinal agents to penetrate the skin. A mixture of potassium carbonate, 15.0 ( $\overline{3} \mathrm{ss}$.) ; sublimed sulphur, 50.0 ( 3 i. 3 v.); and water, 12 liters ( 3 gallons), makes a serviceable application for the milder forms of mange and eczema. The animal should be well washed with the mixture in a tub every second day, and peruvian balsam, oil of cade or tar should be applied daily. Potassium bicarbonate in aqueous solution is serviceable in relieving itching (gr.vo- $\overline{3}$ i.) While probably not so generally efficient as the corresponding sodium salt, it is of benefit when given an hour after feeding to horses subject to colic at that time. It is more 
useful t'ıan sodium bicarbonate in alkalizing an over-acic urine ( $\log \mathrm{s})$, and in dissolving and eliminating uric acid from the blool. Potassium citrate and acetate are indicated in irritation or inflammation of the kidneys and bladder and cause :bsorption of exudations (pleuril effusions, for example), throngh their diuretic power. They are also good uric acid solvents. They are sometimes prescribed in fever on account if slight diarphoretic and powerful dinretic properties, but :re rarely used in veterinary medicine, in comparison with potassinm nitrate, in this condition.

The salts under consideration stimulate bronchial secretion and make it thinner, and are recommended accordingly in bronchitis. They are less efficient than potassinm iodide for these purposes in this affection.

Potassit Nitras. Potassinm Nitrate. $\mathrm{KNO}_{3}$ (U.S. \& B.P.)

Synonym.-Nitre, saltpetre, E.; nitre prismatique, azotate (uitrate) de potasse, Fr.; saltpetersaures kali, kalisalpeter, G.

Derivation.-Saltpetre is formed in the soil in certain regions and climates aul is made artificially, by the putrefaction of animal or vegetable material, in the presence of heat, moisture, oxygen, and alkaline or earthy bases capable of fixing the nitric acid set free in this process, linown as nitrification. The natural conditions for nitrification alle present in some parts of India, and saltpetre is laropuly imported from Calcutta. Artificially, nitre beds are minle of animal and vegetable matter, wood ashes, ant calcareous earth or old plaster from houses. Sodium nitrate is imported extensively from Chili, where it occurs as a mineral product, and is used widely in this country in artificial fertilizers. Chili saltpetre may be converted into nitre by treatment with potash.

Properties.-Colorless, transparent, six-sided, rhombic prisms, or a crystalline powder, odorless and having a cooling, saline and pungent taste. Permanent in the air. Soluble in 3.8 parts of water; very sparingly soluble in alcohol. 
Dose.-H. \& C., Zss.-i. (15.-30.); Sh. \& Sw., 3 ss.-i. (2.-4.); D., gr.v.-xx. (.3-1.3).

Action External.-Refrigerant.

Action Internal.-Stomach and Intestines.-Nitre canses, in lethal doses, violent gastro-enteritis, collapse and depression of the circulation. The pulse becomes both slow and weak. There is muscular weakness and paralysis. Potassium nitrate is one of the most irritating salts of this group, but its toxic effect depends upon its state of concentration, and horses are not so susceptible to the local irritating action as man.

Blood.-Nitre passes into and ont of the blood unchanged. It has been said that the salt diminishes fibrin in the blood because it prevents clotting ontsirle the body. This action probably does not obtain within the body.

Heart.-Nitre is said to be more depressing to the heart. than the other potassium compounds. There is no basis for this belief. Like the potassium salts generally, it exerts a powerful, depressing action upon the heart in large doses.

Lungs. - The respiration is slowed by considerable doses of potassium nitrate, and it exerts an antispasmodic action by depression of the unstriped fibres of the bronchi.

Kidneys.-Potassium nitrate is eliminated unchanged by the kidneys, and in its elimination stimulates the epithelium of the renal tubules, increasing their secretion. The solids are, therefore, augmented in the urine. The local blood pressure is exalted in the Malpighian bodies and the quantity of urine is thus increased.

Potassium nitrate is more frequently prescribed than any other potassium salt in veterinary practice, and is commonly considered one of the best febrifuges. In human medicine it is rarely employed for internal use, and is held to be decidedly inferior to other diuretic remedies.

Slin.-Nitre is a mild diarphoretic.

Summary.-Potassium nitrate is a diuretic, cardiac sedative, and slight diarphoretic.

Alministration.-Nitre is dissolved in a pail of drinking water aud kept constantly at the larger animal's disposal. 
The salt is rendered harmless by dilution; vascular tension and diuresis are increased by the water, and the solution is cooling and grateful to the taste in ferer. Smaller doses ( $\tilde{z}$ ss.) may be given on the food to horses.

Uses.-Nitre, ammoninm chloride, and common salt, each one part, are dissolved in three parts water, ancl sometimes usecl for their refrigerant offect on local inflammatory conditions. Ice poultices are more efficient. The value of nitre is over-estimuted in reterinary practice. There are better heart sedatives (aconite), diuretics and diaphoretics (sweet spirit of nitre). It is recommended in purpura and rhenmatism as alterative. Here again it is less serviceable than ergot in the former, or salicylic acid and alkalies in the latter clisease. Nitre is, however, in common use in such febrile affections as pueumonia and influenza in horses.

In laminitis, the combination of potassinm nitrate (two parts) with potassium chlorate (one part) is of value when given to horses in tablespoonful doses four to fire times daily on the food and in the drinking water.

\section{Potassir Chloras. Potassium Chlorate. $\mathrm{KClO}_{3}$.} (U. S. \& B. P.)

Synonym.-Kali oxymuriaticum, E.; chlorate de potasse, Fr.; chlorsaures kali, G.

Derivation.-Pass chlorine into a mixture of potassium carbonate and calcium hydrate; dissolve the result in boiling water and recover the chlorate by crystallization.

$\mathrm{K}_{2} \mathrm{CO}_{3}+6 \mathrm{Ca}(\mathrm{OH})_{2}+12 \mathrm{Cl}=2 \mathrm{KCl} \mathrm{O}_{3}+\mathrm{Ca} \mathrm{CO}_{3}+$ $5 \mathrm{Ca} \mathrm{Cl}_{2}+6 \mathrm{H}_{2} \mathrm{O}$.

Properties.-Colorless, lustrons, monoclinic prisms or plates, or a white powder, orlorless, and having a cooling, saline taste. Permanent in the air. Soluble in 16.7 parts of water. Insoluble in absolute alcohol, but slightly soluble in mixtures of alcohol and witer. Explodes readily when rubbed with sugar, sulphur, charcoal, glycerin and many other substances.

Dose.-H. \& C., 3 ss.-i. (15.-30.); Slı. \& Sw., 3 ss.-i. (2.-4.); D., gr.v.-xx. (.3-1.3). 
Action Internal.-Potassium chlorate closely resembles the nitrate in its action. Locally, it is an irritant.

Stomach and Bowels.-Medicinal doses have no apparent effect. Lethal doses occasion gastro-enteritis, diarrhœa, cyanosis and depression of the heart.

The Blood.--The blood is unaffected by medicinal doses, but in poisoning the red corpuscles are broken down and erenated. The hæmoglobin is converted into methæmoglobin, which is probibly a mixture of himatin and soluble albumin. Hrmoglobin and disintegratel corpuscles appear in the mine as hrmoglobinuria. The blood is chocolatecolored after death. The liver, spleen, kidneys aud intestines are softened and filled with disorganized blood. It was formerly thonght that potassium chlorate parted with its oxygen in the blood, and it was (and is) prescribed in many disorders as an oxidizing agent. It has been stated that venous blood acquires an arterial hue under the influence of the salt. But potassium chlorate does not part with its oxygen; neither does it oxidize the blood, nor impart to the venous blood an arterial color.

Hecort.-The action is mucl the same as nitre.

Kidneys.-Action similar to nitre in therapentic doses.

Elimination.-Potassium clrlorate is eliminated by all channels; mainly by the urine, but also by the sweat, saliva, etc. Acting locally as a stimulant in the mouth, and then affecting the throat a second time by its elimination in the saliva, potassium clilorate is frequently prescribed in diseases of the mouth and pharynx as a topical stimulant. It is given in electuary, solution, or ball internally.

Summary.-Sialogogue, dinretic and heart depressant.

Uses.-Stomatitis is treated by chlorate of potassium in saturated solution applied on a swab. The salt is valuable in the treatment of pharyngitis in electuary. A favorite combination consists of fluid extract of belladonna, $\bar{z} \mathrm{i}$; potassium chlorate, $₹$ ii.; powdered licorice root, $\overline{3} \mathrm{v}$., with sufficient molasses to make an electuary. One ounce is to be smeared on the teeth of a horse thrice daily. An half ounce 
of a saturated solution of potassium chlorate, with a few drops of laudanum, forms a useful injection for hæmorrhoids in dogs. Otherwise the salt may be-prescribed for the same purposes as nitre.

\section{Potassir Bitartras. Potassium Bitartrate. $\mathrm{KH}_{4} \mathrm{C}_{\mathbf{t}} \mathrm{HO}_{6}$}

(U. S. P.)

Synonym.-Potassii tartras acida, B.P.; cream of tartar, cremor tartari, acid tartrate of potash, E.; tartarus deparatus, P. G.; pierre de vin, Fr.; weinstein, G.

Derivation.-Obtained from crude tartar (argol) deposited on the sides of wine casks during fermentation of grape juice, by purification.

Properties.-Colorless or slightly opaque, rhombic crystals, or a white, son̉ewhat gritty powder; odorless and having a pleasant, acidulous taste. Permanent in the air. Soluble in about 201 parts of water; very sparingly soluble in alcohol. Reaction acid.

Dose.-H. \& C., 3 ss.-i. (15.-30.); Sh. \& Sw., zss. (15.); D., 3 ss.-i. (2.-4.).

Action internal.-Intestines.-Potassium bitartrate is a non-irritating purgative in large doses. It is a hydragogue cathartic and has a strong affinity for water; abstracting it from the blood vessels in the bowels, holding the same in solution, and thus flushing out the intestines.

Blood.-Potassium bitartrate is in part decomposed, converted into the carbonate, and absorbed as such into the blood. The greater part is excreted by the bowels unchanged. A portion of the latter may have been absorbed and eliminated by the intestines.

Kidneys. - Potassium bitartrate is an active diuretic and renders the urine more alkaline, but for some reason it is not ordinarily employed in veterinary practice. Nevertheless, it is the best and safest diuretic which can be used by the veterinarian in the treatment of the horse and smaller animals.

Summary.-Diuretic in small doses. Hydragogue 
cathartic in large doses. It should be given in solution and is useful in dropsies, more particularly of renal origin; also in catarrhal jaundice, and as a lixative for foals and calves. In cases where the mine of the lrorse is thick, stringy and high-colored, potassium bitartrate will canse it to regain its normal state. It may be easily administored in either food or drinking water, and its diuretic effect is enlanced when the salt is given with a large amonnt of water.

\section{Sodium.}

(The metal is not employed in medicine.)

Soda. Sodid. NaOH. (U. S. P.)

Synonym.-Soda caustica, B. P. ; sodiun hydrate, caustic soda, sodium hydroxide, watrum causticum, S. hydricum, E. ; soude cuustique, Fr. ; natron ætznatron, G.

Derivation.-It is made from liquor sodæ by evaporation, and rum in moulds.

Properties.-Dry, white, translucent pencils, or fused masses, showing a crystalline fracture; odorless, and having an acrid and caustic taste. Exposed to the air it rapidly deliquesces, absorbs carbon dioxide, and becomes covered with a dry coating of carbonate. Soluble in 1.7 parts of water; very soluble in alcohol.

Liquor Sode. Solution of Soda. (U. S. \& B. P.)

An aqueous solution of sodium hydrate, containing about 5 per cent. of lhydrate.

Synonym.-Solution of sodium hydrate, E.; natrum hydricum solutum, soude caustique liquide, Fr.; liquor natri caustici, P. G.; ætznatron lange, G.

Derivation.-An aqueous solution of sodium carbonate is boiled with calcium hydrate, and the supernatant liquid is siphoned off.

$\mathrm{Na}_{2} \mathrm{CO}_{3}+\mathrm{Ca}(\mathrm{OH})_{2}=2 \mathrm{NaOH}+\mathrm{CaCO}_{3}$.

Properties.-A clear, colorless liquid, odorless, having a very acrid and canstic taste, and a strongly alkaline reaction. 
Dose.-H. \& C., 亏ss.-i. (15.-30.); Sh. \& Sw., 3 ss.-i. (2.-4.); D., १ $\mathrm{v}_{\text {.-xx. }}(.3-1.3)$.

Action and Uses._Soda and liquor sodæ resemble similar compounds of potassium, but are used chiefly for chemical and pharmacentical purposes.

Liquor sodie should be given largely diluted with water. In poisoning by the caustic alkalies or. soap lye, use emetics or stomach pump; and give vinegar, diluted acetic acid, lemon juice and demulcents.

\section{Sodir Carbonas. Sodium Carbonate. $\mathrm{Na}_{2} \mathrm{CO}_{3} 10 \mathrm{H}_{2} \mathrm{O}$.}

$$
\text { (U. S. \& B. P.) }
$$

Synonym.-Washing soda, sal soda, carbonas sodicus, E.; natrum carbonicum crudum, P. G.; carbonate de soude, Fr:; kohlensaures natron, soda, $G$.

Derivation.-Made by Leblanc's process. Three steps:

1st. Salt and sulphuric acid heated together. $2 \mathrm{Na} \mathrm{Cl}+$ $\mathrm{H}_{2} \mathrm{SO}_{4}=\mathrm{Na}_{2} \mathrm{SO}_{4}+2 \mathrm{HCl}$.

2nd. Sodium sulphate is heated with carbon. $\mathrm{Na}_{2} \mathrm{SO}_{4}+$ $4 \mathrm{C}=\mathrm{Na}_{2} \mathrm{~S}+4 \mathrm{CO}$.

3rd. Sodium sulphide heated with chalk. $\mathrm{Na}_{2} \mathrm{~S}+\mathrm{Ca}$ $\mathrm{CO}_{3}=\mathrm{Na}_{2} \mathrm{CO}_{3}+\mathrm{Ca} \mathrm{S}$.

Properties. - Colorless, monoclinic crystals, odorless and having a strongly alkaline taste. In dry air the salt effloresces, -loses about half its water of crystallization and becomes a white powder. Soluble in 1.6 parts of water and in 1.02 parts of glycerin. Reaction alkaline.

Sodil Carbonas Exsiccatus. Dried Sodium Carbonate.

$$
\mathrm{Na}_{2} \mathrm{CO}_{3} \text {. (U. S. \& B. P.) }
$$

Synonym.-Natrum carbonicum siccum, P. G.; carbonate de soude, Fr.; getrocknete soda, G.

Derivation.-Sodium carbonate (200 $\mathrm{gm}$.) is broken into small fragments and allowed to effloresce in dry air. It is then heated to drive off water of crystallization, till it weighs $100 \mathrm{Gm}$. 
Properties.-A loose, white powder, corresponding in solubility to sodium carbonate. Reaction alkaline. Action of sodium carbonate similar to the hydrate, but infrequently employed in medicine.

Dose.-H. \& C., 3 ii.-vi. (8.-24.); Sh. \& Sw., gr.xx.-xl. (1.3-2.6); D., gr.v.-xx. (.3-1.3). Given in large amount of water.

Sodil Bicarbonas. Sodium Bicarbonate. $\mathrm{Na} \mathrm{HCO}_{3}$. (U. S. \& B. P.)

Synonym.-Soda, baking soda, sodium sesquicarbonate, natrum carbonicum acidulum, bicarbonas sodicus, sodium hydrocarbonate, bicarbonate of soda, E.; natrum bicarbonicum, P. G.; bicarbonate de soude, sel digestive de vichy, Fr.; doppelkohlensaures natron, $\mathrm{G}$.

Derivation.-Pass $\mathrm{CO}_{2}$ through a solution of sodium carbonate. $\mathrm{Na}_{2} \mathrm{CO}_{3}+\mathrm{CO}_{2}+\mathrm{H}_{2} \mathrm{O}=2 \mathrm{Na} \mathrm{HCO}_{3}$.

Properties.-A white, opaque powder, odorless, and having a cooling, mildly alkaline taste. Permanent in dry, but slowly decomposed in moist air. Soluble in 11.3 parts of water, insoluble in alcohol and ether. Slight alkaline reaction.

Incompatibles.-Decomposed by acid and acid salts, as bismuth subuitrate.

Dose.-H., żss.-ii. (15.-60.); Sh. \& Sw., 3 ss.-i. (2.-4.); D., gr.v.-xxx. (.3-2.).

GENERAL ACTION OF SODIUM SALTS.

Sodium and its salts are not nearly so poisonous as similar potassium compounds, because they are less depressing to the circnlatory, muscular and nervous systems, but death has occurred after enormous doses. The salts possess a local paralyzing action on nerve and muscular tissue. They are absorbed and eliminated more slowly than the corresponding potassium compounds. Sodium salts alkalize the blood and urine, but are only slightly diuretic. Sodium 
carbonate, phosphate, and sulphate diminish the solids in the bile and, therefore, increase its fluidity.

\section{Sodium Bicarbonate.}

Action External.-Sodium bicarbonate lessens irritability of the skin in itching and burns.

Action Internal.- When sodium bicarbonate is given in generous doses after eating it neutralizes the acid products of indigestion and fermentation. If ingested in small doses before eating, sodium bicarbonate increases the secretion of gastric juice and hydrochloric acid. The constant administration of sodinm bicarbonate weakens the digestive powers and creates anæmia, general cachexia, and scorbutic symptoms. Sodium bicarbonate liberates carbonic dioxide in the stomach, and thus exerts a sedative effect. It dissolves mucus and thins the biliary secretion. It is, therefore, useful in catarrh of the gastro-intestinal tract.

Blood.-.The blood is made more alkaline.

Kidneys.-The urine is alkalized, but the salt is only feebly ciiuretic.

Uses External.-In aqueous solution (1-50) sodium bicarbouate relieves itching in urticaria, prurigo and chronic eczema. It also allays the pain of slight burns and of acute rhenmatism. For this purpose cloths should be soaked in saturated solutions and placed upon the affected parts. Added to water ( 3 i.-Oi.) in which instruments are to be boiled, it prevents rusting.

Uses Internal.-Sodium is one of the most useful remedies in gastric or intestinal indigestion associated with acidity, flatulence, and distress. It does not always remove the canse of indigestion, however, and, therefore, shonld be combined with agents which do: e.g., cathartics, antiseptics, carminatives and stomachics. For this reason sodinm bicarbonate is often prescribed to dogs witlı bismuth subcarbonate, salol or beta naphtol; to horses, with gentian or un romica and ginger.

Sodium bicarbonate is of value in alkalizing the blood 
in acute rheumatism, when it is thought to increase oxidation and tissue waste. The salt assists the action of calomel, with which it should be generally conjoined, since it stimulites the flow of bile and aids the alkaline intestinal juices in transforming the inactive chloricle into the active oxide. Sodium bicarbonate is occasionally given in acute bronchitis, but it is distinctly inferior to the corresponding potassinm salt in thimning and increasing bronchial secretions.

This salt is highly recommended in the treatment of hæmoglobinæmia (azoturia) in horses, when given in quantities of 10-30 ounces daily. Theoretically, sodium bicarbonate is of benefit in this disease, by nentralizing acid products of metabolism which lead to solution of the hæmoglobin.

Administration.-Sodium bicarbonate may be given in solution or on the tongue or food in the pure state.

Sodir Sulphas. Sodium Sulphate. $\mathrm{Na}_{2} \mathrm{SO}_{4} 10 \mathrm{H}_{2} \mathrm{O}$. (U. S. \& B. P.)

Synonym.-Glauber's salts, sulfas sodicus (natricus), sal mirabile Glauberi, sulphate of soda, E.; sulfat de sonde, sel de Glauber, Fr.; Glaubersalz, G.

Derivation.-Nentralize the residue left in the manufacture of $\mathrm{HCl}$ from salt, with sodium carbonate. $2 \mathrm{Na} \mathrm{HSO}_{4}+$ $\mathrm{Na}_{2} \mathrm{CO}_{3}=2 \mathrm{Na}_{2} \mathrm{SO}_{4}+\mathrm{CO}_{2}+\mathrm{H}_{2} \mathrm{O}$.

Properties.-Large, colorless, transparent, monoclinic prisms, or granular crystals, odorless, and having a bitter, saline taste. The salt effloresces rapidly in the air, and finally loses all its water of crystallization. Soluble in 2.8 parts of water; insoluble in alcohol; soluble in glycerin.

Dose.CC., Hbi.-iss. (500.-750.) ; H. (laxative), $\overline{3}$ ii.-iv. (60.-120.); Sl.., 3 ii.-iv. (60.-120.).

Action Internal.-Digestive Tract.-Sodium and magnesium sulphate rapidly abstract water from the blood vessels of the alimentary canal through the intestinal glands (succus entericus) until a 5-per-cent. solution is reached. This solution is but slowly reabsorbed so that it sweeps out the 
contents of the bowels. They are hydragogue cathartics. These salts also stimulate peristaltic action to some extent by their physical properties, or throngh their action in angmenting the mass of intestinal contents. Glauber's salt is said to be slower in action than Epsom salts.

Liver:-Glauber's salt increases the secretion of bile and prevents its absorption in the bowels by expelling it from the tract.

Kidneys.-C Small doses of sodium sulphate are absorbed and alkalize the blood and urine. It is a slight diuretic.

Administration.-Glanber's salt is given to cattle in solution with ginger and molasses.

Uses.-Sodium sulphate is a decided cholagogue and more active in this respect than magnesium sulphate. In human medicine, Glauber's salts prove irritating and cause griping, so that Epsom salts are preferred. In veterinary practice, on the other hand, sodium sulphate acts more effectively, and, in fact, is the best saline cathartic we possess. Sodium sulphate is the principal ingredient of Carlsbad salts, which have recently come into vogue with veterinarians, although long valued in human medicine. The formula for the artificial Carlsbad salts (Sal carolinum factitium), which is the preparation commonly used, is as follows:

$\mathrm{P}$

Sodii sulphatis exsiccati.......4 40.0

Sodii bicarbonatis............ 35.0

Sodii chloridi............. 15.0

Potassii sulphatis ........... 2.0

M. et fiat pulvis.

Sig. One to two heaping tablespoonfuls on the food two or three times daily for horses.

Sodium sulphate is thus given as Carlsbad salts or alone in small doses as a laxative, but in constipation associated with indigestion and malnutrition, gentian, powdered rhubarb, iron and other stomachics and tonics are often combined; while, in fever, small doses of Glauber's salts are useful in promoting the activity of the skin and kidneys, 
and for this purpose may be given with spirit of nitrous ether, to which diluted sulphuric acid and the compound tincture may be added to improve appetite and digestion. Glauber's salts are useful in aiding the action of peristaltic ageuts, as aloes. Small doses are given to horses in their drinking water while the aloes is acting.

Sodium sulphate is of benefit in the treatment of diarrhœa, dysentery and overloaded and impacted colon of horses when given in frequent and repeated doses in connection with linseed oil.

An enema, consisting of one pound of Glauber's salts in a quart of water, to which two ounces of oil of turpentine and four ounces of glycerin may with advantage be added, should be injected high into the bowel to secure rapid purgation in horses suffering with colic.

Glauber's and Epsom salts are the most common purgatives given to ruminants. It is therefore impossible to enumerate special indications for their employment in the case of these animals. To assist the action, one teaspoonful each of ginger and molasses are given with each onnce of Glauber's or Epsom salts, and often an equal weight of sodinm chloride. When a speedy action is desired, Glanber's salts should be administered with a large amount of water and thirst should be encouraged by the addition of common salt. When, on the other hand, it is essential to remove fluid and morbid effusions from the body, the purgative salt should be exhibited in concentration and the patient should be deprived of water to a considerable extent. Glauber's and Epsom salts are not used so commonly in canine practice as calomel and castor oil, but find more favor with German than English-speaking veterinarians.

Sometimes salts are serviceable in irritable states of the bowels in dogs (piles, duodenitis and intestinal catarrh) in teaspoouful doses; and when given every second day, as a laxative, in eczema. Vomiting, however, not uncommonly follows the ingestion of salts by dogs.

Sodium and magnesium sulphate are autidotes to lead 
aud barium salts forming insoluble sulphates; also they are the best antidotes to carbolic acid, giving rise to the harmless sulphocarbolates.

Sodir Chloridurr. Sodium Chloride. $\mathrm{NaCl}$.

(U. S. \& B. P.)

Synonym.-Common or table salt, muriate of soda, sal commune or culinare, chloruretum sodicum, E.; natrium chloratum purum, P. G.; chlorure de sodium, sel commun, sel de cuisine, Fr.; chlornatrium, kochsalz, G.

Derivation.-Mined in native state and obtained by evaporation of brine, spring or sea water.

Properties.-Colorless, transparent, cubical crystals, or a white, crystalline powder, odorless, and having a purely saline taste. Permanent in dry air. Soluble in 2.8 parts of water; almost insoluble in alcohol; insoluble in ether or chloroform. Reaction neutral.

Dose.-Cathartic, C., Hoss.-i. (250.-500.); Sh., §i.-ii. (30.-60.).

Action External.-Salt is a stimulant to the skin when applied in concentrated solution.

Action Internal. - Salt is an essential constituent of food, and necessary to the composition of blood plasma, from which it is constantly eliminated in the urine. Herbivorous animals require sodium chloride in addition to that contained in their food ; for blood is rich in common salt, while vegetables abound more especially in potassium salts. The potrssium salts, according to Bunge, on entering the blood bring about a chemical reaction, whereby sodium chloride circulating in the plasma is split; the chlorine in sodium chloride combines with potassium, while the acid set free from the potassium salt unites with sodium, and both products are swept away in the urine, thus removing sodium chloride from the blood. This is only replaced by that 
taken as food. Animals deprived of salt suffer from anæmia, general weakness and oedema.

Stomach and Intestines. - Salt has caused gastro-enteritis and death in enormous doses. Large doses occasion emesis in dogs. Salt combines with lactic acid in the stomach to form sodium lactate, and in the process sets free hydroclloric acid. Hydrochloric acid, in its turn, promotes the formation of pepsin from pepsinogen of the gastric tubules. Salt, therefore, improves gastric digestion. Salt acts in the bowels as a mild hydragogue purgative. It is unfit as a cathartic for loorses or dogs, but is useful for cattle and sheep when combined with magnesium or sodium sulphate. Salt creates thirst and, therefore, promotes the ingestion of water. A large supply of water flushes the system and removes deleterious and imperfectly oxidized matters.

Blood. - The red corpuscles are augmented by salt.

Metabolism. - Salt increases tissue change and tends to elevate temperature. The appetite is also sharpened owing to the ingestion of water and metabolic activity.

Summary.-Emetic, cathartic, digestive, alterative.

Uses External.-A solution consisting of one ounce each of salt, nitre and sal ammoniac, in one quart of water, may be used on bruises and sprains as a stimulant and refrigerant lotion. Severe hæmorrhage, collapse and surgical shock are treated most successfully by injections of hot normal salt solution into a vein, under the skin, or into the rectum. The solution maintains the proper salinity of the blood, replaces the mass of blood lost, and supplies heat. The solution is made by adding one heaping teaspoonful of salt to a quart of boiled water at a temperature of $100^{\circ}$ to $105^{\circ}$ F. From 2 to 4 quarts may be given to horses, and from $\frac{1}{2}$ to 1 pint to dogs. (See p. 732.) Salt is an efficient antidote externally and internally to silver nitrate.

Uses Internal.-Salt is a serviceable emetic for dogs, when zine sulphate is not at hand, in emergencies and poisoning. One teaspoonful may be stirred into a cup of lukewarm water with a tablespoonful of mustard. It is a useful 
addition to Epsom salts, since it increases thirst and the ingestion of water, and assists purgation in overloaded conditions of the firnt and third stomachs of ruminants. One-half pound of salt is atministered to cattle with onehalf to one pound of Epsom salts, one-quarter pound of ginger and a pint of molasses in two quarts of water. The habitual ingestion of salt is prejudicial to ascarides and diminishes the secretion of mucus in which they live. It is even more efficacions in destroying oxyurides inhabiting the lower bowels. Enemata containing 1 to 2 tablespoonfnls of salt to the pint of water are employed for this purpose. Salt should be constantly kept in the feed boxes of horses and cattle.

Auimals convalescing from acute diseases, and those with feeble digestion, need salt particnlarly. Horses are commonly given a bran mash once a week, with plenty of salt to enhance its laxative and hygienic action. Dogs usually procure sufficient salt in their ordinary food, but it should be added to their diet in the treatment of obesity. Repeated doses of salt aid the absorption of serous pleural effusions.

Sodi Phospras. Sodium Phosphate. $\mathrm{Na}_{2} \mathrm{HPO}_{4}+1 \kappa \mathrm{H}_{2} \mathrm{O}$. (U. S. \& B. P.)

Synonym.-Phosphas sodicns (natricus), sal mirabile perlatum, phosphate of soda, E.; natrum phosphoricum, P. G.; phosphate de soude, Fr:; phosphorsaures natrum, G.

Derivation.-Digest bone ash with sulphuric acid. $\mathrm{Ca}_{3} 2 \mathrm{PO}_{4}+2 \mathrm{H}_{2} \mathrm{SO}_{4}=\mathrm{Ca}_{4} \mathrm{H}_{4} \mathrm{PO}_{4}$ (acid calcium phosphate) $+2 \mathrm{Ca} \mathrm{SO}_{4}$. Filter and add sodium carbonate to filtrate.

$\mathrm{Ca} \mathrm{H}_{4} 2 \mathrm{PO}_{4}+\mathrm{Na}_{2} \mathrm{CO}_{3}=\mathrm{Na}_{2} \mathrm{H} \mathrm{PO}_{4}+\mathrm{H}_{2} \mathrm{O}+\mathrm{CO}+$ $\mathrm{Ca} \mathrm{HPO}_{4}$. Evaporate, and sodium phusphate crystallizes out.

Properties.-Large, colorless, monoclinic prisms, odorless, and having a cooling, saline taste. The crystals effloresce in the dry air and gradually lose 5 molecules of 
their water of crystallization. Soluble in 5.8 parts of water; insolnble in alcohol; slightly alkaline reaction.

Dose.-Same as sodium sulpliate; D., 3i.-ii. (4.-8.), as laxative and cholagogue cathartic.

The phosphate resembles the sulphate, but is a milder purgative and more efficient lepatic stimulant. It is indicated in jaundice, and as a laxative for foals and calves. It is occasionally prescribed in rickets as a source of phosphorus, but the calcium phosphate is more appropriate.

SodI Sulphis. Sodium Sulphite. $\mathrm{Na}_{2} \mathrm{SO}_{3} 7 \mathrm{H}_{2} \mathrm{O}$. (U. S. \& B. P.)

Synonym.-Natrum sulfuriosum, sulfis sodicus (natricus), sulphite of soda, E.; sulfite de sonde, Fr.; schwefligsaures natron, G.

Derivation.-Saturate a solution of sodium carbonate or hydrate with sulphurons anhydride gas. $\mathrm{Na}_{2} \mathrm{CO}_{3}+\mathrm{SO}_{2}=$ $\mathrm{Na}_{2} \mathrm{SO}_{3}+\mathrm{CO}_{2}$.

Properties.-Colorless, transparent, monoclinic prisms, odorless, and having a cooling, saline, sulphurous taste. In air the salt effloresces, and is slowly oxidized to sulphate. Soluble in 4 parts of water; sparingly soluble in alcohol; Neutral or feebly alkaline.

Dose.-H. \& C., zi. (30.); Sh. \& Sw., 3 ss.-i. (2.-4.); D., gr.v.-xxx. (.3-2.).

Sodi Bisulphite. Sodium Bisulphite. $\mathrm{NaHSO}_{3}$. (U. S. P.)

Derivation.-Obtained from sodium carbonate or bicarbonate and sulphurous anlydride gas.

Properties.-Opaque, prismatic crystals, or a granular powder, exhaling an odor of sulphur dioxide and having a disagreeable, sulphurous taste. Exposed to the air the salt loses sulphur dioxide and is gradually oxidized to sulphate. Soluble in 4 parts of water and in 72 parts of alcohol ; reaction acid.

Dose.-Same as sodium sulphite. 
Sodir Hyposulphis. Sodium Hyposulphite. $\mathrm{Na}_{2} \mathrm{~S}_{2} \mathrm{O}_{3} 5 \mathrm{H}_{2} \mathrm{O}$. (U. S. \& B. P.)

Synonym.-Sodium thiosulphate, hyposulphate of soda, E.; natrum subsulfurosum (hyposulfurosum), P. G.; liyposulphis sodicus, hyposulfite de soude, sulfite solfuré de sonde, Fr.; unterschwefligsaures natron, G.

Derivation.-Dissolve sulphur in a boiling aqueous solution of sodium sulphite.

Properties.-Colorless, transparent, monoclinic prisms, odorless, and having a cooling, afterwards bitter, taste. Permanent in air below $33^{\circ}$ C. $\left(91.4^{\circ}\right.$ F.), but efflorescent in dry air above that temperature. Soluble in 0.65 part of water; insoluble in alcohol; slightly soluble in oil of turpentine; reaction neutral.

Dose._Same as sodium sulphite.

Administration.-The sulphites are given in solution, or may be added in powder to the food of horses.

\section{ACTION OF THE SOLPHITES.}

Action External.-The sulphites are antiseptics, deodorizers and parasiticicles externally.

Action Internal.-The sulphites are decomposed by the acids in the stomach, and sulplut dioxide $\left(\mathrm{SO}_{2}\right)$ is evolved. The bisulphite and hyposulphite are richer in sulphur and, therefore, are more powerful. Sulphur dioxide gas is an antiseptic.

Blood.-If any part of the sulphites escapes decomposition in the digestive tract, it is absorbed unchanged.

Summary.-Antiseptics, deodorizers and parasiticides externally. Autiseptics in the alimentary tract.

Uses.-A 15 per cent. solution of the sulphites is used in parasitic skin diseases. The salts are indicated in indigestion with fermentation, flatulence and foul-smelling freces. They have been greatly lauded as remedies in general septic conditions, but have proved as useless as most other drugs in such cases. 
The cther sodium salts are of no particular value in reterinary practice except sodium bromide. See Bromine, p. 210.)

\section{Ammonium.}

The metal is not emplored in medicine. Ammonia $\left(\mathrm{NH}_{3}\right)$ exists in the free state as a gas, and is used in medicine in solution in water or alcohol.

\section{Aqua Amonie. Ammonia Water. (U. S. P.)}

Symonym.-Liquor ammonire, B. P.; spirits of hartshorn, E.; liquor ammonii caustici, P. G.; spiritus salis amrnoniaci cansticus, ammonia aqua solnta, ammoniaque liquide, eau (solution, liqueur) d'ammoniaque, Fr.; salmiakgeist, ætzammoniak, ammoniakflüssigkeit, G. An aqueous solution of ammonia $\left(\mathrm{NH}_{3}\right)$, contailing 10 per cent, by weight, of the gas.

Properties.-A colorless, transparent liquid, having a very pungent odor, an acrid, alkaline taste, and a strongly alkaline reaction. Spec. gr.0.960.

Derivation. - Evolve ammonia gas by heating ammonium chloride with calcinm hydrate, and pass it into water.

$2 \mathrm{NH}_{4} \mathrm{Cl}+\mathrm{Ca}(\mathrm{OH})_{2}=2 \mathrm{NH}_{3}+2 \mathrm{H}_{2} \mathrm{O}+\mathrm{Ca} \mathrm{Cl}_{2}$.

Dose.-H. \& C., 3 ss.-i. (15.-30.); Sh. \& Sw., 3 i.-ii. (4.-8.);

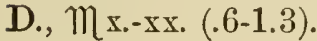

PREPARATION.

Linimentum Ammonice. Ammonia Liniment. (U. S. \& B. P.) Ammonia water, 350; cottonseed oil, 600; alcohol, 50. (U. S. P.)

Aqua Ammoniæ Fortis. Stronger Ammonia Water. (U.S.P.)

Synonym.-Liquor ammonix fortior, stronger solution of ammonia, B. P.; ean d'ammoniaque forte, Fr.; starker salmiakgeist, G.

An aqueous solution of ammonia $\left(\mathrm{NH}_{3}\right)$, containing 28 per cent., by weight, of the gas.

Derivation.-Same as aqua ammonix.

Properties.-A colorless, transparent liquid, having an 
excessively pungent odor, a very acrid and alkaline taste, and a strongly alkaline reaction. Spec. gr.0.901.

Dose.-H. \& C., 3 ii.-vi. (8.-24.); Sh. \& Sw., ji. (4.); D., m.

\section{PREPARATION.}

Spiritus Ammonice. Spirit of Ammonia. (U. S. P.)

Synonym.-Spiritus ammoniaci caustici dyondii, ammoniated alcohol, E.; liquor ammonie caustici spirituosus, P. G.; alcoolé d'ammoniaque, liqueur d'ammoniaque vineuse, Fr.; weingeistige ammoniakflussigkeit, G.

An alcoholic solution of ammonia $\left(\mathrm{NH}_{3}\right)$, containing 10 per cent, by weight, of the gas.

Properties. - A colorless liquid, having a strong odor of ammonia, and a spec. gr. of about 0.810 .

This preparation combines the stimulating properties of ammonia and alcohol.

Dose.-H. \& C., 3 ss.-i. (15.-30.); Sh. \& Sw., 3 i.-ii. (4.-8.); D., $m x_{*}-$ $\mathrm{xx} .(.6-1.3)$.

\section{AMMONIA PREPARATIONS.}

Action External.-Ammonia is a powerful irritant im stronger solution, or gas. If it is applied for a sufficient time, hyperæmia, vesication and sloughing ensue.

Action Internal.-Stomach.-If swallowed in concentrated solution death may take place instantaneously from oedema. and spasm of the glottis. Otherwise, more or less extensive inflammation of the alimentary caual will follow, according to the amount ingested. Diluted vinegar and lemon juice, together with the white of egg, or sweet oil, should be given as antidotes. Tracheotomy may be indicated, if there is glottic obstruction. Ammonia, in passing through the mouth, throat, gullet and stomach, reflexly stimulates the heart and respiration before absorption can occur.

Ammonia is an antacid in the stomach, but should not be employed in gastric irritability.

Respirctory Tract.-Inhalation of stronger ammonia through the nostrils causes reflex stimulation of the heart and respiration by irritation of the nasal branches of the fifth nerve. Care must be exercised to prevent inflammation 
of the air passages. Ammonia stimulates the respiratory centre when it is absorbed through any channel.

Circulation.-Ammonia is a rapid but transient heart stimulaut, making the ventricular contractions stronger and more frequent, by stimulation of the accelerator nerres and heart muscle. Ammonia also stimulates the vasomotor centre, probably, and, becanse of this fact, and the large amount of blood prmperl into the ressel-, vascular tension is heightened. Lethal doses paralyze the cardiac muscle. Ammonia differs from alcohol in being more evanescent as a stimulant, in not affecting the brin nor metabolisw, and in not acting as a fool.

Blood.-The normal blood contains ammonia, which is supposed to aid in maintaining its fluidity. When a poisonous quantity enters the circulation, anmonia has a damaging effect on the red corpuscles; not only clepriving them of their oxygen, but incapacitating them from absorbing more. Ammonia is thought to prevent coagulation of blood within the vessels in conditions fitvorable to thrombosis.

Nervous System.-An intravenous injection of a lethal dose of ammonia occasions tetanic convulsions in animals, owing to stimulation of the reflex and motor functions of the cord. Medicinal doses excite the spinal reflex and motor centres. Where ammonia is applied directly to nerve tissue it excites in dilute solution, but paralyzes functional activity in concentration. The brain is unaffected by the therapentic administration of ammonia.

Elimination.-Ammonia combines with acid in the stomach and is absorbed into the blood. Its fate is unknown. Probably it is oxidized in part and escapes as nitric acid, possibly to some extent as urea and uric acid. The acidity of urine is increased by its elimination.

Summary.-Heart and respiratory stimulant and antacid. Externally, rubefacient, vesicant, and escharotic.

Uses.-The indications for ammonia are closely in accord with its physiological actions.

Externally.-It is frequently used in stimulating lini- 
ments. One part each of water of ammonia and oil of turpentine, may be combined to advantage with 4 to 6 parts of camphor liniment. Ammonia water is one of the best remedies to relieve pain and antagonize the action of insect bites, as stings of bees and wasps. It should be applied directly to the poisoned part.

Internally.-Ammonia is indicated when rapid stimulation of the heart and respiration is desirable. In emer- , gencies it may be given intravenously (aq. ammon. fort. 1; water, 4 parts); or by inhalation. It is serviceable in the treatment of prussic acid and aconite poisoning, syncope, collapse and shock following surgical operations; also in snake bites. The spirit of ammonia may be prescribed in fevers as a stimulant. Ammonia is inferior to ammonium carbonate or the aromatic spirit in the care of colic and tympanites.

Ammonit Carbonas. Ammonium Carbonate. $\mathrm{NH}_{4} \mathrm{HCO}_{3}$ $\mathrm{NH}_{4} \mathrm{NH}_{2} \mathrm{CO}_{2}$. (U. S. \& B. P.)

Synonym.-Volatile salt, sal volatile siccum, carbonas ammonicus, E.; ammonium carbonicum, P. G.; carbonate d'ammoniaque, alkali volatil concret, sel volatil d'Augleterre, Fr.; flüchtige, laugensalz, reines hirchhornsalz, kohlensäures ammonium, G.

Derivation.-A mixture of ammonium chloride or sulphate, and calcium carbonate, is sublimed and resublimed. Ammonium carbonate, so-called, is a mixture of ammonium carbonate and bicarbonate.

$4 \mathrm{NH}_{4} \mathrm{Cl}+2 \mathrm{CaCO}_{3}=\mathrm{NH}_{4} \mathrm{HCO}_{3} \cdot \mathrm{NH}_{4} \mathrm{NH}_{2} \mathrm{CO}_{2}+2$, $\mathrm{CaCl}+\mathrm{NH}_{3}+\mathrm{H}_{2} \mathrm{O}$.

Properties. - White, hard, translucent, striated masses, having a strongly ammoniacal odor without empyreuma, and a sharp saline taste. On exposure to the air the salt loses both ammonia and carbonic dioxide, becoming opaque, and is finally converted into friable, porous lumps, or a white powder. Soluble in about 5 parts of water. Alcohol only dissolves the carbonate $\left(\mathrm{NH}_{4} \mathrm{NH}_{2} \mathrm{CO}_{2}\right)$. 
Dose.-H., 3 ii. (8.); C., J iii.-vi. (12.-24.); Sh. \& Sw., gr.xv.-xl. (1.-2.6); D., gr.iii.-x. (.2-.6); D., emetic, gr.xr. (1.).

\section{PREPARATION.}

Spiritus Amimonice Aromaticus. Aromatic Spirit of Ammonia.

(U. S. \& B. P.)

Synonym.-Sal volatile, alcoolat anmoniacal aromatique, Fr.; aromatischer ammoniakgeist, $\mathrm{G}$.

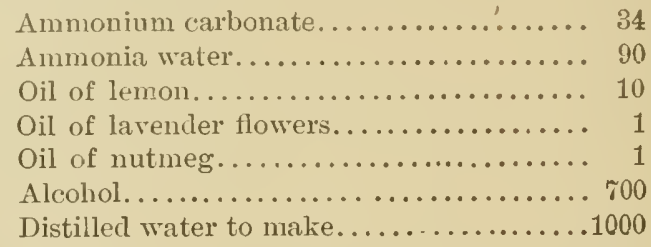

Properties.-A nearly colorless liquid when freshly prepared, but gradually acquiring a somewhat darker tint. It has a pungent; ammoniacal odor and taste. Spec. gr.0.905.

Dose.-H. \& C., รु i.-ii. (30.-60.); Sh. \& Sw., 3ii.-iv. (8.-15.); D., 3 ss.-i. (2.-4.), well diluted.

The aromatic spirit of ammonia nearly resembles ammonium carbonate in action, but the alcohol and volatile oils add to the stimulant qualities of ammonia and ammonium carbonate.

Action Internal.-Ammonium carbonate is decomposed by acid in the stomach, but its ultimate fate is nuknown. It stimulates gastric secretion, vascularity and motion, and also excites intestinal peristalsis. It is, therefore, a stomachic and carminative. It is also an antacid, and, in iarge loses, an emetic to dogs. The action of ammonium carbonate is almost identical with that of ammonia water in stimulating the heart and respiration, but it has more power in augmenting the bronchial secretions.

Summury.-Cardiac and respiratory stimulant, expectorant, stomachic and emetic.

Administration.-Ammonium carbonate is given in ball, or in solution in cold water, to avoid irritating fumes; also, with syrup or gruel. It is often prescribed with other stimulants and antispasmodics, as alcohol, capsicum, camphor and asafoetida. 
Uses.-The indications for the administration of ammonium carbonate are much the same as those for ammonia preparations. It is a more useful expectorant, however, and it and the aromatic spirit are more serviceable in the treatment of flatulence. For the latter condition in horses with colic a ball containing ammonium carbonate 3 ii., and capsicum 3 i., with sufficient linseed meal and molasses to make the proper bulk and consistencr, is often efficient. We may use ammonium carbonate alone in the case of acute or chronic bronchitis, or it may be combined with other stimulants, or expectorants, as ammoninm chloricle. In electric shock, so common an accident in the larger cities from "live" wires, the administration of full doses of aromatic spirit diluted with half a pint of water will prove a most effective heart stimulant when the animal can swallow.

Ammonir Chloridum. Ammonium Chloride. $\mathrm{NH}_{4} \mathrm{Cl}$.

(U. S. \& B. P.)

Synonym.-Sal ammoniac, ammonia muriatica or hydrochloratum, chloruretum ammonicum, sal ammonicum, ammoniæ hydrochloras or murias, muriate of ammonia, E.; chlorure d'ammonium, sel ammoniæ, muriate d'ammoniaque, Fr.; salmiak, chlorammonium, G.

Properties.-A white, crystalline powder without odor, having a cooling, saline taste, and permanent in the air. Soluble in three parts of water; almost insoluble in alcohol. Reaction neutral.

Dose.-H., 3 ii. (8.); C., 3 iii.-vi. (12.-24.); Sh. \& Sw., gr.xv.-xl. (1-2.6); D., gr.iii.-x. (.2-.6).

Action Internal.-Sal ammoniac is a feeble heart and respiratory stimulant, and is not comparable to the ammonia compounds, or ammonium carbonate, in this respect. It is eliminated in great part unchanged by the mine, but also by the other channels. In its excretion it stimulates the mucous membranes, increases their secretions generally, 
and is thought to improve their nutrition. Hence it has been termed an alteratire. Ammonium chloride both excites the secretion of the bronchial mucous membrane and renders it less viscid in inflammatory conditions. It is mildly diarphoretic, diuretic and cholagogne.

Summary.-Externally refrigerant, internally expectorant, alterative, feebly diarphoretic, diuretic, and chologogue.

Uses.-Four ounces each of nitre and sal ammoniac may be dissolved in two quarts of water as a refrigerant lotion. Sal ammoniac is indicated more especially in the second stage of acute bronchitis, in chronic bronchitis, and in chronic intestinal catarrh with diarrhœe. Ammonium chloride may be given to dogs with glycerin and chloroform water as a cough mixture. If cough is excessive, codeine or morphine sulphate can be added to this prescription with advantage.

Liquor Ammonir Acetatis. Solution of Ammonium Acetate. (U. S. \& B. P.)

Synonym.--Spirit of Mindererus, spiritus Mindereri, acetas ammonicus liquidus, E.; liquor ammonii acetici, P. G.; acetate d'ammoniaque liquide, esprit de Mindererus, Fr.; essigsaure ammonium-flüssigkeit, $\mathrm{G}$.

An aqueous solution of ammoninm acetate $\left(\mathrm{NH}_{4} \mathrm{C}_{2} \mathrm{H}_{3} \mathrm{O}_{2}\right)$ containing about 7 per cent. of the salt, together with small amounts of acetic acid and carbon dioxide.

Derivation.-Ammonium carbonate is gradually added to cold, dilnted acetic acid until the latter is nentralized.

Properties.-A clear, colorless liquid, free from empyreuma, of a mildly saline, acidulous taste, and an acid reaction.

Incompatibles.-Acids and alkalies.

Dose.-H. \& C., 3 ii.-iv. (60.-120.); D., 3 ii.-viii. (8.-30.).

Action.-Spirit of Mindererus stimulates the secretory cells of the kidneys and sudoriparous glands. In the stomach it exerts a mild, antacid action. 
Summary.-Externally, refrigerant; internally, diuretic, diaphoretic and antacid.

Uses.-Liquor ammonii acetatis is an exceedingly feeble medicine and is often employed as a vehicle with more powerful agents of its class, e.g., spiritus rtheris nitrosi. It is useful as a febrifuge.

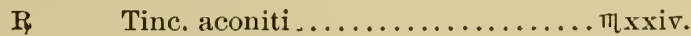

Sodii bromidi................. 3 ii.

Sp'r. ætheris nitrosi........... 3 ss.

M. Liquor. ammonii acetatis ad....... 3 iv.

S. Teas. every hour.

The foregoing prescription is a good palliative combination for dogs suffering with fever and restlessness. The solution of ammonium acetate is frequently conjoined with sweet spirit of nitre and ammonium chloride or potassinm iodide, in the treatment of acute respiratory diseases of horses, e.g., influenza, bronchitis, and pneumonia. It may be added to the drinking water withont rendering it unpalatable.

\section{Lithium.}

(The Metal is not Used in Medicine.)

Lithin Carbonas. Lithinm Carbonate. $\mathrm{Ii}_{2} \mathrm{Co}_{3}$. (U. S. \& B. P.)

Synonym.-Carbonas lithicus, carbonate of lithia, E.; lithium carbonicum, P. G.; carbonate de lithine, cartbonate lithique, Fr.; kohlensaures lithion, G.

Derivation.-Made by action of lithium chloride on acid ammonium carbonate.

$2 \mathrm{LiCl}+\mathrm{NH}_{4} \mathrm{HCO}_{3} \doteq \mathrm{Li}_{2} \mathrm{CO}_{3}+\mathrm{NH}_{4} \mathrm{Cl}+\mathrm{HCl}$. Purified by washing with alcohol and drying.

Properties.-A light, white powder, odorless, and having an alkaline taste. Permanent in the air. Soluble in 80 parts of water; insoluble in alcohol. Reaction alkaline.

Dose.-D., gr.iii.-х. (.2-.6). 


\title{
Lithis Citras. Lithium Citrate. $\mathrm{Li}_{3} \mathrm{C}_{6} \mathrm{H}_{5} \mathrm{O}_{7}$.
}

\author{
(U. S. \& B. P.)
}

Synonym.-Lithium citricnm, citrate of lithia, E.; citrate de lithine, Fr.; citronsaures lithion, G.

Derivation.-Made by action of citric acid on lithinm carbonate.

$$
2 \mathrm{H}_{3} \mathrm{C}_{6} \mathrm{H}_{5} \mathrm{O}_{7}+3 \mathrm{Li}_{2} \mathrm{CO}_{3}=2 \mathrm{Iii}_{3} \mathrm{C}_{6} \mathrm{H}_{5} \mathrm{O}_{7}+3 \mathrm{H}_{2} \mathrm{O}+3 \mathrm{CO}_{2} \text {. }
$$

Recovered by evaporation and crystallization.

Properties.-A white powder, odorless, and having a cooling, faintly alkaline taste; deliquescent on exposure to air ; soluble in 2 parts of water; almost insoluble in alcohol or ether. Reaction neutral.

$$
\begin{aligned}
& \text { Dose.-D., gr.v.-xx. (.3-1.3). } \\
& \text { ACTION of LITHIUM saLts. }
\end{aligned}
$$

Lithium salts are said to form soluble compounds with uric acid in the blood, and so assist its elimination in the urine. As lithium combines more readily with acid sodium phosphate in the blood than with uric acid, it is doubtful whether it is a very efficient uric acid* solvent in the body. The lithium salts alkalize the urine and notably increase its secretion.

Summary.-Lithium salts are diuretics, and uric acid solvents in some degree. The carbonate may be given in powder or pill; the citrate in solution.

Uses.-Lithinm compounds are of little value in veterinary medicine. They are serviceable, however, in the treatment of dogs with a very acid urine of high specific gravity; with uric acid calculus, or those affected with chronic rheumatism. The salts will not dissolve calculi in the body, but prevent their formation.

Water is extremely useful in such conditions. Lithium citrate may be placed in the drinking water. Thirst should be encouraged by the administration of salt on the food, and high rectal injections may be given to create absorption of water by this channel. Lithium salicylate is thought to be

\footnotetext{
* Uric acid can not exist as such in blood, which is an alkaline fluid.
} 
the better salt for rheumatism. It is probable that treatment with salicylic acid and lithinm citrate would be more satisfactory.

\section{SECTION III.}

Alkaline Earth Metals: Calcium, Barium, Magnesidm, Aluminum and Cerrum.

\section{Calcium.}

(The Metal Calcium is not Employed in Medicine.)

Creta Preparata. Prepared Chalk. $\mathrm{Ca} \mathrm{Co}_{3^{\circ}}$. (U. S. \& B. P.)

Synonym.-Drop chalk, E.; craie préparée, Fr.; präparirte kreide, G.

Derivation.-Made from chalk by levigation, elutriation and dessication.

Properties.-A white, amorphous powder, often moulded into conical drops; odorless and tasteless; permanent in the air. Almost insoluble in water; insolnble in alcohol.

Incompatibles.-Sulphates and acids.

Duse.-H., 亏i.-ii. (30.-60.); C., 亏ii.-ir. (60.-120.); Sh. \& Sw., 3 ii.-iv. (8.-15.); D., gr.x.- 3 i. (.6-4.).

\section{PREPARATIONS.}

Pulvis Cretce Compositus. Compound Chalk Powder. (U. S. P.)

Chalk, 30; acacia, 20; sugar, 50.

Dose.-D., gr.x.- z i. (.6-4.).

Mistura Cretre. Chalk Mixture. (U. S. P.) 1000.

Compound chalk powder, 200 ; cinnamon water, 400 ; water to make

Dose.-D., こ̇i.-ii. (30.-60.).

Trochisci Cretce. Troches of Chalk. (U. S. P.)

Contain .25gm., or gr.iv., each.

Dose.-D., same as prepared chalk. 
Calcil Carbonas Precipitatus. Precipitated Calcium Carbonate. $\mathrm{CaCO}_{3}$. (U. S. P.)

Synonym.-Calcis carbonas precipitata, B. P.; precipitated carbonate of lime, creta præcipitata, carbonas calcicus præcipitatus, E.; calcaria carbonica præcipitata, P. G.; carbonate de chaux précipité, craie précipitée, Fr.; præcipitirten kohlensanren kalk, G.

Derivation. - Obtained by precipitation of calcinm chloride with sodium carbonate. tate.

$\mathrm{CaCl}_{2}+\mathrm{Na}_{2} \mathrm{CO}_{3}=\mathrm{CaCO}_{3}+2 \mathrm{NaCl}$. Dry the precipi-

- Properties.-A fine, white powder, without odor or taste, and permanent in the air. Nearly insoluble in water; insoluble in alcohol. The solubility is increased by presence of carbon dioxide. Permanent in air.

Dose.-H., „i.-ii. (30.-60.); C., ङii.-iv. (60.-120.); Sh. \& Sw., 3 ii.-iv. (8.-15.); D., gr.x.- 3 i. (.6-4.).

\section{Calcium Carbonate.}

Action External. - Dessicant and slightly astringent powder; also protective.

Action Internal.-Alimentary Canal.-Calcium carbonate is the slowest acting antacid, becanse of its comparative insolubility, and is of value when it can exert its longcontinued influence throughout the digestive tract. It resembles bisuuth in mechanically coating or protecting inflamed or irritable surfaces. It is not so astringent nor antiseptic as the bismuth salts, and these are generally preferable to chalk for the smaller animals. It is excreted unchinged in the freces.

Administration.-Calcinm carbonate may be given to dogs. in troches, pills or powder ; to other animals in powder, balls or electuary. Chalk is commonly prescribed suspeuded in flour gruel, milk or mucilage to the larger animals. The official preparations are suitable for dogs.

Uses.-Chalk forms a dusting powder for moist eczema, 
slight burns and intertrigo. Zinc oxide and starch (1 to 4 ) is, however, a better preparation. Chalk is the most useful antacid for diarrhoea accompanied by fermentation of the intestinal contents, while its local astringent and protecting influence assist in overcoming the trouble. It is especially good for foals and calves given in flour gruel, and often combined with catechu, ginger and opium. The following prescription is appropriate for dogs with diarrhœa:

Tinc. kino, tinc. catechu co. and tinc. opii camphor. āa zss.; mistura cretæ to make $\tilde{j}$ iv. S. Teaspoonful every three hours. A serviceable ball for horses with diarrhoea contains: Creolin, chalk and ginger, āa $\overline{\bar{j}} \mathrm{ss}$; powdered opium, $3 \mathrm{i}$.

\section{Calx. Lime. CaO. (U. S. \& B. P.)}

Synonym.-Burned lime, quicklime, calcaria, calx viva, calx usta, oxydum calcicum, E.; calcaria usta, P. G.; chaux, chanx vive, Fr.; kalk, gebrannter kalk, G.

Derivation.-Prepared by burning white marble, oyster shells, or the purest varieties of natural calcium carbonate, to expel carbon dioxide.

Properties.-Hard, white, or grayish-white masses, which in contact with air gradually attract moisture and carbon dioxide and fall to a white powder; odorless; of a sharp, caustic taste. Reaction intensely alkaline. Solubie in about 750 parts of water; insoluble in alcohol.

PREPARATIONS.

Liquor Calcis. Solution of Lime. (U. S. \& B. P.)

A saturated, aqueous solution of calcium hydrate.

Synonym.-Lime water, solution of calcium hydrate, aqua calcariæ ustæ, aqua calcis, calcaria solnta, oxydum calcicum, aqua solutum, E.; aqua calcariæ, P. G.; eau (liquor) de chaux, Fr.; kalkwasser, G.

Derivation.-Dissolve lime in water. The percentage of calcium hydrate $\left[\mathrm{Ca}(\mathrm{OH})_{2}\right]$ varies with the temperature, being somewhat over 0.17 per cent. at $15^{\circ} \mathrm{C}$. $\left(59^{\circ} \mathrm{F}\right.$.), and diminishing as the temperature rises.

Properties. - A clear, colorless liquid without odor, and having a saline and feebly caustic taste. It absorbs carbon dioxide from the air, so that a pellicle of calcium carbonate forms on the surface of the liquid. Reaction strongly alkaline. 
Dose.-H. \& C., żiv.-vi. (120.-180.); Calres, 3 ii. (60.); D., 3 i.-viii. (4.-30.).

Syrupus Calcis. Syrup of Lime. (U. S. P.)

Lime, 65; sugar, 400; water to make 1000.

Dose.-Calves and dogs, ¿ ss.-i. (2.-4.). Weil diluted with water or milk.

Linimentum Calcis. Lime Liniment. (U. S. \& B. P.)

Symonym.-Carron oil.

Solution of lime and linseed oil, of each one rolume.

Dose.-Foals and calres, $\overline{3}$ ii.-iv. (60.-120.).

\section{ACTION OF LIME AND SOLUTION OF LIME.}

External.-Lime is canstic, but less so than potassium or sodium hydrate. It is an irritant. The dust of quicklime will cause conjunctivitis. If inhaled, it will cause inflammation of the air passages; if swallowed, irritation of the digestive tract. The hydrate is a canstic also, but is not so active as lime. Solution of lime is a sedative and astringent.

Internal.-Neither lime nor slaked lime (calcium hydrate) are used internally, except in the official preparations. Solution of lime acts as a sedative, antacid, and astringent in the stomach. It dissolves mucous secretions. Lime water is also a mild astringent in the bowels.

Uses.-Lime is employed outside of the body to destroy putrefying organic matter by combining with water and forming slaked lime, which absorbs many of the products of decomposition. Whitewash, a mixture of slaked lime and water, is not a disinfectant, although it covers sources of infection. It may be made so by combination with 'sufficient crude carbolic acid to make a 2 per cent. solution. Linimentum calcis is one of the most satisfactory applications for superficial burns. Old clean cotton or linen cloths are soaked in it and spread over the burned surface of the body. This preparation has been facetionsly called "carrion" instead of carron oil, becanse it is not germicidal. Antiseptic applications are of course desirable in burns giving rise to a raw surface, and the addition of two per 
cent. of carbolic acid will not only render the preparation antiseptic, but more or less anæsthetic as well. When the burn is extensive, boric acid with raseline (1 to 8 ) will be safer. Orthoform (see p. 253) is the most comfortable application which can be made on burns, but is expensive. A mixture of slaked lime and charcoal, equal parts, makes a nsefnl stimulant, absorbent, dessicant, and antiseptic dressing powder for wounds and ulcers in horses. Lime water is serviceable in relieving itching in skin diseases, and dries up moist surfaces throngh its astringent properties. With carbolic acid ( 1 to 50) lime water is most efficient in allaying pruritus. Lime water is inimical to apthous ulcerations and may be employed to swab out the mouth in this disease. Enemata of lime water destroy pin worms. Solution of lime is one of the best remedies in the treatment of romiting in dogs. It is a direct sedative to the stomach, and, mixed with milk, equal volumes, prevents the rapid coagulation of the casein, lessens the formation of large, tough curds in the stomach, and assists the retention and digestion of milk. Syrup of lime is twenty-four times stronger in calcium hydrate than lime water, and is more astringent. It may be given to foals and calves suffering from indigestion and diarrhœa. It should be administered in a considerable quantity of milk.

Lime water may be given as an antidote in poisoning by acids. Carron oil is a good, mild laxative and antacid for horses with "heaves." It is given on the food. Furthermore, it is an excellent purgative for foals and calves in the treatment of diarrhœa and indigestion.

Calci Phosphas Precipitatus. Precipitated Calcium Phosphate. $\mathrm{Ca}_{3}\left(\mathrm{PO}_{4}\right)_{2}$. (U. S. P.)

Synonym.-Calcis phosphas præcipitata, B. P.; precipitated phosphate of lime, phosphas calcicus præcipitatus, E.; calcaria phosphorica, P. G.; phosphate de chaux liydrate, Fr.; phosphorsaure kalkerde, G. 
Derivation.-Obtained from bone ash (impure calcium phosphate) by solution in hydrochloric acid and purified by precipitation with ammonia water and by washing with water.

Properties.-A light, white, amorphous powder; odorless and tasteless and permanent in the air. Almost insoluble in cold water; insoluble in alcohol; easily soluble in hydrochloric or nitric acids.

Dose.-H., 3 ii.-iv. (8.-15.); C., 3 ss.-i. (15.-30.); Sh. \& Sw., 3 i.-ii. (4.-8.); D., gr.v.-xx. (.3-1.3).

Syrupus Calcil Lactophosphatis. Syrup of Calcium Lactophosphate. (U.S. P.)

Precipitated calcium carbonate........ 25

Lactic acid..................... 60

Phosphoric acid.................. 36

Orange flower water.............. 25

Sugar....................... 700

Water, a sufficient quantity to make.....1000

Dose.-Foals and calves, 3 ss.-i. (15.-30.); D., 3 i.-iv. (4.-15.)

\section{Calcium Phosphate.}

Action and Uses.-Lime phosphate is a constituent of bone to the extent of 50 per cent. and of the soft tissue in less amount. It is, therefore, essential to the body as a tissue builder, and is a food rather than a medicine. Disease of bones (rickets) can be artificially produced by withholding calcium phosphate from the food. Other bone diseases (fragilitas ossium and caries) occur naturally in animals receiving an insufficient diet. These same diseases disappear on treatment with calcium phosphate. The value of the salt in these disorders, therefore, appears to be conclusive. Moreover, in wasting affections phosphates are eliminated in considerable amount by the urine. A reasonable deduction would lead us to use phosphates in such conditions. The diseases most appropriately treated with calcium phosphate, according to the foregoing, are rickets, osteomalacia, delayed union in fractures; also anæmia, 
malnutrition, and weakness in young animals. Here the drug should be given with iron.

Administration.-Precipitated calcium phosphate may be given on the food, but is more readily absorbed if it is administered in the syrup of calcium lactophosphate.

\section{Calci Chloride. Calcium Chloride. $\mathrm{CaCl}_{2}$}

Derivation.-Nentralize hydrochloric acid with calcium carbonate and evaporate: $2 \mathrm{H} \mathrm{Cl}+\mathrm{CaCO}_{3}=\mathrm{CaCl}_{2}+\mathrm{CO}_{2}$ $+\mathrm{H}_{2} \mathrm{O}$. Fusion at the lowest possible temperature renders the salt anhydrous.

Properties.-White, translucent, hard fragments; odorless; having a sharp, saline taste, and very deliquescent. Soluble in 1.5 part of water, in 8 parts of alcohol.

Dose.-H. \& C., 3 ss.-i. (15.-30.); D., gr.5-20 (.3-1.3).

Actions and Uses.-Calcium chloride has been used of late extensively both to prevent and arrest hemorrhage. Outside of the body, calcium chloride causes blood to coagulate more quickly and firmly than usual, and the same kind of action appears to obtain-i.e., rendering blood more coaguable-when the salt is given interually. Calcium chloride is an efficient hemostatic in hematemesis, hemoptysis, purpura hemorrhagica and in all conditions giving rise to hemorrhage. When administered by the mouth several days before surgical operations in maximum doses it may lessen hemorrhage in cases likely to be attended by much bleeding. Two per cent. solutions of gelatin in normal salt solution have been given by the mouth, rectum and subcutaneously for the same purposes, and it is thought that the gelatin owes its potver in arresting hemorrhage to the calcium ( 0.6 per cent.) contained iu it. Unless gelatin is sterilized fractionally for half an hour on three to five successive days, tetanus may ensue when it is injected under the skin, and numerous cases have been reported following such use in human surgery. The simplest manner of employing gelatin to arrest hemorrhage internally is by 
injections per rectum; $1 \frac{1}{2}$ ounces for small animals and 10 onuces to a pint for larger animals of the following aqueous solution, given at body temperature after the bowel has received a cleansing enema of boiled water. The solution is made of gelatin to the amount of 6 per cent. and calcium chloricle to the amount of 1 per cent., to which a little laudanum may be added to prevent expulsion. It should be repeated every five hours as long as there is danger of hemorrhage. Recent experiments by H. C. Wood, Jr., appear to show that gelatin may be given cullibitum by the mouth and retain its hemostatic action; therefore gelatin should be given freely in this way as well. Aqueous solutions of gelatin 10 per cent. with calcium chloride 1 per cent., have been used locilly to arrest bleeding in wounds and cavities of the body, but considering the danger of tetanus and the fact that adrenalin chloride is a better local hemostatic, such a use of gelatin is inadvisable.

Calix Chlora'ta. (See Clllorime, p. 237.)

\section{Barium.}

(Barinm is not used in the metallic state.)

Barit Chloridum. Barinm Chloride. $\mathrm{BaCl}_{2}$. (Non-official.)

Derivation. - Native barium sulphate is fused with charcoal. The resnlting sulphide is treated with hydrochloric acid. $\mathrm{BaSO}_{4}+2 \mathrm{C}=\mathrm{BaS}+2 \mathrm{CO}_{2} \cdot \mathrm{BaS}+2 \mathrm{HCl}$ $=\mathrm{BaCl}_{2}+\mathrm{H}_{2} \mathrm{~S}$.

Properties. - Occurs in colorless, glistening rhombic plates; taste bitter and disagreable; permanent in dry air; soluble in 2.5 parts of cold water' reaction neutral.

Dose.-H., 亏 i.-ii. (4.-8.); intratracheally, gr.vii.-xv. (.5-1.).

Action Intemal.-Barinm chloride is an intense irritant. if swallowed in considerable amount and in insufficient dilution. Large medicinal doses stimulate the muscular coat of the bowels and ciuse increased peristalsis and 
purging. Evacuations from the bowels follow in horses one-half to one hour after the salt is given in drench; in one to two hours after administration to these animals in ball; in a few minutes after intravenous injection. Subcutaneous application will occasion abscess. The drug resembles physostigmine in its action on the intestines.

Heart and Blood Vessels.-Barium chloride makes the ventricular contractions of the heart stronger and slower. The salt acts entirely on the heart muscle and does not influence the vagi. Lethal doses are followed by slower and slower ventricular contractions, succeeded by peristalsis of the cardiac muscle, and, finally, by stoppage of the heart in systole.

Barium chloride also directly excites the musenlar walls of the capillaries, and, like physostigmine, increases vascular tension in small doses.

Muscles. - Barium chloride stimulates muscular contraction when it is applied locally. The potassium salts antagonize the action of barium chloride on the circulation and muscles.

Summary.-Circulatory stimulant, and purgative in large medicinal doses.

Uses.-Barium chloride is a new remedy in veterinary medicine. Many practitioners are afraid to use barium chloride, but repeated use of the drug has convinced us that it is absolntely safe when given in the dose of 1 gram or 15 grains intravenously, or 8 to 15 grams ( 2 to 4 drachms) by the mouth in solution for the horse. The intravenous dose of 1 gram, or the dose per orem of 8 grams, may be repeated with safety in one hour if necessary. Barium chloride is of the greatest value in colic and obstinate constipation of horses. It may for the time cause some increase of pain, but not nearly as much as follows the use of eserine or arecoline, and it quickly passes off when the bowels are moved. Great care should be exercised to avoid introduction of the barium solution into the connective tissue while injecting it into the vein. The needle of the hypodermic 
syringe should be introduced into the jugular about midway of the neck, and, after some drops of blood have exuded from the vein, the syringe is attached and slowly emptied, when the plunger is withdrawn until some blood enters the syringe. Then the needle may be removed with safety. It is rarely necessary to repeat the dose of barium chloride, and small doses of fluid extract of cannabis indica may be given to lessen colic while barium is acting, when it is giren by the mouth.

Extensive experiments of Muir* with barium chloride lead him to conclude that the salt may be administered intravenously in the amount of 1.0 to $2.0 \mathrm{gm}$. (15 to $30 \mathrm{gr}$.) in 1 to 2 drachms of sterile water; that $2 \mathrm{gm}$. is a safe dose by the intrajugular method for a horse of ordinary weight and fair condition; that the drug acts promptly when given in this way, catharsis being produced within one to nine minutes, and that even volvulus may be relieved; that there are no unpleasant symptoms following the smaller dose $(1 \mathrm{gm}$.), and that even after the $2 \mathrm{gm}$. dose there are only slight signs of pain and sweating; that while the passages from the bowels are few in number the total amount of feces is large; finally, that the drug has the advantage of being cheap.

That the toxic line is closely approached in giving barium chloride in the dose of $2 \mathrm{gm}$. intravenously, is shown by experiment 31 , in which a gelding weighing 900 pounds received two $2 \mathrm{gm}$. doses intrajugularly about $2 \frac{3}{4}$ hours apart, when death occurred from heart failure in nine minutes after the second dose.

* Jour. of Comp. Med. and Vet. Archives, Jan, and Feb, 1899. 


\section{Magnesium.}

(The metal is not used in medicine.)

Magnesil Solphas. Magnesium Sulphate. $\mathrm{MgSO}_{4}+7 \mathrm{H}_{2} \mathrm{O}$.

(U. S. \& B. P.)

Synonym.-Epsom salt, sal amarum, sal Epsomense, sal anglicum, sulfas magnesicus, etc., E.; magnesia sulfurica, P. G.; sulfate de nugnésie, sel d'Epsom, sel de sedlitz, sel 1 amer, Fr.; bittersalz, schwefelsanre magnesia, G.

Derivation.-It is obtained from native dolomite, a double carbonate of magnesium and calcium, or magnesite $\left(\mathrm{MgCO}_{3}\right) . \quad \mathrm{IgCO}_{3}+\mathrm{H}_{2} \mathrm{SO}_{4}=\mathrm{MgSO}_{4}+\mathrm{H}_{2} \mathrm{O}+\mathrm{CO}_{2}$.

Properties. - Small, colorless, rhombic prisms, or acicular crystals, without odor, and having a cooling, saline and bitter taste; slowly efflorescent in dry air; soluble in $\mathbf{1 . 5}$ parts of water; insoluble in alcohol ; reaction neutral.

Incompatilles.-Lime water, alkaline carbonates, phosphoric acid, phosphates, silver nitrate and lead acetate.

Dose.-H., laxative, ₹ii.-iv. (60.-120.); C., purgative, \#bi.-ii. (500.-1,000.); laxative, $₹$ iii.-iv. (90.-120.); Calves, 亏ii.-iii. (60.-90.); Sh., 3 iv.-vi. (120.-180.); D., 3 i.-iv. (4.-15.).

Action Internal.-Epsom salt is the best purgative for general purposes in the treatment of cattle and sheep. Its mode of action is similar to that described under sodium sulphate. Briefly, magnesium sulphate causes purgation by increasing intestinal secretion, retarding absorption of fluid from the bowels, and probably by stimulating peristalis. The salt moves the bowels, in the case of the larger animals, usually within twelve or fifteen hours. It is indirectly a cholagogue, by sweeping bile out of the intestines.

Epsom salt is absorbed to some extent, and is eliminated by the kidneys and sweat glands, increasing the secretions of these organs, especially when the dose is small.

Summary. - Hydragogue and cholagogue cathartic. Feeble diuretic and diaphoretic.

Uses.-For uses the reader is referred to Sodium Sulphate (p. 136), as they are almost identical, although the latter salt is usually preferable in veterinary medicine. 


\section{Magnesil Carbonas. Magnesium Carbonate.}

$$
\left(\mathrm{MgCO}_{3}\right)_{4} \cdot \mathrm{Mg}(\mathrm{OH})_{2}+5 \mathrm{H}_{2} \mathrm{O} \text {. (U. S. P.) }
$$

Synonym.-Magnesii carbonas ponderosa vel levis, B. P.; magnesia alba, magnesia hydrico-carbonica, carbonas magnesicus, carbonate of magnesia, E.; magnesia carbonica, P. G.; carbonate de magnésie, magnésie blanche, Fr.; weisse magnesia, G.

Derivation.-Mix concentrated, boiling, aqueous solutions of magnesiun sulphate and sodium carbonate, and evaporate.

$5 \mathrm{MgSO}_{4}+5 \mathrm{Na}_{2} \mathrm{CO}_{3}+\mathrm{H}_{2} \mathrm{O}=\left(\mathrm{MgCO}_{3}\right)_{4} \mathrm{Mg}(\mathrm{OH})_{2}$ $+5 \mathrm{NA}_{2} \mathrm{SO}_{4}+\mathrm{CO}_{2}$. Purified by digestion with water, filtration and drying.

Properties.-Slight, white, friable masses (heavy magnesium), or a light, white porvder (light magnesium), withont odor, and having a slightly earthy taste; permanent in the air ; almost insoluble in water, to which, however, it imparts a slightly alkaline reaction; insoluble in alcohol.

Dose.-Foals and calves, 3 i.-ii. (4.-8.); D., gr.vo-3i. $(.3-4$.$) .$

\section{Magnesia. Magnesia. MgO. (U.S. P.)}

Synonym.-Magnesia le vis, light magnesia, B.P.; calcined magnesia, magnesia calcinata, E.; magnesia usta, P. G.; magnésie, magnésie calcinée, Fr.; gebrannte magnesia, G.

Derivation.-Heat magnesium carbonate. $4\left(\mathrm{MgCO}_{3}\right)$. $\mathrm{Mg}(\mathrm{OH})_{2}+5 \mathrm{H}_{2} \mathrm{O}=5 \mathrm{MgO}+6 \mathrm{H}_{2} \mathrm{O}+4 \mathrm{CO}_{2}$. Water and carbon dioxide are driven off and magnesia $(\mathrm{MgO})$ is left.

Properties.-A white, very light, very fine powder, withont odor, and having an earthy, but not a saline taste. On exposure to the air it absorbs moisture and carbon dioxide; almost insoluble in water; insoluble in alcohol. (.3-4.).

Dose._Foals and calves, 3 i.-ii. (4.-8.); D., gr.v.- 3 i. 
Magnesta Ponderosa. Heavy Magnesia. MgO. (U.S. P.)

Derivation.-Made from light magnesia by trituration with alcohol, drying and pulverizing.

Properties. A white, dense, very fine powder. Only differs in tests from light magnesia in that it does not readily unite with water to form a gelatinous liydrate.

Dose.-Foals and calves, ji.-ii. (4.-S.); D., gr.v.- żi. (.3-4.).

ACTION OF MAGNESIUN CARBONATES AND OXIDES.

Internal.-Alimentary Canal.-These salts are antacid and neutralize abnormal acidity, due to fermentation, when exhibited after a meal. The carbonate also exerts a sedative action in liberating carbon dioxide in the stomach, and both the carbonate and oxide unite with the gastric juice to form chlorides, lactates and bicarbonates. These compounds are mild, saline purgatives.

Blood and Urine.-The oxide and carbonate of magnesium alkalize the blood and urine, and are slight diuretics. They resemble potassinm and sodium bicarbonates, as antacids, but are milder because feebly absorbed.

Uses.-Phillip's milk of magnesia is a good laxative and antacid preparation for puppies. One or more teaspoonfuls may be added to milk, which will be taken voluntarily. Magnesia is a nseful remedy for foals and calves affected with intestinal indigestion, tympanites and acid diarrhøe. It may be given to advantage in powder: magnesia and rhubarb, 2 drachms each; with ginger, one drachm. This dose should be administered in milk or flour gruel. Mragnesia may give rise to intestinal concretions if its use is persisted in for a considerable period.

Magnesium carbonate and oxide are antidotes to mineral acids, oxalic acid, salts of mercury, arsenic and copper, and alkaloids, by alkalizing the gastric contents and rendering these bodies insoluble. Arsenic antidote is kept on hand at drug stores and is made by adding solution of ferric 
sulphate to an aqueous mixture of magnesia (see ferri oxidum hydratum cum magnesia, p. 196).

\section{Aluminum.}

(The metal is not used as medicine.)

Alumen. Alum. $\mathrm{Al}_{2} \mathrm{~K}_{2}\left(\mathrm{SO}_{4}\right)_{4}+24 \mathrm{H}_{2} \mathrm{O} . \quad$ (U. S. \& B. P.)

Synonym.-Potassium alum, aluminum and potassium sulphate, sulphate of aluminium and potassium, E.; alun, sulphate d'alumine et de potasse, Fr.; alaun, kalialann, G.

Derivation.-From alum slate, clay, shale or schist, a native mixture of aluminium silicate and iron sulphide. This is roasted and exposed to the air, when the sulphur is oxidized into sulphuric acid and combines in part with aluminium and iron to form sulphates. The mass is lixiviated with water, and aluminum and iron sulphates together with sulphuric acid are recovered in solution. The solution is concentrated and to it is added potassium chloride. The double sulphate of potassium and aluminum (alum) is formed, which crystallizes out on cooling, while potassium sulphate and ferric chloride remain as byeproducts. Alum is purified by recrystallization.

Properties.-Large, colorless, octohedral crystals, sometimes modified by cubes, or in crystalline fragments; withont odor, but having a sweetish and strongly astringent taste. On exposure to the air the crystals are liable to absorb ammonia and acquire a whitish coating. Soluble in 9 parts of water. It is also soluble in warm glycerin; insoluble in alcohol ; reaction acid.

Incompatibles.-Iron, lead and mercury salts, alkalies, lime, tartrates and tannic acid.

Dose.-H. \& C., 3 ii.-iv. (8.-15.); Sh. \& Sw., gr.xx.-3i. (1.3-4.); D., gr.v.-x. (.3-.6); emetic, D., 3 i. (4.). 
Alumen Exsiccatum. Dried Alum. $\mathrm{Al}_{2} \mathrm{~K}_{2}\left(\mathrm{SO}_{4}\right)_{4}$. (U.S.\& B. P.)

Synonym.-Alumen ustum, burnt alum, E.; alum calcine (desseché brulé), Fr.; gebrannter alaun, G.

Derivation.-Heat $100 \mathrm{Gm}$. of alum moderately until aqueous vapor ceases to be disengaged, and the product is reduced to $55 \mathrm{Gm}$.

Aldmini Hydras. Aluminum Hydrate. $\mathrm{Al}_{2}(\mathrm{OH})_{6}$. (U. S. P.)

Synonym.-Aluminum hydroxide, hydrated alumina, E.; alumine, Fr.; thonerdehydrat, reine thonerde, G.

Derivation.-Alum, $100 \mathrm{Gm}$; sodium carbonate, $100 \mathrm{Gm}$;; water, a sufficient quantity. Mix hot, boiling solutions of alum and sodium carbonate. Precipitate strained, washed, and dried.

Properties.-A white, light, amorphous powder; odorless and tasteless; permanent in dry air; insoluble in water or alcohol.

Dose._Same as alum.

Alumini Sulphas. Aluminum Sulphate. $\mathrm{Al}_{2}\left(\mathrm{SO}_{4}\right)_{3}+16 \mathrm{H}_{2} \mathrm{O}$. (U. S. P.)

Synonym.-Sulphate of alnminum, E.; sulphate d'alumine, Fr.; schwefelsäure thonerde, G.

Derivation.-Aluminum hydroxide $\left[\mathrm{Al}(\mathrm{OH})_{3}\right]$ is dissolved in diluted sulphuric acid, and the solution is filtered and evaporated to dryness.

Properties.--A white, crystalline powder, without odor, having a sweetish and afterwards astringent taste; permanent in the air; soluble in 1.2 parts of water; insoluble in alcohol ; reaction acid.

Dose.-Same as alum. 


\section{Aluminum Salts.}

Action External.-Dried alum is a superficial caustic, in contact with raw surfaces, on account of its affinity for water. It is only used externally. Alum has no action on unbroken skin, but applied to mucous membranes or denuded parts it is antiseptic and astringent; coagulates albumin of discharges; combines with albumin of the tissues, which it coagulates; squeezes blood ont of the vessels; reduces inflammation and makes the part whiter, tongher and denser. Alum is an hrmostatic, stopping bleeding by compression of the structures surrounding the vessels, and by causing blood to clot. Alum coagulates casein and gelatin in the presence of an alkali.

Action Internal. - Stomach and Intestines. - Enormous doses of alum produce gastro-enteritis, while large doses cause vomiting in carnivora. All the secretions are diminished in the alimentary canal, and constipation ensues, unless the dose is excessive. Traces of alum are said to appear in the urine, but since the sult coagulates albumin, absorption cannot occur to any extent. Alum does not, therefore, occasion any astringent action in the body outside of the digestive tract, and is excreted by the bowels.

Uses External. - Alum is employed mainly for local surgical purposes. In arresting slight hemorrhages it may be applied in saturated solution on absorbent cotton pledgets, or in the form of burnt alum dusted upon the bleeding surface. Epistaxis may be controlled by the injection of a strong solution into the nostrils, or by iusufflation of burnt alum. Alum is sometimes used on granulating surfaces of indolent ulcers, or wounds, as a slight caustic, stimulant and antiseptic. It can be employed alone, or as a dusting powder, containing: alum, 1 part; charcoal, 4 parts; and salicylic acid, 2 parts. Alumen exsiccatum will often prevent the escape of synovia from small punctured wounds when applied to their apertures. Solutions

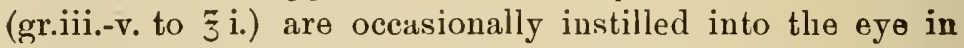
conjunctivitis, but alum is not generally so satisfactory as 
boric acid, zinc sulphate, or silver nitrate, in this disease. Alum erystals may be applied with profit to granular lids. Alum is used more frequently in the treatment of stomatitis, or apthous sore mouth. It is also beneficial in ptyalism. A 5 per cent. solution may be utilized to touch the inflamed oral parts by means of a swab. A spray of the same strength is serviceable for the cure of laryngitis and bronchitis in dogs.

A 2 per cent. solution is appropriate as an injection for otorrhœa, or canker of the ear, attacking dogs. A similar solution will relieve leucorrhœa, pruritus vulvæ, and prolapsus ani. The following combination, containing dried alum, forms an excellent preparation for application to dead tissue. It causes sloughing of the necrotic mass and is indicated when the use of the knife is inadmissible.

$\mathrm{P}$

Alumenis exsiccati .......... 25.0

Acidi arsenosi.............. 15.0

Acidi carbolici.............. 10.0

Cerati .................... 25.0

M. et fiat unguentum.

Uses Internal.-Alum is a prompt, safe and non-depressing emetic for dogs. It is suitable in poisoning, or when the secretions are excessive in laryngitis or bronchitis. Teaspoonful doses should be given in solution in syrup every 15 minutes in these latter diseases, until vomiting occurs. Aluminum hydrate is an antacid and astringent. It combines with acid in the stomach (antacid) and goes into a soluble form when it acts as an astringent in the bowels. It is, therefore, more applicable for internal use in the treatment of diarrhœa and dysentery. Other astringents, such as tannic acid in some form, lead acetate, or copper sulphate, are, however, usually more valuable in diarrhœeal disorders. Aluminum sulphate may be used interchangeably with alum, externally or internally. 


\section{Cerium.}

(Cerium is not employed medicinally.)

Ceril Oxalas. Cerium Oxalate. $\mathrm{Ce}_{2}\left(\mathrm{C}_{2} \mathrm{O}_{4}\right)_{3}+9 \mathrm{H}_{2} \mathrm{O}$.

(U. S. \& B. P.)

Synonym.-Cerous oxalate.

Derivation.-Precipitate a solution of ammonium oxalate with a soluble salt of cerium.

Properties.-A white, granular powder, without odor or taste, and permanent in the air; insoluble in water, alcohol or ether.

Dose.-D., gr.iii.-v. (.18-.3).

Action and Uses.-The physiologrical details concerning the action of cerium are unknown. It is useful in relieving vomiting of a reflex or nervous character, and is often combined with bismuth salts. Cerium oxalate is soluble in the gastric juice.

\section{SECTION IV.}

Plumbum, Argentum, Zincum, Cuprum and Bismuthum.

\section{Plumbum.}

(Lead is not used in the metallic state in veterinary medicine, except as a last resort.)

Plumbi Oxidur. Lead Oxide. PbO. (U. S. \& B. P.)

Synonym.-Litharge, E.; lithargyrum, P. G.; bleiglätte, G. Derivation.-Made by roasting lead in the air.

Properties.-A heavy, yellowish or reddish-yellow powder, or minute scales, without odor or taste. On exposure to the air it slowly absorbs moisture and carbon dioxide. Almost insoluble in water ; insoluble in alcohol. Reaction faintly alkaline. Lead oxide is only valuable for its preparations. 
PREPARATION.

Emplastrum Plumbi. Lead Plaster. (U. S. \& B. P.)

(Diachylon Plaster.)

Lead oxide, 32; olive oil, 60; water, a sufficient quantity. Basis of other preparations.

Plombi Acetas. Lead Acetate. $\mathrm{Pb}\left(\mathrm{C}_{2} \mathrm{H}_{3} \mathrm{O}_{2}\right)_{2}+3 \mathrm{H}_{2} \mathrm{O}$.

(U. S. \& B. P.)

Synonym.-Sugar of lead, E.; sel (sucre) de saturne, Fr.; essigsaures bleioxyd, bleizucker, $\mathrm{G}$.

Derivation. - Heat lead oxide in acetic acid and water.

$\mathrm{PbO}+2 \mathrm{HC}_{2} \mathrm{H}_{3} \mathrm{O}_{2}+2 \mathrm{H}_{2} \mathrm{O}=\mathrm{Pb}\left(\mathrm{C}_{2} \mathrm{H}_{3} \mathrm{O}_{2}\right)_{2}+3 \mathrm{H}_{2} \mathrm{O}$.

Lead acetate crystallizes on cooling.

Properties.-Colorless, shining, transparent, monoclinic prisms or plates, or heavy, white crystalline masses, or granular crystals, having a faintly acetous odor and a sweetish, astringent, afterwards metallic, taste. Efflorescent and absorbing carbon dioxide on exposure to the air. Soluble in 2.3 parts of water and in 21 parts of alcohol. Reaction slightly acid.

Incompatibles.-Hard water, alkalies, mineral acids and salts, potassium iodide, opium, vegetable astringents and albuminous liquids.

Dose.-H. \& C., 3 i. (4.); Sh. \& Sw., gr.xv.-xx. (1.-1.3); D., gr.i.-ii. (.06-.12).

\section{PREPARATIONS.}

Made from lead acetate in which lead exists as the subacetate, $\mathrm{Pb}_{2} \mathrm{O}\left(\mathrm{C}_{2} \mathrm{H}_{3} \mathrm{O}_{2}\right)_{2}$.

\section{Liquor Plumbi Subacetatis. Solution of Lead Subacetate.}

(U. S. \& B. P.)

Synonym.-Goulard's extract, acetum plumbicum, acetum Saturni, plumbum hydrico-aceticum solutum, subacetas plumbicus liquiclus, E.; liquor plumbici subacetici, P. G.; sous-acetate de plomb liquide, extract de Goulard de vinaigre, plomb, Fr.; bleiessig, G.

Composition.-An aqueous liquid, containing in solution about 25 per cent. of lead subacetate (approximately), $\mathrm{Pb}_{2} \mathrm{O}\left(\mathrm{C}_{2} \mathrm{H}_{3} \mathrm{O}_{2}\right)_{2}$.

Liquor Plumbi Subacetatis Dilutus. Diluted Solution of Lead Subacetate. (U. S. \& B. P.)

Synonym.-Lead Water. Lead Subacetate, 3; water to make 100. 
Ceratum Plumbi Subacetatis. Cerate of Lead Subacetate. (U. S. P.) Solution of lead subacetate, 20 ; camphor cerate, 80 .

\section{Plumbi Carbonas. Lead Carbonate. $\left(\mathrm{PbCO}_{3}\right)_{2} \mathrm{~Pb}(\mathrm{OH})_{2}$.} (U. S. \& B. P.)

Synonym.-White lead, E.; céruse, Fr.; bleiweiss, G.

Derivation.-Expose lead to the action of acetic acid vapor and air with carbonic dioxide.

$4 \mathrm{~Pb}+2 \mathrm{HC}_{2} \mathrm{H}_{3} \mathrm{O}_{2}+2 \mathrm{O}_{2}+2 \mathrm{CO}_{2}=\left(\mathrm{PbCo}_{3}\right)_{2} \mathrm{~Pb}$ $(\mathrm{OH})_{2}+\mathrm{Pb}\left(\mathrm{C}_{2} \mathrm{H}_{3} \mathrm{O}_{2}\right)_{2}$.

Properties. - A heavy, white, opaque powder, or a pulverulent mass, without odor or taste. Permanent in air. Insoluble in water or alcohol. Used ouly externally.

Plumbi Nitras. Lead Nitrate. $\mathrm{Pb}\left(\mathrm{NO}_{3}\right)_{2}$ (U. S. \& B. P.)

Synonym.-Salpetersaures bleioxyd, bleisalpeter, G.

Derivation.-Dissolve lead in nitric acid.

Properties.-Colorless, transparent, octohedral crystals, or white, nearly opaque crystals; without odor, and having a sweetish, astringent, and afterwards metallic taste; permanent in the air; reaction acid; soluble in 2 parts of water; almost insoluble in alcohol. Only used externally in 1 per cent. solution as an astringent and deodorant in gangrenous surfaces, etc.

Plumbi Iodidum. Lead Todide. $\quad \mathrm{Pb} \mathrm{I}_{2}$. (U. S. \& B. P.)

Derivation-Treat solution of lead nitrate with that of potassium iodide.

$\mathrm{Pb}\left(\mathrm{NO}_{3}\right)_{2}+2 \mathrm{KI}=\mathrm{PbI}_{2}+2 \mathrm{KNO}_{3}$. Dry the precipitate.

Properties.-A heavy, bright yellow powder, without odor or taste; permanent in the air; soluble in about 2,000 parts of water; very slightly soluble in alcohol; used only externally. 
PREPARATION.

Unguentum Plumbi Iodidi. Ointment of Lead Iodide. (U. S. \& B. P.)

Lead iodide, 10; Denzoinated lard,90. Employed externally to absorb glandular swellings,

\section{ACTION OF LEAD SALTS.}

External.-Lead salts act like other astringents in contracting tissues and vessels, and in coagulating albumin of exndations, but unlike other astringents, they are sedatives and not irritants locally.

Internal.-Soluble salts of lead in concentrated solution -and at times insoluble salts-cause gastro-enteritis in large doses. Lead saits are absorbed in medicinal doses whether taken in a soluble or insoluble state. The chemical form of the lead componnd at the time of absorption is unknown. Lead is deposited in the tissues and very slowly eliminated in the urine and intestinal mucus. Lead salts have a marked astringent action on the entire digestive canal and diminish secretions accordingly. It is doubtful whether lead salts remotely contract blood vessels after absorption. There are other agents-notably ergot-which possess much greater power for this purpose.

Toxicology.-Poisoning not infrequently occurs in animals at pasture, from eating paint, sheet lead, or products of lead works. The drinking water may be contaminated with lead, especially from new pipes, or lead receptacles in which water has been standing. Hard water is not affected by lead pipes, since an insoluble crust of lead phosphate and sulphate is deposited upon the interior of the pipes. There is an acute and chronic form of poisoning. The first is caused by single large doses of solinble lead salts, and is characterized by gastro-enteritis and colic; sometimes convulsions, coma, paralysis and death. The frees are colored black with lead sulphide; the vomitus is white from lead chloride. Three groups of symptoms may be briefly tabulated, which occur to a greater or less degree in chronic lead poisoning: 


\section{DIGESTIVE SYMPTOMS.}

Lead line on gums.

Thirst.

Colic.

Abdomen retracted, or "tucked

Constipation.

Anorexia.

up."

NERVOUS SYMPTOMS.

Paralysis of tendons-extensorsof extremities.

Animals stand on knees before.

Animals stand on toes behind.

Convulsions.

General paralysis.

Wasting of muscles.

Coma.

Delirium.

Amaurosis.

GENERAL SYMPTONS.

Dyspnoea.

CEdema.

Pulse accelerated.

Emaciation.

General debility.

Anæmia.

Animals die in chronic poisoning from paralysis of the respiratory muscles, or in convulsions. The lead line on the margin of the gums, at their junction with the teeth, is due to sulphuretted hydrogen in the mouth, acting upon the lead deposited in the gums, and forming lead sulphide. The treatment consists in remoring the calise, relieving the symptoms and in hastening elimination. Potassium iodide eliminates lead in a soluble form into the bowels and kidneys. Magnesium sulphate converts lead into an insoluble sulphate in the intestines and then sweeps out the salt. The stomach pump or emetics should be employed in acute poisoning. Alum is the best emetic. This treatment should be followed by the administration of opium and Epsom salts.

Administration.-Lead acetate is given to the larger animals in solution or ball; to the smaller patients in pill; to young animals in solution in milk.

Uses External.-Lead acetate is useful in the treatment of skin diseases, as weeping eczema and erythema, and in excoriations, blistered surfaces, bruises, strains, and burns. An efficient lotion having an astringent and sedative action in such conditions, contains : laudanum, 1 part; Goulard's extract, 4 parts ; and oil, glycerin or water, 16 parts. The 
"white lotion" of veterinary medicine is made by adding 3 drichms each of lead acetate and zinc sulphate to a pint of water. It is a favorite astringent, sedative and antiseptic application for strains and "scratches." The stronger solution of lead subacetate should not be employed extensively on raw surfaces or mucous membranes undiluted. The diluted solution of lead subacetate may be used as an injection for leucorrhoea. Lead acetate should be diluted with 20 to 40 parts of vinegar or water. Lead acetate is not suitable for collyria, if there is any ulceration of the cornea, because a permanent film may be deposited and obscure the sight. Lead iodide, in 10 to 20 per cent. ointment with petrolatum, has proven of service in aiding resolution of induration or caked condition of the udder in acute mammitis if applied twice daily with thorough massage before suppuration has set in.

Uses Internal.-Lead acetate is serviceable in the treatment of diarrhoa, dysentery, and hemorrhage from the stomach and bowels. It is frequently prescribed in these diseases with opium.

Argentum.

Argenti Nitras. Silver Nitrate. $\mathrm{AgNo}_{3} \cdot \quad$ (U. S. \& B. P.)

Derivation.-Dissolve silver in nitric acid with heat.

$3 \mathrm{Ag}_{2}+6 \mathrm{HNO}_{3}=6 \mathrm{AgNO}_{3}+3 \mathrm{H}_{2}$. Evaporate and crystallize.

Properties.-Colorless, transparent, tabular, rhombic crystals, becoming gray, or grayish-black on exposure to light in the presence of organic matter; without odor, but having a bitter, caustic and strongly metallic taste; reaction neutral; soluble in 0.6 part of water and 26 parts of alcohol.

Incompatibles. - Alkalies and their carbonates, acids (except nitric and acetic), chlorides, potassium iodide, astringent infusions and solutions of arsenic. 
Dose.-H. \& C., gr.v.-x. (.3-.6); Sh. \& Sw., gr.i.-ii. (.06-.12); D., $\operatorname{gr} \cdot \frac{1}{8}-\frac{1}{2}(.008-.03)$.

Argentr Nitras Dilutus. Diluted Silver Nitrate. (U. S. P.)

Synonym.-Argenti et potasii nitras, B. P.; mitigated caustic, E.; argentum nitricum crystallizatum, P. G.; azotas (nitras) argenticus, azotate d'argent, nitre lunaire, Fr.; salpetersaures silberoxyd, silbersalpeter, G.

Derivation.-Melt silver nitrate, 30, with potassium nitrate, 60 , in a crucible at as low a temperature as possible. Mix and cast into suitable moulds.

Properties.-A white, hard solid, generally in the form of pencils or cones of a finely granular fracture; becoming gray or grayish-black on exposure to light in presence of organic matter; odorless, having a canstic, metallic taste, and nentral reaction. Each of its constituents soluble in water and alcohol to the extent mentioned under Argenti Nitras and Potassii Nitras. Used only externally.

Argexti Nitras Fusus. Moulded Silver Nitrate. (U. S. P.)

Synonym.-Lunar caustic, lapis infernalis, azotas (nitras) argenticus fusus, E.; argentum nitricum fusum, P. G.; azotate d'argent fondu, pierre infernale, Fr.; höllenstein, geschmolzenes salpetersaures silberoxyd, G.

Derivation. - Melt silver nitrate, 100, with hydrochloric acid, 4 , at as low a temperature as possible. Mix and pour into suitable moulds.

Properties.-Practically same as above. Used only externally. Silver oxide, cyanide, and iodide are official, but unimportant in veterinary medicine.

\section{ACTION OF SILVER NITRATE.}

External.-Silver nitrate is more caustic in action than any of the lead, copper or zinc salts (except the chloride). When applied externally in the pure state to a mucous membrane, or a raw surface, it forms a white coating of coagrulated albumin. This coating limits the further action 
of the salt, so that lunar caustic is always superficial and localized in its effect. Silver nitrate is the caustic in most common use, since it produces a more healthy condition in a granulating wound after its application and separation of the eschar. In acting thus more favorably than other agents of its kind, it may be said to possess a local alterative effect. In dilutiou, silver nitrate is stimulant, astringent, antiseptic and caustic, according to its strength.

Internal.-Silver nitrate is probably precipitated to it considerable extent by the hydrochloric acid of the gastric juice, as the chloride. Some of it is absorbed, howerer, possibly in combination with albumin and peptones. Silver is deposited in the tissues in the form of the oxide, causing dark staining of the skin in man. These stains, occurring when silver nitrate comes in direct contact with the skin, can be removed by a solution containing potassium cyanicle, $2 \frac{1}{2}$ drachms; iodine, 15 grains ; and water, 3 ounces. Large doses of silver nitrate canse gastro-enteritis with nervons symptoms - paralysis and couvulsions - and death from. depression of the respiratory centres. Common salt is the antidote, both externally and internally, forming the insoluble chloride. In addition to salt, opium and demulcents should be exhibited in acute poisoning. Silver nitrate in medicinal doses has probably a local stimulating, astringent and alterative action. on the mucous membrane of the stomach; to a less degree on the bowels. Elimination occurs in part throngh the agency of the liver and intestines. A chronic form of poisoning by silver nitrate (argyrism), is seen in man, following its continned use, and is accompanied by pigmentation of the skin, marasmus, chronic indigestion, with wasting of the testes and mammary glands. A similar condition has been produced in animals, associated with anorexia, weakness, anæmia and emaciation.

Uses External.-An aqueous solution (gr.iii. to 3 i.) is most valuable in the treatment of catarhal conjunctivitis, while a stronger preparation (gr.x. to $\bar{j}$ i.) is employed for purulent 
conjunctivitis, as a stimulant, astringent and antiseptic collyrium. When strong solutions, like the latter, are used, the eye should immediately be flooded with a solution of common salt and water to precipitate the excess of silver nitrate as the insoluble chloride and thus prevent further irritation. Lunar caustic is applied in pencil form to ulcerated surfaces. When these surfaces are touched lightly the canstic stimulates sluggish granulations; when more lieavily, it destroys exuberant granulations. In 2 to 4 per cent. solution, silver nitrate is canstic to mucous menbranes; in $\frac{1}{2}$ per cent. solution it is stimulant and astringent to mucous membranes.

Boils may be aborted by painting them with a saturated solution of silver nitrate. Pruritus ani, or vulvæ, is relieved by painting the parts several times daily with a 1 per cent. solution. A solution (gr.iii. to $\bar{j} i$.) may be used in the form of spray in the treatment of pharyngitis and laryugitis in the dog. In catarrh of the external ear, so common in dogs, the canal should be swabbed with a 5 per cent. watery solution of silver nitrate after thorough eleansing with sther, or alcohol and naphtha, to remove dirt and sebaceous inatter.

Fissures in the skin occurring in sore teats of cows are cured by the application of fused silver nitrate.

Uses Internal.-The crystals should only be employed internally, to insure purity. Silver nitrate is not of much value for internal use except in the digestive tract. Pills containing the silver salt are sometimes given to dogs with diarrhœa and ulcer of the stomach. Dysentery may be treated by enemata containing 12 grains of silver nitrate to the ounce of water. If this treatment is followed by much irritation, injections of salt and water should be used afterwards. 


\section{Protargol. (Non-official.)}

Protargol was first introduced into medicine by Prof. Neisser, in 1897, as a local remedy for gonorrhœa in man. It is a fine, yellowish-brown, soluble powder, a combination of a protein substance with silver; odorless, and possessing a strong metallic taste.

Protargol has recently superseded silver nitrate (which contains 63.5 per cent. of silver) to a considerable extent in medicine because, containing less silver ( 8.3 per cent.). protargol is decidedly less irritating, is not precipitated by albumin or solutions of sodium chloride, does not discolor the skin and more than equals silver nitrate in certainty and.efficiency of action.

Protargol is particularly applicable in veterinary medicine as a bland but powerfully penetrating antiseptic and mild astringent in the treatment of inflammatory conditions of the conjunctival membranes. The drug does not cause the pain, redness, swelling and lachrymation which follow the use of silver nitrate; nor does it lead to the formation of fibrinous coagula and the production of false membranes and opacities of the cornea seen after the application of silver nitrate.

A 10-per-cent. solution of protargol induces less flushing of the eye and discomfort than a 1-per-cent. solution of silver nitrate, and the irritation of a 2 or 4 per cent. solution is not, as a rule, more than would be produced by a one-half grain to the ounce solution of zinc sulphate (Cheney).

Protargol is indicated in acute catarrhal and purulent conjunctivitis in from one-half to 10 per cent. aqueous solutions; usually in one-half per cent. solution in the catarrhal form, two or three times daily, applied with a camel's hair brush or by instillation; and in the purulent variety, in 2 or 4 per cent. solution with a pledget of absorbent cotton on a probe, or with a camel's hair brush, in conjunction with frequent boric acid irrigations. This new silver combina- 
tion has also been used with reported success (and the use might apply to canine practice) in human medicine as a non-irritating astringent and antiseptic agent internally in $.5 \mathrm{gm}$. doses, twice or thrice daily, in pills, for the relief of hæmorrhages and ulcerations of the alimentary canal, in diarrhoa and in purulent inflammation of the genito-uriuary tract.

\section{Argyrol.}

Argyrol represents one of the latest of the numerous organic silver compounds, this preparation containing as much as 30 per cent. of the metal combined with a proteid substance obtained from wheat. It occurs as a brownish powder, soluble in less than its own weight of water, forming dark-brown solutions which stain clothing black, but the stains may be removed by ordinary lamndry processes. Like protargol, it is not precipitated by the salts of the tissues, nor does it coagulate albumin, so that its action is not neutralized by the tissnes-as is the case with silver nitrate, and thus, mlike the latter, it possesses a penetrating power when applied locally. Argyrol is used in from 2 to 20 per cent. aqueous solntions for the same purposes to which protargol is adapted. It bids fair to supersede the latter, however, since it contains more silver and is therefore a more powerful antiseptic and yet non-irritating.

\section{Soluble Silver.}

Soluble silver, known al'so as Colloidal Silver, or more commonly as Collargol, is an allotropic form of metallic silver wholly soluble in water, and discovered by Lea abont 1890. It may be used intravenously, subcutaneonsly, by inunction (as Crede's ointment, see below), and by the mouth, if first dissolved in the proportion of five parts of collargol with one part of white of egg in one hundred parts of water; or it may be giren in pill with sugar of milk. When given intravenously-which is the most effective mode of administration-one injection may suffice, but if it 
does not cause immediate improvement in the symptoms, several doses may be thus given at six-hour intervals. Soluble silver has recently proven successful in many cases of general iufection, where it appears to either kill or inhibit the growth of staphylococci and streptococci. It is certainly worthy of trial in veterinary medicine in this field, where it has accomplished notewortlyy results.

Puerperal septicæmia, mastitis, extensive cellulitis, fetid bronchitis, pneumonia, influenza, ento and pericarditis, deep suppurations, phlebitis, suppurating nasal sinusitis, empyema and other bacterial infections have yielded to the systemic and local influence of soluble silver in lunman medicine. Its expense is the only objection to its free employment in animal practice, and this applies to all the new organic silver compounds. A rigor often occurs from one to four hours after the injection of collargol, but no other ill effects have been noted. When the silver can be used locally (in local infections) it is also effective and may or may not be at the same time given intravenously, its desirability by the latter mode depending on the degree of general infection. The dose intravenously is $5 \frac{1}{2}-1$ (2.-4.) for horses; dogs, そr.1-2 (0.6-0.12), given in 2 to 5 per cent. aqueous solution. It is injected into the tissues as in abscess, in 1 per cent. solutions. It is soluble in 20 parts of water, which should be distilled or boiled, and solutions in water may be kept for months in brown bottles. Solutions, from having a clear, brown color, become gray and turbid when decomposed. 1-3000 aqueous solutions are appropriate for use on mucous membranes or cavities of the body.

Crede's Ointment, made by incorporating collargol with lard and wax to the extent of 15 per cent., has given good results when rubbed for thirty minutes into the skin-which has previonsly been scrubbed with soap, water and alcolool -in the treatment of local and even general infectious. It often arrests the formation of boils, threatened suppuration of glands, lymphangitis, phlebitis, cellulitis and mistitis. The dose by inunction is $\frac{1}{2}$ to 1 ounce for horses, $\frac{1}{2}$ to 1 
drachm for dogs. Collargol appears to be non-toxic when given intravenously or by innnction, if used with reasonable care.

\section{Zincum.}

(Zinc is not used in Medicine in the metallic state.)

\section{ZiNci Chloridum. Zinc Chloride. $\mathrm{ZnCl}_{2}$ * (U.S. \& B. P.)}

Derivation.-Dissolve zinc in hydrochloric acid by boiling. The solution contains the zinc chloride with chlorides of iron and lead as impurities. These are precipitated by adding first nitric acid then zinc carbonate. Filter and finally evaporate. $\mathrm{Zn}_{2}+4 \mathrm{HCl}=2 \mathrm{Zn} \mathrm{Cl}_{2}+2 \mathrm{H}_{2}$.

Properties.-A white, granular powder, or porcelainlike masses, irregular or moulded into pencils ; odorless ; of such intensely caustic properties as to make tasting dangerous unless the salt be dissolved in much water, when it has an astringent, metallic taste; very deliquescent; reaction acid; soluble in about 0.3 part of water; very soluble in alcohol.

Liquor Zinci Chloridi. Solution of Zine Chloride.

(U. S. \& B. P.)

Derivation.-Made as above with the addition of water. It contains about 50 per cent., by weight, of zinc chloride.

Properties.-A clear, colorless liquid, odorless, having a very astringent, sweetish taste and an acid reaction. Spec. gr. about 1,535 at $15^{\circ} \mathrm{C}$. (59 $9^{\circ} \mathrm{F}$.).

Toxicology.-Zinc chloride is a powerful irritant if swallowed in any degree of concentration, and will, therefore, produce gastro-enteritis. Emetics or the stomach pump should be used, followed by demulcents and sodium bicarbonate.

Uses.-Zinc chloride is employed in a paste made into small pieces with flour; or on lint soaked in a saturated 
solution, dried, and introduced under the skin about the base of tumors to cause their destruction by sloughing. It is employed in the form of pencils on unhealthy, granulating surfaces, as in "foot rot," and injected in strong solution into fistulons tracts to destroy their walls. It is not nsed internally.

\section{ZincI Suliphas. Zinc Sulphate. $\mathrm{ZnSo}_{4}+7 \mathrm{H}_{2} \mathrm{O}$.}

(U. S. \& B. P.)

Derivation.-Prepared by dissolving zinc in sulphuric acid. $\mathrm{Zu}_{2}+2 \mathrm{H}_{2} \mathrm{SO}_{4}=2 \mathrm{ZuSO}_{4}+2 \mathrm{H}_{2}$.

Iron and tin exist as impurities, and are removed by chlorine solution and zinc carbonate.

Properties. - Colorless, transparent, rhombic crystals, withont odor, and having an astringent, metallic taste. Efflorescent in dry air; reaction acid ; soluble in 0.6 part of water, in 3 parts of glycerin; insoluble in alcohol.

Incompatibles.-Lead acetate, silver nitrate, lime water, alkalies and carbonates, vegetable decoctions or infusions, and nilk.

Dose.-H. \& C., 3 i.-ii. (4.-8.); Sh. \& Sw., gr.x.-xx. (.6-1.3); D., gr.ii.-iii. (.12-.2); Emetic, D., gr.x.-xv. (.6-1.).

Znnci Carbonas Precipitatus. Precipitated Zinc Carbouate. (U. S. P.)

Synonym.-Zinci carbonas, B. P.; precipitated zinc carbonate, kohlensaures zinkoxyd, $\mathrm{G}$.

Devivation.--Solutions of nearly equal weight of sodium carbonate and zinc sulphate are boiled together; dry precipitate. $9 \mathrm{ZuSO}_{4}+8 \mathrm{Na}_{2} \mathrm{CO}_{3}+2 \mathrm{H}_{2} \mathrm{O}=2(\mathrm{Zn} \mathrm{CO})_{3} \mathrm{Zn}$ $(\mathrm{OH})_{2}$ ? (liydrated basic zinc carbonate) $+8 \mathrm{Na}_{2} \mathrm{SO}_{4}+2$ $\mathrm{CO}_{2}$. This salt is in reality a mixture of zinc carbonate and oxide, in varying proportions, with water of crystallization.

Properties.-An impalpable white powder, of somewhat variable chemical composition, withont odor or taste ; insoluble in water or alcohol. 


\section{Zinci Oxidum. Zinc Oxide. Zn O. (U. S. \& B. P.)}

Derivation.-Heat the carbonate to redness.

$2(\mathrm{Zn} \mathrm{CO})_{3} \mathrm{Zn}(\mathrm{OH})_{2}=8 \mathrm{ZnO}+2 \mathrm{H}_{2} \mathrm{O}+6 \mathrm{CO}_{2}$.

Properties.-An amorphous, white powder, withont odor or taste. It gradnally absorbs carbon dioxide from the air. Insoluble in water or alcohol.

Dose.--H. \& C., 3 i.-ii. (4.-8); D., gr.v.-x. (.3-.6).

\section{PREPARATION.}

Uuguentum Zinci Oxidi. Ointment of Zinc Oxide. (U.S. \& B. P.)

Zinc oxide, 200; benzoinated lard, 800 . (U. S. P.)

Zinci Acetas. Zinc Acetate. $\mathrm{Zn}\left(\mathrm{C}_{2} \mathrm{H}_{3} \mathrm{O}_{2}\right)_{2}+2 \mathrm{H}_{2} \mathrm{O}$. (U. S. \& B. P.)

Derivation.--Dissolve zinc oxide in diluted acetic acid and boil.

$\mathrm{ZnO}+2 \mathrm{HC}_{2} \mathrm{H}_{3} \mathrm{O}_{2}=\mathrm{Zn}\left(\mathrm{C}_{2} \mathrm{H}_{3} \mathrm{O}_{2}\right)_{2}+\mathrm{H}_{2} \mathrm{O}$. Evaporate and erystallize.

Properties.-Soft, white, six-sided monoclinic plates, of a pearly lustre, having a faintily acetous odor, and an astringent metallic taste. Exposed to the air the salt gradually effloresces and loses some of its acid; reaction acid; soluble in 2.7 parts of water and in 36 parts of alcohol.

Incompatibles.--Same as sulphate.

Dose.-Same as sulphate.

ACTION OF THE ZINC SALTS.

External.-The salts of zinc (except the chloride) have an astringent action on raw surfaces and mucous membranes, notably the sulphate and acetate. Absorption is not followed by poisoning. They resemble other astringents, but do not possess the sedative qualities of the lead salts, nor the local alterative properties of silver nitrate.

Internal.-In the alimentary tract very large doses of the sulphate or acetate may induce gastro-enteritis, which is to be treated with demulcents and alkaline carbonates in 
order to form insoluble compounds. Vomiting will relieve carnivora; otherwise the stomach pump must be resorted to.

Zinc salts apparently prodnce no remote effects upon the body in medicinal doses, althongh in man chronic poisoning is said to occur anong workers in zinc. The condition is manifested by digestive disturbances, nervous symptoms and paralysis. In therapeutic doses the zinc salts are astringent, diminishing secretion in the digestive tract.

\section{USES OF ZINC SALTS.}

External.-Zinc sulphate is in common nse as an astringent collyrium for subacnte conjunctivitis (gr.ss.-ii. to $う$ i.). It is also employed in the treatment of canker of the ear in dogs (gr.x. to $₹ \mathrm{i}$.), or as "white lotion" (see Plumbi Acetas), in this affection.

The salt is likewise serviceable as a stimulant and astringent solution (gr.ii.-v. to $\bar{j}$ i.) in moist eczema, ulcers, atonic inflammations of mucous membranes and lencorrhoa.

Zinc carbonate is a much milder astringent than zinc sulphate or acetate. The impure carbonate (calamine) is an ingredient of the popular astringent and antiseptic, "pink ointment" of veterinary medicine used for the cure of "scratches" in horses.

R Zinci carbonatis (impure) .......... $3 \mathrm{ii}$.

Alumenis.....................

Calcii carb. præcip..............

Creasoti

Ceræ flavi.................... $\bar{a} \bar{a} \bar{z}$ iss.

M.

Adipis....................... ${ }^{3} \times \mathrm{xv}$.

S. External use.

The lard and wax are first melted together and then the other ingredients are stirred in. Calamine in the form of a lotion is a more cleanly application for honse dogs and pets than in ointment or paste. The following is useful in dermatitis, erythema and moist eczema attended with itching. The carbolic acid may be omitted when the lotion is 
applied over a large surface to avoid poisoning by absorption or from the acid being licked off by the patient.

Bx

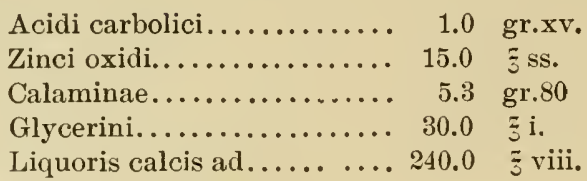

I. et fiat lotio (shake).

Sig. External use.

Zinc ointment is used externally in the form of a dusting powder, ointment or paste. In eczema, erythema and scratches, the zine oxide ointment is valuable and can be combined with carbolic acid (gr.x. to $\xi i$.) to great advautage, when itching is a prominent symptom. Still better than zinc ointment is a paste containing zinc oxide, 2 parts; starch and vaseline, each 3 parts. Zinc acetate can be userl in all cases as a substitute for zine sulphate.

Internal.-Zine sulphate is the best and most prompt. emetic for dogs in many conditions, as poisoning. It should be given in tepid water. Zinc oxide is occasionally prescribed in diarrhoea, ani empirically as a tonic and antispasmodic in chorea and epilepsy. It has also been recommended for its remote astringent action, to prevent excessive sweating and profuse bronchial secretion. Zinc oxide may be given in powder, pill, or dissolved in alkaline solutions.

\section{Cuprum.}

(Copper is not used in the metallic state in Medicine.)

Cupri Sulphas. Copper Sulphate. $\mathrm{Cu} \mathrm{So}_{4}+5 \mathrm{H}_{2} \mathrm{O}$. (U. S. \& B. P.)

Synonym.-Cupric sulphate, blue vitriol, blue stone, sulfas cupricus, cuprum vitriolatum, E.; cuprum sulfuricum purum, P. G.; vitriol bleu, sulfate de Cuivre, Fr.; kupfervitriol, blauer-vitriol, schwefelsaures kupfer (kupferoxyd), G. 
Derivation.-Boil metallic copper and sulphuric acid together. $2 \mathrm{Cu}+2 \mathrm{H}_{2} \mathrm{SO}_{4}=2 \mathrm{CuSO}_{4}+2 \mathrm{H}_{2}$. Dissolve product in hot water and crystallize.

Properties.-Large, trausparent, deep blue, triclinic crystals; odorless, of a nauseous, metallic taste; slowly efflorescent in dry air; soluble in 2.6 parts of water; almost insoluble in alcohol; reaction acid.

Incompatibles.-Mineral salts (except sulphates), alkalies and their carbonates, iodides, lime water and vegetable astringents.

Dose.-H. \& C., ji.-ii. (4.-8.); Sh. \& Sw., gr.xx.-xl. (1.3-2.6); D., gr.i.-ii. (.06-.12); Lmetic, D., gr.vi.-хx. (.36-1.3).

ACTION OF COPPER SULPHATE.

External.--Copper sulphate is stimulant, astringent or caustic to mucous membranes or raw surfaces, according to the strength applied.

Internal.-In poisonous doses copper sulphate causes salivation, vomiting, gastro-enteritis, and nervous symptoms (convulsions, paralysis and delirium), and finally, respiratory arrest. The blood corpuscles are broken down, and there is fatty degeneration of the liver and hromoglobinuria. The treatment consists in emptying the stomach in animals which cannot, or do not, vomit, and the use of yellow prussiate of potash, together with demulcents and opium. Large doses are emetic to the dog, but should not be used except in phosphorus poisoning. Smaller doses are astringent in the digestive tract.

Uses external.-Copper sulphate is employed in the solid, crystalline form in granular conjunctivitis, by rubbing the stick over the affected surfaces of the lids.

A solution (gr.ss.-ii. to $\bar{z}$ i.) is dropped into the eye for simple conjunctivitis. Copper sulphate is similar in action to zinc sulphate, but more powerful. On ulcerated and granular surfaces it is used as a stimulant and astringent, as in the following mixture, a combination of cupric sulphate and zinc sulphate, of each $2 \frac{1}{2}$ drachms (10 gm.), with 
solution of lead subacetate, 5 drachms ( $20 \mathrm{gm}$.), which is of value in thrush and as an application for chronic sores and unlealthy, indolent granulating surfaces. It may also be applied locally with an equal part of drierl alum in the form of powder for the treatment of thrush. The disappearance of the moistnre and foul odor will soon herald recovery.

Uses internal.-Copper sulphate is prescribed for its local effect with opinm in diarrhoen, and injected into the bowel in 2 per cent. solution in ulcerated conditions of the rectum. Copper sulphate is not a safe emetic, as it may cause poisoning if it is not quickly expelled. The sulphats of copper is a tonic remedy in anæmia and nervons conditions. It is thonght to resemble arsenic and to stimulate locally the tissues, heart and bloodvessels; thus increasing the number of corpuscles, firmness of flesh and amount of fat. Copper sulphate is often used as a tonic in the treatment of Inmbricoid worms and ozœna, combined with iron. Copper sulphate in $1 \mathrm{drachm}$ doses $(4.0 \mathrm{gm}$.), with powdered charcoal and fenugreek, of each 1-1 $\frac{1}{2}$ drachms (4.0-6.0 gm.), given to the horse night and morning for eight to ten days and followed by a brisk cathartic of aloes and linseed oil, will canse the expulsion of ascarides. It is recommended in purpura, and is given to dogs in the form of arsenite of copper for chorea and epilepsy. Glanders and farcy are tre:ted with copper sulphate, but these diseases are rarely curable and are too dangerous to the community to warrant drug treatment.

\section{Cupri Acetas. Copper Acetate. (Non-official.)}

Synonyms. - Verdigris, cupric subacetate, E.; acetate de cuivre, vert-de-gris, F.; grunspan, G.

Derivation.-Exposure of copper plates to pomace or residue resulting from expression of juice from grapes in wine making, or to immersion in pyroligneous acid.

Properties.-Pale-green masses of minute, acicular crysstals, sometimes of bright blue hue. Verdigris is the 
impure article; the pure salt is known as Crystals of Venus. The taste is coppery and odor vinegar-like. Soluble in water.

Dose.-H. \& C., gr.15-30 (1.-2.); Sh. \& Sw., gr.5-10 $(.3-.6)$.

Action External.-It is astringent, stimulant and escharotic according to the strength and whether applied to the unbroken skin or to mucous membranes or raw surfaces.

Action Internal.-This salt is an efficient vermifuge for the expulsion of ascarides from the horse. It should be given in doses of gr.15-30 (1.-2. gm.) twice claily with powdered gentian and charcoal, 1 drachm of each $(1.0 \mathrm{gm}$.), for a week and then be followed by a cathartic dose of aloes. It is a poison in large doses, causing gastro-enteritis, convulsions and death. The antidotes are milk, raw eggs and soap.

\section{Bismuthum.}

(Bismuth is not employed medicinally in the metallic state.)

Bismuthi Subcarbonas. Bismuth Subcarbonate. $(\mathrm{BiO})_{2} \mathrm{CO}_{3^{\prime}}$ $+\mathrm{H}_{2} \mathrm{O}$ ? (U. S. P.)

Synonym.-Bismuthi carbonas, B. P.

Derivation - Made by dissolving pure metallic bismuth in diluted nitric acid, precipitating with ammonia water, and redissolving in nitric acid. This :olution is treated with ammonium carbonate, or a solution of sodium carbonate.

$2 \mathrm{Bi}\left(\mathrm{NO}_{3}\right)_{3}+3 \mathrm{Na}_{2} \mathrm{CO}_{3}+\mathrm{H}_{2} \mathrm{O}=(\mathrm{BiO})_{2} \mathrm{CO}_{3}+\mathrm{H}_{2} \mathrm{O}$ $+2 \mathrm{CO}_{2}+6 \mathrm{NaNO}_{3}$. The precipitated bismuth subcarbonate is filtered and washed.

Properties.-A white, or pale yellowish-white powder, of somewhat varying chemical composition; odorless and tasteless, and permanent in the air. Insoluble in water or alcohol, but completely soluble in nitric or hydrochloric acid, with copions efflorescence.

Dose.-H., 3 ii.-iv. (8.-15.); D., gr.x.-xxx. (.6-2.). 
Bismuthi Subnitras. Bismuth Subnitrate. $\mathrm{BiO} \mathrm{NO}_{3}+\mathrm{H}_{2} \mathrm{O}$ ? (U. S. \& B. P.)

Synonym.-Bismuthum subnitricum, P. G.; bismuthum lydriconitricum, magisterium bismuthi, subazotas (s. subnitras) bismuthicus, sous-azotate de bismuth, Fr.; basisches salpetersaures bismuthoxyd, $\mathrm{G}$.

Derivation.-Dissolve pure metallic bismuth in diluted nitric acid. First reaction- $\mathrm{Bi}_{2}+6 \mathrm{HNO}_{3}=2 \mathrm{Bi}\left(\mathrm{NO}_{3}\right)_{3}$ $+3 \mathrm{H}_{2}$. Final reaction- $\mathrm{Bi}\left(\mathrm{NO}_{3}\right)_{3}+\mathrm{H}_{2} \mathrm{O}=\mathrm{BiONO}_{3}+2$ $\mathrm{HNO}_{3}$. Evaporate; add water; wash and dry precipitated bismuth subnitrate.

Properties.-A heavy, white powder of somewhat varying chemical composition; odorless and almost tasteless, and permanent in the air. Almost insoluble in water and insoluble in alcohol, but readily soluble in nitric or hydrochloric acid.

Dose.-Same as subcarbonate.

Bismuthi Salicylas. Bismuth Salicylate. (Non-official.)

Properties.-White, soft powder; insoluble in water, ether, alcohol or chloroform; soluble in acids.

Dose.-D., gr.v.-x. (.3-.6).

\section{Bismuth Subgallate. (Non-official.)}

Synonym.-Dermatol.

Properties.-A fine, yellow powder; permanent in the air and odorless; used externally as a substitute for iodoform; it is antiseptic aud astringent; occasionally given internally.

ACTION OF BISMUTH SUBNITRATE AND SUBCARBONATE.

External.-The insoluble salts of bismuth have a protecting, sedative, astringent and antiseptic action on raw 
surfaces. If applied over very extensive areas for a considerable length of time, they may cause absorption and poisoning. Bismuth has no action on the unbroken skin.

Internal.- The salts of bismuth are absorbed and eliminsted to some extent. When administered continuously in enormous doses, they have produced poisoning characterized by stomatitis, colic, diarrhoa, general weakness, black discolorations of the mucons membrane begiuning in the mouth, sometimes nephritis, and death from exhaustion. An odor of garlic appears in the breatl after the continuous exhibition of bisunth, owing to traces of tellurium contained in the bismuth.

Medicinally used, the salts of bismuth are absolutely harmless, although formerly poisoning was not infrequent from their contamination with arsenic. The tongue and frees are stained black by bismuth salts, which are transformed into the sulphide. Bismuth, locally and mechanically, by reason of its weight and insolubility, protects and coats the mucous membrane of the digestive tract, and thus exerts a sedative, astringent and antiseptic action throughont the canal. Bismuth must, therefore, be given, to be effective, in large and freguent doses, and when the stomach is empty. For this reason the drug is not of much value in the treatment of the horse, as a sufficient quantity cannot be used economically.

USES OF BISMUTH SUBNITRATE AND SUBCARBONATE.

External.-Bismuth subnitrate is a very good dusting powder on sores, and for moist skin diseases and ulcerated surfaces. It may be combined with zinc oxide and salol, or used in the form of an ointment in the proportion of 1 to 4. The following is an effectual combination to apply to superficial wounds and raw surfaces attended with much secretion :

R. Bismuthi subnitratis....... $50.0 \quad \frac{\pi}{3} \mathrm{i} .3 \mathrm{v}$.

Acidi tannici .............. 25.0 3 vi.

Iodoformi .................. $15.0 \quad 3 \mathrm{iv}$

Carbo ligni.................. 100.0 3ii. 3 ii.

M. et fiat pulvis.

Sig. Dust on surface. 
It may be employed to advantage in coryza and ozena, by insufflation into the nostrils. Dermatol (bismuth subgallate) is even more efficient than the subnitrate alone as an antiseptic and astringent dusting powder.

liternal.-Bismuth is one of the best agents to relieve romiting in dogs, owing to the soothing and sedative effect upon inflamed mucons membranes. It may be given alone upon the tougne or in combination with oxalate of cerium. It is also a very efficient agent in diarrhoea in the dog, being astringent, sedative and antiseptic. Its use should be preceded by the artministration of oil or calomel, in diarrhoea. Bismuth is given for diarrhoen in powder with salol; or in suspension with gnm arabic and water, with one drop of carbolic acid to each dose of bismuth; or better, in capsules, dispensing one grain of rarbolic acid and five grains of bismuth. The sedative effect upon the stomach is increased by giving the subcarbonate of bismuth with bicarbonate of sodium, while the sedative effect upon the bowels is enhanced by combining morphine with bismuth subnitrate. It is generally immaterial whether the subnitrate or subcurbonate of bismuth be selected in any given case. Bismuth salicylate is more powerful as an antiseptic than the other salts. It is useful in diarrhœil, intestinal fermentation and indigestion of dogs. The drug should be given in capsules.

\section{SECTION V.}

\section{Ferrum.}

Metallic iron is official in the form of fine, bright and non-elastic wire, from which are made iron preparations and reduced irou.

Ferrum Reductum. Reduced Iron. (U. S. \& B. P.)

Synonym.-Iron by hydrogen, Quevenne's iron, ferrum hydrogenio reductum, ferrum ope hydrogenii paratum, E.; 
ferrum redactum, P. G.; fer réduit par l'hydrogène, Fr:; reducirtes eisen, $\mathrm{G}$.

Derivation.-Hydrogen gas is passed over freshly made and carefully washed ferric oxide in a lot and closed tube. $\mathrm{Fe}_{2} \mathrm{O}_{3}+3 \mathrm{H}_{2}=\mathrm{Fe}_{2}+3 \mathrm{H}_{2} \mathrm{O}$.

Properties. - A very fine grayish-black, lustreless powder, without odor or taste; permanent in dry air; insoluble in water or alcohol.

Dose.-H., 3 i.-ii (4.-8.); C., उ ii.-iv. (8.-15.); Sh. \&. Sw., gr.xx.-хxx. (1.3-2.); D., gr.i.-v. (.06-.3).

Ferri Sulphas. Ferrous Sulphate. $\mathrm{Fe} \mathrm{So}_{4}+7 \mathrm{H}_{2} \mathrm{O}$.

$$
\text { (U. S. \& B. P.) }
$$

Synonym.-Copperas, green vitriol, vitriolum martis purum, sulfas ferrosus, ferrum vitriolatum purum, E.; ferrum sulphuricum purum, P. G.; sulfate de fer, sulfate ferreux, Fr.; schwefelsaures eisenoxydul, G.

Derivation.-Iron wire is dissolved by boiling in diluted sulphuric acid. $\mathrm{Fe}_{2}+2 \mathrm{H}_{2} \mathrm{SO}_{4}=2 \mathrm{Fe} \mathrm{SO}_{1}+2 \mathrm{H}_{2}$.

Properties. - Large, pale, blueish-green, monoclinic prisms, withont odor, and having a saline, styptic taste; efflorescent in dry air. On exposure to moist, air the crystals rapidly absorb oxygen and become coated with brownishyellow, basic ferric sulphate; soluble in $1 . \varepsilon$ parts of water; insoluble in alcohol.

Dose.-H., 3 i.-ii. (4.-8.); C., 3 ii.-iv. (8.-15.); Sh. \& Sw., gr.xx.-xxx. (1.3-2.); D., gr.i.-v. (.06-.3).

Ferri Sulphas Exsiccatus. Dried Ferrous Sulphate.

$$
2 \mathrm{FeSO}_{4}+3 \mathrm{H}_{2} \mathrm{O} \text {. (U. S. \& B. P.) }
$$

Synonym.-Ferrum sulfuricum siceum, P. G.; sulfate de fer desséché, Fr.; entwässerte schwefelsanres eisenoxydul, G.

Derivation.-Allow ferrous sulphate, 100 , to effloresce at a temperature of $104^{\circ} \mathrm{F}$. Then heat on a water bath till the product weighs 65 .

Properties.-A grayish-white powder, soluble in water.

Dose.-Same as sulphate. 
Ferri Sulphas Granulatus. Gronulated Ferrous Sulphate. $\mathrm{Fe}_{2} \mathrm{SO}_{4}+7 \mathrm{H}_{2} \mathrm{O}$. (U. S. \& B. P.)

Derivation.-Dissolve ferrous sulphate, 100, in distilled water, 100, and add sulphuric acid, 5. Evaporate till the product weighs 150. Pour alcohol, 25, upon it and dry.

Properties.-Pale, bluish-green, crystalline powder.

Dose.-Same as sulphate.

Ferri Carbonas Saccharatus. Saccharated Ferrous Carbonate. (U. S. \& B. P.)

Synonym. - Ferrum carbonicum saccharatum, P. G.; carbonas ferrosus saccharatus, saccharure de proto-carbonate de fer, Fr.; zückerhaltiges kohlensaures eisen, G.

Derivation.-Ferrous sulphate, 50 ; sodium bicarbonate, 35 ; sugar and distilled water. Made by solution, precipitation and washing.

Properties. - Greenish-brown powder, without odor; sweetish taste; becomes oxidized on exposure to the air.

Dose.-Twice that of iron sulphate.

Massa Ferri Carbonatis. Mass of Ferrous Carbonate. (U. S. P.)

Dose.-D., gr.i.-v. (.06-.3) in pill.

*Syrupes Ferri Íodidi. Syrup of Ferrous Iodide.

$$
\text { (U. S. \& B. P.) }
$$

Contains 5 per cent., by weight, of ferrous iodide $\left(\mathrm{FeI}_{2}\right)$.

Properties._Transparent, pale green liquid; sweet, ferruginous taste.

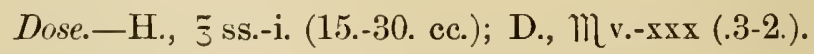

Ferri Chlonidur. Ferric Chloride. $\mathrm{Fe}_{2} \mathrm{Cl}_{6}+\mathrm{H}_{2} \mathrm{O}$.

(U. S. T.)

Synonym. - Ferrum sesquichloratum, P. G.; ferrum muriatum oxydatam, chlcridum seu chloruretum ferricum,

* The U.S. P., September 1905, changes composition of the syrup of ferrous iodide so that it now contains five instead of ten per cent. of ferrous iodide. 
ferri perchloridum, sesquichloride (perchloride) of iron, E.; perchlorure de fer, chlorure ferrique, Fr.; eisenchlorid, G.

Derivation.--Iron, $15 \mathrm{gm}$.; hydrochloric acid, nitric acid and water, of each a sufficient quantity. Made by solution with heat.

Properties.--Orange yellow, crystalline pieces, odorless, or having a faint odor of hydrochloric acid, and a strong styptic taste; deliquescent; soluble in water and alcohol; reaction acid; not used internally.

\section{Liquor Ferri Chloridi. Solution of Ferric Chloride.} (U. S. P.)

Synonym.-Liq. ferri perchloridi, B. P. An aqueous solution of ferric chloride $\left(\mathrm{Fe}_{2} \mathrm{Cl}_{6}\right)$ ' containing about 37.8 per cent. of the anhydrous salt; 62.9 per cent. of the crystallized salt, or about 13 per cent. of metallic iron.

Derivation.-Dissolve iron wire, 150 , in hydrochloric acid, 870 ; nitric acid and water to make 1,000. U. S. P.

First reaction.- $\mathrm{Fe}_{2}+4 \mathrm{HCl}=2 \mathrm{Fe} \mathrm{Cl}_{2}+2 \mathrm{H}_{2}$.

Second reaction. $-6 \mathrm{FeCl}_{2}+6 \mathrm{HCl}+2 \mathrm{HNO}_{3}=3 \mathrm{Fe}_{2}$ $\mathrm{Cl}_{6}+2 \mathrm{NO}+4 \mathrm{H}_{2} \mathrm{O}$.

Properties.-A reddish-brown liquid, having a faint odor of hydrochloric acid; an acid, strongly styptic taste and an acid reaction.

Dose.-H. \& C., 3 ii.-iv. (8.-15.); Sh. \& Sw., Mx.-xx. (6.-1.3); D., M ii.-x. (.12-.6).

\section{PREPARATIONS.}

Tinctura Ferri Chloridi. Tincture of Ferric Chloride. (U.S. P.)

Solution of ferric chloride, 250; alcohol to make 1,000 .

Dose.-H. \& C., z i.-ii. (30.-60.); Sh. \& Sw., आ $x x .-x \times x .(1.3-2.) ; D .$, mv.- 3 i. $(.3-4$.

Contains 13.6 per cent. of the anhydrous salt, or 4.69 per cent. of metallic iron.

Tinctura Ferri Perchloridi. Tincture of Iron Perchloride. (B. P.)

Dose.-Same as Tinctura Ferri Chloridi, U. S. P. 
Liquor Ferri Subsulphatis. Solution of Ferric Subsulphate. (U. S. P.)

Synonym.-Solution of basic ferric sulphate, $\mathrm{FeO}\left(\mathrm{SO}_{4}\right)_{5}$, Monsel's solution, solution of persulphate of iron, E.; liquor hémostatique de Monsel, Fr.; basischschewefelsaures eisenoxydlösung, Monsel's eisenlösung, G. Contains about 13.6 per cent. of metallic iron.

Derivution.-Ferrous sulphate, 675 ; sulphuric acid, 65; nitric acid and distilled water, of each a sufficient quantity to make 1,000 .

Properties.-A dark, reddish-brown liquid, odorless, or nearly so; of an acid, strongly styptic taste and an acid reaction; miscible with water and alcohol.

Dose.-H. \& C., żss. (15.); Sh. \& Sw., M x.-xx. (.6-1.3);

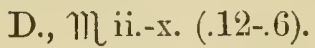

It has no value for internal use.

Ferri Oxidum Hydratum Cum Magnesia. Ferric Hydrate with Magnesia. (U. S. P.)

(Arsenic Antidote.)

Solution of ferric sulphate, 50 Cc.; water, $100 \mathrm{Cc}$. Magnesia, 10 gm.; water, 750 Cc. Keep solutions separate till ready for use; then mix.

Uses.-This preparation is used as a chemical antidote to arsenic, whereby the arsenic mass is mechanically enwrapped and converted into the insoluble arsenite. The administration of the arsenic antidote should be followed by emetics, or the stomach pump.

Dose.-Large quantities shonld be repeated frequently ad libitum.

Ferri ex Potassir Tartras. Iron and Potassium Tartrate. (U. S. P.)

(Potassio-Ferric Tartrate.)

Synonym.-Ferrum tartaratum, B. P.; tartarus ferratus, 
P. G.; ferri potassio-tartras, ferrum tartarizatum, tartras ferrico-kalicus, etc., E.; tartrate de fer et de potasse, tartre martial, Fr.; weinsaures eisenoxyd-kali, eisenweinstein, G.

Derivation.-Solution of ferric sulphate, $100 \mathrm{Cc}$; tartaric acid, $29 \mathrm{gm}$.; distilled water, $200 \mathrm{Cc}$; ammonia water and water, of each a sufficient quantity. Made by solution and precipitation.

Properties.-Thin, transparent scales, varying in color frow garnet-red to reddish-ibrown; without odor, and having a sweetish, slightly ferruginons taste; slightly deliquescent in the air; very soluble in water; insoluble in alcohol.

Dose.—D., gr.v.-x. (.3-.6).

Ferri et Ammonir Citras. Iron and Ammonium Citrate. (U. S. \& B. P.)

Synonym.-Ferrum citricum ammoniatum, P. G.; ferri ammonio-citras, ferro-ammonium citricum, ammonio-citrate of iron, E.; citrate de fer et d'ammoniaque (de fer ammoniacal), citrate ferrique ammoniacal, Fr.; citronensaures eisenoxyd-ammonium (ammoniak), G.

Derivation.-Solution of ferric citrate, $100 \mathrm{Cc}$.; ummonia water, 40 Cc. Evaporate. U. S. P.

Properties.-Thin, transparent, garnet-red scales, without odor, and having a saline, mildly ferruginous taste; deliquescent in moist air; soluble in water; insoluble in alcohol.

$$
\text { Dose.-D., gr.ซ.-x. (.3-.6). }
$$

Ferri et Quinine Citras. Iron and Quinine Citrate. (U. S. \& B. P.)

Synonym.-Chininum ferro-citricum, P. G.; citras ferricoquinicus, citrate de fer et de quinine, Fr.; citronensaures eisen chinin, G.

Source.-Ferric citrate, 85 gm.; quinine, 12 gm.; citric acid, $3 \mathrm{gm}$.; water, a sufficient quantity to make $100 \mathrm{gm}$. U.S. P. 
Properties.-Thin, transparent scales, of a reddishbrown color, without odor, and having a bitter, mildly ferruginous taste; slowly deliquescent in damp air; slowly but completely soluble in cold water and but partially soluble in alcohol.

Ferri et Quinine Citras Solubilis. Soluble Iron and Quinine Citrate. (U. S. P.)

Occurs in thin, greenish-yellow, transparent scales. Very rapidly and completely soluble in cold water.

Dose.—D., gr.v.-x. (.3-.6).

GENERAL ACTION OF IRON AND ITS SALTS.

External.-Soluble salts of iron, especially the chloride, sulphate, subsulphate and nitrate, are strongly astringent. They contract tissue when applied to raw surfaces or mucous membranes, by coagulating albumin, and through this reans, by compressing the blood ressels from without and plugging them from within with clotted blood, arrest hæmorrhage. Iron-in the form of liquor ferri chloridi or liquor ferri subsulphatis-is the most powerful of the metallic hemostatic agents we possess.

Internal.-Alimentary Canal.-Iron is a food rather than a medicine. It exists as a natural constituent of vegetable foods and of the body, and is found particularly in the hremoglobin of the blood-to the extent of about half an ounce in that of the horse. There is a sufficient quantity in the food to support healthy animals. If iron is given to a normal animal, it lias little effect unless continued for a long time in considerable quantity, when it may produce indigestion and constipation.

The iron salts and iron itself have practically the same physiological action, but some preparations are more irritating and astringent in the digestive tract than others. Ferric chloride and ferrous sulphate are particularly consti- 
pating; while reduced iron, the oxide, carbonate, and salts of the vegetable acids, are slightly so. Iron may blacken the tongue from formation of the sulphide. In the stomach all forms of iron are converted into ferric, and, to a slight extent, ferrous chloride, by the gastric juice. Strongly acid salts are decomposed and the combined acid in the salt is set free, owing to the formation of the chloride. This acid may prove irritating to the mucous membrane of the stomach. Acid salts, as the snlphate, are, therefore, more suitable for the horse than for the dog, as the latter is more susceptible to the irritating action. Ferric salts being astringent, so all the iron salts possess some astringency when they are converted into ferric chloride in the stomach. But those preparations most astringent outside of the body, are also most astringent in the digestive canal, from the fact that probably only part of the dose is changed in the stomach.

The iron salts, on entering the bowels, come into contact with an alkaline medinm. Ferric chloride conning from the stomach is converted into ferric oxide-which is held in solution by organic compounds in the intestines-and ferrous chloride is transformed into soluble ferrons carbonate. In the lower bowels, owing to the presence of nascent hydrogen, sulphides and tannic acid, these compounds are converted into ferrons sulphide and tannate and excreted as such in the feces, which are by them colored black. Iron is naturally absorbed from the organic compounds of the metal existing in the nucleoalbumins of food, but it is probable that the inorganic salts given as medicine are taken up from the digestive tract in minute quantities by the lencocytes of the blood and stored in the spleen, lympl nodes, bone-marrow and liver, in which latter organ it is ntilized in the formation of hematin and similar bodies and erentually transformed into hemoglobin, the essential component of the red corpuscle. It is in this way that inorganic iron administered as medicine becomes of benefit. The numerous compounds of iron now manufactured by 
pharmaceutical concerns under the name of albuminates and peptonates, and supposed to imitate the natural organic forms of iron found in the blood and liver, have not proved superior to the inorganic salts, but, on the contrary, many are practically worthless.

Blood.-In anæmia, iron indirectly increases the amonnt of hæmoglobin in the blond, and also the number of reil corpuscles. The latter effect is not so marked as with arsenic. In regard to iron increasing the number of corpuscles, we may say that the blood-forming organs, especially the red marrow, are stimulated, or supplied with blood-making material by iron. Iron increases the power of the red corpuscles to hold and carry oxygen from the lungs to the tissues, and to transform it into ozone. Iron is then indirectly an oxidizing agent, stimulating tissue change and vital activity. On account of the astringent properties of iron, when applied externally to the tissues, it is thought that its tonic action internally depends, in part, upon its stimulating the contractility of the muscles and vessels, and, therefore, improving tone.

Elimination.-Iron is chiefly excreted by the intestinal mucous membrane, however alministered, yet it is also eliminated to a slight extent by the urine, bile, saliva, sweat and tears.

Summary.-Iron is essentially a blood tonic and restorative, increasing the number of red blood corpuscles, the amount of hæmoglobin, and aiding nutrition. Externally it is an astringent, styptic and stimulant.

Uses External.-Liquor ferri chloridi and liquor feri subsulphatis are frequently used to stop bleeding from wounds or natural cavities of the boily. They may be injected, applied by swab, or on absorbent material, which is packed into the wound or cavity. As a local application in pharyngitis, we use 1 part of the solution of ferric chloride with 4 parts of glycerin. In the same strength, diluted with water, the chloride may be injected into the uterus to stop hemorrhage. Again, a 
solution, in the strength of 2 drachms to the pint of water, is employed as an enema to destroy ascarides. The objection to these solution.s of iron is that they form heavy, nasty, tenacious clots, when employed to arest hamorrhage, and the clots are apt to decompose and favor sepsis. Therefore they should not be used if other means, as ligature, pressure, heat or cold, or adrenalin chloride can be utilized.

Internal.-Reduced iron is one of the best preparations for dogs. It is commouly administered in pill, and often with other tonics, as strychnine, quinine, and arsenic. Reduced iron is non-irritating; non-astringent, and nonconstipating. It may be placed on the tongue in the form of powder.

Ferrous sulphate is one of the two most valuable forms of iron which can be prescribed to the larger animals. The other form is the tincture of ferric chloride. The sulphate is more astringent and irritating than some of the other iron salts, but does not usually cause constipation in the horse. Indeed, when constipation is due to loss of tone in the lower bowel, small doses, by their local stimnlant action, may actually assist peristalsis. The dried ferrous su'phate is prescribed to horses in anæmia, and is the most common constituent of tonic powders. It is frequently combined with powdered gentian, nux vomica, arsenic, and bicarbonate of sodium. Sodium bicarbonate is useful in indigestion and lessens the astringent action of sulphate of iron in neutralizing the acid set free from the salt. Nux vomica relieves constipation. A common and useful preparation for the horse is as follows:

Px

Sodii bicarbonatis.

Pulv. nucis vomicæ ............āà 3 ii.

Ferri sulphatis ex-icc............. 3 i.

M. et f. pulv. 1. Dispense pulv. tales no. xxx.

S. Give one powder on the feed three times daily.

Ferrous sulphate is given in anæmia secondary to chronic 
indigestion, intestinal parasites, leucorrhœa, ozœna, albuminuria, and in convalescence from acute diseases. Ferrous sulphate is in itself an anthelmintic, but, to get its full effect when used for this purpose in the treatment of round worms in the horse (ascarides), it shonld be given twice daily on the food for ten days, and then a pint of linseed oil containing three ounces of oil of turpentine is to be administered to complete the cure. In convalescence, iron may well be preceded by alcohol and bitters. Large doses of iron sulphate are indicsied in hæmorrhage from the bowels, if unassociated with acute inflammation, as in purpnra. In the latter disease, snlphuric acil is a synergistic remedy.

The saccharated ferrons carbonate may be given horses if they will not voluntarily take the sulphate of iron on their food. It is a nseful, mild, non-astringent preparation for dogs, and may be given in powder, or the mass may be dispensed in pills.

The iodiae of iron is thonght to be of benefit in man in scrofulous conditions, but as these states do not eommonly occur in horses and dogs, the drug is chiefly of value, in the form of the syrup, in rickets, and acts almost as a specific in that form of polynria afflicting horses during hot weather, and also in mill cases of anasarca and dropsy. The syrup should be preseribod undilnted and water shonld be added just before administering the preparation. If prescribed with water, the syrup will undergo decomposition if allowed t) stand for any length of time. The syrnp must be a fresh preparation, else free iodine is formerl in it, which will blacken the bucal mucons membrane. When the action of iodine and iron is desirable, it is often better to prescribe them separately.

The tincture of ferric chloride is a very powerful preparation. It contains free hydrochloric acid. Alcohol constitntes three-qnarters of its bnlk, and there are also some traces of ether. It was formerly thought to be hydrochloric ether, arising from the action of the contained muriatic acid on the alcohol of the preparation; but Weir 
Mitchell has shown it to be nitrous ether. Ferric chloride is of itself diuretic, apart from any action of nitrous ether in the tincture. The free acid aids digestion in the stomach. The tincture of ferric chloride is locally stimulant and astringent, and generally aids digestion; is diuretic, and in large doses, owing to the alcohol which it contains, is somewhat stimulating. The tincture is, accordingly, particularly valuable in anæmia, dependent upon chronic indigestion in horses and cattle, and in that occurring in convalescence from acute diseases. In such conditions, the preparation stimulates appetite, digestion and reual activity. When given by the mouth, the tincture of the chloride of iron is of local benefit in pharyngitis, combined with chlorate of potash, glycerin and water. It is also prescribed in intestinal hemorrhage (dried ferric subsulphate or MIonsel's salt given in 1-2 drachm closes in gelatine capsnles is more effective for this purpose in the larger animals), but there is no remote astringent or styptic effect exerted upon the vessels or tissues. Small doses of the tincture of ferric chloride may be safely dropped, undiluted, upon the tongue of horses or cattle from a small bottle used a measure. It is Rreguently conjoined with alcohol and mineral acids.

Iron and ammonium citrate and iron and potassium tartrate are mild, non-astringent preparations, suitable for dogs and given in pill.

Iron and quinine citrate is a useful combination for dogs, dispensed in pill. It is often employed in canine distemper and chorea.

\section{Manganum.}

(Manganese is not used in Medicine in the metallic state.)

Potassil Permanganas. Potassinm Permanganate. $\mathrm{KMnO}_{4}$. (U. S. \& B. P.)

Synonym.-Kali hypermanganicum crystallisatum, P. G.; hypermanganas potassicus S. kalicus, permanganate of 
potash, E.; permanganate de potasse, Fr.; uebermangansaures kali, G.

Derivation.-Caustic potash, chlorate of potassium and black oxide of manganese are fused together. $6 \mathrm{KHO}+$ $\mathrm{KClO}_{3}+3 \mathrm{MnO}_{2}=3 \mathrm{~K}_{2} \mathrm{MnO}_{4}+\mathrm{KCl}+3 \mathrm{H}_{2} \mathrm{O}$. The manganate of potassium is boiled with water till the color changes to purple and the permanganate is formed. $3 \mathbf{K}_{3}$ $\mathrm{MnO}_{4}+2 \mathrm{H}_{2} \mathrm{O}=2 \mathrm{KMnO}_{4}+4 \mathrm{KHO}+\mathrm{MnO}_{2}$. The liquid is neutralized with carbonic dioxide gas and evaporated.

Properties.-Slender, monoclinic prisms, of a dark purple çolor, odorless, and having a taste at first sweet, but afterwards disagreeable and astringent; permanent in dry air; soluble in 16 parts of water; undergoes decomposition with alcohol; reaction neutral.

Incompatibles.-It is very readily deoxidized in the presence of organic matter.

Dose.-H., gr.xv.-xx. (1.-1.3) in one pint of water; D., gr.i.-ii. (.06-.12), in pill, or tablet, with kaolin.

\section{ACTION OF POTASSIUM PERMANGANATE.}

External. - Potassium permanganate, like hydrogen dioxide, is a powerful oxidizing agent. It quickly parts with its oxygen in contact with organic matter, largely in the form of ozone, and is broken up into black oxide of manganese and potassa. Solutions, which are of a purple hue, change into a dark brown color when this transformation occurs, and are no longer of any medicinal value. This action is exceedingly rapid and transient, and its effects correspondingly so on the tissues. For this reason, and because bacteria are so combined with organic matter in the tissues, its action is largely exerted on the latter, and potassium permanganate is, therefore, a better antiseptic tlian disinfectant. Outside of the body, permanganate of potash is a disinfectant, but it is too expensive for general purposes. In powder it is slightly caustic, owing to the potassa set free in its decomposition; and in solution is stimulant to the tissues. A solution of permanganate of 
potash is a deodorizer when in contact with putrid and decomposing matter, bnt is not of the slightest value as a deodorizer and disinfectant to premises when simply standing in vessels, as frequently advised. There is no dinger from absorption of potassium permanganate when applied to the body.

Uses Internal.-Potassinm permanganate is occasionally nsed in human medicine in dyspepsia and flatnlence, for it: antiseptic action, aud in obesity. It is supposed to resemlle iron in its effects, and has been used in ammenorrho: associated with auæmia. Moor, of New York, his shown that potassium permanganate is the best chemical antidote for morphine or opium, chemically destroying them by oxidation; 10 to 15 grains may be given to dogs in 8 ounces of water immediately after poisoning. Horses may be given 2 drachms of permanganate of potash in 5 pints of water. In case morphine has been swallowed, solntions of potassium permanganate should be accidulated with vinegar, or diluted sulphuric acid, in order to form soluble compounds in the digestive tract. After morpline or opium have been absorbed in to the blood, it is said that potassium pernanganate is also antidotal when injected subcutaneously. It is difficult to see, theoretically, how this can be the case, and practically has been proven not to be so.

Uses External.-Potassium permanganate is a valuable antiseptic and deodorizer in solutions, varying in strength from one-tenth of 1 per cent. to 4 per cent., and is used in the treatment of sores, wounds, ulcers, abscess, caries, gangrene, fetid ozœna, otorrhœa, and leucorrhœi. In the stronger solution it is stimulant, as well as antiseptic. It is a useful agent in stomatitis and sore throat; when applied locally by means of a swab. The powder is employed as a caustic upon ulcers. Potassium permanganate is one of the best agents with which to sterilize the hands before operating. A saturated solution is to be recommended for this purpose, and the stains may be removed from the hands 
by washing them in a saturated solution of oxalic acid, or in a dilute solution of hydrochloric acid.

Potassium permanganate is a test for impure water in changing color in the presence of organic matter. Two ounces of a 1 per cent. solution will clarify and deodorize 100 gallons of stale and putrescent rain water.

\section{SECTION VI.}

\section{Hydrargyrum.}

Mercury. Quicksilver.

Synonym.-Mercurius vivas, argentum vivum, E.; mercure, vif-argent, Fr.; quecksilber, G.

Derivation.-Cinnabar, the native sulphate, is roasted or distilled with lime, and condensed.

Properties.-A shining, silver-white metal, without odor or taste; liquid at ordinary temperatures, and divisible into spherical globules; insoluble in the ordinary solvents; boils at $675^{\circ} \mathrm{F}$., and is completely volatilized; spec. gr., 13.5584 . When cooled to $38.88^{\circ} \mathrm{F}$, it forms a ductile, malleable mass.

\section{PREPARATIONS CONTAINING METALLIC MERCURY.}

1.-Hydrargymum cum Creta. Mercury with Chalk. (U. S. \& B. P.)

Mercury, 38 gm.; honey, 10 gm.; prepared chalk, 57 gm.; water, sufficient quantity to make $100 \mathrm{gm}$. (U. S. P.)

Mercuric'oxide becomes developed by keeping, making the powder more active.

Properties.-A light gray, rather damp powder, free from grittiness, without odor, and having a slightly sweetish taste. Contains mercury in fine division by shaking the ingredients together.

Dose.-Foals and calves, gr.x.-xv. (.6-1.); D., gr.i.-x. (.06-.6).

\section{II.-Massa Hydrargyri. Mass of Mercury. (U. S. P.)}

Synonym.-Pilula hydrargyri, B. P.; blue mass, blue pill, pilulæ coruleæ, E.; pilule de mercure, Fr.; mercurial pillen, G.

Mercury, $33 \mathrm{gm}$.; glycyrrhiza, $5 \mathrm{gm}$; althæa, $25 \mathrm{gm} . ;$ glycerin, 3 gm.; honey of roses, $34 \mathrm{gm}$. Contains 33 per cent. of mercury in a state of fine division. (U.S. P.)

Dose.-D., gr.i.-x. $(.06-.6)$. 
III.-Unguentum Hydrargyri. Mercurial Ointment. (U.S. \& B. P.) (Blue Ointment.)

Synomym. - Pommade mercurielle, pommade Napolitaine, Fr.; graue quecksilbersalbe, $\mathrm{G}$.

Mercury, $500 \mathrm{gm}$.; lard, $250 \mathrm{gm}$.; suet, $230 \mathrm{gm}$.; oleate of mercury, $20 \mathrm{gm}$. Contains 50 per cent. of mercury. (U. S. P.)

Hydrargyr Oxidum Rubrum. Red Mercuric Oxide. $\mathrm{HgO}$. (U. S. \& B. P.)

Synonym. - Hydrargyrum oxydatum rubrum, P. G.; hydrargyri-nitrico-oxydum, mercurius corrosivus (præcipitatus) ruber, oxydum hydrargyricum, peroxide of mercury, red precipitate, mercuric oxide, E.; deuto-oxyde (peroxyde) de mercure, oxyde mercurique, précipité rouge, poudre de Jean de Vigo, Fr.; rothes quecksilberoxyde, rother präcipitat (quecksilber-präcipitat), G.

Derivation.-Dissolve mercury in diluted nitric acid.

$3 \mathrm{Hg}_{2}+16 \mathrm{HNO}_{3}=6 \mathrm{Hg}\left(\mathrm{NO}_{3}\right)_{2}$. (Mercuric nitrate) $+4 \mathrm{NO}+8 \mathrm{H}_{2} \mathrm{O}$.

Rub mercuric nitrate with metallic mercury and heat.

$2 \mathrm{Hg}\left(\mathrm{NO}_{3}\right)_{2}+\mathrm{Hg}_{2}=4 \mathrm{HgO}+2 \mathrm{~N}_{2} \mathrm{O}_{4}$.

Properties.-Heavy, orange-red, crystalline scales, or a crystalline powder; odorless, and having a somewhat metallic taste; permanent in the air; almost insoluble in water; insoluble in alcohol.

PREPARATION.

Unguentum Hydrargyri Oxidi Rubri. Ointment of Red Mercuric Oxide.

(U. S. \& B. P.)

Synonym.-Red precipitate ointment, red mercuric oxide, 10; castor oil, 5; ointment, 85. (U.S. P.)

Hydrargyri Oxidum Flatum. Yellow Mercuric Oxide. HgO. (U. S. \& B. P.)

Synonym.-Hydrargyrum oxydatum via humida paratum, P. G.; hydrargyrum oxydatum præcipitatum (vel 
flavum), precipitated oxide of mercury, E.; oxyde de mereure jaune (précipité), Fr.; prücipitirtes (Gelbes) quecksilberoxyde, $\mathrm{G}$.

Derivation.-Precipitate an aqueous solntion of mercuric cllloricle, 100, with caustic soda, $40: \mathrm{HgCl}_{2}+2 \mathrm{NiOH}=$ $\mathrm{HgO}+2 \mathrm{NaCl}+\mathrm{H}_{2} \mathrm{O}$.

Properties.-A light orange-yellow, amorphous, heavy, impalpable powder; odorless, and lraving a somewhat metallic taste; permanent in the air, but turuing darker on exposure to the light; almost insoluble in water ; insoluble in alcohol.

PREPARATIONS.

Unguentum Hydrargyri Oxidi Flavi. Ointment of Yellow Mercuric Oxide. (U. S. P.)

Yellow mercuric oxide, 10; ointment, 90.

Oieitum Hydrargyri. Oleate of Mercury. (U. S. \& B. P.)

Yellow mercuric oxide, 200; oleic acid, 800 . (U. S. P.)

Hydrargyri Chloridum Corrosivun. Corrosive Mercuric Chloride. $\quad \mathrm{Hg} \mathrm{Cl}_{2}$. (U. S. P.)

(Corrosive Chloride of Mercury, Corrosive Sublimate.)

Synonym.-Hydrargyri perchloridum, B. P.; hydrargyrum birchloratum corrosivum, P. G.; hydrargyrum muriaticum corrosivum, hydrargyri bichloridum, sublimatus corrosivus, sublimatum corrosivum, mercurius sublimatus corrosivus, chloruretum (chloretum) liydrargyricum, perchloride of mercury, bichloride of mercury, E.; Deutochlorure de mercure, sublimé corrosif, chlorure mercurique, Fr.; retzendes quecksilberchlorid, retzender quecksilbersublimat, G.

Derivation.-Heat a mixture of mercuric sulphate, 20; sodium chloride, 16 ; manganese dioxide, $1 . \mathrm{Hg} \mathrm{SO}_{4}+2$ $\mathrm{NaCl}+\mathrm{MnO}_{2}=\mathrm{HgCl}_{2}+\mathrm{Na}_{3} \mathrm{SO}_{4}+\mathrm{MnO}_{2}$. The bichloride sublimes and is condensed.

Properties.-Heavy, colorless, rhombic crystals, or crys- 
talline masses; odorless, and having an acrid and persistent metallic taste; permanent in the air; soluble in 16 parts of water and in 3 parts of alcohol.

Incompatilles.-It is incompatible with most substances.

Dose.-H. \& C., gr.v.-vii. (.3-.5); Sh. \& Sw., gr.ii. (.12); D., gr. $\frac{1}{30}-\frac{1}{3}(.002-.008)$.

Hydrargyri Chlopidum Mite. Mild Merenrons Chloride. $\mathrm{Hg}_{2} \mathrm{Cl}_{2}$. (U. S. P.)

(Culomel, Mild Chloride of Mercur\%)

Synonym.-Hydrargyri subchloridum, B. P.; hydrargyrum chloratum mite, P. G.; hydrargyri chioridum, hydrargyrum chloratum (muriaticum) dulce, mercurius dulcis, calomelas chloruretum (chloretum) hydrargyrosum, subchloride (protochloride) of mercury, E.; protochlorure (sousmuriate) de mercure, calomèle, Fr.; quecksilberchlorür, calomel, G.

Derivation. - Heat mercurous sulpliate and sodium chloride. Calomel sublimes. $\mathrm{Hg}_{2} \mathrm{SO}_{4}+2 \mathrm{NaCl}=\mathrm{Hg}_{2}$ $\mathrm{Cl}_{2}+\mathrm{Na}_{2} \mathrm{SO}_{4}$.

Properties.-A white, impalpable powder; odorless and tasteless; permanent in the air; insoluble in water or alcohol. When strongly heated it is wholly volatilized. without melting.

Dose.-H., 3 ss.-i. (2.-4.); C., 3 v.-vi. (20.-24.); D., gr.ss. (.03), in divided doses; D., gr.iii.-v. (.2-.3) in single doses.

PREPARATION.

Pilutce Cathartice Compositce. Compound Cathartic Pills. (U. S. P.)

Compound extract of colocynth, 80; calomel, 60 ; extract of jalap, 30; gamboge, 15; water, Q. S. to make 1,000 pills.

Dose.-D., pill 1 to 3.

Hydrargyri Iodidum Rubrum. Red Mercuric Iodide. $\mathrm{HgI}_{2}$.

(U. S. \& B. P.)

(Binodide of Mercury, Red Iodide of Mercury.)

Synonym. - Hydrargyrum biiodatum rubrum, P. G.; 
deutoioduretum (biniodidum) hydrargyri, mercurius iodatus ruber, iduretum hydrargyricum, E.; deut-iodure (bi-iodure) de mercure, iodure mercurique, Fr.; rothes jodquecksilber, quecksilberjodid, .G.

Derivation.-Mix aqueous solutions of corrosive mercuric chloride, $40 \mathrm{gm}$., and potassium iodide, $50 \mathrm{gm}$. The red iodide is precipitated. Filter, wash and dry. $\mathrm{HgCl}_{2}+2 \mathbf{K I}$ $=\mathrm{HgI}_{2}+2 \mathrm{~K} \mathrm{Cl}$.

Pioperties. -A scarlet-red, amorphous powder ; ndorless and tasteless; permanent in the air; almost insoluble in water; soluble in 130 parts of alcohol.

Unguentum Hydrargri Nitratis. Ointment of Mercuric

Nitrate. (Citrine Ointment).

Mercury, 70 gm.; nitric acid, 175 gm.; lard oil, $760 \mathrm{gm}$. Properties.-A lemon-yellow ointment.

Hydrargyrum Ammoniatum. Ammoniated Mercnry. $\mathrm{NH}_{2}$ $\mathrm{Hg}$ Cl. (U.S. \& B.P.)

(White Precipitate, Mercuric Ammonium Chloride.)

Synonym.-Hydrargyrum præcipitatum album, P. G.; hydrargyrum amidato-bichloratum (ammoniato-muriaticum), hydrargyri ammonio-chloridum, mercurius præcipitatus albus, E.; oxychlorure ammoniacal de mercure, mercure précipité blanc, Fr.; weisse quecksilber-präcipat, quecksilberchloridamidid, G.

Derivation.-Mix an aqueous solution (1-20) of corrosive mercuric chloride, 200 , with ammonia water, 15.

$\mathrm{HgCl}_{2}+2 \mathrm{NH}_{4} \mathrm{OH}=\mathrm{NH}_{2} \mathrm{Hg} \mathrm{Cl}+\mathrm{NH}_{4} \mathrm{Cl}+2 \mathrm{H}_{2} \mathrm{O}$. Filter, wash with dilnted ammonia water (1-20), and dry the precipitated ammoniated mercury.

Properties.-White, pulverent pieces, or a white, amorphous powder, without odor, and having an earthy, afterwards styptic and metallic, taste; permanent in the air; almost insolulule in water or in alcohol. 
PREPARATION.

Unguentum Hydrargyri Ammoniati. Ointment of Ammoniated Mercury. (U. S. \& B. P)

Synonym.-White precipitate ointment. Ammoniated mercury, 10; benzoinated lard, 90. (U.S. P.)

GENERAL ACTION OF MERCURY AND ITS 3ALTS.

External.-The salts of mercury are germicidal, irritant, and, in some cases, caustic, when applied externally. Corrosive sublimate is the most powerful antiseptic in general use. It unites with albuminous fluids in, or on, the tissues, to form insoluble albuminates of mercury, and its antiseptic properties are then lost. This action is prevented by adding 5 parts of tartaric acid to 1 part of corrosive sublimate, and such combination is provided in the tablets manufactured for surgical purposes.

Hydrochloric acid, or salt, may be employed to obtain the same result. Corrosive sublimate is a more powerful germicide than carbolic acid, yet it cannot be used to disinfect metallic instruments, as the bichloride is decomposed and mercury deposited upon them. The salts of mercury kill the lower forms of animal as well as vegetable life, and are valuable in the treatment of parasitic skin diseases. As a rule, antiseptics relieve itching, and the mercury salts are often used to combat this condition. Mercury and its salts are absorbed when rubbed into the unbroken skin, particularly when in combination with oil or grease.

Metallic mercury and its salts (notably the iodide), when rubbed well into the skin with fat, are thought to aid the absorption of inflammatory exudates in underlying parts.

Internal.--The irritant salts of mercury, as the bichloride, iodide, nitrate, and some of the oxides, in large doses, produce gastro-enteritis, romiting, colic, bloody diarrhœea, collapse and death. The white of egg is an antidote to corrosive sublimate, forming an insoluble albuminate. 
Emetics, or the stomach pump, should be used in case romiting is not spontaneous.

The use of mercury, or any of its compounds, if continued for any considerable time, either internally or externally, in such a way as to lead to absorption, may canse a chrovic form of poisoning or mercurialism. This condition is characterized by fetor of the breath and soreness of the gums, making mastication painful. The gums are swollen and bleed easily; the tongue swells and salivation ensnes. The teeth become loosened, the salivary and parotid glands enlarge, the temperature is elevated, and if the condition continues, there are: ulceration of the mouth (mercurial stomatitis), necrosis of the jaw, general weakness, a watery condition of the blood, œedema, anæmia and cachexia, prostration and death. Local poisoning, as exhibited by paralysis of the hand and forearm, has occurred in a man who applied the ointment of red iodide of mercury to cattle. There is a tendency for mercury to accumulate in the system, when given in large doses, or in smaller doses when continued for a considerable period.

The prevailing fashion of administering calomel in small and repeated doses, may lead to mercurialism if purgation does not occur.

Stomach and Intestines.-Mercury and the mercurous salts, especially calomel, are mildly irritant and act as purgatives. The upper part of the intestinal tract, notably the duodenum, is influenced, and peristalsis and secretion are stimulated so that the bile is hurried along together with the other ingesta, and expelled. Cillomel and mercury are called cholagogus cathartics, yet they do not increase the amount of bile secreted, but diminish it by retarling its absorption in the bowels. Corrosive sublimate, on the other hand, is an hepatic stimulant and augments the amount of bile secreted.

The purgative action of calomel and mercury in the small intestines is assisted by salines, which increase the amount of fluid in the bowels, and aid in the expulsion and 
prevent the absorption of mercury. The saline should be given four hours after the administration of calomel to cattle.

Blood.-Nercury is generally thought to circulate in the blood as an albuminate. It is stated that metallic mercury is ahsorbed nnchanged and circulates in this condition in the blood; while it is also held that corrosive sublimate finds its way into the blood as an albuminate of the oxide of mercury, in combination with sodium chloride. It will be perceived that there is no exact knowledge concerning this matter. It is supposed that mercury diminishes the fibrin in the blood, and the production of exudations, especially in connection with inflammation of serous membranes, and even hastens the absorption of inflammatory deposits. Mercury is, therefore, said to be antiphlogistic in combating the effects of inflammatiou. A part of this result may be attributed to the antiseptic action of the salts of mercury in the intestines by preventing fermentation and absorption of toxic material. For want of a better term to explain the beneficial actions of mercury on the tissues, that vague term "alterative" is frequently applied. Mercury (and calomel in particular) is somewhat diuretic, stimulating the secreting cells, or nerves of the kidneys, and increasing the amount of urine.

Elimination. - Mercury is eliminated slowly by the kidneys, liver, salivary glands, and, in fact, by every conceivable channel. In thus stimulating the eliminative activities of the various glands, mercury has been termed a deobstruent. It has been surmised that its alterative effect depends, in part, upon this action in stimulating-to use the old term-the emunctories.

SUMMARY OF ACTIONS OF MERCURY AND ITS SALTS.

External.-Antiseptic, germicide, irritant, caustic, parasiticide, antipruritic and sorbefacient.

Internal.-Antiseptic, purgative, cholagogue, antiphlogistic, and alterative diuretic (calomel). 
Internal.-Antiseptic, purgative, cholagogue, antiphlogistic, and alterative diuretic (calomel).

\section{TOXICOLOGY.}

\section{(See action internal.)}

\section{USES OF MERCURY AND ITS SALTS.}

Hydrargyrum cum ereta is similar to calomel in its effects, but very much milder, unless it contains the black oxide of mercury, when its action is much intensified. The same may be said of massa hydrargyri. Either preparation may be giren dogs as a laxative in indigestion with romiting and diarrhœa; or to foals and calves with iutestinal indigestion and diarrloen, particularly if accompanied with jaundice. The oleatum or nnguentum liydrargyri are rubbed into the skin to canse resolution of chronic inflammatory swellings, and also to kill animal and vegetable parasites. As the former action is due to absorption, large quantities will lead to poisoning when applied over an extensive surface. We can use other and safer remedies, as creolin, tar or sulphur ointment, for parasiticides. It is to be remembered in this connection that grease alone will kill lice and other parasites on the skin. On accuunt of their sorbefacient properties, the oleate and blue ointment of mercury are applied over chronically enlarged glands, swollen joints, and thickened tendons. In view of their parasitic action, these preparations are employed to kill the fungus of farus and ringworm, and to destroy lice and the acari of mange, when inhabiting circumscribed areas. Itching in skin diseases, as chronic eczema and psoriasis, is relieved by either blue ointment or the oleate of mercury.

HYDRARGYRI OXIDUM RUBRUM, OR FLAVUM.

The official ointments of the red and yellow mercuric oxides are prescribed, as stimulant and antiseptic preparations, in chronic conjunctivitis, granular lids and scaly skin diseases. They are also employed on indolent ulcers, 
swollen glands and old granulating surfaces. W' $W^{1}$ en used on mucous membranes, or raw surfaces, the official ointments should be diluted with equal parts of lard.

\section{HYDRARGYRI CHLORIDUM CORROSIVUM.}

External.-Corrosive sublimate is of value mainly as an antiseptic on the unbroken skin. It is germicide in solutions containing 1 part to 500 , or 1,000 , of water. Applied to mucons membranes, or raw surfaces, it is antiseptic in solutions varying in strength from $1-10,000$ to $1-1,000$.

In the larger carities of the boly, as the vagina, solutions should not be used in strength greater than 1-5000 or 1-3000. Recent experiments by Harrington and Walker go to show that corrosive sublimate is much less active than commonly believed. A 1-1000 solution requires more than ten minutes' contact to kill common forms of pus cocci, so that dipping the hands for a few seconds in such solutions does more harm than gond in indncing a false security which does not exist. They conclude by saying that, as the result of their experiments, "corrosive sublimate in any of the strengths commonly employed is a much overrated disinfectant, and under the best of conditions is so uncertain in its action that it would be of advantage to abandon its use altogether in surgery." These men are kuown to be careful and trustworthy investigators and their findings agree with the results obtained hy many great surgeons. In view of the ease with which corrosive sublimate combines with albumin, it is best not to rely upon this agent for wound disinfection, but to employ normal salt solntion for cleansing, followed by hydrogen dioxide. For hand disinfection, alcohol in 60 to 70 per cent. strength is much more effectire.

Mercuric bichloride, even in the weaker solution, is ton damaging to the serous membrane of the peritoneal cavity, and there is too much danger of absorption to warrant us in using it in intra-abdominal operations. As a canstic, 
saturated solutions are injected into fistulous tracts: e.g., fistulæ of the withers, "quittor" and "poll evil," to destroy their so-called pyogenic membranes, and lasten repair. There is not much danger of absorption when used in this way.

Corrosive snblimate is very useful as a parasiticide, in destroying lice, ringworm and the fungus of favus, in solution (1-500) on the mbroken skin. It also relieves itching in pruritus, prurigo and urticaria, but is generally inferior to carbolic acid in this respect. Bichloride solutions may be used in the septic uterus (after removing placental or membranous remains, with the hands, forceps or curette), in the strength of 1-3000 or 1-5000. Apart from the body, corrosive sublimate in solution (1-500 or 1-1000) is one of the cheapest and most effective disinfectants for premises infected with the contagion of glanders, anthrax, etc. The walls and floors of stables (after thorough cleansing and washing with soft soap and boiling water), clothing and all paraphernalia, not metallic, can be disinfected by washing or'soaking in solntions of bichloride. Before operations, the operative field should be sterilized by scrubbing with green soap and then with Harrington's solution* after the hair has been shaved from the part. The operator's hands can be also sterilized to best advantage with 60 per cent. solution of alcohol in water. During an operation, irrigation with corrosive (1-3000) solution or boiled normal salt solution is commouly practised. In epizöotic abortion, in addition to quarantining the diseased animals, their discharges and the premises shonld be disinfected, and both the well and sick female animals should be washed twice daily about the genital regions with a solution of corrosive sublimate. Yellow wash, made by

* Harrington's solution consists of: Commercial alcohol (94 per per cent.), 640 c.c.; commercial hydrochloric acid, 60 c.c.; corrosive sublimate, $0.8 \mathrm{gm}$. It is the most powerful preparation for skin disinfection known, rendering the skin sterile in most cases after application for two minutes. 
the addition of $30 \mathrm{gr}$. of mercuric bichloride to 1 pint of lime water, is sometimes employed as a stimulant application in chronic eczema, and to relieve itching. It contains the yellow oxide of mercury. In purulent conjunctivitis, frequent irrigation with a 1-1000 solution of corrosive sublimate is of the greatest service.

Internal.-Corrosive sublimate is of valne in minute doses as a blood tonic, and is recommended as an antiphlogistic agent in diminishing fibrinous exudation in inflammatory diseases of serous membranes, as pleuritis, meningitis, and arthritis. It is probably inferior to calomel for this purpose. We at least know that calomel is an efficient cathartic in inflammatory diseases.

Mercuric bichloride is employed as an intestinal antiseptic and hepatic stimulant, in the treatment of dysentery and diarrhœa with mucous or vile smeling discharges. In these conditions, irrigation of the rectum with a $1-5000$ solution is of advantage. This solution should be drained off through the rectal tube and followed by an injection of plain boiled water.

Administration.-Corrosive sublimate is given in the form of a pill or ball. If exhibited in solution to the large animals, it must be diluted with 2 quarts of water.

HYDRARGYRI CHLORIDUM MITE.

External.-Calomel is of use in chronic eczema when applied over small patches in its pure state, or as "black wash." The latter consists of one drachn of calomel in one pint of lime water, forming the black oxide of mercury, and is a very efficient preparation to relieve itching and promote recovery in chronic eczema, by mild stimulation. Calomel is of benefit when blown into the eye once or twice a week, stimulating and hastening absorption of opacities of the cornea following keratitis. It is the best agent to arrest thrush when worked up into the commisure of the hoof, between the frog and the bars, aud retained in place by oakum packing. 
Internal.-Calomel is a purgative, intestinal antiseptic, cholagogue, diuretic and alterative. It is also used for its remote antiplilogistic effects. It is particularly adapted to dogs, and is given in a single dose, or often, to better advantage, in half-grain doses, repeated every two hours till purgation occurs. For diarrlıea or vomiting in dogs, calomel is useful in removing the source of irritation, in being antiseptic and easily borne by an irritable stomach. Calomel is partially transformed, by the alkaline secretions of the bowels, into the mercurous oxide, and it is to this salt that its purgative action is due. Combination with sodinm bicarbonate assists in this transformation, and is commonly practised. The administration of calomel should be followed by oil, salines or other cathartics, if purgation does not occur within twenty-four hours after its ingestion, otherwise mercurialism may occur.

In heptogenons jaundice, with light-colored frees, owing to hepatic congestion, gastro-duodenitis or constipation, calomel is a valuable remedy for $\operatorname{dog} \mathrm{s}$, followed by the use of nitro-muriatic acid. In the jaundice occurring as a form of influenza in horses, nitro-muriatic acid is more effective. Calomel is one of the best remedies for the treatment of dysentery, unless there is great weakness. It should be continued in repeated small doses till the character of the discharge changes. Foals and calves, with indigestion and diarrhoea, may be given calomel to advantage to remove the source of irritation in the digestive tract. Calomel must be combined with a small dose of aloes, or with linseed oil, to form an effective cathartic for the horse. As aloes acts on the large, and calomel on the small, intestines, the above combination secures a general purgative influence.

Cattle are given calomel, followed by the administration of salines, to produce free catharsis. As a remedy for round worms, $\frac{1}{4}$ to $\frac{1}{2}$ grain each of santonin and calomel, with 5 grains of sugar of milk, are administered to dogs four times, at half hour intervals, and followed by castor oil. Lumbricoid worms in the horse, may be treated by conjoining 2 
drachms of santonin with 1 drachm of calomel, given in the form of a ball to the fasting animal, and repeated once on the following day if necessary. Calomel was formerly very frequently used, and is occasionally prescribed to this day in the treatment of pleuritis, meningitis, peritonitis, pericarditis, and iritis, for its antiphlogistic and alterative action in diminishing inflammatory exudations.

At the present time these actions are very much questioned, and any beneficial effects accruing from the use of calomel in inflammatory diseases are now ascribed to its action as a purgative and intestinal antiseptic in destroying and eliminating toxins from the bowels.

Calomel is of value in inflammatory diseases when given at the onset of the attack. In dropsy (ascites of dogs), calomel sometimes acts as a useful diuretic, when combined with digitalis and squill in pill form.

Administration.-Calomel is given to cattle on the tongue or in gruel; to horses in ball, on the food, or on the tongue; to dogs in pill, tablet or on the tongue. The compound cathartic pill is a good purgative preparation for occasional nse. Two to three pills for large dogs; one to two pills for smaller animals.

\section{HYDRARGYRI IODIDUM RUBRUM.}

The red mercuric iodide is a favorite remedy in veterinary practice. It causes absorption of morkid exudations through its counter-irritant, local absorbent and alterative effect, in combining the action of iodine and mercury. It is employed with 8 to 10 or 12 parts of lard or vaseline, and is of value in the treatment of periostitis with osseous deposits, especially for splints. Spavin and ringbone are treated with red iodide of mercury alone, but are generally cured more effectively by rest, firing, and blistering. The red iodide of mercury ointment is also of use for enlarged glands, chronic swelling abont tendons, joints or bursi; and applied about the throat in chronic laryngitis and "roaring." The ointment is rubbed on splints every third 
day, or until vesication is produced, and the hair begins to drop ont, when its use is stopped for a time. Like other mercury preparations, the red iodide must not be employed in large quantities over an extensive surface. It is much more irritant locally than blue ointment.

\section{UNGUENTUM HYDRARGYRI NITRATIS.}

Citrine ointment is similar to unguentum liydrargyri ammoniati (wlite precipitate ointment), but more powerful, and should be diluted with equal parts of lard. These preparations are used for their stimulant action in granular lids, chronic eczema, psoriasis, pityriasis, and for their anti-parasitic effect in ringworm.

\section{SECTION VII.}

\section{Arsenum.}

Arsenic is not used in the metallic state in medicine.

Arseni Trioxidum (U. S. P.)

Aciduy Arsenosum. Arsenous Acid. $\mathrm{As}_{2} \mathrm{O}_{3^{2}}$.

Synonym.-Acidum arseniosum, B. P.; acidum arsenicosum, P. G.; arsenic trioxide, white arsenic, arsenicum album, arsenic, arsenious anhydrid, E.; acid arsenieux, arsenic blanc, fleurs d'arsenic, Fr.; arsenicsäure, weisser arsenic, G.

Derivation.-Arsenical ores are roasted and purified by sublimation.

Properties.-A heavy solid, occurring either as an opaque, white powder, or in irregular masses of two varieties; the one amorphous, transparent and colorless, like glass; the other crystalline, opaque, or white, resembling porcelain. Both are odorless and tasteless. The glassy variety dissolves slowly in 30 parts of water; the porcelain-like in 80 parts of water. Arsenous acid is sparingly soluble in alcohol, but soluble in glycerin, hydrochloric acid and solutions of the 
alkali hydrates and carbonates. When heated to $424^{\circ} \mathrm{F}$., arsenous acid is completely volatilized without melting.

Incompatibles.-Lime water, salts of iron and maguesia.

Dose.-H., gr.i.-v. (.06-.3); Sh. \& Sw., gr.i.-ii. (.06-.12); D., gr. $\frac{1}{30}-\frac{1}{10}(.002-.006$. $)$

Usual dose for H., gr.ii.-iii. (.12-.2).

Liquor Potasit Arsenitis. Solution of Potassium Arsenite.

$$
\begin{gathered}
\text { (U. S. P.) } \\
\text { (Foutler's Solution.) }
\end{gathered}
$$

Synonym.-Liquor arsenicalis, B. P.; liquor kali arsenicosi, P.G.; solutio atrsenicalis Fowleri, kali arsenicosum solutum, arsenical solution, E.; liqueur arsenicale de Fowler, Fr.; Fowlers'che tropfen, G. Arsenous acid, $10 \mathrm{gm}$.; potassium bicarbonate, $20 \mathrm{gm}$.; compound tincture of lavender, $30 \mathrm{Cc}$; distilled water to make 1000 Cc. Strength, 1 part of arsenous acid in 100.

Dose.-H. \& C., 弓ii.- 3 i. (8.-30.); Sh. \& Sw., 3i.-ii. (4.-8.); D., Mii.-文. (.12-.6).

Usual dose for H., $\overline{3}$ ss. (15.).

Liquor Acidi Arsenosi. Solution of Arsenous Acid.

$$
\text { (U. S. P.) }
$$

Synonym.-Liquor arsenici hydrochloricus, B.P.; hydrochloric solution of arsenic, E.; liqueur arsenicale hydrochlorique, Fr.; chlorarsenik-lösung, G.

Arsenous acid, $10 \mathrm{gm}$.; diluted hydrochloric acid, $50 \mathrm{Cc}$; distilled water, a sufficient quantity to make $1000 \mathrm{Cc}$. Strength, 1 part of arsenous acid in 100.

Dose.-_Same as Fowler's solution.

$$
\text { ACTION OF ARSENICAL COMPOUNDS. }
$$

External.-Arsenous acid acts as a canstic on raw surfaces and mucous membranes. It produces considerable pain, and may lead to poisoning. In frogs poisoned by 
arsenic the epidermis peels off very readily, owing to degeneration of its lower layers.

Internal.-Digestive Tract.-Arsenic, when given in minute doses, improves the appetite, and increases both the motion and secretions of the stomach and duodenum. In larger amounts, arsenic is an irritant, causing loss of appetite, nausea and digestive disturbance. In toxic doses arsenic produces gastro-enteritis.

Blood.-Arsenic is absorbed into the blood, and in some forms of anæmia increases notably the number of red corpuscles, and to some extent the hæmoglobin.

Circulation.-It is said that arsenic stimulates the pulse rate when given in minute doses. In large doses it has a local depressing action on the heart-and probably a local action on the vessels-lowering the force and frequency of the heart and reducing blood pressure. The nerve endings, ganglia, and muscle of the heart are alike paralyzed, and this action takes place when the heart is removed from the body.

Respiration.-In small doses arsenic stimulates the respiratory centre, and probably the peripheral vagi; whereas in lethal amounts it is said to paralyze only the latter.

Nervous System.-The nervous apparatus is powerfully influenced by arsenic. Toxic doses canse paralysis of the spinal tracts, in frogs, with loss of sensation, motion, and reflex action. There is conflicting testimony relating to which centres succumb first. The nerve trunks are affected in the higher animals. There is peripheral neuritis and trophic changes occur. Medicinal doses of arsenic are stimulant to the nervous system generally.

Metabolism. - Therapeutic doses probably diminish tissue change and the elimination of urea and carbonic dioxide. Large doses, on the other hand, increase metabolic processes and the escape of nitrogenous waste.

Elimination.-Arsenic is eliminated slowly by most channels. It is found in the mine, fæces, milk, sweat, tears and saliva. It exists in, and can be recovered from, the 
bodies of animals years after their death from toxic amounts of arsenic.

Summary.-Arsenic is unfortunately one of the drugs whose physiological action-so far as we kuow it-does not throw any light, in many instauces, upon its therapeutic effects. In altering the condition of the patient for the better, in some diseases, it is described by that rague and otherwise indefinable term, "alterative."

Toxicology.-The lower animals, as the horse and cow, are proportionately not nearly so susceptible to the poisonous effects of arsenic as the human subject. Two grains is the smallest fatal dose reported in man. Amounts larger than a drachm appear to be required to cause death in the horse, although much sinaller quantities have produced death when repeated a number of times. Mild toxic action is seen following therapentic doses of arsenic when the physiological limit is reachel. This condition is characterized by loss of appetite (nausea and vomiting in dogs), watery discharge from the nose and eyes, pnffiness of the eyelids, indigestion with mild colic, and diarrhœa. The pulse may be accelerated and harder than normal.

Acute Poisoning begins with bilious, mucous, or bloody purging and colic. There is vomiting in dogs. Thirst is excessive; the urine is ligh-colored and albuminons; the pulse is feeble, small and frequent; the respiration is rapid and difficult from abdominal pain ; the extremities are cold, and there is great weakness of the limbs. Collapse, with convulsions and coma, often close the scene in from five to twenty hours to three days.

A sub-acute form of poisoning occasionally occurs after a remission from the acute attack, only to be followed by death in from two to five days. In the interim, cutaneous eruptions may appear. Rarely, death takes place within an hour or two, in coma, collapse or convulsions.

Chronic Poisoning, such as is seen in the hmman subject living in apartments furnished with arsenical wall paper or fabrics, or in those working in arsenic, is rarely observed 
in animals and wonld not be likely to occur save in those living in the immediate vicinity of chemical works. In this condition there are symptoms similar to those noted above as ncenring in the milder form of arsenic poisoning, together with gradual loss of strength and flesh, local paralysis or parap!egia, and anesthesia. Fatty degeneration of the liver, kidneys, heart, stomach and muscles, in cases of chronic arsenical poisoning, is fomd after death.

The post-mortem changes observed after acnte poisoning, are as follows: The gastric mucons membrane, especially the villons portion in horses, is swollen, softened and covered with patches of a deep erimson or dark brown color. There is rarely ulceration. The upper portion of the small intestines, and in horses sometimes the whole of the intestinal tract, is similarly affected with that of the stomach. There is generally a wide-spread fatty degeneration of the internal organs and muscles.

The treatment of acnte poisoning depends mainly upon the use of the official freshly prepared arsenic antidote (ferri oxidum hydratum cum magnesia) in large quantities. If this can not be obtained, an antidote can be prepared by precipitating Monsel's Solution, or the tincture of the chloride of iron, with sodium bicarbonate or ammonii. Dialyzed iron may be precipitated with an alkali. In either case the precipitate should be washed in a filter of muslin and given in large anounts. If vomiting has not occurred, zine sulphate should be given, or the stomach pump or siphon resorted to, and the stomach well washed out. The after-treatment is carried out with castor oil, demulcents, opinm and external heat. Sweet spirit of nitre is to be prescribed, with considerable water, to flush out the kidneys.

Uses External.-A paste containing 1 part each of arsenous acid and gnm arabic, with 5 parts of water, is used to destroy warts and morbid growths. Arsenous acid, dilnted with 5 parts of lard, may be employed to slough out fistulous tracts. In any case, there is danger of poisoning. through absorption, if a sufficient amount of arsenic is used; 
but, on the other hand, the danger is slight if a large enough quintity is applied to canse rapirl slonghing. Arsenic is the principal constituent of so-cilled "sheep-dips" employed to kill ticks and other parasites in the wool. Finlay D Dm recommends $2 \frac{1}{2} \mathrm{lbs}$. of arsenous acid with an ecpual amount of pearl ash, soft soap, and sulphur, dissolred in 10 gallons of boiling water and added to 90 gallons of cold water. This quantity will suffice for dipping 100 sheep. The sheep are submerged, except their heads, for a few seconds, and placed on a grating to drain into a tub, from which the water flows back into the first receptacle. The excess of water in their fleeces is sqneezed ont with the hands and a scraper. Sheep have been poisoned after dipping, by eating grass and fodder on which they have drained; therefore the sheep should always be kept on clean floors or yards in the open air and smilight nutil they have become thoroughly dry. Sulpho-naphtol (1-100) or creolin are preferable for this purpose, since they are as effective and safer.

Uses Internal.-Arsenic is of the greatest service in the treatment of indigestion in horses associated with malnutrition and staring coat. In this condition, arsenons acid is: often combined with sodium bicarbonate and nux vomica, and may be given in powder on the food. It is also of value in atonic diarrhœa, and is nsed in both the serous and dysenteric varieties. In diminishing tissue change, and in acting as a blood tonic, arsenic is believed to improve the condition, endurance, and wind in horses, and is popularly prescribed by dealers and others.

The classical case of the arsenic-eating peasants of Styria seems to corroborate this view. These people appear to be very robust and healthy. Five grains of arsenic was given experimentally to one of them withont prodncing any untoward effect. Arsenic seems to influence farorably diseased mucous menibranes of the respiratory tract, to improve their nutrition, and hasten absorption and repair in diseases of the air passages. Coryza, ozœna, chronio 
cough, asthma, emphysema and "broken wind" are greatly benefitted by a course of arsenic, and in chronic conditions the treatment should be sustained for months in small doses.

"Thick wind" and convalescence from acute bronchitis, pneumonia or influenza are favorably influenced by arsenic. Arsenic is one of the best agents we can prescribe in general debility and anæmia and may be conjoined with bitters or iron.

In dry, scaly skin diseases, arsenic is the most successful internal remedy, but should not be prescribed in moist conditions associated with a proliferation of new cells, or exudate of serum or other liquid. It is particularly useful in psoriasis; also in chronic squamous, or papular eczema, lichen, acne and chronic urticaria, when given for a considerable length of time in small doses.

In the human subject, arsenic is almost a specific in chorea, but does not seem to yield such good results in that disease in dogs, usually associated with distemper. Fowler's Solution should be given to dogs with chorea, in doses of two or three drops three times daily, and gradually increased till the physiological limit is reached. Large single doses of arsenous acid ( $3 \mathrm{ss}$. ) are sometimes given with calomel ( 3 i.) and aloes ( 3 iv.), in a ball to horses to kill round worms.

Administration.-Arsenic is given to horses as Fowler's Solution, or arsenous acid, on the food. If continued for a long time, arsenic must be prescribed in small doses once daily, or in larger doses once in two or three days. Arsenic is exhibited to dogs in tablet or pill, and as Fowler's Solution. The administration of Fowler's Solution secures more rapid and accurate results than that of white arsenic. 


\section{Antimonium.}

(The metal antimony is not used in medicine.)

Antimonit Sulphidum. Antimony Sulphide. $\mathrm{Sb}_{2} \mathrm{~S}_{3}$. (U. S. P.)

Synonym.-Antimony trisulphide, E.; stibium oxydatum, oxydum antimonicum vel stibicum; oxyde d'antimoine, Fr.; antimonoxyd, G.

Derivation.-Native antimony sulphide, purified by fusion, and as free from arsenic as possible.

Properties.-Steel-gray masses of a metallic lustre and a striated, crystalline fracture, forming a black, or grayishblack, lustreless powder; without odor or taste, and permanent in the air. Insoluble in alcohol or water.

\section{Antimonin Solphidum Purificatum. Purified Antimony}

$$
\text { Sulphide. } \mathrm{Sb}_{2} \mathrm{~S}_{3} \text {. (U. S. P.) }
$$

Synonym.-Antimonium nigrum purificatum, B.P.; purified antimony trisulphide, E.

Derivation.-Antimony sulphide, 100 ; purified by maceration in ammonia water, 50 ; washing and drying. U. S.P.

Properties.-A heavy, grayish-black, lustreless powder ; without odor or taste, and permanent in the air. Insoluble in water or alcohol.

Dose.-The antimony sulphides should not be used in medicine. Dose would be twice or thrice that of tartar emetic.

Antimonium Sulphuratum. Sulphurated Antimony.

(U. S. \& B. P.)

Synonym.-Kermes Mineral. Chiefly antimony trisulphide $\left(\mathrm{Sb}_{2} \mathrm{~S}_{3}\right)$, with a very small amount of antimony trioxide.

Derivation.-Boil purified antimony sulphide, 100, with solution of soda, 1200. (U. S. P.) $\mathrm{Sb}_{2} \mathrm{~S}_{3}+4 \mathrm{NaOH}=\mathrm{Na}$ 
$\mathrm{SbO}_{2}+\mathrm{Na}_{3} \mathrm{SbS}_{3}+2 \mathrm{H}_{2} \mathrm{O}$. Strain and add sulphnric acid to the hot liquor as long as precipitation occurs. $\mathrm{NaSbO}_{2}+$ $\mathrm{Na}_{3} \mathrm{SbS}_{3}+2 \mathrm{H}_{2} \mathrm{SO}_{4}=\mathrm{Sb}_{2} \mathrm{~S}_{3}+2 \mathrm{Na}_{2} \mathrm{SO}_{4}+2 \mathrm{H}_{2} \mathrm{O}$.

Properties. - An amorphous, reddish-brown powder, becoming lighter in color on exposure to the light, and having neither odor or taste. Insoluble in water or alcohol.

\section{Golden Sulphur. (Non-official.)}

A yellow, insoluble powder, is another compound of antimony, consisting of a mixture of antimony trisulphide and trioxide.

Dose.-Neither kermes mineral nor golden sulphur are suitable for internal use. The dose would be twice or thrice that of tartar emetic.

Antrmoni Oxidum. Antimony Oxide. $\mathrm{Sb}_{2} \mathrm{O}_{3}$. (U. S. P.) (Antimony Trioxide.)

Derivation.-Pour a solution of chloride of antimony into water. The oxychloride of antimony is precipitated. $\mathrm{Sb} \mathrm{Cl}+\mathrm{H}_{2} \mathrm{O}=\mathrm{Sb} \mathrm{O} \mathrm{Cl}+2 \mathrm{H} \mathrm{Cl}$.

The precipitate is treated with carbonate of sodium, and the result is washed and dried. $2 \mathrm{Sb} \mathrm{O} \mathrm{Cl}+\mathrm{Na}_{2} \mathrm{C} \mathrm{O}_{3}$. $=\mathrm{Sb}_{2} \mathrm{O}_{3}+2 \mathrm{NaCl}+\mathrm{C} \mathrm{O}_{2}$.

Properties.-A heavy, grayish-white powder, without odor or taste, and permanent in the air. Almost insoluble in water and insoluble in alcohol.

Dose.-Would be twice that of tartar emetic, but the oxide is inappropriate for internal use.

Antmonil ex Potassin Tartras. Antimony and Potassium

Tartrate. $2 \mathrm{~K}(\mathrm{SbO}) \mathrm{C}_{4} \mathrm{H}_{4} \mathrm{O}_{6}+\mathrm{H}_{2} \mathrm{O}$. (U. S. P.)

Synonym.-Antimoninm tartaratum, B.P.; tartar emetic, tartarated antimony, tartarus emeticus, stibio-kali tartaricum, E.; antimonii potassio-tartras, antimoninm tartarizatum, tartarus stibiatus, P.G.; tartrate de potasse et d'antimoine émétique, tartre stibié, Fr.; brechweinstein, G. 
Derivation.-Make a paste with cream of tartar, antimony trioxide, and water. Set aside 24 hours, boil in water 15 minutes and crystallize. $2 \pi \mathrm{HC}_{4} \mathrm{H}_{4} \mathrm{O}_{6}+\mathrm{Sb}_{2} \mathrm{O}_{3}=2 \mathrm{~K}$ ( $\mathrm{SbO}) \mathrm{C}_{4} \mathrm{H}_{4} \mathrm{O}_{6}+\mathrm{H}_{2} \mathrm{O}$.

Properties. - Colorless, transparent crystals of the rhombic system, becoming opaque and white on exposure to the air; or a white, granular powder, without odor, and having a sweet, afterwards disagreeable, metallic taste. Soluble in 17 parts of water; insoluble in alcohol.

Dose.-H. \& C., 3 ii.-iv. (8.-15.); emetic, pigs, gr.iv.-x. (.24-.6); D., gr. $\frac{1}{10}-\frac{1}{2}$. (.006-.03); emetic, D., gr.i.-ii. (.06-.12).

\section{PREPARATIONS.}

Vinum Antimonii. Wine of Antimony. (U. S. P.)

Synonym.-Vinum antimoniale, B.P.

Antimony and potassium tartrate, $4 \mathrm{gm}$.; boiling distilled water, $65 \mathrm{Cc}$; alcohol, 150 Cc.; white wine to make 1000 Cc. (U. S. P.)

Dose for Dogs. 一 m v.- 3 i. (.3-4.).

Syrpus Scillo Compositus. Compound Syrup of Squill. (U. S. P.)

Synonym.-Hive syrup. Fluid extract of squill, $80 \mathrm{Cc}$; fluid extract of senega, $80 \mathrm{Cc}$; antimony and potassium tartrate, $2 \mathrm{gm}$.; precipitated calcium phosphate, $10 \mathrm{gm}$; sugar, $750 \mathrm{gm}$.; water to make $1000 \mathrm{Cc}$.

Dose for Dog. - $\Pi \nabla .-\mathrm{xxx} .(.3-2$.

\section{ACTION OF ANTIMONII ET POTASSIL TARTRAS.}

Tartar emetic is the only antimony compound suitable for internal use. The sulphide and oxide of antimony are insoluble, save in the hydrochloric acid of the gastric juice, and are not so certain or reliable in their action as tartar emetic. Kermes mineral and golden sulphur, containing variable amounts of antimony trisulphide and trioxide, are even more uncertain and unreliable than antimony sulphide or oxide.

External.-Tartar emetic is irritant, and when rubbed into the skin produces a pustular eruption and often sloughing and destruction of tissue.

Internal.-Stomach and Bowels.-Tartar emetic is a gastro-intestinal irritant, causing salivation and nausea in 
small doses, vomiting and diarrhœa in large quantities; while toxic amounts are followed by vomiting (in carnivora), serous or bloody purging, great depression of the circulation and respiration, muscular weakness, collapse and death.

Uneasiness, nausea, colic and death have been reported in horses only after enormous doses of tartar emetic by the month. The horse, and ruminants are comparatively insusceptible to the action of tartar emetic. The writer has observed a cow, however, in which nausea and actual vomition occurred, following a therapeutic dose of kermes mineral in electuary.

Tartar emetic is a powerful but slowly acting emetic (attended with a good deal of nausea) in dogs. Tartar emetic has been recovered in the first vomitus following its intravenons injection. It also expels the contents of a. bladder artificially replacing the normal stomach. These results go to show that tartar emetic acts both as a specific. emetic upon the vomiting centre, and locally as an emetic upon the mucous membrane of the stomach. Tartar emetic is eliminated in great part by the mucous membrane of the alimentary canal.

Circulation.-The principal action of antimony is exerted upon the heart and vessels. The heart muscle is weakened and vascnlar tension markedly lowered by large doses of tartar emetic. This action depends upon the influeuce of antimony on the cardiac muscle itself, and possibly upon the vagus nerve-endings in the heart. Whether the action upon vascnlar tension is clne to a centric or peripheral vasomotor depression, or is owing to relaxation of the vessel walls themselves, remains undecided. The pulse is reduced in force and frequency by large doses of tartar emetic. Following lethal amounts, the heart becomes flabby and relaxed, and death occurs in diastolic arrest. The preceding remarks apply only to the action of tartar emetic upon carnivora.

Antimony was formerly a very popular drug when general depressant and depletant treatment was in vogue, 
because of its powerfully depressing action upon the circulation.

Respiratory Organs.-Small doses of antimony increase secretion of bronchial mucus. Toxic amounts weaken the respiratory movement by lowering the functional activity of the respiratory and vagus centres, and canse a copious ontpouring of serous and mucous secretion into the bronchial tubes, which has the effect of drowning an animal in his own secretions. This action is only seen in horses after intravenous injection of toxic quantities of tartar emetic.

Nervous System.-Large doses of antimony depress the functional activity of the brain and sensory tract of the spinal cord. Larger doses prodnce loss of reflex action and anæsthesia, owing to the influence of antimony upon the sensory side of the cord ; while in toxic amounts, antimony is a general paralyzant to all the spinal centres and to the motor nerves. This action applies to man and carnivora, and not to horses or ruminants.

Muscles.-In carnivora and man, antimony lessens muscular strength and relaxes spasm through its depressing action upon the motor nerves and muscular tissue.

Elimination.-Antimony is mainly eliminated by the bowels, but also by the kidneys, bronchial mucous membrane, and other channels.

Toxicology.-'The symptoms are those described under "Action on the Stomach and Bowels." The pulse is first slow and then rapid and weak. The freal discharges in man are copious and of the rice water appearance characteristic of Asiatic cholera. If romiting is not free, zine sulphate shonld be given, or the stomach washed out. Tannic acid should be administered as a chemical antidote, together with the use of external heat, alcohol, strychnine and morphine subcutaneously, and demulcents by the moutli:

Uses External.-Tartar emetic is used in ointment, in the strength of $1-4$, over chronically enlarged and rheumatic joints of cattle. It is also employed over the sides of the 
chest in cattle, to produce counter-irritation and pustulation in the strength of 1 part to 12 of lard.

Internal.-The therapentic value of tartar emetic is limited mainly to canine practice. Antimony is still prescribed largely by the Germans as a general and circulatory depressant and expectorant for horses. General depressant, or anti-stimulant treatment is not ordinarily indicated in inflammatory affections, and even if it were, antimony does not exert such an action in any considerable degree upon horses or ruminants.

Aconite is a much more valuable and eficient circulatory depressant than antimony for the horse. There are three indications for antimony in canine practice: 1st, as an emetic; 2nd, as a general depressant in inflammatory diseases and in strong patients; 3rd, as an expecturant in acute bronchitis. The first indication is generally attained more promptly and safely by zinc sulphate. The second and third indications may be combined by prescribing antimony in the first, or dry stage of acute bronchitis in dogs, in the form of hive syrup. For example:

Ix.

Syr. Scillæ Co.

Sp't's Ather. Nitrosi................ āā $\tilde{z} s s$.

M. Liq. Ammon. Acetatis .............. ad $\xi \mathrm{iv}$.

S. Teaspoonful every 2 hours.

Antimony may be employed as an emetic in bronchitis to clear the stomach and upper part of the respiratory tract of secretions, and to reduce the force and frequency of the heart. Ipecac is, however, a better and safer agent for this purpose, and antimony is generally counter-indicated in the second, or exudative stage of bronchitis.

The Germans prescribe tartar emetic very commonly to horses as a parasiticide against round worms and tape worms. Four or five drachms are given in aqueous solution to the fasting animal, and followed by the administration of a dose of oil. 


\section{SECTION VIII.}

\section{Phosphorus.}

Phosphorus. Phosphorus. P. (U. S. \& B. P.)

Derivation.-Digest bones in sulphuric acid, or treat hone ash with sulphuric acid; filter and eraporate. $\mathrm{Ca}_{3}$ $\left(\mathrm{PO}_{4}\right)_{2}$ (bone ush) $+2 \mathrm{H}_{2} \mathrm{SO}_{4}=\mathrm{Ca} \mathrm{H}_{4}\left(\mathrm{PO}_{4}\right)_{2}$ (acid calcium phosphate) $+2 \mathrm{Ca} \mathrm{So}_{4}$.

Heat acid calcium phosphate, charcoal, and sand together, and distil over phosphorns into water.

Heat breaks up $\mathrm{Ca} \mathrm{H}_{4}\left(\mathrm{PO}_{4}\right)_{2}$ into $\mathrm{Ca}\left(\mathrm{PO}_{3}\right)_{2}$ (calcium metaphosphate) $+2 \mathrm{H}_{2} \mathrm{O}$.

Then : $2 \mathrm{Ca}\left(\mathrm{PO}_{3}\right)_{2}+2 \mathrm{SiO}_{2}+10 \mathrm{C}=\mathrm{P}_{4}+2 \mathrm{Ca} \mathrm{Si} \mathrm{O}$ $+10 \mathrm{C} \mathrm{O}$.

Properties.-A translucent, nearly colorless solid, of a waxy lustre, having at ordinary temperature about the consistency of bees' wax. By long keeping, the surface becomes red and occasionally black. It has a distinctive but disagreeable odor and taste. It should not be tasted except in a state of great dilution. When exposed to the air it emits white fumes which are luminous in the dark, and have an odor somewhat resembling garlic. On long exposure to the air, it takes fire spontaneously. Insoluble in water, or nearly so ; soluble in 350 parts of alcohol, in 80 parts of ether, in about 50 parts of any fatty oil, and very soluble in chloroform and carbon disnlphide. Besides the official form there are sereral other allotropic forms of phosphorus, including the red, or amorphous, the black, and the crystallized metallic phosphorus.

Red phosphorus is non-poisonous, and althongh it has heen commonly tanglit that it is inert, some recent experiments have tended to show that its therapeutic value is equal to that of the yellow phosphorus.

Dose.-H., gr.i.-ii. (.06-.12); C., gr.ii.-iii. (.12-.18); Sh. \& Sw., gr: $\cdot \frac{1}{100}-\frac{1}{20}(.0006-.003) ; \mathrm{D} ., \mathrm{gr}^{\circ} \cdot \frac{1}{100}-\frac{1}{20}(.0006-.003)$. 


\section{PREPARATIONS.}

Oleun Phosphoratum. Phosphorated Oil. (U. S. \& B. P.)

Phosphorus, $1 \mathrm{gm}$.; expressed oil of almond and ether, of each a sufficient quantity to make $100 \mathrm{gm}$. (U. S. P.)

Properties.-A clear, yellowish liquid, having the odor of phosphorus and ether. The ether in this preparation evaporates in time and the strength is proportionately, and perhaps dangerously, increased.

Dose.-H., 3 ii.-iii. (8.-12.); D., mi.-v. (.06-.3).

\section{Spiritus Phosphori. Spirit of Phosphorus. (U. S. P.)}

Phosphorus, $1.2 \mathrm{gm}$; absolute alcohol, a sufficient quantity to make 1000 Cc. Teaspoonful $={ }_{10}^{\frac{1}{0}}$ of a grain.

Dose.-D., Tlvii.-xxx. (.42.2.).

A saturated solution of phosphorus in alcohol may be made by dissolving . 312 parts of phosphorus in 100 parts of absolute alcohol.

Dose.-H., $\tilde{\text { ss. }}$ i. (15.-30.).

\section{ACTION OF PHOSPHORUS.}

Internal.-All that is certainly known of phosphorus concerns its effect upon the growth of bone. It has been proven experimentally that phosphorus stimulates the formation of bone, making the spongy tissue in the long and short bones thicker, and the compact tissue denser. If lime is abstracted from the food, the new growth of bone is of a gelatinous consistency.

Phosphate of lime increases more markedly the earthy constituents of bone. Phosphorus exists to a considerable extent as a component part of nerve tissue, as well as bone, and is to a less degree a constituent of other tissues. It is thought that phosphorus acts as a stimulant to the nutrition of those tissnes of which it is a constitnent part. In man, large therapentic doses of phosphorns frequently occasion sexual excitement, increased force and rapidity of the pulse, mental exhilaration and capacity for work. There is abundant clinical evidence to indicate that phosphorns is a nerve stimulant and tonic, but whether it is a general diffusible stimulant is not so certain. The therapentic action of phosphorus differs decidedly from its toxic effect. Phosphorus enters the blood in part as phosphorus, in the 
form of vapor, and in solution with fatty matters obtained in the digestive tract. It has everywhere a tendency to unite with oxygen, both within and without the body. It is probably converted in part in the intestiues and blood vessels into phosphoretted hydrogen, and becomes further oxidized into phosphoric acid, so that after the ingestion of phosphorus, phosphoretted hydrogen, phosphoric acid and phosphorus may be found in the blood. Neither phosphoric acid, the phosphates nor hypophosphites represent in any sense the action of phosphorus. Phosphoric acid is said to stimulate gastric digestion and resembles in action the phosphates, to which, however, it is therapentically inferior. The hypophosphites are probably converted in the digestive tract into phosphates. The phosphates generally differ from phosphorus in not being so stimulant to the nervous system, but serve as restoratives in supplying the constituents essential for the well-being of the various tissues. Phosphate of iron is a constituent of the blood corpuscles, sodium phosphate of blood plasma, potassium phosphate of nerve tissue, maguesium phosphate of muscular tissue, and calcium phosphate of bone. They tend to promote growth and nutrition of these tissues.

Toxicology.-The symptoms of poisoning do not ordinarily appear until some hours after ingestion of toxic doses. Then abdominal pain, uausea and vomiting (in those animals in which it is possible) and purging occur. The breath, vomitus and fæcal discharges may be luminous, aud have the odor of phosphorus. There is fever, anorexia and thirst. This condition is followed by an intermission in which the patient appears to be recovering, only to be succeeded by jaundice, nerrous symptoms, as delirium, coma and convulsions, and death. The urine rarely becomes albuminous in animals, but contains lencin and tyrosin.

Phosphorus is largely eliminated in the urine as hypophosphoric acid. There is general fatty degeneration of the viscera and muscles. The blood is disorganized, and there are widespread ecchymoses. Jaundice follows closure of 
the common, or hepatic duct, or smaller biliary tubules, and disorganization of the blood. There is rapid atrophy of the liver, and phosphorus poisoning in man is often indistinguishable during life from acnte yellow atrophy of the liver. Chronic poisoning, attented with necrosis of the jaw and other symptoms, and occurring among workers in phosphorus, is unlikely to occur in the lower animals. Acute poisoning is treated by emptying the stomach with a stomach tube or copper sulphate; the latter forming an insoluble phosphide of copper. Cathartics should also be administered. Permanganate of potash or hydrogen dioxide should be employed as antidotes, for their oxidizing action. Old turpentine is usually recommended as the antidote, but only the French variety is of any value, and that is generally unobtainable. Demulcents and opium are in order after evacuant and antidotal treatment has been carried out, but oil should never be given in phosphorus poisoning, as it assists the solution and absorption of the poison.

Uses.-Phosphorus is indicated as a stimulant to the growth of bone in rhacitis and osteomalacia, as a nerve stimulant and tonic in conditions of nervous exhaustion and impaired vitality, due to excessive activity of the sexual organs or otherwise. It is used empirically in treatment of boils, acne, and psoriasis, and has been prescribed with alleged advantage as a general stimulant in pneumonia.

Administration.-Phosphorus may be given in pill or ball, with cacao butter, or in the official preparations to dogs, and in the saturated alcoholic solution to horses. Phosphide of zinc represents the action of phosphorus, and yields phosphoretted hydrogen in its decomposition in the body. It may be given to dogs (gr. $\frac{1}{10}$ ) in the form of pills. 


\section{SECTION IX.}

\section{Chlorine.}

\section{Chlorum. Chlorine. Cl.}

The gas is not official.

\section{Aqua Chlorr. Chlorine water. (U.S. P.)}

Synonym.-Liquor chlori, B.P.; chlorum solutum, aqua oxymuriatica, solntion of chlorine, E.; aqua chlorata, P.G.; eau chlorée, chlore liquide, Fr.; chlor-wasser, G.

An aqneons solution of chlorine $(\mathrm{Cl})$, containing at least 0.4 per cent. of the gas.

Derivation.-Generate chlorine gas with hydrochloric acid, 35; water, 25; and manganese dioxide, 10. Heat in flask and pass gias into water.

\section{$4 \mathrm{H} \mathrm{Cl}+\mathrm{Mn} \mathrm{O}_{2}=\mathrm{Cl}_{2}+2 \mathrm{H}_{2} \mathrm{O}+\mathrm{MnCl}_{2}$.}

Properties.-A clear, greenish-yellow liquid, having the suffocating odor and disagreeable taste of chlorine, and leaving no residne ou evaporation. Incompatible with salts of silver and lead. The preparation deteriorates on keeping.

Dose.-Not nsed internally.

The B. P. preparation contains 0.6 per cent. of chlorine.

\section{Calx Chlorata. Chlorinated Lime. $\mathrm{Ca}_{2} \mathrm{Cl}_{2}, \mathrm{Ca}_{2}$.}

$$
\text { (U. S. P.) }
$$

Synonym.-Calx chlorinata, B. P.; calcaria chlorata, P. G.; chloris calcicus, chloruretum calcis, calcii hypochloris, chloride of lime, hypochloride of calcium, bleaching powder, E.; chlorure de chanx, poudre de Temnant, on de Knox, Fr.; chlorkalk, bleichkalk, G.

A preparation often improperly called "chloride of lime." It should contain not less than 35 per cent. of available chlorine.

Derivation.-Pass chlorine gas over calcium hydrate, 
when chlorinated lime, a mixture of calcium chloride and hypochlorite, results. $2 \mathrm{Ca} \mathrm{O}_{2} \mathrm{H}_{2}+2 \mathrm{Cl}_{2}=\mathrm{Ca} \mathrm{Cl}_{2} \mathrm{O}_{2}, \mathrm{Ca}$ $\mathrm{Cl}_{2}+2 \mathrm{H}_{2} \mathrm{O}$. It may also be regarded as a mixture of lime and chlorine. $2 \mathrm{Ca} \mathrm{O}_{2} \mathrm{H}_{2}+2 \mathrm{Cl}_{2}=2 \mathrm{Ca} \mathrm{O}, 2 \mathrm{Cl}_{2}+2 \mathrm{H}_{2} \mathrm{O}$.

Properties.-A white, or grayish-white granular powder, exhaling the odor of hypochlorous acid, having a repulsive saline taste, and becoming moist and gradnally decomposing on exposure to air. In water or in alcohol it is only partially soluble. It evolves chlorine on exposure to the air or on addition of an acid. Chlorinated lime possesses an alkaline reaction and bleaching properties. .

Dose.-Only of value externally.

PREPARATION.

Liquor Calcis Chlorinatce. Solution of Chlorinated Lime. (B. P.)

Contains $1 \mathrm{lb}$, to the gallon of water, or 13 grains of chlorine gas to the ounce.

Liquor Sod Chlorate. Solution of Chlorinated Soda.

(U. S. P.)

Synonym.-Liquor sodæ chlorinatæ, B. P.; Labarraque's solution, E. An aqueous solution of several chlorine compounds of sodium, containing at least 2.6 per cent., by weight, of available chlorine.

Derivation.-A solution of sodium carbonate, 150 ; chlorinated lime, 75 ; in water to make 1,000 .

Properties.-A clear, pale, greenish liquid, having a faint odor of chlorine and a disagreeably alkaline taste.

Dose.-Only of value externally.

ACTION AND USES OF CHLORINE.

Chlorine gas, in the presence of organic matter and moisture, unites with the lyydrogen of water and sets free nascent oxygen. When chlorine comes in contact with sulphuretted hydrogen, it removes and destroys the compound. Chlorine is thus a powerful oxidizing disinfectant agent and deodorizer. One-quarter of 1 per cent. of chlorine in solution is an effective germicide. When chlorine gas is 
iuhaled undiluted, it is an irritant to the respiratory tract, producing sometimes spasm of the glottis, or severe bronchitis, and at other times a condition of narcotism, with death from paralysis of the respiratory center. In dilute form it is stimulant, antiseptic, and deodorant in relation to the body. Chlorine gas may be generated from salt and black oxide of manganese, 1 part each; with commercial sulphuric acid and water, 2 parts each.

Bacteria are killed after three hours' exposure to a moist atmosphere containing 1 per cent. of chlorine gas. Chlorine may be used to advantage in this manner as a substitute for sulphur fumigation. Chlorine water is employed, well diluted, for the same purposes and with the same results as the solution of chlorinated soda. Chlorinated lime varies much in strength. To be of any value it should be so irritating to the eyes that it cannot be held near the face. It owes its medicinal value to the hypochlorite of lime which it contains. If the componnd is very moist, it is because calcium chloride preponderates. Chlorinated lime is often employed as a deodorizer, standing about premises in vessels, but is of no practical value unless it comes directly in contact with bacteria or sulphuretted compounds which it is desirable to destroy. It is the best and cheapest germ destroyer we possess for disinfecting premises and other appurtenances, apart from the body, as walls and floors of buildings, fæcal and other discharges, sewers, privies and cesspools. A 10 per cent. solution is to be employed on the floors, walls and other parts of buildings. The pure compound may be mixed with manure and discharges. Even a 1 per cent. solution is germicidal, and may be employed to wash blankets, harness and other paraphernalia. It is said not to harm woolen or cotton fabrics, in the latter solution. One or two pounds of chlorinated lime is a useful disinfectant when put in privy vaults once a week.

One or two ounces of chlorinated lime will render drinkable 65 gallons of putrid, bad smelling water, after a 
few hours hare elappsed for precipitation following the use of the compound. Upon the body, a 2 or 3 per cent. solution of chlorinated lime is employed as a stimulant, deodorant, and antiseptic, for decubitus, foul-smelling and gaugrenous sores, severe burus and indolent ulcers. It is used in 10 per cent. solution as a parasiticide in ringworm aud sciblies. A 1 per cent. solution forms a valuable wash in ulcerative stomatitis. Chlorinated lime may be prescribed, with an equal amonnt of lard, upon ulcers when a stimulating action is desired. Its iuterual administration is mudesirable. The solution of chlorinated soda is a slight caustic, deodorizer and antiseptic preparation on indolent, sloughing, foul-smelling surfaces. It mily be prescribed in sore throat, or ozœua, as a spray, or injected into the uterus, vagina or rectum. It is commonly diluted with 8 to 10 parts of water.

\section{Bromine.}

Bronum. Bromine. Br.

Derivation.-From seaweed and mineral springs.

Properties.-Heavy, dark, brownish-red liquid, volatilizing with the production of an irritating vapor. Soluble in 30 parts of water, and readily soluble in alcohol and ether. Of no value in veterinary medicine.

\section{Potassin Bromidum. Potassium Bromide. $\mathrm{K} \mathrm{Br}$.}

\section{(U. S. \& B. P.)}

Synonym.-Kalinm bromatum, P.G.; bromkalium, G.

Derivation.-Obtained from liquor potassa, bromine, and charcoal by the same process described in making potassium iodide (p. 247).

Properties. - Colorless, or white, cubical crystals, or grauules; odorless, and having a pungent, saline taste. Permanent in the air. Soluble in about 1.6 parts of water and in 200 parts of alcohol. 
Dose.-H. \& C., §i.-ii. (30.60.); Sh. \& Sw., 3 ii.-iv. (8.-15.); D., gr.v.- 3 i. (.3-4.).

Sodil Bromidum. Sodinm Bromide. Na Br.

(U. S. \& B. P.)

Synomym.-Bromure de sodium, Fr.; bromnatrinm, G.

Derivation. - Similar to potassium bromide. Liquor sodx is used instead of liquor potassz.

Properties.-Colorless, or white, cubical crystals, or a white, granular powder; odorless, and lhaving a saline, slightly bitter taste. From air the salt attracts moisture without deliquescing. Soluble in 1.2 parts of water, and in 13 parts of alcohol.

Dose.-Same as potassium bromide.

ACTION OF THE BROMIDES.

\section{External.-None.}

Internal. - In a large, single dose, the bromides canse in horses muscular weakness, dulness and staggering gait. The pulse is weakened and the respiration slowed. The urine is increased in quantity and sexual desire diminisled. Bromism may be produced in man, or the lower animals, by: the continnons administration of the bromides. This condition is characterized by general weakness and unsteady gait, mental dulness, indigestion, fetid breath, cutaneous anæsthesia, loss of sexual power, and occasionally an acneform eruption. Deatl has never been cansed in man by the bromides.

Nervous System. - The bromides are essentially depressant to nerve tissue. Therapentioally, this depressing action is seen particularly in relation to the motor centres of the cerebral cortex, and in lessening reflex action. The whole nervons system is depressed, but the motor tract in the brain and the sensory nerves are the first to succumb to the influence of the bromides. Intellection is clouded, and dulness and mental apathy are observed in man after large 
amounts. Reflex action is diminished owing to the depressing effect of the bromides upon the afferent nerves and reflex centres. Finally, with the continuous administration of large doses the motor area of the spinal cord, the motor nerves, and muscles fall under the depressing action of these agents.

Circulation.-Potassium bromide is a powerful depressant to the heart in toxic doses. In the smaller animals, therapentic amounts exert no appreciable effect npon the circulation. The depressing action of potassium bromide upon the heart is due partly to potassium and partly to bromine. The other bromides are less depressant, and it is safer to administer sodium bromide when large doses of these salts are desirable. The old idea that the beneficial action of the bromides, in relieving nervous excitability and in causing sleep, was due to the production of vasomotor spasm and cerebral anæmia, is now exploded.

Temperature.-The temperature falls, following the action of toxic amounts of the bromides, owing to vasomotor paralysis, vascular dilatation, and consequent loss of heat.

Krdneys.-Both the amount of urine and the quantity of urea are increased by large doses of bromides.

Sexual Organs.-The bromides diminish sexual desire and power. In so doing they either act upon the spinal centres or lessen peripheral sensibility of the genito-urinary tract.

Metabolism. - Carbonic dioxide elimination is notably decreased by the action of large doses of potassium bromide, while nitrogenous elimination from the kidneys is increased. It is therefore doubtful whether tissue change is increased or diminished.

Elimination.-Tho bromides are eliminated unchangerl by all channels and are fonnd in the sweat, mine, milk, salivi, intestiual secretions, etc.

Uses Internal.-The bromides, being particularly useful in the treatment of functional nervons diseases, do not possess nearly the value in veterinary medicine that they have 
in human practice. Moreover, their use is limited mainly to canine disorders, as bromides have little influence upon diseases of horses.

Bromides are especially indicated in irritation of the molor area of the cerebral cortex (convulsions), in general nervous excitability, and in conditions due to exalted reflex action in dogs.

The bromides are indeed the best agents we can use to I cure fits or convulsions in dogs. They should be combined with chloral and given, if necessary, per rectum. The bromides are useful in canine chorea, in connection with Fowler's Solution. With chloral the bromides are antidotes to strychnine poisoning. Sexual excitement in dogs may be allayed by the bromides. The bromides are occasionally of value in reflex cough, palpitation of the heart, and asthma, but are inferior to other agents in these disorders.

Potassium bromide is recommended in the treatment of tetunus of the horse, but opium, belladona and cannabis indica are generally more effectual. If the bromides are used they should be given with chloral.

\section{Iodine.}

IoDUM. Iodine. I. (U. S. \& B. P.)

Synonym.-Iodinum, U. S. P. 1870; iodum, P. G.; iode, Fr.; jod., G.

Derivation.-Iodine is a non-metallic element, existing in combination in the animal, vegetable and mineral kingdoms. It occurs in seaweed, from which it is obtained by distillation. It is also mined in the form of iodates and iodides.

Properties.-Iodine occur's in heavy, bluish-black, dry and friable rhombic plates, having a metallic lustre, it distinctive odor, and sharp, acid taste. It is soluble in 5,000 parts of water, and in 10 parts of alcohol; very soluble in ether aud in solutions of potassinm iodide. Iodine volatilizes on heating, with the formation of a 
a purple vapor. With starch, iodine forms au insoluble blue compound.

Dose.-H. \& C., 3 ss.-i. (2.-4.); Sh. \& Sw., gr.x.-xx. (.6-1.3).

Not often used in solid state.

PREPARATIONS.

Liquor Iodi Compositus. Compound Solution of Iodine. (Lugol's Solution.) (U. S. P.)

Iodine, 5; ppotassium iodide, 10; water to make 100. (1-20).

Dose.-H. \& C., zii-iv. (8.-15.) D., mii-x. (.12-.6). Should be given in one quart of water to the larger animals.

Tinctura Iodi.

(U.S.P.)

Iodine, 70; alcohol to make 1,000 .

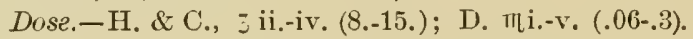

\section{Tinctura Iodi. (B. P.)}

Dose.-Same as U. S. P. tincture.

Unguentum Iodi. 1-25. (U. S. P.)

Too weak for most veterinary purposes.

Action External.-Iodine is an irritant upon the skin and mucous membranes. The yellow stain produced by iodine may be removed by ammonia water, alkalies and sodium hyposulphite. It is exceedingly doubtful whether iodine is absorbed through the unbroken skin, but it is thought to have a special resolvent and alterative action over and above that of other counter irritants. Whether this be true or not, its easy mode of application makes it a very convenient irritant for local uses.

Action Internal.-Iodine produces gastro-intestinal irritation and inflammation in large doses; and in toxic quantities indnces colic, vomiting-ir animals capable of the act, - purging and salivation. The pulse becomes rapid and weak; there is often suppression of urine, and occasionally nephritis. Widespread fatty degeneration has been found after fatal poisoning in the lower animals. If there is much starchy material in the bowels, the frcal discharges may 
be of a bluish color. Aphrodisiac action has been noted in man, following small doses of iodine. The treatment of acute poisoning is embraced in the use of starch by the mouth, or raw eggs, exterual heat; strychnine, alcohol, and atropine subcutaneously. Chronic poisoning by iodine, or iodism, in man, commonly causes symptoms analogous to a severe cold in the head, with pain over the frontal sin:s, sore thoat, running at the eyes and nose, gastric indigestion, together with an acneform, and occasionally purpuric, or furuncular eruption.

These more frequent symptoms of iodism occur as well after the administration of potassinm iodide as after that of iodine, but other rarer effects, such as wasting of the testes and mammary glands, and nervous disorders, e.g., neuralgic pains and convulsions, are hardly ever observed following the use of potassium iodide. It is evident that the physiological effects of iodine and potassium iodide are somewhat different; and clinical experience shows that the therapentic value of the metalloid differs in some respects from that of the salt. It is not definitely known in what form iodine exists in the body, after its ingestion, although it is usually taught that it is converted into an iodide. Both iodine and potassium iodide are readily absorbed from mucous membranes, and are found in all the tissues and fluids of the body. Iodine is eliminated, to a great extent as an iodide, probably of sodium, by the usual channels, as well as by the mucous membranes. The kidneys eliminate the greater amount, but iodine is found in the saliva and gastric juice after it has ceased to be present in the urine. Experiments concerning the action of iodine upon the kidneys and circulation, yield conflicting results, although the nitrogenous elements in the urine usually appear to be increased by its administration.

Uses External.--Iordine is of most value applied externally, or locally, while potassium iodide is administered internally because it is not irritating to the digestive organs. Although potassium iodide does not exactly represent the 
action of iodine, yet it is usually preferable for the reason just stated. Potassium iodide renders iodine soluble and prevents its precipitation in fluids within and without the body; it is therefore combined with iodine, when concentrated solutions are desirable. Six parts of potassinm iodide and twelve parts of iodine are added to one hundred parts of water, or ointment, to make a suitable counter-irritant. preparation for the horse. A useful tincture for veterinary purposes contains 15 parts of iodine and 18 parts of potassinm iodide in 100 parts of alcohol. Iodine is employed in aqueous and alcoholic solution, or in ointment, as above, either painted upon or rubbed into the skin over enlarged glands, rhenmatic swellings about the joints, or upon the chest in chronic pleuritis. It is also of value in strains, bruises, periostal inflammation and muscular rheumatism. In the horse, severe sprains and inflammation of joints, bones, and periostenm are treated more satisfactorily by blistering agents. Iodine is a valuable disinfectant and parasiticide, particularly in ringworm and favus in dogs, when the tincture is applied locally. Iodine is applied externally, in the form of the tincture, on patches of chrouic mange, eczema, and psoriasis.

Iodine is often recommended for erysipelas, but is inferior to ichthyol for this purpose. Iodine is injected into joints, synovial sacs, abscesses, and cavities of the body to promote healing through its antiseptic and irritant action; to cause adhesive infiammation, and in this manner to close cavities and to prevent the accumulation of fluids in them. The official tincture is commonly used for injections.

The tincture of iodine may be injected undiluted directly into the substance of enlarged glands, in amounts varying from 15 to 30 drops, to assist their absorption. If the tincture is injected into the subcutaneous tissue, abscess may ensne. Ozœna may be treated to advantage by irrigation with a solution containing one drachm of the tincture of jodine to the pint of normal salt solution. In inflammation of the upper air passages, iodine is sometimes beneficial as 
a stimulant and antiseptic inhalation, which is produced by adding one-half a drachm of iodine to the pint of boilingr water.

Uses Internal.-Iodine is thonght to act more satisfactorily than potassium iodide in the treatment of dialuetes insipidus or polyuria of the horse, in which it often appears to be a specific.

Administration.-Iodine may be combined with gentian and iron in the form of a ball, as recommended by Finlay Dun, or better, as Lugol's Solution, which is less irritating and more active.

Potassin Iodidum. Potassinm Iodide. K I. (U.S. \& B. P.)

Synonym.-Jodkalium, G.

Derivation.--Iodiue is dissolved in hot liquor potasse. $6 \mathrm{I}+6 \mathrm{~K} \mathrm{OH}=5 \mathrm{KI}+\mathrm{KIO} \mathrm{O}_{3}+3 \mathrm{H}_{2} \mathrm{O}$. The evaporated residue is heated with chircoal to remove $O$ from $K \mathrm{I}$ $\mathrm{O}_{3}$ (Potassinm Iodate). $\mathrm{KIO}_{3}+3 \mathrm{C}=\mathrm{KI}+3 \mathrm{C} \mathrm{O}$. The result is purified by crystallization.

Properties. - Potassium iodide occurs in colorless, transpareut or translucent, cubical crystals; or as a white, granular powder, having a slight odor of iodine, and a bitter, saline taste. Permanent in dry air, but slightly deliquescent in moist air. It is soluble in 0.75 part of water ; in 18 parts of alcohol, and 2.5 parts of glycerin.

Dose.-H., ¿ii.-iv. (8.-15.); C., j vi. (24.); STh. \& Sw., gr.xv.-xxx. (1.-2.); D., gr.ii.-x. (.12-.6).

\section{PREPARATION.}

Unguentum Potassii Iodidi (12 per cent.) (U.S. P.)

Unimportant in reterinary practice.

Sodi Iodidux. Sodium Iodide. Ta I. (U. S. \& B. P.

Synonym.-Iodure de sodium, Fr.; jodnatrum, G.

Derivation.-Made from sodinm hydrate in the sime manner as potassium iodicle. 
Properties.-Occurs in colorless, cubical crystals, or as a white, crystalline powder, without odor, and having a bitter, saline taste. Soluble in .6 part of water and in 3 parts of alcoliol.

Dose.-Same as potassium iodide.

Syrupus Acid Hydriodici. Syrup of Hydriodic Acid.

$$
\text { (U. S. P.) }
$$

Contains 1.3 per cent. of $\mathrm{H} \mathrm{I}$.

Derivation.-Potassium iodide, 13 ; potassium hypophosphite, 1; tartaric acid, 12; water, 15; diluted alcohol and syrup, of each a sufficient quantity to make 1,000.

Properties.-A colorless, odorless, syrupy liquid, of a sweet, acid taste.

Dose.-D., Mxv.- 3 i. (1.-4.)

Action External.-Potassium iodide is not absorbed unless rubbed into the skin with fat, and is not a local irritant, and therefore posseses very little value as an external application.

Action Internal.-Potassium iodide and iodine are both described by that unsatisfactory term, alterative. In certain diseases, as in rheumatism, iodine and the iodides alter nutrition and cause absorption of exudates in some unknown manner; hence the term alterative. In addition to this action, potassium iodide forms soluble compounds with the lead and mercury salts, and hastens elimination in poisoning by these metals. It is often taught that potassium iodide liberates iodine in the tissues, and that the latter forms soluble compounds with albumin, which are then readily eliminated; thus explaining the effect of potassium iodide in aiding resolution of morbid exudation and inflammatory thickenings. This action has yet to be definitely determined, and we do not know certainly how either iodine or potassium iodide exert their influence upon nutrition. Potassium iodide is absorbed and eliminated within a few minutes. 
Uses Internal.-Potassium iodide is useful in causing absorption of enlarged, lymphatic glands, and its action should be assisted by the application of iodine or red mercuric iodide externally. Potassium iodide, in small doses, diminishes congestion and increases the fluidity and amount of secretion in acnte laryngitis, acute and subacute bronchitis, and appears to possess an alterative action in improving the condition and untrition of the bronchial mucous membranes. It resembles ammonium chloride in the latter respect. It is also of value in chronic bronchitis, nuassociated with copious secretion. Chronic plenritis, pericarditis, and ascites are treated with potassium iodide, which assists absorption, and occasionally exerts a diuretic effect. Tardy resolution of pnenmonic cousolidation is lastened by potassium iodide. Endocarditis with cardiac hypertrophy is said to be benefited by potassium iodide and digitalis.

Champignon, or scirrhus cord of horses, is sometimes cured by the sorbefacient powers of potassium iodide in full doses. "Roaring" and "thick wind" may be cured by the administration of potassium iodide. Potassinm iodide is the best remedy known for actinomycosis. Is should be given to the larger animals in doses of 3 drachms daily, or in the same dose as Lugol's Solution. Potassium iodide is one of the many remedies prescribed in chronic rheumatism.

Summary.-Iodine and potassium iodide resemble one another in many respects. Iodine is a local irritant, potassium iodide is not. The known physiological action of potassium iodide and iodine does not explain their medicinal uses. In combating certain diseases, in an inexplicable manner, they are known as alteratives. In man, iodine is superior to potassium iodide in the treatment of scrofula. In the horse, iodine is considered of more value in the treatment of diabetes insipidus; while in both man and 
the lower animals, potassium iodide is regarded as more valuable in chronic rhenmatism. The action of iodine in benefiting local disorders, when applied externally, is due to its counter irritant effect, rather than to absorption.

Administration.-Potassium iodide is given in solution. Sodium iodide and syrup of hydriodic acid are simply substitutes for potassium iodide.

\section{Iodoformum. Iodoform. $\quad \mathrm{CH} \mathrm{H}_{3}$. (U. S. \& B. P.)}

Derivation.-Alcohol, potassium carbonate, iodine, and water are heated together. $\mathrm{C}_{2} \mathrm{H}_{6} \mathrm{O}+2 \mathrm{KHCO}_{3}+8 \mathrm{I}=2$ $\mathrm{CHI}_{3}+2 \mathrm{KI}+2 \mathrm{CO}_{2}+3 \mathrm{H}_{2} \mathrm{O}$.

Ioloform contains over 90 per cent. of iodine.

Properties.-Small, lemon-yellow, lustreless crystals of the hexagonal system; having a peculiar and very penetrating persistent odor, somewhat resembling that of saffron and iodine, and an unpleasant and slightly sweetish and iodine-like taste. Very slightly soluble in water; solnble in 52 parts of alcohol, and in 5.2 parts of ether. Very soluble in chloroform, benzine, fixed and volatile oils.

PREPARATION.

Unguentum Iodoformi. Ointment of Iodoform (1-10).

(U. S. \& B. P.)

Action External.-Iodoform is an antiseptic, local aursthetic, stimulant, protective, and dessicant agent. While many forms of bacteria will grow in iodoform, it is nevertheless a very ralnable antiseptic. The antiseptic properties seem to be bronglit ont by its decomposition when iodine is set free, and this decomposition goes on more readily in the presence of bacterial growth-in animal tissues and fluidsattended by the formation of toxins. Iodoform is particularly useful in septic and suppurating wonnds. When applied over extensive surfaces absorption and poisoning mily occur. The symptoms of iodoform poisoning are most diverse. The writer has had the fortune, or misfortune, 
to observe a case of fatal poisoning following abdominal section and packing of the pelvic cavity with iodoform gauze.

Symptoms of gastro-intestinal irritation are seen in poisoning in dogs, as romiting and diarrhœa, together with nervous symptoms, as convulsions, stupor and sleep. The pulse may be very rapid or infrequent; the temperature high or normal. Iodine is eliminated in the urine in iodoform poisoning and can easily be discovered by the starch test. General fatty degeneration of the interual organs is found after death. The local dessicant effect of iodoform on raw surfaces assists the antiseptic action, and the local anæsthetic properties combine to make iodoform the best antiseptic powder we possess, barring the odor.

Action Internal.-The internal action of iodoform possesses no therapeutic value. Care must be observed to prevent animals licking off iodoform from the surface of the body. One drachm will poison a dog weighing ten pounds. Elimination.-Iodoform is eliminated in the form of iodine, iodates, and iodides, by all the secretions.

Uses External. - Iodoform is chiefly of value applied over suppurating and septic surfaces, sores, and ulcers, where it hinders the growth of bacteria, stimulates unhealthy granulations, relieves pain, possibly neutralizes toxins, and certainly produces a vile odor. For this reason one of its substitutes should be employed when an antiseptic powder is desirable for use upon dogs living in or about dwellings. Iodoform is commonly employed in its purity. It may be mixed in any proportion with boric acid, or with taunic acid (1-8), for its astringent effect. Combined with collodion (1-15), it forms a useful dressing for sealing small wounds or abrasions upon the hands. The anresthetic action of iodoform is taken advantage of to relieve pain in fissure of the rectum, and hiemorrhoids. Zuill recommends the following combination by insufflation in the early stages of inflammation of the frontal sinuses:

Iodoform, Magnesia, Silver Nitrate-equal parts. 
Three grains may be used in suppositories for the smaller animals. Iodoform may cause healing in abscessinjected into the cavity with glycerin-(1-10), using 2-4 drachms of the mixture for smaller animals, or 1 ounce for the larger animals. Iodoform is very efficacious in the treatment of local tubercular lesions. Iodoform combined with lard or oil (1-10), is an excelient agent for burns which are not so extensive as to endanger the patient through absorption and iodoform poisoning.

\section{Iodolum. Iodol. $\mathrm{C}_{4} \mathrm{I}_{4} \mathrm{NH}$.}

(Non-official.)

Synonym.-Tetraiodopyrrol.

Derivation.-Action of iodine on pyrrol in solution in alcohol. Contains 88.9 per cent. of iodine.

Properties.-Crystalline, shining, yellow-brown powder. Tasteless and odorless. Practically insoluble in water; soluble in 3 parts of alcohol, and in ether and fatty oils. The surgical use of iodol has led to poisoning through absorption, but the latter is so slow that the danger is exceedingly slight. Iodol is suitable for all purposes in which iodoform is indicated. It is too expensive for general use, but is preferable for application to $\operatorname{logs}$, on account of its lack of odor.

\section{Aristolum. Aristol. $\mathrm{C}_{20} \mathrm{H}_{24} \mathrm{O}_{2} \mathrm{I}_{2}$. (Non-official.)}

Synonym.-Dithymol diiodide.

Derivation.-Made by the action of thymol in a solution of sodium liydroxide, on iodine, dissolved in a solution of potassium iodide. Contains 45.8 per cent. of iodine.

Properties.-Light reddish, or tawny-brown crystalline powder. Possesses peculiar iodine-like odor, and is insoluble in water, soluble in ether, fats, and slightly soluble in alcohol. Aristol is inferior as an antiseptic to either 
iodoform or iodol. It is nsed with some benefit in dry skin diseases, as psoriasis, in powler or oirtment. Other antiseptic dusting powders include acetanilie, bismnth sul)nitrate, salol and boric acid. Acetanilid has been slown to be a good antibacterial agent. It is cheap and may be applied pure, and it is an eflicient substitnte for iodoform. A few cases of poisoning have been reported following its extensive surgical nse. Bismuth subnitrate and salol may indnce poisouing when nsecl over large surfaces. They are dessicants and feeble antiseptics. Boric acid is harmless and mildly antiseptic.

\section{Orthoform. Orthoform.}

Orthoform is the methyl-erster of meta-amido-paraoxy-benzoic acid. It occurs as a white or dirty yellow, light. powder, sparingly soluble in water and alcohol but more so in glycerin and solutions of the mineral acids. It may be combined with iodoform, aristol, horic acid, salicylic acid, carbolic acid, turpentine and iodine withont incompatibility.

Action.-Externally, orthoform exerts a powerful anesthetic effect on raw surfuces, but has little action on intact mucons membranes and none on the unbroken skin. It is also a mild antiseptic, of abont the same valne as boric acid. It is but feebly toxic and poisoning does not ocenr nnless large quantities are applied over abraded surfaces. In the dog, 15 grains per 2 pounds of body weight have proved toxic when given by the mouth; and $7 \frac{1}{2}$ grains per 2 pounds of live weight are required to indnce fatal poisoning. It appears to be a cerebro-spinal paralysant in these large doses. The anesthetic action of medicinal doses is usually prolonged, varying from a few hours to two or three days. Orthoform has been nsed extensively in human medicine for the past few years, and cases of poisoning have been of rare occurrence and none fatal, although as much as two or three omnces have been applied on ulcerated surfaces in a 
week's time. Occasionally the drug produces an exythema or dermatitis owing to pecnliar susceptibility of the patient. On the other hand, orthoform has been used successfully $i_{1}$ the treatment of dermatitis following iry poisoning in the human.

Uses.-Orthoform is valuable chiefly for its power in relieving pain when applied to raw surfaces. It is an excellent application for burns. In superficial burus, orthoform may be combined to advantage with ichthyol, of each 10 per cent., in lanolin. In bums of the second and third degree, the use of orthoform and boric acid, equal parts, forms an excellent remedy. Orthoform is serviceable in Jelieving pain and irritation of nlcers, hemorrhoids and fissures of the rectum. In human practice it is largely employed in connection with diseases of the nose, ear and throat to arrest pain after operations, and in ulceration and iuflammation of these parts, as sore throat. Nasal gleet in horses should be benefited if not cured by the insufflation of the powder. Orthoform is commonly used in ointment containing 10 to 20 per cent. of the drug. It may be blown pure into cavities or applied as a saturated solution in collodion. It may be sprayed on a part with an atomizer in 5 per cent. solntion with equal parts of alcohol and water. It has been injected into the bladder in cystitis mixed with water. In ulceration and other painful affections of the stomach, orthoform will give relief owing to its local anesthetic action. It may be administered mixed with water and syrip in the dose of $0.5-1.0$ ( $7 \frac{1}{2}-15 \mathrm{gr}$.) for dogs. It is an expensive drug at present. 


\section{SECTION $\mathrm{X}$.}

\section{Sulphur.}

\section{Official Varietries.}

Sulphur Sublimatum. Sublimed Sulphur. S. (U.S. \& B. P.)

Synonym. - Flowers of sulphur, flores sulphuris, E; fleurs (crème) de soufre, Fr.; schwefelblumen, schwefelblüthe, $\mathrm{G}$.

Derivation.-Obtained from native sulphur by sublimation.

Properties.-A fine, yellow powder, having a slightly characteristic odor and a faintly acid taste. Insoluble in water; slightly soluble in absolute alcohol; more readily soluble in benzine, benzol, oil of turpentine and many other oils; also in ether, chloroform, and in boiling, aqueous solutions of alkaline hydrates.

\section{PREPARATIONS.}

Sulphur Lotum. Washed Sulphur. S. (U. S. \& B. P.)

Synonym.-Sulphur depuratum, flores sulphuris loti, P. G.; soufre lavé, Fr.; gereinigte schwefelblumen, G.

Derivation.-Obtained from sublimed sulphur which is treated with diluted ammonia water to wash out sulphurous and sulphuric and other impurities.

Properties._A fine, yellow powder, without odor or taste. Solubility, same as sublimed sulphur.

Pulvis Glyeyrrhiza Compositus. Compound Powder of Glycyrrhiza.

$$
\text { (U. S. P.) }
$$

Senna, 180; glycyrrhiza, 236; washed sulphur, 80; oil of fennel, 4; sugar, 500.

Dose.-1)ogs (laxative), 3 ss.-i. (2.-4.).

Ungnentum Sulphuris. Sulphur Ointment. (U. S. P.)

Washed sulphur, 300; lenzoinated lard, ro0. 
Uuguentum Sulphuris. (B. P.) (Ialf strength of U.S. P.)

Sulphur Precipitatum. Precipitated Sulphur. S. (U. S. \& B. P.)

Synonym. - Lac (magisterium) sulphuris, milk of sulphur, E.; soufre précipité, lait de soufre, Fr.; Schwefelmilch, G.

Derivation.-Obtained from a molution of sublimed sulphur, 100; in boiling calcium hydrate, 50; by precipation with hydrochloric acid. Calcium sulphide and hyposulphite are formed. $12 \mathrm{~S}+3 \mathrm{Ca} \mathrm{O}_{2} \mathrm{H}_{2}=$ $2 \mathrm{Ca} \mathrm{S}_{5}+\mathrm{Ca} \mathrm{S}_{2} \mathrm{O}_{3}+3 \mathrm{H}_{2} \mathrm{O}$. Then: $2 \mathrm{CaS}_{5}+\mathrm{Ca} \mathrm{S}_{2} \mathrm{O}_{3}+6 \mathrm{H} \mathrm{Cl}=$ $3 \mathrm{Ca} \mathrm{Cl}_{2}+12 \mathrm{~S}+3 \mathrm{H}_{2} \mathrm{O}$.

Dose (of sublined, washed or precipitated sulphur).-H. \& C., 亏̈ii.-iv. (60.-120.); Sh. \& Sw., 亏̈i.-ii. (30.-60.); D., 3 ss.-iv. (2.-15.).

Larger doses laxative; smaller for constitutional action.

Precipitated sulphur is the best preparation for internal use, as it occurs in a finer state of division and is more readily acted upon by the digestive juices. It may contain traces of sulphides or sulphuretted hydrogen, owing to the method of preparation.

Action External.-Sulphur has no action upon the skin when applied in the pure state. The ointment is the most effective agent in destoying acari which produce mange, grease, and scab. Sulphur does not kill acari as readily as a sulphide which is formed by the addition of an alkali. When sulphur ointment is rubbed into the skin it causes considerable irritation and an artificial eczema. Sulphur is converted into sulphuretted hydrogen and sulphurous acid by living tissue, and this transformation may occur to some extent when sulphur is rubbed into the skin with fat.

Action Internal.-Digestive Organs.-Sulphur is not acted upon by the stomach, but is somewhat dissolved by the alkaline intestinal juices, and converted in part into sulphicles and sulphuretted hydrogen. The sulphides, together with sulphuretted hydrogen, are absorbed into the blood. Minute traces of sulphuretted hydrogen are eliminated by the lungs and skin, while oxidation of sulphur 
also occurs in the body, as there is an increased secretion of urinary sulphuric acid and sulphates. The sulphides and sulphuretter hydrogen act as laxatives, and in all probability sulphur itself exerts a mild, mechanical irritation upon the bowels. Peristaltic motion and intestinal secretions are both slightly increased. The frecal discharges are soft and pasty, and offensive flatus containing sulphuretted hydrogen escapes from the intestines after the administration of sulphur.

Constitutional Action.-Dulphne acts remotely during its elimination in stimulating the functions of the skin and respiratory mucous membranes,--so-called alterative action. Sulphur causes disorganization of the blood, and depresses and paralyzes nerrous and muscular tissue after the continuous administration of colossal doses. Ordinary therapentic quantities of sulphur have no effect of this kind.

Uses External.-Sulphur is mainly of service externally as a local stimulant and parasiticide in skin diseases. The female acarus, which produces mange (acariasis), bores under the epidermis and lays her egess in the burows, while the male insect remains upon the surface. It is essential, therefore, to remove the epidermis in order to expose the ova. and female to the action of sulphur. The hair should be first shaved and the parts soaked and scrubbed with green soap and water. All cloths or brushes used in this operation should be burned or disinfected by boiling. Sulphur acts most efficiently in ointment, as fat fills up the burrows and deprives the insects of air. The official ointment is used in mange, but a combination with an alkali is more serviceable, unless there is active irritation of the skin, or eczema. The following ointment is recommended:

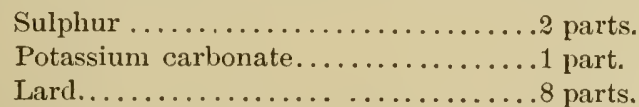

In obstinate cases of ringworm or mange, the preparation mentioned by Brunton is of value. This consists of 
one part each of sulphur and oil of cade, with two parts each of green soap and lard. True mange in the dog, if extensive and of long duration, is practically incurable by any drug. Sulphur ointment is of benefit in chronic eczema and acne, for its local stimulant and alterative action.

Uses Internal.-Sulphur is commonly used for its laxative action in the treatment of young animals and dogs suffering with constipation and hæmorrhoids. It may be of service in chronic bronchitis with copions secretion. Sulphur is frequently prescribed intermally for its action in chronic diseases of the skin, and is thought to exert a beneficial alterative action. Sulphur is also recommended in chronic rhenmatism.

Disinfectant Action.- When sulphur undergoes combustion, sulplurous anliydride $(\mathrm{SO})_{2}$ is evolved, and the latter combining with water forms sulphurons acid. $\mathrm{SO}_{2}+\mathrm{H}_{2} \mathrm{O}$ $=\mathrm{H}_{2} \mathrm{SO}_{3}$.

Sulphurous acid, and, in a less degree, sulphurous anlyydride, are germicidal, and are in common use as disinfectants. Bacteriological experiments, however, show that sulphur fumigation, as ordinarily employed for disinfection, is of little worth for destroying disease germs. Disinfection by formaldehyde or chlorine gas is to be preferred. Sole reliance should never be placed npon sulphur fumigation, but premises shonld be treated as recommended in the section on antiseptics and disinfectants (p. 718).

In employing sulphur as a disinfectant, animals must be removed from the premises and communication with the onter air shonld be excluded as far as possible. Three pounds of roll or flowers of sulphur, mixed with 2 ounces of alcohol, should be employed for every 1,000 cubic feet of air space to be disinfected. The premises which are being disinfected should be closed for six hours. Alcohol is employed to ignite the sulpluur and also to form steam by its combustion. The latter mites with sulphurous anliydride to make the more potent sulphurous acid. 
If steam can be introduced into the apartment, together with sulphur fumes, the effect is vastly more efficient. The sulphur and alcohol may be placed in an iron or earthen. ware ressel; floating in a tub of water to avoid danger of fire. The sulphur not infrequently fails to burn freely, and combustion is secured more certainly by placing flowers of sulphur with alcohol in an iron vessel which should rest upon a stand in a large iron pot. A lighted alcohol lamp is then put under the smaller iron vessel, containing the sulphur, and the lamp is ignited. The lamp insures complete combustion of the sulphur and the outer iron pot prevents the surrounding premises from catching fire.

Sulphurous anhydride has been employed for its local antiseptic and stimulant action, in inflammatory diseases of the upper air passages in liorses. For this purpose it is burned in such quantities that the vapor is capable of being inspired because largely diluted with air. With such dilution the antiseptic action is lost, and there is danger of producing considerable irritation, and the procedure is of loubtful value. The same treatment has been pursued in verminous bronchitis of lambs and calves, caused by the Strongylus filaria and S. micrurus. Local treatment, with tracheal injection, is more efficient.

\section{Acidum Sulphurosum. Sulphurons Acid. $\mathrm{H}_{2} \mathrm{SO}_{3}$.}

$$
\text { (U. S. \& B. P.) }
$$

Synonym.-Acide sulfureux, Fr.; schwefelige säure, G. Derivation.-Charcoal, 20, and sulphuric acid, 80, are heated together and the sulphurous anhydride evolved is passed into water. $4 \mathrm{H}_{2} \mathrm{SO}_{4}+2 \mathrm{C}=4 \mathrm{SO}_{2}+2 \mathrm{CO}_{2}+4 i$ $\mathrm{H}_{2} \mathrm{O} .4 \mathrm{SO}_{2}+4 \mathrm{H}_{2} \mathrm{O}=4 \mathrm{H}_{2} \mathrm{SO}_{3}$.

Properties. - A colorless liquid of the characteristic odor of burning sulphur, and of a very acid, sulphurous taste. It should contain not less than 6.4 per cent., by weight, of sulphurous anhydride, and not more than 93.6 per cent. of water. 
Dose.-H. \& C., ङi.-ii. (30.-60.); Sh. \& Sw., zi.-ii. (4.-8.); D., 3 ss.-ii. (2.-8.).

Actions cunt Uses. - Sulphurons acid decomposes organic matter, abstracts oxygen, and is transformed into sulphuric acid. The amonnt of sulphuric acid formed from the medicinal solntion is not sufficient to interfere with its action in or mpon the body. Sulphurous acid is a deodorant, deoxidizer, parasiticide and disinfectant externally. Solntions (1.4) are used as local stimnlant and antiseptic applications to wounds, ulcers, or sores with foul discharges, and to kill the fungus growths producing ringworm.

Sulphurous acid is employed as an antiseptic in indigestion, associated with flatulence, and has the same effect and value as the sulphites and hyposulphites which are used for the same purposes.

Potassa Sulphurata. Sulphurated Potassa. (U.S. \& B.P.)

Synonym.-Potassii sulphuretum, potassii sulphidum, liver of sulphur., E.; foie de sonfre, Fr.; kalischwefelleber, G.

Derivation.-Powdered and dried potassium carbonate, 200 , is mixed with sublimed sulphur, and heated in a crncible. Potassa sulplurata is a mixture of potassium liyposulphite and sulphide. $3 \mathrm{~K}_{2} \mathrm{C} \mathrm{O}_{3}+8 \mathrm{~S}=\mathrm{K}_{2} \mathrm{~S}_{2} \mathrm{O}_{3}+2 \mathrm{~K}_{2} \mathrm{~S}_{3}$ $+3 \mathrm{CO}_{2}$.

Properties. - Irregular pieces of a liver-brown color, which by exposure to the air gradually absorb moisture, oxygen, and carbonic dioxide, and change to greenish-yellow and finally to a gray mass containing potassium carbonate, hyposulphite and sulphate. The compound has a faint odor of hydrogen sulphide, and a bitter alkaline tiste. Soluble in 2 parts of water, with the exception of a small residue.

Dose.-H. \& C., 3 ii.-iv. (8.-15.); D., gr.ii.-x. (.12-.6).

Action Internal. - Sulphurated potassa has practically the same action as sulphur, internally and externally, although more stimulating locally. Large doses have caused gastro-intestinal inflammation, and the remote effects ob- 
served after repeated enormous doses of sulphur: i.e., disorganization of the blond and paralysis of nerves and muscles. It has been administered internally as a substitute for sulphur, but is only of value externally.

Action and Uses External.-Sulphurated potassa is one of the most serviceable agents we possess in the treatment of chronic forms of skin diseases, as psoriasis, lichen, but particularly eczema of the lorse and dog. It is locally stimulant, alterative (?), and parasiticide. There is only one drawback to its general use, which is its exceedingly disagreeable odor. Peruvian balsam is frequently substituted for this reason in the treatment of skin diseases in dogs. A solution, "yellow lotion," is used in different strengths (1-8 to 1-15), according to the amount of stimulation which the skin will endure. The "yellow lotion," is a good agent for killing lice upon the skin. The following prescription will be found of benefit in canine practice.

R

Potassæ Sulphuratæ.............. 3 ii.ss.

Chloralis.......................

Ol. Anisi ..................mii.

M.

Aquæ ad........................

S. External use.

The chloral relieves itching and the anise disguises to some extent the odor of hydrogen sulphide. 


\section{SECTION XI.}

\section{Acids.}

Acidum Hydrochloricum. Hydrochloric Acid. $\mathrm{H} \mathrm{Cl}$. (U.S. \& B. P.)

\section{(Muriatic Acid.)}

Synonym.-Acidum hydrochloratum, S. chlorhydricum, E.; acide chlorlydrique S. muriatique, Fr.; salzsaüre, G.

A liquid composed of 31.9 per cent., by weight, of absolute hydrochloric acid, and 68.1 per cent. of water. (U.S.P.)

Derivation.-Distil together sulphuric acid, sodinm chloride and water. The resulting hydrochloric acid gas is passed into distilled water, while acid sodium sulphate remains in the retort and is further acted upon by sodium chloride. $2 \mathrm{NaCl}+\mathrm{H}_{2} \mathrm{~S} \mathrm{O}_{4}=\mathrm{H} \mathrm{Cl}+\mathrm{Na} \mathrm{HSO}_{4}$; then : . $\mathrm{Na} \mathrm{HSO}_{4}+\mathrm{NaCl}=\mathrm{H} \mathrm{Cl}+\mathrm{Na}_{2} \mathrm{SO}_{4}$.

Properties.-A colorless, fuming liquid, of a pungent odor and an intensely acid taste. Spec. gr., about 1.163 at $15^{\circ}$ C. $\left(59^{\circ}\right.$ F.) Miscible, in all proportions, with water and alcohol.

Incompatibles.-Alkaline and other carbonates, and lead and silver salts.

PREPARATIONS.

Acidum Hydrochloricum Dilutum, Acidum Nitrohydrochloricum, Acidum Nitrohydrochloricum Dilutum.

Acidum Hydrochloricum Dilutum. Diluted Hydrochloric Acid.

(U. S. \& B. P.)

(Diluted Muriatic Acid.)

Derivution.-Hydrochloric acid, 100; distilled water, 219. Diluted hydrochloric acid contains 10 per cent. of absolute hydrochloric acid. (U. S. P.)

Properties.-It does not fume in the air and is without odor. Spec. gr., 1.050. Otherwise corresponds to hydrochloric acid. (U. S. P.)

Dose.-H., 3 i.-ii. (4.-8.); C., zii.-iv. (8.-15.); Sh., 3 ss.-i. (2.-4.); Sw. \& D., $\pi x \cdot-\mathrm{xxx} .(.6-2$.$) .$ 


\title{
Acidum Sulphuricom. Sulphuric Acid. $\mathrm{H}_{2} \mathrm{SO}_{4}$.
}

\author{
(U. S. \& B. P.)
}

Synonym.-Oil of vitriol, E.; acide sulphurique, huile de vitriol, Fr.; schwefelsäure, vitriolöl, G.

A liquid composed of not less than 92.5 per cent. by weight of absolute sulphuric acid, and not more than 7.5 per cent. water.

Derivation.-Sulphurous anhydride (U.S.P.), generated by roasting iron pyrites, or sulphur, is passed into leaden chambers. Nitric acid is introduced with steam, and the sulphurous anhydride undergoes oxidation and hydration. $2 \mathrm{HNO}_{3}+2 \mathrm{SO}_{2}+\mathrm{H}_{2} \mathrm{O}=2 \mathrm{H}_{2} \mathrm{SO}_{4}+\mathrm{N}_{2} \mathrm{O}_{3}$.

The nitrous acid combines with oxygen and water in the air, and is re-transformed into nitric acid, acting continually as a carrier of oxygen to sulphurous anhydride.

Properties.-A colorless liquid, of oily consistence, inodorous, and very canstic and corrusive. Spec. gr. not below 1.835. Miscible, in all proportions, with water and alcohol, with evolution of so much heat that the mixing requires great caution. (U.S. P.)

Incompatibles. - Alkalies and carbonates, calcinm and lead salts.

\section{PREPARATIONS.}

Acidum Sulphuricum Dilutum, Acidum Sulphuricum Aromaticum.

Acidum Sulpluricum Dilutum. Diluted Sulphuric Acid.

(U. S. \& B. P.)

Derivation.-Sulphuric acid, $100 \mathrm{gm}$; distilled water, $825 \mathrm{gm}$.

Properties. - Diluted sulphuric acid contains 10 per cent., by weight, of absolute sulphuric acid. Spec. gr. about 1.070 . (U. S. P.)

Dose.-H., 3 i.-ii. (4.-8.); C., 3 ii.-iv. (8 -15.); Sh., 3 ss.-i. (2.-4.); Sw. \& D., $\eta \mathrm{x}_{\cdot}-\mathrm{xxx} .(.6-2$.$) .$

Acidum Sulphuricum Aromaticum. Aromatic Sulphuric Acid.

(U. S. \& B. P.)

Synonym.-Tinctura aromatica acida, P. G.; elixir vitrioli Mynsichti, elixir of vitriol, E.; élixir vitriolique, teinture (alcool) aromatique sulphurique, Fr.; säure aromatische tinctur, Mynsicht's elixir, G. 
Derivation.-Sulphuric acid, $100 \mathrm{Cc}$; tincture of ginger, $50 \mathrm{Cc}$; oil of cinnamon, 1 Cc.; alcohol to make 1,000 Cc. (U. S. P.)

Properties.-Aromatic sulphuric acid contains about 20 per cent., by weight, of official sulphuric acid, partly in form of ethyl-sulphuric acid. Spec. gr. about 0.939 . (U. S. P.)

Dose.-H., 3 ss.-i. (2.-4.); C., 3 i.-ii. (4.-8.); Sh., Mxv.-xxx. (1.-2.); Sw. \& D., mv.-xv. (.3-1.).

\section{Acidum Nitricum. Nitric Acid. $\mathrm{HNO}_{3}$ (U. S. \& B. P.)}

Synonym.-Acide azotique, Fr.; salpetersäure, G.

A liquid composed of 68 per cent., by weight, of absolute nitric acid, and 32 per cent. of water.

Derivation.--Seven parts of sodium or potassium nitrate are distilled with four parts of sulphuric acid and water. $\mathrm{KNO}_{3}+\mathrm{H}_{2} \mathrm{SO}_{4}=\mathrm{KHSO}_{4}+\mathrm{HNO}_{3}$.

Properties.-A colorless, fuming liquid, very caustic and corrosive, and having a peculiar, somewhat suffocating odor. Spec. gr. about 1.414. (U. S. P.)

Incompatibles.-Alkalies and carbonates, iron sulphate, lead acetate and alcohol.

\section{PREPARATIONS.}

Acidum Nitricum Dilutum, A CIDUM Nitrohydrochloricum, ACIDUM NitrohydrochloricUM DILUTUM.

I. Acidum Nitricum Dilutum. Diluted Nitric Acid. (U. S. \& B. P.)

Derivation.-Nitric acid, 100; distilled water, 580. Diluted nitric acid contains 10 per cent, by weight, of absolute nitric acid. Spec. gr. about 1.057. (U. S. P.)

Dose.-H., 3 i.-ii. (4.-8.); C., 3 ii.-iv. (8.-15.); Sh., 3 ss.-i. (2.-4.); Sw. \& D., $m v_{\bullet} \cdot \mathrm{xxx} \cdot(.3-2$.$) .$

II. Acidum Nitrohydrochloricum. Nitrohydrochloric Acid. (U.S. P.) (Nitromuriatic Acid.)

Derivation.-Nitric acid, 180 ; hydrochloric acid, 820. Chemical composition uncertain.

Properties.-A golden yellow, fuming and very corrosive liquid, having a strong odor of chlorine. The strong acid should always be freshly prepared and should be used in preference to the diluted acid. It may be made off hand by mixing 4 parts of vitric acid with 16 parts 
of hydrochloric acid. The mixture should remain in an open bottle not more than half full, until the fumes pass off.

Dose.-H., $\Pi \mathrm{xx} .-\mathrm{xl} .(1.3-2.6) ; \mathrm{D} ., \mathrm{miii} .-\mathrm{v} .(.2-.3)$.

III. Acidum Nitrohydrochloricum Dilutum. Diluted Nitrochloric Acid.

$$
\text { (U.S. \& B. P.) }
$$

\section{(Diluted Nitromuriatic Acid.)}

Synonym.-Acidum chloro-nitrosum, P. G.; aqua regia S. regis, acide chloro-azotique S. chloro-nitreux, eau Regales, Fr.; salpetersalzsäure, königswasser, G.

Derivation. - Nitric acid, 40; hydrochloric acid, 180; distilled water, $\% 80$.

Properties. - A colorless, or pale yellowish liquid, having a faint odor of chlorine and a rery acid taste. Completely volatilized by heat. (U. S. P.)

Dose.-H., 3 i.-ii. (4.-8.); C., zii.-iv. (8.-15.); Sh., 3 ss.-i. (2.-4.); Sw. \& D., $\pi \mathrm{v} .-\mathrm{xxx}$. (.3-2.).

\section{Acidum Phosphoricum. Phosphoric Acid. $\mathrm{H}_{3} \mathrm{P} \mathrm{O}_{4}$.}

$$
\text { (U. S. \& B. P.) }
$$

A liquid composed of not less than 85 per cent., by weight, of absolute orthophosphoric acid $\left(\mathrm{H}_{3} \mathrm{P} \mathrm{O}_{4}\right)$, and not more than 15 per cent. of water. (U. S. P.)

Derivation.-Heat phosphorus with diluted nitric acid till uitrous fumes cease. $\mathrm{P}_{3}+5 \mathrm{HNO}_{3}+2 \mathrm{H}_{2} \mathrm{O}=3 \mathrm{H}_{3}$ $\mathrm{PO}_{4}+5 \mathrm{NO}$.

Properties.-A colorless liquid, without odor, but having a strongly acid taste. Spec. gr. not below 1.710. Miscible, in all proportions, with water or alcohol. (U. S. P.)

\section{PREPARATION.}

Acidum Phosphoricum Dilutum. Diluted Phosphoric Acid.

Derivation.-Phosphoric acid, 100; distilled water, 750. (U. S. P.) Diluted phosphoric acid contains 10 per cent., by weight, of absolute orthophosphoric acid. Spec. gr, about 1057.

Dose.-H., 3 i.-ii. (4.-8.); C., 3 ii.-iv. (8.-15.); Sh., 3ss.-i. (2.-4.); D., $\dddot{\nabla} .-\mathrm{xxx}_{\text {: }}(.3 \cdot 2$. 


\title{
Acidum Aceticum. Acetic Acid. $\mathrm{H}_{2} \mathrm{H}_{3} \mathrm{O}_{2}$.
}

\author{
(U. S. \& B. P.)
}

Synonym.-Acetum purum, - acetum destillatum, P.G.; acide acétique dilné, Fr.; reiner essig, $G$.

A liquid composed of 36 per cent., by weight, of absolute acetic acid, and 64 per cent. of water.

Derivation.-Distillation of dry sodium acetate with sulphuric acid and crystallization of the distillate. $\mathrm{Na} \mathrm{C}_{2}$ $\mathrm{H}_{3} \mathrm{O}_{2}+\mathrm{H}_{2} \mathrm{SO}_{4}=\mathrm{H} \mathrm{C}_{2} \mathrm{H}_{3} \mathrm{O}_{2}+\mathrm{Na} \mathrm{H} \mathrm{SO}_{4}$.

Properties.-A clear, colorless liquid, having a strong, vinegar-like odor, a purely accir taste and a strongly acid reaction. Spec. gr. about 1.048. Miscible with water or alcohol in all proportions.

\section{PREPARATION.}

Acidum Aceticum Dilutum. Diluted Acetic Acid. (U. S. \& B. P.)

Synomym.-Acetum concentratum, - acide acétique, Fr.; verdünnte essigsäure, $\mathrm{G}$.

Derivation.-Acetic acid, 100; distilled water, 500. Diluted acetic acid contains 6 per cent., by weight, of absolute acetic acid. Spec gr. about 1008 . Not employed internally except in the form of official aceta. Vinegar is impure diluted acetic acid, made by destructive distillation of wood, or by acetous fermentation and oxidation of alcoholic solutions, as cider.

$\mathrm{C}_{2} \mathrm{H}_{5} \mathrm{OH}+\mathrm{O}_{2}=\mathrm{H} \mathrm{C}_{2} \mathrm{H}_{3} \mathrm{O}_{2}+\mathrm{H}_{2} \mathrm{O}$. A temperature of $80^{\circ} \mathrm{F}$., and the presence of the ferment or mould (Mycoderma aceti), are necessary.

Acidum Aceticum Glaciale. Glacial Acetic Acid. $\mathrm{H} \mathrm{C}_{2} \mathrm{H}_{3} \mathrm{O}_{2}$.

$$
\text { (U. S. \& B. P.) }
$$

Symonym. - Acidum aceticum, P. G.; acidum aceticum concentratum, acetum glaciale, - acide acétique concentrée, esprit de vinaigre, vinaigre glacial, Fr.; essigsäure, eissesig, G.

Derivation.-Same as acetic acid.

Iroperties.-A clear, colorless liquid of a strong vinegarlike odor, and a very pungent, purely acid taste. Contaius about 99 per cent. of absolute acid. Not used internally. 
Acidum Tartaricum. Tartaric Acid. $\mathrm{H}_{2} \mathrm{C}_{4} \mathrm{H}_{4} \mathrm{O}_{6}$.

(U. S. \& B. P.)

Synonym.-Sal essentiale tartari, - acide tartrique, acide de tartre, Fr.; weinsäure, weinsteinsäure, G.

Devivation. - Boil acid potassium tartrate or argol (incrustation in wine casks) with chalk to form calcium tartrate. $2 \mathrm{~K} \mathrm{H} \mathrm{C}_{4} \mathrm{H}_{4} \mathrm{O}_{6}+\mathrm{Ca} \mathrm{C} \mathrm{O}_{3}=\mathrm{Ca} \mathrm{C}_{4} \mathrm{H}_{4} \mathrm{O}_{6}+\mathrm{K}_{2} \mathrm{C}_{4} \mathrm{H}_{4} \mathrm{O}_{6}$ $+\mathrm{H}_{2} \mathrm{O}+\mathrm{C} \mathrm{O}_{2}$. Add calcium chloride, which precipitates more calcinm tartrate, and decompose with sulphuric acid. $\mathrm{H}_{2} \mathrm{~S} \mathrm{O}_{4}+\mathrm{Ca} \mathrm{C}_{4} \mathrm{H}_{4} \mathrm{O}_{6}=\mathrm{H}_{2} \mathrm{C}_{4} \mathrm{H}_{4} \mathrm{O}_{6}+\mathrm{Ca} \mathrm{S} \mathrm{O}_{4}$.

Evaporate solution. Calcium sulphate crystals separate and are removed, while tartaric acid crystallizes on further evaporation.

Properties. -- Colorless, translucent, monoclinic.prisms, or crystalline crusts, or a white powder; odorless, having a purely acid taste, and permanent in the air. Soluble in about.8 part of water, and in 2.5 parts of alcohol. Not commonly used in Veterinary medicine.

Dose.-H., 3 ii.-iv. (8.-15.); D., gr.x.-xxx. (.6-2.).

Acidum Citricum. Citric Acid. $\mathrm{H}_{3} \mathrm{C}_{6} \mathrm{H}_{5} \mathrm{O}_{i^{*}} \quad$ (U.S. \& B. P.)

Synonym.-Acidum citri S. limonum, S. limonorum, acide citrique, acide du citron, Fr.; citrouensäure, G.

Derivation.-Usually prepared from the lemon (Citrus lemonum), or lime (Citrus bergamia). Boiling lemon juice (containing 7 per cent. of citric acid) is treated with chalk to form calcium citrate. $2 \mathrm{H}_{3} \mathrm{C}_{6} \mathrm{H}_{5} \mathrm{O}_{7}+3 \mathrm{Ca} \mathrm{C} \mathrm{O}_{3}=\mathrm{Ca}_{3}$ $\left(\mathrm{C}_{6} \mathrm{H}_{5} \mathrm{O}_{7}\right)_{2}+3 \mathrm{C} \mathrm{O}_{2}+3 \mathrm{H}_{2} \mathrm{O}$.

Calcium citrate is boiled with sulphuric acid and the resulting citric acid is obtained by filtration, evaporation and crystallization. $\mathrm{Ca}_{3}\left(\mathrm{C}_{6} \mathrm{H}_{5} \mathrm{O}_{7}\right)_{2}+3 \mathrm{H}_{2} \mathrm{~S} \mathrm{O}_{4}=2 \mathrm{H}_{3} \mathrm{C}_{6} \mathrm{H}_{5}$ $\mathrm{O}_{7}+3 \mathrm{Ca} \mathrm{S} \mathrm{O}_{4}$.

Properties. - Colorless, translucent, right - rhombic prisms; odorless, having an agreeable, purely acid taste; efflorescent in warm air and deliquescent when exposed to 
moist air. Soluble in 0.63 parts of water and in 1.61 parts of alcohol.

$$
\begin{gathered}
\text { Dose.-H., } 3 \text { ii.-iv. (8.-15.); D., gr.x.-xx. (.6-1.3). } \\
\text { PREParation. }
\end{gathered}
$$

Syrupus Acidi Citrici. Syrup of Citric Acid. (U. S. P.)

Citric Acid, 10; water, 10; spirit of lemon, 10; syrup to make 1000. Dose._Ad lib.

\section{Acidum Lacticum. Lactic Acid. $\mathrm{H}_{3} \mathrm{H}_{5} \mathrm{O}_{3}$.}

$$
\text { (U. S. \& B. P.) }
$$

Synonym.-Acide lactique, Fr.; milchsäure, G.

An organic acid, nsually obtained by subjecting milksugar or grape-sugar to lactic fermentation. Composed of 75 per cent., by weight, of absolute lactic acid, and 25 per cent. of water.

Properties.-A colorless, syrupy liquid, odorless, of a purely acid taste. Spec. gr. about 1.213. Freely miscible with water, alcohol, or ether.

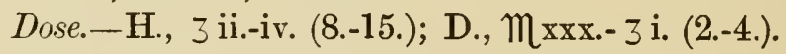

Other acids to be found in other sections.

Action External. - The concentrated mineral acids are powerful escharotics, but in dilution are stimulant, astringent, rubefacient, or vesicant, according to their streugth. Acids have a great affinity for the alkaline juices of the tissues and blood, and weak acid solutions are thus neutralized. Strong acids coagulate albumin, probably by combining with alkalies which hold albumin in solution, forming dense compounds with the albuminoids of the tissue. When mineral acids are present in abundance the albumin first coagulated is afterwards dissolved (except nitric acid), and the corrosive action of the acids is extensive. Acids further destroy tissue by combining with water, for which they have also a great affinity, particularly sulphuric and phosphoric acids. The former withdraws water to such an extent that the tissues are carbonized and blackened. Sulphuric acid is more destructive of tissue and acts more extensively than 
the other mineral salts. Nitric acid is less caustic, and hydrochloric acid is the least corrosire. Nitric acid stains the parts yellow, and hydrochloric, white. The vegetable acids, as citric and tartaric acids, are slightly caustic, but are irritant to the skin, and still more so to raw surfaces and mucous membranes. An ounce of tartaric acid has caused death in man through its local effect. The action of the caustic alkalies is more widespread than that of the mineral acids. Diluted sulphuric and nitric acids are astringents, because of their power of condensing tissues, and also hemostatics in causing compression of blood ressels by contraction of the tissues about them. Nitric acid is commonly employed externally, becanse its effect is limited by its own eschar, which is not dissolved by an excess of acid.

Hydrochloric acid has no astringent effect and is not used externally for its canstic properties. The acids are antiseptic, but are less appropriate than other agents in most cases, on account of their irritant action.

Action Internal.-Alimentary Canal.-Acids stimulate the normal alkaline secretions in the body, particularly those entering the month and intestines. The flow of saliva, bile and intestinal juices is therefore increased. Diluted acids are called refrigerants in imparting a sense of coolness to the skin and mucous membrane of the mouth, and, by augmenting the secretion of saliva, relieve thirst in fever. In opposition to the action of acids in stimulating alkaline secretions, exists the fact of their checking acid secretions, ${ }^{*}$ such as the gastric juice and sweat, and if the administration of acids is prolonged for several weeks, gastric digestion may be impaired in consequence, unless the gastric juice is abnormally deficient. The acids exert a local stimulant and astringent action upon the intestinal canal. Nitric acid is particularly a stimulant, sulphuric acid an astringent, while nitric and nitrohydrochloric acids are especially cholagogues. Hydrochloric acid assists digestion in supplying a deficiency in the uormal hydrochloric acid of the gastric juice, in aidiug the conver-

* With the exception of hydrochloric acid. 
sion of proteids into peptones by pepsin, and in stimulating the formation of the latter. Mineral acids are essentially digestive tonics, promoting gastric digestion $(\mathrm{H} \mathrm{Cl})$, increasing the secretion of the liver and intestinal glands, and improving the tone and blood supply of relaxed intestinal mucous membrane.

Constitutional Action.-Acids are absorbed, and in considerable doses make the blood less alkaline, the urine more acid, and appear to exert a slight astringent action upon the skin and mucons membranes during their elimination. We possess no knowledge to explain the remote effects of the acids. Some authorities state that the acids are converted into salts in the blood, as sulphates, nitrates and phosphates, which are eliminated by the urine, skin and bowels. Others teach that much of the acid is eliminated in the urine in the form of ammonia compounds. Brunton says that the acids cause a modification of tissue change in the liver so that the production of urea is lessened by the passage of the acids through that organ, and that the ammonia furnished by the acids is not converted into urea, as normally happens, but is eliminated in the urine as such. Both these suppositions are apparently fallacions, because if the acids were transformed into salts they woukd not produce remote astriugent effects, and if they were converted into ammonia compounds they would not acidify the urine. The probable explanation is that in small doses acids are more prone to decomposition, while large doses are eliminated in part unchanged by the kidneys, bowels and skin, and so acidify the urine and exert a remote astringent action.

Acidity of the bloor canses death in depressing the heart, and becanse an alkaline or nentral medium (normal blood) is essential to carry away carbouic dioside from the tissues. Vegetable acids are thought more especially to lower the force of the heart, which they critainly do in poisonous quantities, and possibly in malicinal doses. In cousiderable amount, the vegetable acils increase the acidity of the urine, but in small doses alkalize this secretion by 
undergoing decomposition in the blood with the prouluction of alkuline carbonates. The regetable acids are infrequently used in veterinary medicine.

Diluted phosphoric acid relieves thirst and forms an agreeable cooling drink in fevers. It is extremely doubtful whether phosphoric acid acts either as a tonic or reconstituent, by supplying phosphates to the blood or tissues.

Poisoning.-If acid is spilled on the skin, alkalies should be applied, and in case of sulphuric acid the excess of acid should first be rubbed off, and then large quantities of very dilute alkaline solutions or soapsuds should be employed to avoid evolution of heat when the acid combines with water. When acids are swallowed, there is excoriation and sloughing of mucous membranes, difficulty in swallowing, vomiting of dark brown material and mucus (in animals capable of the act), severe colic, pain on movement, constipation, or, rarely, bloody diarrhœa. Oscusionally some acid flows into the laryux during deglutition ancl oelema and suffocation rapidly ensue. There is inflammation of the upper part of the digestive canal, thirst, ant collapse, with weak pulse and cold extremities. Softening, slonghs, hremorrhage and perforation of the mucous membrane of the month, gullet and stomach and small intestines are found post mortem.

Treatment.-Soapsuds, sodium bicarbonate, lime water, magnesia, or other alkalies. Demulcents, as milk, white of egg, gum arabic and linseed tea. Opinm and stimulants. Wash ont the stomach with a large amount of alkaline solution.

Uses External. - Strong mineral acids are used as caustics. One part of sulphuric acid is mixed with three parts of sulphur, or asbestos, to form a paste for the destruction of morbid growths.

The application of sulphuric acid is somewhat dangerous, as it is difficult to limit the action, and it is not by any means the best escharotic, nor so gool as nitric acid, which produces less extensive destruction of tissue, and is a 
useful agent for the removal of tumors, for the canterization of bites infiicted by rabid animals, and for the treatment of fonl, sloughing wounds or foot rot.

The action of nitric acid may be limited by surrounding the part to which the strong acid is applied with oil, or by washing the acicl off with soapsuds. Glacial acetic acid is freguently employed to remove warts and small excrescences. It is not so powerful as sulphuric or nitric acid.

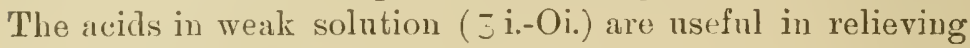
irritation of the skin, as in urticaria, for their astringent action upon piles, and to stop slight hæmorrhages. Vinegar diluter with 3 parts of water may be used for the same purposes. Nitric acid ( $M$ v.-xxx. to $\xi \mathrm{i}$. of water) is a good antiseptic, stimulant and astringent application to indolent ulcers, wounds, or, in the month, for ulcerative or mercurial stomatitis.

Uses Internal. - The acids are all of value in digestive disorders. Hydrochloric acid is useful in fever, to relieve thirst and aid digestion, when a few drops may be put in the drinking water. Hydrochloric acid is especially indicated for gastric indigestion with deficiency in the secretion of gastric juice, and for fermentation and tympanites in chronic gastritis. Hydrochloric acid should be given after feeding, and is often combined with bitters. It acts as an antiseptic in addition to aiding digestion. Hypersecretion of hydrochloric acid, or hyperchlorhydria, is said to be characterized by acid reaction of the secretion in the month, and a desire to lick alkaline earthy matters. It is best treated by alkalies after feeding. All the acids are serviceable in the treatment of diarrhoa and intestinal indigestion. Aromatic sulpluric acid (with opinm) is more particularly valuable as a remedy for watery purging. Nitric and nitrohydrochloric acids are of more use in diarrhoea with indigestion, jamdice, and disordered hepatic functions. The latter acid is often combined with nux vomica in the treatment of intestinal indigestion, and is a. vilnable remedy in catarrhal jaundice of dogs. 
Sulphuric acid is utilized in acute lead poisoning, as an antidote, to form insoluble sulphates in the bowels. The acids are given for their remote astringent action in arresting or preventing hæmorrhage (purpura) from internal organs, and in diminishing excessive sweating and mucous discharges; but are inferior to other agents in the treatment of these conditions.

Administration. - The acids should all be thoroughly diluted with water for internal use.

\section{Acidum Boricum. Boric Acid. $\mathrm{H}_{3} \mathrm{~B} \mathrm{O}_{3}$. (U.S.\& B. P.)}

Synonym.-Boracic acid, E.; acide borique, Fr.; acidum boracicum, sal. sedativum Hombergü, börsäure, G.

Derivation._Made by evaporation and crystallization of solutions obtained by passing steam issuing from rocks in volcanic regions of Italy, through water; or by the action of hydrochloric or sulphuric acids upon borax. $\mathrm{Na}_{2} \mathrm{~B}_{4} \mathrm{O}_{7}$ (borax) $+10 \mathrm{H}_{2} \mathrm{O}+2 \mathrm{H} \mathrm{Cl}=4 \mathrm{H}_{3} \mathrm{~B} \mathrm{O}_{3}+2 \mathrm{NaCl}+5 \mathrm{H}_{2} \mathrm{O}$. Recovered by filtration and recrystallization.

Propertieș.-Transparent, colorless scales, of a somewhat pearly lustre, or, when in perfect crystals, six-sided triclinic plates, slightly unctuous to the touch; odorless, having a faintly bitterish taste, and permanent in the air. Soluble in 25.6 parts of water and in 15 parts of alcohol; also soluble in 10 parts of glycerin. It is feebly acid.

Dose.-Foals and calves., gr.xx.-xxx. (1.3-2.).

PREPARATION.

Glyceritum Boroglycerini. Glycerite of Boroglycerin. (U. S. P.)

Synonym.-Glycerite of glyceryl borate, solution of boroglyceride. Boric acid, 310 ; glycerin to make 1,000 . Solution prepared by heat $\left(302^{\circ} \mathrm{F}\right.$.)

Sodil Boras. Sodium Borate. $\mathrm{Na}_{2} \mathrm{~B}_{4} \mathrm{O}_{r^{*}} \quad$ (U. S. \& B. P.)

Synonym.-Borax, natrum biboricum, boras, boras sodicus, E.; borate de soudre, Fr.; börsäures natron, G. 
Derivation.-Natural deposits of the crude article occur as incrustations on shores of lakes in Nevada and California, which are purified by calcination and crystallization. Borax is also made by boiling boric acid with sodium carbonate. $4 \mathrm{H}_{3} \mathrm{~B} \mathrm{O}_{3}+\mathrm{Na}_{2} \mathrm{C} \mathrm{O}_{3}=\mathrm{Na}_{2} \mathrm{~B}_{4} \mathrm{O}_{7}+\mathrm{C} \mathrm{O}_{2}+6 \mathrm{H}_{2} \mathrm{O}$.

Properties.-Colorless, transparent, monoclinic prisms, or a white powder, inodorous and having a sweetish, alkaline taste. Slightly efflorescent in warm, dry air. Solnble in 16 parts of water at $59^{\circ} \mathrm{F}$., and in 0.5 part of boiling water; insoluble in alcohol. At $176^{\circ} \mathrm{F}$. it is soluble in one part of glycerin. Borax is slightly alkaline.

\section{ACTION OF BORIC ACID AND BORAX.}

Boric acid and borax are essentially mild, non-irritating antiseptics. These agents are practically harmless, as ordinarily employed, yet death in man has been reported from absorption of a 5 per cent. solution of boric acid nsed for irrigation of the large cavities of the body, and fatalities lave resulted from the ingestion of quantities of boric acid.

Three drachms of boric acid may be given daily to dogs, withont causing any untoward effect. In man, boric acid poisoning has been exhibited by feeble pulse, subnormal temperature, vomiting, ery thema and swelling of superficial parts, involuutary evacuations, coma, and disordered respiration. Boric acid is eliminated by the urine, sweat. saliva and fæces. It is said to be diuretic, and, in large doses, to induce acute parenchymatous nephritis.

Boric acid and borax, like other antiseptics, relieve itching and lestroy parasites upon the skin. Boric acid exerts an antiseptic action upon the contents of the digestive tract and upon the urine. It is thonght to possess some emmenagogne action.

\section{USES OF BORIC ACID AND BORAX.}

The value of boric acid and borax is chiefly surgical. They are employed more commonly upon the mucous mem- 
branes of the eyelids, mouth, nose, vagina, urethra and bladder, for their non-irritating, antiseptic properties, and also to relieve itching and to destroy parasites upon the skin. Boric acid is in more frequent use than any other agent in simple catarrhal conjunctivitis, and may be combined to advantage with cocaine as follows:

R

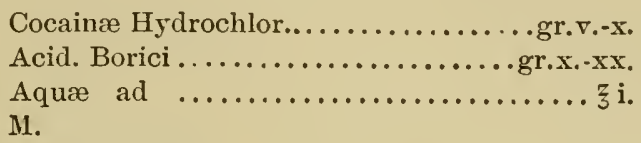

M.

S. Eye lotion.

Borax is perhaps more appropriate in the mouth, being alkaline. It is applied by swab, in saturated solution, for aphthous and other forms of stomatitis. A saturated solution (4 per cent.) of boric acid is useful as an injection for ozœna, eystitis and vaginitis. Borax in saturated solution assuages pruritus ani and vulvæ, and is employed as an application for ringworm. Boroglyceride is indicated for burns and wounds.

A serviceable antiseptic dressing is made by soaking gauze in a boiling saturated solution of boric acid. The dried ganze contains boric acid, which crystallizes upon it as the solution cools. One part of boric acid in combination with four parts of zinc oxide forms a soothing, protective dusting powder for chafed surfaces, intertrigo, ery thema and moist eczema in dogs. Boric acid is occasionally prescribed in 10 per cent. vintment for eczema and psoriasis. It is perhilps the best remedy for canker of the ear in dogs (otitis externa). The ear should be syringed out with a 2 per cent. solution, dried with absorbent cotton, and powdered with pure boric acid. The internal uses of boric acid are unimportant. It has been recommended and given as an emmenagogue, and as an intestinal antiseptic in fermentative dimrhoen of foals and calves. Boric acid is often administered in human medicine to acidify the urive and disinfect the genito-urinary tract, although not so efficient as urotropin. 


\section{SECTION XII.}

\section{Class 1.-Carbon.}

Carbon is represented officially as follows:

Carbo Animalis. Animal Charcoal. (U. S. \& B. P.)

Synonym. - Bone black, E.; charbon, Fr.; thier (or) knochen-kohle, G.

Derivation.-Bones freed from fat are exposed to great heat in close iron cylinders till ammoniacal vapors pass off. The resulting charcoal is pulverized and contains abont 10 per cent. of carbon and 88 per cent. of calcium phosphate and carbonate.

Properties.-Dull, black, granular fragments, or a dull black powder; odorless, nearly tasteless, and insoluble in water or alcohol.

Dose.-H. \& C., 3 i.-ii. (30.-60.); Sh. \& Sw., 3 ii.-iv. (8.15.); D., gr.xx.- 3 i. (1.3-4.).

\section{PREPARATION.}

Carbo Animalis Purificatus. Purified Animal Charcoal. (U.S. \& B. P.)

Derivation.-Animal charcoai, 100, is boiled with hydrochloric acid, 300, and water to remove ash. Result filtered and residue ${ }^{\circ}$ n filter paper washed and dried.

Properties. - A dull, black powder, odorless and tasteless; insoluble in water, alcohol or other solvents

Dose.-Same as above.

\section{Carbo Ligni. Charcoal. (U. S. \& B. P.)}

Synonym.-Wood charcoal.

Derivation.-Soft wood is charred by piling it in leaps, igniting, and covering it with sand and turf to prevent rapid combustion.

Properties.-A black, odorless and tasteless powder, free from gritty matter. Insoluble in water or alcohol.

Dose.-Same as animal charcoal.

Action External.-Charcoal is an oxidizing antiseptic 
and deodorizer. It has the power, when dry, to absorb liquids and gases, condensing the latter within its pores. It thus both absorbs oxygen and gives up oxygen when in contact with oxidizable substances.

In dessicating and oxidizing decomposing organic matter, charcoal deprives germs of their proper moisture and food requirements for growth, but is not a true germ destroyer or disinfectant. Cliarcoal absorbs noxious and foul-smelling gases, and is thus a deodorant.

Action Internal.-Charcoal has proved serviceable in certain digestive troubles. Since charcoal loses its absorptive properties so soon as it becomes thoroughly wet, it seems probable that the beneficial action in such conditions is due to the charcoal scraping off mucus from the walls of the stomach and bowels, or to increasing their vascularity and peristalsis, rather than to its absorbing gases. If administered continually in large quantities charcoal may produce mechanical obstruction in the bowels, and it is therefore employed in conjunction with laxatives.

Uses External.--Charcoal is applied as a dusting powder with astringents and antiseptics upon ulcers, galled and raw surfaces. The following combination is useful: charcoal, 4 parts; salicylic acid, 2 parts; burnt alum, 1 part. It is also used in flaxseed meal ponltices upon foul, sloughing parts. Animal charcoal is better than wood charcoal as an absorbent, although the unpurified bone black is said to be more efficient, since the mineral matters separate the carbon particles and aid its absorptive power.

Uses Internal.-Charcoal is employed occasionally in indigestion, chronic gastric and intestinal catarrh, tympanitis and diarrhcea, accompanied by uncous discharges. Charcoal possesses the power of attracting and holding alkaloids in its subtance, and so may be used in large doses as an antidote in poisoning by alkaloidal drugs, as opinm, nux romica and aconite. Its action is slow, and other means, as tannic acid, emetics and the stomach tube, are more efficieut. 
Carbonei Disulphidum. Carbon Disulphide. $\mathrm{C} \mathrm{S}_{2}$. (U.S. P.)

Synonym.-Carbonii bisulphidum, bisulphide of carbon, carbon sulphide, E.; carboneum sulphuratum, alcohol sulfuris, P. G.; sulfure de carbone, Fr.; schwefelkohlenstoff, G.

Derivation. - Obtained from carbon and sulphur by distillation.

Properties.-A clear, colorless, highly refractive liquid; very cliffusive, having a strong, characteristic, but not fetid odor, and a sharp, aromatic taste. Soluble in 535 parts of water; very soluble in alcohol, ether, chloroform, and fixed volatile oils. Spec. gr. 1.268 to 1.269. Carbon disulphide vaporizes readily and is highly inflanmable.

Actions and Uses.-Carbon disulphide is a rapid and powerful anæsthetic when inhaled, and also locally, when applied in spray upon the skin. It produces muscular weakness, coma, and, rarely, convulsions in toxic doses. It has been used as a counter-irritant in the form of vapor, to cause absorption of enlarged lymphatic glands. Favorable results have been reported by Perroncito, with carbon disulphide given in gelatine capsules to horses to destroy the larve of the bot fly (CEstrus equi). Three capsules, containing 10 grams each, for a horse, or 8 grams. each for a colt, should be given at hour intervals, and followed in 12 hours by a pint of oil.

\section{Class 2.-Alcohol, Ether and Chloroform.}

Alconol. Alcohol. $\mathrm{C}_{2} \mathrm{H}_{5} \mathrm{O} \mathrm{H}$.

(U.S. P.)

Contains varying amounts of absolute alcohol.

Synonym.-Spiritus rectificatus, B. P.; spiritus vini rectificatissimus, alcohol vini, rectified spirit, E.; alcool, Fr.; weingeist, G.; spiritus, P. G. 
Alcohol is derived directly from fruit sugar, and indirectly from starch. The grains, as wheat, rye, corn; and potatoes, supply starch most economically. The starch in these substances is converted into glucose by heating with very dilute sulphuric acid, or by fermentation with malt. Glucose is further acted upon by yeast containing the Toruli cerevisia, which converts 15 per cent. of glucose into alcohol and carbonic dioxide. $\mathrm{C}_{6} \mathrm{H}_{12} \mathrm{O}_{6}=2 \mathrm{C}_{2} \mathrm{H}_{5} \mathrm{OH}+2 \mathrm{C} \mathrm{O}_{2}$.

The weak alcoliol resulting is subjected to repeated distillation until sufficiently pure and concentrated. In the natural fermentation of fruit sugar in grape juice, during the formation of wine, the amount of alcohol is self-limited to 15 , rarely 20 per cent., since the ferment is killed by an amount of alcohol greater than this.

Derivation.-The official alcohol is derived from rectified spirit ( 84 per cent. alcohol, by weight), by maceration, first with anhydrous potassium carbonate, then with freshly fused calcium chloride, and finally by distillation.

Properties.-A liquid composed of ahout 91 per cent., by weight, or $9 \pm$ per cent., by rolume, of ethyl alcohol $\left(\mathrm{C}_{2} \mathrm{H}_{5} \mathrm{O}\right.$ $\mathrm{H})$, and about 9 per cent., by weight, of water. A transparent, colorless, mobile and volatile liqnid, of a characteristic, rather agreeable odor, and a burning taste. Spec. gr. abont .820 at $15^{\circ} \mathrm{C}$. $\left(59^{\circ} \mathrm{F}\right.$.). Miscible witl water in all proportions and without any trace of cloudiness. Also miscible with ether or chloroform. It is readily volatile at low temperatures, and boils at $78^{\circ} \mathrm{C}$. (172.4 $\mathrm{F}$.). It is inflammable and burns with a blue flame.

Dose.-H. \& C., 3 i.-iii. (30.-90.); Sh. \& Sw., 3 ii.-iv. (8.-15.); D., 3i.-ii. (4.-8.).

\section{PREPARATION.}

Alcohol Dilutum. Diluted Alcohol. (U. S. P.)

Synonym.-Spiritus tenuior, B. P.

A liquid composed of about 41 per cent., by weight, or about 48.6 per cent., by volume, of absolute ethyl alcohol $\left(\mathrm{C}_{2} \mathrm{H}_{5} \mathrm{O} \mathrm{H}\right)$, and about 59 per cent. of water. (U. S. P.)

Derivation.-Alcohol, 500; distilled water, 500 . 
Alcohol Absolutum. Absolnte Alcohol. $\mathrm{C}_{2} \mathrm{H}_{5} \mathrm{O} \mathrm{H}$. (U. S. P.)

Synonym.-Alcohol ethylicum, B. P.

Ethyl alcohol, containing not more than 1 per cent., by weight, of water.

Derivation.-Percolation of the purest alcohol through quicklime, out of contact with the air, and redistillation in vacuo.

Properties.-Transparent, colorless, mobile and volatile liquid, of a characteristic, rather agreeable odor, and a burning taste. Very hydroscopic. Spec. gr. not higher than 0.797 at $15^{\circ} \mathrm{C} .\left(69^{\circ} \mathrm{F}\right.$. $)$

Alconol Deodoratum. Deodorized Alcohol. (U. S. P.)

A liquid composed of about 92.5 per cent., by weight, or 95.1 per cent., by volume, of ethyl alcohol $\left(\mathrm{C}_{2} \mathrm{H}_{5} \mathrm{O} \mathrm{H}\right)$, and about 7.5 per cent., by weight, of water.

Derivation.-Distillation of alcolol with about 2 per per cent. of pure fused sodium acetate.

Properties.-Similar to alcohol, except as regards odor.

\section{Spiritus Frumenti. (U. S. P.)}

Synonym. - Eau-de-vie de grains, Fr.; whiskey kornbranntwein, G.

Derivation.-An alcoholic liquid obtained by the distillation of the mash of fermented grain (usually of mixtures of corn, wheat and rye), and at least two years old.

Properties.-An amber-colored liquid, having a distinctive odor and taste, and a slightly acid reaction. Its specific gravity should not be more than 0.930 , nor less than 0.917 , corresponding, approximately, to an alcoholic strength of 44 to 50 per cent., by weight, or 50 to 58 per cent., by volume. Contains no more than traces of fusel oil. The alcoholic liquors owe their flavor or bouquet to ethers which are only dereloped in course of time. The amylic alcohol, 
or fusel oil in whiskey is therefore converted into ethers, which give the characteristic flaror to whiskey.

Dose.-H. and C., 亏3ii.-iv. (60.-120.); Sh. \& Sw., 亏i.-ii. (30.-60.); D., 3 i.-iv. (4.-15.).

\section{Spiritus Vini Galdict. Brandy. (U. S. \& B. P.)}

Synonym.-Spirit of French wine, E.; eau-de-vie, cognac, Fr.; Frantzbranntwein, G.

Derivation.-An alcoholic liquid obtained by the distillation of the fermented unmodified juice of fresh grapes, and at least four years old.

Brandy is somewhat astringent and is often not made from the distillation of wine, but is a factitious preparation. Native brandy is said to be purer, but is usually inferior in Hivor to that of foreign mannfacture. Brandy contains 3947 per cent. of absolute alcohol by weight; $46-55$ per cent. ly volume.

Dose.-Same as that for whiskey.

Spiritus Juniperi Compositus. Compound Spirit of Juniper.

$$
\text { (U. S. P.) }
$$

Derivation.-Oil of juniper, 8 ; oil of caraway, 1 ; oil of fennel, 1; alcohol, 1,400; water to make 2,000.

Compound spirit of juniper is similar to gin in its therapeutic action. Contains about 15 per cent. more alcohol. Gin is made by distillation of fermented malt and juniper berries. Gin differs from the other alcoholic preparations therapeutically in being more diuretic.

Dose.-Same as that for whiskey.

\section{Rum. (Not official.)}

Rum is made from a fermented solution of molasses by distillation. It contains, by weight, from 40 to 50 per cent. of absolute alcohol. Rum does not differ physiologically from alcohol. There is no authoritative Latin name for rum. Dose.-Same as that for whiskey. 


\section{Vinuar Album. White Wine. (U. S. P.)}

Derivation.-An alcoholic liquid made by fermenting the juice of fresh grapes, the fruit of Vitis vinifera (nat. ord. vitacex), free from seeds, stems, and skins.

Properties. - A pale amber or straw-colored liquid, having a pleasant odor, free from yeastiness, and a fruity, agreeable, slightly spiritnons taste, without excessive sweetness or acidity. The Pharmacopoei directs that the wine should contain from 10 to 14 per cent., by weight, of absolute alcohol. California Hock and Reisling, Ohio Catawba, Sherry, Muscatel, Madeira and the stronger wines of the Rhine, Mediterranean, and Hungary. come within the pharmacopœial limits. Wines containing more than 14 per cent. of alcohol, are usually fortified, i. e., have alcolol or brandy added to them, and much imported Sherry and Madeira contain 15 to 20 per cent., by weight, of absolute alcohol.

\section{Vinum Rubrum. Red Wine. (U. S. P.)}

Derivation.-An alcoholic liquid made by fermenting the juice of fresh colored grapes, the fruit of Vitis vinifera (nat. ord. vitacex), in presence of their skins.

Properties. - A deep-red liquid, having a pleasant odor, free from yeastiness, and a fruity, moderately astringent, pleasant and slightly acidulous taste, without excessive sweetness or acidity. Shonld contain mot less than 10, nor more than 14 per cent., by weight, of alcohol. Native Claret, Burgundy, Bordeaux, and Hungarian wines may be included within the pharmacopoial limits of rinum rubrum. Port (vinnm portense) is fortified with brandy during fermentatiou, and contains 15 to 25 per cent., by weight, of absolute alcohol. Port is astringent from tamnic acid in the grapes, skins and stalks, or the astringency may be due to logwood.

Red wines are said to be rough, contain tannic acid and therefore are astringent. Dry wines are those which contain little sugar. The wines develop ethers with age, and these improve their flavor and action. 
Champagne contains about 10 per cent. of absolute alcohol and carbonic acid gas, which acts as a local sedative upon the stomach. Ale, stout and beers contain from 4 to 8 per cent. of alcohol, together with bitters and malt extracts. Cider contains 5 to 9 per cent. of absolute alcohol. Imported Sherry (vinum xericum, B. P.), 15 to 20 per cent. of absolute alcohol. Alcohol is the solvent most commonly employed in pharmacy, dissolving alkaloids, resins, volatile oils, balsams, oleo-resins, tannin, sugar, some fats and fixed oils.

Action External.-Alcohol is a germicide. When applied in dilution to mucous membranes, raw surfaces or wounds, alcohol is a stimulant, antiseptic and local anesthetic; while in concentration, it is irritant and even caustic, coagulating mucus and albumin. If alcohol is allowed to evaporate from the unbroken skin, cooling of the surface and contraction of the superficial blood vessels ensue, with diminished secretions of sweat; but when alcohol is rubbed into the skin, it is absorbed, takes up water, hardens the integument and causes temporary vasular dilatation. Alcoholic aqueous dilutions containing 60 to 70 per cent. of absolute alcohol are among the most valuable skin disinfectants. Absolnte :llcohol, or the undiluted commercial alcohol, have no gormicidal action on dry bacteria, and alcohol in less thin 40 per cent. strength is too weak.

Action Internai.-Alcohol acts locally upon the mucous membrane of the alimentary canal, as described above, and if swallowed in concentration it produces congestion and white patches in the month by coagulating albumin upon the mucous membrane. The secretion of saliva is increased. In small doses the more powerful alcoholic liquors, as whiskey and brandy, aid digestion by stimulating locally the gastric circulation, secretion, movement and appetite.

During and immediately after ingestion of undiluted stronger alcoholic liquors, and before there is time for absorption to occur, the heart is reflexly stimulated by the irritant action of the alcolol upon the sensory nerve endings in the mucous membrane of the mouth, gullet and stomach. 
In large amounts, alcohol destroys the action of the peptic ferment, causes inflammation of the walls of the stomach and perverts the normal secretiou into a mucous discharge. Alcohol in physiological doses may be slightly decomposed in the stomach with the formation of aldeliyde and acetic acid, which may precipitate some of the proteids, peptones and pepsin. 'This action is not sufficient to interfere with the stomachic effect of alcohol. Alcohol is slightly astringent in the digestive tract, and may relieve pain by its local anæsthetic action upon the stomach, and spasm, by stimulating the nervous mechanism controlling the stomach and bowels, and so coördinating the peristaltic movements.

Circulation. - Alcohol is absorbed into the blood and undergoes decomposition in the body. When alcohol is mixed with blood, outside of the body, coagulation occurs, and separation of hæmoglobin from the corpuscles. The blood does not, under these circumstances, yield its oxygen readily to reducing agents. In rapid alcoholic poisoning the corpuscles have been found shrivelled and containing a yellow precipitate of hæmoglobin in their substance. Alcolol diminishes oxidation in the body by hindering the oxyhæmoglobin from giving up its oxygen, but this action does not occur until large quantities have been absorbed.

Alcohol is essentially a heart stimulant. It makes the heart beat more forcibly and rapidly, and also increases blood pressure, despite the fact that normally alcohol causes dilation of the arterioles. It is evident, however, that the vascular dilation is not general, for if it involred the splanchnic area so much blood would be diverted to this region that the skin would be pale instead of flushed, as can be seen in man. In weakened bodily conditions, with vascular relaxation, alcohol may increase vascular tonicity and diminish the rate of the heart-beat by stimulation of the cardiac inhibitory apparatus. The heart and blood ressels are paralyzed by poisonous doses of alcohol, and blood tension falls tremendously. Evidence is contradictory in relation to the 
exact physiological action of alcohol upon the nervous mechanism controlling the heart and blood ressels. The action upon the heart is thought by some experimenters to be due to the stimulation of the accelerator nerves (Dogiel); by others to stimulation of the cardiac motor ganglia; while Wood refers the action to excitation of the heart muscle. It is also uncertain whether the action npon the vessels is due to the effect of alcohol upon the vasomotor system entirely, or in part to direct action upon the vessel walls. The fact that the heart is reflexly stimulated by the irritant action of large doses of concentrated alcoholic liquors upon the stomach, has already been noted. Primary vascular contraction may follow the reflex stimulation of alcohol before absorption and vascular dilatation occur.

Respiration. - The respiratory centre is stimulated by medicinal doses, but depressed and paralyzed by toxic amounts of alcohol.

Temperature. - The temperature, both in health and fever, may be lowered by alcohol, because of its action in hindering the ozonizing functions of the red blood corpuseles and thus diminishing oxiclation in the body, and becanse alcohol causes relaxation of the peripheral blood vessels and loss of heat by radiation from the skin. The larger the quantity of alcohol ingested, the greater the fall of temperature. In alcoholic narcosis, vasomotor paralysis leads to great loss of heat, particularly if the animal be at the same time exposed to cold. In small doses, alcohol may sometimes increase the temperature by stimnlating the heart, but the sensation of warmth perceived in man following the use of alcohol is generally fallacious, and is simply due to flushing of the vessels of the skin aud stomach. Some of the lowest temperatures ever observed have been in drunken persons exposed to cold.

Tissue Change.-Metabolism is diminished by alcohol. Experiments conducted to determine quantitatively the elimination of carbonic dioxide are conflicting in their 
results, after the ingestion of small doses of alcohol, but when considerable quantities have been absorbed the elimination of carbonic dioxide is decidedly diminished. The explanation of the preceding is clear when it is considered that many other circumstances may obscure the action cf small amounts of alcolol upon carbonic dioxide elimination. The nitrogenous products in the urine, particularly urea resulting from tissue change, are also lessened by alcohol.

Nervous System.-Moderate doses of alcohol stimulate, while excessive quantities depress and paralyze the nervous system. This action is similar to that exerted upon the circulatory organs. The local effect of alcohol upon the peripheral nerves resembles the action after absorption npon the system generally. The nervons system is affected in nearly the same order and manner as by anæsthetics, and the same stages may be observed. The stages include the stimulant, depressant and paralytic. The law of dissolntion is demonstrated by alcohol, as the more highly organized centres, and those more recently developed in the process of erolution, are the first to succumb, and following ont this order, the medulla, the first of the higher centres to be developed, is the last to be influenced by the drug. In accordance with this law the cerebrum is first acted upon. The period of excitement is brief and is due in a considerable degree to the increased cerebral circulation and flushing of the brain. It is essential to emphasize the fact that by far the most apparent and decided action of alcohol is one of depression upon the nervous system as a whole. The stimulating influence of alcohol upon the spinal centres is more marked in the lower animals than in man, because the brain is proportionately small and poorly developed in the former. The primary stimulating effect of alcohol is shown in man by increased mental activity and apparent brilliancy, but acute reasoning and judgment are not enhanced, and in many cases there is almost immediate mental confusion and drowsiness induced. In man there is emotional excitement and the functions of speech and imagination are stimulated 
in the primary stage, but depression is soon noted in the loss of jndgment and reasoning power, emotional control, lecent restraint and speech. The patient cries, shonts, sings or laughs and talks incoherently. In the lower animals the stimulation of the higher and sensory psychical cerebral centres, with exhilaration, is rarely observed, but repression is seen in stupor and muscular incoürdination. Stimulation of the cerebral motor centres is shown by motor excitement.

In min, following the symptoms described above, there is incoördination of muscular movements, first of those more highly and recently organized, such as are employed in writing, and then the muscular movements more remotely ileveloped are affected, and the person is unable to will, and finally there is complete paralysis of the motor centres and muscles. The staggering and uncertain gait of drunken people occurs not only becanse the cerebral motor and cerebellar centres are depressed by alcohol, but also becanse. of loss of sensation and touch, or muscular sense, which is essential in maintaining the equilibrium. In relation to the spinal cord, primary stimulation of the reflex centres is more marked in animals than in man, as has been pointed out. In animals this stimulation causes motor excitement, so that the patient trembles, jumps about, or strikes out with the feet. Depression of the reflex centres occurs in the latter stage of poisoning, and is exhibited by involuntary defreation and micturition; sensation and voluntary motion are lost. The motor nerves and muscles are not generally paralyzed, except by the local action of alcohol. The medulla finally becomes depressed and paralyzed, so that respiration, which is first stimulated, now fails, and the heart muscle becomes paralyzed and stops beating.

The action of alcohol upon the nerrous system may be summarized with a fair degree of accuracy, as stimulation and then depression of the parts enumerated below, and in the following order : 
Cerebral psychical centres.

Cerebral sensory centres.

Cerebral motor centres.

Cerebellum.
Spinal centres.

Sensory, reflex and motor.

Medullary centres.

Vasomotor (early depression).

Respiratory.

Horses and dogs are comparatively susceptible to alcohol, rumiuants slightly so. An amount of alcohol equivalent to a pint of whiskey has killed a sound horse, while four ounces of whiskey will canse death in dogs if vomition be prevented by ligature of the cesophagus.

Primary motor excitement is followed by mustealy, stagrgering gait, and coma in fatal cases.

Skin.-Alcohol dilates the peripheral vessels, and therefore brings more blood to the sudoriparons glauds, and excites their functional activity. It is thus a diaphoretic.

Kidneys. - Alcohol acts as a diuretic by increasiugr general blood tension, and probably by angmenting local vascnlar tension in the kidney in dilating the afferent vessels supplying the Malpighian bodies.

Nutrition.-Alcohol is a food, and, like other carbohydrates, supplies heat and energy in its decompcsition, but does not directly furnish tissue elements. We are ignorant of the fate of alcohol after absorption, but we do know that the greater portion is decomposed and is not eliminated. Alcohol is most advantageous as a food in fever, or in conditions associated with weak digestion, since it is readily absorbed and assimilated. Alcohol causes dulness and lessened power for mental or physical work in man, and in normal conditions is not a desirable food unless there is a deficiency in the ordinary diet. In diminishing oxidation in the body, alcohol assists the accumulation of fat.

Elimination. - When alcolool is ingested in ordinary doses it is practically all consumed, and none but the most. trivial amount is eliminated, $i$. e., two to three per cent. The greater the quantity absorbed the larger the amount. eliminated by the mine, breath, sweat and freces, both relatively and absolutely; but under no circumstances after the most enormous doses does the elimiuation exceed 25 per cent. of the quantity ingested. 
Summary.-Alcohol is externally refrigerant, astringent, anhidrotic and antiseptic, and if applied so that absorption occurs, it is rubefacient. On raw surfaces it is slightly anæsthetic. Alcohol is internally a stomachic, carminative and slight local anrsthetic in the digestive tract. It stimnlates the heart reflexly before and directly after absorption, and excites the respiratory centre. Alcohol is a narcotic, first stimulating and then depressing the nervous system. Alcohol forms a componnd with hæmoglobin which gives up oxygen less easily, and so diminishes oxidation and tissuo change. It supplies force and is a food. Alcohol is a dimretic, diaphoretic, and antipyretic in lessening tissue change and dilating peripheral vessels, and in causing sweating.

Acute Poisoning.-In coma and muscular relaxation, the treatment consists in the external application of heat and counter-irritants; while strychnine, digitalis and atropine should be given subcutaneously and followed up with ammonia by the mouth.

Uses External.-Alcohol is applied to the unbroken skin, on cotton or lint, to bruises, for its local refrigerant and astringent action in relieving pain and congestion. Diluted* to 70 per cent. strength, alcohol forms an antiseptic and local anæsthetic application to wounds, and like most. antiseptics, relieves itching, particularly when combined. with 1 to 2 per cent. of carbolic acid.

Uses Internal. - Digestive Tract.-Alcohol is a usefuI remedy to promote appetite and assist digestion. The drug. should be given immediately before eating or with the food, properly diluted, and often advantageously with egg and milk during ferer or convalescence from acute diseases. Alcohol is frequently combined with bitters, as compound tincture of gentian, when employed as a stomachic.

Acute Diseases.-Alcohol finds its greatest field of usefulness in the treatment of febrile diseases, notably influeuzir

* Alcohol diluted with one-third part of water makes one of the best known agents for hand-disinfection. It is relied upon entirely in some of the best hospitals in the country, in addition to thorough scrubbing in soap and water. 
and pneumonia in horses. In such conditions it overcomes the paralysis of the splanchnics caused by the toxins and flushes the vessels of the skin, in this way equalizing the circulation and allowing the heart to receive more blood. A ligh temperature does not contra-indicate the use of alcohol, but it is not desirable in the first stages of acute juflammatory disorders. Alcohol is particnlarly serviceable in asthenic diseases and in continued fevers associated with general depression, as septicæmia. Alcohol is more readily burned up in fever, aids digestion, lessens oxidation and tissue waste, supplies force and is a food. Alcohol stimulates the nervous system and bridges over a period when artificial stimulation is necessary. This drug may reduce temperature, but larger doses are needed for this purpose (i.e., to cause vasomotor depression and interference with the ozonizing functions of the blood) than is generally desirable, since the respiratory centre and heart may be depressed, and digestion retarded. In certain conditions, however, as septicæmia, alcohol exerts a leciled antipyretic action. Alcohol furthermore favors antipyresis throngh its diarphoretic and dinretic properties, in causing increased radiation of heat and elimination of toxic products. The most essential action of alcohol in acute diseases consists in stimulating the heart and respiration, in eqnalizing the circulation by overcoming internal congestions through the action on tlie heart, combined with that causing vascular dilatation. Alcohol naturally increases the force and frequency of the normal heart, and may act likewise in fevers. This effect is nndesirable, and for this reason alcohol is contra-indicated in the first stage of sthenic diseases, but in fevers associated with weakness of the heart, alcohol often decreases its rapidity by stimulation of its inhibitory apparatus, although increasing the cardiac force. This action may be dne to invigoration of the weakened organ. Since alcohol does not always act uniformly upon the heart in febrile conditions, we must be guided by its effect in each case. The pulse, respiration, skin 
and nervous system are our guides, and the object is to briug the functions into a more normal condition. Alcohol should therefore reduce the frequency of the pulse and respiration, when they are too rapid, and should make the skin moister and the animal quieter. If these results are obtained, the use of the drug should be persisted in; if otherwise, administration should be stopped. Small and repeated doses are more appropriate in fever.

Alcolol is one of the inost valuable agents at our command in the treatment of surgical shock, collapse, exhaustion, severe hemorrhage, and following exposure to cold. In these conditions it should be given hot and only slightly diluted. In poisoning by drugs which depress the circulation, or by toxines resulting from bacterial infection, alcohol is an invaluable remedy. Alcohol is a chemical antidote in cirbolic acid poisoning, and besides overcomes the shock produced by the latter; whiskey or brandy may be used.

Administration.-Veterinary practitiouers are fortunately exempt from any moral considerations in relation to their medicinal use of alcohol. Rum, gin and whiskey are more commonly employed than the other alcoholic preparations, althongh diluted alcohol is practically as valuable. Gin is indicated when a diuretic action is important. Brandy, being more astringent, is given to dogs with diarrhoa, and, combined with cracked ice in small quantities, relieves vomiting. Sherry may also be administered to dogs with advantage. The dose of the various alcoholic liquids depends upon the quantity of alcohol contained in them.

Animals will usually take alcoholic preparations voluntarily if largely diluted with water, milk or gruel. Whiskey should be diluted with about 4 parts of water when given in drench, unless the reflex action is desired, when it is administered in considerable doses with an equal amount of hot water.

Diluted alcohol, undiluted whiskey or brandy are injected subcutaneously when a rapid action is imperative. 
Æither. Ether. $\left(\mathrm{C}_{2} \mathrm{H}_{5}\right)_{2} \mathrm{O}$. (U. S. P.)

Synonym. - Æther purus, B. P.; æther fortior, pure ether, E.; éther hydrique pur, Fr.; reiner æther, G.

A liquid composed of about 96 per cent., by weight, of absolute ether or ethyl oxide $\left[\left(\mathrm{C}_{2} \mathrm{H}_{5}\right)_{2} \mathrm{O}\right]$, and abont 4 per cent. of alcohol containing a little water.

Derivation.-Obtained by distillation of alcohol with sulphuric acid. There are two steps in the production of ether; sulphovinic acid and water are formed in the first step. $\mathrm{H}_{2} \mathrm{~S} \mathrm{O}_{4}+\mathrm{C}_{2} \mathrm{H}_{5} \mathrm{OH}=\mathrm{C}_{2} \mathrm{H}_{5}, \mathrm{H} \mathrm{S} \mathrm{O}_{4}+\mathrm{H}_{2} \mathrm{O}$. Sulphovinic acid is then further acted upon by alcohol.

$\mathrm{C}_{2} \mathrm{H}_{5}, \mathrm{H} \mathrm{S} \mathrm{O}_{4}+\mathrm{C}_{2} \mathrm{H}_{5}, \mathrm{O} \mathrm{H}=\left(\mathrm{C}_{2} \mathrm{H}_{5}\right)_{2} \mathrm{O}+\mathrm{H}_{2} \mathrm{~S} \mathrm{O}_{4^{*}}$ The distillate is freed from water by agitation with calcinm oxide and chloride, and subjecterl to redistillation.

Properties. - A transparent, colorless, mobile liquid, having a characteristic odor and a burning and sweetish taste. Spec. gr. .725 to .728 at $59^{\circ}$ F. Soluble in about 10 times its volume of water at $59^{\circ} \mathrm{F}$. Miscible in all proportions with alcohol, chloroform, benzine, benzol, fixed and volatile oils. Ether boils at about $37^{\circ} \mathrm{C}$. $\left(98.6^{\circ} \mathrm{F}\right.$.), and it should therefore boil when a test tube, containing some broken glass and half filled with it, is held for some time in the hand. Ether is highly volatile and inflammable; its vapor, when mixed with air and ignited, explodes violently. The color of light blue litmus paper moistened with water shonld not be changed when the paper is immersed in ether for 10 minutes. Upon evaporation ether should leave no residue. Ether is a solvent for fats, oils, alkaloids, resins, gutta percha and gun cotton. Ether vapor is heavier than air, and, consequently, etherization should never be done above a light or fire.

Dose.-H. \& C., 3 i.-ii. (30.-60.); Sh. \& Sw., 3 ii.-iv. (8.-15.); D., $m_{x}-3$ i. (.6-4.).

\section{PREPARATIONS.}

Spiritus AEtheris. Spirit of Ether. (U. S. \& B. P.)

Ether, 325; Alcohol, 675. (U. S. P.)

Dose.-Same as for ether. 
Spiritus Atheris Compositus. Compound Spirit of Ether.

(U. S. \& B. P.)

Synonym.-Hoffman's anodyne. Ether, 325; alcohol, 650; etherial oil, 25. (U. S. P.)

Dose.-Same as for ether.

Action External.-Ether evaporates rapidly from the skin, and abstracts so much heat in the process that the superficial parts are cooled, benumbed, and even frozen. This action is taken advantage of in spraying ether from an atomizer upon the skin (with or withont cocaine injection) to cause local anæsthesia in minor surgical operations, as opening abscesses. The spray should not be applied more than a few minutes, or freezing, damage to the tissues, and retardation of the healing process will ensue. If ether is applied with friction, or if evaporation from the skin is prevented by bandaging, it will act as a rubefacient.

Action Internal.-Digestive Organs.-Ether is an irritant to the mucous membrane of the digestive tract, and should be given only when considerably diluted with water. Ether stimulates secretion, motion, and increases local blood supply of the stomach. Ether, in concentration, resembles alcohol and ammonia in stimulating the heart reflexly, by its irritant action upon the alimentary canal, before it has time to be absorbed. It relieves pain and spasm in the digestive tract by coördinating or restoring nervous control over the stomach and bowels.

Circulation.-Ether stimulates the motor ganglia of the heart, and increases its force and frequency. It also excites the vasomotor centres and increases vascular tension. In poisonous doses, or after prolonged inhalation, the circulation becomes depressed and weak.

Nervous System. - Ether exerts a primary, transient, stimulating action upon the brain and lower nervous centres, but rapidly succeeding this, ether depresses and abolishes the functions of all the great nerve centres in the following order, and with the following results :

1. The cerebrum (with loss of consciousness). 
2. Sensory spinal tract (loss of sensation).

3. Motor spinal area (loss of motion and partial loss of reflex action).

3. Sensory medullary centres.

5. Motor medullary centres (failure of respiration).

Ether does not affect the nerves or muscles when inhaled or ingested. Ether depresses the action of the nerves, however, when applied locally.

Respiration. - The respiratory centre is stimulated by inhalation or ingestion of therapentic quantities of ether. Paralysis of the respiratory centre occurs in the last stage of ether poisoning. Ether vapor is irritant to the respiratory mucous membrane, and causes conghing and cloking during inhalation. It is not an appropriate anæsthetic, therefore, in inflammatory conditions of the respiratory tract. Ether vapor excites the peripheral ends of the trifacial nerve in the face, and the vagus nerve in the lumgs, so that temporary arrest of respiration often occurs when a fresh supply of ether is added during inhalation.

Temperature. - The temperature of the body may be raised during the stage of excitement and struggling, but frolonged etherization leads to loss of heat, owing to evaporation of ether from the lungs, and general depression of the nervous system.

Elimination.-Ether is eliminated principally from the lungs, and to some extent by the kidneys. Recent experiments indicate that ether causes contraction of the renal arteries, with diminution in the size of the kidneys, suppression of urine and albuminuria.

Uses.-Apart from its value as an anæsthetic, ether is mainly of service for two purposes. First: in collapse, syncope, or "sinking spells," due to poisoning, or watural causes. Ether may be given by the mouth, or subcutaneously into the muscular tissue, to avoid abscess. It is to be compared with, and is an efficient substitute for, nitroglycerin, or amyl nitrite, as a rapid, circulatory stimulant. Ether should not be given as a stimulant, however, 
when such an agent is required during or following anæsthesia. Second: In spasmodic, or mild, flatulant colic, ether relieves pain, spasm and flatulence. The following prescription will be found useful for the horse:

$\mathrm{B}$

Etheris.............................

Chloroformi........................ 3 ii.

Tinc. opii........................

M. et f. haustus.

S. Gire at once in a pint of cold water.

Ether is a good antispasmodic remedy for hiccough or "thumps," in the lorse, and has a narcotic action upon tape and Inmbricoid worms. When used for the latter purpose, ether should be followed by a purge. Ether may be employed in enema to narcotize and remove the Oxyuris curvula of the horse.

Administration.--Ether should be given in gelatine capsules; mixed with two parts of cracked ice and water; or with equal parts of brandy or whiskey, to avoid undice irritation of ether and its vapor upon the mucous membraues during deglutition.

\section{Chloroformum. Chloroform. $\mathrm{C} \mathrm{H} \mathrm{Cl}_{3}$. (U. S. \& B. P.)}

Synonym.-Chloroformum purificatum, purified chloroform, E. ; chloroformium, P. G.; formylum trichloratum, chloroforme pur, Fr.; reines chloroformum, $G$.

A liquid consisting of 99 to 99.4 per cent., by weight, of absolute chloroform, and 1 to 0.6 per cent. of alcohol.

Derivation.-Alcohol and water are heated in a still to $37.7^{\circ}$ C. $\left(100^{\circ}\right.$ F.), when chlorinated lime is added and chloroform is evolved. The chemical action is very complicated. Chloroform, free from chlorinated compounds, is also made from acetone and clilorinated lime by distillation. Calcium acetate, hydrate aud chloride result as bye products.

$2 \mathrm{C}_{3} \mathrm{H}_{6} \mathrm{O}+6 \mathrm{CaO} \mathrm{Ol}_{2}=2 \mathrm{CH} \mathrm{Cl}_{3}+\mathrm{Ca}\left(\mathrm{C}_{2} \mathrm{H}_{3} \mathrm{O}_{2}\right)_{2}$ $+2 \mathrm{Ca} \mathrm{O}_{2} \mathrm{H}_{2}+3 \mathrm{Ca} \mathrm{Cl}_{2}$. 
For tests and purification, vil. U. S. P.

Properties.-A heavy, clear, colorless, mobile and diffusible liquid, of a characteristic, ethereal odor, and a buruing, sweet taste. Spec. gr. not below 1.490 at $15^{\circ}$ C. (59 F.). Soluble in about 200 times its volume of cold water, and in all proportions in alcohol, ether, benzol, benzine and the fixed and rolatile oils. Chloroform is volatile even at a low temperature, and boils at $60^{\circ}$ to $61^{\circ} \mathrm{C}$. $\left(140^{\circ}\right.$ to $141.8^{\circ} \mathrm{F}$.). It is not inflammable, but its vapor in the presence of a naked flame undergoes decomposition with the formation of noxious gases, chiefly chlorine. This has caused death during chloroform inhalations. Chloroform is a solvent for fats, resins, oils, balsams, gutta percha, wax and many alkaloids.

Dose.-H. \& C., 3 i.-ii. (4.-8.); Sh. and Sw., Mxx.-xxx. (1.3-2.); D., \ii.-xx. (.12-1.3).

\section{PREPARATIONS.}

Aqua Chloroformi. (U.S. \& B. P.)

(Saturated Solution.)

Used as vehicle in cough and diarrhœe mixtures for dogs.

\section{Linimentum Chloroformi. (U. S. \& B. P.)}

Chloroform, 300; soap liniment, 700. (U. S. P.)

Spiritus Chloroformi. (U. S. \& B. P.)

Chloroform, 60; alcohol, 940. (U. S. P.)

Dose.-H. \& C., 3 i.-ii. (30.-60.); Slı. \& Sw., 3 ii.-iv. (8.-15.); D., 3 ss.-i. (2.-4.).

Action External.-Chloroform acts as a rubefacient if rubbed into the skin, or prevented from evaporation by bandaging. In evaporating from the surface it produces mild refrigeration. Chloroform penetrates the skin more easily than many other agents, and is employed for this reason to aid the absorption of belladonna and other drugs used in liniments. Chloroform possesses some local anæsthetic action upon mucous membranes, raw surfaces, or when rubbed into the skin. 
Action Internal.-Digestive Tract.-Chloroform is an irritant in concentration, but, properly diluted, stimulates the flow of saliva and increases the secretions, motion, and blood. supply of the stomach. Chloroform has a local anæsthetic and antiseptic action in the alimentary canal, and by its stimulant effect in restoring the normal state of nervous and muscular tissue, relieves spasm, pain and flatulence in the stomach and small intestines.

Circulation.-Chloroform, unlike ether, does not stimulate the heart and vasomotor centres, except for a very brief period. On the contrary, ignoring the primary and transitory stimulant action just noted, chloroform exerts an ever increasing depressing influence upon the heart muscle, its ganglia, and the vasomotor centres.

Vasomotor depression leads to dilatation of the arterioles and leaking of blood into the veins, with consequent venous engorgement (particularly of the abdomen), and arterial anæmia. Failure of respiration in chloroformization is secondary to cerebral anæmia, and chloroform kills, primarily by vasomotor depression. (Leonard Hill and Hare.)

The ventricles dilate and all cardiac muscular contractility is lost in fatal poisoning. Death, however, occurs almost invariably in healthy animals during chloroform inhalation from respiratory failure associated with circulatory depression. The heart nsually continues to beat after ressation of breathing. Death from syncope occurs occasionally, and circulatory depression is greater and occurs more suddenly than with ether. As chloroform anæsthesia proceeds, the pulse becomes rapid from the depressing effect of chloroform upon the vagus centre.

Respiration.-Chloroform does not markedly affect the respiration, when inhaled for an ordinary period, or when ingested in small doses, but after long continued inhalation, or when large quantities have been swallowed, depression and paralysis of the respiratory center ensues. Since asphyxia leads to inhibition and heart failure-besides 
being dangerous in itself-the respiration should always be watched as carefully during chloroform as in ether inhalation.

Nervous System. - Chloroform influences the nervons system as described under ether; that is, the cerebrum, sensory and motor spinal tract, and sensory and motor centres of the medulla. Chloroform, although generally following the order noted, affects the nervous system more rapidly, irregularly and persistently than ether, and therefore is more dangerous. It occasionally happens that some of the reflexes are abolished by chloroform before sensation has been annulled, and irritation of a sensory nerve produced during operation may reflexly stimulate the inhibitory centres (instead of increasing vascular tensiou as in health), and so cause leart failure. Operations should never be performed, therefore, under chloroform until complete anæsthesia is secured. The nerves are not influenced by the constitutional action of chloroform, although the latter is a local irritant and anæsthetic to them.

Temperature.-The temperature is reduced by the continual inhalation of chloroform through its depressing action on the heat producing centres, and because heat loss is increasea by evaporation of chloroform from the lungs.

Antiseptic Action.-Chloroform prevents the growth of micro-organisms, but does not influence that of the unorganized digestive ferments. Chloroform's antiseptic properties may be ntilized in preserving solutions for subcutaneous injection. Saturated aqueous solutions are used for this purpose.

Elimination. - Chloroform is eliminated by the lungs and kidneys, but is also decomposed in part in the body.

Uses External.-Chloroform is employed in liniment to relieve pain and produce counter-irritation in muscular rheumatism and strains.

Uses Internal.-Chloroform is of service internally in the treatment of four conditions: 1. Intestinal colic. 2. Flatulence. 3. Diarrhœea. 4. Cough. Chloroform alleviates. 
pain in colic by restoring the functions of the nervous and muscular mechanism of the bowels, by relieving spasm, and also by its local anæsthetic action. It stimulates motion and secretion; it is an antiseptic in the digestive tract, and, in these ways, overcomes fiatulence.

Chloroform should be combined with opium in diarrhoa and colic. It is administered in spirit or water to assnage congh.

Administration.-Chloroform may be administered in 3 parts of glycerin ; in emulsion with white of egg or accacia; or as the spirit, diluted with water.

\section{ANÆSTHESIA.}

Anresthesia is commonly divided into three stages: the stimulant, anæsthetic and paralytic. The so-called "law of dissolntion" is illustrated by anæsthetics, as the more highly organized (cerebral) centres are the first to succumb, while the lower centres (medulla) are the last to be affected. The anæsthetics resemble other narcotics in producing a primary condition of stimulation of the nervous system, which is followed by depression. In the first stage of anæsthesia there is often struggling and excitement. This is partly due to the physiological action of the drug and partly to fright. In this stage the functions of the brain are stimnlated and then depressed. The higher functions are the first to be excited, and the effect produced is very similar to that of beginning alcoholic intoxication. The lower motor functions are next stimulated, inducing struggling and motor excitement. In the first stage of etherization, the local irritant action of the vapor causes choking and conghing, and also induces struggling. The respiratory and cardiac centres are temporarily stimulated, the pulse and respiratory movements are increased in force and frequency, and blood tension is raised. The smaller animals may vomit during the first stage of anrsthesia. A sub-division of the first stage, sometimes described as the anodyne stage, occurs when sensation is lost, before consciousness and voluntary 
motion. Short operations, as extraction of teeth, have been done by men upon themselves in the anodyne stage of anæsthesia, without pain. The anæsthetic stage is that condition characterized by absence of consciousness, sensation, motion, and partial loss of reflex action, and is that state suitable for operations. The stimulating action of the anæsthetic has ceased and there is now depression of the cerebral functions, the motor and sensory tract of the cord, and, to a partial extent, the reflex centres. The muscles are completely relaxed, and the patient lies absolutely motionless. The conjunctive fail to respond to irritation, i.e., winking is not produced when the conjunctival membrane is lightly toncherl with the finger. Occasionally the muscles are seen to be rigid and twitching during this stage of etherization, although sensation and conscionsness are absent. The respiration and pulse should not be particularly altered during the anæsthetic stage, unless danger threatens. In the last stage, narcosis, or poisoning, is beginning, and there in depression of the three great medullary centres, controlling the heart, respiration and vascular tension, and also the lowest reflex centres of the cord, so that the urine and fæces are passed involuntarily. Micturition frequently occurs in the first stages of anæsthesia and should not of itself be considered a danger signal. The pulse becomes rapid, feeble and irregular; the breathing is at first stertorous, and then the respiratory movements become shallow and weak, with considerable intervals intervening between them. The pupils are often dilated. The pupil is said to be a guide during anæsthesia, especially with chloroform, since it is contracted during the anæsthetic stage and dilates quickly when danger approaches. Death, however, occurs in animals with either dilated or contracted pupils. With the former, probably from asphyxia; with the latter, from syncope; and the pupil should not be regarded as an unfailing sign. The foregoing stages are conventional, and are not in any case so clearly defined in practice as they are described theoretically upon paper. The first stage may be either absent or prolonged, and the last stage should not be reached at all. 


\section{COMPARISON OF ETHER WITH CHLOROFORM.}

ETHER.

More diffusible.

Inflammable.

Irritating; may induce bronchitis and nephritis.

Administered slightly diluted with air.

Stimulant to heart, except in enormous quantities.

Stimulant to vasomotor centres, except as above.

Respiratory centres not so easily depressed as by chloroform.

Larger quantity required.

Less rapid: " stage of struggling and excitement longer.

More expensive.

Fatal from respiratory failure.

\section{CHLOROFORM.}

Vapor heavier.

Less irritating.

Not inflammable.

Hore danger from shock during imperfect anæesthesia.

Depresses powerfully, heart, respiratory and vasomoter centres in large doses.

Acts more quickly, profoundly, and persistently.

Srıaller quantity required.

Cheaper.

Proportion of deatlis to inhalations, 5 times greater than with ether.*

Death occurs from respiratriry failure combined with circulatory depression.

Jccasionally fatality results from syncope.

It will be seen that all the advantages are in favor of chloroform, except that of safety. "Ether is more expensive than chloroform, but cheaper than a funeral." (Edes.)

\section{ACCIDENTS AND DANGERS ATTENDING ANESTHESIA.}

Respiratory failure and asphyxia may occur from giving too much of the anæsthetic and from mechanical obstruction in the air passages. The tongue may fall back upon the epiglottis and prevent the free entrance of air. The latter accident is obviated by pulling the tongue forward with the hand, or, in the case of the smaller animals, by means of forceps, or suture passed through the tongue. Mucus, blood or vomitus may obstruct the mouth, pharynx, larynx, or trachea, and should be remored if possible by swabbing with absorbent cotton. To prevent excessive secretion of mucus in the air passages, it is wise to give atropine subcutaneously fifteen minutes before etherization. The head should be extended and the lower jaw of the patient held forward during anæsthesia, and no impediment to the free movement of the chest is allow-

* Mortality about 1 in 3,500 chloroformizations, about 1 in 20,000 etherizations in human practice. 
able. Struggling is to be avoided, as far as possible, since it leads to irregular respiration and asphyxia, and causes the anæsthetizer to push the inhalation, strains the heart, and favors cardiac dilatation, with chloroform. Struggling may be prevented to some extent by giving the anæsthetic well diluted with air at the outset. Asphyxia results also from tetanic fixation, or relaxation of the respiratory muscles. The signs of asphyxia include cyanotic mucous membranes, muscular twitchings, shallow, feeble, slow and irregular breathing, with long intervals between the respiratory movements, and dilation of the pupils. If death occurs, the heart continues to beat after the breathing stops. The condition of the diaphragm during etherization is an important guide. At first the contractions of the diaphragm are so vigorous that the viscera are forced backward, and the abdominal wall bulges outward during inspiration. As the inhalation is prolonged the diaphragm becomes flaccid and powerless, the respiratory movements are shallow, and the breathing thoracic. The abdominal contents may then be drawn forward during inspiration into the thoracic cavity, so that the abdomen is retracted (Hare). When the latter condition is seen, etherization should be stopped instantly.

Heart failure occurs more frequently with chloroform, but does occasionally result from the action of ether upon animals with a fatty or otherwise weak heart. Cardiac depression is shown by a weak, irregular and rapid pulse, and pallor of the mucous membranes. The respiratory movements are sustained after the heart ceases to beat in fatal cases. Operations should never be done under chloroform before the patient is fully under the influence of the anæsthetic, as irritation of a sensory nerve caused by even a slight surgical procedure may cause death by reflexly stimulating the vagus and inhibiting the heart. such an accident is not likely to occur with ether, and minor operations are often done with safety during the first stage of anæsthesia (primary anæsthesia) when con- 
scionasness and sensation are lost and the muscles begin to $1 \leftrightarrow l i x$.

Shock from hemorrhage, or prolonged and severe surgical operations, may lead to fatalities during anresthesia, and it sometimes occurs when an operation is begun before the patient is fully under the influence of an anrstheticparticularly chloroform-as described above. In practice, these canses of danger, i.e., respiratory failure, circulatory depression and shock, are usually combined.

We have already seen that asphyxia leads to circulatory disorder, and both may be associated with surgical shock. The following embraces the proper treatment of all these conditions, and should be followed in every case where danger threatens during anæsthesia :

1. Remove the anæsthetic and allow plenty of fresh air.

2. See that there is no mechanical obstruction to the movements of the chest, or to the free entrance of air. Pull forward the tongue and lower jaw and extend the head.

3. Invert small animals, particularly with chloroform, to allow blood to flow to the brain.

4. Use forced, artificial respiration with bellows and rubber tube introduced into laryns. Practice ordinary artificial respiration in small animals by carrying the forelegs ontward and forward till they meet in front of the head, and then bring them back till they touch, and compress the sides of the chest. These movements should be repeated twenty times a minute. Artificial respiration may be done in the horse by two persons compressing intermittently one side of the chest with the knees and hands. Rhythmical traction of the tongne, at intervals of five seconds, has proved successful in restoring respiration. Dash hot or cold water or ether upon the chest and epigastrinm; or use the faradic battery to stimulate respiration by moving the electrodes over the chest and abdomen. Stretch the sphincter ani apart by placing the thumbs in rectum anul reparating them with force. This is a powerinl reflex. stimulus to heart and respiration. 
5. Give subcutaneous injections of strychnine and digitalis. Administer nitroglycerin on the tongue, or inhalation of amyl nitrite. In chloroformization, give strychnine and atropine hypodermatically; and, in shock, saline infusion as below. Do not use alcohol or ether as stimulants, for their action resembles that of the anrsthetics.

6. Use hot $\left(115^{\circ}\right.$ F.), high, rectal injec'ions of salt solution (one heaping teaspoonful to the quart) in case of surgical shock with feeble pulse and subnormal temperature resulting from hemorrhage or other cause. Two to four quarts for large animals, and one pint to one quart for smaller animals. Saline infusion is still more efficient. (See "Saline Infusion," p. 732.)

CHOICE OF AN ANESTHETIC.

Ether and chloroform are the only anæsthetics of any importance in veterinary medicine. The A.C. E. mixture, containing alcohol (one part), chloroform (two parts), and ether (three parts), possesses no particular value, and is not so safe as ether. While chloroform is inferior to ether in the matter of safety, it may be given to horses by an experienced and careful person. without much danger. It is commonly the most suitable anæsthetic for the horse, for the following reasons: It is less expensive; whereas several pounds of costly ether are required to produce anæsthesia, a few ounces of chloroform will accomplish the same result. Chloroform inhalation is much easier of administration, more rapid, and canses less struggling. It may be used without any special inhaler, and may be given to animals in the upright position.

Chloroform may be employed for all animals during parturition, as it is less dangerous in this condition and because only enough is needed to produce relaxation of parts to relieve pain, to assist dilatation of the os and mannal operations done to rectify faulty position of the foetus.

Chloroform is indicated in all cases where the actual 
cantery is used in the neighborhood of the mouth. Ether is the most suitable anæsthetic for cats and dogs, unless the animal is suffering from bronchitis, emphysema or asthma, when chloroform is more appropriatc. Chloroform, being more rapid and less irritating, may in any case be employed to begin anæsthesia, which should then be maintained by ether.

\section{PRACTICAL ANZETHESIA.}

The horse should be starved for twelve hours, and should receive a cathartic twenty-four hours before anæsthetization, in order to afford more room for the respiratory movements and prevent accidents in casting.* A specially constructed inhaler, or nose bag, strapped about the head, may be employed for convenience. Harger recommends placing a sponge wrapped loosely in flammel, in the upper nostril, as the animal lies upon his side upon the ground, and after a few minutes a similar sponge is introduced into the lower nostril.

Chloroform is then administered frequently in drop doses from a bottle having a cork nicked upon the side sufficiently to allow the anæs thetic to flow out in this small quantity. The nostrils should be previously smeared with vaseline to afford protection from the irritation of the chloroform. If the operation is prolonged, anæsthesia may be carried on with ether. It is not essential to cast a horse before chloroformization, but the animal should be controlled with side lines, and a twitch should be placed upon the nose, unless an inhaler is used. Since there is commonly more or less struggling, it is, however, more satisfactory to cast an animal before anæsthesia is begun. The writer has given chloroform in several instances to horses in the standing position until they fell or were pushed over upon a straw bed, with the aid of only one assistant. In thus producing anæsthesia, a sponge covered with a towel was used, and this was saturatel with about half an onnce

* Hspodermic injections of atropine fifteen minutes before anæsthesia is begun lessens mucous secretion a id is often combined to alvantage with morphine, which diminishes excitement and allows of the use of a smaller amount of
the anæesthetic. 
of chloroform, and held at first three inches from the animal's nose, in order that the vapor should be thoroughly diluted with air. From half a drachm to a drachm of chloroform should be added from time to time, always allowing plenty of air, and inhalation may be continued for an hour with comparative safety.

The anæsthetizer should give his whole attention to the work, and watch carefully the respiration, pulse and pupil, for any sign of danger. If any arise, the anæsthetic should be removed and treatment pursued as recommended in the previous section.

Dogs should be fasted for twelve hours before etherization, in order to avoid vomiting during anæsthesia. It is necessary to muzzle dogs before anæsthesia is begun. This may be done by tying a strong tape about the nose, bringing both ends up between the ears, over the top of the head, and then tying them together in a knot, and finally carrying the ends down, one on either side of the neck, and fastening them underneath. A wire muzzle may be used to control the dog more conveniently, and the ether is then poured upon a sponge within the muzzle and the muzzle is covered with cloth to keep out the air. If the extemporaneons tape muzzle is employed, ether is administered by means of a cone made out of stiff cardboard, or newspaper covered with a towel, or a straw cuff may be utilized. The cone should be tight, and the ether is then poured upon a sponge, or absorbent cotton, and introduced within the cone. If the muzzle obstructs the breathing, it can be loosened after anæsthesia is secured. While it is essential to obtain a free supply of air in chloroform inhalation, it is as desirable that air should be somewhat excluded by means of the cone during etherization. One-half ounce, or more, of ether is added from time to time as required. If larger quantities are employed, it is wasteful, but not dangerous, as with chloroform.

Dogs may also be anasthetized by placing them in a covered pail, tight box or barrel, or by driving them into 
their kennels, and dropping in cloths, sponges, or absorbent material saturated with chloroform, while excluding the outer air: The smaller animals can be destroyed in a humane and satisfactory manner by this method.

\section{USES OF ANESTHESIA.}

Anæsthetics are not employed as frequently as is desirable in veterinary medicine. Anæsthesia entails skilled assistance, increased expense, and danger; but, on the other hand, facilitates rapidity and asepsis during operations by lessening struggling, and should be employed to relieve suffering where a local anæsthetic is impracticable. The owner of an animal should be made to understand the extra risk and expense attending operations under anæsthesia, and his consent should be secured before using ether or chloroform.

The general indications for anæsthetics embrace all severe, prolonged, and delicate operations. The more special indications are as follows: In abdominal operations, as ovariotomy, herniotomy and reduction of hernia. In operations for retdined testicle, scirrhus cord, castration, and in dystocia to cause dilatation of a rigid and otherwise undilatable os, to assist the operator in remedying faulty positions of the fotus by relaxation of the parts, and to facilitate instrumental delivery in bitches. Anæsthesia is also indicated in removal of tumors, in arytenectomy, excision of the eyeball or parts of the hoof, extraction of teeth, reduction of dislocations, setting of fractures, and to relieve severe pain in colic, and to overcome spasm in chorea, or convulsions due to poison or natural causes.

Chloroform is used to destroy sick, injured or aged horses, but is not so rapid, convenient, or effective as the 44 calibre revolver, or rifle. The bullet should be directed toward a point upon ihe forehead at the intersection of two imaginary lines drawn from either eye up to the root of the opposite ear. 


\section{Class 3.-Nitrites.}

Spiritus સtheris Nitrosi. Spirit of Nitrous Ether.

$$
\text { (U. S. \& B. P.) }
$$

Synonym.-Sweet spirit of nitre, spiritus nitri dulcis, spiritus nitrico-athereus, E.; éther azoteux alcoolisé, liqueur anodine nitreuse, Fr.; versüsster sallpetergeist, $G$.

An alcoholic solution of ethyl nitrite $\left(\mathrm{C}_{2} \mathrm{H}_{5} \mathrm{NO}_{2}\right)$, yielding, when freshly prepared, not less than 11 times its own volume of nitrogen dioxide (NO).

Derivation.-Dissolve sodium nitrate, 770 , in water; add deodorized alcohol, 550; introduce sulphuric acid, 520, previously diluted with water; distil in flask and condense. Wash distillate with ice water to remove alcohol, with cold solution of sodium carbonate to remove traces of acid; agitate with potassium carbonate to remove traces of water; filter, and add sufficient deodorized alcohol to make the mixture weigh 22 times the weight of the nitrous ether to. which it was added.

Froperties. - A clear, mobile, volatile, inflammable liquid, of a pale-yellowish or faintly greenish-yellow tint, having a fragrant ethereal and pungent odor, free from acidity, and a sharp, burning taste. Spec. gr. 0.820. Mixes freely with water and alcohol.

Dose.-H. and C., z i.-iv. (30.-120.); Sh. and Sw., 3 ii.-iv. (8.-15.); D., $\eta_{x .-3}$ i. (.6-4.).

Smaller doses every two hours, diarphoretic. Larger doses, repeated three times daily, diuretic.

\section{Amyr Nitris. Amyl Nitrite. $\mathrm{C}_{5} \mathrm{H}_{11} \mathrm{NO}_{2}$. (U. S. \& B. P.)}

Synonym. - Amylum nitrosum, amylæther nitrosus, amylo-nitrous ether, E.; azotite d'amyl, Fr.; amylnitrit, G.

A liquid containing abont 80 per cent. of amyl (principally iso-amyl) nitrite, together with variable quantities of undetermined compounds. 
Derivation.-Obtained through distillation of nitric acid and amylic alcohol. Distillate purified hy sodium carbonate. $\mathrm{HNO}_{3}+\mathrm{C}_{5} \mathrm{H}_{11} \mathrm{OH}=\mathrm{C}_{5} \mathrm{H}_{11} \mathrm{NO}_{2}+2 \mathrm{H}_{2} \mathrm{O}$.

Properties.-A clear, yellow or pale-yellow liquid, of a peculiar ethereal, fruity (banana) odor, and a pungent, aromatic taste. Spec. gr. 0.870 to 0.880 .

Dose (by inhalation).-H., 3 ss.-i. (2.-4.); D., Mii.-v. (.12-.3).

When given internally the smaller doses should be used dissolved in alcohol.

Spiritus Glonorni. Spirit of Glonoin. $\mathrm{C}_{3} \mathrm{H}_{5}\left(\mathrm{~N} \mathrm{O}_{3}\right)_{3}$. (U.S. \& B. P.)

Synonym.-Propenyltrinitrate, glonoin trinitrate, spirit of nitroglycerin, trinitrate of glyceryl, trinitin, E.

An alcoholic solution containing one per cent., by weight, of nitroglyceriv. It is probably decomposed in the blood with the formation of potassium and sodium nitrite.

Derivation. - Nitroglycerin is obtained by dropping pure glycerin upon a mixture of sulphuric and nitric acids, kept cool by ice, and purified by washing with water. The official one per cent. solution is not explosive unless it becomes concentrated by evaporation to an extent exceeding 10 per cent.

Properties. - A clear, colorless liquid, possessing the odor and taste of alcohol. Caution should be exercised in tasting it, since even a small quantity is liable to produce a violent headache. Spec. gr. .826 to .832.

Dose.-H., 3 ss.-i. (2.-4.); D., Mi.-ii. (.06-.12).

\section{ACTION OF THE NITRITES.}

External.-Spirit of nitrous ether, like ether, produces a cooling and local anæsthetic action, owing to its evaporation upon the skin. Amyl nitrite has a slight depressing action upon the peripheral ends of the sensory nerves.

Internal.-The actions of spirit of nitrous ether, amyl 
nitrite and nitroglycerin are essentially the same. Spirit of nitrous ether should contain 4 per cent. of ethyl nitrite. Analyses of 68 samples taken at random from drug stores, resulted in showing that a teaspoonful of the best specimen contained as much ethyl nitrite as a pint of the worst, with all manuer of variations between these extremes. To be of value as a nitrite, sweet spirit of nitre should be freshly prepared by a reliable chemist.

Circulation.-The important action of the nitrites centres upon the heart and blood vessels. The arterioles all over the body become relaxed and blood tension is lowered by the nitrites. This action is no doubt peripheral, but whether due to the impression upon the vasomotor ganglia or muscular fibres in the walls of the vessels is uncertain. The heart beats more rapidly in consequence of the lessened vascular resistance and direct depression of the inhibitory centre and becanse a diminished blood pressure indirectly depresses the vagus centre and often stimulates the accelerator. The arterioles being dilated in the heart, as elsewhere, there follows an increased cardiac blood supply, nutrition and force. It is probable that the nitrates also stimulate the cardiac ganglia, and increase the force as well as the rapidity of the heart. This is shown by the fact that in small doses blood pressure is raised by the nitrites, despite the vascular dilatation.

Nervous System.-The nitrites depress, especially, the spinal motor centres, and, in a less degree, the activity of the motor nerves. Reflex excitability is thus lessened.

Muscles.-Amyl nitrite paralyses muscular tissue when applied locally; and the nitrites, when administered internally, relieve spasm of muscular tissue by their depressing action upon the motor nerves and muscles.

Kidneys. - The nitrites augment the amount of urine by dilating the afferent branches of the renal arteries which supply the glomeruli, and so increase the tension within them. The other functions of the body are not affected in any degree by medicinal doses. 
Blood.-In poisoning by the nitrites, the oxidizing power of the blood is lost. Both the venous and arterial blood become of a chocolate hue from the conversion of the normal oxy-hæmoglobin into meth-hæmoglobin. The latter yields up oxygen very sparingly.

Respiration. - The respiratory movements may be increased both in force and frequency by medicinal doses of nitrites, but paralysis of the respiratory centre and asphyxia occurs in fatal poisoning.

nummary.-The nitrites dilate arterioles, increase the rate of the heart-beat, depress the spinal motor area, and lessen reflex action. They relieve spasm and increase the secretion of urine.

Poisoning. - Fatal poisoning is extremely rare as an accidental occurence. Piecovery from a quantity 120 times greater than the normal dose of nitroglycerin, has been reported in human practice. The physiological effects of the nitrites are more noticeable in man, owing to his bare skin. Immediately following the inhalation of amyl nitrite, the face becomes extremely flushed, and throbbing of the vessels of the head, with a feeling of tremendous pressure and headache, ensue. These symptoms are due to vascular dilatation. There is dizziness, the heart is rapid and violent, and the pulse full, frequent and easily compressible. The respiratory movements are increased. These symptoms occur after a full medicinal dose.

In poisoning there is pallor, vomiting in man, trembling, weakness, cyanosis and failure of respiration and heart. The treatment is included in the administration of ammoniacal and alcoholic stimulants, together with the subcutaneous injection of ergotin, strychnine and atropine, to restore the vascular tone.

The nitrites differ sufficiently to call for a word concerning their individual characteristics. It is important to emphasize the fact that their action, as a whole, is transient. Amyl nitrite diminishes vascular tension, as shown by the sphygmograph, within a minute of its inhalation, and this 
condition lasts for 2 to 4 minntes, with variations of from 10 to 30 minntes. The same action of nitroglycerin occurs within 6 minutes and lasts from half to, rarely, an hour and a half.

Good spirit of nitrous ether lowers tension from 45 to 60 minutes. In addition to this difference in degree, spirit of nitrous ether differs somewhat in kind of action. It is more stimulant to the heart, and more diuretic, owing to the ether it contains. For the same reason sweet spirit of nitre increases the secretions and motion of the upper part of the digestive tract, relieves spasm and is of some value in indigestion and mild colic. In stimulating the activity of the sweat glands, following its action in dilating peripheral vessels, sweet spirit of nitre is a useful diarphoretic and mild febrifuge.

It has been pointed out that spiritus ætheris nitrosi is far from being a reliable preparation as a nitrite, and therefore nitroglycerin or amyl nitrite are preferable where rapid and certain vascular dilatation is essential.

USES OF THE NITRITES.

Internal.-Respiratory Diseases.-No drug is more efficient than spirit of nitrous ether, in the treatment of acute diseases of the respratory tract, as coryza, pharyngitis, laryngitis and bronchitis. Its value lies in its power of dilating peripheral vessels, equalizing the circulation and preventing local congestions.

In assisting diarphoresis and diuresis, sweet spirit of nitre hastens elimination of toxines and cools the body; and in both ways is useful in abating fever. The following prescription may be of service in canine practice in febrile conditions :

$\mathrm{R}$

Tinc. aconiti...................mxxiv.

Spiritus ætheris nitrosi ............ 3 vi.

Potassii bromidi.................

M.

Liq. Ammonii acetatis ad .............

Sig. Teaspoonful in water every 2 hours. 
In asthenic and febrile diseases, as influenza, sweet spirit of nitre is of worth, combined with tonic doses of quinine and alcoholic stimulants.

The nitrites are the most successful remedies in relieving dyspnœa, when due to spasm of the bronchial tubes, or congestion of the lungs. They relax the bronchioles and avert congestion by vascular dilatation and equalization of the circulation. Thus the dyspnoea occurring in pneumonia, acute pulmonary œdema, asthma and chronic bronchitis, are advantageously treated by half-hourly or hourly doses of nitroglycerin.

Cardiac Diseases. - These are comparatively rare in the lower animals; but, in general, it may be said that no remedy is more useful for its transient action in the severe dyspnœa of cardiac diseases than nitroglycerin. The passing engorgement of the right heart and lungs is relieved by nitroglycerin, which tends to distribute the blood about the body in its proper channels, and thus takes the load off the heart temporarily.

Nervous Diseases. - Nitrite of amyl is invaluable in warding off epileptic seizures in man, when warning of their approach is given the patient. As this warning cannot be detected in the lower animals, the nitrites are of less value, but may be combined with the bromides as prophylactic agents. The nitrites exert their favorable effect in this condition by preventing cerebral vasomotor spasm, which is thought to occur in epilepsy.

Diseases of Urinary Organs.-Spirit of nitrous ether is useful as a diuretic in carnivora, when the urine is concentrated and irritating to the bladder. It is also a valuable remedy, for the same reason, in acute cystitis of all animals, when it may be combined to advantage with potassium citrate, or acetate and tincture of aconite.

Summary.-We may summarize the therapeutical indications for the nitrites as follows:

1. To dilate peripheral arterioles and equalize the circulation in internal congestions. 
2. To stimulate the heart.

3. To relieve spasm of vascular, nervous, or muscular origin.

4. To increase the secretion of urine.

Administration.-Sweet spirit of nitre is given by the mouth, diluted with water, and often combined with alcoholic stimulants (whiskey), diarphoretics (liq. ammon. acetatis), diuretics (potassium nitrate), and bitters (quinine).

Amyl nitrite is administered usually by inhalation to the larger animals, from a sponge; or two or three drops are given by inhalation from a bit of linen or cotton to the smaller animals. It may be given internally on sugar to the smaller animals, or in spirit to the larger patients. The spiritus glonoini is the only preparation of nitroglycerin in use. It may be dropped undiluterl upon the tongne of the conscions or unconscions animal. The tongue of the smaller animals may be frequently moistened with a small stick dipped in the solntion, or it may be given in pill or tablet.

\section{Class 4.-Chloral.}

\section{Chloralum Hydratcir. (U. S. P.)}

Chloralum Hidratum. Chloral. $\mathrm{C}_{2} \mathrm{H} \mathrm{Cl}_{3} \mathrm{O}+\mathrm{H}_{2} \mathrm{O}$.

Synonym.-Chloral hydras, B. P.; hydrate of chloral, E.; chloratum hydratum crystallisatum, P. G.; hydrate de chloral, Fr.; chloralhydrat, G.

A crystalline solid, composed of trichloraldehyde, or chloral with one molecule of water.

Derivation.-Dry chlorine gas is passed into absolnte alcohol mutil the latter is saturated. Aldehyde and hydrochloric acid first result, $\mathrm{C}_{2} \mathrm{H}_{5} \mathrm{OH}+2 \mathrm{Cl}=\mathrm{C}_{2} \mathrm{H}_{4} \mathrm{O}+2 \mathrm{H} \mathrm{Cl}$. The chlorine gas then acts upon the aldehyde, abstracting 3 atoms of hydrogen and replacing 3 atoms of chlorine, and so forms chloral. $\mathrm{C}_{2} \mathrm{H}_{4} \mathrm{O}+6 \mathrm{Cl}=\mathrm{C}_{2} \mathrm{H} \mathrm{Cl}_{3} \mathrm{O}+3 \mathrm{H} \mathrm{Cl}$.

Chloral is purified first by distillation with sulphuric acid, and then with lime, and when mixed with water forms chloral hydrate $\left(\mathrm{C}_{2} \mathrm{H} \mathrm{Cl}_{3} \mathrm{O}+\mathrm{H}_{2} \mathrm{O}\right)$. 
Properties.-Separate, rhomboidal, colorless and transparent crystals, having an aromatic, penetrating and slightly acrid odor, and a bitterish, caustic taste. Slowly rolatilized when exposed to the air. Freely soluble in water, alcohol or ether; also in chloroform, benzol, benzin, carbon disulphide, fixed and volatile oils. It liquifies when triturated with about an equal quantity of camphor, menthol, thymol or carbolic acid. Chloral is decomposed by caustic alkalies, alkaline earths and ammonia, chloroform being formed, and a formate of the base produced.

Dose.-H. \& C., 3 i.-ii. (30.-60.); Sh. \& Sw., 3 i.-ii. (4.-8.); D., gr.v.-xx. (.3-1.3).

Action External.-Chloral is a strong irritant applied locally in concentration to the skin and mucous membranes, and if injected under the skin may cause abscess and sloughing. It is a powerful antiseptic, and relieves itching, especially in combination with camphor.

Action Internal.-Alimentary Canal. - Chloral produces severe irritation of the mucous membrane in concentrated solution (20 per cent. or over), and large doses may cause vomiting in dogs. The writer has seen intense glossitis and stomatitis follow the breaking of a gelatine capsule, containing chloral, in the mouth of a horse.

Blood.-Chloral is absorbed into the blood unchanged. It was formerly thought that the action of chloral was due to chloroform produced by the decomposition of the former in the alkaline blood. $\mathrm{C}_{2} \mathrm{H} \mathrm{Cl} \mathrm{Cl}_{3} \mathrm{O} \mathrm{KH} \mathrm{O}=\mathrm{CH} \mathrm{Cl}_{3}+$ $\mathrm{K} \mathrm{C} \mathrm{H} \mathrm{O}$ (formic acid).

It is now known that the blood is not sufficiently alkaline to decompose chloral, and that chloroform is not found in the blood, tissues or excretions, except in the case of the urine, when it is strongly alkaline. Moreover, chloral acts as usual upon a frog when the blood of the batrachian is replaced by a neutral saline solution.

Heart and Blood Vessels.-Chloral in large doses depresses the action of the heart muscle, its contained ganglia, and the vasomotor centres. It also produces local paralysis of the vascular walls. Blood pressure is therefore lowered. 
In small medicinal doses the circulation is not influenced materially, but in poisoning the pulse at first is accelerated and then becomes slow, weak and irregular, and the heart is arrested in diastole.

Nervous System-The salient action of chloral is exerted upon the brain and cord. Like other narcotics, the depressing effect may be preceded by a transient and unimportant excitation of the brain and cord; but this commonly passes unnoticed, and the prominent action of chloral consists, in ordinary doses, in depressing the higher functions of the brain, and in larger doses, the motor tract of the cord. Moderate therapentic doses cause, therefore, dulness and sleepiness (with contracted pupils) in the lower animals, while doses approaching the toxic point produce insensibility, coma, paralysis of the inferior cornua, with loss of reflex action and muscular power, so that the animal falls; paraplegia, dilated pnpils and anæsthesia. These symptoms may occur and be followed by recovery. The anæsthesia is of spinal origin. Neither the sensory nerves, motor nerves, nor muscles are affected except in the later stages of poisoning.

Insensibility to pain is said, by Brunton, to follow the action of chloral upon the gray matter of the cord, by preventing the transmission of painful sensations through this tract. It is uncertain whether chloral acts as an hypnotic by its direct depressing influence upon the brain tissue, or by inducing cerebral anæmia in causing the blood to be withdrawn from the cerebrum into the dilated peripheral arterioles.

Respiration.-The respiration is not interfered with by moderate medicinal doses of chloral, but toxic quantities depress and paralyze the respiratory centre. The respiratory movements become deep, regular, accelerated (26) and full, with large therapentic doses, but with toxic doses, slow, irregnliır and shallow. Death occurs more commonly from arrest of respiration, yet primary lieart failure, or both combined, may lead to a fatal result. 
Temperature.-The temperature may be elevated at first, but soon falls, owing to diminished heat production and increased loss, through heart failure and viscular dilatation.

Elimination.-Chloral is eliminated by the urine, in part unchanged and in part in an altered condition.

Summary.-Chloral is a local stimulant and antiseptic, and relieves itching. It is a powerful depressant to the cerebrum, vasomotor and respiratory centres, inferior cornua, heart muscle and its ganglia.

Acute Poisoning. - Large doses produce insensibility, coma, and complete loss of muscular power, so that the animal falls. There is general anæsthesia, and the pupils dilate. The pulse is weak, at first frequent, later infrequent. and irregular. The respiration may be primarily qnickened, but subseqently becomes slow, shallow and irregular. The animal sweats, sways, gapes and trembles and sometimes falls to the ground, the sphincters are relaxed and involuntary defæcation occurs, but recovery commoniy follows. With doses greater than 4 ounces, horses die in a generally anæsthetic and paralyzed state. In man, death has followed the ingestion of 10 grains of chloral, and several fatalities have occurred after doses of 20 or 25 grains, although these are exceptional cases. The fatal dose for dogs is said to be from 2 to 6 drachms.

Treatment.-Emetics and the stomach tube, shouting at and beating the animal, external heat. Five times the ordinary dose of strychnine and atropine subcutaneously. Strong, hot coffee and alcohol by the rectum. Amyl nitrite inhalations.

Administration.-Chloral has been given intravenously, subcutaneously, and intratracheally, as well as by the mouth and rectum. The common way of administering it is in solution per orem or rectum. It uray canse abscess if injected under the skin, or thrombi when thrown into a rein. Experiments of Dr. Muir* appear to show that chloral may

* Jour. Compar. Med. and Vet. Archives, April, May, 1900. 
be safely given intrajugularly; one ounce dissolved in two cunces of sterile water and repeated once in an hour if desirable. If given in ball, the chloral may prove too irritating in the digestive tract. It should be diluted at least 10 tmes, and is given by the moutl $l_{2}$ witl glycerin and water, or weak syrup, or with boiled starch solution by the rectum.

Uses External.-Chloral may be employed as a stimulant, antiseptic, and slight local anæsthetic, in solution ( 1 to 4 ), upon ulcers and wounds. It may be used with an equal part of camphor diluted with 8 parts of ointment, to relieve itching. A 4 to 8 per cent. aqueous solution forms an excellent antiseptic preservative for anatomical specimens, and chloral may be added to urine for this purpose.

Uses Internal.-Chloral is used for three purposes in veterinary practice :

1. First and foremost, to relieve spasm.

2. To assuage pain.

3. To procure sleep.

In human medicine chloral is mainly employed as an hypnotic, but a purely soporific action is rarely required for the lower animals. The spasmodic conditions benefited by chloral include colic, convnlsions, chorea, epilepsy, asthma, canine distemper, spasmodic cough, rigidity of the uterine os in the first stage of labor, hysteria, tetanus, and strychnine poisoning. In spasmodic colic, chloral is inferior to opium as an anodyne, but has the advantage of not inducing constipation. It may be employed in colic, by the rectum, combined with morphine given under the skin, as recommended for procuring anæsthesia. It is the best remedy we possess for the treatment of convulsions in dogs, apart from anæsthetics. Ether may be administered during the convulsion, and ehloral given simultaneously, or between the attacks, in gr.v.-xx., per rectum, and repeated if necessary. Chloral is only indicated in chorea when the movements are so severe that the animal cannot secure sleep or rest. In distemper in dogs, chloral is used for the same purpose, when there is excessive cough and restlessness. Chloral is 
given per rectum in tetanus, so as to keep the animal conti. nually narcotized, and may be employed in conjunction with tetanus antitoxin.

Spasm of the os uteri is relieved by chloral when given per rectum in frequently repeated doses, until the safe physiological limit is reached. Chloral is inferior to ether or chloroform as an anæsthetic, because it is not so safe, nor is the anæsthesia so complete, but it relieves pain effectually, and is more easily administered. It is combined, in order to produce anæsthesia, with small doses of morphine, which decidedly enhance the anodyne action of chloral.

To prepare a horse for surgical operation, 3 grains of morphine sulphate and 1 grain of atropine sulphate may be injected under the skin, and followed in 10 minutes by an enema containing 1 ounce of chloral.

\section{Class 5.-Antipyretics and Analgesics.}

Acetanilidum. Acetanilid. $\mathrm{C}_{6} \mathrm{H}_{5} \mathrm{~N} \mathrm{H}_{2} \mathrm{H}_{3} \mathrm{O}$. (U. S. \& B. P.)

Synonym.-Phenylacetamide, antifebrin.

An aceíyl derivative of aniline.

Derivation.-Glacial acetic acid and aniline are distilled together, and the residue is purified by repeated crystallization. $\mathrm{H} \mathrm{C}_{2} \mathrm{H}_{3} \mathrm{O}_{2}+\mathrm{C}_{6} \mathrm{H}_{5} \mathrm{~N} \mathrm{H}_{2}=\mathrm{C}_{6} \mathrm{H}_{5} \mathrm{~N} \mathrm{H} . \mathrm{C}_{2} \mathrm{H}_{3} \mathrm{O}+\mathrm{H}_{2} \mathrm{O}$.

Properties.-White, shining micaceous crystalline laminæ, or a crystalline powder, odorless, having a faintly burning taste, and permanent in the air. Soluble in 194 parts of water, and in 5 parts of alcohol; also soluble in 18 parts of ether, and easily soluble in chloroform.

Dose.-H., 3 i.-ii. (4.-8.); Sh. \& Sw., 3 ss.-i. (2.-4.); D., gr.iii.-vii. (.2-.5).

Phenacetinum. Phenacetin. $\mathrm{C}_{10} \mathrm{H}_{13} \mathrm{NO}_{2}$ (178.63). (B.P.)

(Non-official, U. S. P.)

Synonym.-Para-acetphenetidin, $\mathrm{C}_{6} \mathrm{H}_{4} \mathrm{O} \mathrm{C}_{2} \mathrm{H}_{5} \mathrm{~N}$ H $\mathrm{C}_{2} \mathrm{H}_{3} \mathrm{O}$ $+\mathrm{H}_{2} \mathrm{O}$ (178.63). 
Derivation.-Obtained by the action of glacial acetic acid upon paraphenetitin, a phenol derivative.

$\mathrm{H} \mathrm{C}_{2} \mathrm{H}_{3} \mathrm{O}_{2}+\mathrm{C}_{6} \mathrm{H}_{5} \mathrm{O} \mathrm{C_{2 }} \mathrm{H}_{5} \mathrm{~N} \mathrm{H}=\mathrm{C}_{6} \mathrm{H}_{4} \mathrm{O} \mathrm{C}_{2} \mathrm{H}_{5} \mathrm{~N} \mathrm{HC}_{2} \mathrm{H}_{3} \mathrm{O}$ $+\mathrm{H}_{2} \mathrm{O}$.

Properties. - Glistening, colorless, tasteléss, odorless, scaly crystals. Practically insoluble in water (1-1700), soluble in 30 parts of alcohol, and in glycerin, chloroform and acetic acid.

Dose.-H. \& C., 3 ii.-iii. (8.-12.); D., gr.v.-x. (.3-.6).

Antipyrinum. Antipyrin. $\mathrm{C}_{6} \mathrm{H}_{5}\left(\mathrm{C} \mathrm{H}_{3}\right)_{2} \mathrm{C}_{3} \mathrm{H} \mathrm{N} \mathrm{N}_{2} \mathrm{O}$. (Non-official, U. S. P.)

Synonym.-Phenazonum, B. P.; phenyl-dimethyl-pyrazolone.

Derivation.-Phenyl-hydrazine is acted upon by acetoacetic ether, when phenyl-monomethyl-pyrazolone, ethyl alcohol and water result.

$\mathrm{H}_{2} \mathrm{~N} \mathrm{~N} \mathrm{H} \mathrm{C}_{6} \mathrm{H}_{5}+\mathrm{C} \mathrm{H}_{3} \mathrm{C} \mathrm{O} \mathrm{C} \mathrm{H}_{2} \mathrm{C} \mathrm{O} \mathrm{O} \mathrm{C}_{2} \mathrm{H}_{5}=\mathrm{C}_{6} \mathrm{H}_{5}$ $\left(\mathrm{C} \mathrm{H}_{3}\right) \mathrm{C}_{3} \mathrm{H}_{2} \mathrm{~N}_{2} \mathrm{O}+\mathrm{C}_{2} \mathrm{H}_{5} \mathrm{OH}+\mathrm{H}_{2} \mathrm{O}$. Then $\mathrm{C}_{6} \mathrm{H}_{5}\left(\mathrm{C} \mathrm{H}_{3}\right)$ $\mathrm{C}_{3} \mathrm{H}_{2} \mathrm{~N}_{2} \mathrm{O}+\mathrm{C} \mathrm{H}_{3} \mathrm{I}$ (methyl iodide) $=\mathrm{C}_{6} \mathrm{H}_{5}\left(\mathrm{C} \mathrm{H}_{3}\right)_{2} \mathrm{C}_{3} \mathrm{H} \mathrm{N}_{2} \mathrm{O}$ $+\mathrm{H}$ I.

Properties.-Colorless, odorless, scaly crystals, of a bitterish taste and alkaline reaction. Soluble in about its own weight of water, alcohol and chloroform.

Incompatibles.-Spirit of nitrous ether, iron sulphate, chloride and iodide; salicylates, tannin, chloral, calomel, and a large number of drugs.

Dose.-H. \& C., 3 iii.-iv. (12.-15.); Sh. \& Sw., 3 i. (4.); D., gr.v.-xx. (.3-1.3).

ACTION OF ACETANILID, ANTIPYRIN AND PHENACETIN.

External. - Acetanilid and antipyrin are antiseptics. Solutions of the latter contract vessels and exert a hæmostatic action.

Internal.-These substances exert no action upon the digestive tract, but acetanilid possesses a decided antiseptic influence upon bacteria within the alimentary canal. 
Brnod.-These agents liave no iufluence upon the blood, in moderate medicinal doses, but in large doses they dimiuish the ozonizing power of the blood, reduce the hremoglobin of the red corpuscles, change it to methæmoglobin, and alter the color of the blood to a brownish-red hue. In large toxic quantities, administered continuously, they cause disintegration of the red corpuscles and elimination of the blood-coloring matter in the urine.

Heart and Blood Vessels.-In ordinary therapentic doses these drugs do not alter the normal condition of the heart or blood vessels, but in large medicinal doses they depress the force of the heart by action (probably) upon the heart muscle. Phenacetin is the least, and acetanilid the most depressant. Antipyrin is said to stimulate the heart and increase blood pressure in minute quantities. These three antipyretics decidedly diminish blood tension in large medicinal doses, owing to depression of the vasomotor apparatus.

Nervous System.-Usual therapentic doses of these substances exert a sedative action upon the sensory nerves and sensory tract of the spinal cord. They are therefore anal-. gesics, although not comparable in this respect to opium. Poisonous quantities of these drugs diminish muscular power, lessen reflex action and cause paralysis. Experi-ments apparently show that acetanilid paralyzes the motor nerves, antipyrin the motor nerve endings, while motor depression seems to be of spinal origin in the case of phenacetin. The brain is undoubtedly influenced by these agents, as evidenced by coma and convulsions in poisoning, but exact knowledge is wanting in relation to the action: upon the brain. The functions of the cerebral cortex are. thought to be depressed by antipyrin, and the special senses. to be first stimulated and then paralyzed by this drug.

Temperature.-Acetanilid, antipyrin and phenacetin are essentially antipyretics. While they do not invariably lower temperature, even in large doses in normal animals, they do so very materially in animals suffering from fever. They apparently depress the activity of the calorefacient centres 
(probably in the corpora striata), and therefore diminish heat production. Testimony is at variance in regard to their action upon heat loss. They frequently induce diarphoresis, but it is generally accepted that heat dissipation is increased to a greater extent than would be accounted for by sweating, and that it occurs even when diarphoresis does not take place. Wood teaches that these agents act solely by lessening heat production, and this is no doubt their main action.

Respiration.-The respiratory functions are unaffected by therapentic doses of these medicines. In lethal doses respiration is quickened, owing to the greater work thrown upon the respiratory centre by the altered condition of the blood, and this vital centre is ultimately paralyzed.

Kidneys.-The drugs under consideration produce slight diuresis in moderate medicinal duses. In poisoning, the urine may become dark-colored by the hæmatin escaping from the disintegrated red blood corpuscles. Antipyrin lessens the nitrogenous products of tissue waste in the urine, and also diminishes the amount of that secretion. Acetanilid, on the other hand, increases the excretion of urea.

Elimination.-Antipyrin is rapidly eliminated unchanged in the urine. Acetanilid escapes in part unchanged, and in part in the same manner as aniline, $i$.e., para-amido-phenolsulphate, while phenacetin is chiefly eliminated as such.

Poisoning.-Toxic doses of these drugs cause, in the lower animals, nervous excitement and convulsions, and sometimes coma, loss of consciousness, staggering gait, muscular failure, sweating, rapid, feeble respiration, weak pulse, eyanosis, occasional vomiting in dogs, fall of temperature and general paralysis.

Treatment.-External heat, alcoholic stimulants by the mouth, rectum, or under the skin; strychnine, and atropine subcutaneously.

Administration.-Antipyrin is given in solution by the mouth, rectum, or under the skin. Acetanilid and phenacetin can be administered in powder, tablet, pill or ball; or 
in solution in alcoholic liquor. Acetanilid is to be preferred for horses on account of its much greater cheapness. The average dose of acetanilid is one drachm for a horse, and three to five grains for a dog; and the dose of phenacetin is twice, and of antipyrin three times greater than that of acetanilid.

Uses External.-Acetanilid is employed as an antiseptic dusting powder undilnted. A ten per cent. solution of antipyrin may be applied as a hæmostatic upon bleeding surfaces.

Uses Internal.-There are three indications for the use of these agents: 1. To lower temperature in fever. 2. To relieve pain. 3. To lessen motor excitement and spasm.

They are not so valuable in veterinary practice as in human medicine, since the lower animals rarely suffer from neuralgic pain, which is the special variety of suffering alleviated by these drugs.

Phenacetin is the most serviceable for dogs, as it is less toxic, more sedative, and more permanent in its antipyretic action than antipyrin or acetanilid. Dogs suffering from distemper are greatly relieved by small and repeated doses of phenacetin, which lessen fever, cough and restlessness. In acute diseases, as preumonia, these antipyretics may be occasionally employed to advantage when the temperature rises above $104^{\circ}$ Far., and remains there any length of time. They are generally inadvisable in asthenic, febrile diseases, since they are too depressant in their action upon the heart and have no effect in removing the cause of the disease. The hyperpyrexia of insolation may be treated with these agents, in combination with cold, externally and per rectum. For the relief of pain their scope is limited in veterinary practice to that of a rheumatic character. Motor disturbances, including convulsions, chorea and spasm, may be abated by the antipyretics, but they are usually inferior to chloral, opium, or other antispasmodics.

Phenacetin, combined witl codeine or heroin, in powder or tablet, is a useful remedy for cough in dogs. 


\section{Class 6.-Antiseptics.}

\section{Acidum Carbolicum Crudum. Crude Carbolic Acid.}

Synonym.-Acide phénique cru, Fr.; rohe carbolsäure, G.

Derivation.-A liquid consisting of various constituents of coal tar, chiefly cresol and phenol, obtained by fractioual distillation at a temperature between $302^{\circ} \mathrm{F}$. and $392^{\circ} \mathrm{F}$., and twice rectified at a temperature between $338^{\circ} \mathrm{F}$. and $374^{\circ} \mathrm{F}$.

Properties.-A nearly colorless, or reddish-brown liquid of a strongly empyreumatic and creasote-like odor, having a benumbing, blanching and caustic effect upon the skin or mucous membrane, and gradually turning darker on exposure to the air and light. Soluble in 15 parts of water. The aqueous solution has a slight acid reaction.

\section{Phenol (U. S. P.)}

*Acidum Carbolicum. Carbolic Acid. $\mathrm{C}_{6} \mathrm{H}_{5} \mathrm{O}$ H. (B. P.)

Synonym.-Phenic acid, phenol, phenyl alcohol, phenylicum crystallisatum, E.; acide phénique, acide carbolique, hydrate de phényle, Fr.; carbolsäure, phenylsäure, phenyl alkohol, G.

Derivation. - Obtained from crude carbolic acid by agitation with caustic soda, heating to $338^{\circ} \mathrm{F}$., and adding hydrochloric acid. Then by agitation with sodium chloride, digestion with calcium chloride, and distillation at a temperature between $336^{\circ} \mathrm{F}$. and $374^{\circ} \mathrm{F}$., and finally by crystallization.

Properties.-Colorless, interlaced, or separate, needleshaped crystals, or a white crystalline mass, sometimes acquiring a reddish tint; having a characteristic, somewhat aromatic odor, and when copiously diluted with water, a. sweetish taste with a slightly burning after-taste. Deliquescent on exposure to damp air. Soluble in abont 15 parts of water, and very soluble in alcohol, ether, chloroform, glycerin, fixed and volatile oils. Faintly acid reaction.

-The U.S. P., 1905, recognizes only "Phenol" as the official name for carbolic acid. 
Phenol crystals melt when heated, but solidify again on cooling. A 95 per cent. solution of carbolic acid crystals, in alcohol, remains fluid at the ordinary temperature. The crystals are also liquified by the addition of about 8 per cent. of water.

Dose.-H. \& C., gr.xv.-xxx. (1.-2.); Sh. \& Sw., gr.v.-x. (.3-.6); D., gr.ss.-i. (.03-.06).

PREPARATIONS.

1.-Unguentum Acidi Carbolici. Ointment of Carbolic Acid.

(U. S. \& B. P.)

Carbolic acid, 5; ointment, 95 . (U. S. P.)

2.-Glyceritum Acidi Carbolici. Glycerite of Carbolic Acid.

(U.S. \& B. P.)

Carbolic acid, 20; glycerin, 80. (U. S. P.)

3.-Acidum Carbolicum Liquefactum. Liquified Carbolic Acid.

(B. P.)

Carbolic acid liquified by addition of 10 per cent. of water.

Dose.-Same as acidum carbolicum.

Action External. - Carbolic acid-causes burning pain when annlied to the skin or mucous membranes, and this action is followed by local aursthesia and the production of a dry white spot. If used in sufficient quantity, it leads to sloughing, but the escharotic effect is superficial, since the acid coagulates albumin, which fornus a protective coating to the underlying parts. Carbolic acid is an antiseptic and disinfectant, and, in proper solution, acts as a sedative upon the peripheral sensory nerves, and is one of the most efficient agents in relieving itching. It checks the growth of both organized (bacteria) and unorganized (digestive) ferments. Strong solutions (1-2 per cent.) kill most bacteria, but a considerable time is required to destroy the organisms of certain diseases and those relating to putrefaction. Some hours are required to kill authrax spores, by even a 5 per cent. solution. Two per cent. solutions destroy the digestive ferments. The lower forms of vegetable parasites, growing upon the skin, perish by the application of carbolic acid. 
Action Internal.-Alimentary Canal.-Carbolic acid exerts a local, anæsthetic action upon the sensory nerve endings in the stomach, and may act to a certain extent in the digestive tract as an antiseptic, hindering abnormal fermentation, but is, in this respect, inferior to creolin, napthol and napthalin. It is probably converted into a snlpho-carbolate in the stomach. In concentration, carbolic acid is a powerful gastro-intestinal irritant.

Blout.-Carbolic acid is absorbed into the blood and probably circnlates in part as an alkaline carbolate of sodium and potassinm.

Heart and Blood Vessels.-Phenol, in poisonons doses, paralyzes the vasomotor centre and later depresses the heart. The effect upon the vessels is the more important and prominent, but neither action is observed after medicinal doses.

Respiration.-Therapentic doses do not influence the respiratory functions, but toric quantities make the respiratory movements rapid and shallow at first, owing to stimulation of the respiratory centre and peripheral vagi, while death occurs after lethal amounts from paralysis of the respiratory centre.

Nervous System. - The brain is depressed by toxic doses of carbolic acid, and sutupor and coma occur. The convulsions appearing in carbolic acid poisoning are due to primary stimulation of the spinal motor area, which is finally depressed and paralyzed. When locally applied, carbolic acid depresses, and paralyzes the peripheral sensory nerves.

Temperature.-Carbolic acid, in mediciual doses, slightly lowers temperature both in health and fever, but is not sufficiently antipyretic to be suitible for such a purpose in practice. It depresses heat prodnction and increases heat loss.

Elimination.-Carbolic acid is eliminated by all ordinary channels, but mainly by the kidneys. The urine becomes dark colored-a very characteristic sign-even after large medicinal doses. The sole cause of this urinary coloration has not yet been fully determined. Phenol normally occurs in small quantities in the urine of man and animals. Three 
grains have been recovered from the urine passer in 24 hours by a horse, and is thought to be a product of intestinal fermentation. In large toxic doses some carbolic acid is eliminated in the urine unchanged. In smaller quantities, part of the acid is decomposed, and part eliminated as sulphocarbolates of potassium and sodium, and a substance called glycuronic acid, while a portion is oxidized in the system into two bodies, hydroquinone and pyrocatechin. The latter accounts, in part, for the dark coloration of the urine, for pyrocatechin can only exist in an alkaline urine. The normal sulphates are absent in the urine following carbolic acid poisoning.

Toxicology-Carbolic acid ranks, as one of the most powerful poisons-together with prussic acid and nitrobenzole-in existence. Several cases of death in man have occurred after the ingestion of one-half an ounce of carbolic acid; and the smallest fatal human dose on record appears to be about one drachm. One or two drachins are fatal to dogs, and a dose as small as 15 grains is said to have caused the death of a doog, while the lethal amount for the horse is abont one ounce. Many cases of accidental poisoning have occurred from absorption of carbolic acid when applied externally for surgical purposes in dressings or solutions upon raw surfaces. The symptoms are the same as when absorption occurs from the digestive tract. Extensive local sloughing, after continuous treatment with moist carbolic applications, is occasionally seen. Dogs and cats are particularly susceptible to the action of phenol. The milder symptoms of poisoning include dulness, loss of appetite, nuscular weakness and trembling, and dark-colored urine having the odor of carbolic acid. After lethal doses death may be instantaneous throngh respiratory arrest, the heart continuing to beat for a time. The mcre ordinary symptoms in severe poisoning in all animals are: trembling, rarely vomiting and purging, restlessness, salivation, loss of muscular power (animal reels and falls), diminution of sensibility, anæsthesia, dyspnoa; the breathing is rapid, 
shallow and stertorous; the pulse is weak, irregular, and usually frequent; the temperature is lowered, and there aro the usual symptoms of collapse, with iusensibility, coma, loss of reflex action, general paralysis, occasional conrulsions and death. Sometimes hæmaturia, albuminuria and hæmoglobinuria have been observed. The condition resembles apoplexy, but the mucous membrane of the month is stained white in patches after ingestion of pure acid, dark with crude acid, and the odor of the poison lingering about the animal, together with the dark, green-colored urine, are characteristic of phenol poisoning. The urine may be clear when first roided, but becomes dark on standing.

The absence of carbolic acid in the urine affords certain evidence that the case is not one of poisoning by this drug. Post-mortem examination reveals hard, whitish or brownish or black patches and sloughs upon the mucous membrane of the mouth, gullet, stomach, and even the small intestines. The blood is dark from asphyxia, and imperfectiy coagulated. There is occasionally fatty degeneration of the liver. and kidut ys. The odor of the acid remains not longer than twenty-funr hours.

Treatinent.-Emetics are usually valueless on account of the anæsthetic condition of the mucous membrane of the stomach. We use, therefore the stomach pump or tube, and, as antidotes, pure whisky or brandy, to avert the local escharotic effect and as a stimulant, also Epsom or Glauber's salts, forming insoluble and harmless sulphocarbolates in the digestive tract aud blood; and these are indicated in every stage of the poisoning. For collapse, heart and respiratory failure, digitalis, strychnine, atropine, ether, brandy subcutaneously, are to be employed, together with heat externally. Mucilaginous driuks are also useful. The local caustic action of carbolic acid on the skin or mucous mem. branes can be prevented by the immediate application of strong (96 per cent.) alcohol to these parts. Of late years alcohol has also been regarded and widely given as a physiological and chemical antidote in carbolic poisoning. In 
experiments, cited by Thornton,* on dogs with mixtures of toxic doses of carbolic acid and alcohol, and with the administration of poisonous doses of the acid followed by alcohol, the results go to show that alcohol does not in any way lessen or alter the poisonous effect of carbolic acid except in preventing the corrosive action on the stomach. His conclusions are somewhat weakened, however, by the fact that doses of alcohol were used which in themselves might be toxic ( 4 to 9 ounces). It is certainly well to give pure whiskey or brandy in a large dose by the mouth after carbolic acid has been swallowed, for two reasons: to prevent the corrosive action of the acid on the mucous membranes, and to act as a circulatory stimulant, eren if there is not any other specific effect produced.

Administration.-Carbolic acid is commonly giren internally, diluted several hundred times with water.

Uses External.-A solution of carbolic acid (1-20) is frequently used in surgery to disinfect the unbroken skin, while a weaker solution (1-50) is more suitable as an antiseptic upon raw surfaces and mucous membranes. While corrosive sublimate has enjoyed chief popularity as a surgical antiseptic for many years on account of its cheapness and supposed superior bactericidal properties, recent experiments (see p. 215) have shown that the value of corrosive sublimate is much over-estimated, so that carbolic acid has again resumed almost the importance it originally had in Listerian days in surgical work. Pure carbolic acid is occasionally used as a caustic to destroy small growths, as warts, and the lining membrane of fistulæ of the poll, withers, or lateral cartilages; to swab out a septic uterus, and as a local anæsthetic upon the skin. A drop of pure acid, or a line drawn with a brush along a proposed path of incision, may render a hypodermic puncture or superficial incision painless. Carbolic acid with glycerin (1-16) is one of the most excellent preparations for applying to sluggish

* Progressive Medicine, p. 345, Dec., 1901. 
ulcers and old sinuses and fistulæ. The glycerin appears to entirely offset the corrosive action of the acid, and the result is a stimulation of the pyogenic membrane and promotion of healing which often can not be obtained by any other remedy. Injection of ten to thirty drops of a two per cent. solution into the substance of boils, glandular swellings, erysipelatous inflammatory patches, poisoned wounds, joints affected with chronic synovitis, and inflamed bursa, will of ten assist recovery and may abort the lesion.

In acute inflummation, the injections are made twice daily; in chronic conditions, once every other day; and if there is a large extent of surface involved, several injections are done at one time.

Bacelli's treatment of tetanus with carbolic acid has met with remarkuble success of late. One drachm of the pure acid in solution ( 5 to 10 per cent.) should be injected in the region of the neck and shoulders of the horse every two hours during the first 32 ho:rrs, and less frequently afterward. As much as 36 drachms may be given to the horse in 24 hours, for there appears to be a special tolerance for carbolic acid acquired in tetanus.

One of the best agents which can be used in the treatment of septic wounds is carbolic acid in $\frac{1}{4}$ to $\frac{1}{2}$ of 1 per per cent. aqueous solution. Aseptic gauze or absorbent cotton are wrung ont in the solution and applied to the part and covered with oil paper, silk or other waterproof material and so kept contimuonsly wet.

Instruments are frequently placed in carbolic acil solution (1-40) during surgical operations, althongh it is sufficient to boil them in water for ten minutes and keep them in the boiled water, or place them in a pure atmosphere upon boiled towels. Carbolized gauze is prepared from unbleacled cotton gauze medicated with half its weight of a mixture consisting of carbolic acid, 1 ; resin, 3; and paraffin, 4 parts. Plain ganze, sterilized by baking at a temperature of $140^{\circ} \mathrm{C}$. for two hours, is preferable, and avoids the danger of absorption and poisoning. The gly- 
cerite of carbolic acid is employed as a local application in stomatitis, upon the ulcerations of actinomycosis with iodine, and also upon the skin to destroy ringworm. It is. inferior, however, to tincture of iodine for the latter purpose. Two per cent. solutions are recommended to kill lice and the acari of scab and mange. Carbolic acid is the most serviceable remedy we possess to relieve itching. Two per cent. solutions may be employed upon the unbroken skin, but the strength should not be greater than half this amount upon excoriated surfaces. In sub-acute moist eczema of dogs, carbolic acid with zinc ointment (gr.5- $\overline{3}$ i.), or the following prescription, will be found of value in relieving itching and promoting recovery:

$\mathrm{P}$

Calaminae............................. $\xi$ ss.

Zinci oxidi............................ 3 ii.

Acid. Carbol............................gr.xx.

II.

Liq. Calcis ad........................

Sig. External use. (Shake.)

Care should always be taken not to apply carbolic preparatious over any considerable extent of raw surface, and to muzzle dogs in the event of an opportunity being afforded them to lick off any undue amount of the acid. A solution (1-50) in boiling water forms an efficient antiseptic and sedative inhalation for horses suffering from catarrh of the upper air passages. One of the most excellent remedies for burns consists of a two per cent. solution of carbolic acid in carron oil. It relieves pain and lessens suppuration, although carbolic acid in oil possesses little antiseptic property. Good results have been reported with intratracheal injections in verminous bronchitis of foals and calves, consisting of the following:

Pr

Acidi carbolici ..................m $m x x$.

Ol. terebinthinae.................. jii.

M.

Chloroformi...................... 3 ss.

Sig. Give at one injection intratracheally. 
Crude carbolic acid may be used to disinfect infected buildings and their contents, and, in two per cent. solution with whitewash, can be applied to walls after cleaning.

Uses Internal.-Evidence has been accumulating of late as to the value of carbolic acid in general diseases of bacterial origin. Not only has Bacelli's treatment proven successful in many cases of tetanus, but in human medicine numerous favorable reports have been made upon the use of carbolic acid when given in large doses in dilution by the mouth in the treatment of surgical sepsis, influenza, erysipelas, etc. Moreover, the subcutaneous injection of 2 drachms ( $8 \mathrm{cc}$.) of a 3 per cent. aqueous solution of carbolic acid every ten days into all pregnant cows during the prevalence of epizootic abortion is a valuable prophylactic measure. Locally, carbolic acid may exert an antiseptic and anæsthetic action in the stomach. Carbolic acid is sometimes of service in relieving vomiting and gastric pain caused by flatulence in dogs, and in counteracting intestinal fermentation and diarrhoea in all animals. In diarrhoea of dogs, grain doses are combined to advantage with bismuth subnitrate in powder, capsule, or pill. 
Crfosotum. Creosote. (U. S. \& B. P.)

Synonym.-Kreosotum, P.G.; kréosote, Fr.; kreosot, G. Derivation.-A mixture of phenols, chiefly guaiacol and cresol, obtained during the distillation of wood tar, preferably of that derived from the beech, Fagus sylvatica Linué. (Nat. ord. cupuliferæ.)

Properties. - An almost colorless, yellowish or pinkish, highly refractive, oily liquid, having a penetrating, smoky odor, and a burning, caustic taste. Usually becoming darker in tint on exposure to the light. Spec. gr: not below 1.070 at $59^{\circ} \mathrm{F}$. Soluble in about 150 parts of water, but without forming a perfectly clear solution. Freely soluble in alcohol, ether, chloroform, acetic acid, fixed and volatile oils.

Dose.-H., Mxv.-xxx. (1.-2.); C., 3 ss.-i. (2.-4.); Sh. and Sw., Mv.-xv. (.3-1.); D., Mss.-ii. (.03-.12).

Action and Uses.-The action of creosote upon animals is practically the same as that of carbolic acid, both in therapentic and toxic doses. The antidotes (soluble sulphates) and treatment of poisoning are also similar. Externally, creosote is as effective a germicide as carbolic acid, but the latter is usually preferred, being much cheaper. Creosote may be applied in the same strength for its local antiseptic, parasiticide, and local anæsthetic action. Internally, creosote is administered, as is carbolic acid, to check vomiting and to act as an intestinal antiseptic. Ontside of of the body, creosote is employed in inhalation in inflammatory diseases of the upper portion of the respiratory tract, and to kill parasites in the air passages. Intratracheal injection may be substituted for inhalation in the latter condition.

Creolindm. Creolin. (Non-official).

Derivation.-Obtained from soft coal by dry distillation. Composition very complex. Is said to contain cresol and higher homologues of phenol. 
Properties.-Dark-brown, syrupy, alkaline liquid, of a tarry taste and odor. Nearly soluble in alcohol; soluble in chloroform and ether. When added to water, creolin forms a white emulsion containing in suspension as much as 12 per cent. of the drug.

Dose.-H. and C., 3 ss.-i. (15.-30.), in single dose. For continuous use-H. and C., 3 i.-ii. (4.-8.); D., Mi.-v. (.06-.3).

Action External.-Creolin is a powerful and useful disinfectant, antiseptic, and parasiticide. It forms a slippery coating upon the skiv. Strong solutions are not caustic, but may canse a dermatitis when continuously applied. Creolin generally represents carbolic acid, but is much more efficient as a germicide, less irritating, and does not endanger animal life from absorption.* Aqueous solutions (emulsions), containing from $\frac{1}{2}$ to 1 per cent., are employed for antiseptic purposes.

Action Internal.-Creolin is eliminated by all channels, giving the secretions a tarry odor, and coloring the urine brown. One or two drachms of creolin (a lethal dose of carbolic acid), when given daily to dogs for weeks at a time, produce no bad effects. It is a good intestinal antiseptic, and better than carbolic acid.

Uses.-Creolin is employed mainly outside of the body, and is a useful general antiseptic for surgical purposes in 1 per cent. solution. Antiseptic poultices, so valuable upon septic slonghing parts, are best made by soaking clean gauze in a $\frac{1}{2}$ per cent. aqueous solution of creolin, and applying the same, covered by a waterproof protective. Creolin solutions are not to be recommended for instruments during operations, as the fluid is so turbid that they cannot be seen by the operator. A 2 per cent. solution is useful for a vaginal or uterine injection; a 1 per cent. solution for irrigation of the bladder in eystitis, or eye in keratitis and conjunctivitis; and a $\frac{1}{2}$ per cent. solntion for intestinal irrigation in dysentery. As a parasiticide, 2 per cent. solutions, or 10 per cent. ointments or soaps, may be used to kill lice and acari of seab and mange.

* Creolin, to avoid toxic effects, should be pure. That made by Merck is a reliable article. 
Sheep are dipped to advantage in 2 per cent. solutions, to destroy ticks, instead of the more dangerous arsenical liquids. Creolin (of Merck) may be used internally, as an intestinal antiseptic and anthelmintic. One ounce given on an empty stomach, in a quart of water, is one of the most effective vermifuges for the horse.

\section{Lysolum. Lysol. (Non-official.) •}

Derivation. - From that part of tar oil which boils between $190^{\circ}$ and $200^{\circ}$ C., by dissolving in fat and saponifying in alcohol.

Properties. - A clear, brown, oily liquid, of a feeble, creosote-like odor. Soluble in water, forming a clear, frothy, soapy fluid, and in alcohol, chloroform, and glycerin. Lyso contains 50 per cent. of cresol.

Lysol is used as a substitute for creolin, in $\frac{1}{2}$ to 2 per cent. aqueous solution. It is a powerful antiseptic, and is stated to be more efficient and half as poisonons as creolin, and ouly $\frac{1}{8}$ as toxic as carbolic acid. Lysol solutions do not obscure instruments, nor damage the hands of the operator. The drug is undoubtedly a very useful one.

\section{NAPHToL. Naphtol. $\mathrm{C}_{10} \mathrm{H}_{7} \mathrm{O}$ H. (U. S. P.)}

Synonym.-Beta maphtol.

A pheuol occurring in wood tar, but nsually prepared artificially from naphthalin.

Derivation.-Naphthalin is treated with strong sulphuric acid. B-naphthalin sulphonic acid is formed $\left(\mathrm{C}_{10} \mathrm{H}_{7} \mathrm{H} \mathrm{SO}_{3}\right)$. The latter acid is dissolved in water with milk of lime, and the resulting calcium salt is recovered by crystallization. The crystals are then dissolved in water and treated with sodium carbouate, when sodium naphthalin-sulphonate $\left(\mathrm{C}_{10}\right.$ $\mathrm{H}_{7} \mathrm{SO}_{3} \mathrm{Na}$ ) results. The latter is mixed with fused sodiun hydroxide, wheu sodium naphtol $\left(\mathrm{C}_{10} \mathrm{H}_{7} \mathrm{O} \mathrm{Na}\right)$ and sodium sulphite are obtained. Hydrochloric acid is added to the 
former, and naphtol results, which is further purified by sublimation and recrystallization.

Properties. - Colorless, or pale buff-colored, shining, crystalline laminæ, or a white, or yellowish-white, crystalline powder; having a faint phenol-like odor, and a sharp and pungent but not persistent taste. Permanent in the air. Soluble in about 1,000 parts of water, and in 0.75 parts of alcohol; also very soluble in ether, chloroform, or solutions of caustic alkalies. .

Dose.-H., 3 ii.-iii. (8.-12.); D., gr.i.-x. (.06-.6).

Actions and Uses.-Naphtol is a powerful disinfectant, antiseptic and parasiticide. It is a useful application externally in 10 per cent. ointment, for mange and ringworm.

Internally it is employed to kill round and tape worms, and as an antiseptic in intestinal fermentation. It should be given in keratin coated pill (to avoid irritating the stomach), or capsules, to dogs, and in ball to horses.

\section{Naphtalinum. Naphtalin. $\mathrm{C}_{10} \mathrm{H}_{8 \cdot}$ (U.S. P.)}

Synonym.-Naphtalene.

Derivation.-A hydrocarbon obtained from coal tar by distillation between $356^{\circ} \mathrm{F}$. and $482^{\circ} \mathrm{F}$. The impure naphtalin resulting is treated with sulphuric acid and sodium hydroxide, and is further purified by distillation with steam, and then by mixture with strong sulphuric acid, and finally by redistillation.

Properties. - Colorless, shining, transparent laminæ, having a strong characteristic odor resembling that of coal tar, and a burning aromatic taste; slowly volatilized on exposure to the air. Insoluble in water; soluble in 15 parts of alcohol; very soluble in ether, chloroform, carbon disulphide, and fixed and volatile oils.

Dose.--H., 3 ii.-iv. (8.-15.); D., gr.i.-xx. (.06-1.3).

Actions and Uses. - Naphtalin, like other coal tar products, is an antiseptic and parasiticide. It may be used as: an antiseptic dressing powder upon wounds, or in 10 per 
cent. ointment for parasitic skin diseases. Naphtalin is almost jusoluble in the digestive tract, and acts as an antiseptic, therefore, thronghont this canal. It is of service in intestinal fermentation, diarrhœa, dysentery, and, as a vermicide, in combination with castor oil. Niplitalin is administered to dogs in starch wafers or gelatine capsules; and to horses in ball or electuary.

\section{Resorcinum. Resorcin. $\mathrm{C}_{6} \mathrm{H}_{4}(\mathrm{O} \mathrm{H})_{2}$. (U. S. P.)}

Synonym.-Resorcinol. Metarliosybenzol.

Derivation.-A diatomic phenol formed by the action of fuming sulphuric acid upon benzine, whereby benzine metadisulphonic acid $\left[\mathrm{C}_{6} \mathrm{H}_{4}\left(\mathrm{H} \mathrm{S} \mathrm{O}_{3}\right)_{2}\right]$ result.. The latter is neutralized by milk of lime; calcium sulphate is expressed, and sodium carbonate is added. The process is continued by filtration, and the filtrate evaporated to dryuess. The residue is heated with solium hydrate, with the formation of sodium resorcin $\left[\mathrm{C}_{6} \mathrm{H}_{4}(\mathrm{O} \mathrm{Na})_{2}\right]$.

Sulphurons acid is driven off from sodium resorcin by boiling, and the result is extracted with ether; impure resorcin is recovered by distillation, and is purified by sublimation or by recrystallization from water.

Properties.-Colorless, or faintly reddish, needle-shaped crystals, ol rhombic plates; having a faint, peculiar odor, and a disagreeable, sweetish, and afterward pungent taste. Resorcin acquires a reddish or brownish tint on exposure to light and air. Soluble in 0.6 part of water, and in 0.5 part of alcohol; readily solnble in ether or glycerin, aud. very slightly soluble in chloroform.

Dose.-H., ji.-ii. (4.-8.); Foals and Calves, 3 ss.-i. (2.-4.); D., gr.ii.-v. (.12-.3).

Action and Uses.-Resorcin was originally employed as an antipyretic, but is not now used for this action, being too depressing to the heart. It is an efficient antiseptic, externally and internally; possesses a slight local anæsthetic effect, and is not nearly so irritating topically as phenol. 
Externally it is of value in scaly skin diseases, as psorıasis, in solution (1-4), in glycerin. Internally, resorcin is of worth in fermentation and indigestion, given in a large amount of water an hour or two after eating

\section{Formaldehyde. $\mathrm{C} \mathrm{H} \mathrm{O} \mathrm{H.}$ \\ (Non-official.)}

\section{Synonym.-Formic aldehyde.}

Derivation.-Obtained by partial combustion of wood alcohol, without ignition, by evaporation of the spirit in contact with a hot, platinized, asbestos plate. $2 \mathrm{C} \mathrm{H}_{3} \mathrm{O} \mathrm{H}$ $+\mathrm{O}_{2}=2 \mathrm{CHOH}+2 \mathrm{H}_{2} \mathrm{O}$.

Properties.-Formaldehyde is a pungent gas, having a spec. gr. of 1.6 ; soluble in water, forming a clear, colorless, stable solution when kept in glass-stoppered bottles, but volatilizing on exposure to the air. Formalin is the commercial name for an aqueous solution containing 40 per cent. of formaldehyde gas.

Action and Uses. - Formaldehyde and formalin are powerful microbicides. A 1 per cent. solution of formalin will kill Staphylococcus pyogenes aureus in about an hour; B. typhosus in 40 to 50 minutes; B. coli communis in 30 to 40 minutes; B. anthracis and S. choleræ in less than 15 minutes. Clothes soaked in cultures of B. typhosus, S. choleræ and Staphylococcus pyogenes anreus, and then for 24 hours in a 1 per cent. solution of formalin, were found to be completely sterile (Slater). Trillat and Robinson have apparently shown that formaldehyde gas has wonderful disinfectant and penetrating properties, destroying all pathogenic bacteria in ordinary rooms containing the microorganisms buried under mattresses, between blankets, in in clothing and other articles, in the air, dust, and morbicl secretions. Harrington has proved, however, that the penetrating power of formaldehyde is nil in the case of moist substances, and that sterilization is not always complete when micro-organisms are imbedded in, or covered by, dry, 
pervious material. Still, formaldehyde is the best practical disinfectant now known for the sterilization of infected premises. It is as yet comparatively expensive. The gas is most effective between $60^{\circ}$ and $70^{\circ} \mathrm{F}$., but will act at other temperatures. It is not so operative in damp air, and the premises should be closed tight from the outer air, in order that the disinfection may be thorough. One-half a liter (about one pint) of pure wood alcohol will yield a $2 \frac{1}{2}$ per cent. formaldehyde atmosphere in 1,000 cubic feet of air space, and this is the proper proportion of alcohol necessary for disinfection. The premises containing the gas should be sealed from 4 to 24 hours. Formaldeliyde vapor is extremely pungent and irritating to the mucous membranes, causing running of the nose and eyes in those exposed to its influence; but some experimenters have subjected animals to formaldehyde vapor (of disinfectant strength) for hours without causing their death. In Harrington's experiments two rabbits were killed by formaldehyde in the disinfection of a room, and exhibited the following post-mortem appearances: Congestion and hemorrhage of the buccal mucous membrane; intense bronchitis with hyperæmia, and consolidation of the lung with a purulent and slightly fibrinous exudate. There was also congestion of the abdominal organs, including the liver, kidneys and spleen. As death may occur, it is certainly unwise to attempt the disinfection of premises with formaldehyde, when inhabited by men or animals. The smaller insect pests and animal parasites are sometimes killed by formaldehyde disinfection, but not invariably so.

Formic aldehyde vapor is not injurious to plants, clothing, metals, or other like articles, as are sulphurous anhydride or chlorine gas, and it bids fair to supersede all other agents for the gaseous disinfection of premises infected with pathogenic bacteria. Formalin may be diluted with $\frac{2}{3}$ of its bulk of water and evaporated ly heat to generate formaldehyde gas, without any special apparatus.

Harrington states that the evaporation of 110 Cc. of 
formalin is sufficient to kill all pathogenic micro-organisms within $2 \frac{1}{2}$ hours, in 1,000 cubic feet of air space. Sheets saturated with formalin will efficiently disinfect premises when reasonably air-tight, by simple evaporation, the sheets being hung $u p$ and sprinkled with formalin through an ordinary watering pot. The objection to this method is the discomfort and irritation which the gas produces on the eyes and breathing apparatus, even thongh the process be conducted as rapidly as possible. A better procedure consists in the evaporation of formalin from a vessel-as a still - placed over a lamp outside of the premises to be disinfected, the vapor being conducted through a key-hole or other aperture. By either method, eight ounces of formalin are required for each 1,000 cubic feet of air space in the room or building to be disinfected.

It is necessary to employ a generator or special appliance to convert wood alcohol into formic aldehyde, but these are not expensive.

Formalin, in $\frac{1}{4}$ to 2 per cent. solution, is perhaps the most powerful antiseptic that has been nsed for surgical purposes, but, when used in such strength 'upon raw surfaces and mucous membranes it produces pain and irritation and coagulates albumin so as to shut off the underlying parts from participating in the antiseptic action. These stronger solutions, althongh formerly eimployed for surgical uses, shonld be confined to skin disinfection or where an escharotic action is desired on slonghing tissues. Ordinarily the strength of an aqueous solution should not exceed 1-2000, or at most 1-1000, for application to raw surfaces or mucons membranes, and even in this dilution applications sometimes produce considerable pain.

Catarrhal inflammations of the mucous membrane of the nose, eyes, month, urethra and other parts are sometimes much benefited by formalin. Success is reporterl from the use of formalin on sloughing surfaces of malignant growths and foul ulcers. A 4 per cent. solution is increased 
to 10 per cent. and finally to pure formalin, the solutions being applied on cotton saturated with the drug and retained on the part for thirty minntes each day. A 5 per cent. solution of formalin is serviceable for sterilizing catheters, instruments and sutures, for the preservation of pathologic specimens, for the disinfection of stables, and in the treatment of canker of the feet in horses.

PREPARATIONS.

Urotropin (Hexametlyylentetramen). $\mathrm{C}_{6} \mathrm{H}_{12} \mathrm{~N}_{4}$.

Urotropin occurs in colorless, transparent crystals, soluble in 1.2 parts of water and in 14 parts of alcohol; odorless, of a sweet, afterward bitter taste, and slight alkaline reaction. It is made by combining solutions of ammonia and formaldehyde, and was first introduced into medicine by Nicolaier in 1895 . Urotropin appears to be decomposed in the kidneys with the liberation of formaldehyde, and thus disinfects the urinary tract. It is, in fact, considered the best urinary antiseptic in human medicine and has been used with the greatest success in the treatment of all infectious diseases of the urinary passages, especially acute and chronic pyelitis and cystitis. It renders normal a putrid urine containing pus or mucus, is a solvent for uric acid and is slightly diuretic. These actions should be of value in similar diseases of animals, especially of dogs. The drug may be given in from 3 to 5 grain doses thrice daily in solution, in canine practice.

\section{Glutol (Formalin Gelatin).}

Glutol is a coarse, white powder, without odor or irritating properties, and is prepared by dissolving gelatin in water and drying the solution in formalin vapor. It is a most valuable antiseptic powder in liberating formaldelyyde gas in contact with living cells. Glutol forms a scab when dusted over fresh wounds, preventing infection, and is serviceable in the treatment of infected wounds, abscesses, boils (after paracentesis), sinuses and other surgical conditions. It was first brought into use by Dr. C. L. Schleich, in 1896. 


\section{Class 7.-Miscellaneous Carbon Compounds.}

\section{Acidum Hydrocyanicum Dilutum.}

Diluted Hydrocyanic Acid. H C N. (U. S. \& B. P.)

Synonym. - Prussic acid, acidum hydrocyanatum, s. borussicum, E.; ${ }^{*}$ acide cyanhydrique, s. hydrocyanique, Fr.; cyanwasserstoffsäure, blansäure, $\mathrm{G}$.

Derivation.-A liquid composed of 2 per cent., by weight, of absolute hydrocyanic acid, and 98 per cent. of water. Obtained by distillation of potassinm ferrocyanide, 20 ; sulphuric acid, 8 ; and water, 65 ; into distilled water. The following reaction first occurs: $K_{4} F_{e} C_{6} N_{6}+2 H_{2} S$ $\mathrm{O}_{4}=2 \mathrm{~K}_{2} \mathrm{~S} \mathrm{O}_{4}+\mathrm{H}_{4} \mathrm{Fe} \mathrm{C}_{6} \mathrm{~N}_{6}$; then on the application of heat, the hydroferrocyanic acid resulting in the first reaction reacts with the remaining potassium ferrocyanide and sulphuric acid, as follows: $\mathrm{H}_{4} \mathrm{Fe}_{6} \mathrm{~N}_{6}+\mathrm{K}_{4} \mathrm{Fe} \mathrm{C}_{6} \mathrm{~N}_{6}+\mathrm{H}_{2} \mathrm{~S}$ $\mathrm{O}_{4}=6 \mathrm{H} \mathrm{CN}+\mathrm{K}_{2} \mathrm{~S} \mathrm{O}_{4}+\mathrm{K}_{2} \mathrm{Fe}\left(\mathrm{Fe} \mathrm{C}_{6} \mathrm{~N}_{6}^{*}\right)$.

Diluted hydrocyanic acid can also be made by mixing hydrochloric acid, 5 ; with distilled water, 55 ; silver cyanide, 6. Shake together in a glass-stoppered bottle. $\mathrm{Ag} \mathrm{C} \mathrm{N}+$ $\mathrm{H} \mathrm{Cl}=\mathrm{H} \mathrm{CN}+\mathrm{AgCl}$. When the precipitate of silver chloride falls, pour off the clear, supernatant fluid.

Properties.-A clear, colorless liquid, of a characteristic taste and odor, resembling those of bitter almond. It is very unstable and is apt to be inert as obtained from ordinary drug stores. It should be kept in inverted glass-stoppered dark bottles.

Incompatibles.-Salts of iron, copper and silver; sulphides and red mercuric oxide.

Dose.-H. \& C., Mxx.-xl. (1.3-2.6); Sh., Mx.-xv. (.6-1.); Sw., Mii.-v. (.12-.3); D., Mi.-iii. (.06-.2).

Action External. - Prussic acid is absorbed to some extent through the unbroken skin; paralyzes the peripheral seusory nerves, aud acts as a local anæsthetic. If the finger

* Scheele's prussic acid contains 4 to 5 per cent. of the pure hydrocyanic acid. 
is held over a bottle containing the acid, it soon becomes anæsthetized. Upon mucous membranes, or raw surfaces, prussic acid is rapidly absorbed and exhibits its usual constitutional action.

Action Internal.-Alimentary Canal.-Hydrocyanic acid exerts a sedative effect upon the mucons membrane of the stomach and upper portion of the digestive tract. It is absorbed into the blood, but we are ignorant of its fate or mode of elimination.

Blood.-In poisoning, the blood becomes first of a bright arterial hue, and later assumes a dark, venous color. The first condition is due to the fact that the blood does not give up its oxygen for some reason. Brunton suggests that it is because the blood is hurried so rapidly through the dilated peripheral vessels that it does not have time to yield up its oxygen. The dark color of the blood is probably owiug to asphyxia and acsumulation of carbonic dioxide, following the paralytic action of prussic acid upon the respiratory centre. A substance called cyanolımoglobin is formed ontside the body by hydrocyanic acid when shaken with blood. The acid appears to deoxydize the normal oxyhæmoglobin, and blood thus treated has no ozonizing property. Cyanohæmoglobin was thought to account for the action of prussic acid, but it does not exist within the body in the blood of poisoned animals. The red bloor corpuscles are altered in shape by the action of prussic acil upon blood withdrawn from the ressels. They generally become rounder, then granular, and finally disintegrate and liquefy. But these changes do not occur in the blood during life. The general action of prussic acid is altogether independent of any influence upon the blood, since the same toxic effect is produced upon the bloodless, or "salt frogr" (vessels containing normal salt solution), as upon the normal batrachian.

Nervous System and Muscles.-Prussic acid has an essentially depressing action upon the nervous system as a whole. The brain, cord and nerves become paralyzed by large doses. 
The convulsions occurring in poisoning are shown by experiments to be dne probably to altered cerebral circulation, although they have been attributed to the direct influence upon the brin substance, and, to asphyxia. They are, however, present during that period of poisoning when the blood is of a bright arterial hue. The spinal cord is paralyzed at a period after coma and conrulsions have appeared. The peripheral nerves and muscles are paralyzed directly by toxic doses, and not through the mediation of the central nervous apparatus. This is proved by shutting off the blood supply containing the drug, from a frog's leg, and leaving the nervous connections intact, when no effect of prussic acid is observed upon the limb.

Heart and Blood Vessels. - Death sometimes occurs instantaneonsly from large lethal doses of prussic acid, owing to diastolic arrest of the heart. This action is due probably to paralysis of the heart and its contained ganglia, and also to irritation of the vagus centre. Moderate nontoxic doses stimulate the vagus centre of the medulla, and slow the pulse withont diminishing the force of the heart. When the vagi are previously divided, this action does not occur, but after large doses slowing of the heart is observed whether the vagi are cut or not; thus showing that the heart muscle, or its ganglia, are directly influenced. Moderate doses of prussic acid first stimulate, and then depress the vasomotor centre. Arterial pressure is therefore primarily raised considerably, but this is followed by a fall to, or below, the normal.

Toxic doses stimulate the vasomotor centre very briefly, and this action is succeeded by profound depression and paralysis of the centre, accompanied by a great diminution of blood tension.

Respiration. - Inhalation of the pure acid will cause death in a confined atmosphere, and even inhalation of the medicinal solution will induce the physiological symptoms of the drug. The respiratory centre is usually depressed from the beginning, by prussic acil, and the respiratory 
movements are therefore lessened in frequency throughout the period of its action. Rarely, there is a primary transient stimulation of the centre, so that the breathing is increased in frequency. In the latter stage of poisoning, the breathing is feeble and shallow, and only occurs at loug intervals. If death does not supervene immediately from diastolic arrest of the heart, it comes on more slowly by asphyxia. The respiratory movements become less frequent and forcible, the animal giving an occasional gasp, until finally the breathing ceases altogether, while the heart continues to beat for a time.

Summary.-Prussic acid in any considerable dose exerts a general paralyzaut action upon the system, including the brain, spinal cord, nerves, muscles, and the three great medullary centres controlling the heart, respiration and vessels. Topically applied, hydrocyanic acid also paralyzes nerves and muscles, and acts therapeutically as a local sedative and anæsthetic.

Toxicology.-Prussic acid is one of the most powerful poisons in existence. Death may be instantaneous, or life may be prolonged for over an hour after a lethal dose. More commonly the animal survives for a few minutes, and we observe the following symptoms in dogs: The animal falls, froths at the mouth, the respiration is of a gasping character and occurs at infrequent intervals. There is unconscionsuess, the pupils become dilated, there are muscular tremblings, and clonic or tonic spasms. Defrecation and micturition occur, and erections often ensue in the male. Respiration ceases before the cardias pulsations.

Three stages may be distinguished in fatal poisoning. First: a very short period elapses before the symptoms appear. There are giddiness, diffiault breathing, and slow pulse in this stage. Second: the pupils dilate, vomiting may occur, and the animal utters lond cries. Spasmodic defæcation, micturition and erections may be present, with convulsions and unconsciousness. Third: the last stage is characterized by collapse, spasms, general paralysis and 
death. The subacute form of poisoning may ensue and prove fatal, or, owing to the volatile character of the drug, complete recovery may take place within one-half or threequarters of an homr. Occasionally dogs continue to be paralyzed for several days and get well. The minimum fatal dose recorded in man is $\frac{9}{10}$ of a grain of pure acid, or abont 50 drops of the medicinal solution. Four to five drachms of the diluted acid frequently, but not invariably, canse subacute poisoning and death, in horses, within an hour. One to two drachms of the pharmacopœial preparation usually kills clogs within ten minutes.

Prussic acid is commonly used to destroy the domestic animals. Two to four drachms of the medicinal acid are to be given to dogs and cats of the ordinary size, aud certain, painless, and rapid death will occur if a fresh preparation of the drug cau be obtained. The nnopened, half-ounce vial, kept by druggists, is recommended. Big dogs, horses, and the other larger animals are not killed rapidly, nor sometimes at all, by great quantities of the diluted acid. Hence, shooting is a more humane and preferable mode of death for them. In the experience of the writer, one to two drachms of prussic acid saturated with potassinm cyanide, failed to kill a horse, when injected directly into the jugular vein. The odor of the acid lingers abont the animal for a few hours after death; the eyes are fixed and staring; the pupils dilated; the teeth are clinched tight and covered with froth, while the blood is of a very dark color. The treatment embraces emptying the stomach by large doses of promptly acting emetics, or by the stomach tube, or pump; atropine, ether, and brandy subentaneously, and inhulations of ammonia, together with artificial respiration, and hot and cold douches upon the chest.

Uses.-Prussic acid is indicated for three therapeutic purposes:

1. To relieve gastric pain and romiting, by its paralyzing action upon the peripheral sensory nerves of the stomach. 
2. To stop coughing.

3. To allay itching by means of its local, sedative action upon the cutaneous sensory nerve-endings.

It is mainly useful in veterinary practice as a cough remedy, when the symptom is of reflex origin, or is caused by chronic or verminous bronchitis; and the acid is often conjoined with chloroform, or opium, in some form. Prussic acid is a dangerous remedy to apply to the skin, as absorption may occur, or the acid may be lapped off by the patient. Solutions containing, of the diluted acid, 3 ss.-i. to $\bar{j}$. of water, are, however, sometimes employed to relieve pruritus.

Potassi Cyanidum. Potassium Cyanide. $\mathrm{K} \mathrm{C} \mathrm{N.}$

$$
\text { (U. S. \& B. P.) }
$$

Synonym.-Cyanure de potassium, Fr.; cyankalium, G.

Derivation.-Made by heating together potassium ferrocyanide and carbonate.

Properties.-White, opaque, amorphous pieces; or a white, granular powder, odorless when perfectly dry, but in moist air exhaling the odor of hydrocyanic acid. Taste sharp and alkaline, and in moist air the salt deliquesces. Reaction very strongly alkaline. Solutions stain and destroy clothing. Soluble in about 2 parts of water; sparingly soluble in alcoliol.

Dose.-H., gr.i.-ii. (.06-.12); D., gr·立.(.006).

Action and Uses.-Potassium cyanide is transformed, in the stomach and blood, into prussic acid, and resembles the latter in its action, but is much slower. Death has been caused in man by 5 grains of the salt.

\section{Argenti Cyanidum. Silver Cyanide. Ag C N.}

A white, insoluble, tasteless, odorless powder, used for making prussic acid. 
Prunus Virginiana. Wild Cherry. (U. S. P.)

Synonym.-Écorce de cerisier de Virginie, Fr.; wildkirschenrinde, G.

The bark of the wild cherry, indigenons in the United States, contains a ferment (emulsin) which, in the presence of water, acts on a glucoside (amygdalin, $\mathrm{C}_{20} \mathrm{H}_{27} \mathrm{~N} \mathrm{O}_{11}$ ) contained in the bark, with the formation of hydrocyanic acid, glucose and a volatile oil. A fluid extract, infusion and syrup of prunus virginiana are official. The latter preparation is sometimes employed in cough mixtures for dogs, on account of its sedative action. The entire value of the drug depends upon the minute amount of prussic acid formed in it. The official hydrocyanic acid is more reliable, but syrupus pruni virginianæ (U.S.P.) may be used as a vehicle for more efficient remedies.

\section{Petrolatum. (U. S. P.) \\ (Threo varieties.)}

1.--Petrolatum Liquidum. Liquid Petrolatum. (U. S. P.)

Derivation.-A mixture of hydrocarbons, chiefly of the marsh-gas series, obtained by distilling off the lighter and more volatile portions from petroleum, and purifying the residue when it has the desired consistence.

Properties. - A colorless, or more or less yellowish, oily, transparent liquid, without odor or taste; or giving off, when heated, a faint odor of petroleum. Spec. gr. about 0.875 0.945. Insoluble in water; scarcely soluble in cold or hot alcohol, or cold absolute alcohol; but soluble in boiling absolute alcohol, and readily soluble in ether, chloroform, carbon disulphide, oil of turpentine, benzin, benzol, and fixed and volatile oils.

2.-Petrolatum Molle. Soft Petrolatum. (U. S. P.)

Synonym.-Vaseline, cosmoline.

Derivation.-A mixture of hydrocarbons, chiefly of the 
marsh-gas series, obtained by distilling off the lighter and more volatile portions from petroleum, and purifying the residue when it has reached the desired melting point. When petrolatum is prescribed or ordered without further specification, soft petrolatum is dispensed.

Properties.-A fat-like mass of about the consistence of an ointment, varying from white to yellowish or yellow; more or less fluorescent when. yellow, especially after being melted; transparent in thin layers, completely amorphons, and without odor or taste; or giving off, when lieated, a faint odor of petroleum.

The melting point of soft petrolatum ranges about $40^{\circ}$ and $45^{\circ} \mathrm{C}$. (104 ${ }^{\circ}$ and $113^{\circ} \mathrm{F}$.). In other respects soft petrolatum has the characteristics of liquid petrolatum.

\section{3.-Petrolatum Spissum. Hard Petrolatum. (U. S. P.)}

Derivation.-A mixture of liydrocarbons, chiefly of the marsh-gas series, obtained by distilling off the lighter and more volatile portions from petroleum, and purifying the residue when it has reached the desired melting point.

Properties.-A fat-like mass of about the consistence of an ointment, varying from white to yellowish or yellow; more or less fluorescent when yellow, especially after being melted; transparent in thin layers, completely amorphous, and without odor or taste; or giving off, when heated, a faint odor of petrolem. The melting point of hard petrolatum ranges about $45^{\circ}$ and $51^{\circ} \mathrm{C}$. $\left(113^{\circ}\right.$ and $125^{\circ} \mathrm{F}$.). In other respects hard petrolatum has the characteristics of liquid petrolatum.

Action and Uses.-Petrolatum is a valuable emollient. It soothes, protects and softens parts to which it is applied, and is superior to animal and vegetable fats and oils in not becoming rancid, and therefore irritant and malodorous. Petrolatum may be used alone, or as an excipient in the preparation of ointments, but does not aid the absorption of drugs (as do alcohol, glycerin, chloroform, and animal oils 
and fats), for it is not itself absorbed even when administered internally. Petrolatum exerts a demulcent action upon the mucous membrane of the alimentary tract, and may be prescribed in electuary or capsule in inflammation thereof. Petrolatum is sold universally under the proprietary names of vaseline and cosmoline, and is often combined with antiseptics for medicinal and surgical purposes in skin diseases and upon inflamed mucous membranes, blistered and abraded surfaces and sores. It is one of the most useful agents in lubricating instruments, protecting metal from rust, preserving leather, and is sometimes employed as a vehicle for electuaries.

\section{Rhigolene. (Non-official.)}

Rhigolene is a petroleum product prepared by repeated distillation until the liquid boils at $64.4^{\circ} \mathrm{F}$. It evaporates at a lower temperature than any other substance, except cymogene, and is employed as a spray to induce numbness and local anæsthesia of a part in minor surgical operations, such as paracentesis of an abscess or the use of the actual cautery. 


\title{
PART II.
}

\section{VEGETABLE DRUGS}

\section{SECTION I.-DRUGS ACTING UPON THE BRAIN.}

\section{Class 1.-Depressing the Brain.}

\author{
Opiur. Opium. (U. S. \& B. P.)
}

Derivation.-The concrete, milky exudation obtained by incising the unripe capsules of Papaver somniferum Linue (Nat. ord. Papaveraceæ), and yielding in its normal, moist condition, not less than 9 per cent. of crystallized morphine, when assayed by the official process (U.S. P.) Opium is procured from Turkey, Asia Minor, Persia, India and Egypt. The Smyrna, or Turkey opinm is the more common variety used in the United States. It occurs in irregular, globular masses, covered with poppy leaves and eapsules of a species of dock, weighing from $\frac{1}{2}$ to 1 pound.

Properties.-Irregular, or sub-globular cakes, with the remuants of poppy leaves and fruits of a species of rumex adhering to the surface; plastic, or of a harder consistency; chestnut-brown or darker, and somewhat shining; internally showing some tears and fragments of vegetable tissue. It has a sharp, narcotic odor, and a peculiar, bitter taste. It yields its medicinal properties to water, alcohol, and diluted acids, forming dark brown solutions. Ether extracts its principles in part.

Constituents. - There are nineteen or more alkaloids; the three first are used in human medicine, but narceine is of no value in veterinary medicine.

\begin{tabular}{|c|c|c|c|c|c|}
\hline Morphine. & $2.5-22.8$ & per cent. & Thebaine. & $.15-1$ & per cent. \\
\hline Codeine. & $.2-\quad .7$ & 66 & Narcotine. & $1.3-10$. & 66 \\
\hline Narceine. & $.1-.7$ & " 6 & Papaverine. & 1. & 66 \\
\hline
\end{tabular}


In addition to these, the following exist in minute quantity, but some are merely "pharmaceutical curiosities":-

Protopine.

Cryptopine.

Oxynarcotine.

Hydronarcotine.

Laudanosine.

Laudaine.

Phoeadine.

Codamine.

Meconodine.

Gnoscopine.

Lanthopine.

Water. 15.-25, per cent.

Neutral Bodies.

Meconin.

Meconoisin.

Impurities.-Starch, molasses, leaves, fruit, stones and water.

Incompatibility.-Solutions of lead acetate and subacetate, and of copper and arsenic salts, precipitate meconates, sulphates and coloring matters, but the opium remains physiologically active. Ferric chloride produces a deep red color with opium, by its union with meconic acid. Tannin compounds precipitate codeine tannate. Alkalies, their carbonates and ammonia precipitate morpinine and narcotine.

Dose.-H., 3 i.-ii. (4.-8.) ; C., 3 ii.-iv. (8.-15.) ; Sh., gr.x.ххx. (.6-2.); Sw., gr.v.-xx. (.3-1.3); D., gr.ss.-iii. (.03-.2).

\section{OpiI Pulvis. Powdered Opium. (U. S. P.)}

Opium dried at a temperature not exceding $85^{\circ} \mathrm{C}$. $\left(185^{\circ}\right.$ F.), and reduced to a fine powder. Powdered opium, for pharmaceutical or medicinal purposes, when assayed should yield not less than thirteen (13) nor more than fifteen (15) per cent. of crystallized morphine. Any powdered opium of a higher percentage may be brought within these limits by admixture with powdered opium of a lower percentage in proper proportions. Powdered opium is the base of all the other pharmacopœial preparations of opium. 
Only those are mentioned here which are applicable to veterinary practice.

Dose.--Same as for opium, but preferable to the crude drug.

\section{PREPARATIONS.}

Extractum Opii. Extract of Opium. (U. S. \& B. P.)

Powdered opium. 100 istilled water, 1000 ; sugar of milk, a sufficient quantity. Wrade by trituration, filtration, and evaporation. (U. S. P.)

Dose.-About one-half that of powdered opium. H., z ss.-i. (2.-4.); C , 3 i.-ii. (4.-8.) ; Sh., gr.v.-xv. (.3 1.); Sw., gr.ii.ss.-x. (.15-.6); D., gr. $1 / 4^{-i . s s . ~}(.015-.09)$.

Pulvis Ipecachuanhce et Opii. Powder of Ipecac and Opium. (U. S. P.)

Synonym.-I)over's powder, E. ; pulvis ipecacuanbæ opiatus, s. pulvis Doweri, P. G.; poudre de Dower, Fr.; Dower'sches pulver, G.

Ipecac, 10 ; powdered opium, 10 ; sugar of milk, 80 . The most diarphoretic compound of opium.

Dose.-H., 5 ss.-i. (15.-30.); D., gr.iii.-xv. (.2-1.).

Tinctura Ipecacuanhe et Opii. Tincture of Ipecac and Opium. (U. S. P.)

Synonym.-Liquid Dover's powder.

Tincture of deodorized opium, 100; fluid extract of ipecac, 100; diluted alcohol, a sufficient quantity to make 1000 . Made by evaporation and filtration.

Dose.-Same as Dover's powder.

Tinctura Opii. Tincture of Opium. (U. S. \& B. P.)

Synonym.-Laudanum, tinctura meconii, tinctura thebaicæ, E.; tinctura opii simplex, P. G.; teinture d'opium, teinture thébaïque, Fr.;. einfache opiumtinktur, G.

Powdered opium, 100 ; alcohol, 400 ; water, 400 ; diluted alcohol. to make 1000 . Made by trituration, maceration with precipitated calcium phosphate, and percolation. (U. S. P.)

Dose.-H., zुi.-ii. (30.-60.); C., ₹ zii.-iii. (60.-90.); Sh. \& Sw.. 3 ii.-vi. (8.-24.); D., miii.-xx. (2-1.3).

Tinctura Opii Camphorata. Camphorated Tincture of Opium.

(U. S. P.)

Synonym.-Tinctura camphoræ composita, B. P.; paregoric, elixir paregoricum, paregoric elixir, E.; tinctura opii benzoica, P. G.; élixir parégorique, Fr.; benzöesaurehaltige opiumtinktur, G. 
Powdered opium, 4 ; benzoic acid, 4 ; camphor, 4 ; oil of anise, 4 ; glycerin, 40; diluted alcohol to make 1000. Made by maceration and filtration. (U. S. P.)

Dose.-D., z i.-iv. (4.-15.).

Opium Deodorutum. Deodorized Opium. (U. S. P.)

Synonym.-Opium denarcotizatum.

Powdered opium (containing 13-15 per cent. of morphine), 100 ; ether, 1400 ; sugar of milk to make 100 Made by repeated maceration and agitation with ether; evaporation and trituration with sugar of milk. The ether removes narcotic and odorous principles, which cause nausea and disagreeable after-effects in opium:

Dose.-Same as powdered opium.

Tinctura Opii Deodorata. Tincture of Deodorized Opium. (U. S. P.)

Powdered opinm, 100 ; ether, 200 ; alcohol, 200 ; water to make 1000. Made by trituration and maceration with precipitated calcium phosphate, 50 ; percolation and evaporation.

Dose.-Same as tincture of opium, but less nauseating.

Extractum Opii Liquidum. (B. P.)

(Gr. 22 extract of opium to $z i$.)

Dose.-Same as laudanum.

Vinum Opii. (U. S. \& B. P.)

Dose.-Same as laudanum.

Morphina. Morphine. $\mathrm{C}_{17} \mathrm{H}_{19} \mathrm{~N} \mathrm{O}_{3}+\mathrm{H}_{2} \mathrm{O}$.

(U. S. \& B. P.)

Derivation.-An alkaloid obtained from opium.

1. Macerate opium in cold water, forming a solution of morphine meconate. 2. Add calcium chloride to precipitate calcium meconate and resins. 3. Evaporate solution remaining, which contains morphine hydrochlorate, till it crystallizes; press in flannel to remove narcotine and coloring matter; redissolve; filter; evaporate and crystallize repeatedly. 4. Decolorize by digestion with charcoal. 5. Precipitate with ammonia and wash, when pure morphine is separated from codeine.

Properties.-Colorless or white, shining prismatic crystals, or fine needles, or crystalline powder; odorless and 
having a bitter taste; permanent in the air ; soluble in 4,350 parts of water.

Dose.-Same as salts of morphine, but the latter are preferable on account of their solubility.

Morphinz Hydrochloras. Morphine Hydrochlorate.

$$
\mathrm{C}_{13} \mathrm{H}_{19} \mathrm{~N} \mathrm{O}_{3} \mathrm{H} \mathrm{Cl}+3 \mathrm{H}_{2} \text { O. (U. S. \& B. P.) }
$$

Derivation. - Morphine is stirred with hot distilled water, to which hydrochloric acid is gradually added. Morphine hydrochlorate crystallizes ont on cooling.

Properties.-White, feathery needles of a silky lustre; or minute, colorless, needle-shaped crystals; odorless and having a bitter taste. Permanent in the air. Soluble in 24 parts of water and in 62 parts of alcohol. Very slightly soluble in ether or chloroform.

Incompatibility.-Incompatible with all agents containing tannin, alkaline carbonates, lime water, salts of copper, mercury, zinc and lead; and with Fowler's solution of arsenic.

Dose.-H. \& C., gr.iii.-x. (.2-.6); Sh., gr.ss.-ii. (.03-.12); Sw., gr. $\frac{1}{10}-\frac{1}{2}(.006-.03)$; D., gr. $\frac{1}{\frac{1}{3}-\frac{1}{2}}(.008-.03)$. $.02)$.

Subcutaneously.-H., gr.iii:-iv. (.2-.24); D., gr. $\frac{1}{8}-\frac{1}{3}$ (.008.-

124 parts of morphine hydrochlorate correspond to 100 parts of morphine.

Morphine Acetas. Morphine Acetate.

$$
\mathrm{C}_{17} \mathrm{H}_{19} \mathrm{~N} \mathrm{O}_{3} \mathrm{C}_{2} \mathrm{H}_{4} \mathrm{O}_{2}+3 \mathrm{H}_{2} \mathrm{O} \text {. (U. S. \& B. P.) }
$$

Derivation.-Morpline is dissolved in acetic acid and water, and the solution evaporated and crystallized.

Properties.-A white, or faintly yellowish-white, crystalline, amorphous powder, having a faint, acetous odor aud bitter taste. It slowly loses acetic acid on exposure to the air, and should be kept in dark, amber-colored, well-stoppered vials. Soluble, when freshly prepared, in 2.5 parts of water, and in 47.6 parts of alcohol.

Dose.-Same as morphine hydrochlorate. 
Morphine Sulphas. Morphine Sulphate. $\left(\mathrm{C}_{17} \mathrm{H}_{19} \mathrm{~N} \mathrm{O}_{3}\right)_{2} \mathrm{H}_{2} \mathrm{~S} \mathrm{O}_{4}+5 \mathrm{H}_{2} \mathrm{O}$. (U. S. \& B. P.)

Derivation.-Morphine is stirred into boiling distilled water; diluted sulphuric acid is added until neutralization is attained, and the sulphate crystallizes out on cooling.

Properties. - White, feathery, acicular crystals of a silky lustre; odorless and having a bitter taste. Permanent in the air. Soluble in 21 parts of water, and in 702 parts of alcohol.

Dose.-Same as hydrochlorate. 125 parts of morphine sulphate correspond to 100 parts of the pure alkaloid.

The official salts of morphine may be used interchangeably. The acetate is more soluble, but less stable, than the sulphate, which is sufficiently soluble for practical purposes, and is in more common use.

Liquor Morphin mydrochloratis. (B. P.) (Gr. $4 \frac{1}{2}-\overline{3}$ i.)

Dose.-H., 3 vi.- 3 ii. (24.-60.); D., Mx.- 3 i. (.6-4.).

Liquor Morphine Acetatis. (B. P.)

Same strength and dose as above.

Injectio Morphine Hypodermica. (B. P.)

(Gr.i. morphine acetate in $m_{x}$.)

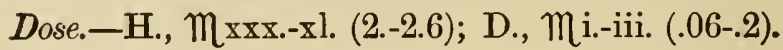

Suppositoria Morphinж. (B. P.)

(G1: $\frac{1}{2}$ morphine.)

Dose.-Dog, 1 per rectum.

Codeina. Codeine, $\mathrm{C}_{18} \mathrm{H}_{21} \mathrm{NO}_{3}+\mathrm{H}_{2} \mathrm{O}$. (U. S. \& B. P.)

Derivation. - Án alkaloid obtained from opium by evaporation of the ammoniacal liquid, after the precipitation of 
morphine. The residue is added to water, precipitated by potassium hydrate, and redissolved in ether, from which codeine crystallizes ont on evaporation.

Properties. - White, or nearly transparent, orthorhombic prisms, or octohedral crystals; odorless, and having a faintly bitter taste; slightly efflorescent in warm air. Soluble in 80 parts of water, and in 3 parts of alcohol; also soluble in 30 parts of ether and in 2 parts of chloroform.

Dose.-D., gr. $\frac{1}{4}$-ii. $(.015-.12)$.

\section{HeroIN.}

\section{(Diacetylmorphine.)}

This remedy, derived from morphine, was first introduced by Dreser, in 1898, and is now used extensively in human medicine as a substitute for morphine and codeine. Heroin occurs as a white, odorless, crystalline powder, possessing a slightly bitter taste and alkaline reaction. Practically insoluble in water, but readily soluble in weak acidulous solutions.

Heroin hydrochloride is a white, crystalline, odorless powder, soluble in 2 parts of water. Heroin surpasses both morphine and codeine therapeutically in many ways. It increases markedly the inspiratory and expiratory force, while lessening the number of the respiratory movements, and exerts a special sedative influence on the respiratory mucous membranes. The drug acts also as a general motor depressant hypnotic and analgesic, but is not comparable to morphine in these respects. The after-effects of small medicinal doses (nausea, constipation, etc.) are slight. Heroin is particularly valuable in the treatment of all varieties of cough affecting the human subject, and should prove useful in canine practice.

Heroin may be administered in powder, pill or tablet, the hydrochloride in solution, every few hours.

The dose of either is, for the dog, gr $\frac{1}{24}-\frac{1}{6}(.0025-.01)$. 


\section{OpIUM AND Morphine.}

The action of morphine and opium is practically the same, with some exceptions to be noted.

Action External.-Opium may be absorbed to a slight extent from the unbroken skin, and causes a mild, anodyne action. Absorption readily occurs from mucous membranes and raw surfaces, with resulting characteristic effects.

Action Internal.-Digestive Tract.-Opium diminishes the two principal activities of the digestive organs, namely, secretion and motion. Secretions all over the body are decreased, except that of sweat. The action upon the alimentary tract in lessening secretion, is partly a local one and partly constitutional, following the absorption of the drug. The mouth is made dry, thirst is increased and appetite impaired. Opium is absorbed rather slowly from the stomach and bowels, and stimulates the splanchnic nerve centre of the sympathetic system, which inhibits the movements of the stomach and intestines, and thus lessens peristaltic action of these organs. Opium is directly opposed to belladomna in this respect, as the latter drug paralyzes the intestinal inhibitory apparatus (splanchnic endings), and so increases peristalsis.

In diminishing both secretion and motion, opium causes constipation in health, but is most useful in relieving vomiting and diarrhoea. In toxic doses, opium may induce diarrhœa from paralysis of the splanchnic inhibitory centre.

Blood and Elimination. - Morphine circulates in the blood as such. The greater part of opium is decomposed in the body, a portion being destroyed by the liver, while some is probably burned up in the blood. The smaller part is eliminated, chiefly by the kidneys, but also in the other secretions. It is found in the gastric juice after hypodermic injection, and therefore washing out the stomach assists elimination and recovery in opium poisoning.

Nervous Syslem.-The most important action of opinm is exerted upon the nervous system. It is necessary to 
study the drug from the comparative standpoint in order to obtain a full understanding of its effects. The brain of man, being more highly developed and sensitive, in comparison with other parts of the nervous system, than the brain of the lower animals, it follows that this organ is more powerfully influenced in man, while the spinal cord is often mainly impressed in the lower animals.

We may take the action of opium on the frog, at one end of the scale, as exhibiting the most active spinal symptoms; while in man, at the other end of the scale, cerebral phenomena predominate. The other animals occupy an intermediate position; the action upon the horse and rumi. nants is something between that exerted upon the frog and man, and the influence upon dogs approaches more nearly that seen in human beings, only that a relatively greater dose is required to produce the same result, as the brain is not so highly organized or sensitive to the action of medicines. The brain of the horse is only one-twelfth as large, in proportion to their respective body-weights, as that of man, and it follows that the spinal cord of the horse is more readily affected by opium, in accordance with the general law that the more highly developed a part is, the more easily is it influenced by therapentic agents.

Opium exerts first a stimulating, and then a depressing action upon the brain and spinal cord, and in studying the action comparatively it will be noted that the influence upon the cord in the frog, horse, ruminant, and to some extent in the dog, preponderates frequently over the effect of the drug upon the brain, for the reasons stated above.

Action Upon the Frog.-In minute non-poisonous doses, sleep is produced, followed by a period of reflex excitement. Toxic doses of one or two grains of morphine, injected under the skin, cause at first a condition where convulsions occur, if the animal is artificially irritated; later they come on spontaneously. This state is followed by general paralysis, respiratory failure and death. The convulsions are shown to be due mainly to irritation of the spinal reflex centres, 
partly to action upon the motor tract of the lrain, and finally proceed from direct local stimulation of the motor nerve endings and muscles.

Action Upon Horses.-Three grains of morphine, injected subcutaneously, occasion sometimes drowsiness, and at other times produce no visible effect. Four to six grains, given in the same way, canse restlessness, a rapid pulse, aud moisture of the skin. The animal paws the ground and walks in a rhythmical manner abont the stall. The pupils are dilated. Large doses (12 grains) are followed by increased excitement aggravated by noises, sweating, ptyalism, muscular rigidity, statgrering gait, trembling and delirium; while still larger dose's (four drichms of the extract of opium) cause violent trembling, convulsions, insensibility to pain and exterual irritation, without coma; or (morphine, gr. 36 under the skiu), rarely, stupor for several hours (3 hours), dilated pupils and blindness, followed by delirium and restlessness, continuing for a longer time ( 7 hours) and ending in recovery. Horses have recovered from an ounce of opium, but $2 \frac{1}{2}$ ounces of the drug, and 100 grains of morphine have proved fatal. The action of opium upon the horse differs from that upon man and dogs in the more frequent occurrence of restlessness and motor excitement due to stimulation of the cerebral and spinal motor centres; and in dilatation instead of contraction of the pupil. The rationale of the latter phenomenon has not been discovered.

Action Upon Ruminants.-These animals are comparatively insusceptible to opium. Ounce doses of the drug cause, in cattle, restlessness, excitement, hoarse bellowing, dry mouth, nausea, indigestion and tympanites. Sheep are affected in much the same mauner. One to two drachins of morphine have led to fatality in cattle. Fifteen to thirty grains of the alkaloid comprise a lethai dose for sheep: Swine are variously influenced; sometimes excited, sometimes dull and drowsy.

Action Upon Birds.-Birds, as represented by chickens, ducks and pigeons, are exceedingly insusceptible to opium. 
Pigeons cannot be given enough opinm liy the month to cause death, but 8 to $10 \mathrm{grs}$. of morphine per orem, or 2 or 3 grs. hypodermatically, will prove fatal. The toxic symptoms in birds are exhibited by unsteadiness, difficult breathing and failure of respiration, convulsions and death. The pupils are unaffected, and sleep does not ensue.

Action Upon Dogs.-When 8 or 10 grains of morphine are given to a moderate sized log, coma comes ou, from which the animal may recover. One half a grain injected under the skin of a dog weighing $25 \mathrm{lbs}$, canses nausea, vomiting and perhaps purging, sleep deepening into coma, contracted pupils, and shallow breathing; the condition Jasting for several hours and followed by recovery. Opinm rarely exerts an hypnotic action upon cats, but rather motor excitement. Lethal doses (average, 4 grains of morphine sulphate subcutaneously to the pound, live weight, for dogs; 2 to 3 grains sometimes kill small dogs), increase the frequency of the pulse, cause vomiting, unsteadiness, contracted pupils, motor excitement, as twitching of the limbs and convulsions, followed by coma, respiratory failure and death. Recovery from full doses of opium is accompanied in dogs by general physical and mental depression and lassitnde, as in man. There are muscular weakness, loss of natural spirit, timidity, and nausea, lasting for several hours. The action of opinm upon dogs differs from that upon man only in degree. The dose required is proportionately larger. There is often more preliminary excitement and symptoms of reflex irritation, as muscular twitching. These animals do not sweat, and the pupils are not so continuously contracted in poisoning. Failure of the drug to produce sleep, and the presence of nausea, retching, dreams, delirium, hallucinations, occasionally observed in dogs, are common to man. Convulsions rarely occur in either men or dogs.

Action Upon Man.-In man, a small dose of morphine $\left(\frac{1}{8}\right.$ gr.) causes nsually a sense of well-being, together with itching of the nose, and later, of the skin generally, dryness of the mouth (occasionally there may be nausea, vomiting 
and faintness), followed by sleep, or a pleasant, dreamy state. After-effects may be absent, or consist of nausea, headache, coated tongue and constipation. If the dose is larger, sleep comes on quickly, the pupils are contracted, the respiratory movements and pulse become slow, and the skiu is moist.

With lethal doses, sleep deepens in to coma, from which the patient can at first be aroused; the coma becomes profound, the pulse feeble and rapid, the respiration stertorous, slow and imperfect. The mucons membranes are cyanotic, the face livid, the pupils dilate, and the surface is covered with clammy sweat. Death occurs from respiratory failure, occasionally preceded by convulsions. One-eighth of one grain of morphine is the smallest fatal human dose recorded. The action of opium upon man, as compared with that upon the horse and ruminants, is characterized by its predominant depressing effect upon the higher mental functions. The motor centres of the brain and cord are only slightly influenced.

General Action of Opium Upon the Nervous System.-The action of opium upon the nervous system may be summarized in primary central stimulation, followed by clepression and paralysis. In man and the dog, the cerebral depression is more prominent; in the horse, the stimulant action upon the motor centres of the brain and spinal cord is more marked; while considerable depression only appears in the later stage of poisoning.

Opium illustrates the law of dissolution in the order of its action, i. e., in the more highly organized functions being the first to succumb; while the lower centres are the last to be influenced. Stimulation of the cerebrum is exhibited by exalted intellectual power in man; by motor excitement in animals. This stage, comparatively short in man and dogs, is succeeded by depression of the intellectual functions and, to a less extent, of the cerebral motor centres. Depression is exhibited by sleep and insensibility to sound, light, external irritation and pain. Relief of suffering often occurs without sleep, and is due to the depressing action of the 
drug upon the brain, and not to any direct influence upon the sensory nerves, which are unaffected except in the later stage of poisoning. The unique and inestimable value of opium depends upon its anodyne action. Depression of the brain in relation to the cortical centres, is not sufficient to cause paralysis in man, except in poisoning. Muscular weakness is present in man and dogs, but even this evidence of depression may be absent in horses and ruminants, yet. pain be effectually relieved. Contraction of the pupil, in man and dogs, is due to stimulation of the oculomotor nerve, probably through excitation of the pupillary centre.

Dilatation, which occurs in poisoning, is due to paralysis of this centre. Initial stimulation of the vomiting centre may cause emesis; but, as depression of the centre rapidly ensues, the act becomes later improbable. The motor tract of the spinal cord is first stimulated and reflex excitability increased. This action, brief and slight in men and dogs, is more prolonged and prominent in horses and ruminants. Depression of the spiual motor cells succeeds excitation, and reflex action is abolished. The excitability of the motor and sensory nerves is slightly increased, but otherwise the nerves are not affected except in poisoning, when the sensory, and later the motor nerves are paralyzed. The muscles remain uninfluenced. Depression of the medulla closes the scene, with paralysis of the respiratory centre.

Respiration.-Opium does not influence the respiratory functions in small therapeutic doses, but large doses make the respiratory movements slower and feebler, and death occurs from the direct depressing and paralyzing action of the drug upon the respiratory centres in the medulla.

Circulation.-Small doses commonly produce little effect upon the heart. Large doses first increase the force and frequency of the heart's action, while toxic quantities depress the orgau. This result is due to a two-fold action upon the circulation, including the heart muscle (or its ganglia) and the inhibitory apparatus. The cardiac muscle is primarily 
stimulated, with acceleration of the pulse, but depression soon follows more or less synchronously with stimulation of the vagus centre and endings, so that the pulse becomes iufrequent. Finally, before deatl, depression of the inhibitory apparatis occurs, and this, coexisting with depression of the lieart itself, produces a feeble, rapid pulse, clraracteristic of the last stage of opium poisoning. Death occurs with diastolic arrest of the heart owing to failure of the cardiac muscle, although fatality is mainly due to the more powerful effect of the drug upon the respiratory centre.

The action of opium upon the vasomotor system is unimportant. Immediately after the administration of large doses there is a slight primary stimulation, followed in the toxic stage by some depression of the vasomotor centres in the spinal cord and medulla.

Pupil.-The pupil of the horse is widely dilated by large doses of opium. The pupil in the dog occasionally remains unchanged, and often dilated before undergoing contraction. Contraction of the pupil is a charactistic physiological effect of large doses of opium in man and the dog. In birds the pupil is unaffected. These varions contradictory phenomena are at present inexplicable.

In man, contraction of the pupil is brought about by stimulation of the pupillary centre in the floor of the aqueduct of Sylvius, and through it, the oculomotor nerves. Dilatation, preceding death, occurs from depression of the centre.

Kidneys and Metabolism.-The excretion of urea appears to be increased by opium in man, but varies greatly in animals. Temporary retention of urine may follow the administration of a considerable dose of opium, owing to diminished sensibility of the bladder. The amount of urine voided may be greater or less than normal; more commonly the latter. Opium lessens the secretion of bile.

The elimination of carbonic dioxide is diminished by the hypnotic action of opium, but is increased if there is 
general excitement and muscular activity following the use of the drug.

Skin.-Opium induces mild diarphoresis in man; occasionally sweating occurs in horses, but not at all in dogs.

Temperature.-The bodily temperature may be slightly increased by large medicinal doses of opium, but is diminished by toxic quantities.

Toxicology.-The symptoms of poisoning have already been snfficiently described in previous sections. The treatment embraces irrigation of the stomach, or the use of emetics, as apomorphine hydrochlorate under the skin, and the subcutaneous injection of strychuine, enemata of hot, strong, black coffee; leading the animal about, slapping him, or using the faridic current. Dr. Moor, of New York, has apparently found in potassium permanganate the most efficient antidote for opinm and morphine. Ten to fifteen grains, dissolved in eight ounces of water, should be given by the mouth, to large dogs. One to two drachms of potassium permanganate may be administered to horses in two or three pints of water. Permanganate solution oxidizes and destroys morphine, and should be acidulated with a little vinegar or diluted sulphuric acid, after the ingestion of morphine salts. The antidote has been recommended to be given subcutaneously after absorption, or hypodermic injection of morphine, but this is not of the slightest use.

Morphine Contrasted with Opium.-Opium is more constipating, more sudorific, and more apt to disturb the digestion than morphine. Morphine is more anodyne and soporific; more readily absorbed and more suitable for use under the skin.

Synergists.-Belladonna aids the action of opium and yet is antagonistic to it. It assists opium in its anodyne action and lessens nausea, indigestion, and constipation following the action of the latter drug. Belladonna and atropine are antagonistic to opium in stimulating the brain and respiration, and increasing peristalsis. Small doses of 
belladonna combined with opium do not interfere with the soporific action of the latter, notwithstanding the exciting influence of the former upon the brain. The bromides also promote the sedative and hypnotic action of opium, and lessen the depression and nausea following its administration. Opium often acts profoundly when combined with chloral, and this combination is occasionally used to induce anæsthesia (p. 301). Chloroform and ether are frequently prescribed with opium to secure an additional antispasmodic and anodyne action in colic. The astringents and mineral acids enhance the effect of opium in diarrbœal disorders.

Codeine.-Codeine in large doses causes motor excitement and convulsions in dogs and cats, but physiological experiments conducted by various observers have otherwise yielded diverse results. This is explicable, since so-called codeine is frequently in part morphine. Codeine is inferior to morphine as an anodyne and hypnotic, but is a useful sedative in relieving bronchial irritation and cough, and in the doses commonly employed does not induce indigestion, nausea or constipation. The writer would particularly recommend it for dogs suffering with bronchitis, combined with phenacetin in powder or tablet. The other alkaloids of opium are not of sufticient therapeutic value to warrant their consideration in this work.

Administration.-Morphine sulphate is employed under the skin where an immediate effect is required. The preparations more frequently used in veterinary practice include laudanum and the deodorized tincture, powdered opium, extract of opium, and the salts of morphine. One-quarter grain of the latter is equivalent to one grain of opium. Paregoric is useful in canine practice for cough mixtures. Dover's powder combines the expectorant and diarphoretic action of ipecac with the sedative, antiphlogistic and sudorific influence of opium, but the former drug has little effect upon the horse. The preparation may be serviceable, however, in the first stage of catarrhal affections of the respirarory tract in dogs. 
Fluid preparations are generally desirable in securing more rapid absorption, but opium produces the best result in diarrhœa, when given in pill or ball. Enemata composed of thin, boiled starch solution and laudanum at the body temperature are to be recommended in diarrhœea of the young, dysentery and pain arising from strangury or disease of the genito-urinary organs. Opium suppositories are of value in canine practice for the same purposes, and will relieve irritation and pain caused by piles.

Uses External.-Opium is applied externally in various ways. In the form of laudanum it is sprinkled on poultices and prescribed in liniment (laudauum and soap liniment, equal parts) for its auodyne action, but has probably little medicinal virtue upon the unbroken skin. On raw surfaces, sores and ulcers, opium does relieve pain, and for this purpose laudanum may be conjoined advantageously with lead water (1-25).

\section{INDICATIONS FOR THE INTERNAL USE OF OPIUM.}

1. To relieve pain and spasm.

2. To lessen secretions.

3. To allay motor excitement, diminish muscular action, and prevent hemorrhage.

4. To abort inflammation.

5. To act as a stimulating and supporting agent.

The sudorific action of the drug upon the lower animals is slight and comparatively unimportant. Among the preceding indications the first three naturally follow from the physiological action of opium, while the latter two are deduced solely from clinical experience.

1. Although the anodyne aud sedative action of opium is not so marked in its influence upon veterinary patients as in human subjects, yet it is by far the most valuable agent we possess for relieving pain of any description, particularly when combined with atropine. In spasmodic colic of horses, opium arrests pain by preventing irregular and violent peristaltic action. It may be given as morphine 
(with atropine), subcutaneously; or as laudanum, with ether and chloroform in a drench, simultaneously with an aloes ball. In this affection opium actually assists the action of the purgative by overcoming spasm. Pain directly antagonizes the effect of opium, and repetition of the dose is both justifiable and necessary until relief is obtained. Hypodermic medication is therefore safer when the dose has to be repeated, in enabling the practitioner to decide that failure to relieve pain is due to insufficient dosage rather than to delayed absorption from the digestive canal.

Opium is indicated in all forms of pain, unless it be caused by congestion or inflammation of the brain substance (cerebritis). The pains and spasmodic contractions resulting from acute or tranmatic meningitis are benefited by opium; also neuralgic and rheumatic pains. The afterpains of parturition are relieved by opium in causing relaxation of the unstriped uterine muscle. The spasms of tetanus are eased when opinm is combined with chloral in enema, or when morphine is injected under the skin. Clonic spasm of the diaphragm in horses ("Thumps") is also treated successfully with opium.

2 and 3. Opium is invaluable in lessening secretion, motion and pain in various digestive disorders. Gastric digestion is iuhibited by the action upon secretion and motion, and opium should not be administered immediately after the ingestion of food, unless the demand for it at that. time is imperative.

Excessive romiting in dogs may be combated with opium and bismuth, or with morphine hypodermatically. Opium quiets peristalsis and secures rest of the canal in gastritis and gastro-enteritis. In superpurgation and in all forms of diarrhœa and dysentery, opium is the remedy par excellence. (Landanum in dose of 5-10 drops for large birds is an efficient remedy for diarrhœe in poultry). Its administration in these disorders should be accompanied, or preceded, by an oleaginons (horse), siline (herbivora), or mercurial (horse and dng) purgative; and its action may be assisted 
by astringents, alkalies, mineral acids, and intestinal antiseptics in various combinations suited to the particnlar case.

Peritonitis, enteritis, and acute obstruction of the bowels are treated most successfully with opium, which quiets the bowels, relieves pain and facilitates the vis medatrix naturæ, besides acting as an antiphlogistic in the first-mentioned diseases. By preventing muscular activity and allaying general excitability, opinm is the most effective hæmostatic in all internal hemorrhages.

Cough, as a symptom of irritation within the respiratory tract, is more commonly treated by some form of opium than by any other drug. When cough is irritative or excessive, and is not remedial in removing secretion, then it is very properly controlled by opium. If, on the other hand, respiratory movements are weak, or cyanosis threatens from retained secretions, opium is distivetly contraindicated, since it depresses the respiratory centres and lessens the irritation produced by the secretions in the bronchial tubes, which would otherwise cause conghing and expulsion of the exudate.

Opium, especially when combined with belladonna, notably diminishes secretions, so that this combination is peculiarly appropriate in the treatment of cough and exudation, and it is only when increasing moist rales are found to exist during this medication that it should be stopped. Plemritic cough causes intense pain and accomplishes nothing, so that opium here affords great relief without inducing bad results.

4. Opium possesses antiphlngistic action in aborting and combating inflammation. R R flex excitability is lessened by opinm, and therefore irritation of nerve centres, which would otherwise canse vasculiur dilatation, stasis, and inflammation, is prevented by the drug. This is at least the theory. Opinm and quinine are the two remedies laving the most popular clinical reputation for aborting colds and iuflammation, and the latter agent also diminishes reflex axcitability. Inflammation of sen nus membranes is thought 
to be that form most favorably influenced by opium, as peritonitis, enteritis wnd meningitis, for which purpose the drug is frequently combined with calomel. But opium is also an extremely useful antiphlogistic remedy in coryza, bronchitis, puenmonia and pleurisy, and in inflammations of the mucous coat of the digestive canal, as gastritis and dysentery. A single full dose should be given at the earliest possible stage of these disorders, and the patient should be kept as quiet as possible to secure the best result.

5. Opium stimulates and supports the system in a manner not explicable on physiological grounds. It often conserves life in a remarkable way in patients weakened by long continued disease, and in those suffering from loss of blood following surgical operation, parturition, or other natural causes.

Contra-indications.-In respiratory diseases associated with cyanosis or excessive exudation, in cerebritis, in very high fever and obstinate constipation. The drug must be used with caution in the treatment of the aged and very young.

Apomorphine Hydrochloras. Apomorphine Hydrochlorate. $\mathrm{C}_{17} \mathrm{H}_{17} \mathrm{~N} \mathrm{O}_{2} \mathrm{H} \mathrm{Cl}$. (U. S. \& B. P.)

Derivation.-The hydrochlorate of an artificial alkaloid, obtained by heating morphine (or codeine) in hermetically closed tubes, with an excess of pure hydrochloric acid. The morphine thus loses one molecule of water; $\mathrm{C}_{17} \mathrm{H}_{19} \mathrm{~N} \mathrm{O}_{3}=$ $\mathrm{C}_{17} \mathrm{H}_{17} \mathrm{~N} \mathrm{O}_{2}+\mathrm{H}_{2} \mathrm{O}$.

Properties. - Minute, grayish - white, shining, acicular crystals, withont odor, having a faintly bitter taste, and acquiring a greenish tint upon exposure to light and air. Soluble in about 45 parts of water, and about 45 parts of alcohol; very little soluble in ether or chloroform. It should be kept in small, dark, amber-colored vials. (U. S. P.)

Dose.-Emetic.-D., gr: $\frac{1}{30}-\frac{1}{10}(.002-.006)$, subcutaneously; D., by the mouth, grr- $\frac{1}{8}-\frac{1}{5}$ (.003-.012); D., expectorant, gr. $\frac{1}{40}-\frac{1}{25}$ (.0015-.0024), by the month. 
PREPARATION.

Injectio Apomorphince Hypodermica. (B. P.)

( 2 grains in 100 minims camphor water.)

Dose.-D., mii.-г. (.12-.3).

Action Internal.-Small doses (gr. $\frac{1}{60}-\frac{1}{30}$ ) cause vomiting in dogs, while larger doses produce salivation and trembling in addition to vomition. Very large quantities (gr.4-5) occa- । sion first great excitement; the dog howls, runs and jumps about, the pupils are dilated and the slightest noise excites great alarm. Then the animal weakens in the hind legs, becomes paraplegic, falls and goes into convulsions. The breathing, at the beginning rapid, becomes weak and slow. Death ensues from respiratory failure.

Nervous System. - The drug primarily stimulates the brain and indnces delirium and excitement, but secondarily canses cerebral paralysis. The origin of the convulsions is not ascertained. Apomorphine is a direct local paralyzant to the muscles, acting upon their substance or upon the motor nerve endings.

Circulation.-Medicinal doses do not alter the force, but may increase the rate and tension of the pulse by stimulation of the cardiac accelerator nerves and vasomotor centres. Toxic doses paralyze the heart muscle and lower blood pressure.

Respiration. - The respiratory movements are at first markedly increased by large doses of apomorphine. The reason for this is uncertain. Lethal doses depress and paralyze the respiratory centre. The breathing then becomes feeble and infrequent. The agent causes a copious outpouring of a watery fluid from the blood vessels of the respiratory mucous membrane, and is, therefore, an expectorant.

Vomiting Centre. - This is stimulated by therapeutic doses of apomorphine, but paralyzed by toxic doses, so that vomiting may not occur in poisoning. Apomorphine does not act locally upon the stomach. 
Uses.-Apomorphine is a reliable, prompt and powerful emetic. The alkaloid is generally given uuder the skin and can be administered aloug with ziuc sulphate or other emetic in poisoning. In narcotic poisoning, as with chloral or opium, apomorphine-like other emetics-may fail to act efficiently. In the first stage of acute bronchitis, apomorphine is useful in canine practice, and again in the later stage, when the animal becomes choked with exudation. The drug, in a mild emetic dose, will aid recovery by causing violent expiratory efforts during vomition, and these tend to expel secretions, which is furthermore assisted by the action of the alkaloid in rendering the secretions less viscid. Chronic dry bronchitis of dogs is likewise benefited by apomorphine.

The alkaloid decomposes in crystal, and rapidly in solution, becoming toxic and of a green hue. Solutions should be freshly prepared and are said to be preserved by the addition of a few drops of lydrochloric acid.

\section{Class 2.-Stimulating the Brain.}

Belladonne Folia. Belladonna Leaves. (U. S. \& B. P.)

Synonym.-Deadly nightshade, E.; folia S. herba belladonnæ, feuilles de belladomne, Fr.; tollkrant, tollkirschen blätter, wolfkirschen-blätter, G.

Derivation. - The leaves of Atropa Belladonna Linné (Nat. ord. solanaceæ). Leaves from 10 to $15 \mathrm{~cm}$. loug, from 5 to $10 \mathrm{~cm}$. broad; broadly ovate, equilaterally narrowed into a petiole, tapering at the apex, entire on the margin, smooth, thin; the upper surface brownish-green, the lower surface greyish-green; both surfaces whitish punctate; odor slight; taste iitterish, disagreeable.

Constituents.-Two alkaloids; 1 , atropine, the chief one, representing the action of belladonna; and, 2, hyoscyamine.

Atropine is now considered to be an artificial product of hyoscyamine, and therefore the latter to be the natural 
alkaloid. Belladonna leaves contain about 0.46 per cent. atropine.

$$
\begin{gathered}
\text { Dose.-H. \& C., } 3 \text { ss.-i. (15.-30.); D., gr.i.-v. (.06-.3). } \\
\text { Preparations. }
\end{gathered}
$$

Extractum Belladonnce Foliorum Alcoholicum.

Alcoholic Extract of Belladonna Leaves. (U. S. \& B. P.)

Made by percolation and evaporation to pilular consistence. Used in preparing the unguentum bellarlonnæ.

Dose.-H. \& C., gr.x.-xx. (.6-1.3); Sh. \& Sw., gr.ii.-iv. (.12-24.); D., gr. $\frac{1}{8}-\frac{1}{2}(.008-.03)$.

Tinctura Belladonnce Foliorum. Tincture of Belladonna Leaves.

(U. S. \& B. P.)

Belladonna leaves, 150; diluted alcohol to make 1,000. Made by maceration and percolation. (U. S. P.)

Dose.-D., m.

Unguentum Belladonnce. Belladonna Ointment. (U. S. \& B. P.)

Alcoholic extract, 10 ; diluted alcohol, 5 ; benzoinated lard, 85 . (U. S. P.)

Belladonne Radix. Belladonna Root.

Synonym.-Racine de Belladonne, Fr.; wolfskirschenwurzel, tollkirschen-wurzel, G.

Derivation.-The root of Atropa Belladonna Linné (nat. ord. solanaceæ). In cylindrical, somewhat tapering, longitudinally wrinkled pieces, 10 to $25 \mathrm{~mm}$. or more in thickness; externally brownish-gray, internally whitish; fracture nearly smooth and mealy, not radiating or showing medullary rays in the thicker roots, only in the layer near the bark; nearly inodorous, taste sweetish, afterwards bitterish and strongly acrid.

Constituents.-Same as leaves. Contains 0.2 to 0.6 per cent. atropine.

\section{PREPARATIONS.}

\section{Extractum Belladonnce Radicis Fluidum.}

Fluid Extract of Belladonna Root. (U. S. P.)

Made by percolation with alcohol and water, and evaporation. One Cc. of the extract $=$ one $\mathrm{Gm}$. of belladonna root. The most reliable preparation. 
Dose.-H., 3 i.-ii. (4.-8.); C., 3 ii.-iii. (8.-12.); Sh. \& Sw., mx.-xv. (.6-1.); D., Mi.-iii. (.06-.2).

Linimentum Belladonnce. Belladonna Liniment. (U. S. \& B. P.)

Camphor, 50; fluid extract of belladonna to make 1,000. (U.S.P.)

Atropina. Atropine. $\mathrm{C}_{17} \mathrm{H}_{23} \mathrm{~N} \mathrm{O}_{3}$. (U. S. \& B. P.)

An alkaloid obtained from belladonna. As it occurs in commerce, it is always accompanied by a small proportion of hyoscyamine extracted along with it, from which it cannot readily be separated.

Derivation.-Atropine is obtained from a strong tincture of the root. Slaked lime is added, which splits up atropine malate and precipitates lime malate. The excess of lime is precipitated by sulphuric acid, and coloring matters by potassium carbonate, which also sets free atropine. The atropine is dissolved in chloroform, recovered on evaporation, and purified by digestion with warm alcohol and charcoal.

Properties.-White, acicular crystals, or a more or less amorphous white powder; without odor, having a bitter, acrid taste, and gradually assuming a yellowish tint on exposure to air. Soluble in 130 parts of water, 3 parts of alcohol, 16 parts of ether, 4 parts of chloroform, and about 50 parts of glycerin. At about $108^{\circ}$ C. $\left(226.4^{\circ}\right.$ F.) it melts, forming a colorless liquid. At abont $140^{\circ}$ C. $\left(284^{\circ} \mathrm{F}\right.$.) it begins to give off white, acrid funes, and when ignited, it is consumed without leaving a residue. It has a markedly alkaline reaction; its saturated aqueous solution acquires a pink color upon the addition of a drop of phenolphtalein.

Incompatibles. - Decomposed by sodium or potassium Inydrate.

Dose.-H., gr.ss.-iss. (.03-.09); average dose, gr.i. (.06); C., gr.i.-ii. (.06-.12); Sh. \& Sw., gr. $\frac{1}{20}-\frac{1}{12}(.003-.005) ;$ D., $\mathrm{grr} \cdot \frac{1}{120}-\frac{1}{60}(.0005-.001)$. 
Atropinæ Sulphas. Atropine Sulphate. $\left(\mathrm{C}_{17} \mathrm{H}_{23} \mathrm{NO}_{3}\right)_{2} \mathrm{H}_{2} \mathrm{SO}_{4}$. (U. S. \& B. P.)

Derivation. - Atropine is dissolved in sulphuric acid and treated with ether, when the insoluble sulphate settles out.

Properties. - A white, indistinctly crystalline powder, without odor; having a very bitter, nauseating taste, and permanent in the air. Soluble in 0.4 parts of water, 6.2 parts of alcohol, 2,270 parts of ether, or 694 parts of chloroform. At $187^{\circ} \mathrm{C}$. $\left(309^{\circ} \mathrm{F}\right.$.) the salt melts, forming a brownish-yellow liquid. When ignited, it chars, emits acrid vapor, and is completely consumed. The salt is neutral to litmus paper.

Dose.-H., gr.i.-iss. (.06-.09) ; C., gr.i.-ii. (.06-.12) ; Sh. \& Sw., gr. $\frac{1}{15-\frac{1}{12}}(.004-.005)$; D., gr. $\frac{1}{120}-\frac{1}{30}(.0005-.002)$, average dose, $\mathrm{gr}^{-\frac{1}{100}}(.0006)$.

\section{ACTION OF BELLADONNA AND ATROPINE.}

External.-Belladonna is ordinarily not absorbed from the unbroken skin, but when applied to raw surfaces and mucous membranes, or, to a less extent, when rubbed into the skin with camphor (linimentum belladomnæ), chloroform, alcohol, etc., it depresses the sensory nerve endings and produces a local anodyne action. Thus applied it also depresses the peripheral motor nerve fibres, and is sometimes injected into spasmodically contracted muscles for relief of spasm. The vessels are said to be first contracted and then dilated by the local action of the drug, and the secretions of the skin are diminished.

Internal.-Digestive Tract.-The action of belladonna, if any, on the secretory functions of the stomach and bowels is unknown. Small doses of the drug depress the peripheral terminations of the intestinal inhibitory nerve (splanchnic), so that peristalsis is increased by the normal stimuli. It is stated that both minute and large doses apparently lessen intestinal peristalsis; the former by primary stimulation of 
the splanchnic nerve endings, the latter by direct depressant action upon the unstriated muscle of the intestinal wall.

Civculation.-Belladonna is readily absorbed into the blood, but has no particular action npon this fluid within the body. Dilute solutions of atropine paralyze and stop corpuscular movement in the blood withdrawn from the ressels. The characteristic action of belladonna upon the circulation consists in depression of the peripheral pnenmogastric fibres in the heart, so that the frequency of its pulsations is increased. There is also, probably, a slight stimulation of the cardiac muscle, or its contained ganglia. Belladonna is therefore a heart stimulant, by increasing the number of its beats without diminishing their force. Large doses sometimes give rise to primary slowing of the pulse, owing to stimulation of the inhibitory apparatus. Blood pressure is exalted synchronously with the increased frequency of the heart, and is due to cardiac stimulation and also to stimulation of the spinal and medullary vasomotor centres, with constriction of blood vessels.

In poisoning, vascular teusion is considerably lowered because of paralysis of the vasomotor centres, smooth muscles (or ganglia) of the vessel walls (with vascular dilation), and the heart muscle itself.

Nervous System.-Cerebrum-Belladonna is classed as a delirifacient by some authorities, - notably Wood. It stimulates the brain incoördinately, and large doses produce restlessuess, nervous excitement and delirium in man, and occasionally delirium in the lower animals. Stimulation is succeeded by exhaustion and some depression, with stupor rather than coma.

Spinal Cord-Belladonna appears to exert a double action (stimulant and depressant) upon the spinal cord. The spinal vasomotor and respiratory centres are stimulated. Large doses cause complete loss of motion and reflex action in the frog, lasting for several days, and followed by reflex excitability and convulsions. Poisoning in mammals is exhibited by less paralyzant action accom- 
panied by more convulsive movements. Various explanations have been offered to reconcile these phemomena. The prevalent theory is that, following complete paralysis of the spinal cord, the motor and sensory tracts recover before the inhibitory centres, so that normal stimuli pass through the latter uurestrained and result in convulsions.

Nerves-The action of belladonna upon the nerve endings is extremely important, and represents, to a considerable degree, the therapentic value of the drug. The peripheral motor nerve terminations, and, to a less extent, their trunks, are depressed and paralyzed. This is never so complete, however, but that there is some voluntary power left in an animal fatally poisoned. Depression and loss of function of the afferent nerves come on more slowly, and exist to a less degree. Belladonna, then, when applied locally or given internally, is an anodyne, but is far inferior in this respect to opium; and, whereas opium acts centrally, belladonna acts peripherally. The terminations of all secretory nerves are also depressed and paralyzed by belladonna, and secretions are therefore diminished.

Secretions.-Dryness of the mouth is one of the first physiological symptoms following the administration of belladonua. This is due to paralysis of the peripherat terminations of the secretory nerve (chorda tympani) of the submaxillary gland, and of the secretory nerve endings of the other salivary and mucous glands in connection with the mouth. Another characteristic effect of belladonna consists in the production of dryness of the skin, which follows the action of the drug in paralyzing the peripheral nervous filaments supplying the secretory cells of the sudoriparous glands. In the same manner the tracheal and bronchial secretions are diminished, and also the secretion of milk (anti-galactagogue action), by depression of the terminations of the secretory nerves. The drug does not exert any decided influence upon the secretions of the aigestive tract, as before stated. The amount of urine is sometimes increased after the ingestion of small doses of 
belladonna, but is considerably lessened, or suppressed, in poisoning by the drug. Experiments upon man show that the urinary solids are increased: urea and uric acid to a slight extent; sulphates and phosphates to a considerable degree.

Mruscles and their Nerves.-Atropine is an antispasmodic in its effect upon involuntary muscle. It depresses both the unstriped fibres and their motor nerve endings. Toxic doses cause paralysis of the smooth muscles. Voluntary muscles are not affected by any doses of atropine, although the motor nerves controlling them are depressed by toxic amounts of the alkaloid. In regard to the effect of the drug upon the intestines, experimental and clinical evidence appear to yield quite contradictory results. It seems probable that any dose of atropine will depress, to a greater or lesser extent, the activity of the intestinal muscle, but, as there is at the same time depression of the inhibitory nerve endings (splanchnics), there is also a tendency to increased peristalsis through the greater sensitiveness of the gut to stimuli. As the result of careful experiments, it appears that minute doses of atropine stimulate the splanchnics and lessen peristalsis; that moderate doses increase markedly peristalsis by paralyzing the splanchnics; and that large doses stop all movement of the bowel by paralyzing the intestinal musculature. Other experiments by reliable men and clinical experience negative these conclusions.

Atropine has been used successfully to produce three diverse actious on the bowel: (1) to quiet intestinal movement in inflammation of the bowels; (2) to relieve spasm in colic and spastic conditions generally; (3) very commonly to aid catharsis in sluggish states of the intestines. In practice, small doses will of ten move the bowels effectively, while, on the other hand, enormous doses (gr.1-12, instead of the usual dose of gr.1-100) have recently proved successful in moving the bowels in obstinate constipation (in 
human patients) due to obstruction, , when all other means failed. Conversely, it is, and has been, the common practice to give atropine with morphine to prevent peristalsis in peritonitis and with apparently satisfactory results. It is at present impossible to reconcile these apparently conflicting facts, but it is readily conceivible that the resultant of the opposing actions of atropine on tho bowels in either stimnlating peristalsis by lepression of the inlibitory apparatus, on the one hand, or, in lessening peristalsis by depression of the muscle, on the other, mily depend as much upon the condition of the gnt as upon the closage, and that, according to the physiological or pathological state, one or the other of these opposing actions will preponderate.

The action upon the inhilitory nerve of the lieart is similar to that exerted upou the inhibitory nerve of the bowels. The pneumogastric terminations are depressed in the heart by moderate doses, while the heart muscle is paralyzed by large quantities of belladonna.

A like depressing influence is believed to be exerted upon the efferent nerve endings of the unstriped muscles of the bladder, urethra, uterus and vagina, as well as upon the muscles of these organs. Belladonna acts medicinally as an antispasmodic in relation to the muscles.

Respiration.-Small doses of atropine do not affect the respiration. Large therapeutic doses make the respiratory movements quicker and deeper, by stimulation of the medullary and spinal respiratory centres. Fatal doses produce respiratory failure and asphyxia, owing to paralysis of the respiratory centre and the peripheral vagus filaments concerned with the respiratory movements. Belladouna also paralyzes the peripheral fibres of the pneumogastric nerve in the bronchial tubes and acts therapentically as follows: 1. As a respiratory stimulant; the drug is generally iuferior to strychnine in this respect. 2. As an antispasmodic, by

* It is probable in these cases that the obstruction was due to spasm, and not mechanical. 
depressing the efferent vagus endings in the bronchial tubes and relaxing spasm of the smooth muscle of their walls. 3. As a sedative, by depressing the afferent vagus fibres and diminishing the irritation produced by secretion, so that cough is allayed. 4. As an agent lessening secretion.

Temperature.-Moderate doses of belladomna cause a rise of temperature, while fatal doses lessen bodily heat. The first plenomenon is produced by stimulation of the spinal thermogenic centres, while the latter effect follows the vasomotor paralysis which occurs after lethal doses.

An elevation of $2-5.4^{\circ} \mathrm{F}$. has been noted in dogs after full doses of atropine, while a greater fall of temperature has been observed in the same animal in fatal poisoning. Elevation of temperature is accompanied by increased heat loss, caused by radiation from the dilated cutaneous vessels. This latter condition is evidenced by a scarlatina-like eruption in man.

Eye.-The action of belladonna upon the eye affords another illustration of the depressing action of the drug upon the nerve terminations. The oculomotor nerve endings are paralyzed by belladonna, and therefore dilation of the pupil ensues. The mydriatic action is exhibited, whether the drug be given by the mouth or dropped directly into the eye. In the former case, the drug does not act through the nervous system, but locally upon the peripheral filaments of the third nerve through the medium of the blood. The muscular fibres of the iris are unaffected by belladonna. The terminations of the sympathetic and and the trigeminus may be stimulated, which would also produce dilation of the pupil. These latter actions are not definitely determined, while it is known that the dominant effect consists in depression of the oculomotor nerve endings, as before stated. Paralysis of accommodation follows paralysis of the terminations of the third nerve in the ciliary muscle, and therefore vision is disturbed. Intraocular tension is increased by large and continuous dosage 
of belladonna, and an artificial glancoma may be thus produced.

Elimination.-Belladonna is probably eliminated entirely by the kidneys.

Summary.-It will be observed that belladonna, generally speaking, first stimulates and then depresses the nerve centres, while it chiefly paralyzes the motor nerve termiuations, including the inhibitory (vagus and splanchnic), the secretory (chorda tympani, etc.), and, to a less extent, the sensory nerves. Secondary depression of the cerebrum is not so profound as that of the great medullary centres, especially the respiratory centre, and there is sometimes a slight and brief stimulation of the motor nerves of the smooth muscles, viz., vagus, splanchnic, and possibly vasomotor nerves.

Full medicinal doses depress the peripheral filaments of the inhibitory and secretory nerves and the motor nerves of the unstriped inuscles, as well as tise muscles themselves, lessen the functional activity of the voluntary motor system, and, to a less degree, that of the afferent nerves. The pulse becomes quickened because of paralysis of the peripheral vagus endings and stimulation of the heart or its ganglia; the blood tension is angmented because of the increased cardiac action and stimulation of the vasomotor centres; and the respiration is accelerated because of excitation of the respiratory centres. The temperature is elevated owing to the circulatory exaltation and stimulation of the heat-producing centres. Slight delirium may be present from the exciting action of the drug upon the cerebral motor centres.

The spinal cord is unaffected by therapeutic doses. Locally applied, belladonna is a direct paralyzaut to nerves, muscles, vessels and cells.

\section{PHYSIOLOGICAL RELATIONS OF BELLADONNA TO OTHER DRUGS.}

A medicinal dose of atropine stimulates, while morphine depresses, the brain, respiratory functions, and intestinal peristalsis. This antagonism ceases when poisonous doses 
of the two drugs are combined, and therefore atroping should be given with caution in the treatment of opium narcosis, so as not to aggravate the already existing central nervous depression, particularly of the respiration. Morphine relieves pain, causes sweating, and contracts the pupil centrally. Atropine dilates the pupil, produces dryness of the skin, and depresses the functions of sensory nerves through its peripheral action. Atropine antagonizes physostigma in so far as the latter stimulates the peripheral oculomotor nerve fibres, the vagi, diminishes blood pressure, depresses the respiratory centres, and stimulates the unstriated muscle of the intestines, and the secretions of the stomach, bowels and bronchial tubes.

Atropine is antagonistic-in part-to pilocarpine, which stimulates secretory nerve terminations in the sweat and salivary glands, the peripheral oculomotor filaments, and the nerve endings in the involuntary muscle of the heart, stomach, intestines and uterus. Atropine counteracts the influence of aconite and muscarin (the poison of fungi) upon the heart, and the action of the latter principle in producing spasm of the pulmonary vessels.

Toxicology.-Toxic doses of belladonna canse in animals dryness of the mouth, increased frequency of the pulse and respiration, elevation of temperature, dilation of the pupil and partial blindness, restlessness, nervousness, delirium, twitching of the muscles (occasionally erythema), and frequent micturition. These symptoms are succeeded, in fatal poisoning, by fall of temperature, retention of urine, muscular weakness, staggering gait, partial anæsthesia, convulsions and paralysis (one preponderating over the other), weak, slow, irregular respiration, feeble, rapid pulse, paralysis of the sphincters, stupor and death. Death occurs mainly from asphyxia, but is due in part to cardiac failure. The physiological test consists in placing a drop of the urine (secreted by the poisoned animal) into the eje of a healthy animal, when mydriasis should follow if the case be one of belladonna poisoning. Three-quarters of a grain of 
atropine under the skin has proved fatal to dogs. Two grains of atropine produce mild toxic symptoms in the horse. Small dogs are slightly poisoned by gr. $\frac{1}{80}$ of atropine; medium sized dogs by gr. $\frac{1}{60}$, given hypodermatically. Cattle are as susceptible as horses, although herbivora are not so easily influenced as carnivora. The pulse in dogs is greatly accelerated, sometimes as high as 400 , while the pulse rate of the horse is not generally more than doubled. Rodents, as guinea pigs and rabbits, and pigeons, are particularly insusceptible to belladonna, in regard to its effect upon the pupil, circulation, etc.

The treatment of poisoning includes the use of the stomach pump, emetics, cardiac stimulants, and pilocarpine under the skin. Also external heat, general faradism and artificial respiration.

Post-Mortem Appearances. - These are not generally characteristic, except of asphyxia. The blood is dark and poorly coaguable. There is congestion of the lungs, general passive hyperæmia, and sometimes ecchymoses in the brain, cord, and their membranes. Congestion of the retina is said, however, to be pathoguomonic of belladonna poisoning.

Administration.-The fluid extract of belladonna root is the official preparation more commonly used for horses. The alcoholic extract of the leaves is given in pill or suppository to dogs, aud in electuary to horses. Atropine sulphate is employed under the skin when a rapid effect is desired.

Uses External.-Local applicatiou of belladonna is more effective when combined with internal medication of the same drug. Belladonna is the best remedy for mammitis, applied by massage in the form of liniment, and given by the month. It relaxes spasm, contracts the blood vessels, and lessens inflammation and congestion; paralyzes the secretory nerves and so diminishes the amount of milk, vascular tension, pain and glandular activity. In fissure of the rectum, and in hæmorrhoids, belladouna (with opium) in ointment or suppository, allays spasm and pain. Lini- 
ment of belladonna is useful in rheumatic or neuralgic pain, and rubbed upon the throat, in cases of pharyngitis and laryngitis, affords a serviceable application, when combined with the internal administration of the drug.

Uses in Connection With the Eye.-In examination of the fundus of the eye, the media, or lens, for cataract, the pupil may be dilated to advantage with a weak solution (gr. $\frac{1}{20}-\overline{3}$ i.) of atropine sulphate. A drop will suffice, and no trace of its effect will remain after the second day. A strong solution (gr.iv.- $亏$ i.) is essential to completely paralyze the iris and ciliary muscle. In the normal animal, accommodation is paralyzed and vision disturbed for 8 or 12 days after the use of this solution. Atropine is particularly useful in keratitis and iritis. In the former disease, photophobia and blepharospasm are diminished by the paralyzing action of atropine upon the trigeminus, and pain, congestion and inflammation are diminished by contraction of the peripheral blood vessels. In central perforating ulcer of the corvea, with protrusion of the iris, atropine, by dilating the pupil, draws the iris away and prevents its permanent adhesion (anterior synechia) to the cornea, while the perforation is becoming filled with lymph and the anterior chamber is being restored. Strong solutions of atropine instilled at frequent intervals, are useful in iritis by (1) paralyzing and securing rest of the iris and ciliary muscles; (2) in lessening local blood supply, congestion and inflammation, and in preventing adhesions of the posterior surfaces of the iris to the anterior capsule of the lens (posterior synechiæ), which both limits the normal variation in the pupillary diameter and interferes with the nutrition of the lens, and so predispose to cataract. Atropine is contraindicated in glaucoma.

Uses Internal.-The general indications follow directly from our knowlerge of the physiological action of the drug. They are as follows:- -1 . To stimulate the respiration and circulation. 2. To diminish secretion. 3. To relieve spasm and pain. 
1. Acute diseases, as pueumonia, bronchitis, influenza, cerebro-spinal meningitis, etc., are frequently treated at the outset with belladonna, with the intent of cutting short the inflammatory process by prociucing general vascular contraction. There does not seem to be sufficient physiological or clinical evidence to warrant this practice (except in pharyngitis and laryugitis), and there are other drugs (ergot) which accomplish the purpose, were it desirable, more efficiently. In the second stage of acute diseases, as pueumonia, belladonna is a valuable agent in combination with strychnine, to stimulate respiration, prevent effusion and vasomotor and cardiac depression. Belladonna is of service also as a respiratory, cardiac and vasomotor stimulant in poisoning by varions drugs, including opium, chloroform, ether, aconite, prussic acid, physostigma and pilocarpine. Recent experiments by Reichert (Therapeulic Mlonthly, May, 1901) and others show that atropine, while stimulating the respiratory centre exerts a powerful depressing action on the pulmonic motor fibres of the vagi, and that in opinm poisoning atropine, instead of strengthening, actually lessens respiratory power. Strychnine undoubtedly is a much better antidote in this condition. In surgical shock, with low temperature, owing to vasomotor paralysis and vascular dilatation, atropine is a most potent remedy, combined with digitalis.

2. Belladonna is employed therapeutically to diminish excessive sweating and salivation, mercurial or otherwise. It is recommended in serous, or watery diarrhoea. Etema of the lungs is combated most successfully with atropine (combined with strychnine) subcutaneously. In the second stage of acute respiratory diseases, as bronchitis, influenza, canine distemper, and pneumonia, belladouna diminishes secretion, irritability and cough, and stimnlates the heart and respiration. It may be associated with opium to increase the sedative effect.

3. Belladonna does not have much influence over spasm of the voluntary muscles, unless injected (atropine) into their 
substance. Rheumatic lameness, and cramps and spasm due to injury of nerves, may be treated in this manner. Spasm of involuntary muscle is, however, more easily overcome, and this action is of exceeding therapeutic importance. Intestinal spasmodic colic of horses succumbs most readily when atropine is given with morphine under the skin. In peritonitis and enteritis, full and repeated doses of atropine, with morphine, assist in paralyzing the smooth muscular fibres of the intestines, and in quieting peristaltic movement. Paradoxical as it may seen, large doses of atropine have been recently used in human medicine, and with remarkably good results, in the treatment of intestinal olstruction from impacted feces, and even in invagination and $t$ trist, on the assumption that the inhibitory splanchnic fibres of the gut are paralyzed by these large quantities given under the skin (see "Action on Muscles and Nerves").

Cough, stridulus breathing, and spasm, associated with acute pharyngitis and laryngitis, are influenced favorably by belladonna, in the first stage. The drug acts locally to paralyze the ends of the motor nerves in the throat, relieves spasm, and also contracts the peripheral vessels and overcomes congestion and inflammation. It may be given to horses in electuary, and also applied in liniment or ointment externally. The following prescription is suitable for horses suffering from pharyngitis or laryngitis.

B

Extract. Belladonnæ Rad. Fl........... $\mathbf{z}^{\mathbf{i}}$.

Pulv. Potassii Chloratis.............. 3 ii.

Pulv. Glycyrrhizæ................

Syrupi Fusci .................... S.

M. et $f$, electuarium.

Sig. Give $\frac{1}{2}$ ounce every two hours. (Furnish $\frac{1}{2}$ ounce for sample dose.)

In bronchitis and asthma, belladonna also allays spasm and lessens secretion, irritation and cough. Other spasmodic conditions benefited by belladonna are, "thumps," lead colic, spasm of the rectum owing to fissure or other causes, 
and incontinence of urine due to spasm of the neck of the bladder. Palpitation of the heart is relieved by the sedative action of the mydriatic upon the unstriated cardiac muscle. Tetanus is favorably influenced by extract of belladonna given in electuary. In this section may be included the effect of small doses of belladouna in paralyzing the inhibitory (splanchnic) nerve terminations in the bowels, and thus assisting the action of peristaltic stimulants, as aloes and stryclınine. Pills containing aloin (gr. $\left.\frac{1}{4}\right)$, extractum belladounæ alcoholicum foliorum (gr. $\frac{1}{8}$ ), and extractum cascaræ sagradæ (gr. $\left.\frac{1}{2}\right)$, form a useful laxative combination in chronic constipation of dogs.

\section{Hroscyamus. Hyoscyamus. (U. S. P.)}

Synonym. - Hyoscyami folia, B. P.; henbane, herba hyoscyami, E.; feuilles de jusquiame noire, Fr.; bilsenkrant, G.

The leaves and flowering tops of Hyoscyamus niger Linné (nat. ord. solanacer), collected from plants of the second year's growth.

Habitat. - Indigenous to England, the Contiuent, and Asia, and naturalized in the northern part of the United States.

Description.-Leaves ovate, or ovate-oblong, up to 25 cm. long and $10 \mathrm{~cm}$. broad; sinuate-toothed; the teeth large, oblong or triangular, grayislı-green, and, particularly on the lower surface, glandular-hairy; midrib prominent; flower's nearly sessile, with an urn-shaped, five-toothed calyx, and a light yellow, purple-veined corolla; odor heavy, narcotic; taste bitter and somewhat acrid.

Constituents.-Two alkaloids : hyoscyamine $\mathrm{C}_{17} \mathrm{H}_{23} \mathrm{~N} \mathrm{O}_{3}$, and hyoscine. The first resembles atropine in composition and action. Impure, amorphous, commercial hyoscyamine is a dark-brown fluid and contains as its active principle mainly hyoscine.

Incompatibility. - Incompatible with caustic alkalies and 
vegetable acids, lead acetate and silver nitrate. Hyoscyamus may be given in pill with the two latter mineral salts.

Dose.-H. \& C., 3 ss.-i. (15.-30.); D., gr.v.-xv. (.3-1.).

PREPARATIONS.

Extractum Hyoscyami. Extract of Hyoscyamus.

(U. S. \& B. P.)

Made by maceration and percolation with diluted alcohol, and evaporation to pilular consistency.

Dose.-H. \& C., gr.xx.- 3 i. (1.3-4.); D., gr.ss.-ii. (.03-.12).

Extructum Hyoscyami Fluidum. Fluid Extract of Hyoscyamus.

(U. S. P.)

Made by maceration, percolation with alcohol and water, and evaporation, so that $1 \mathrm{Cc} .=1 \mathrm{Gm}$. of hyoscyamus.

Dose.-H. \& C., 3 ss.-i. (15.-30.); D., आlv.-xv. (.3-1.).

Tinctura Hyoscyami. Tincture of Hyoscyamus. (U. S. \& B. P.)

Hyoscyamus, 150 ; diluted alcohol to make 1,000. Made by maceration and percolation. (U.S. P.)

Dose.-D., 3 i.-iv. (4.-15.).

The preparations of hyoscyamus are of rather uncertain strength. The dose is generally two to four times that of similar belladonna preparations.

Succus Hyoscyami. Juice of Hyoscyamus. (B. P.)

Dose.-Same as tincture.

Hyoscyamine Sulphas. Hyoscyamine Sulphate. $\left(\mathrm{C}_{17} \mathrm{H}_{23} \mathrm{~N} \mathrm{O}_{3}\right)_{2} \mathrm{H}_{2} \mathrm{~S} \mathrm{O}_{4}$.

The neutral sulphate of an alkaloid obtained from hyoscyamus.

Derivation.-Hyoscyamine is derived from hyoscyamus seed. The latter are treated with ether to extract fat, and then distilled with alcohol and sulphtric acid. The liquid residue is neutralized with canstic soda and precipitated with tannic acid. The precipitate is mixed with lime and extracted with alcohol. The resulting alcoholic solntion is. first treated with sulphuric acid, then with caustic soda, and 
finally with ether, which dissolves the alkaloid. The latter is recovered by distillation.

Properties. - White, indistinet crystals, or a white powder, without odor, and haring a bitter, acrid taste. Deliquescent in damp air. Soluble in 0.5 part of water, and in 2.5 parts of alcohol; very slightly soluble in ether or chloroform.

Dose.-H., gr.i.-ii. (.06-.12); D., gr. $\frac{1}{60}-\frac{1}{30}(.001-.002)$.

Hyoscyamine Hydrobromas. Hyoscyamine Hydrobromate. $\mathrm{C}_{17} \mathrm{H}_{23} \mathrm{~N} \mathrm{O}_{3} \mathrm{H}$ Br. (U. S. P.)

The hydrobromate of an alkaloid obtained from hyoscyamus.

Properties. - A yellowish-white, amorphous, resin-like mass, or prismatic crystals, having, particularly wlien damp, a tobacco-like odor, and an acrid, nauseous and bitter taste. Deliquescent on exposure to the air. Soluble in about 0.3 part of water, 2 parts of alcohol, 3,000 parts of ether, or 250 parts of ehloroform.

Dose.-H., gr.i.-ii. (.06-.12); D., gr. $\frac{1}{60}-\frac{1}{30}(.001-.002)$.

Hyoscine Hydrobromas. Hyoscine Hydrobromate. $\mathrm{C}_{17} \mathrm{H}_{21} \mathrm{~N} \mathrm{O}_{4} \mathrm{H}$ Br. (U. S. P. \& B. P.)

The hydrobromate of an alkaloid obtained from hyoscyamus.

Properties. - Colorless, transparent, rhombic crystals ; odorless and having an acrid, slightly bitter taste. Permanent in the air. Soluble in 1.9 parts of water, and in 13 parts of alcohol; very slightly soluble in ether or chloroform.

Dose.-H., gr. $\frac{1}{6}-\frac{1}{4}(.001-.015)$; D., gr. $\frac{1}{150}-\frac{1}{100}(.0004-.0006)$. Action Internal.-The action of hyoscyamus is a resultant of that of its two alkaloids, hyoscine and hyoscyamine. The latter is practically atropine, except that its mydriatic action is shorter. Hyoscine, in poisonous doses, is a power- 
ful depressant to the cerebrum, respiratory centre, spinal reflex centres, and motor tract. It differs from atropine in being a cerebral sedative, and in its greater paralyzant action upon the spinal cord. The tetanic stage succeeding spinal paralysis, observed in atropine poisoning, does not ensue with hyoscine. The latter alkaloid slightly depresses and slows the heart, and does not paralyze the vagus terminations, nor depress the motor and sensory nerves or muscles. The circulation is but slightly influenced, and vasomotor depression only occurs in the latter stage of lethal poisoning. Death occurs from paralysis of the respiratory centres. Poisoning in auimals is exhibited by loss of muscular power, slowing and failure of respiration, dryness of the mouth, stupor and asphyxia. The pulse may be infrequent, the pupils are dilated and the skin is muist, rather than dry. Delirium and convulsions sometimes occur in man. The effect of the combined action of hyoscyamine and hyoscine in liyoscyamus is shown when we compare the drug with belladonna. Hyoscyamus is more of a cerebral sedative and hypnotic, and less of a heart and respiratory stimulant. It is said to possess more power in overcoming spasm, and griping of cathartics, and in aiding intestinal movement. Hyoscyamus is also thonght to exert a more pronounced antispasmodic action than belladonna upon the smooth muscles of the bladder and urethra.

Uses.-Hyoscyamus is generally applicable in the same disorders for which belladonna is indicated. In relieving some sorts of spasm, hyoscyamus is more efficient than belladonna, as in spasmodic colic, spasm of the bladder, and griping caused by cathartics. Tetauus, chorea, and epileptic convulsions in dogs, are benefited by henbane, but the drug does not possess a curative actiou. Hyoscyamine may be combined to advantage with strychnine, subcutaneously, in impaction of the bowels in horses. The former drug depresses the inhibitory nerve endings (splanchnic), and assists the stimulant action of strychnine upon the intestinal muscle. Repeated small doses of oil will facilitate peristal- 
sis in this condition. Hyoscine lydrobromate is indicated in spasmodic affections and in nervous and sexual excitement. It is a powerful drug and should be employed at the outset in small doses. To avoid contamination with hyoscyamine is difficult, and the preparation of Merck is to be recommended. Hyoscine has not been employed to any extent in veterinary practice. It is used in human medicine as a hypnotic and sedative in mania and delirium of the insane. It causes no unpleasant after-effects.

\section{Cannabis Indica. Indian Cannabis. (U. S. \& B. P.)}

The flowering tops of the female plant of the Cannabis sativa Linné (nat. ord. urticaceæ), grown in the East Indies.

Synonym.-Indian hemp, E.; chanvre indien, Fr.; indischer hanf, G.; herba cannabis indicx, P. G.

Haschisch is a confection of the drug. Arabian habitués of this preparation are frequently impelled by its influence to deeds of violence. They are called "haschaschins"; hence the English, assassins. Gunjab, or ganga, is the dried plant used in India for smoking. Churrus is the resin and epidermis scraped off the leaves. Bhang consists of the young leaves, flowering and fruiting tops and resin resulting from the first season's growth.

Habitat.-The official cannabis is indigenous to Asia, but the common hemp plant (Cammabis sativa) grows in America and many other parts of the world.

Description. - Branching, compressed, brittle, about 5 $\mathrm{cm}$. or more long, with a few digitate leaves, having linearlanceolate leaflets, and numerous, sheathing, pointed bracts, each containing two small pistillate flowers, sometimes with the nearly ripe fruit; the whole more or less agglutinated with a resinous exudation. It has a brownish-green color, a peculiar narcotic odor, and a slightly acrid taste.

Incompatibility.- Water precipitates the active resinous principles. Lemon juice and other vegetable acids are the most efficient antidotes. 
Constituents.-Resins, including: (1) cannabiu and (2) cannabinon; the first is stated to be very active; (3) tetanocannabine (in which is contained a substance, choline, $\mathrm{C}_{5} \mathrm{H}_{15} \mathrm{~N} \mathrm{O}_{z}$ ), and, with alkalies, forms (4) cannabine. None of these substances is a practical substitute for the crude drug.

\section{PREPARATIONS,}

Extractum Cannabis Indicre. Extract of Indian Cannabis.

(U.S. \& B. P.)

Made by maceration and percolation with alcohol, and by evaporation to a pilular consistence.

Dose.-H., 3 i.-ii. (4.-8.); D., gr.1/4-i. (.015-.06).

Extractum Cannabis Indicoe Fluidum.

Fluid Extract of Indian Cannabis. (U. S. P.)

Made by maceration and percolation. The alcohol is distilled off from the last part of the percolate, and the residue is dissolved in the first part (700 Cc.), and enough alcohol is added to make $1 \mathrm{Cc}$. of the fluid extract equal $1 \mathrm{gm}$. of the crude drug.

Dose.-H., 3 iv .-vi. (16.-24.); D., mii.-x. (.12-.6).

Administration.-The extract is given in ball, pill, electuary or suppository ; or the fluid preparations may be used.

Tinctura Cannabis Indice. Tincture of Indian Cannabis.

(U. S. \& B. P.)

Indian cannabis, 150 ; alcohol to make 1,000 Cc. Made by maceration and percolation. (U. S. P.)

Dose.-D., Mxx.-xxx. (1.3-2.).

Action External.-Cannabis indica exerts a considerable irritant action when applied to mucous membranes. This is followed by local anæsthesia.

Action Internal.-Indian hemp is a distinct depressant to the functions of the brain and cord in poisonous doses, although therapeutically stimulating these organs in small quantities, and producing in man a kind of intoxication and mild delirium. The reflex activity of the spinal cord is primarily increased, but this is succeeded by diminution of reflex movement, and, after large doses, by anæsthesia and loss of voluntary motion. The seusory tract (centres and 
nerves) is depressed by considerable therapentic doses. Our knowledge of the detailed physiological action of the drug is imperfect. The action of cannabis comes on slowly and continues for a long time. A horse receiving, under the direction of the writer, at the Harvard Veterinary Hospital, $\frac{1}{2}$ ounce of the solid extract, became drowsy. Sleep after a few hours deepened into stupor, and stupor into coma. The respiration became slow, the pulse slightly accelerated, and the animal so anæsthetic that amputation of the penis was done on the following day without producing the slightest pain or struggling. The animal had to be supported in slings, and only recovered after three days. Frequently neither constipation, anorexia, or other deleterious aftereffects follow the action of this drug.

The following are extracts from experiments of Muir* with cannabis indica :

Gelding, 8 years old; condition poor. Gave two doses of fluid extract (Park \& Davis, normal liquid) undiluted of $12.5 \mathrm{cc}$. each (about 3 drachms) intrajugularly, twenty minutes apart. Became rapidly nervous and excitable, increased by sounds or touch. In half an hour from first dose he became sleepy and stupid. A third dose of $15 \mathrm{cc}$. ( $1 / 2$ ounce) was injected into the jugular about an hour after the first and caused sweating and a sleepy condition, in which the animal jerked and twitched his head as if dreaming. The temperature dropped to $96^{\circ} \mathrm{F}$., and the sleepy state, alternating with excitement when annoyed, lasted three hours, when delirium supervened and continued for six hours, at which time all the symptoms disappeared.

Pnny, 575 pounds: condition fair. Received $15 \mathrm{cc}$. ( $1 / 2$ ounce) of the fluid extract intravenously. In two minutes became delirious : in ten minutes was asleep against stall. In half an hour fell to floor and slept there for eighteen hours and could not be aroused. Temperature fell to $925^{\circ} \mathrm{F}$. Respiration regular; pulse from 42 to 30 during experiment.

Gelding. 1050 pounds; in good condition. Given $45 \mathrm{cc}$. of fluid extract iutravenously. In three minutes rearing. kicking, snorting a:sd going on like one mad. He ran back and forth along one side of his stall like a caged tiger with sweat rolling off him and cutting and bruising himself, being apparently anesthetic. He endeavored to bite

* Jour. Comp. Med. and Vet. Archives, April and May, 1900. 
or strike anyone approaching and remained delirious and excitable for twelve to twenty-four hours.

Gelding, 650 pounds. $30 \mathrm{cc}$. of fluid extract injected into jugular. In four minutes became unsteady; in twelve minutes he was asleep; in half an hour he fell and so slept for eighteen hours. Temperaturs dropped to $91.8^{\circ} \mathrm{F}$. from normal; the pulse was accelerated and the respiration slightly so.

Muir deduces from his experiments that as much as 50 cc. ( $\xi$ iss.) of the fluid extract may be given with safety intravenously.

In the human being, cannabis induces very peculiar mental phenomena, including hallucinations, a sense of double consciousness, and great prolongation of time, so that minutes are drawn out into hours, and hours into days. Sometimes sexual excitement, exaltation, and hilariousness are exhibited; at other times a dreadful premonition of impending death seizes the human subject. The drug is not fatal, except in colossal doses, but the effects may appear alarming. Intrajugular injection into a small dog, of five drachms of the fluid extract $(10$ minims of which proved active in man) only caused death after several hours (Hare). Preparations of Indian hemp vary greatly in strength, many being entirely inert, and this fact constitutes one of the principal objections to its use. The practitioner is recommended to experiment until he secures a reliable preparation, and use no other thereafter.

Uses.-Cannabis is indicated for the relief of: 1, pain; 2 , spasm; 3 , nervous irritability. It is not comparable to morphine as an analgesic, on account of the uncertainty and slowness of its action, and because anæsthesia is only produeed by an unwarrantably large dose. It is only superior to opium in not cansing coustipation, anorexia and incligestion, and is therefore sometimes given in colic to horses, for the relief of pain and spasm. The permanency of the action of Indian hemp suggests its use in conditions of long continued pain or spasm. In thirty-five cases of tetanus in the haman being, treated with cannabis, twenty-one recovered and fourteen died. The results reported in veter- 
inary practice have been almost as favorable; over half the cases have recovered when subjected to this medication. Medication has, however, usually very little influence in tetauns. Recovery appears to be largely a matter of chance, even with antitoxin. Cannabis Indica is occasionally employed as a sedative for irritable cough, and to relieve the spasms of chorea.

Caffeina. Caffeine. $\mathrm{C}_{8} \mathrm{H}_{10} \mathrm{~N}_{4} \mathrm{O}_{2}+\mathrm{H}_{2} \mathrm{O}$. (U. S. \& B. P.)

Synonym.-Theine, guaranine, E.; coffein, G.; caféine, Fr.

A feebly basic, proximate principle, obtained from the dried leaves of Thea sinensis Linné (nat. ord. ternstrœemiaceæ), or from the dried seeds of Coffea arabica Linné (nat. ord. rubiaceæ), and found also in other plants.

Habitat.-Indigenous to Africa, and cultivated in other tropical countries.

Derivation.-Crushed coffee is treated with successive portions of boiling water, and the resulting solution is precipitated with lead acetate and filtered. Hydrogen sulphide decomposes the excess of lead acetate remaining in the filtrate. The latter is then concentrated by evaporation and neutralized with ammonia water. Caffeine crystallizes on cooling. The alkaloid is purified by charcoal and recrystallization.

Properties.-Fleecy masses of long, flexible, white crystals, possessing a silky lustre, without odor, having a bitter taste, and permanent in the air. Soluble in 80 parts of water, 33 parts of alcohol, 555 parts of ether, or 7 parts of chloroform. Also soluble in abont 2 parts of boiling water, and very soluble in boiling alcohol. Caffeine is neutral to litmus paper. Caffeine exists, curionsly enough, as the active principle of the national non-alcoholic beverages in use all over the world. Coffee contains .6-2.2 per cent. of caffeine, together with glucose, fat, vegetable casein, tannic acid, and an empyreumatic oil developed by roasting, $i . e$.; 
caffeone. Tea contains 1.36-3. per cent. of caffeine, or theine, and also a volatile oil and tannic acid. Guarana (the seeds of Paullinia cupana) contains 4 tc 5 per cent. of caffeine; maté, or Paraguay tea (the leaves of Ilex paragnayensis), contains .45-1.2 per cent. Caffeine is found in kola nut (the fruit of Sterculia acuminata), used as a beverage in Africa, and now as a nervons stimulant in human medicine, and is. allied to theobromine, occuring in chocolate beans, and to cocaine, existing in coca leaves.

Incompatibility.-Caffeine is incompatible with mercury salts, potassium iodide and tannic acid.

Dose.-H., gr.vii.-xv. (.5-1.); D., gr.ss.-iii. (.03-.2), according to size.

\section{Caffeina Citrata. Citrated Caffeine. (U.S. P.)}

Derivation.-Dissolve citric acid (50) in hot, distilled water; add caffeine (50) and evaporate the resulting solution, on a water bath, to dryness, constantly stirring towards the end of the operation. Reduce the product to a fine powder. aud transfer it to well-closed bottles.

Properties.-A white powder, odorless, having a purely acid taste and acid reaction. One part of citrated caffeine forms a clear, syrupy solution, with about 3 parts of water. Upon dilution with water this yields a white precipitate (caffeine), which redissolves when about 25 parts of water have been added. It is also soluble in a mixture of two volumes of chloroform and 1 volume of ether.

Dose.--H., gr.xv.-xxx. (1.-2.); D., gr.i.-vi. (.06-.36).

Action Internal.-Caffeine has no particular action upon the digestive tract, except in large quantities, when it may cause gastro-intestinal irritation. The effect of caffeine and raw coffee is identical, but when the latter is roasted, aromatic oils, or an empyreumatic oil, are developed (caffeone). It is impossible to separate completely the action of caffeine from caffeone in boiled coffee. Recent investigations appear, to show that the oil (caffeone), of which there is from one to 
one-half drachm in a cup of the beverage, has no physiological action except to disturb digestion and cause biliousness. This result is not in accord with the general belief, and it is probable that caffeone possesses a transient action in stimulating intestinal peristalsis, the cerebral functions, and in lessening vascular tension.

Circulation.-Caffeine increases blood pressure by making the heart beat more forcibly and rapidly. The systolic contractions are longer and stronger. Experimental eridence concerning the physiological details of its action upon the heart is contradictory. The vasomotor centres are not influenced by the drug except in poisoning, when both the heart muscle and vasomotor system are depressed, and the heart stops in diastole after cessation of respiration.

Nervous System. - Caffeine is a certain and direct stimulant to the cerebral cortex. It produces wakefulness and restlessuess, and stimulates the reasoning and imaginative faculties in man. In the lower animals caffeine of ten causes the most intense cerebral excitement and mauia in large doses. The alkaloid also stimulates the spinal notor area, and toxic quantities prodnce restlessness, increased reflex excitability and convulsions in the lower animals. It is. undetermined whether the convulsions are of cerebral or spinal origin. The motor and sensory nerves are unaffected by medicinal doses. Alcohol and opium stimulate the brain, but do so incoördinately. This is nct the case with caffeine.

Kidneys.-The kidneys primarily contract for two or three minutes with corresponding diminution in the flow of urine, after the subcutaneous injection of small doses of caffeine. This effect is independent of the general vascular condition, and is followed by an increase in the volume of the kidneys, with accompanying angmentation of the urinary secretion, as shown by experiments with Roy's oncometer. The renal cells are directly stimulated. The drug is therefore a local diuretic and one of the most efficient and powerful.

Respiration.-The respiration is not affected to any 
considerable extent by caffeine, save by toxic doses, when it is depressed.

Metabolism.-The action of caffeine (and coffee) upon tissue change is inconstant. Sometimes it restrains nitrogenous consumption with corresponding diminution of urea elimination. Caffeine is entirely decomposed in the body, unless ingested in large quantities, when it is eliminated in part in the urine.

Toxicology.-Caffeine is a spinal and muscle poison to the frog. Tetanic convulsions occur in the batrachian similar to those produced by strychnine, but there is also muscular rigidity. These phenomena follow the direct stimulation of the spinal motor tract and muscles, and are succeeded by paresis. In man, stimulation of the cerebral cortex occurs, while both stimulation of the brain and cord are observed in the domestic animals. The symptoms of poisoning in dogs, cats and mammals generally, are restlessness, occasionally vomiting in dogs, rapid breathing, primary reduction followed by rise in temperature, clonic or tonic convulsions, muscular weakness, and general paresis. Death occurs from respiratory failure. The minimum fatal dose is about $1 \mathrm{gr}$. to $1 \mathrm{lb}$. of live weight in the cat.

Administration. - Caffeine and its ordinary salts are decomposed by water and therefore should not be given subcutaneously. The alkaloid is best administered in solution with sodium salicylate, or benzoate, for use under the skin as follows :-

$\mathrm{B}$

Caffeinæ...........................

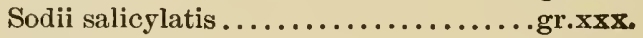

Aquæ..........................

M.

S. Give fifteen drops hypodermically to horse.

Three drops to dog.

or :-

B

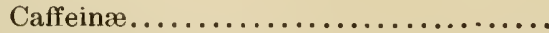

Sodii benzoinatis.............. āā gr.xv.

M.

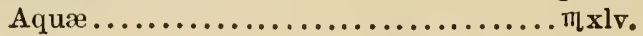

S. Give one-half, under the skin, to horse. 
The soluble citrate may be given internally, but it is not a defiuite or reliable preparation, and inferior to caffeine, which may be administered in solution with a little citric acid, or as above.

Uses.-Three indications for the use of caffeine may be deduced from our knowledge of its physiological action. 1. To stimulate the brain. 2. To stimulate the heart. 3. To canse diuresis:

1. Its application as a cerebral stimulant is very limited in veterinary medicine. It acts thus as an antidote to opium poisoning. One and a half grains of caffeive will save cats poisoned by the minimum fatal dose (gr.1 $\frac{7}{8}$ ) of morphine.

2. Caffeine possesses particular value in the treatment of dropsy of cardiac origin in dogs, because of its diuretic action, as well as stimulant effect upon the heart. The therapentic indications are much the same as for digitalis, but caffeine differs from digitalis in the following particulars:

Does not prolong diastole. Is not cumulative.

Does not slow the heart. More transient in its effect.

Nor regulate the leart.

Caffeine, in combination with acetanilid and other modern coal tar products, is useful in preventing their depressing action upon the heart. Furthermore, caffeine, tea and coffee are serviceable in the treatment of acute asthenic diseases in the horse (influenza), dog (distemper), and in foals and calves, acting as nervous and cardiac stimulants and perhaps restraining tissue waste.

3. The diuretic property of caffeine renders it appropriate in aiding the absorption of pleuritic effusion, ascites, and dropsies, particularly of cardiac and renal origin, and in the hepatic form as well. 


\section{SECTION II.-DRUGS ACTING ON THE SPINAL CORD.}

\section{Class 1.-Stimulating the Inferior Cornua.}

Nux Vomica. Nux Vomica. (U. S. \& B. P.)

Synonym.-Semen nucis vomicæ, poison nut, Quaker button, E.; noix vomique, Fr.; krähen-augen, brechnuss, G.

The seed of Strychnos nux-vomica Linné (nat. ord. Loganiaceæ).

Description. - Abont $25 \mathrm{~mm}$. in diameter, orbicular, grayish, or greenish-gray, soft, hairy, of a silky lustre, with a slight ridge extending from the centre of one side to the edge; internally horny, somewhat translucent, very tough, with a large circular cavity into which the heart-shaped, nerved cotyledons project. It is inodorous and persistently bitter.

Constituents.-Two alkaloids. 1. Strychnine, 0.2-0.6 per cent. 2. Brucine $\left(\mathrm{C}_{23} \mathrm{H}_{26} \mathrm{~N}_{2} \mathrm{O}_{4}\right), 0.5-1.0$ per cent. Similar in action to strychnine, but weaker and slower. Both alkaloids exist in combination with igasuric acid. Brucine occurs in rectangular octohedral crystals; is soluble in alcohol, in 7 parts of chloroform, and possesses a bitter taste. With sulphuric and nitric acids a beautiful blood-red color is developed.

Dose.-H. \& C., 3 i.-ii. (4.-8.); Sh., gr.xx.-xl. (1.3-2.6); Sw., gr.x.-xx. (.6-1.3); D., gr.i.-ii. (.06-.12).

\section{PREPARATIONS.}

Extractum Nucis Vomice. Extract of Nux Vomica. (U. S. \& B. P.)

Made by maceration with alcohol, water and acetic acid; percolation with alcohol and water; distillation to remove alcohol, and evaporation. The liquid extract is treated with ether, and sufficient sugar of milk is added to make the content of alkaloids equal to 15 per cent. of of the dry extract. (U. S. P.)

Dose.-H. \& C., gr.vii.-xv. (.5-1.); Sh., gr.ii.ss.-v. (.15-.3); Sw., gr.i.-ii. (.06-.12); D., gr. $\frac{1}{8}-\frac{1}{4}(.008-.016)$. 
Extractum Nucis Vomica Fluidum. Fluid Extract of Nux Nomica.

(U. S. P.)

Made by digestion and percolation with alcohol, water and acetic acid. The alcohol is distilled off and the solution evaporated. Alcohol and water are added so that $100 \mathrm{Cc}$. of the fluid extract shall contain Gm. 1.5 of total alkaloids.

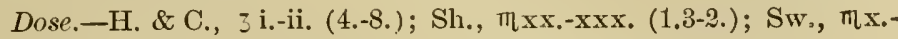
xx. (.6-1.3); D., mi.-ii. (.06-.12).

Tinctura Nucis Vomica. Ticture of Nux Vomica. (U. S. \& B. P.)

Made by solution of the extract of nux vomica, 20, in alcohol, and water to make 1,000 . (U. S. P.)

Dose. -D., $\Pi \mathrm{l} .-\mathrm{x} \cdot(.3-.6)$.

\section{Strychnina. Strychnine. $\mathrm{C}_{21} \mathrm{H}_{22} \mathrm{~N}_{2} \mathrm{O}_{2^{*}}$ (U. S. \& B. P.)}

An alkaloid obtained from nux vomica, and also obtainable from other plants of the natural order Loganiaceæ.

Derivation. - Nux vomica seeds are powdered and strychnine is extracted with water acidulated with hydrochloric acid. The solution is concentrated and strychnine precipitated with lime. It is then redissolved in boiling alcohol and the crystals are deposited npon concentration of the solution.

Properties.-Colorless, transparent, octohedral, or prismatic crystals, or a white, crystalline powder; odorless, and having an intensely bitter taste, perceptible in even highly dilute ( 1 in 700,000$)$ solution. Permanent in the air. Soluble in 6,700 parts of water, and in 110 parts of alcohol; in 2,500 parts of boiling water, and in 12 parts of boiling alcohol; also soluble in 7 parts of chloroform, but almost. insoluble in ether. Upon ignition, it is consumed, leaving no residue. No coloration is produced with nitric or sulphuric acid, but a drop of the latter and a grain of potassium bichromate, added to a crystal of strychnine upon a porcelain plate, yields a beautiful violet, changing into purplishred and cherry-red, and fiually to orange or yellow.

Dose.-Same as strychnine sulphate or nitrate (minimum quantities), which are preferable on account of their greater solubility. 
Strychninæ Sulphas. Strychnine Sulphate.

$$
\left(\mathrm{C}_{21} \mathrm{H}_{22} \mathrm{~N}_{2} \mathrm{O}_{2}\right)_{2} \mathrm{H}_{2} \mathrm{~S} \mathrm{O}_{4}+5 \mathrm{H}_{2} \mathrm{O} \text {. }
$$

(U. S. \& B. P.)

Derivation.-Made by the action of sulphuric acid on strychnine.

Properties.-Colorless, or white, prismatic crystals, odorless, and having an intensely bitter taste, perceptible in highly dilute $(1$ in 700,000) solution. Efflorescent in dry air. Soluble in 50 parts of water and in 109 parts of alcohol; 2 parts of boiling water and in 8.5 parts of boiling alcohol. Almost insoluble in ether. Upon ignition, it is consumed, leaving no residue. On adding potassium or sodium hydrate to an aqueous solution of the salt, a white precipitate is thrown down, which should conform to the test for strychnine.

Dose.-H., gr.ss.-i.ss. (.03-.09); C., gr.i.-iii. (.06-.18); Sh., $\mathrm{gr}^{1} \frac{1}{4}-\frac{1}{2}(.015-.03) ; \mathrm{D} ., \mathrm{gr} \cdot \frac{1}{120}-\frac{1}{40}(.0005-.0015)$.

The dose of strychnine should be proportioned to the weight in the case of all animals, but more particularly dogs. Otherwise, convulsive attacks or a fatal result may occur. The dose can be accurately determined, according to Feser, by the following data:

Dose per kilo (2 lbs. $3 \mathrm{oz} .119 .9 \mathrm{gr}$, avoirdupois) live weight.

Horse, sheep and cattle.... .0001-.0002 ( $\left(\mathrm{gr} \cdot \frac{1}{600}-\frac{1}{30} \overline{0}\right)$

Swine................0002.0003 ( $\left.\mathrm{gr} \cdot \frac{1}{300}-\frac{1}{2} 0 \overline{0}\right)$

Dogs....................

In accordance with the foregoing figures, dose for

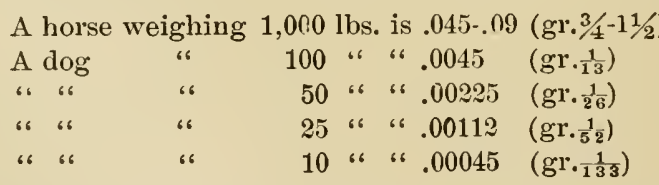

Moreover, these doses may be given every three hours withont producing poisoning by their cumulative action. Notwithstanding the foregoing figures, caution should be exercised in prescribing strychnine to dogs, as these animals 
appear sometimes extremely susceptible to strychnine, and it is therefore advisable to begin with a minimum dose (gr. $\frac{1}{120}$ ) in small animals.

The dose of one grain should not be ordinarily exceeded in the case of horses, as one and one-half grains proved fatal in an exceptional case. If animals are depressed by disease or shock, they will often bear much larger doses than could ordinarily be administered with safety.

Action External.-Strychnine is a powerful antiseptic, but is of no practical value on account of its poisonous properties. Brucine is a local anæsthetic.

Action Internal.-Digestive Tract.--Strychnine and nux vomica act as bitter stomachics in increasing vascularity, appetite, gastric secretion and motion. In addition to this they improve the local tone of the alimentary canal; probably by exciting the various spinal centres. Strychnine also stimulates the intestinal muscular tunic and therefore increases peristaltic action.

Circulation.-Strychnine excites the heart muscle and motor ganglia, and thus makes the cardiac pulsations more forcible. Large doses diminish the frequency of the frog's heart, and produce diastolic pauses. Blood tension is heightened by medicinal doses, through stimulation of the medullary vasomotor centres. Toxic quantities cause vasomotor paralysis and fall of arterial tension. Experiments conducted with blood (Harley) containing strychnine, shaken with air for 24 hours, resulted in the blood yielding about one-third more oxygen and one-half less carbonic dioxide, than blood, minus strychnine, similarly treated. Harley concluded that strychnine arrests oxidation in the body, but there is no physiological evidence that such is the case in life.

Nervous System and Muscles.-Strychnine is essentially a spinal stimulant. Many ingenious experiments conclusively prove that the principal effect of strychnine (convulsions) is produced by direct stimulation of the cells of the inferior cornua. The alkaloid also appears to cause dilatation of the arterioles in the spinal cord. 
Two experiments only may be presented. The first proves that the action of strychnine in producing convulsions is of spinal origin; the second that the alkaloid acts upon the inferior cornua.

1. When strychnine is placed directly upon the spinal cord of a frog, after the blood has been withdrawn from the body, and allowed to diffuse through its substance, convulsions occur. These are limited, at first, to those muscles whose nerves have their insertion nearest the point of application of the poison, and then they progress as the alkaloid continnes its path of absorption throngh the cord.

2. When every part of a frog below the second cervical vertelura has been removed, save the nerves, muscles and bones, and the inferior cornua be cut under the third cervical vertebra and the tissues entirely separated, so that the fore part of the frog is connected with the hind part only by the superior columns, then strychnine dropped in the mouth produces tetanic spasm solely in the fore legs. This shows that the cells of the inferior columns only were those affected. Furthermore, it is probable that other centres of the entire spinal tract, including the medulla, are stimulated to a less degree. Thus the respiratory centres, the vasomotor centres, as well as the reflex and trophic centres, are excited; but. the medullary cardiac centre is but slightly, if at all, affected by moderate doses. The cerebrum is not influenced by the drug, as consciousness is retained throughont the period of poisoning until asphyxia supervenes. The muscles and sensory nerves are nnaffected by any dose of strychnine. But the motor nerves are paralyzed at once by enormous doses of the alkaloid; or are paralyzed in the later stages of poisoning by less amounts. Therefore, convulsions do not appear at all, or cease when this condition is reached. An experiment performed upon a frocr, poisoned with strychnine, in which all the tissues of one hind leg were previously ligated, except the sciatic nerve, showed that stimulation of the intact leg caused spasms only of the ligated leg, since in the latter the motor nerve fibres had not been reached by the drug- 
(because of the ligation), and therefore were not paralyzed as were those in the limb left intact.

Respiration.-It is probable that strychnine, in therapentic doses, is the most reliable and powerful respiratory stimulant at our command. The breathing is quickened and deepened by the slimulating action of the alkaloid on the medullary respiratory centres.

Organs of Special Sense.-The eye-sight and hearing are rendered more acute by strychnine.

Elimination. - Strychnine escapes to some extent $\mathrm{mu}-$ changed in the urine, while a portion of it is converted into strychnic acid. The alkaloid' is found in the urine within half an hour of its ingestion, and is said to be entirely eliminated within forty-eight hours. Strychnine is one of the drugs that is supposed to possess a cumulative action, $i$. e., which exhibits sudden and powerful effects after its continuous administration. This is said to be due to the fact that it is not eliminated as rapidly as it is ingested, owing to contraction of the renal vessels, and that it accumulates in the spinal cord. The cumulative effect is not seen when the drug is given subcutaneously; on the contrary, the system becomes so habituated to it that ordinarily poisonous doses can then be given with safety. Therefore, when a cumulative action is observed, it is probably owing to delayed absorption.

Administration.-For tonic purposes, strychnine may be given to dogs in pills or tablets, and to horses in solution dropped on the tongue. Nux vomica is given to horses upon the food in the form of powder, or in fluid extract upon the tongue; while the tincture is more appropriate for dogs. When large doses of strychnine are used, or an immediate action is desired in acute diseases and emergencies, the alkaloid should be given under the skin.

Toxicology. - One-twentieth of a grain of strychnine nitrate, injected subcutaneously by the writer into a dog weighing 25 lbs., caused uneasiness and excitement, with protrusion of the eye-balls, and in the space of ten minutes, 
tetanic convulsions. The breathing was shallow and almost imperceptible, the pulse rapid and irregular, the lips were covered with foam, the tail was stiff and exteuded, the ears laid back, and there was general muscular :igidity, the animal lying on his side in a state of opisthotonos. This condition lasted about three minutes, and was followed by a period of relaxation. But the slightest noise or intritation of the skin brought on convulsions. The convulsions became less frequent and violent, and ceased altogether within half an hour. The same animal was given gr. $\frac{1}{40}$ of th:s 2 ilkaloid on the following day, but without producing any appreciabla result. One-tenth of a grain, given on another cay and in the same manner, caused immediate uneasiness and restlessness, and in ten minutes induced a severe convulsion, lasting for three minntes, in which the animal was so rigid that he conld be lifted bodily without bending. The ears were drawn back, the limbs were extended and stiff, the tail was straight and rigid, and there was twitching of the muscles of the jaw and limbs. The corners of the mouth were drawn back (risus sardonicus), the mouth was covered with foam, and there was some trismus. The breathing was nearly suppressed, owing to tetanic spasm of the respiratory muscles. Following this convulsion, the jaw dropped, the muscles relaxed and another attack could not be produced by noises or external irritation. Some twitching of the temporal muscles persisted. Evidently the second stage of poisoning had ensued, and the motor nerves and cells of the inferior cornna had become paralyzed. Death occurred in general paralysis within half an hour, and without any recurrence of convulsions or tetanic condition. Death takes. place more commonly in strychnine poisoning from asphyxia, during a convulsion, and is caused by spasm of the respiratory muscles, or, more rarely, by spasm of the glottis. Sometimes a fatal result ensues from exhaustion, between the paroxysm, and occasionally death appears to follow the intense action on the nervous system, and depression of the heart. 
The lethal dose for dogs has been set at gr. $\frac{1}{6}$-gr. $\frac{1}{3}$ (Kaufmann). This is much too large, as evidenced by the experiment mentioned above. The fatal amount varies greatly in accordance with the weight of an animal; probably less than gr. $\frac{1}{20}$ would kill toy terriers, and cases are reported where they have been destroyed by $\mathrm{gr}^{-\frac{1}{60}}$ of the alkaloid. The therapentic dose should therefore be proportioned as advised, to the weight of the animal. Five to eight grains of nux vomica will kill dogs.

The minimum fatal dose of strychnine for man is onehalf a grain. Usually four to seven grains constitute a lethal quantity, but recovery has ensued following the ingestion of 22 grains, after \& full meal.

Horses.-The toxic symptoms in horses resemble those already described in the dog. They do not appear for some time (20 minutes to 6 hours), depending on the rapidity of absorption when the drug is swallowed, and include excitement, muscular spasm and convulsions, increased frequency of the pulse, and difficult respiration. Death occurs in convulsions or in the interim between them. The minimum fatal dose of strychnine, when given under the skin, is about $1 \frac{1}{2}$ to 3 grains, and when swallowed, 3 to 5 grains of the alkaloid, or 1 to 2 ounces of nux vomica.

Cattle are similarly affected with horses and dogs. There are exhibited muscular spasms, frequent pulse, difficult respiration, sensitiveness to light, sounds and external stimuli, protrusion of the eye-balls and convulsions. The fatal dose, by the month, varies greatly owing to difficulty of absorption in the complicated and capacions digestive apparatns of these ruminants. This is true of all medicines. When given under the skin, the lethal dose is a little larger than that for horses. The fatal dose for swine is said to be from $\mathrm{gr} . \frac{1}{6}-\mathrm{gr} \cdot \frac{3}{4}$. Chickens are comparatively insusceptible; also guinea pigs and some monkeys.

Strychnine poisoning differs from tetanus in the fact that muscular rigidity is continuous in the latter, but disanpears to a considerable degree, if not completely, in the 
periods between the convnlsions, in the case of strychnine poisoning. Moreover, in tetanus the body and limbs are less, and the jaw more affected; while in strychnine poisoning the condition is reversed.

Treatment.-The treatment embraces the nse of chemical antidotes, as iodine or its salts, or tannic acid; animal charcoal and emetics or the stomach tube, before absorption has occurred. The best physiological antidote is chloral in large doses per rectum. Chloroform and nitrite of amyl may also be given by inhalation, and quiet and rest enforced. Artificial respiration is of no service on account of the muscular spasms, unless air be forcibly driven into the trachea through a cannla. Calabar bean and gelsemium both depress the inferior cornua, but neither are of much value in strychnine poisoning.

Post-Mortem Appearances.-These are simply those of asphyxia, with the usual congestive lesions and dark fluid blood, except that sometimes spinal hyperæmia is observed.

Uses Internal.-The indications for the employment of strychnine may be directly deduced from its physiological actions. The indications are as follows:

1. To stimulate the heart and respiration.

2. To stimulate the spinal cord; more particularly the motor cells of the inferior cornua.

3. To stimulate appetite, digestion, and intestinal peristalsis in atonic conditions.

1. Strychnine is the best remedy we can employ to stimulate the heart, respiration and nervous system, and to promote appetite and digestion in acute diseases. No better drug can be used in the treatment of pneumonia and influenza in horses. Strychnine should not be usol continuously, but only to bridge over a period of weakness or collapse. It may be combined in the first stages of pneumonia with nitrites, as spirit of nitrous ether, or nitroglycerin. The latter dilate arterioles, equalize the circnlation by preventing venous stasis, and engorgement of the right heart; while 
strychnine is preferable to digitalis as a heart stimulant, because it does not so powerfuly contract the arterioles. Strychnine is useful in influenza by counteracting nervous depression and improving the appetite and digestion. In collapse and shock, following surgical operations, or in ether and chloroform poisoning, strychnine is invaluable as a cardiac and respiratory stimulant, given in large doses subcutaneously. In chronic heart disease, strychnine is a useful remedy. The alkaloid has proved extremely serviceable in chronic brouchitis, emphysema, and in that combination of these clisorders, often associated with asthma and known as "broken wind" or "heaves," in horses.

The beneficial effect in these diseases is explained by the stimulating action of the drug on the respiratory centres, and the favorable influence exerted on digestion.

2. In mild cases of cliorea in dogs, strychnine is prescribed to advantage with Fowler's solution. In accordance with its physiological action, strychnine is indicated in varions paralyses of spinal origin, but should not be used where there is irritation or inflammation of the spinal motor tract. It is employed in hemiplegia and paraplegia, resulting from hæmorrhage, after the lapse of several weeks, when inritation prodnced by the clot has passed away. Strychnine is sail to ive efficacious in the after-treatment of cerebrospinal meningitis of horses, and also in the paraplegia of cattle (from parturient fever), and that of dogs resulting from varions and often undiscovered canses. Strychnine is serviceable in the paralysis of lead poisoning, and in that form caused ly traumatism or toxæmia following influenza, distemper in rlogs, and rheumatism. When injected into the muscular tissue, the alkaloid is believed to stimulate the peripheral nerves and muscular fibres, and is employed with benefit in Jocalized paralyses affecting groups of muscles before atrophy has occurred, as in "roaring" in horses.

Either retention or incontinence of urine, resulting from atony or paralysis of the bladder, may be relieved by strych-. nine; also prolapse of the rectum induced by similar causes. 
Strychnine has proved curative in some cases of amaurosis, when injected in the region of the temple.

3. Strychnine or nux vomica, associated with iron and arsenic, form the most generally satisfactory tonic combination for the horse and dog, particularly with reference to the digestion. Atonic forms of indigestion and constipation, the accompaniments of anæmia, are those amenable to the inflnence of the drug, which stimulates peristalsis, and therefore hinders fermentation, prevents relaxation, and so oversecretion from loss of vascular tone.

Strychnine is a valuable remedy in overloaded rumen and chronic tympanitis of cattle: or in overloaded and impacted large intestines of the horse. It should be given subclitaneously in these conditions, and trequently in cosnbination with pilocarpine and eserine.

Constipation, and, on the other hand, diarrhoea, are benefited by strychnine when they are due to atony of the intestival muscular coat. It is more commonly in the former disorder that the drug finds its usefulness. The aloin, strychnive and belladonna pills, with cascara sagrada, are useful in habitual constipation in dogs, but the weaker formula, containing $\mathrm{gr}^{-\frac{1}{120}}$ of strychnine, should be employed. The value and application of strychnine in anorexia and atonic digestive disorders has been sufficiently treated above.

\section{Class 2.-Depressing the Inferior Cornua.}

\section{Physostigma. Physostigma. (U. S. P.)}

Synonym.-Physostigmatis semen, B. P. ; Calabar or ordeal bean, faba physostigmatis, S. faba Calabarica, E. The seed of Physostigma venenosum. Balfour (nat. ord. leguminosæ).

Habitat.-Calabar and the region of the mouth of the Niger, in Western Africa.

Description.-About 25 to $30 \mathrm{~mm}$. long, 15 to $20 \mathrm{~mm}$. 
broad, and 10 to $15 \mathrm{~mm}$. thick; oblong and somewhat reniform; testa granular, chocolate-brown, with a broad, black groove extending over the entire length of the convex edge. Embryo with a short, curved radicle and two large, white, concavo-convex cotyledons; inodorous; taste bean-like.

Constituents. - The principal constituent is the alkaloid plyysostigmine, or eserine. There are also the alkaloids calabarine, a product resulting from the decomposition of eserine, and eseridine, similar in action to eserine, but weaker; and a neutral principle, physoterin, resembling cholesterine.

Calabar Bean Dose.-H., gr.xv.-xxx. (1.-2.); D., gr.1-i. (.015-.06).

The official preparations are the fluid extract and tincture of physostigma, but physustigmine is solely used in veterinary medicine, since it is more certain and generally free from calabarine, which produces, in toxic doses, a tetanic condition followed by paralysis.

Physostigmine Salicylas. Physostigmine Salicylate.

$$
\mathrm{C}_{10} \mathrm{H}_{21} \mathrm{~N}_{3} \mathrm{O}_{2} \mathrm{C}_{7} \mathrm{H}_{6} \mathrm{O}_{3} \text {. (U. S. P.) }
$$

Synonym.-Eserine salicylate. The salicylate of an alkaloid obtained from physostigma.

Derivation.-Physostigmine is obtained from the alcoholic extract of Calabar bean by dissolving the extract in water, adding sodium bicarbonate, shaking the mixtnre with ether, and evaporating the ethereal liquid. The salicylate of the alkaloid-the most stable salt-is made by adding physostigmine to a solution of salicylic acid in boiling. distilled water, when the salt crystallizes on cooling.

Properties.-Colorless, or faintly-yellowish, shining, acicular, or short, columnar crystals; odorless, and having a bitter taste. Soluble in 150 parts of water, and in 12 parts of alcohol; in 30 parts of boiling water, and very soluble in boiling alcohol. (U. S. P.)

Dose. - H. \& C., gr.i.ss.-iii. (.09-.18); D., gr. $\frac{1}{100}-\frac{1}{30}$ (.0006-.002). 
Given usually intravenously or subcutaneously to. horses.

Physostigminz Sulphas. Physostigmine Sulphate.

U. S. P.)

Synonym.-Eserine sulphate.

The sulphate of an alkaloid obtained from physostigma.

Properties.-A white, or yellowish-white, micro-crystalline powder, odorless, and having a bitter taste. It is very deliquescent when exposed to moist air, and gradually turns, reddish by exposure to air and light. Very soluble in water and in alcohol.

Dose.-H. \& C., gr.i.ss. - iii. (.09-.18); D., gr. $\frac{1}{100}-\frac{1}{30}$ (.0006-.002).

Given usually intravenously or subcutaneously to horses.

\section{PHYSOSTIGMINE.}

Action Internal.-Alimentary Tract.-The flow of saliva is at first stimulated, whether by direct excitation of the 'salivary cells or not, is undetermined. Salivation ceases when the gland is deprived of blood by general vascular contraction. The peristaltic action of the stomach and bowels is increased by the direct local action of the alkaloid on the muscles or ganglia of their walls. There are three stages, in reference to this action, observed in poisoning. First there is stimulation of peristaltic action, then tetanic contraction and diminution of the intestinal calibre, and finally relaxation and dilatation of the bowels. The secretions in the digestive tract are augmented with the expulsion of considerable mucus per rectum. When the alkaloid is given to horses, under the skin, within the trachea or intravenously, defæcation and expulsion of gas commonly occurs in the space of half an hour, occasionally in a few minutes, and is often considerable in amount (11-20 lbs. of fæces).

Circulation.-Eserine is readily absorbed, but exerts' no 
inflnence on the blood. Moderate doses render the cardiac pulsations slower and more forcible, and increase vascular tension. Large toxic doses cause the heart to beat more rapidly and less forcibly. The first effect is due probably to stimulation of the peripheral vagi and heart muscle, and possibly the involuntary muscular fibres in the vessel wails. The second phenomenon follows depression of the heart and peripheral vagi, and terminates in cardiac paralysis and diastolic arrest. The action on the circulation is entirely subordinate to the influence of the drug upon the nervous system, and is unimportant from a therapeutical standpoint.

Nervous System and Mruscles.-The essential physiolngical action of physostigmine cousists in depressing the cells of the inferior cornua. This has been abundantly and directly proved by application of the alkaloid to the spinal cord; anl, indirectly, by diviling the posterior from the inferior portion of the cord, by stopping the blood supply to the latter part, when the drug cansed loss of motor power and abolition of reflex action in the inferior portion, while joflex phenomena were undisturbed in the superior portion (Frizer). The superior columus are finally depressed; perception of pain is wanting, but that of tonch persists. The sensory nerves are not affected, and the motor nerve trunks but slightly. The muscular tremors are dne to stimulation of the voluntary muscles themselves, or to the motor nerve "nclings. These are characteristic of physostigma poisoning in animals-not in man-and are probably due to calabarine, which shonld not be present in pure physostigmine. The involuntary muscles throughont the body are stimulated, including those of the stomach, intestines, bionchial tubes, heart, blood vessels (?), spleen, uterus, bladder and iris. In the case of some of these organs, it has not been decided whether the muscles themselves, or the motor nerve terminatious, are affected.

Respiration.-The respiration is not disturbed by medicinal doses. Toxic quantities at first quicken, and then 
retard the respiratory movements, and death occurs from. asphyxia, before cessation of the heart, owing to paralysis of the medullary and spinal respiratory centres. The acceleration of breathing is due to the stimulation of the pulmonary vagal endings, and possibly to constriction of the unstriped muscle of the bronchial tubes.

Secretions.-Secretion is generally increased, including that of the salivary, gastric, intestinal, sudoriparous and lachrymal glands. In this respect eserine is antagonistic to atropine.

Eye.-Physostigmine is a myotic, applied locally or administered internally. Intraocular tension is diminished and there is spasm of accommodation. In all probability contraction of the pupil is brought about by stimulation of the oculomotor nerve endings simultaneously with paralysis of the terminations of the sympathetic nerve in the iris. Eserine is thus directly antagonistic to atropine in its effect upon the eje, but they resemble each other in that they both exert a local action and do not affect the irides of birds. Euormous doses of physostigmine paralyze the oculomotor nerves and dilate the pupil.

Elimination.-Eserine is rapidly absorbed and eliminated, mainly by the urine, but also in the other secretions.

Toxicology.-Physostigma has been called "ordeal bean," becanse native Africans suspected of crime are given the crude drug. Vomiting it, they are proved innocent and survive the ordeal. Retaining it, they die, and so are properly and primitively punished. Animals poisoned by Calabar bean exhibit muscular tremors which continue throughout the toxic period, and are often so violent as to simulate convulsions. Soon there is loss of muscular power and the animal falls or lies down. The respiration becomes rapid, labored, and stertorous; the pulse is increased in frequency by large toxic doses, and the temperature slightly elevated. There are salivation and sweating. The pupil is sometimes contracted and, when enormous lethal doses have been injected, dilated. Vomiting occurs in animals capable of 
the act, and loud peristaltic noises are heard, followed by the expulsion of fæces, mucus and flatus, with colicky pains and tenesmus. Reflex action is diminished or abolished, but sensation is preserved until late in the toxic period. The muscles are completely relaxed and powerless, notwithstanding the tremors which afflict them. The breathing becomes weak and irregular, and death occurs from respiratory failure.

In experiments of Winslow and Muir conducted independently and at different times the following cases may be of interest :

A healthy gelding, weighing 1,050 lbs., was strapped upon the dissecting table and given three grains of eserine sulphate intrajugularly. Within a few minutes slight muscular tremors appeared in the neck; the pulse rose to 120 , the respiration was 24 , and the temperature normal. There was slight sweating. The pulse soon fell to 60 , and was strong and hard, while the respiration became rapid and labored. No other symptoms developing within twenty-five minutes after the first dose, three grains of the alkaloid were administered in the same manner as before. The pulse became more frequent (78), the respiration (48) was difficult, and the muscular tremors increased in violence till they resembled convulsions. Sweating was profuse, while șaliva dropped freely from the mouth. The respiration and pulse were now reduced in frequency and became weaker. The pupils were unaffected, but the eyes were staring. Half an hour after the second dose increased peristaltic action was evidenced by loud noises and the expulsion of gas and dung. Recovery began in two hours from the exhibition of the second dose. It is possible that the severe muscular tremors were caused by contamination of the eserine with calabarine, as the former was not a fresh preparation. An aged express horse, suffering for a week from impaction of the colon, was given twelve minims of a commercial extract of calabar bean. He fell down almost immediately, perspired freely, exhibited muscular tremors, and expired within a few minutes. The writer is unable to state the minimum fatal dose for horses, but eserine should be used with caution in weak subjects.WINSLOW. 
A gelding, $800 \mathrm{lbs}$. poorly nourished, temperature normal, pulse 60 , was given physostigmine sulphate gr.11/2 (0.1) in $1 / 2$ drachm of water intrajugularly. Muscular tremors and colic came on in seven minutes; in eleven minutes defæcation occurred, the heart became more rapid and colic increased. Passages from the bowels were frequent for two hours-about fifteen in all-the frees weighing twenty pounds in the aggregate. All symptoms abated after three hours from the time of receiving the drug.-Muir.*

A gelding, thirteen years old, in good condition and weighing 1,205 lbs., temperature and pulse normal, was given 3 grains $(0.18)$ of eserine sulphate under the skin. After thirty-two minutes elapsed, defæcation began and continued with the expulsion of considerable mucus and flatus until six passages had occurred within one hour and twenty minutes. During this time the pulse had increased in frequency and the animal was restless and pawing the ground. An hour and a half after the first dose, a second dose of 3 grains (0.18) was given in the same way. In twenty minutes muscular tremors and weakness became marked, for the first time, and great distress was evident, the patient appearing to be in much pain; the respiration short, jerky and labored, and nostrils distended and working. The evacuations continued until twenty-three had been passed from the time of receiving the first dose and until one and one-half hours after the second dose. The fæces were quite liquid and contained much mucus and during passage were attended with considerable straining. The weight of fæces aggregated 17 lbs. Four hours after the last dose all symptoms disappeared.MUIR.*

Summary. - Full medicinal doses of physostigmine (gr.1 $1 \frac{1}{2}-3$ ), given subcutaneously or intravenously, produce slight to considerable colic, increased peristalsis and mucous secretion, muscular tremors and weakness, and frequent defæcation-beginning in ten to twelve minutes after intravenous use, about thirty minutes or more after injection under the skin-and being twice as copious when the

* Jour. of Comp. Med. and Vet. Archives, Jan. and Feb., 1899. 
former mocie of administration is employed than after the latter. Moreover, the disagreeable accompaniments of purging last twice as long (five hours) after the hypodermic method than after the intravenous injection (two and a half hours). Muir deduces from his experiments that physostigmine is a safe and active cathartic for the horse and is free from evil after-effects; that along with increased peristalsis and mucous evacuation from the bowels there are colic and more rapid pulse-rate with muscular tremors and weakness; that eserine acts more quickly and effectively when injected into the external jugular vein, and that it may be safely given in this manner in a dose of 3 grains dissolved in 30 minims of water, to horses in fair condition and of average weight.

The toxic line is closely approached, however, when physostigmine is used in this way, and we should be careful not to cross it by exceeding the dose. Barium chloride acts as rapidly and withont the unpleasant accompaniments of purgation caused by eserine, so that the former is generally preferable as a cathartic for use by the intravenous method.

The minimum fatal dose is stated to be from $\mathrm{gr} \cdot \frac{1}{15}-\frac{1}{12}$ for dogs, and $\operatorname{gr} \frac{1}{30}-\frac{1}{20}$ for cats. The treatment should be pursued with emetics, or the stomach pump, and the antidote, atropine. The latter will prevent death in rabbits when given five minutes after the administration of three times a minimum fatal dose. Atropine should be given under the skin, together with alcoholic stimulants, digitalis, and ammonia by the mouth. Artificial respiration should be practised and external heat applied. The essential action of atropine in antagonizing eserine, consists in stimulation of the respiratory centres, while it depresses the peripheral cardiac vagi, and so, to a certain extent, counteracts the primary influence of eserine on the heart. Large doses of atropine may only exaggerate the secondary depression of eserine upon the peripheral vagi in the heart, and so hasten 
a fatal result. Strychnine is antagonistic to eserine in stimulating the respiratory centres and inferior cornua.

Uses External.-Physostigmine has been injected empirically, but it is asserted with good results, into chronic dropsical effusions of joints and bursæ of tendons in horses, after removal of the fluid by aspiration. From .05-.1 gm. is used, dissolved in 5.-10. gm. of sterile water ; and this treatment is followed by cold applications for several days afterwards.

Uses Internal.-Three physiological actions of eserine are put to therapeutical uses.

1. The myotic action on the eye.

2. Stimulation of the involuntary muscles.

3. Depression of the inferior cornua.

1. Eserine is useful, in alternation with atropine, to break up adhesions to the iris and to lessen intraocular tension in glaucoma, and, by this action, to prevent prolapse of the iris and staphyloma, after wounds and ulcers of the cornea. It is also employed to contract the pupil and shut ont the light in photophobia, and to antagonize the influence of atropine on the eye. The action of eserine is, however, not nearly so powerful or persistent as that of atropine on the eye, and is somewhat painful. It is employed in 1 per cent. solution.

2. Physostigmine is mainly of value for its action in rapidly stimulating the unstriated muscles of the alimentary canal. In obstiuate constipation of horses it is particularly useful; $1 \mathrm{gr}$. of eserine sulphate being given intravenously with $3 \mathrm{grs}$. of pilocarpine sulphate; the latter to increase the intestinal secretions. Strychnine sulphate (gr.1) may be added to counteract the depressing action of eserine on the respiratory centres and inferior cornua.

Physostigmine is prescribed in obstruction of the bowels, from twist or intussusception in the horse, and while there is danger that the condition may be aggravated by the increased intestinal movement, yet it may be employed when high rectal injections and elevation of 
the hind quarters of the patient fail. The alkaloid, in combination with pilocarpine, is serviceable for its speedy action in flatulent colic and impaction of the cæcum, colon, and rectum in horses, and has been employed to expel calculi and foreign bodies from the intestines.

Physostigmine is contraindicated in spasmodic colic, when it may increase the trouble by exciting intense peristaltic contractions. Its employment is also attended with some danger in overloaded or greatly distended stomach or bowels, in view of possible rupture of these organs. The drug, like other purgatives, may aggravate the damage produced by twist or intussusception.

In atonic conditions of the stomach and bowels, and in indigestion due to chronic intestinal catarrls, small and repeated doses of eserine are sometimes of benefit. There have recently been reported many successful cases of parturient apoplexy in cows treated with eserine. One or two grains are injected intravenously or subcutaneously to cause a rapid movement of the bowels. In these patients the drug is followed by strychnine subcutaneously to combat paralysis (see p. 409).

Eserine has been employed in chronic bronchitis, asthma and emphysema, to improve the tone of the bronchial mucous membranes and expel secretions.

3. Eserine is one of the drugs commonly used in the treatment of tetanus in human and veterinary practice, with only a moderate degree of success. It must be employed early, given every three or four hours, and pushed to the physiological limit. Chorea and epilepsy have been treated. with eserine without any pathological basis, as far as epilepsy is concerned, and with little therapentic advantage in either instance. In some cases of paraplegia, resulting from myelitis, a favorable effect has been obtained in man. Eserine is an appropriate purgative (hypodermatically) in acute encephalitis.

Physostigmine and pilocarpine are the two best antidotes in atropine poisoning. Eserine has been used in 
strychnine poisoning, but is inferior to chloral, bromides and anrsthetics, and while it is antagonistic to a cortain extent, and alters the character of strychnine convulsions, yet animals die more quickly when poisoned by both strychnine and eserine, than by strychnine alone.

Gelsemium. Gelsemium. (U. S. \& B. P.)

Synonym.-Radix gelsemii, yellow jessamine, E.; racine de jasmin jaune, Fr.; gelber jasmin-wurzel, G. The rhizome or roots of Gelseminm sempervirens (Linné) Persoon (nat. ord. Loganiaceæ).

Habitat.-Sonthern United States.

Description.-Cylindrical, long, or cut in sections, mostly from 5 to $15 \mathrm{~mm}$. and occasionally $3 \mathrm{~cm}$. thick, the roots much thinner; externally light yellowish-brown, with purplish-brown longitudinal lines; tongh; fracture splintery; bark thin, with silky bast-fibre closely adhering to the pale, yellowish, porous wood, which has fine medullary rays, and in the rhizome a thin pith; odor aromatic, heavy; taste bitter.

Constituents. - The most important is the first alkaloid. 1. Gelsemine, $\mathrm{C}_{54} \mathrm{H}_{69} \mathrm{~N}_{4} \mathrm{O}_{12}$. A colorless, crystallizable, bitter principle, soluble in alcohol and ether, and slightly in water. 2. Gelseminine, a brown, amorphous, bitter alkaloid. 3. Gelseminic acid. 4. A volatile oil.

Dose.-H., 3 i.-ii. (4.-8.); D., gr.v.-x. (.3-.6).

\section{PREPARATIONS.}

Extractum Gelsemii Fluidum. Fluid Extract of Gelsemium.

(U. S. P.)

Made by maceration, percolation and evaporation, so that $1 \mathrm{Cc}$. $=1 \mathrm{Gm}$. of the crude drug.

Dose.-H., 3 i.-ii. (4.-8.); D., Mv..x. (.3-.6).

Tinctura Gelsemii. Tincture of Gelsemium. (U. S. \& B. P.)

Gelsemium, 150; alcohol and water to make 1,000. Made by maceration and percolation. (U. S. P.)

Dose.-H., 3 ss.-i. (15.-30.); D., $\prod \times x$.- 3 i. (1.-4.). 
Gelsemina. Gelsemine.

(Non-official.)

Dose.-H., gr. $\frac{1}{4}-\frac{1}{2}(.015-.03)$; D., gr. $\frac{1}{60}-\frac{1}{3} 0(.001-.002)$.

Action External.-None.

Action Internal.-Gelseminm exerts no action on the digestive apparatus, or on the blood, after its absorption; neither does it affect the circulation in medicinal doses, but in toxic quantities it directly depresses the heart. The influence of the drug on the vasomotor system is unknown.

Nervous System and MIuscles. - The prominent effect of gelseminm is directed on the nervous system, as evidenced by paralysis and convulsions after large doses. Both the convulsant and paralyzant action are of spinal origin. That the convulsions are not cerebral is shown by the fact that they occur below the point of section in mammals (under the influence of the drug), with divided spinal cord. That they are not peripheral is proved by their occurrence in the hind extremities when the posterior aorta is tied before the animal is poisoned. The paralyzant action of gelsemium is due to direct depression of the cells of the inferior cornua, and this is followed, in the later stages of poisoning, by depression of the spinal sensory tract and general anæsthesia.

The cause of the secondary convulsions is undetermined. A tetanizing principle in gelsemium, antagonistic to gelsemine, such as we find in physostigma, has been thought to be the cause of the convulsions. The muscles, motor (except those of the face) and sensory nerves are unaffected by gelsemium.

Respiration.-Gelsemium, in toxic doses, progressively weakens and paralyzes the medullary and spinal respiratory centres, and death occurs from asphyxia.

Eye.-In poisoning, in animals, there is dilatation of the pupil produced in the latest stages of the toxic period. In man, there is, in addition to this, paralysis and drooping of the eyelids (ptosis), and paralysis of the recti muscles with 
strabismus. These symptoms have been attributed with apparent reason to paralysis of the motor cells in the floor of the fourth ventricle and aqueduct of Sylvius, since they are a prolongation of the spinal motor tract. As the pupil is dilated, however, by the application of much smaller doses than are required by the mouth, it seems probable that mydriasis results from local paralysis of the oculomotor nerve endings.

Toxicology.-Poisoning in animals is exhibited by muscular weakness, especially in the fore legs, staggering gait and falling. These symptoms are followed by convulsive movements of the muscles of the head, fore legs, and sometimes of the hind legs. 'The respiration is slow and difficult, the pulse feeble; temperature is reduced, and there is sweatung. Consciousness is preserved until the occurrence of asphyxii. Death takes place from respiratory failure, with almost simultaneous cardiac arrest. Morphine subcutaneonsily has proved a successful antidote. Respiratory and heart stimulants should be employed in poisoning by gelsemium, such as strychnine, atropine, alcohol, and digitalis, together with artificial respiration, after evacuation of the stomach.

Uses.-Gelsemium possesses little value in veterinary medicine. There is no therapeutic use to which it has been put that cannot be filled to better advantage by some other remedy. Thus, gelsemium has been employed as a cardinc depressant and antipyretic in acute diseases (pneumonia and pleurisy), but its other actions are disadvantageons and it is inferior to aconite, veratrum viride, or the modern antipyretics. In spasmodic diseases, irritable cough, vesical irritation, tetanus, chorea, etc., it is less satisfactory as a motor depressant than opium, belladonna, chloral, and bromides. Zuill, however, reports very successful results in horses from gelsemine $(.08 \mathrm{Gm}$.) in tetanus. The dose should be given under the skin every half hour till muscular relaxation occurs. The drug lias been used considerably to relieve rheumatic and neuralgic pains, but these succumb more 
readily to opium, coal tar products, etc. As a mydriatic, gelsemine is not to be compared with atropine for general purposes, but its action is more transient. A solution (gr.8$3 i$ ) is instilled (in man) in drop doses every fifteen minutes for one hour, and then every thirty minutes for two hours, to secure wide dilatation of the pupil ; or discs, containing gr. $\frac{1}{300}$ of gelsemine (with gelatine) are used for application to the eye.

\section{SECTION III.-DRUGS ACTING ON THE SPINAL CORD AND MOTOR NERVES.}

\section{Class 1.-Depressing the Inferior Cornua and Motor Nerves.}

\section{Tabacum. Tobacco.}

\section{Synonym.-Tabaci folia, B. P.}

The commercial dried leaves of Nicotiana Tabacum Linné (nat. ord. solanaceæ).

Habitat.-Tropical America. Cultivated in various temperate and tropical parts of the earth.

Description.-The leaves are up to $50 \mathrm{Cm}$. long, oval or ovate-lanceolate, acute, entire, brown, friable, glandularhairy, of a heavy, peculiar odor and a nanseous, bitter and acrid taste.

Constituents.-Chiefly nicotine, $\mathrm{C}_{10} \mathrm{H}_{14} \mathrm{~N}_{2}$ (0.7-5.-10 per cent.). A colorless, volatile, oily alkaloid, resembling tobacco in odor and taste. Freely soluble in alcohol and ether; less so in water. Nicotine is decomposed by heat and therefore tobacco smoke contains none of it, but in its stead, pyridine $\mathrm{C}_{5} \mathrm{H}_{5} \mathrm{~N}$, and varions allied alkaloids, viz.: picoline, $\mathrm{C}_{6} \mathrm{H}_{7} \mathrm{~N}$; lutidine, $\mathrm{C}_{7} \mathrm{H}_{9} \mathrm{~N}$; rubidine, $\mathrm{C}_{11} \mathrm{H}_{17} \mathrm{~N}$; coridine, $\mathrm{C}_{10} \mathrm{H}_{15} \mathrm{~N}$; parvoline, $\mathrm{C}_{9} \mathrm{H}_{13} \mathrm{~N}$; and collidine, $\mathrm{C}_{8} \mathrm{H}_{11} \mathrm{~N}$; together with small amounts of sulphur, creosote, acetic and hydrocyanic acids and carbon compounds. Pyridine resembles nicotine in depressing the spinal motor tract and 
in paralyzing respiration, and is said to be formed more in pipe smoke, while, in the smoke of cigars, the less harmful collidine is produced by dry distillation. Nicotine exists to a very slight degree in Turkish tobacco.

Dose.-Nicotine, H. \& C., gr: $\frac{1}{60}-\frac{1}{20}(.001-.003)$.

ACTION OF TOBACCO AND NICOTINE.

Action Externcl.-Tobacco is a local anodyne, antiseptic and parasiticide.

Action Internal. - Digestive Tract. - The physiological effect of tobacco is due to nicotine. Nicotine increases peristaltic action and, in large doses, causes tetanic spasm of the intestines, even when it is injected into the blood. In toxic quantities nicotine is a powerful gastro-intestinal irritant, and produces the usual symptoms of pain, vomiting (in animals capable of the act), purging and collapse.

Cinculation.- When nicotine is added to blood recently withdrawn from the body, this fluid assumes a dark hue, and the corpuscles quickly disintegrate. The blood, in poisoning, becomes dark colored, owing to asphyxia, but regains its arterial tint when removed from the body and shaken with air. It is only in poisoning by enormous quantities that changes in the red blood corpuscles can be detected microscopically, although the spectrum of hæmoglobin is altered in poisoning, proving that the corpuscles are in some way affected. Experiments with nicotine upon frogs show that there is produced a primary fall in blood pressure and pulse rate, followed by a rise in both, only to be succeeded by a return to the original depressed condition caused by the alkaloid.

The exact physiological data accounting for these circulatory phenomena have not been worked out, although they are probably dependent upon stimulation followed by depression of the inhibitory apparatus and vasomotor system.

Nervous System and Muscles.-Nicotine first stimulates, and then paralyzes the cells of the inferior cornua and motor 
nerves, so that in poisoning couvulsions are succeeded by paralysis. The cerebrum is unaffected; likewise the muscles, but the function of the sensory nerves is somewhat depressed.

Respiration.-The respiration is depressed in some unknown manner.

Eye.-Nicotine, in moderate toxic doses, or when applied locally, contracts the pupil. This action is exceptionial for a member of the solanaceæ. Myosis is produced in the eye, recently removed from the head, so that the action must be a local one. It is due to paralysis of the endings of the cervical sympathetic nerve and, probably, to stimulation of the peripheral oculomotor nerve termination as well.

Elimination.-Nicotine is eliminated in the urine, which is increased in amount thereby.

Toxicology.-Nicotine is one of the most powerful and rapidly acting poisons. When swallowed, it causes, in animals, local irritation and pain in the throat and stomach; muscular tremors and weakness, on account of which the animal falls. These symptoms are followed, first, by severe tonic and clonic convulsions, and then by abolition of voluntary motion and quietude. The pupils are contracted, and there is vomiting (in the case of some animals), purging and micturition. The respiration is at first shallow and rapid, but becomes weaker and slower, and death occurs from respiratory failure and general collapse. The pulse is primarily slow and intermittent, but later becomes rapid. The treatment of poisoning consists in evacuation of the stomach; the use of tannic acid; respiratory and heart stimulants, as strychnine, atropine, and alcohol; together with external heat and artificial respiration. The minimum lethal dose is about one drachm of tobacco, or one minim of nicotine, for small dogs. For horses, five to ten drops of nicotine or one-half pound of tobacco.

Uses.-Tobacco is not a particularly valuable medicinal agent. Its internal action is often violent, and causes great 
nausea. Absorption and poisoning may follow its external application in the smaller animals. The drug may be employed for four purposes, as follows:

1. As a local sedative.

2. As a parasiticide.

3. As a motor depressant.

4. As a cathartic.

External.-1. Tobacco is an efficient sedative in decoction (1-40), for relieving pruritus ani and vulvæ. It must be remembered that absorption and poisoning may occur when larger amounts are used externally than can be administered with safety by the mouth.

2. The latter remark applies also to the use of tobacco decoctions in killing parasites on the skin, such as the acari of mange and scab, together with lice and fleas. There are other agents which are as efficient and less dangerous (creolin). A decoction can be made by boilincr tobacco (2.5) with water (130), salt (5), and wood ashes (10), for three hours, as recommendel by Ostertag.

Internal.-3. Tobaceo has been employed as a motor depressant in spasmodic disorders, such as asthma, tetanus (given by the rectum or under the skin), and strychnine poisoning, but it is inferior to, and more dangerous than, other drugs.

4. The Germans prescribe tobacco to stimulate peristalsis in ruminants, in doses of 2 ounces, with one-half pound of coummon salt and one pound of Glauber's salts for cattle; and for sheep, $\frac{1}{2}$ onuce, with 2 ounces of salt and 3 ounces of Glauber's salts. Tobacco was given formerly in colic and intestinal obstruction, but this use is obsolete. The decoction (1-2 per cent.) may be jnjected into the rectum of horses, in non-toxic quantities, to kill oxyurides and ascarides, and to excite peristalsis and relieve spasm in colic.

Tobacco smoke is sometimes used in the same manner to destroy worms in the lower bowels. 


\section{Coniur. Conium.}

Synonym.--Conii folia, B. P.; hemlock fruit, E.; fruits de grande ciguë, Fı; schierlingsfrüchte, G.

The full grown fruit of Conium maculatum Linné (nat. ord. umbelliferæ), gathered while yet green.

Habitat.-Indigenous to Europe and Asia, but naturalized in the United States.

Description.-About $3 \mathrm{~mm}$. long; broadly ovate; laterally compressed; grayish-green; often divided into the two mericarps, each with five crenate ribs, without oil-tubes, and containing a seed which is grooved on the face; odor and taste slight. When triturated with a solution of potassium or sodium hydrate, conium gires off a strong, disagreeable, mouse-like odor. Conium fruit resembles carraway and anise seed, but these have oil-tubes or vittæ.

Constituents. - There are two essential principles in conium: conine, or coniine, and metlyyl-coniine.

1. Conine, $\mathrm{C}_{8} \mathrm{H}_{17} \mathrm{~N}$, is a yellowish, oily, volatile liquid alkaloid, of an odor resembling that of monse urine, and acrid taste. It is freely soluble in alcohol and ether, and is soluble in 100 parts of water, with which it forms a hydrate. It undergoes decomposition when exposéd to air and heat, and becomes first brown aud then resin-like. For this reason the alkaloid is uncertain in its physiological action, but its isalts (the hydrobromate and acetate) are more stable and reliable. Conine may be obtained by distillation of the fruit with an alkaline water.

2. Methyl-coniine, $\mathrm{C}_{8} \mathrm{H}_{19} \mathrm{C} \mathrm{N}$, is a colorless liquid.

3. There is also a nearly.inert and crystalline alkaloid, conliydrine.

Incompatibility.-Vegetable acids, caustic alkalies and astringents are incompatible with conium.

Conium Dose.-H. \& C., 3 i.-ii. (4.-8.); Sh. \& S.w., gr.x.тx. (.6-1.3); D., gr.ii.-v. (.12-.3).

Conince Hydrobromas.-H. \& C., gr. $\frac{2}{4}-1 \frac{1}{2}$ (.045-.1); Sh. \& Sw., gr. $\frac{1}{5}-\frac{2}{5}(.012-.024) ;$ D., gr. $\frac{1}{60}-\frac{1}{30}(.001-.002)$.

Dissolved in alcohol. 
PREPARATIONS.

Extractum Conii. Extract of Conium. (U. S. \& B. P.)

Made by maceration and percolation with acetic acid and diluted alcohol, and evaporation to pilular consistence. (U.S. P.)

Duse.-H. \& C., gr.xii.-xxiv. (.72-1.5); Sh. \& Sw, gr.ii.-iv. (.12-.24); D., gr. $1 / 4$-i. $(.015-.06)$.

\section{Extractum Conii Fluidum. Fluid Extract of Conium. (U. S. P.)}

Made by maceration and percolation with acetic acid and diluted alcohol, and evaporation so that $1 \mathrm{Cc} .=1 \mathrm{Gm}$. of the crude drug.

Dose.-H. \& C., 3 i.-ii. (4.-8.); Slı. \& Sw., $\pi x-x x .(.6-1.3)$; D., mii.-v. $(.12-.3)$.

\section{ACTION OF CONIUM AND CONINE.}

Exterral. - Conine is an irritant applied to mucous membranes. Conium is thought to act as a local sedative upon raw surfaces. Physiological experiments show that the sensory nerves are but slightly depressed.

Internal.-Circulation.-Conium has little effect upon the digestive apparatus, except in toxic doses, when it may cause irritation, vomiting and diarrhoea. It is absorbed and produces primary acceleration of the pulse, probably owing to paralysis of the pueumogastric, followed by a fall in the pulse rate. The action on the circulation is insignificant compared to that on the nerves.

Nervous System and Muscles.-The predominant action of conium consists in paralyzing the voluntary and involuntary muscles, with loss of motion but without loss of consciousness or sensation. That this effect is due to paralysis of the motor nerres has been proved by poisoning frogs with conium after ligature of the aorta, so that the blood supply to the hind legs was cut off. Failure of motion and reaction to galvanism occurs in the fore legs, but irritation of the paralyzed fore legs causes reflex contraction of the posterior extremities. This experiment shows that the motor nerves are paralyzed in the fore legs, but that the sensory nerves and spinal cord retain their functional activity. The afferent nerves are somewhat depressed by enormous doses. The brain and spinal cord are slightly 
affected by conium. The convulsions occurring in conium poisoning appear, nevertheless, to be of cerebral origin, althongh consciousness is retained until the stage of asphyxia. The special senses are somewhat interfered with (sight). The motor cells of the inferior cornua are slightly depressed by methyl-coniine, which influences the cord as well as the motor nerves, but it is considerably less active than conine. The muscles are primarily unaffected.

Eye.-The pupil is dilated (not constantly) and ptosis is observed in poisoning by conium, owing to paralysis of the oculomotor nerve eudings. If conine is dropped into the eye, primary contraction, due to reflex irritation, is soon succeeded by dilatation of the pupil.

Respiration. - The spinal and medullary respiratory centres are finally depressed by lethal doses of conium, and this result, together with paralysis of the respiratory muscles, causes death by asphyxia.

Elimination.-Conine is excreted by all channels, but. mainly in the urine.

Toxicology.-The minimum fatal dose of conium is uncertain, owing to the proneness of the alkaloid to decomposition, and to the volatility and the variable amount contained in the crude drug. A few drops of the alkaloid will kill small cats and dogs. Herbivora, as goats, sheep and horses, are less susceptible than carnivora.

The domestic animals occasionally become accidentally poisoned by eating hemlock at pasture. The symptoms exhibited are dulness, loss of muscular power (at first in the hind legs), stumbling and falling, or lying down. We observe, also, nansea, salivation (sometimes amaurosis), dilatation of the pupil and ptosis, sweating, and often muscular tremors and clonic convulsions. The pulse becomes slow and feeble, the breathing faint, the surface cold and clammy, and the animal often lies as still as though dead, so complete is the paralysis. Death finally occurs from asphyxia, frequently associated with coma. The respiration ceases before the heart-beat. The urive of poisoned animals may 
be used as a physiological test in frogs, to decide doubtful cases. The treatment of poisoning consists in evacuation of the stomach and the use of tanuic acid, artificial respiration, external heat, and respiratory and cardiac stimulants, as strychnine, atropine and alcohol. The post-mortem appearances are those of asphyxia, with sometimes evidences of gastro-intestinal irritation.

Administration.-Conium is best given in the form of the fluid extract or hydrobromate of the alkaloid. The English use the sucens conii, of which the dose for the horse is 3 ii.-iv.; dog, 3 ss.-i.; but it is inferior to the fluid extract and often inert. The initial dose of any preparation should be small, on account of the variation in strength, but should be rapidly increased until physiological effects are evident.

Uses External.-Conium is occasionally applied externally as a poultice of the leaves, or in ointment (equal parts of cerate and extract of conium), to relieve pain of sores, ulcers, malignant growths, rheumatism and neuralgia. It is thought to be both resolvent and curative on cancers and tumors, but without sufficient basis. It relieves pain when pain is due to spasm.

Uses Internal. - Conium is rarely used in veterinary medicine on account of the uncertainty of its preparations and natural therapeutic limitations. Conium has been employed as a motor depressant in many diseases, but should only be prescribed for spasm due to irritation of nerve trunks or endings; not for spasmodic conditions of central origin. In tetanus and strychnine poisoning, it is valueless, and is not wholly antagonistic (tremors and convulsions) in convulsi ve disorders. When an animal poisoned with strych-' nine is given paralytic doses of conium, the tetanic spasms of strychnine still persist. The drug has beeu prescribed in chorea, however, and with temporary benefit, when the convulsive movements were so severe as to threaten life. 


\section{SECTION IV.-DRUGS ACTING ON THE SENSORY NERVES.}

\section{Class 1.-Depressing the Sensory Nerves.}

Cocaine Hydrochloras. Cocaine Hydrochlorate.

$$
\mathrm{C}_{17} \mathrm{H}_{21} \mathrm{~N} \mathrm{O}_{4} \mathrm{H} \mathrm{Cl} \text {. (U. S. \& B. P.) }
$$

The hydrochlorate of an alkaloid obtained from coca (or cuca) leaves. These are derived from the Erythroxylon coca Lamarck (nat. ord. Lineæ). Cultivated in Peru and Bolivia, and introduced into medicine by Koller in 1884 .

Derivation. - Cocaine hydrochlorate is recovered by agitating an acidulated alcoholic solution of coca leaves with ether. The ethereal liquid is made alkaline with sodium carbonate and evaporated. The residue is purified, decolorized, neutralized with hydrochloric acid, and finally crystallized.

Description.-Colorless, transparent crystals, or a white, crystalline powder; without odor; of a saline, slightly bitter taste, and producing upon the tongue a tingling sensation, followed by numbuess of some minutes' duration. Permanent in the air. Soluble in 0.48 part of water, and in 3.5 parts of alcohol; very soluble in boiling water and in boiling alcohol; also soluble in 2,800 parts of ether, or in 17 parts of chloroform. The prolonged application of heat to the salt, or to its solution, induces decomposition. The salt is neutral to litmus paper.

$$
\text { Dose.-H., gr.v.-x. (.3-.6); D., gr. } \frac{1}{8}-\frac{3}{4}(.008-.045) \text {. }
$$

Action External.-Solutions of cocaine (4-10 per cent.), applied to mucous membranes, produce perfect local anæsthesia by paralyzing the sensory nerve endings. The functious of the nerves of special sense are abolished before those of ordinary sensibility. Stronger solutions paralyze the motor nerve terminations. The local application of cocaine causes pallor of the mucous membrane, which is succeeded by redness and congestion. The first condition 
follows constriction of the superficial blood vessels, which is said to be due to tonic contraction of the smooth muscnlar fibres of the vessel walls. The secondary vascular relaxation, and tendency to congestion in parts subjected to cocaine anxthesial, lessens the resistance of the tissues (especially of sensitive structures, as the eye) to the irritation of antiseptics and bacterial products. Therefore, surgical operations performed under cocaine anæsthesia are somewhat more prone to be followed by inflammation.*

Action Internal.-Digestive Tract.-Cocaine exerts a local anasthetic action upon the gastric mucons membrane, and in this way lessens the appetite and sometimes stops vomiting. Intestinal peristalsis is increased by moderate doses, but is decreased and destroyed by the paralytic action of large doses of cocaine.

Nervous System.-Ordinary medicinal doses produce no marked effect upon the nervons system, except some mental exhilaration. Full doses of cocaine stimulate the psychical functions of the brain and cause intoxication, which is followed, in poisoning, by stupor and convulsions. The convulsions are mainly of cerebral origin, but may be due in part to irritation of the spinal reflex centres. These latter centres are first stimulated by toxic doses, but depression and paralysis of the spinal cord follows; the sensory before the motor tract. The sensory nerves are paralyzed and the motor nerves depressed by toxic doses. The voluntary muscles are stimulated by medicinal doses of cocaine, and the alkaloid relieves fatigue and, experimentally, more than donbles the response to stimuli in wornout muscles. Voluntary muscles are paralyzed by the local application of large quantities of cocaine.

Circulation.-The action of cocaine upon the heart and vessels is not very marked, except in poisoning. The alkaloid is, however, a slight cardiac stimulant in moderate doses, increasing the pulse-rate and tension. The action upon the heart is catused by depression of the cardio-inhibitory contres, and sometimes as well by depression of the cardiac

* Nefers to use of strong solutions. 
inlibitory ganglia. Vascular tension is increased because of stimulation of the medullary vasomotor centres, smooth muscle of the ressel walls, and becanse of the increased action of the heart. On. the other hand, both minute and large doses may diminish the pulse rate.

Respiration. - Cocaine is a respiratory stimulant in medicinal doses, but a paralyzant in toxic amounts. The respiratory centres are first stimulated and the breathing is made deeper and quicker. Depression and paralysis of the respiratory centres follow; cyanosis supervenes, and the respirations are shallow and irregular. Death occurs from asphyxia.

Temperature.-The body heat is elevated, sometimes to an excessive degree, by poisonous doses of cocaine. Medicinal doses do not affect the temperature. The rise of temperature is said to follow increased heat production.

Kidneys. - The greater part of the cocaine absorbed is oxidized within the body. The smaller part is eliminated by the kidneys. Experimental evidence concerning the influence of the alkaloid npon the secretion and composition of the urine is conflicting and indefinite.

Toxicology.-Moilerate doses produce in dogs mental exhilaration and joyousuess, so that they bark and jump about with delight. Poisoning with large doses (gr. $\frac{1}{4}$ of cocaine to $2 \mathrm{lbs}$. of live weight) may be dirided into three stages. In the first stage, there is restlessness, anxiety and terror, with rhythmical movements. Noises frighten the animal and he fails to recognize his master. The second stage is characterized by a joyous condition, in which dogs bark, dance about and lick people's hands. In the third stage, weakness and nervous phenomena appear,-als muscular twitching, rhythmical movements, a pendulum-like motion of the head, convulsions and stupor. There is dyspnœa, feeble pulse ancl failing respiration. In an experiment upon a Newfoundland dog, weighing about 100 lbs., conducted by the writer, there were no symptoms produced by 3 grains of cocaine under the skin, except dilation of the 
pupils and a constant lapping with the tongue. In man, an amount of cocaine exceeding gr. $\frac{3}{4}$ should not be employed under the skin, or upon mucous membranes, and death has occurred in susceptible patients from even smaller doses. The most powerful action follows the use of cocaine in very vascular parts, as about the face. One-half a grain of cocaine given subcutaneously to a girl eleven years old, was followed by a fatal result in 40 seconds, and the writer has seen violent convulsions produced by the instillation of a few drops of a 2 per cent. solution into the eye of a man. On the other hand, spontaneous recovery has obtained in the human subject after the ingestion of $22 \mathrm{grs}$. of the alkaloid. In the horse, a toxic dose of cocaine ( 3 i.) causes restlessness and excitement, dilated pupils and salivation, culminating within an hour in a state of acute mania and intense excitement. These symptoms are followed by gradual recovery after the lapse of a few hours. Three grains of cocaine given under the skin, will sometimes induce nervous excitement in susceptible horses. The treatment of dangerous forms of cocaine poisoning, with respiratory and heart failure, consists in the use of opium and rapidly acting stimulants, as morphine sulphate under the skin, nitroglycerin upon the tongue, and strychnine, atropine and brancly subcutaneously.

Uses External.-Cocaine and its synthetic chemical substitutes are the most valuable agents we possess to cause complete local anæsthesia for surgical purposes.* The operations most suitable for the hypodermatic application of cocaine are included in the following:

Removal of tumors.

Docking and pricking the tail.

Tarsal tenotomy.

Firing.

Plantar neurectomy.
Opening of abscess.

Injuries and operations upon the eyeballs and eyelids.

Operations about the feet in horses. Operations upon mucous membranes.

The alkaloid may also be employed to dilate the pupil for examination of the eye, and to detect lameness. In a case of doubtful foot lameness in the horse, injection of

\footnotetext{
* Cocaine in surgery can often be combined most advantageously with adrenalin. (See p. 639.)
} 
woviline into the plantar nerve trunk, on either side of the leg aud a little above the point of selection for plantar neurectomy, will often completely abolish sensation in the foot. This fact may be determined by pricking the soft parts above the hoof. If there is complete anæsthesia of the foot, and the seat of lameness be situated therein, the hor'se will go sound while the anrsthesia lasts. This method may be taken advantage of in the diagnosis of localized lameness elsewhere. If cocaine is injected over an area (suspected to be the canse of lameness), and the animal goes sound while the cocaine anæsthesia lasts, the site of lameness becomes certain.

It has been discovered that injections of powerful cocaine solutions into a sensory nerve trunk will paralyze its sensibility throughout its peripheral distribution (regional anæsthesia). When ligation of a limb, or part, can be secured between the operative field and the heart, the anæsthetic action of cocaine is more profound and toxic symptoms are less liable to occur, since the drug is drained away in the blood during the operation. Many operations can be performed under cocaine, as neurectomy, firing, tenotomy, etc., without casting the horse. In using cocaine for the removal of tumors, or opening of abscess, the solution is injected at several points in a circle about the base of the tumor or abscess, and not in the inflamed tissue of the latter. Following the first injection, the succeeding applications may be made painless by inserting the needle within the area made anæsthetic by the previous injection (circumferential anæsthesia).

The amount of cocaine solution to be injected is of importance. This depends upon the strength of the solution, the weight and species of the animal, and the seat of application. A solution stronger than 4 per cent. is irritating to the eye. The stronger cocaine solutions (5 to 10 per cent.) are more powerful paralyzants to the sensory nerves, and are advisable when they can be used with safety, but $a$ 4 or 2 per cent., or even a much weaker solution, will ordin- 
arily produce anrsthesia when introduced-under the skin. In the horse, subentuneons injection of a 5 or 10 per cent. solution may be made to the amount of 2 drachms of the former, or 1 drachm of the latter solution (cocaine, gr.6). Larger doses may induce restlessness, excitement, etc., which, although not indicative of danger, may interfere with operative procednres. When larger quintities are desirable, $2 \frac{1}{2}$ drachms of a 4 per cent. solution, or 5 drachms of a 2 per cent. solution, may be employed (equivalent to 6 grs. of the alkaloid), or Schleich's solution may be ntilized. Not more than 7 to 15 drops of a 10 per cent. solution should be injected into dogs. This is equivalent to $\mathrm{gr} \cdot \frac{3}{4}$ and $\mathrm{gr} \cdot 1 \frac{1}{2}$ of the alkaloid. Three-quarters of a grain of cocaine is a safe limit of dosage for small dogs. If a 2 or 4 per cent. solution is employed, $30 \mathrm{MI}$. of the former, or $15 \mathrm{MI}$. of the latter solntion (equivalent to gr. $\frac{3}{5}$ of cocaine) may be injected. When larger amounts of the anasthetic solution are indicated for dogs, Schleich's method of infiltration is appropriate (infiltration anæsthesia).

This method depends upon the fact that the pressure of saline solutions injected into the tissues causes a benumbing of the sensory nerves, and also upon the anæsthetic action of minute doses of cocaine and morphine. Schleich's solution may be used in any amount and can be made conveniently from the tablets prepared by chemists. The ordinary solution contains :

1 part of cocaine hydrochlorate.

2 parts of common salt.

0.1 part of morphine hydrochlorate.

In 1,000 parts of sterile water.

NotE.-Since writing the above, it has been found that the morphine in Schleich's solution is an irritant rather than a local anæsthetic, and when large quantities of cocaine or eucaine are to be used under the skin-or danger is, for any reason, to be anticipated-the writer would strongly advise the employment of a normal salt solution ( 4 grains sodium chloride to 1 drachm of water), containing from one-tenth to one per cent. of cocaine or encaine. These weaker solutions will usually cause complete and safe local aniesthesia. The solutions should always be war'm-at body temperature-and not over twenty-four hours old, as acid develops. which interferes with the anæsthetic action. They are most conveniently made. by solution of cocaine tablets in salt solution at the time of operation. 
Local applications to mucous membranes may be reapplied, once or twice, at intervals of five minutes, to secure perfect anæsthesia. A larger quantity of cocaine than recommended above for hypodermatic use, should not be employed. Cocaine, as already stated, produces a primary astringent action, followed by secondary irritation and vascular relaxation; yet, when properly diluted and applied in the first stage of inflammation, it may prove a valuable abortive and sedative agent.

Hæmorrhage from mucous membranes can be arrested by its topical application; coryza aborted, and hæmorrhoids relieved by this method. Pruritus, about the anus and vagina, is allayed by cocaine. In relation to the eye, 5 to 10 drops (horse) of cocaine solution (1 to 4 per cent.) are employed for various purposes, embracing examination, removal of foreign bodies, operations, and the relief of suffering in acute inflammation resulting from natural causes or mechanical irritation. The following prescription is of value in superficial inflammatory and painful conditions of the eye :

B

Cocainæ hydrochlor...................gr.v.

Acid. borici........................gr.x.

- M.

Aq. dest. ad......................

S. Instil a few drops into the eye hourly.

Injection into the eyeball is preferable to instillation for enucleation.

Solutions of cocaine should be freshly made, and must not be sterilized by boiling, although they should be made with sterile water, or, better, normal salt solution. The tablets supplied by pharmaceutical chemists are convenieut for hypodermatic use. Ten grains of boric acid will preserve an ounce of cocaine solution for a month.

Uses Internal.-Cocaine may be administered in aqueous solutions for the relief of persistent vomiting in dogs. Otherwise, the drug finds no indication in veterinary medicine. The alkaloid is occasionally used as a stimulating 
and supporting agent in asthenic fevers and adynamic conditions of the human patient.

The most wonderful recent advauce in surgery consists in the production of anæsthesia in the lower (posterior) half of the body by injection of cocaine solution into the lumbar region of the spinal canal. This method has been successfully used in men and dogs in a large number of cases, rendering parturition and operations on the abdominal and pelvic organs and lower (posterior) extremities painless. The anæsthesia lasts one to five hours, and, in man, from gr. $\frac{1}{6}$ to gr. $\frac{1}{3}$ of cocaine hydrochlorate in aqueous solution are injected into the spinal canal between the fourth and fifth lumbar vertebra. The procedure is not devoid of danger, and in man is frequently followed by headache, vomiting, sweating, slight chills and rise of temperature. The injection must be conducted with absolute aseptic precautions.

\section{Eucainz Hydrochloras. Eucain Hydrochlorate.}

$$
\mathrm{C}_{19} \mathrm{H}_{27} \mathrm{~N} \mathrm{O}_{4} \mathrm{H} \mathrm{Cl} \text {. (Non-official.) }
$$

This is a laboratory product, formerly known as encain Hydrochlorate "B." Eucain is used in 2 per cent. aqueous solution in the eye, and in 10 per cent. on mucous membranes, and is said to be harmless in any ordinary amount. It is employed as a substitute for cocaine in the same manner and for the same purposes, but with the following. advantages :-

Safer, 5 times less toxic.

Cheaper.

Does not decompose on keeping in solution.
Can be sterilized by boiling.

Less irritating.

Does not dilate the pupil.

Is a slight antiseptic.

Ophthalmologists find that the drug does dilate the pupil after several instillations, and that it does irritate the already inflamed eye. It, moreover, does not contract vessels when locally applied, and does sometimes produee poisoning like cocaine, but much less frequently. 


\section{HoLocain.}

This is a synthetic compound allied to phenacetin, and it is claimed to be superior to cocaine for application to the eye. The anæsthetic effect appears within fifteen seconds and lasts fifteeu minutes. It is stated that holocain produces no local irritation of the eye, nor dilatation of the pupil, and does possess an inherent antiseptic action. One to two drops of a one per cent. solution are employed to anresthetize the eye. Holocain is too toxic for hypodermatic application.

\section{SECTION V.-DRUGS ACTING ON THE SECRETORY NERVES.}

\section{Class 1.-Pilocarpus and Pilocarpine.}

\section{Pilocarpus. Pilocarpus. (U. S. P.)}

Synonym.-Jaborandi, B. P.; the leaflets of Pilocarpus Selloanus Engler (Rio Janeiro Jaborandi), and of Pilocarpus Jaborandi Holmes (Pernumbuco Jaborandi, nat. ord. Rutaceæ).

Habitat.-Brazil, in the neighborhood of Pernambuco.

Description.-Abont 10 to $15 \mathrm{~cm}$. long, and 4 to $6 \mathrm{~cm}$. broad; short stalked, oval or ovate-oblong, entire and slightly revolute at the margin, obtuse and emarginate, unequal at the base, dull green, coriaceous, pellucid-punctate, mostly smooth; when bruised, slightly aromatic; taste somewhat bitter and pungent.

Constituents. - 1 . Pilocarpine, $\mathrm{C}_{11} \mathrm{H}_{16} \mathrm{~N}_{2} \mathrm{O}_{2}(.25-.5$ per cent.), is the alkaloid to which jaborandi owes its principal effect. 2. Jaborine, $\mathrm{C}_{22} \mathrm{H}_{32} \mathrm{~N}_{4} \mathrm{O}_{4}$, an alkaloid resembling atropine in its action on the heart, pupils, intestines and salivary glands. It occurs occasionally as an impurity in commercial pilocarpine, to which it is antagonistic. It is soluble in alcohol. 3. Pilocarpidine, $\mathrm{C}_{10} \mathrm{H}_{14} \mathrm{~N}_{2} \mathrm{O}_{2}$, an alkaloidal product of the 
decomposition of pilocarpine, which it resembles in action, but is weaker. It is soluble in alcohol. 4. Jaborandine is another alkaloid resulting from the decomposition of pilocarpine, and is similar to atropine. These atropine-like principles are never present in sufticient quantity to wholly antagonize the predominant action of pilocarpine in jaborandi. 5. A peculiar acid. 6. A volatile oil.

Dose.-H. \& C., 3 ii.-iv. (8.-15.); Slı. \& Sw., 3 ss.-i. (2.-4.); D., gr.v.- 3 i. (.3-4.).

\section{PREPARATIONS.}

Extractum Pilocarpi Fluidum. Fluid Extract of Pilocarpus.

$$
\text { (U. S. P.) }
$$

Made by maceration and percolation with diluted alcohol, and evaporation, so that $1 \mathrm{Cc} .=1 \mathrm{Gm}$. of the crude drug.

Dose.-H. \& C., 3 ii.-iv. (8.-15.); Sh. \& Sw., 3 ss.-i. (2.-4.); D., mv. -3 i. $(.3-4$.).

Extractum Jaborandi. (B. P.)

Dose.-H., gr.xl.- 3 i.ss. (2.6-6.); D., gr.ii.-x. (.12-.6).

Pilocarpina Hydrochloras. Pilocarpine Hydrochlorate. $\mathrm{C}_{11} \mathrm{H}_{16} \mathrm{~N}_{2} \mathrm{O}_{2} \mathrm{H} \mathrm{Cl}$. (U. S. P.)

Derivation.-The hydrochlorate of an alkaloid obtained from pilocarpus, with alcohol and hydrochloric acid by distillation and evaporation. The residne is dissolved in a slight excess of ammonia and chloroform, shaken with water, and neutralized with hydrochloric acid. Crystals of the lydrochlorate form on evaporation. The salt is purified by recrystallization.

Properties.-Small, white crystals, odorless and having a faintly bitter taste; deliquescent on exposure to damp air. Very soluble in water and in alcohol; almost iusoluble in ether or in chloroform.

Dose.-H., sialogogue, gr.i.-ii. (.06-.12); cathartic, gr.ii.-v. (.12-.3); C., cathartic, gr.v.-x. (.3-.6); H., diarphoretic, gr.vi -xii. (.36-.72), daugerous; Sh., gr.i. (.06); D., gr. $\frac{1}{10}-\frac{1}{3}$ $(.006-.02)$. 
Pilocarpine Nitras. Pilocarpine Nitrate. (B. P.)

Dose.-Sime as for hydrochlorate.

ACTION OF PILOCARPUS AND PILOCARPINE.

Internal. - Alimentary Canal. - Pilocarpine increases enormonsly salivary secretion, and, in a less degree, the gastric and intestinal secretions. It stimulates peristaltic action of the stomach and bowels as well, and acts as a purgative. Salivation is dne to direct excitation of the secretory nerve (chorda tympani) endings and the gland cells. Salivation occurs when pilocarpine is injected in to the gland and prevented from entering the general circulition; also when the secretory nerves are severed. The action on the salivary glands is set aside by atropine. The parotid, submaxillary, and sublingual glands, become somewhat tense and tender under the influence of pilocarpine, and the saliva is rich in salts and ptyaline, and contains a slight excess of urea. The unstriped muscle of the stomach and intestines is stimulated by pilocarpine through its action on the efferent nerve endings and muscular tissue.

Circulation. - Pilocarpine is absorbed, but exerts no action on the blood. In toxic doses the alkaloid is a heart depressant, but in moderate amount slows the pulse rate. In man the pulse is quick and the blood tension lowered. The effect on the domestic animals is probably due to stimulation of the peripheral vagi or inhibitory cardiac ganglia, although Ringer's experiments proved that the ventricular contractions of the frog's heart were slowed by pilocarpine when freed from the inhibitory garglia, and he attributes its action to stimulation of the heart muscle and motor ganglia situated therein. In poisoning, the heart is enfeebled and slow, and there is vasomotor paralysis. Atropine antagonizes the action of pilocarpine on the heart.

Respiration.-The respiration is not directly affected by the drug, but the bronchial secretions are greatly increased, and in poisoning there are, in consequence, odema of the lungs and dyspnœa. 
Nervous System and Muscles.-Medicinal doses do not cause any functional disturbance of the nerrous system, but very large doses excite the spinal motor tract and reflox centres and cause convulsions in frogs, succeeded by spinal depression and paralysis. The latter is due in part to au influence on the muscles themselves. Tremors occur occasionally in man and the domestic animals in poisoning. The nerves escape unscathed. The involuntary muscles are stimulated throughout the body, owing to the direct action of the drug upon them and their motor nerve terminations.

Skin. - Moderate doses of pilocarpine stimulate but slightly the secretion of sweat in the lower animals, but in man the secretion is enormous ( $1 \mathrm{pt}$.). The salivary secretion appears to supplant that of the skin in the domestic animals, unless very large doses are employed (H., gr.vii.xii.), which cause diarrhoea, salivation and loss of body weight (40 to $60 \mathrm{lbs}$.), and may entail pulmonary œedema. and heart failure. The secretory nerve terminations and glandular cells are both stimulated. The secretion of tears, nasal mucus and milk are slightly increased in the same manner, and the growth of hair is rendered more luxurious.

Temperature.-The temperature is reduced by evaporation from the skin, if there is much sweating.

Genito-Urinary Organs.-Pilocarpine exerts a slight and uncertain oxytocic action on the pregnant uterus and has sometimes precipitated parturition in pregnant animals at full term. The unstriped muscle of the spleen and bladder is stimulated, and micturition is frequent. Pilocarpine, in repeated small doses, angments the flow of urine and probably increases tissue waste and the excretion of urea by its general action on the secretions. It is eliminated nnchanged in the urine.

Eye.-Pilocarpine contracts the pupil when applied to the eye; it also increases tension of the eyeball and impairs the sight temporarily, owing to action on the retina. The myosis is due to stimulation of the peripheral oculomotor nerve endings. When the alkaloid is given internally it 
may contract the pupil, but jaborandi, or the fluid extract, are less likely to do so on account of the opposing alkaloid (jaborine), which tends to dilate the pupil.

Summary. - Pilocarpine possesses two important actions. 1. To increase secretions (stomach, intestines, salivary glands, sudoriparous, lachrymal and mammary glands, kidneys, bronchial and nasil mucous membranes, and ear). 2. To stimulate the involuntary muscles (stomacl, intestines, heart, nterus, bladder, spleen, vessels and iris). Both actions are peripheral and are probably exerted on the gland cells and muscle fibres, as well as on the efferent nerve terminations.

Administration.-Pilocarpine is given usually when an immediate effect is desired. Therefore the hydrochlorate or nitrate are employed subcutaneously. If prescribed in combination with eserine, the sulphate of both alkaloids. may be used, or eserine sulphate and pilocarpine hydrochlorate may be injected separately.

Toxicology.-Symptoms appear in five or ten minutes after the subcutaneous injection of pilocarpine, and in fifteen to twenty minutes after the injection of jaborandi. Salivation alone occurs after small doses, but with toxic quantities there are present salivation, accompanied by more or less sweating, intestinal colic, purging and perhaps vomiting, a slow, weak pulse, and dyspnœa. Muscular tremors are observed sometimes in man, and convulsions in frogs, but spasmodic movements are uncommon in the domestic animals. Dogs have been killed by gr. $\frac{3}{4}$ of pilocarpine. The administration of an amount larger than 5 grs. of the alkaloid to horses, subcutaneously, is attended with danger. Atropine is the physiological antagonist of pilocarpine in relation to the heart, secretions, pupils, and, in large doses, probably to the intestines.

Atropine should be given along with alcoholic. stimulants, or ammonia, in jaborandi or pilocarpine poisoning.

Uses Internal.-The chief value of pilocarpine in veterinary medicine consists in its use as a purgative to stimulate 
secretion, and, to a certain extent, peristaltic action-in combination with physostigmine (vid. "Pliysostigmine")-in obstinate constipation of horses. It is also given in colic, and in obstruction from twist and intussusception, with physostigmine. * The alkaloid is a good substitute for eserine for application to the eye (in 1 or 2 per cent. solution), and is less painful. Pilocarpine is the most efficient antidote to atropine, and should be administered in amount equal to 4 times that of atropine. Jaborandi is employed to remove waste matters from the blood and system (urea and effusions), but is of little value in veterinary practice, compared with its efficiency. in human medicine, on account of its feeble sudorific action. It has been recommended in dropsy of cardiac origin, not uncommon in dogs, but is dangerous, since it tends to produce pulmonary œdema and heart weakness. For the same reason it is inadvisable in pleuritic effusions and renal dropsy, and in all three conditions it is inferior to purgatives. Pilocarpine is highly recommended by the Germans in cerebral and spinal meningitis, to assist absorption of effusion. Pilocarpine stimulates the skin in its elimination, and is sometimes of service in chronic eczema, psoriasis, prurigo, and chronic urticaria. It is recommended in chronic rheumatism as an eliminative, and in acute inflammation of the brain, and in laminitis without rational basis. Small doses of pilocarpine have been employed successfully to stimulate a failing milk secretion, and to prevent excessive sweating in general debility. Jaborandi relieves dry throat and excessive thirst. Obesity, in robust dogs, may be treated with pilocarpine under the skin, in one-half grain doses daily. Success sumetimes attends this method. The drug is contraindicated when there is impairment of the respiratory functions, a weak or fatty heart, and in unconsciousness, when excessive secretion may obstruct the air passages.

* The lacter use of the drug is attended with danger of increasing the obstruction, if not successful. 
SECTION VI--DRUGS ACTING ON THE HEART.

\section{Class 1.-Increasing the Force and Decreasing the Frequency of the Heart.}

\section{Digitalis. Digitalis.}

Synonym.-Digitalis folia, B. P. ; foxglove, digitalis leaves, E.; digitale, feuilles de digitale pourprée (de grande digitale), Fr.; fingerhutkraut, G.

The leares of Digitalis purpurea Linné (nat. ord. scrophularineæ), collected from plants of the second year's growth.

Description.-From 10 to $50 \mathrm{~cm}$. long; ovate or ovateoblong, narrowed into a petiole, crenate, dull green, densely and finely pubescent, wrinkled above, paler and reticulate beneath, midrib near the base broad; odor slight, somewhat tea-like; taste bitter, nauseons.

Constituents. - The active principles of digitalis are four glucosides; the three first represent its stimulant action. 1. Digitoxin, the most poisonous and active. Said to be cumulative. It occurs in crystals, soluble in alcohol and chloroform, slightly in ether, and insoluble in water. 2. Digitalein, an amorphous, bitter substance, soluble in water and alcohol and nou-cumulative. Dose.-H., gr. $\frac{1}{8}-\frac{1}{4}$ (.008.015); D., gr. $\frac{1}{100}(.0006)$.

3. Digitalin, a bitter, crystalline body, soluble in alcohol, and sparingly solnble in water and ether. 4. Digitonin, $\mathrm{C}_{27} \mathrm{H}_{44} \mathrm{O}_{13}$, resembling, or identical with, saponin of senega. Soluble in water. It is a heart depressant, muscular paralyzant and powerful irritant, besides being antagonistic to digitalis. In addition to these principles, there are: 5. Digitin, an inert body. 6. Digitalic and antirrhinic acids. 7. Tannin, coloring matters, starch, sugar, gum, a volatile oil, salts, etc., common to most vegetables.

Two substances are found in commerce: 1. Nativelle's digitalin, $\mathrm{C}_{25} \mathrm{H}_{40} \mathrm{O}_{15}$, occurring in white crystalline tufts com- 
posed of needles. It is bitter, and soluble in alcohol and chloroform; insoluble in water or ether. It contains digitoxin, digitalin, digitalein and digitonin, but mainly digitoxin, and is cumulative. Dose.-H. \& C., gr. $\frac{1}{4}-\frac{1}{2}$ (.015-.03); D., $\operatorname{gr} \cdot \frac{1}{60}-\frac{1}{30}(.001-.002)$.

2. Homolle's or Quévenne's digitalin, an amorphous, whitish powder, or small scales; very bitter, inodorous, and soluble in 2,000 parts of water. It is composed largely of digitalin, with a little digitoxin. Dose.-H. \& C., g1. $\frac{1}{4}(.015)$ $=$ gr. 221 of digitalis leaves; D., gr. $\frac{1}{60}-\frac{1}{30}(.001-.002)=$ gr. $1 \frac{1}{2}-3$ of digitalis leaves.

Neither Nativelle's nor Homolle's digitalin form a complete substitute for digitalis, and their use is not recommended. Schmiedeberg's digitalin is, however, said to be a reliable substitute, by eminent authority.

Incompatibility.-Digitalis is incompatible with tannic acid, lead acetate, cinchona and ferric salts.

Digitalis Folia Dose.-H., gr.x.- 3 i. (.6-.4); C., 3 ss.-i.ss. (2.-6.); Sh. \& Sw., gr.v.-xv. (.3-1.); D., gr.ss.-iii. (.03-.2).

\section{PREPARATIONS.}

Extractum Digitalis. Extract of Digitalis. (U.S. P.)

Made by maceration and percolation with alcolıol and water; distillation of the alcohol and evaporation to pilular consistence.

Dose.-H. \& C., gr.v.-xx. (.3-1.3); D., gr.1 -1. (.008-.06):

Extractum Digitalis Fluidum. Fluid Extract of Digitalis. (U. S. P.)

Made by maceration and percolation with alcohol and water, and evaporation, so that $1 \mathrm{Cc}$. $=1 \mathrm{Gm}$. of the crude drug.

Dose.-H., $\prod_{\mathrm{x} .-}$ - i. (.6-4.); C., 3 ss.-i.ss. (2.-6.); Sh. \& Sw., $\prod_{\mathrm{v}} \cdot-\mathrm{xv}$. (.3-1.); D., Mss.-iii. (.03-.2).

Tinctura Digitalis. Tincture of Digitalis. (U. S. \& B. P.)

Made by maceration and percolation of powdered digitalis (150), with sufficicient alcohol and water to make 1,000. (U. S. P.)

Dose.-H. \& C., 3 ii.-vi. (8.-24.); Sh. \& Sw., $m x x x .-3$ i.ss. (2.-6.); D., $\operatorname{m} v \cdot-\operatorname{xxx}$. (.3-2.).

Infusum Digitalis. Infusion of Digitalis. (U. S. \& B. P.)

Digíalis, 15 ; alcohol, 100 ; cinnamon water, 150 ; boiling water, 500 ; cold water to make 1,000 . By maceration. (U. S. P.) 
Dose.-H. \& C., zँ ii.-vi. (60.-180.); Sh. \& Sw., ₹ ss.-i. (15.-30.); D., 3 i.-iv. (4.-15.).

ACTION OF DIGITALIS.

External.-None.

Internal.-Alimentary Canal.-Digitalis in large doses is a gastro-intestinal irritant, and in poisoning causes nausea, colic, purging, and vomiting in animals capable of the act.

Circulation.-The dominant action of digitalis is expended upon the heart and blood vessels. After medicinal doses we find the pulse becomes: 1. Slower. 2. Fuller and stronger. 3. More regular in rhythm, if previously irregular. In poisoning, these phenomena are reversed and the pulse is : 1. Rapid. 2. Weak. 3. Irregular. The therapentic effects following moderate doses are due to: 1 . Stimulation of the heart muscle, and perhaps its contained ganglia (pulse stronger and somewhat less frequent). 2. Excitation of the vagus centre and the vagus cardiac terminations (pulse infrequent). 3. Stimulation of the muscular walls of the vessels and vasomotor centres (increased vascular tension). The tension is also augmented by the greater force of the heart-beat, and, on the other hand, the heart is slowed in overcoming the increased resistance in the vessels. In consequence of the action on the heart the ventricular contractions are complete and forcible, and the diastolic period is lengthened; therefore, more blood enters the viscus and more is squeezed out with each contraction. The systolic period is unaltered. Furthermore, the nutrition of the heart is thought to be promoted by: 1. Increase of its blood supply during the prolonged diastole. 2. Stimulation of the trophic nerves (vagi?) of the heart. As diastole is the rest period of the heart, increasing it conserves the strength of the organ. In poisoning, the symptoms noted above follow: 1. Paralysis of the peripheral vagi (pulse rapid). 2. Insufficient filling of the vessels and paralysis of the vessel walls (low tension). 3. Forcible contractions contending against prolonged dilatation of the 
ventricles (pulse irregular). At one time the heart beats slowly and weakly because the heart muscle cain scarcely overcome the tendency of the excited vagi to cause ventricular dilatation; at another time the heart beats rapidly and sirongly, when powerful systolic contractions overmaster the inhibitory influence of the vagi. The heart, during this irregular toxic period, is seen to be unequally affected, in that one portion (the apex) may be firmly contracted while the rest of the ventricle is dilated. Moreover, the auricles and ventricles do not act synchronously and the heart assumes various peculiar shapes (hour-glass, etc.). Death usually occurs from cardiac arrest in systole, in which condition it remains pale, firm and contracted, and unresponsive to mechanical or electrical stimulation. Rarely (men and dogs) the heart stops in diastole. The action of digitalis on the heart is more pronounced in dogs and sheep than in horses and cattle. The characteristic effect of digitalis is observed when it is applied locally to the isolated nervefree apex, or when the vagi are previously cut or paralyzed by atropiue, and when the spinal cord is destroyed. These facts show that the heart muscle is influenced. That the peripheral vagi are stimulated, is shown by the fact that an amount of galvanic stimulation of the vagi, ineffective before poisoning, will, after exhibition of digitalis, cause diastolic arrest of the heart. In regard to the vessels, experiments conducter on the terrapin exhibit the fact that when the vessels are deprived of their nerve supply, the heart excised, and an artificial circulation substituted, even then vascular contraction and retardation of flow will occur under the influence of digitalin added to the factitious blood. The resultant of the various actions of medicinal doses of digitalis is increased work of the heart, so that more blood is pumped throughout the body in any given unit of time.

Respiration. - The respiratory centres are unaffected save by toxic doses, and then through insufficient blood supply, when the respiration becomes weak and imperfect. 
Nervous System and Muscles.-These are not influenced by therapeutic doses of digibalis. Toxic quantities cause loss of reflex action, muscular weakness, and convulsions in the frog. The first two phenomena are dne to primary stimnlation of the inhibitory reflex centres of Setschenow in the medulla, followed by general paralysis of the spinal cord, and direct depression of the motor nerves and muscles; while the convulsions are cansed by circulatory changes in the base of the brain dependant upon the embarrassed heart.

Temperature.-The temperature is unaffected by medicinal doses. Toxic doses reduce temperature. Fever is lowered by large doses of digitalis, but it is rarely safe to use the drug as an antipyretic. Moreover, digitalis is sometimes inoperative as a heart stimulaut in fever, because the functional activity of the vagus centres and peripheral terminations is depressed and insensitive to the action of the drug.

Kidneys.-Metabolism and Elimination.-The influence of digitalis on the amount of urinary secretion is uncertain. It has no special action upon the nucons membrane or secreting cells of the kidney. If general vascular teusion is lowered (cardiac disease), digitalis will exert a diuretic action in consequence of increasing blood pressure. As a rule, it. may be stated that if digitalis increases the systemic vascular tension more than that of the kidney, diuresis follows. The effect of digitalis on tissue waste is nncertain and the mode. of its elimination is unknown. Experiments relative to the composition of the urine are conflicting. The smooth muscle of the uterus is said to be stimulater to contraction by digitalis.

Cumulative Action.-Digitalis and strychnine are said to be cumulative in their action. Evidence is stronger in the case of the former drug than in that of the latter. By cumulative action is meant sudden transition from a therapentic to a toxic effect. This may be due to three causes. 1. Tardy absorption. 2. Increasing susceptibility. 3. Delayed elim- 
ination and accumulation of the drug in the system. The cumulative action of digitalis is chiefly due to the latter cause. It should never be administered in full medicinal doses nninterruptedly for any considerable length of time.

Toxicology. - Poisoning may occur from large single doses within 3 to 10 hours of their ingestion, and last for 16 or more hours with a fatal result; or may appear suddenly after the administration for several days of large medicinal doses (cumulative action). A minimnm fatal dose for the horse is about 3 vi. of digitalis, or gr.i.ss. of Homolle's digitalin. For dogs, 3 i. of digitalis, or gr. $\frac{1}{4}$ of digitalin. The symptoms exhibited are chiefly concerned with the digestion and circulation. They consist in dulness, lassitude, loss of appetite, nausea, flatulence, diarrhoea, infrequent, full pulse (reduced 6-10 beats in the horse), and contracted pupils. There is vomiting in dogs. In fatal cases these symptoms are followed by severe colic and tympanites; rapid, feeble, dicrotic, irregular or intermittent pulse (120-140 in horses), while the heart may be heard and felt beating wildly and strongly, and a systolic blowing murmur can frequently be detected. This is due to mitral or tricuspid regurgitation cansed by irregular contraction of the columnæ carnæ. The pulse is imperceptible because of the failure of the heart to fill the vessels. The extremities are cold, the eye is protruding, and salivation occurs. Bloody diarrhoea is very often present and the urine may be suppressed. The breathing finally becomes difficult and death ensues within a few hours, or as late as several days.

Treatment. - Evacuation of the stomach and bowels. Taunic acid, as a chemical antidote, alcohol, opium, and aconite, which is the physiological antagonist in depressing the action of the heart and lowering blood tension. In addition, external heat should be applied and complete quiet and rest secured.

Administration. - In view of its slow absorption and elimination digitalis should not be given oftener than once in six, eight, or even twelve hours. Very large doses may 
be given, however, repeatedly, in case of threatened heart failure, by the subcutaneous method. The appearance of indigestion, nausea or dulness, and a decided fall in the pulse rate, should be a warning to stop the administration at once. The best preparations are the tincture, infusion, and fluid extract. The former may be injected deep into the muscle to avoid abscess.

Uses External.-Digitalis is occasionally employed as a poultice of the leaves, applied over the loins to promote diuresis, or in local inflammation, to contract vessels.

Uses Internal.-Digitalis is a heart stimulant, although sometimes classified as a heart depressant. It may, however, exert a sedative effect upon a weak, rapid, irregular heart, by increasing the inhibitory and muscular power. Digitalis is indicated: (1) In all conditions where the heart is weak, irregular or intermittent, and the circulation sluggish; (2) as a diuretic, chiefly in dropsy secondary to cardiac disease, but also in that of renal origin; digitalis has also been used (3) as a hæmostatic in internal hæmorrhage, and (4) to reduce temperature in fever; but, in both of the last two instances, with questionable advantage.

1. In syncope following disease, shock, injury or poisoning (aconite), digitalis is invaluable when injected subcutaneously together with alcoholic stimulants. In acute diseases, digitalis is one of the most generally serviceable stimulants. The drug is peculiarly applicable in the second stage of pneumonia, because it strengthens the right ventricle, forces the blood through the obstructed lung, and prevents systemic venous engorgement and arterial anæmia. In other words, it equalizes the circulation. Moreover, in stimulating the peripheral vagi, digitalis improves the tone of the bronchioles and prevents collapse of the air vesicles, and, by the same action, steadies the rhythm of the breathing. The drug is likewise an efficient circulatory stimulant in influenza of horses and distemper of dogs. Digitalis is frequently prescribed in chronic bronchitis and emphysema, 
to strengthen the heart and obviate passive pulmonary congestion and congh. Valvular disease of the heart, in its varions phases, is the most common field of usefulness for digitalis in human medicine, but in veterinary practice these disorders are rarer and the exact lesion difficult or impossible to diagnose. In mitral stenosis and regurgitation and aortic stemosis, with lack of compensatory hypertrophy of the heart and evidence of circulatory disturbances, digitalis is clearly indicated. In these conditions the drug enables the heart to pump more blood into the arteries and prevents engorgement of the right heart and veins and the occurrence of dropsy. Digitalis is counter-indicated in aortic insufficiency, because in prolonging diastole it allows more time for the blood to flow back from the aorta through the leaky valve into the ventricle. As a general proposition, digitalis is inferior to aconite in simple cardiac hypertrophy. But this does not apply when enlargement of the heart is insufficient to compensate for valvular lesions.

Digitalis is extremely successful in palpitation of the heart (horses) following over-exertion, but is not appropriate in palpitation due to nervcusness (logs), or to indigestion. Digitalis may be exhibited to alvautage in rheumatic fever and in endocarditis or pericarditis to quiet the heart and secure rest by prolonging diastole.

2. Digitalis is a valuable diuretic in dropsy of cardiac origin (dogs), by stimnlating the heart and overcoming venous stasis in the kilneys and elsewhere. It is often desirable to combine iron preparations with digitalis. Turbidity results from the action of iron on the tanuic acid contained in digitalis when in solution, but this can be removed by the addition of a little diluted phosphoric acid.

3. Since digitalis contracts the nterus and blood vessels, it has been prescribed in nterine hæmorrhage, and to stop bleeding from other interual parts; but it is inferior to ergot on accomnt of the general rise of blood tension. Hæmoptysis, due to passive congestion of the lungs in mitral disease, is relieved by digitalis. 
4. The use of digitalis in large doses as an antipyretic in fevers is not without danger and is inadvisable.

\section{Strophanthus. Strophanthus.}

$$
\text { (U. S. \& B. P.) }
$$

The seed of Strophanthus hispidus De Candolle (nat. ord. A pocynaceæ), deprived of its long awn.

Habitat.-Tropical Africa. There are eighteen species, and the seeds from at least two are found in commerce. This has led to some confusion, as the chemical and physiological properties of their different products vary to some extent.

Description.-About $15 \mathrm{Mm}$. long and 4 or $5 \mathrm{Mm}$. broad; oblong-lanceolate, flattened and obtusely edged, grayishgreen, covered with appressed, silky hair, one side with a ridge extending into the attenuated, pointed end; kernel white and oily, consisting of a straight embryo, having two thin cotyledons, and surrounded by a thin layer of perisperm; nearly inodorous; taste very bitter.

Constituents. - The chief one is (1) Strophanthin, $\mathrm{C}_{31} \mathrm{H}_{48}$ $\mathrm{O}_{12}$ (8-10 per cent.), a glucoside occurring in white, transparent crystals, and having a very bitter taste. Soluble in water and alcohol, and insoluble in chloroform or ether. Decomposed by sulphuric acid into glucose and strophanthidin. Strophanthin is said to be contained only in S. Kombé. (2) Kombic acid. (3) An alkaloid, Ineine. (4) Tanghinin, occurring in rhombic prisms.

PREPARATION.

*Tinctura Strophanthi. Tincture of Strophanthus. (U. S. \& B. I.)

Made by digestion and percolation of strophanthus(50) with alcohol and water to make 1000 . (U. S. P.)

Dose.-H \& C., 3 i.-iv. (4.-15.); D., mii.-x. (.12-.6). The minimum doses should be used at first, since preparations vary in strength.

* Tincture of strophanthus has been doubled in strength $(100-1000)$ in the 1905 U.S. P. The doses should be therefore half those given above. 


\section{Strophanthinum. Strophanthin.}

(Non-official.)

$$
\text { Dose.-H., gr. } \frac{1}{5}-\frac{1}{2}(.012-.03) ; \mathrm{D} ., \mathrm{gr}_{\cdot} \cdot \frac{1}{10} \overline{0}^{-\frac{1}{6}}(.0006-.001) \text {. }
$$

The glucoside is not always pure and the dose is uncertain and must be given with caution.

Action External.-Strophanthus is a local anæsthetic.

Action Internal.-Strophanthus is a gastro-intestinal irritant in large doses, like digitalis, and produces violent purging and sometimes vomiting. On account of its bitter qualities it may act in small medicinal doses as a stomachic, improving appetite and stimulating gastric secretion and motion.

Circulation.-The action of strophanthus on the circulation is very similar to that of digitalis, but it is a more powerful and uncertain heart stimulant and produces less vascular constriction. By its influence the heart beats are made more forcible, infrequent and regular. Diastole is prolonged and systole is strengthened but unaltered in time. Arterial tension is raised and the pulse wave is increased in volume and force. The physiological details are not so well ascertained as are those of digitalis, but it is known that strophanthus directly stimulates the heart muscle in moderate doses. The vessels are slightly contracted, but not. nearly as much so as by digitalis. Increased blood pressure results mainly from the augmented heart's action. In poisoning, the peripheral vagi are paralyzed and vascular tension falls, owing probably to tetanic contraction of the ventricles. The heart is arrested in systole or diastole.

Nervous System.-Muscles and Respiration.-Strophanthus is a powerful muscle poison. Therapentic doses increase muscular activity and tone, while toxic quantities paralyze voluntary muscles. Medicinal doses not only stimulate the voluntary muscles, but also the unstriped muscle of the heart, and to some extent that of the vessel walls. The nerve centres and trunks are unaffected except by the local application of strophanthus, which paralyzes 
the sensory nerve endings and muscular tissue. The respiration is uninfluenced by therapeutic doses of strophanthus, but in poisoning death sometimes takes place from respiratory failure following paralysis of the respiratory muscles.

Kidneys. - The kidneys are irritated by large doses of strophanthus and the urine is albuminous. Inflammation of the renal tubules with minute hæmorrhages are found post mortem. The renal vessels are not dilated, and the oncometer shows that the size of the kidney is not increased. The drug stimulates the secretory cells of the renal tubules and causes diuresis directly as well as indirectly by increasing general blood pressure. The active principle is eliminated in the urine. A cumulative action in the lower animals and in man has been noted by several observers.

Uses Internal.-In general, it may be stated that the indications for strophanthus are identical with those for digitalis, but the former is not so certain in its effects. It is of value as a substitute for digitalis when this medicine is not well borne, and to replace digitalis temporarily in order to avoid its cumulative action. On account of the stimulant and diuretic properties of strophanthus, it is useful in mitral disease, cardiac dropsy, pericardial and pleural effusions, pulmonary oedema, and chronic nephritis; but in heart disease digitalis should be tried first.

\section{Convallaria. Convallaria. (U. S. P.)}

Synonym.-Lily of the Valley, E.; muguet, Fr. The rhizome and roots of Convallaria majalis Linné (nat. ord. Liliaceæ).

Habitat.-United States, in the Alleghany Mountains, Europe and northern Asia.

Description. - Of horizontal growth and somewhat branched; about $3 \mathrm{Mm}$. thick, cylindrical, wrinkled, whitish, marked with few circular scars; at the annulate joint with about 8 or 10 long thin roots; fracture somewhat fibrous, 
white; odor peculiar, pleasant; taste sweetish, bitter and somewhat acrid.

Constituents.-Two glucosicles; (1) Convallamarin $\left(\mathrm{C}_{46} \mathrm{H}_{44}\right.$ $\mathrm{O}_{24}$ ), the active principle. A white powder, having a sweet, bitter taste, and soluble in water and alcohol. (2) Convallarin $\left(\mathrm{C}_{34} \mathrm{H}_{31} \mathrm{O}_{11}\right)$, occurring in prismatic crystals, solnble in alcohol, sparingly in water, and insoluble in ether. A purgative.

PREPARATION.

Extractum Convallarice Fluidum. Fluid Extract of Convallaria. (Ü. S. P.)

Made by maceration and percolation with diluted alcohol, and evaporation, so that $1 \mathrm{Cc} .=1 \mathrm{Gm}$. of the crude drug.

Dose.-H. \& C., 3 i-iv. (4.-15.); D., Mıv.-x. (.3..6).

Action and Uses Internal. - Convallaria, by virtue of convallamarin, resembles digitalis in its action on the heart, vessels and kidneys, but is not so reliable and powerful. The indications for its use are the same as those for digitalis. In some cases it is successful where the latter drug lias failed. Occasionally purging has resulted owing to the convallarin in convallaria. The fatal dose of convallamarin is stated by Marmé to be gr. $\frac{1}{4}$ to $\frac{1}{2},(.015-.03)$ for dogs, and yet the dose recommended in veterinary text books is gr. $\frac{1}{2}$ to 2 (.03-.12). The glucoside is not a pure principle and therefore varies in strength. For this reason the dose is uncertain and its use undesirable.

\section{Scrlla. Squill. (U. S. \& B. P.)}

Synonym.-Squills, E. ; meerzwiebel, G. ; scille, squille, Fr.; bulbus scillæ, P. G.

The bulb of Urginea maritima (Linné) Baker (nat. ord. Liliaceæ), deprived of its dry, membranaceous outer scales, and cut into thin slices, the central portions being rejected. Habitat.-Southern Europe, on the shores of the Mediterranean. 
Description.-In narrow segments about $5 \mathrm{Cm}$. long, slightly translucent, yellowish-white or reddish, brittle and pulverizable when dry; tough and flexible after exposure to damp air; inodorous; taste mucilagiuous, bitter and acrid.

Constituents. - Various active principles have been recovered, but it is doubtful if any completely represent the action of the drug. All possess some poisonous properties. Merck sells three substances derived from squill: (1) Scillitoxin, a glucoside. (2) Scillipicrin. (3) Scillin. In addition, the drug contains mucilage.

Squill Dose.-H., 3 i.-ii. (4.-8.) ; C., 3 ii.-iv. (8.-15.) ; Sh., gr.xv.-xxx. (1.-2.); D., gr.i.-v. (.06-.3).

\section{PREPARATIONS.}

Extractum Scilloe Fluidum. Fluid Extract of Squill. (U. S. P.)

Made by maceration and percolation with alcohol and water, and evaporation, so that $1 \mathrm{Cc} .=1 \mathrm{Gm}$. of the crude drug.

Dose.-H., ji.-ii. (4.-8.); C., з ii.-iv. (8.-15.); Slı., mxv.-xxx. (1.-2.) ; D., mi.-v. (.06-.3).

Tinctura Scille. Tincture of Squill. (U. S. \& B. P.)

Made by maceration and percolation of squill, 150; with alcohol and water to make 1000 . (U. S. P.)

Dose.-H., 3 vi.- 3 i.ss. (24.-45.); C., 3 i.ss.-iii. (45.-9n.); Sh., 3 i.ss.-iii. (6.-12.); D., $\Pi \mathrm{v} .-\mathrm{xxx}$. (.3-2.).

Syrupus Scillo. Syrup of Squill. (U. S. \& B. P.)

Made by boiling and filtering vinegar of squill, 450 ; adding sugar, 800 ; straining and adding water to make 1000 . (U. S. P.)

Dose.-H. , 3 ss. (15.); D., 3 ss.-i. (2.-4.).

Incompatible with ammonium carbonate.

Syrupus Scilla Compositus. Compound Syrup of Squill.

(U. S. P.)

Synonym.-Coxe's Hive Syrup. Fluid extract of squill, 80 ; fluid extract of senega, 80 ; antimonium and potassium tartrate, 2 ; precipitated calcium phosphate, 10 ; sugar, 750 ; water to make 1000.

Dose.-D., mv.-xxx. (.3-2.).

Action Internal. - Gastro-intestinal Tract.-Therapentic doses do not exert any effect, but toxic quantities cause vomiting and purging in dogs, with fall of temperature, 
stupor, intermittent paralysis, convulsions, suppression or scanty secretion of bloody urine, and death within 12 or 15 hours. Lesions of gastro-enteritis and congestion of the kidneys are found after death.

Circulation.-The action of squill on the heart and vessels is practically identical with that of digitalis.

Respiration.-Clinical experience, rather than physiological experiments, has shown that squill acts as an expectorant during its elimination from the bronchial mucous membrane, thereby increasing secretion and vascularity.

Kidneys.-Squill is eliminated mainly by the kidneys, and in its excretion directly stimulates them and increases the amount of urine. In toxic doses it produces acute parenchymatous nephritis and urinary suppression. Squill is a more powerful diuretic than digitalis.

Uses Internal.-Squill is useful in ascites of dogs, resulting from valvular lesions or otherwise, to stimulate the heart and cause diuresis. It may be given to advantage in pill with digitalis, calomel, and extract of hyoscyamus-one grain of each. Small doses of squill are often exhibited to dogs in the second stage of acute bronchitis, and occasionally to horses, as an expectorant; and in large doses as an emetic for logs in the form of the simple or compound syrup. The drug is indicated in bronchitis with scanty secretion, or when exudation is excessive to improve the tone of the bronchial mucous membrane.

\section{Class 2.-Decreasing the Force and Frequency of the Heart.}

Aconitum. Aconite.

Synonym.-Aconiti radix, B.P.; racine d'aconit, Fr.; tubera aconiti, P.G.; eisenhutknollen, G. The tuber of Aconitum Napellus Linné (nat. ord. Ranunculaceæ).

Habitat.-Northwestern North America, Europe and Asia in mountainous regions, and cultivated in the United States for its showy flowers. 
Description.-From 10 to $20 \mathrm{Mm}$. thick at the crown; conically contracted below; from 50 to $75 \mathrm{Mm}$. long, with scars or fragments of radicles; dark brown externally, whitish internally, with a rather thick bark; the central axis about seven-rayed ; without odor ; taste at first sweetish, soon becoming acrid and producing a sensation of tingling and numbness which lasts for scme time.

Constituents.-The alkaloid representing the action of the drug is aconitine $\left(\mathrm{C}_{33} \mathrm{H}_{42} \mathrm{NO}_{12}\right.$ ? $)$, which is precipitated by ammonia from an aqueous solution of an alcoholic extract of the root of various species. It is a colorless, crystalline, or amorphous, gray powder, almost insoluble in water, and soluble in 40 parts of alcohol or ether, and 1 part of chloroform. Its salts are soluble in water.

Commercial preparations vary in purity and strength, and since it is extremely poisonous (an Indian arrow poison) its internal administration is undesirable. Psendo-aconitine. $\left(\mathrm{C}_{36} \mathrm{H}_{49} \mathrm{NO}_{11}\right)$, aconine $\left(\mathrm{C}_{26} \mathrm{H}_{39} \mathrm{NO}_{11}\right)$, and other alkaloids in combination with aconitic acid $\left(\mathrm{C}_{6} \mathrm{H}_{6} \mathrm{O}_{6}\right)$, have been obtained from aconite, but their identity and chemistry are uncertain.

Aconite Dose.-H. \& C., gr.iii-xx. (.2-1.3); D., gr. $\frac{1}{10}$-ii. (.006-.12).

Aconitina Nitras. (Squibb.) Subcutaneously.

H., gr. $\frac{1}{30}(.002) ; \mathrm{D}$., gr. $\frac{1}{20}-\frac{1}{100}(.0003 \cdot .0006)$.

\section{PREPARATIONS.}

Tinctura Aconiti. Tincture of Aconite. (U.S. \& B. P.)

Synonym.-Teinture de racine d'aconit, Fr.; eisenhuttinktur, G. Made by maceration and percolation of aconite, 350; with alcohol and water to make 1000 . (U. S. P.)*

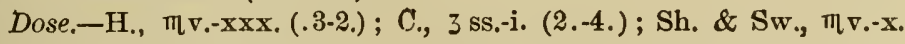
(.3-.6); D., Mlss.-v. (.03-.3).

Extractum Aconiti Fluidum. Fluid Extract of Aconite. (U.S. P.)

Made by maceration and percolation with alcohol and water, and evaporation, so that $1 \mathrm{Cc} .=1 \mathrm{Gm}$. of the crude drug.

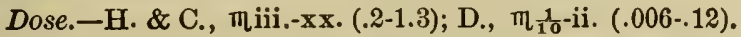

* The U.S. P. (1905) calls for a tincture of aconite having about one-third thestrength of above, or exactly $10 \%$. The doses therefore should be three times those given above. 
Extractum Aconiti. Extract of Aconite. (U. S. P.)

Made by maceration and percolation with alcohol, and evaporation to pilular consistence.

Dose.-H., gr.i..iii. (.06-.2); D., gr. $\frac{1}{10}-\frac{1}{4}(.006-.015)$.

$$
\text { Extractum Aconiti. (B. P.) }
$$

Dose.-H., gr.iii.-vi. (.2-.36); D., gr.ss.-i. (.03-.06).

Fleming's Tincture. (Non-official.) (79 per cent.)

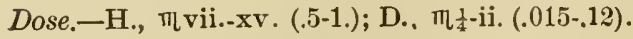

\section{ACONITE AND ACONITINE.}

Action External.-Aconite or aconitine applied to mucous membranes, raw surfaces or the unbroken skin, irritates and then paralyzes the nerves of touch and temperature. This is evidenced in the human subject by a sensation of tingling and burning, followed by unmbness and local anæsthesia.

Action Internal.-Digestive Tract.-Aconite in medicinal doses has no special effect on the digestive organs. Toxic doses produce nausea and retching, and, in animals capable of the act, vomiting.

Circulation.-The chief therapeutic value of aconite depends upon its influence over the heart and vessels. It reduces both the force and frequency of the cardiac pulsations and lowers blood tension. The physiological details accounting for this action are not positively known. It is probable that the slowing of the heart is primarily due to stimulation of the vagus endings in the heart, inhibitory centre in the medulla, and cardiac motor ganglia; but soon both the heart muscle and its contained ganglia are progressively depressed, and, by lethal doses, paralyzed. This local action of aconite on the heart is exhibiced when the drug is applied directly to the organ in situ, or to the cut-out heart, when its pulsations are diminished in force and frequency.

The vasumotor system is only indirectly influenced by depression of the sensory nerves, so that the normal peripheral stimuli do not reflexly stimulate the vasomotor centre and contract the arterioles. Therefore blood pressure is 
lowered for this reason and becanse of the lessened heart's action. In poisoning, the pulse is weak, irregular and intermittent; first slow and then rapid. There is ultimately paralysis of the peripheral vagi, heart muscle and its ganglia, and the vasomotor centres. 'The heart is arresterl in diastole, but death immediately results from respiratory failure.

Nervous System.-The most striking effect of aconite on the nervous system consists in loss of sensation and temperature sense after large medicinal doses. This phenomenon is due to depression of the sensory nerve terminations, and possibly to depression of sensory perceptive centres in the brain, and sensory side of the cort. The drug is not comparable with opium, since doses large enough to produce a general anoilyne action are dangerous.

Poisonous doses of aconite cause loss of motor power as well as loss of sensation. This latter effect follows paralysis of the motor nerve endings and perhaps the spinal motor tract. There is some disturbance of the brain, as exhibited by occasional delirium and convulsions. The latter are supposed to follow interference with the circulation at the base of the brain. Furthermore, in man, dizziness, blindness, deafness and loss of speech sometimes occur in lethal poisoning.

Respiration.-The breathing of animals under the influence of aconite resembles that observed after section of the vagi. The respiration is slow ; the expiration is prolonged, and is succeeded by a considerable interval before the next inspiration. This condition is brought about by depression of the mednllary respiratory centres and terminations of the afferent vagi in the lungs.

Temperature.-The bodily heat is reduced by aconite in fever, after medicinal doses, and in poisoning by the drug. Vascular dilatation, retarded circnlation, and therefore heat dissipation, probably explains the antipyretic action.

Skin.-Aconite sometimes produces slight diarphoresis. The cause of this action is unknown. 
Kidneys and Elimination.-The urinary flow is somewhat increased, especially in fever. The cause is also uncertain; neither is the mode of elimination understood.

Toxicology.-The minimum fatal dose of aconite is about 3 i. for the horse; gr.xx. for medium sized dogs; and gr.v.-vi. for cats. The smallest fatal dose recorded in man is a teaspoonful of tincture of aconite, equivalent to about gr.xxx. of the crude drug. The minimum lethal quantity of aconitine is gr. $\frac{1}{16}$ for man, and about the same for cats. For dogs it is from gr. $\frac{1}{4}$ to $\mathrm{gr} \cdot \frac{1}{2}$. The writer has found that cats will live from fifteen minutes to half an hour after receiving the smaller deadly doses under the skin, but large doses produce death immediately by paralyzing the heart. Large therapeutic doses cause, in horses, restlessuess, pawing the ground, shaking of the head, champing of the jaws, increased secretion of salivary mucus, and attempts at swallowing, probably owing to the peculiar sense of irritation produced by the drug in the throat. Nausea and retching are observed in all animals, while vomiting occurs in dogs and cats. The pulse and respiration are weakened and generally retarded. After lethal doses these symptoms are intensified. We observe violent retching, frequent and difficult attacks of swallowing, ejection of frothy mucus from the mouth, copious sweating in horses; pulse first weak and infrequent, later rapid, running and almost imperceptible; respiration slow, interrupted, and shallow, and reduction of temperature. Death is preceded by muscular twitchings in the horse and loss of strength, so that the subject falls and is unable to rise; or in the case of cats and rabbits, the animals jump vertically into the air, topple over backwards and go into convulsions, lying helpless on their side. The labial muscles are retracted and the lips drawn back, showing the teeth covered with foam. The face is anxions, the eyeballs are retracted or protruded, and the pupils more commonly dilated. Death takes place usually from asphyxia, occasionally from syncope. The post-mortem appearances are simply those resulting from asplyxia. 
Treatment.-Evacuate the stomach by pump or siphon. Enetics are cortraindicated as disturbing the heart. Carsliac and respiratory stimulants are to be given subcutaneously, as alcohol, ammonia, ether, and tincture of digitalis, in addition to inhalation of amyl nitrite. The patient must be kept quiet, and artificial respiration done if practicable and necessary.

Experimental and clinical evidence abundantly prove the antagonism of digitalis as an antidote in stimulating the heart, even when it has stopped beating, in aconite poisoning.

Uses External.-Aconite may be applied in the official liniment (fluid extract of aconite, 40 ; chloroform liniment, 60 ) to relieve pain of an inflammatory, neuralgic, or rheumatic character ; or as aconitine in ointment (2-4 per cent.) for the same purposes. Aconitine is very expensive, however. Care must be exercised to prevent undue absorption and poisoning.

Uses Internal.-Aconite fills certain indications which no other drug does. It is useful in fever because :

1. It diminishes the force and frequency of the heart.

2. It causes arterial relaxation and equalizes the circulation; i.e., it allows the blood to flow away from congested areas into dilated peripheral vessels. It is not so powerful as veratrum viride.

3. It lowers temperature and produces sweating and diuresis.

4. It relieves pain and restlessness to some extent.

Aconite is particularly applicable, conjoined with sweet spirit of nitre, in the first stages of febrile diseases; in those attacking the young; and in those of self-limited and short duration, viz., coryza, laryngitis, pharyngitis, pleuritis, bronchitis and pneumonia nncomplicated with influenza. It is also indicated in the initial period of acute muscular rheumatism, enteritis and peritonitis (combined with opium), and in mammitis, lymphangitis, and laminitis. Spasmodic and painful disorders, as colic, are relieved more success- 
fully by powerful anodynes and antispasmodies (opium and bellarlouna).

In chronic or long continued fevers, the use of aconite should not be persisted in, but it shonld be given at the very outset of fevers and repeated frequently in small doses. Mv. for the horse, and Mss. for the dog, every fifteen minntes for two hours, and afterwards $m_{x}$. for the horse, and $M i$. for the dog, hourly, being governed, however, by th: condition of the pulse and temperature and the physiological effects. Aconite is a useful sedative in some carcliac disturbitucs. It quiets nervous palpitation, and that form resulting from hypertrophy of the heart. It can be admiuistered to alvantage in the first stages of acute pericarditis and endocarditis.

\section{Veratrum Viride. Veratrum Viride.}

Synonym.-Veratri viridis rhizoma, B. P.; American hellebore, green hellebore root, Indian poke root, E.; grüner germer, G.

The rhizome and roots of Veratrum viride Solander (nat. ord. Liliaceæ).

Description.-Rhizome upright, obconical, simple or dividecl, from 3 to $8 \mathrm{Cm}$. long and 2 to 4 or $5 \mathrm{Cm}$. thick; externally blackish-gray, internally grayish-white; showing numerous short, irregular wood-bundles. Roots emanating from all sides of the rhizome, numerous, shrivelled, light yellowish-brown; about 10 to $20 \mathrm{Cm}$. long and $2 \mathrm{Mm}$. thick. Inodorous, but strongly sternutatory when powdered; taste bitterish and very acrid.

Constituents.-1. Jervine $\left(\mathrm{C}_{21} \mathrm{H}_{27} \mathrm{NO}_{3}\right)$, a pure alkaloid, occurring also in veratrum album. 2. Veratroidine or cevadine; an impure alkaloid, non-crystallizable, composed chiefly of mbijervine in combination with resin and other bodies. It is said to be identical with veratrine. 3. Pseudojervine. 4. Traces of rubijervine. 5. A resin, nearly inert, but a gastro-intestinal irritant.

Veratrum Viride Dose.-H. \& C., 3 ss.-i. (2.-4.); Sh. \& Sw., gr.xx.-xxx. (1.3-2.); D., gr. $\frac{1}{10}$-i. (.006-.06). 
PREPARATIONS.

Extractum Veratri Väridis Fluidum. Fluid Extract of Veratrum

Viride. (U.S. P.)

Made by maceration and percolation with alcohol, and evaporation, so that $1 \mathrm{Cc} .=1 \mathrm{Gm}$. of the crude drug.

Dose.-H. \& C., 3 ss.-i. (ఖ.-4.); Sh. \& Sw., Mxx.-xxx. (1.3-2.); D., गI $\frac{1}{10}$-i. $(.006-.06)$.

Tinctura Veratri Liridis. Tincture of Veratrum Viride.*

(U.S. \& B. P.)

Made by maceration and percolation of veratrum viride, 400 ; and alcohol to make 1000 . (U. S. P.)

Dose.-H. \& C., 3 i.-ii. (4.-8.); Sh. \& Sw., mxl.- 3 i. (2.6-4.) ; D., ml $\frac{1}{5}$-ii. $(.012-.12)$.

\section{JERVINE.}

Action Internal.-Digestive Tract.-Large doses cause salivation, but have little effect on the stomach or bowels.

Circulation.-Jervine is a powerful depressant to the heart muscle, its contained ganglia, and the vasomotor centres. Large doses, therefore, weaken and slow the pulse and lower vascular tension.

Respiration.-The alkaloid is also a profound respiratory depressant, and death occurs from asphyxia in poisoning, the heart continuing to beat after cessation of breathing:

Nervous System.-In poisoning by jervine, animals exhibit muscular tremors and weakness, and finally fall from loss of muscular power. Lying prostrate on the ground in a paralytic state, they are attacked by clonic convulsions. The paralysis is attributable to depression of the cells of the inferior cornua, while the convulsions are due to disturbance of the cerebral circulation, or stimulation of the cerebral motor tract. The muscles and nerves are unaffected by jervine, but there is partial auæsthesia of spinal origin observed just before death.

\section{VERATROIDINE.}

Action Internal.-The alkaloid is a decided gastro-intestinal irritant and produces vomiting in animals capable of the act, and often ! n. .

* The U. S. P. (1905) The doses should be font ili. egivilinose. 
Circulation.--Veratroidine mainly influences the cardiac inhibitory apparatus. When artificial respiration is practised, so that the effect of the substance on the respiratory centres will not overcome the action on the circulation, it is found that the alkaloid slows the heart's action in moderate doses by stimulating the pneumogastric centres. Large toxic doses, on the other hand, paralyze the vagus nerve endings in the heart, and the cardiac pulsations therefore become very frequent. The vasomotor centres are uninfluenced by ordinary quantities of veratroidine.

Respiration.-The depressing action of veratroidine on the respiratory functions is more marked than that exerted on the heart, so that, unless artificial respiration is sustained, asphyxia supervenes, and this leads to vasomotor spasm and great rise of blood pressure. Death ensues from respiratory failure.

Nervous System.-The influence of veratroidine upon the nervous system is similar to that of jervine, but the spasmodic phenomena are not so prominent.

\section{VERATRUM VIRIDE.}

Action Internal.-The action of the drug is a resultant of the action of jervine and veratroidine.

Circulation.-The most noteworthy therapentic value of veratrum viride depends upon its effect on the circulation. It first lowers the force (jervine), and, if continued, the frequency (veratroidine and jervine) of the pulse, and also reduces vascular tension. Veratrum viride is considerably more powerful than aconite, which depresses the action of the heart but does not directly lessen arterial action. Veratrum viride is, however, inferior to aconite for general purposes, as it does not possess the anodyne, diarphoretic, or diuretic properties peculiar to the latter drug.

The temperature is reduced several degrees by large doses of veratrum viride.

Toxicology. - The symptoms exhibited in veratrum viride poisoning are: salivation, vomiting, or attempts at vomiting, 
purging, abdominal pain, muscular weakness, difficulty in progression, loss of power and general paralysis, muscular tremors and spasms, and occasionally convulsions. The pulse is unaltered in rate at first, but later becomes infrequent and compressible and finally rapid, thread-like and running. The respiration is shallow, the temperature reduced, the skin is cold and clammy; there is semi-consciousness, loss of sight and death from asphyxia.

Treatment should be pursued with cardiac and respiratory stimulants, as amyl nitrite (by inhalation), alcohol, strychnine and atropine; tannic acid as a chemical antidote; opium to subdue pain; and demulcents to relieve local irritation of the digestive tract. Warm water should be given the smaller animals to wash out the stomach and to assist vomition, and quietude should be enforced. In man, fatal poisoning is rare, since the drug is spontaneously vomited. The same would probably apply to dogs. Recovery has ensued in horses after ingestion of two ounces of veratrum album root.

Administration.-It is advisable to give small doses of the tincture or fluid extract hourly. In the case of the smaller patients the dose should be preceded by the administration of a correspondingly small dose of laudanum $\left(m_{\nabla .}-x.\right)$ to prevent vomiting.

Uses Internal.-The alkaloids of veratrum viride are difficult to obtain in their purity, and are not used in practice.

The drug is applicable as a circulatory sedative at the outset of sthenic diseases aftlicting strong, plethoric animals. Veratrum viride bleeds an animal into its own vessels by causing vascular dilatation. The indications are similar to those applying to venesection, and are therefore limited. In some cases of acute diseases, included within the limits defined above, it may prove of the ntmost service to cut short or even abort the attack.

In this list may be placed stheuic pneumonia, cerebritis, laminitis, puerperal fever, and, when veratrum is combined with opium to obviate stimulation of peristaltic action, 
enteritis, peritonitis, and abdominal wounds and injuries. Veratrum viride is said to relieve pain, lower temperature, and lessen the duration of acute rheumatic fever.

VERATRUM ALBUM.

Synonym.-Veratri albi rhizoma, white hellebore root, E.

Habitat.-Europe (used on the continent).

Constituents.-1. Jervine. 2. Veratralbine, which is allied to veratrine. Veratrum album generally resembles veratrum viride, but it is a more powerful gastro-intestinal irritant. The Germans recommend it to be given as an emetic to swine, in milk, gr.v.-xx. (.3-1.3), and as an emetic to cattle, 3 iv.-vi. (16.-24.). Its use is, however, attended with some danger in these doses.

\section{Veratrina. Veratrine. (U. S. \& B. P.)}

A mixture of alkaloids obtained from the seed of Asagræa officinalis (Schlechtendal et Chamisso) Lindley (nat. ord. Liliaceæ). (U. S. P.)

Derivation.-Obtained from the seed by alcohol, which is recovered by distillation, and the residue is treated with water to precipitate resins ; filtered, and veratrine is precipitated from the filtrate by ammonia. It is further purified by solution in water, decolorization, and reprecipitation.

Properties.-A white, or grayish-white, amorphous, or semi-crystalline powder; odorless, but causing intense irritation and sneezing when even a minute quantity reaches the nasal mucous membrane ; having an acrid taste, and leaving a sense of tingling and numbness on the tongue. Permanent in the air. Very slightly soluble in cold or hot water; soluble in 3 parts of alcohol, and very soluble in boiling alcohol; also soluble in 6 parts of ether, and in 2 parts of chloroform.

Dose.-H., gr.ss.-ii. (.03..12); C., gr.ii.-v. (.12-.3); D., gr. $\frac{1}{50}-\frac{1}{10}(.0012-.006)$.

The smaller doses to be given subcutaneously. 
PREPARATION.

Oleatum Veratrince. Oleate of Veratrine. (U. S. P.)

Veratrine, 4 ; olive oil, 6 ; benzoinated lard, 90 . (U. S. P.)

Action Internal.-Veratrine is a powerful irritant. If inhaled in minutest quantity it causes sneezing and a mucous, or bloody discharge. When injected under the skin, veratrine occasions suffering and restlessness. Rubbed into the skin or applied to a mucous membrane or a raw surface, it produces redness and pain followed by local anæsthesia.

Action Internal.-Here again veratrine is an intense irritant. After ingestion of large doses, there are salivation, violent vomiting, in animals capable of the act; often severe purging, pain, collapse, fall of temperature and other symptoms common to gastro-enteritis.

Nervous System and Muscles.-Veratrine is a direct poison to the motor and sensory nerves and muscles, first exalting and then depressing and paralyzing their functional activity. The cerebrum, and probably the spinal cord, escape its influence. Spasms and convulsions occur in veratrine poisoning, in the stage of motor excitement, while paralysis follows depression of the nerves and muscles. The action of the substance begins and ends sooner in the nerves than the muscles. The effect of veratrine on voluntary muscle is peculiar. A tracing of a muscle during contraction shows that the latent period, and that of ascent, is unaltered; but the amount of contraction is much augmented, as shown by the increased height of the curve; and the time of relaxation is greatly prolonged.

Circulation.-Veratrine affects the heart muscle much as it does the striped muscle, and the vagus nerve similarly to the spinal nerves. The cardiac muscle and vagus nerve (central and peripheral), and probably the vasomotor centres, are primarily stimulated and secondarily depressed and paralyzed. Three conditions have been noted in relation to the pulse, corresponding to different stages in the action of veratrine. 1. A small dose may induce a temporary 
rise of pulse rate, force and tension, by stimulation of the cardiac muscle and vasomotor centres. 2. Large doses are followed by slowing and weakening of the pulse. The vagus is stimulated and the heart muscle depressed. 3. Towards the end of fatal poisoning the pulse becomes weak, thread-like, rapid and irregular from paralysis of the heart muscle, inlibitory apparatus and vasomotor centres. It sometimes happens, however, that the pulse remains weak and slow in this stage because the paralyzing influence of the substance on the heart muscle prevents quickening of the heart even after the removal of inhibitory control. The heart is dilated and irresponsive to galvanism, after death, as are also the voluntary muscles.

Respiration.-Lethal doses paralyze the respiratory centres and death occurs from respiratory arrest. The breathing may be quickened by small doses of veratrine, owing to transient stimnlation of the respiratory centres and vagus nerve endings in the lungs.

Toxicology.-The symptoms of poisoning are referable to the action of veratrine on the digestive, nervous and muscular apparatus, heart, and respiratory organs. They include nausea, salivation, clammy sweating, excessive vomiting in dogs, cats and cattle, attempts at vomiting in the: horse, abdominal pain, severe purging, muscular twitchings or convulsions (excited by external stimuli), loss of muscular power and paralysis. The pulse, at first weak and infrequent, becomes thready, rapid and irregular. The temperature is reduced and the respiration is weak and slow. Death occurs in convulsions or paralysis. One-sixteenth of a grain has cansed alarming symptoms in man. The minimum fatal dose is about one grain for dogs. One grain subcutaneously, or five to six grains by the mouth, produce poisonous symptoms in horses.

Treatment.-External heat; respiratory and cardiac stimulants should be employed subcutaneously. Potassium carbonate and demulcent drinks are to be given internally. 
Uses External.-Veratrine is of trivial value, since its therapeutic application is narrowly limited. It is occasionally useful in the official ointment, or in greater strength (gr.xl. to $\xi i$.) for its local anæsthetic action applied over rheumatic joints. It may be employed also as a simple rubefacient. An aqueous solution of veratrine sulphate, or an alcoholic dilution of the pure alkaloid, is recommended by Ellenberger and other noted Germans in shoulder lameness, myalgia, and chronic rheumatic affections of the horse, to be injected every alternate day, or oftener, into the muscular tissue over the seat of the trouble. The animal should be led about for fifteen or thirty minutes while the pain of the treatment lasts. The initial close is $\mathrm{gr} \cdot \frac{2}{3}(0.4 \mathrm{Gm}$.), to be increased to gr. $1 \frac{1}{2}(0.1 \mathrm{Gm}$.).

Uses Internal.-Veratrine has been employed as a cardiac sedative and antipyretic in pneumonia, acute rheumatism, and in other sthenic disorders, but it is inferior for these purposes to aconite or veratrum viride, and its other effects are undesirable. The remedy is landed by foreign anthorities as an emetic and cathartic for cattle in impaction of the third stomach, and in conditions where it is desirable to quickly unload their digestive apparatus.

P. Cagny advises veratrine, grs. $2 \frac{1}{2}-3(.15-.20)$, subcutaneously, to be followed if necessary by daily doses of grs. $5-7 \frac{1}{2}$, $(0.3-0.5 \mathrm{Gm}$.) in mucilaginons drinks by the month. The same writer finds the drug useful in "broken wind." Veratrine stimulates intestinal secretion and peristalsis in the horse, but is inferior to eserine and pilocarpine in that animal as a quickly acting cathartic.

\section{SECTION VII.-DRUGS ACTING ON THE RESPIRATORY ORGANS.}

IPecacuanha. Ipecac. (U. S. \& B. P.)

Synonym. - Ipécacuanha, racine brésilienne, Fr.; brechwurzel, ruhrwurzel, G. 
The root of Cephaëlis Ipecacuanha (Brotero) A. Richard (nat. ord. Rubiaceæ).

Habitat.-South America from Brazil to Bolivia, and New Grenada. Cultivated in India.

Description.-About $10 \mathrm{Cm}$. long and 4 or $5 \mathrm{Mm}$. thick; mostly simple, contorted, dull grayish-brown or blackisl, finely wrinkled, closely and irregularly annulated, and often transversely fissured; bark thick, brittle, browuish, easily separated from the thin, whitish, tough, ligneous portion; odor slight, peculiar, uauseous; taste bitterish, acrid, nauseating.

Constituents. - 1. The alkaloid Emetine $\left(\mathrm{C}_{30} \mathrm{H}_{40} \mathrm{NO}_{5}\right)$, existing to the extent of 1-2 per cent., and representing in the main the action of the crude drug. It is a white, odorless, uncrystallizable powder, with a bitter, burning taste, and soluble in alcohol and chloroform; less so in ether, and very slightly in water, turning yellow on keeping. The impure commercial alkaloid occurs in brownish-red transparent and deliquescent scales, very soluble in water. 2. An amorphous, bitter glucoside. 3. An astringent, ipecacuanhic acid. 4. A volatile oil, starch, gum, tannin, coloring matter, etc. Hemidesmus exists as an impurity, it is cracked, not annulated; also bitter almond powder, which exhales the odor of prussic acid when wet. Emetine (non-official) is rarely used.

Dose of Powdered Root.-H, 3 i.-ii. (4.-8.) ; C., 3 ii.-iv. (8.-15.); Sh., 3 ss.-i. (2.-4.); D., gr.ss.-ii. (.03-.12).

Emetic.—D. \& Sw., gr.xv.-xxx. (1.-2.).

\section{PREPARATIONS.}

Extractum Ipecacuanha Fluidum. Fluid Extract of Ipecac.

(U. S. P.)

Made by maceration and percolation with alcohol and water, evaporation of a portion of the percolate, solution of the residue in the reserved portion of the percolate, and addition of alcolol and water, so that $1 \mathrm{Cc} .=1 \mathrm{Gm}$. of the crude drug.

Dose.-Same as powdered root. 
Syrupus Ipecacuanhce. Syrup of Ipecac. (U. S. P.)

Fluid extract of ipecac, 70 ; acetic acid, 10 : glycerin, 100 ; sugar, 700 ; water to make 1000 .

Dose.-D. (expectorant), $m \times x v .-3$ i. (1.-4.).

Vinum Ipecacuanhoe. Wine of Ipecac. (U. S. \& B. P.) (U. S. P.)

Fluid extract of ipecac, 100 ; alcohol, 100 ; white wine, 800 .

Dose.-Same as syrup of ipecac.

Pulvis Ipecacuanhce et Opii. (See opium.)

Tinctura Ipecacuanhre et Opii. (See opium.)

Action External.-Ipecac, and still more emetine, are decided irritants when applied to mucous membranes, raw surfaces, or even to the unbroken skin; causing, varionsly, hyperæmia, vesication, pustulation, and ulceration, according to their strength and the mode of application. Ipecac is said to possess some antiseptic properties not resident in emetine.

Action Internal.-Digestive Tract.-Ipecac is naturally a local irritant in the alimentary canal as well as externally. It increases the flow of saliva and gastric juice, besides dilating the blood vessels of the stomach. Small doses are stomachic, improving the digestion and tone of the gastric mucous membrane, and, in some cases, minute quantities are anti-emetic in allaying vomiting. Large doses, on the other hand, cause a little nausea and free vomiting. It is probable that emetine is not, as frequently stated, a specific emetic; that is, it does not act directly on the vomiting centre, although vomiting is produced, when emetine is thrown into the blood, by means of its elimination through the stomach. This is substantiated by the following facts: (1) Emetine is recovered in the first vomitus after its subcutaneous injection. (2) Vomition does not occur so rapidly, and more emetine is required, when the alkaloid is injected in place of being ingested. (3) Furthermore, when the vagus is previonsly divided, vomiting does not follow hyperdermic medication of emetine. The act does, however, follow the administration of apomorphine and tartar emetic under similar 
conditions. Therefore it is probable that in whatsoever way ipecac is exhibited, vomiting proceeds from direct irritation of the stomach or the vagus endings situated therein. Ipecac and emetine do not act so promptly as zinc sulphate or apomorphine (under the skin). The latter are accordingly preferable as evacuants in poisoning. Compared with tartar emetic, ipecac is less persistent and prostrating in its effect. It is probably the mildest emetic at our command. Ipecac and emetine increase secretion and cause hyperæmia and purging, sometimes of a bloody character, in their passage through the bowels. Gastro-enteritis may follow the administration of large quantities to dogs. Experimental evidence has shown that ipecac is an hepatic stimulant (cholagogue), increasing the secretion of bile.

Circulation.-Medicinal doses have no influence on the circulation. Lethal doses paralyze the heart muscle. The act of vomiting, nerertheless, may slightly depress the heart.

Respiratory Organs. - The lungs are often found inteusely hyperæmic after death by ipecac poisoning. Not only this; portions are seen to be collapsed and consolidated. In therapentic doses the pulmonary mucous membrane is stimulated during its elimination and is made more vascular' the secretion of bronchial mucus is increased and congh is reflexly excited. Ipecac is thus strictly a stimulating expectorant in so far as its action on the bronchial mucous membrane is concerued; but it is, perhaps unfortunately and misleadingly, classed by some (Brunton) as a depressing expectorant because of the side influence on the circulation.

Skin.-Ipecac is mildly diarphoretic in addition to and independent of this action common to nauseants in general.

Toxicology.-The following toxic phenomena are exhib. ited by animals: vomiting, loss of muscular power and paralysis, increased, followed by diminished reflex activity, failure of heart and respiration, and post mortem evidences of congestion and inflammation of the lungs and intestines. 
Three ounces of ipecac have killed a horse, and two grains of emetine have proved fatal to a dog.

Uses. - The indications for the therapentic employment. of ipecac may be described under the following heads:

1. Emetic and anti-emetic.

2. Expectorant.

3. Diarphoretic.

4. Empirically in dysentery and chronic diarrhœa.

1. Powdered ipecac is a good agent for dogs, cats and pigs, given in luke-warm water, in repeated doses if necessary, to empty an overloaded stomach. Also in acute bronchitis and laryngitis of dogs and cats, when the patient is endangered by accumulation of secretion, ipecac, by the forcible expulsion attending vomiting, removes secretion from the upper respiratory tract and clears out the stomach of any secretion which may have been swallowed. Ipecac is efficacions in stopping vomiting in cases of acute catarrh of the stomach in dogs, and is given as the wine with tincture of aconite, one drop each in a drachm of ice water, at half hour intervals. The drug is also of service in reflex vomiting, and that due to an atonic or depressed condition of the stomach. Therapentically, ipecac should only be of value in the latter disorder, but it nevertheless is often efficient in the vomiting of irritative dyspepsia, as noted above.

2. Ipecac is prescribed in the first stage of acute bronchitis, when the secretion is scanty, and again in bronchitis of long standing, to stimulate the bronchial mucous membrane. It may be given to dogs and cats in repeated expectorant doses of the wine or syrup, with other expectorants, diarphoretics and diuretics, as syrup of squill and spirit of nitrous. ether, or as Dover's powder, to all animals.

3. Ipecac is a feeble diarphoretic, and inferior to sweet spirit of nitre, aconite, alcohol or external heat, for general sudorific purposes. The combination of opium and ipecac, in Dover's powder, is an appropriate mixture to relieve pain and canse diarphoresis in acute rheumatism, and may cut: short attacks of acute inflammation of the respiratory tract. 
4. Ipecac is probably the best single remedy for the treatment of dysentery. It should be given in the form of powder, bolus, or pill every four hours, combined with opium to prevent nausea or vomiting. Extemporaneous decoctions are likewise useful given in the form of enemata, and may take the place of the drug by the mouth in dysentery or chronic diarrhœa. Ipecac has been used successfully in the treatment of anthrax, in man, by dusting the powdered drug over the external lesion and giving it internally every two hours.

\section{SECTION VIII.-ANTIPYRETIC AND ANTISEPTIC ORGANIC AGENTS.}

\section{Class 1.-Cinchona and its Alkaloids.}

Cinchona. Cinchona. (U. S. P.)

Synonym.-Cinchonæ cortex, B.P. ; cortex chinæ, E.; quinquina, Fr. ; chinarinde, $\mathrm{G}$.

The bark of Cinchona calisaya Weddell, Cinchona officialis Linné, and of hybrids of these and of other species of cinchona (nat. ord. Rubiaceæ), yielding when assayed not less thau 5 per cent. of total alkaloids, and at least 2.5 per cent. of quinine.

Habitat.-Indigenous in South America on the east slope of the Andes, and northwards into Colombia; southwards to the lower part of Bolivia, at altitudes varying from 5,000 to $10,000 \mathrm{ft}$. Cultivated in India, East Indies, Jamaica, and to some extent in South America.

Description.-In quills or incurved pieces, varying in length, and usually 2 or 3 or sometimes $5 \mathrm{Mm}$. thick. The outer surface covered with a gray or brownish-gray cork, usually slightly wrinkled, marked with transverse, and also with intersecting, longitudinal fissures (C. Calisaya), and sometimes with scattered warts and slight, longitudinal 
ridges; inner surface light cinnamon-brown, very finely striate; fracture short and granular in the outer layer, and finely fibrous in the inner layer; powder, light brown or yellowish-brown; odor slight, somewhat aromatic; taste bitter and somewhat astringent.

Constituents.-(a) Four chief alkaloids and three artificial products.

* Isomers

(1) Quinine $\left(\mathrm{C}_{20} \mathrm{H}_{24} \mathrm{~N}_{2} \mathrm{O}_{2}+3 \mathrm{H}_{2} \mathrm{O}\right)$. Solutions of its salts are fliorescent. Turns plane of polarization to left. Gives green color with ammonia and chlorine water. Precipitated from its salts as hydrate.

(2) Quinidine $\left(\mathrm{C}_{20} \mathrm{H}_{24} \mathrm{~N}_{2} \mathrm{O}_{2}\right)$. Solutions of its salts fluorescent. Differs from quinine in turning plane of polarization to right, and in being insoluble in ammonia except in excess.

(Quinicine is an artificial amorphous alkaloid, probably not occurring naturally, and obtained from quinine by heat and excess of a mineral acid. Quinoidine is a cheap brown amorphous substance obtained from the mother liquor after quinine sulphate has crystallized out, and contains a mixture of quinidine, cinchonine and cinchonidine.)

* Isomers

(3) Cinchonine $\left(\mathrm{C}_{19} \mathrm{H}_{22} \mathrm{~N}_{2} \mathrm{O}\right)$. Is not fluorescent. Turns plane of polarization to right. Does not assume a green color with ammonia $\{$ or chlorine water.

(4) Cinchonidine $\left(\mathrm{C}_{19} \mathrm{H}_{22} \mathrm{~N}_{2} \mathrm{O}\right)$ is slightly fluorescent. Turns plane of polarization to left.

Cinchonicine is an artificial alkaloid obtained from cinchonine by heat and an excess of a mineral acid.

Some other alkaloids of no particular importance are :

Quinamina $\left(\mathrm{C}_{19} \mathrm{H}_{24} \mathrm{~N}_{2} \mathrm{O}_{2}\right)$.

Paricina $\left(\mathrm{C}_{18} \mathrm{H}_{18} \mathrm{~N}_{2} \mathrm{O}\right)$.

Aricina, etc.

* Isomers are bodies composed of the same elements, in the same proportions, but possessing different chemical or physical properties. 
(b) Less Important Constituents.-(5) Kinic, or quinic acid $\left(\mathrm{C}_{7} \mathrm{H}_{12} \mathrm{O}_{6}\right)$, occurs in colorless prisms. Related to benzoic acid and eliminated in the urine as hippuric acid. Found in coffee beans and other vegetables.

The alkaloids in cinchona are naturally united with kinic or kinovic acid, and salts of this combination are used in medicine; i.e., quinine kinate, which is soluble and may be employed subcutaneously.

(6) Kinovic, or quinovic acid $\left(\mathrm{C}_{32} \mathrm{H}_{48} \mathrm{O}_{6}\right)$, a white, amorphous substance allied to kinovin.

(7) Kinovin, or quinovin $\left(\mathrm{C}_{30} \mathrm{H}_{48} \mathrm{O}_{8}\right)$, a glucoside readily decomposed into glucose and kinovic acid.

(8) Cincho-tannic acid, or kino-tannic and kinovi-tannic acid (2-4 per cent.). The astringent principle of cinchona. Distinguished from tannic acid in yielding green color with ferric salts.

(9) Cinchona red, the coloring matter of cinchona bark. Nearly insoluble in water.

(10) A volatile oil existing in minute amount.

(11) Starch, gum, resin and salts common to other vegetable matters.

Incompatibility.-Cinchona is incompatible with lime water, ammonia, metallic salts or gelatin.

Dose.-H., 3 ii.-iv. (8.-15.); C., 3 i.-ii. (30.-60.); Sh. \& Sw., 3 i.-iv. (4.-15.); D. \& C., gr.x.- 3 i. (.6-4.).

PREPARATIONS.

Extractum Cinchonce F'uidum. Fluid Extract of Cinchona.

$$
\text { (U.S. \& B. P.) }
$$

Made by maceration and percolation with glycerin, alcohol and water ; evaporation of the last portion of the percolate, and addition of the residue to first portion, with enough water and alcohol to make $1 \mathrm{Cc} .=1 \mathrm{Gm}$. of crude drug. (U. S. P.)

Dose.-Same as cinchona.

\section{Extractum Cinchonce. Extract of Cinchona. (U, S. P.)}

Made by maceration and percolation with alcohol and water; dise tillation of alcohol and evaporation to pilular consistence.

Dose.-H., 3 i.-ii. (4.-8.) ; D., gr.v.- $\mathrm{xxx}$. (.3-2.). 
Infusum Cinchonce. Infusion of Cinchona. (U. S. P.)

Made by percolation of cinchona, 60 ; with aromatic sulphuric acid, 10 ; and water to make 1000 .

Dose.-H., Oi. (500.) ; D., 3 ii-iv. (\$.-15.).

\section{Infusum Cinchonce Acidum. (B. P.)}

Dose.-H., Oi. (500.); D., 3 ii.-iv. (8.-15.).

Tinctura Cinchonce. Tincture of Cinchona. (U. S. \& B. P.)

Made by maceration and percolation of cinchona, 200; with glycerin, 75; and alcohol and water a sufficient quantity to make 1000 . (U.S. P.)

Dose.-H., ऊु̃i.-ii. (30.-60.); D., 3 ss.-ii. (2.-8.).

\section{Cinchona Rubra. Red Cinchona.}

Cinchonæ rubræ cortex, B. P. The bark of Cinchona succirubra Pavon (nat. ord. Rubiaceæ), containing not less than 5 per cent. of its peculiar alkaloids.

Habitat.-Ecuador, west of Chimborazo.

Description.-In quills or incurved pieces, varying in length, and from 2 to 4 or $5 \mathrm{Mm}$. thick; the outer surface covered with a grayish-brown cork, more or less rough from warts and longitudinal, watery ridges, and from few, mostly short, transverse fissures; inner surface more or less deep reddish-brown and distinctly striate; fracture short-fibrous in the inner layer; powder reddish-brown; odor slight; taste bitter and astringent.

Constituents.-Same as cinchona.

Dose.-Same as cinchona.

PREPARATION.

Tinctura Cinchonce Compositu. Compound Tincture of Cinchona.

(U. S. \& B. P.)

Made by maceration and percolation of red cinchona, 100 ; bitter orange peel, 80 ; serpentaria, 20 ; with glycerin, 75 ; and alcohol and water to make 1000 . (U. S. P.)

Dose.-H., 3 ii-iv. (60.-120.); D., 3 ss.-ir. (2.-15.) 
Quinine Sulphas. Quinine Sulphate. $\left(\mathrm{C}_{20} \mathrm{H}_{24} \mathrm{~N}_{2} \mathrm{O}_{2}\right)_{2}$ $\mathrm{H}_{2} \mathrm{SO}_{4}+7 \mathrm{H}_{2} \mathrm{O}$. (U.S. \& B.P.)

Synonym.-Chininum sulfuricum, P.G.; sulfas quinicus, disulphate or basic sulphate of quinia, E.; sulphate de quinine, Fr.; schwefelsaures chinin, G.

Derivation.-Prepared by boiling cinchona bark with hydrochloric acid and adding lime to the filtered decoction to precipitate alkaloids and coloring matter. The precipitate is washed and digested in boiling alcohol to dissolve quinine and cinchonine. The alcohol is distilled off and the residue dissolved in diluted sulphuric acid, boiled with animal charcoal, filtered, and quinine sulphate crystallizes, leaving cinchonine sulphate in solution.

Properties.-White, silky, light and fine needle-shaped crystals; fragile and somewhat flexible, making a very light and easily compressible mass; lustreless from superficial efflorescence after being for some time exposed to the air; odorless, and having a persistent and very bitter taste. The salt is liable to lose water on exposure to warm air, to absorb moisture in damp air, and to become colored by exposure to. light. Soluble at $15^{\circ} \mathrm{C}$. $\left(59^{\circ} \mathrm{F}\right.$.), in 740 parts of water, and in 65 parts of alcohol; in 30 parts of boiling water; also in 40 parts of glycerin ; in about 680 parts of chloroform, and freely in dilute acids.

Incompatibles.-Alkalies and their carbonates, iodine, and tannic acid.

Dose.-H. (tonic), gr.xv.- 3 i. (1.-4.); C., 3 ss.-i.ss. (2.-6.); Sh. \& Sw., gr.v.-x. (.3-.6) ; D. \& Cats, gr.i.-ii. (.06-.12). Antipyretic Dose-H. \& C., 3 ii.-iv. (8.-15.); Sh., gr.xxx.-xl. (2.-2.6); Sw., gr.xv.-xxv. (1.-1.6); D.\& Cats, gr.v.-x. (.3-.6).

Quinine Bisulphas. Quinine Bisulphate. $\mathrm{C}_{20} \mathrm{H}_{24} \mathrm{~N}_{2} \mathrm{O}_{2}$ $\mathrm{H}_{2} \mathrm{SO}_{4}+7 \mathrm{H}_{2} \mathrm{O}$. (U.S. \& B. P.)

Derivation.-Made by the action of sulphuric acid on quinine sulphate suspended in water ; filtration and crystallization. 
Properties.-Colorless, transparent or whitish, orthorhombic crystals or small needles; odorless, and having is very bitter taste ; efflorescing on exposure to the air ; soluble in 10 parts of cold water and in 32 parts of alcohol ; very soluble in boiling water and in boiling alcohol.

Dose.-Practically same as quinine sulphate, but theoretically it should be slightly larger. The salt is very soluble, but is commonly made extemporaneonsly by adding diluted sulphuric acid (Q. S.) to quinine sulphate in preparing solutions for medicinal use. The salt is serviceable for administration in pill; or for hypodermic injection, when it should be given in about one-third smaller dose than that of quinine sulphate by the mouth.

Quinine Hydrobromas. Quinine Hydrobromate.

$$
\mathrm{C}_{20} \mathrm{H}_{24} \mathrm{~N}_{2} \mathrm{O}_{2} \mathrm{H} \mathrm{Br}+\mathrm{H}_{2} \mathrm{O} \text {. (U. S. \& B. P.) }
$$

Derivation.-Made by the action of barium bromide, in solution, on quinine sulphate suspended in water; filtration, evaporation, and crystallization.

Properties.-White, light, silky needles; odorless, and having a very bitter taste. The salt is likely to lose water on exposure to warm or dry air. Soluble in 54 parts of cold water, and in 0.6 part of alcohol; very soluble in boiling water and boiling alcohol; also soluble in 6 parts of ether and in 12 parts of chloroform.

Dose._Same as quinine sulphate.

Quinine Hydrochloras. Quinine Hydrochlorate.

$$
\mathrm{C}_{20} \mathrm{H}_{2 \pm} \mathrm{N}_{2} \mathrm{O}_{2} \mathrm{H} \mathrm{Cl}+2 \mathrm{H}_{2} \text { O. (U. S. \& B. P.) }
$$

Derivation.-Made by the action of hydrochloric acid on quinine, and by crystallization.

Properties. - White, silky, light and fine, neelle-shaped crystals; odorless, and having a very bitter taste. The salt is liable to lose water when exposed to warm air. Soluble in 34 parts of cold water, and in 3 parts of alcohol ; in 1 part 
of boiling water, and very soluble in boiling alcohol; also soluble in 9 parts of chloroform.

Dose.-Same as quinine sulphate.

Quinine Valerianas. Quinine Valerianate. $\mathrm{C}_{20} \mathrm{H}_{24} \mathrm{~N}_{2} \mathrm{O}_{2} \mathrm{C}_{5} \mathrm{H}_{10} \mathrm{O}_{2}+\mathrm{H}_{2} \mathrm{O}$. (U. S. \& B. P.)

Derivation.-Made by decomposition of quinine sulphate with ammonia, followed by the immediate action of valerianic acid, and crystallization from a cold solution.

Properties.-White, or nearly white, pearly, lustrous, triclinic erystals, having a slight odor of valerianic acid, and a bitter taste. Permanent in the air. Soluble in 100 parts of cold water, and in 5 parts of alcohol; in 40 parts of boiling water, and in 1 part of alcohol.

Dose.-D., gr.i.-ii. (.06-.12). Three times daily as tonic.

Quinine Bimuriatis Carbamas. Double Muriate of Quinine and Urea. (Non-official.)

Very soluble and contains about 61 per cent. of quinine.

Dose.-Same as quinine sulphate, but used subcutaneously in 50 per cent. aqueous solution.

Chinoidinum or QuinoIdinum. Chinoidine or Quinoidine.

Brown, resinous mass, containing mixture of quinidine, cinchonine and cinchonidine. A cheap substitute for quinine sulphate.

Dose.-Three or four times that of quinine sulphate.

Quinidine Sulphas. Quinidiue Sulphate. $\left(\mathrm{C}_{20} \mathrm{H}_{24} \mathrm{~N}_{2} \mathrm{O}_{2}\right)_{3}$

$$
\mathrm{H}_{2} \mathrm{SO}_{4}+2 \mathrm{H}_{2} \mathrm{O} \text {. (U. S. P.) }
$$

The neutral sulphate of an alkaloid obtained from the bark of several species of cinchona (nat. ord. Rubiacer).

Synonym.-Sulfate de quinidine, Fr.; schwefelsaures chinidin (cinclinin), G.

Derivation.-Recovered from quinoidine, or from the 
mother liquors after the crystallization of quinine sulphate, by the same method as for quinine.

Properties. - White, silky needles, odorless, and having a very bitter taste; permanent in the air. Soluble in 100 parts of cold water, and in 8 parts of alcoliol; in 7 parts of boiling water, and very soluble in boiling alcohol; also in 14 parts of chloroform and in acidulated water; almost insoluble in ether.

Dose.-One-third larger than quinine sulphate.

Cinchoninæ Sulphas. Cinchonine Sulphate. $\left(\mathrm{C}_{19} \mathrm{H}_{22} \mathrm{~N}_{2} \mathrm{O}\right)_{2}$

$$
\mathrm{H}_{2} \mathrm{SO}_{4}+2 \mathrm{H}_{2} \mathrm{O} \text {. (U. S. \& B. P.) }
$$

Derivation.-Obtained from the mother liquors after the crystallization of the sulphates of quinine, quinidine and cinchonidine, by precipitation with caustic soda, washing with alcohol to free it from other alkaloids, solution in sulphuric acid, and by purification with animal charcoal, and crystallization.

Properties.-Hard, white, lustrous, prismatic crystals, without odor, and having a very bitter taste. Permanent in the air. Soluble in 66 parts of cold water, and in 10 parts of alcohol; in 13.59 parts of boiling water, and in 3.25 parts of boiling alcohol. Also soluble in 78 parts of chloroform, but almost insoluble in ether.

Dose.-One-third larger than that of quinine sulphate.

Cinchonidinæe Sulphas. Cinchonidine Sulphate.

$$
\left(\mathrm{C}_{19} \mathrm{H}_{22} \mathrm{~N}_{2} \mathrm{O}\right)_{2} \mathrm{H}_{2} \mathrm{SO}_{4}+3 \mathrm{H}_{2} \mathrm{O} \text {. (U. S. \& B. P.) }
$$

Derivation.-Procured from the mother liquors, after the crystallization of quinine sulphate, by further con- 1 centration. Purified by crystallization from alcohol, and finally from hot water.

Properties. - White, silky, acicular crystals, without odor, and having a very bitter taste; slightly efflorescent on exposure to air. Soluble in 70 parts of cold water, and in 66 parts of alcohol; in 1.42 parts of boiling water, and in 8 parts 
of boiling alcohol ; also soluble in 1316 parts of chloroform, and almost insoluble in ether.

Dose--One-third larger than that of quinine sulphate.

CINCHONA AND ITS ALKALOIDS AS REPRESENTED BY QUININE.

Action External.-Quinine is a powerful antiseptic and microbicide. A solution (1 to 250) of the alkaloid or its salts are poisonous to the fungi of fermentation and putrefaction. A one per cent. solution quickly destroys bacteria and vibrios, but spores may live in it for some days. Quinine and its salts cause irritation of the denuded skin, or mucous membranes, but exert no effect upon the unbroken skin.

Action Internal.-Digestive Troct.-Quinine, in therapeutic doses, acts as a simple bitter (stomachic), and therefore promotes appetite and gastric digestion. It stimulates the gustatory nerves in the mouth and gastric nerves in the stomach, thus reflexly increasing the flow of saliva and gastric juice, and the vascularity and peristaltic motion of the stomach together with the appetite. Large doses, particularly if the stomach be irritable, may cause vomiting. Quinine becomes dissolved in the gastric juice and is converted into the chloride. A portion unabsorbed finds its way into the bowels and is there precipitated by the alkaline juices and bile, whose acids form insolnble salts with quinine, unless the bile is in great excess. For this reason it is often customary in practice to give a cholagogue cathartic to expel bile before the administration of quinine.

Blood.-Quinine is absorbed into the blood, and would naturally be precipitated in this alkaline fluid; but this is not the case, and it has been shown that quinine is probably 'zeld in solution by the loosely combined carbonic dioxide gas in the blood. Quinine possesses several well-defined and important actions in relation to the blood.

1. White Blood Corpuscles.-Quinine in great dilution lessens the amoboid movements of the white corpuscles in blood removed from the body. When a frog receives large doses of quiniue and its mesentery is irritated, the white 
corpuscles do not collect in the arterioles or migrate through their walls (diapedesis). Again, when inflammation has already begun in the mesentery, quinine stops the transmigration of leucocytes and disperses those already accumulated in the blood vessels. Moreover, large doses appear to actually lessen the number of white corpuscles in the blood. The foregoing actions should make quinine invaluable in checking inflammation. How great this influence may be, when quinine is given in medicinal doses, it is impossible to determine.

2. Red Blood Corpuscles.-Therapentic doses increase the number of red corpuscles. The latter diminish in size in febrile conditions, but, under the action of quinine (and other antipyretic agencies), regain their normal condition. This follows the effect of quinine in lowering temperature, and is not due to any specific power of quinine exerted on the corpuscles themselves.

3. Oxidation.-Quinine hinders the red corpuscles from serving as carriers of ozone and also prevents them from yielding it to the tissues. In this way functional activity of tissue cells is diminished and metabolism decreased. Quinine also lessens the ozonizing power of vegetable juices. The alkaloid prevents the development of acidity in blood outside the body, which is thought to be the product of oxidation.

Heart and Blood Vessels.-Quinine in moderate doses does not affect the heart or vessels appreciably. It is sometimes stated that small doses reflexly stimulate the heart (in stimulating the stomach), or that they indirectly stimulate the heart and ressels by stimulating the whole system. Quinine, in very large doses, is a depressant to the circulation, decreasing the pulse rate, force, and tension. When injected into a vein or directly applied to the cardiac muscle, quinine causes diastolic arrest of the heart by paralyzing the cardiac muscle or its contained ganglia. In poisoning by quinine there is vascular dilatation and great fall of blood pressure preceded by a temporary rise. This phenomenon follows direct action on the blood vessels. Although 
the pulse is at first slowed, the vagus becomes finally paralyzed in poisoning, and the pulse is rapid and imperceptible in the later stages.

Nervous System.-Toxic doses of quinine injected into the carotid artery cause meningitis by direct irritation. Large doses will also produce congestion and extravasation of blood into the middle ear and labyrinth, and will give rise to epileptiform convulsions in the lower animals. In man, ringing in the ears, fulness in the head, and slight deafness commonly follow a large medicinal dose (cinchonism). There is some clinical evidence that quinine in medicinal doses is a cerebral stimulant, but there is no experimental proof of the fact.

Spinal Cord and Nerves.-Quinine, as shown by experiments conducted on the frog, lessens reflex activity after small doses. This condition disappears on section of the medulla. Large doses, however, cause permanent loss of reflex excitability. The same alkaloid first excites and then paralyzes the peripleral sensory nerve endings. Muscular contractility is also diminished by large doses of quinine.

Uterus.-Quinine is thought by many to be an abortifacient. There is no sufficient warrant for this conclusion. There is, nevertheless, considerable testimony affirming that the alkaloid stimulates uterine contractions in inertia during. parturition, but at the same time increases the likelihood of metrorrhagia.

Kidneys, Metabolism and Elimination.-Quivine lessens the secretion of nric acid and, to a somewhat less extent, that of urea. Tissue waste must therefore be reduced. This is in accordance with our knowledge of its power to lesseri oxidation and protoplasmic activity. It is curious to note in this connection that the excretion of carbonic dioxide by the lungs is not notably decreased. Quinine is eliminated chiefly by the kidneys, but more or less is unabsorbed and passes away in the frecs. While its excretion in the urine begins soon after its ingestion, and lasts for some days, the greater amount escapes within forty-eight hours. Quinine is 
found in minute amount in the bile, tears, saliva, milk, etc., after its administration.

Antipyretic Action.-Quinine does not alter the normal temperature of a healthy animal, but does reduce temperature in fever. The amount of reduction depends upon the cause of the fever. An explanation of the antipyretic power of the alkaloid may include :

1. The antiseptic property of quinine. This is most marked in the case of the plasmodium malariæ, which is destroyed by the alkaloid, and the malarial fever is therefore overcome.

2. In lessening reflex excitability. Whatever may be the cause of fever, the nervous system is the potent instrumentality in its production.

3. In diminishing oxidation, activity of cellular protoplasm and beat formation.

4. Direct depressing action on the cerebral calorificient centres.

Administration.-Quinine sulphate is usually given to horses in aqneous solution with sufficient diluted sulphuric acid to dissolve the salt. It may also be administered to these animals in ball, gelatine capsule, enema or subcntaneously. Quinine is exhibited to dogs in pills, solution or suppositories. The alkaloid is not commonly injected under the skin, because local irritation and abscess may follow; but this does not frequently happen in the horse. The bisulphate, hydrobromate and hydrochlorate are most suitable for hypodermic use. The first salt is more soluble, but the latter two are less irritating.

\section{At a temperature of $45^{\circ} \mathrm{C} .\left(113^{\circ} \mathrm{F}\right.$.)}

Quinine bisulphate is soluble in....... 8.8 parts of water.

Quinine hydrobromate is soluble in....45.02 " " " " "

Quinine hydrochlorate is soluble in.....21.4 " " " " " "

This temperature may be used for subcutaneous injection, but the salts should be thoroughly dissolved and one grain of tartaric acid should be added to each five grains of quinine bisulphate, in order that precipitation may not occur in the 
tissues. The dose by the subcutaneous method is one-third less than by the month.

The sulphates of quinidine, cinchonine and cinchonidine are similar in action to quinine, and their relative antipyretic effect is said to be : quinine, 100 ; quinidine, 90 ; cinchonidine, 70 ; cinchonine, 40 . The cinchona compounds are indicated for tonic and stomachic purposes.

Uses External.-Quinine is too expensive for general antiseptic employment externally.

Uses Internal.-It is impossible to draw definite deductions as to the therapentic indications for quinine founded on physiological experiments, since these are only suggestive and not conclusive. For the sake of convenience, we may classify the uses of quinine under the following heads:

1. Tonic Action.-Quinine is of unquestionable value as a tonic, more particularly in relation to digestion, but probably also by increasing the number of red corpuscles and stimulating the nervous system generally. It is in those cases of anorexia and atonic dyspepsia secondary to exhaustion, overwork, auæmia, or following acute diseases, that the drug is indicated. Here, combination with iron is often of service, and the tincture of the chloride is a good preparation because it contains sufficient free muriatic acid to dissolve any of the salts of quinine. The compound tincture of cinchona is a prime, bitter tọnic for dogs ; or quinine may be given in a pill with reduced iron and arsenic as a tonic. Chorea in the human patient has been treated successfully with quinine, but this remedy has failed in dogs. Nevertheless, the alkaloid is an excellent tonic in canine distemper with its accompanying anæmia, but should be combined with iron and arsenic. Quinine is a good tonic for purpura in horses.

2. Antiseptic and Antiphlogistic Effect.-Quinine possesses much less antipyretic power than phenacetin, antipyrin and acetanilid. It acts more favorably with a falling than a rising temperature, and should be given two or three hours before the probable time of maximum temperature. Quinine 
is employed in many acute diseases, such as influeuza, bronchitis and pneumonia of horses. In full doses, at the outset of colds or inflammatory diseases of the respiratory tract, quinine may prove abortifacient. Isater in these diseases the drug may be given as an antipyretic and antiphlogistic (withont much benefit probably), but in the couvalescent stages quinine, in small doses, becomes of great worth as a tonic. Some experiments involving the injection of putrid material into the blood of dogs, appeared to indicate tlat quinine had a restraining influence on the resulting septic state, and, in some cases, saved life.

Puerperal fever and erysipelatous inflammation yield somewhat to quinine, and the drug should be tried in these infections. The alkaloid does not lower the temperature or prove destructive to the micrococci of pyæmia. A vast number of cases of rheumatic fever have been treated with quinine in human practice, but the results are inferior to those obtained by salicylates. In subacute and chronic muscular rheumatism quinine is sometimes useful. It has been injected into the affected muscles in this disorder, in horses, with favorable results.

The alkaloids of cinchona may be used as antipyretics in all acute diseases with the exception of meningitis, cerebritis, gastritis, nephritis and cystitis, where they produce too much irritation. They are also contra-indicated in epilepsy and middle-ear disease.

3. Specific Properties.-Quinine stands preëminent in the treatment of malaria, as it is the only drug which can be relied upon to kill malarial organisms. While periodicity in the febrile attacks is characteristic of malaria, an absolute diagnosis can only be made by the discovery of Laveran's plasmodium in the red blood corpuscles. Malaria but rarely affects the lower animals in this part of the country (New England), although exceedingly common among human beings. Cases are said to occur not infrequently among horses and cattle in India. A single full antipyretic dose of quinine, if given from twelve to six hours before a promised 
malarial attack, will usually prevent it. A single large dose should be given once daily for several days thereafter. When the disease is severe, treatment may also be pursued by the rectal and hypodermic methods at the same time that quinine is given by the mouth. A purge of aloes and calomel should be exhibited prior to the administration of quinine in the treatment of malaria.

\section{Class 2.-Salicylic Acid, Salicin, Salol, Oil of Gaultheria and Methyl Salicylate.}

\section{Acidum Salicylicum. Salicylic Acid. $\mathrm{HC}_{7} \mathrm{H}_{5} \mathrm{O}_{3}$.} (U. S. \& B. P.)

Synonym.-Acid salicylique, Fr. ; salicylsaüre, G.

An organic acid, existing naturally in combination in various plants, but chiefly prepared synthetically from carbolic acid.

Derivation.-Made by passing carbonic dioxide through sodium carbolate at a temperature of $428^{\circ} \mathrm{F} .\left(220^{\circ} \mathrm{C}\right)$. $2 \mathrm{Na} \mathrm{C}_{6} \mathrm{H}_{5} \mathrm{O}$ (sodium carbolate) $+\mathrm{CO}_{2}=\mathrm{Na}_{2} \mathrm{C}_{7} \mathrm{H}_{4} \mathrm{O}_{3}$ (sodium salicylate) $+\mathrm{C}_{6} \mathrm{H}_{6} \mathrm{O}$ (phenol). Sodium salicylate is treated with hydrochloric acid, when salicylic acid is precipitated. $\mathrm{Na}_{2} \mathrm{C}_{7} \mathrm{H}_{4} \mathrm{O}_{3}+2 \mathrm{H} \mathrm{Cl}=\mathrm{HC}_{7} \mathrm{H}_{5} \mathrm{O}_{3}+2 \mathrm{Na} \mathrm{Cl}$.

Properties.-Light, fine, white, prismatic needles, or a light, white, crystalline powder; odorless, having a sweetish, afterward acrid taste, and permanent in the air. Soluble in abont 450 parts of cold water, and in 2.4 parts of alcohol; in 14 parts of boiling water, and very soluble in boiling alcohol. Also soluble in 2 parts of ether, 2 parts of absolute alcohol, and 80 parts of chloroform.

Incompatible.-Spirit of nitrous ether.

Impurities.-In artificial salicylic acid, metacreosotic and orthocreosotic acids.

Dose.-H. \& C., 3 ii.- 5 i. (8.-30.); Sh., 3 i.-iv. (4.-15); Sw., 3 ss.-i. (2.-4.); D., gr.v.-xxx. (.3-2.). 
Salicinum. Salicin. $\mathrm{C}_{13} \mathrm{H}_{18} \mathrm{O}_{7}$. (U. S. \& B. P.)

A neutral priuciple (glucoside) obtained from several species of Salix and Populus (nat. ord. Salicaceæ).

Habitat. -Europe, but cultivated in North America.

Derivation.-Obtained from a decoction of willow bark. Salicin crystallizes on evaporation, after removal of tannin by agitation with lead oxide. It is purified by repeated solution and crystallization.

Properties.-Colorless, or white, silky, shining crystalline needles, or a crystalline powder; odorless, and having a very bitter taste. Permanent in the air. Soluble in 28 parts of water, and in 30 parts of alcohol; almost insoluble in ether or chloroform.

Dose.-H. \& C., 3 ii.- 3 i. (8.-30.); Sh., 3 i.-iv. (4.-15.); Sw., 3 ss.-i. (2.-4.); D., gr.v.-xxx. (.3-2.).

Sodir Salicylas. Sodium Salicylate. $\mathrm{Na} \mathrm{C}_{7} \mathrm{H}_{5} \mathrm{O}_{3}$. (U. S. \& B. P.)

Synonym.-Silicylate de soude, Fr.; natrium salicylicum, G.

Derivation.-Made by the action of salicylic acid on sodium carbonate. $2 \mathrm{HC}_{7} \mathrm{H}_{5} \mathrm{O}_{3}+\mathrm{Na}_{2} \mathrm{CO}_{3}=2 \mathrm{Na} \mathrm{C}_{7} \mathrm{H}_{5} \mathrm{O}_{3}+$ $\mathrm{H}_{2} \mathrm{O}+\mathrm{CO}_{2^{\circ}}$ The solution is filtered, and heated to expel carbon dioxide.

Properties.-A white, amorphous powder; odorless, and having a sweetish, saline taste. Permanent in cool air. Soluble in 0.9 part of water, and in 6 parts of alcohol; very soluble in boiling water or alcohol; also soluble in glycerin.

Dose.--Same as salicin.

\section{SALOL. Salol. $\mathrm{C}_{6} \mathrm{H}_{5} \mathrm{C}_{7} \mathrm{H}_{5} \mathrm{O}_{3}$. (U. S. P.)}

Synonym.-Phenyl salicylate, salicylic ether of phenol.

Derivation.-Made by heating salicylic and carbolic acids with phosphorus pentachloride.

Properties.-A white, crystalline powder; odorless, or having a faintly aromatic odor, and almost tasteless. Per- 
manent in the air. Almost insoluble in water; solnble in 10 parts of alcohol; also soluble in 0.3 part of ether, and readily in chloroform and in fixed or volatile oils.

SALICYLIC ACID, SALICIN, SODIUM SALICYLATE AND SALOL.

Action External.-Salicylic acid, salicin and salol are powerful antiseptics. A solution of salicylic acid (1-60) is equivalent to a solution of carbolic acid (1-22) in destroying some bacteria, but is not generally as useful. Salicylic acid, sodium salicylate and salicin are irritating to the unbroken skin or raw surfaces. Salol is not. The salicylates are not antiseptic, but actually favor fermentation.

Action Internal.-Salicylic acid is an irritant in the digestive tract and in large doses causes nausea and vomiting in dogs. It is converted into salicylates by the alkaline intestinal juices, and is absorbed in this form, chiefly as sodium salicylate. For this reason, and because the latter salt is less irritating than salicylic acid, sodium salicylate is preferred to the acid when a constitutional action is desired. Salicin splits up in the bowels into salicylic acid, salicylous acid $\left(\mathrm{HC}_{7} \mathrm{H}_{5} \mathrm{O}_{2}\right)$, salicyluric acid $\left(\mathrm{HC}_{9} \mathrm{H}_{8} \mathrm{NO}_{4}\right)$, and glucose. Salol is decomposed by the pancreatic juice into salicylic acid (64 per cent.) and carbolic acid (36 per cent.) After large doses the urine takes on the characteristic smoky color produced in poisoning by phenol. Salol is an intestinal antiseptic.

Circulation.-The circulation is not preceptibly influenced by moderate doses of sodinm salicylate, or salicylic acid, but large doses depress the heart force, blood pressure and nervous system. The artificial acid is said to be more depressant than natural salicylic acid obtained from plants, because of orthocreosotic and metacreosotic acids existing as impurities in the former.

Nervous System.-The action of salicylic acid on the nervous system is unknown. Therapeutic quantities often cause, in man (salicylism), ringing in the ears and headache.

Respiration. - The respiratory movements are primarily 
quickened by the stimulation of the peripheral vagi and respiratory centres produced by sodium salicylate and salicylic acid; but after large doses the respiratory ceutres are depressed and paralyzed and death takes place by asphyxia.

Temperature. - Medicinal doses do not influence the normal temperature of healthy animals, but do often lower bodily heat in fever, and frequently induce sweating. The largest therapeutic doses must be given to secure an antipyretic action. The physiological details concerned in the reduction of febrile temperature have not been ascertained.

Kidneys and Elimination.-Salicylic acid, salicin and sodium salicylate circulate in the blood as sodium salicylate and are eliminated in the urine as salicyluric and salicylic acids. This happens in this wise: Some of the salicylic acid of sodium salicylate combines with glycocoll in the body and forms salicyluric acid. $\mathrm{HC}_{7} \mathrm{H}_{5} \mathrm{O}_{3}+\mathrm{C}_{2} \mathrm{H}_{5} \mathrm{NO}_{2}$ (glycocoll) $=\mathrm{HC}_{9} \mathrm{H}_{8} \mathrm{NO}_{4}$ (salicyluric acid) $+\mathrm{H}_{2} \mathrm{O}$; while some of the sodium salt is decomposed by phosphoric acid in an acid urine into salicylic acid. Like quinine, the excretion of salicylic acid begins soon and goes on slowly. Therefore large doses given continuously may accumulate in the body. The quantity of urea and uric acid in the urine is increased very considerably by salicylic acid, but the urine itself may either be increased or diminished in amount. It is made aseptic. by the escaping salicylic acid, or in the case of salol, by both carbolic and salicylic acids. The urine of animals. taking salicylic acid may be rendered green by iudican and pyrocatechin, formed through the action of pancreatic juice, and takes on a purple color with ferric chloride.

Toxicology.-In man, continued large doses give rise to delirium, vomiting, depression of the circulation, epistaxis, hæmaturia, and retinal hæmorrhages. The herbivora are not easily affected by large doses of salicylic acid, or salicylates, but dogs exhibit nansea and vomiting, accelerated respiration, irregular pulse, loss of muscular strength, staggering gait, stupor, and, if death occurs, it is preceded by slow breathing, dilated pupils, dyspnœa, and convulsions due ta 
asphyxia. The minimum fatal dose for a small dog is about one drachm of sodium salicylate, subcutaneously.

Administration.-Sodium salicylate contains 48 grains of the acid to the drachm. Sodium salicylate is used in preference to the acid because it is soluble and unirritating. It is given in solution, or to dogs in pills or tablets. Salicylic acid may be exhibited in solution by warming it with glycerin (gr.iv- 3 i.); or with syrup (1-5), and aquæ ammonia in sufficient quantity to dissolve it, thus forming ammonium salicylate. It may also be administered in pill or ball. Salol is given in pill, powder or mixture with water. The larger doses of salicylic acid and salicylates should not be repeated, and are used for their antipyretic action. A maximum daily dose of one ounce of salicylic acid or sodium salicylate, for horses, or one drachm of either for large dogs, should rarely be exceeded.

Uses External.-Salicylic acid is employed in various forms as an antiseptic. Aqueous solutions (1-300) may be applied to wounds. Stronger solutions are prepared with alcohol, borax, sodium bicarbonate, and ammonium acetate solution. But as salicylates are formed in the (3) latter combinations, the solutions are useless as antiseptics. Salicylic acid may be applied as a dusting powder with zinc oxide (1-8), or in ointment (1-20 or 30), for its stimulant and antiseptic effect on wounds. It is used in the treatment of burns with cottonseed oil (1-8). Salicylic acid is useful in powder or ointment in acute moist eczema (1-50), and in the following formula:

Salicylic acid, one part; zinc oxide, starch and vaseline, each 16 parts. Ringer recommends in pruritus ani and vulvæ-salicylic acid, 3 ii.; ol. theobrom., 3 v.; cetac., 3 iii.; ol. myrist, 3 i.ss.

Antiseptic gauze is prepared by soaking the material in hot, saturated, watery solutions of salicylic acid. Salol is used as an antiseptic dusting powder of uncertain value.

Uses Internal.-The salicylic acid group are specifics in rheumatic fever. They lower temperature, lessen pain, and 
by shortening the attack lessen the danger of cardiac complications. Sodium salicylate should be given every three hours in doses of 3 ii. to horses, and gr.x.-xx. to dogs. But this form of rheumatism is rare in veterinary practice, and salicylic acid is unfortunately not vearly so valuable in the treatment of other varieties. Salicylates are probably worthless in chronic rheumatic arthritis where the local application of heat, stimulating liniments and blisters are serviceable; but they may be used with benefit in acute muscular rheumatism, sciatica, and rheumatic complications of influenza in horses. Salicylic acid, salicin, and salicylates are not comparable with the cold tar products as general antipyretics, and are useless in hyperpyrexia. Sodium salicylate is sometimes prescribed in gastric fermentative dyspepsia when the salicylic acid, set free by the hydrochloric acid of the gastric juice, acts as an antiseptic. Salol is a good intestinal antiseptic in diarrhœea and intestinal indigestion, particularly when combined with bismuth subnitrate in powder or aqueons mixture for dogs. Salol is undissolved in the stomach, is less irritating than either salicylic acid or sodium salicylate, and is prescribed to lessen pain and fever. It may be advantageously given in doses of gr.v. to dogs, with phenacetin gr.v., and codeine gr. $\frac{1}{2}$, for painful rheumatism. It is probably inferior to sodium salicylate, but can be conveniently administered in powder as just noted. Salol is also a local anresthetic and antiseptic in the minary tract (carbolic acid from decomposition), and is useful in cystitis and urethritis, to alleviate pain, to prevent frequent micturition, and to render the urine aseptic. Repeated large doses are likely to canse carbolic acid poisoning. Salicin acts more slowly than salicylic acid or sodium salicylate, and is not in general use.

Oleum Gaultheriz. Oil of Gaultheria. (U. S. P.)

Synonym.-Oil of checkerberry, oil of wintergreen, oil of boxberry. 
A volatile oil distilled from the leaves of Gaultheria procumbeus Linné (nat. ord. Ericaceæ), consisting almost entirely of methyl salicylate $\left(\mathrm{CH}_{3} \mathrm{C}_{7} \mathrm{H}_{5} \mathrm{O}_{3}\right)$, and nearly identical with volatile oil of betula.

Habitat.-North America; west as far as Minnesota, and south to Georgia.

Properties. - A colorless or yellow, or occasionally reddish liquid, hisving a characteristic, strongly aromatic odor, and a sweetish, warm and aromatic taste. Spec. gr. 1.175 to. 1.185 at $59^{\circ} \mathrm{F}$. Solubility same as methyl sulicylate.

$$
\text { Dose.-H., } 3 \text { ii.- zi. (8.-30.); D., mv.-xv. (.3-1.) }
$$

\section{Methyl Salicylate. $\quad \mathrm{CH}_{2} \mathrm{C}_{7} \mathrm{H}_{6} \mathrm{O}_{3}$. (U. S. P.)}

Synonym.-Artificial or synthetic oil of wintergreen.

Derivation.-Prepared by distillation of salicylic acid, or salicylates, with methyl alcohol and sulphuric acid.

Properties.-A colorless or slightly yellowish liquid, having the characteristic, strongly aromatic odor and the sweetish, warm and aromatic taste of oil of ganltheria, with the essential composition of which it is identical. It is wholly ilentical with oil of betula (birch). Spec. gr. 1.1831.185 at $59^{\circ} \mathrm{F}$. Soluble in all proportious in alcohol, glacial acetic acid, or carbon disulphide.

$$
\begin{aligned}
& \text { Dose.-H., } 3 \text { ii.- }- \text { i. }(8 .-30 .) ; \text { D., } \prod_{\text {v.-xv. }}(.3-1 .) \\
& \text { ACTION AND USES OF OIL OF GAULTHERIA AND METHYL } \\
& \text { SALICYLATE. }
\end{aligned}
$$

Oil of wintergreen contains about 90 per cent. of methyl salicylate. Eleven parts of methyl salicylate are equivalent to nearly ten parts of salicylic acid. The oil and methyl salicylate are free from the impurities of artificial salicylic acil, while metlyl salicylate is of more certain composition than the oil. Both behave similarly to salicylic acid therapeutically, although the oil is more of a local irritant, and they a"e used for the same purposes as salicylic acid. Either 
may be given in emulsion, or to dogs in capsules, and in combination with salicylic acid or salicylates.

Oil of wintergreen is serviceable in the following liniment for rheumatism :

$\mathrm{B}$

Tinc. Aconiti.

Ol. Gaultheriæ.

Chloroformi....................... āa $\frac{\tilde{z}}{3} \mathrm{i}$

Lin. Saponis.....................ad. 3 viii.

M.

S. External use.

The external application of methyl salicylate to acutely inflamed rheumatic joints is one of the most efficient forms of treatment, and, since some is absorbed, will take the place of the internal administration of salicylates to a considerable extent. It is usually best to combine this treatment with internal medication, however. Plain ganze, or other absorbent material, is saturated with methyl salicylate, applied to the affected joint, and then covered with oil silk, or rubber protective and bandage.

\section{SECTION IX.-VOLATILE OILS, OR DRUGS CONTAINING THFM.}

GENERAL ACTION OF VOLATILE OILS.

Synonym.-Essential, ethereal, aromatic or distilled oils. Externally, volatile oils cause reddening of the skin (rubefacients), sometimes blistering (vesicants), and often local anæsthesia, notably oil of cloves and peppermint. They are also parasiticide, antiseptic and disinfectant, particularly oil of mustard, which is the most powerful germicicle known.

Internally, these agents stimulate the flow of gastric; salivary, and intestinal secretions, and increase the vascularity and movements of the stomach and borrels. They therefore temporarily improve digestion, overcome flatulence by expelling gas from the intustines, prevent griping pro- 
duced by cathartics, and disguise and offset disagreeable effects and tastes of medicines. In the digestive tract, volatile oils excite reflexly the nervous system and heart, and angment the pulse rate and vascular tension. In large doses, volatile oils are gastro-intestinal irritants. Volatile oils may be absorbed from the skin, bronchial mucous membrane, and stomach. They are eliminated by the skin, bronchial mucous membrane, and kidneys, and occasionally by other channels. In the process of excretion the parts are stimulated; vascularity, secretion, and contractility of the unstriated muscle of the bronchial tubes are increased, and volatile oils thus assist expectoration and coughing. In irritating the kidneys and mucous membrane of the genito-urinary tract, the volatile oils are stimulant and diuretic; while in poisonous doses they produce acute nephritis, strangury, and hæmaturia. Toxic doses, injected into the circulation, lower the force of the heart and the blood pressure, and occasion a sort of intoxication, and sometimes convulsions. To summarize: volatile oils possess the following actions in a greater or less degree: parasiticide, antiseptic, disinfectant, rubefacient, vesicant, local anæsthetic, sialagogue, stomachic, carminative: antispasmodic, stimulant, expectorant, emmenagogue, and diuretic actions.

\section{Class 1.-Used Mainly for their Action on the Skin.}

\section{Terebrnthina. Turpentine. (U. S. \& B. P.)}

A concrete oleoresin obtained from Pinus palustris Miller, and from other species of Pinus (nat. ord. Coniferæ).

Habitat. - Southeastern United States ; from Virginia to the Gulf of Mexico.

Description.--In yellowish, opaque, tough masses, brittle in the cold; crumbly, crystalline in the interior, of a terebinthinate odor and taste.

Oledm Terebinthine. Oil of Turpentine.

A volatile oil distilled from turpentine. 
Synonym.-Spirit of turpentine, E.; essence de térébinthine, Fr.; terpentinöl, G.

Properties.-A thin, colorless liquid, having a characteristic odor and taste, both of which become stronger and less pleasant by age and exposure to the air. Spec. gr. 0.855 to 0.870 . Soluble in three times its volume of alcohol; also soluble in an equal volume of glacial acetic acid.

Solvent for resins (varnish), fats, wax, gutta percha, india rubber, sulphur, phosphorus, iodine, and many alkaloids. It is dextro-rotatory, but the French variety is leavorotatory. Old oil of turpentine and French oil of turpentine (Pinus maratima) are oxidizing agents.

Constituents.-Turpentine contains $20^{\circ}$ to 25 per cent. of oil of turpentine. Oil of turpentine is composed of several isomeric hydrocarbons, called terpenes, and having the formula $\mathrm{C}_{10} \mathrm{H}_{16}$.

Among these terpenes are oil of juniper, savin, cubeb, caraway, cloves, thyme, etc. They differ from each other and turpentine in their boiling points and direction in which they rotate the plane of polarization. The terpenes are oxidized into camphors.

Dose.-Carminative-H. \& C., 3 i.-ii (30.-60.); Sh. \& Sw., 3 i.-iv. (4.-15.); D., $m_{x}$.-xxx. (.6-2.).

Anthelmintic-H. \& C., 3 ii.-iv. (60.-120.); D., 3 ss.-ị. (2.-15.).

Diuretic-H. \& C., 3 ii.-vi. (8.-24.).

PREPARATIONS.

Linimentum Terbinthinc. Turpentine Liniment. (U. S. \& B. P.)

Resin cerate, 650 ; oil of turpentine, 350 ; melt the resin cerate and add the oil of turpentine. (U. S. P.)

Oleum Terebinthince Rectificatum. Rectified Oil of Turpentine.

$$
\text { (U. S. P.) }
$$

Derivation.-Made by shaking oil of turpentine, 1, with lime water, 6; and distillation.

Properties.-A thin, colorless liquid, having the same properties as oil of turpentine. 


\section{Tenebencm. Terebene. $\mathrm{C}_{10} \mathrm{H}_{16}$. (U. S. P.)}

A liquid consisting chiefly of pinene, and containing not more than very small proportions of turpinene and dipentene. Made by action of sulphuric acid on oil of turpentine and by distillation.

Properties.-A colorless, or slightly yellowish, thin liquid, having is rather agreeable, thyme-like odor, and an aromatic, somerwat terebinthinate taste. Spec. gr. about 0.862 . Only slightly soluble in water, but soluble in an equal volume of alcohol, glacial acetic, or carbon disulphide.

Dose.-H. \& C., 3 ii.-vi. (8.-24.); Mv.-xv. (.3-1.).

\section{Terpini Hrdras. Terpin Hydrate. $\mathrm{C}_{10} \mathrm{H}_{18}(\mathrm{OH})_{3}+\mathrm{H}_{2} \mathrm{O}$. (U. S. P.)}

The hydrate of the diatomic alcohol, Terpin.

Derivation.-Rectified oil of turpentine, alcohol and nitric acid are mixed together in shallow, porcelain dishes, and after three or four days terpin hydrate crystallizes out. The crystals are collected, drained, dried on absorbent paper, and purified by recrystallization in alcohol.*

\section{OIL OF TURPENTINE}

Action External.-Oil of turpentine is an irritant to the skin, causing itching, pain and redness, or even vesication, followed by local anæsthesia. It produces intense irritability and restlessness when applied externally to some horses. The oil is antiseptic, disinfectant and parasiticide. Some absorption may occur from the skin.

Action Internal.-Alimentary Canal.-The oil induces a sense of warmth in the stomach (man), and increases gastric secretion, motion and vascularity, but is too disagreeable to be used as a stomachic. In acting similarly in the bowels, particularly in stimulating the muscular coat, oil of turpentine is a iseful carminative by exciting peristalsis and expelling gas in tympanites. Its antiseptic properties also antagonize intestinal fermentation.

* Colorless, odorless crystals, having a somewhat bitter taste. Soluble in 250 parts of water and in 10 parts of alcohol.

Dose.-H. 3 ss.-ii. (2.-8.); D., gr.v.-xx. (.3-1.3). 
Large doses occasion purging and are anthelmintic. Toxic doses create gastro-enteritis and sometrus intestinal nlceration. The irritation of the nerve endings in the digestive tract cansed by turpentine, leads to refler stimul:1tion of the nerrous system and heart.

Circulation.-Turpentine is readily absorbed into the biood. Experimental evidence is at variance with regard to the action of the oil on the circulation. Small doses apparently increase the force and frequency of the heart-beat, and slightly raise blood pressure. The vessels are somewhat contracted and the drug is employed to arrest hæmorrhage in the digestive tract and in remote organs. It is inferior to ergot as an hæmostatic. Large doses of turpentine lower the cardiac force and frequency, and canse vascular dilatation and fall of blood pressure.

Respiration.-Oil of turpentine enhances the strength and rapidity of the respiratory movements, in small doses, but large quantities depress the respiration. The oil is easily absorbed by inhalation and is also eliminated in the breath. Inhalation of the oil stimulates the bronchial mucous membrane, acts as an antiseptic, and excites muscular contraction of the bronchial tubes and cough.

Authorities differ as to the influence of turpentine on bronchial secretion. Rossbach found that the inhalation of air saturated with turpentine diminished secretion, while the topical application of a watery solution increased secretion. The oil is essentially a local stimulating expectorant.

Nervous System.-Medicinal doses occasion mental exhilaration in man. Large doses canse dulness, languor, and unsteady gait in animals; while distinctly toxic doses produce coma, sensory paralysis, loss of reflex activity, and, at times, convulsions.

Kidneys and Genito-Urinary Tract.-The kidneys are very prone to irritation during its elimination. Small doses induce frequent micturition. Large quantities lead to albuminuria, pain in the lumbar region, hæmaturia, and 
constant painful passage of high-colored urine, owing to irritation of the urinary mucous membrane and muscular spasm of the urethra (strangury). Menorrhagia and dysmenorrhoea occur in females under the influence of the oil. Acute nephritis and complete suppression of urine follow great toxic doses. The urine has sometimes the odor of violets.

Elimination.-Turpentine is eliminated in the urine, breath, and, to some extent, in the bile and intestinal mucus; slightly by the skin.

Toxicology.-Turpentine poisoning is not an uncommon occurrence from the administration of large doses (undiluted) by empirics. Post-mortem appearances reveal gastroenteritis, sometimes congestion and inflammation of the lungs, and fatty degeneration of the liver, kidney and muscles, following prolonged use of the oil.

The action of terebene and terpin hydrate is very similar to oil of turpentine.

Administration.-Oil of turpentine is given with eight or more times its volume of cottonseed or linseed oil, gruel, or milk; and in emulsion with acacia or white of egg. An emulsion is made by shaking a single dose with powdered acacia, and adding water or oil. Terebene is administered in a similar manner. Terpin hydrate may be exhibited in pill, ball or alcoholic solution.

USES OF OIL OF TURPENTINE, TEREBENE AND TERPIN HYDRATE.

External.-Oil of turpentine is employed as a stimulant and counter-irritant with two or three parts of cottonseed oil and soap liniment, or as the official liniment in rhenmatism, myalgia, sprains, shoulder lameness, swollen joints, gangrene, frost bites, burns and ulcers. It is serviceable in the same form and for the same actions applied to "sitfasts" and obstinate ulceration about the heels in horses, and in footrot of sheep. As a parasiticide, the oil, diluted two or three times with sweet oil, is painted on the skin to lill ringworm and lice. 
Oil of turpentine is a valuable counter-irritant in relieving pain and inflammation of deep-seated parts. It is particularly useful in tympanitis, flatulent and spasmodic colic and peritonitis. In the first two named disorders, external application is combined with the internal and rectal exhibition of the drug. Oil of turpentine is less frequently employed over the chest in pleurisy and bronchitis. The turpentine stupe is the favorite method of applying the oil in abdominal troubles. A blanket is thoroughly sprinkled with turpentine, folded, and rolled into a cylindrical form which will fit into an ordinary pail. Boiling water is then poured on the blanket until it is saturated. The blanket is quickly wrung out, placed over and around the horse's trunk, covered with rubber protective and dry blankets, and allowed to remain in place fifteen to thirty minutes.

Uses Internal.-Digestive Tract.-Oil of turpentine is of greatest utility in colic and in expelling gas in tympany given internally ( 3 ii.-Oii. of linseed oil) and per rectum. Enemata can also be employed for their stimulant action on the nervous system and circulation, in collapse. One or two ounces of oil of turpentine are dissolved in two or four ounces of cottonseed oil, when used as an enema for horses. Turpentine is an anthelmintic for round and tape worms. From two to four ounces of the oil with one ounce of oleoresin of aspidium in a pint of linseed oil, are recommended for the latter purpose in the case of horses. Oil of turpentine is often advised as an antidote for phosphorus because it contains ozone and forms a harmless, camphor-like body-turpentine phosphoric acid. The French variety only is efficacious; the American oil, so far from being antagonistic to phosphorus, actually assists its solution and absorption. Turpentine is occasionally given in indigestion, cluronic diarrhœe, and dysentery of horses and cattle, as a local stimulant and antiseptic.

Respiratory Organs.-Oil of turpentine is an efficient stimulating and antiseptic expectorant in subacute and chronic brouchitis; aud deodorant in gangrene of the lungs. 
It is administered intervally, and by inhalation in the proportion of one teaspoonful to the quart of boiling water. Terebene is used as a substitute for oil of turpentine, as a stimulating expectorant, and is likewise prescribed as an antiseptic and carminative in flatulence, and as a genitourinary stimulant. Terpin hydrate increases bronchial secretion and is employed in both acute and chronic bronchitis. Oil of turpentine has been found beneficial in verminous bronchitis of calves and lambs (caused by Strongulus micrurus and filaria), injected into the trachea midway in the neck, according to the following prescription :

B

Ol. Terebinthinæ................... 3 i.-ii

Acid. Carbol.......................

Glycerini.........................

M.

Chloroformi........................ āā 3 ss.

S. Inject in one dose.

"Gapes" in fowl, due to Syngamus trachealis, is cured by the same mixture diluted with 5 parts of oil and applied to the throat internally with a feather.

Circulation.-Oil of turpentine is of some worth as a cardiac stimulant and hæmostatic. It is said to have been exhibited in parturient fever and apoplexy of cattle with success. Bleeding from the nose, lungs, digestive tract, uterus, kidneys, and bladder, and hæmorrhages occurring in purpura hemorrhagica, are sometimes stopped by the internal use of turpentine.

Genito-Urinary Tract.-Oil of turpentine is indicated as a stimulant in amenorrhœa, chronic pyelitis and cystitis. The drug is contra-indicated in acute inflammation of the kidneys and alimentary canal.

\section{Pix Burgundica. Burgundy Pitch. (U. S. \& B. P.)}

The prepared, resinous exudation of Abies excelsa Poiret (nat. ord. Coniferæ).

Synonym.-Poix blanche, poix de Bourgogne, Fr.; Burgunder harz (pech), G. 
Habitat.-Southern Europe, mountainous regions.

Properties.-Hard, Jet taking gradually the form of the vessel in which it is kept; brittle, with a shining, conchoidal fracture; opaque or translncent; reddish-brown or yellowishbrown; odor agreeably terebinthinate; taste aromatic, sweetish, not bitter. It is almost entirely soluble in glacial acetic acid, or in boiling alcohol, and partly soluble in cold alcohol.

Constituents. - 1 , resin ; 2 , a volatile oil $\left(\mathrm{C}_{10} \mathrm{H}_{16}\right)$.

Dose.-H. \& C., 亏3i.-iii. (30.-90.); Sh. \& Sw., J i.-ii. (4.-8.); D., gr.xx.-xl. (1.3-2.6).

PREPARATION.

Emplastrum Picis Burgundicce. Burgundy Pitch Plaster.

$$
\text { (Cं. S. P.) }
$$

Burgundy pitch, 800 ; olive oil, 50 ; yellow wax, 150.

\section{Terebinthina Canadensis. Canada Turpentine.}

$$
\text { (U. S. \& B. P.) }
$$

A liquid oleoresin obtained from Abies balsamea (Linné), Miller (nat. ord. Coniferæ).

Synonym.-Canada balsam, balsam of fir, balsamum Canadense, E. ; baume de Canada, Fr. ; Canadischer terpentin, G.

Habitat.-Canada and N. United States; west to Minnesota, south on mountains to Virginia.

Properties.-A yellowish or faintly greenish, transparent, viscid liquid, of an agreeable terebinthinate odor, and a bitterish, slightly acrid taste. When exposed to the air it gradually dries, forming a transparent mass. It is completely soluble in alcohol, chloroform or benzol.

Constituents.-1, volatile oil, 20-30 per cent.; 2, a resin; 3 , a soluble bitter principle.

Dose.-H. \& C., 3 i.-iii. (30.-90.); Sh. \& Sw., 3 i.-ii (4.-8.); D., gr.xx.-xl. (1.3-2.6). 
ACTION AND USES OF BURGUNDY PITCH AND CANADA TURPENTINE.

Burgundy pitch is slightly stimulating to the skin and is used as a mild counter-irritant (in plaster) in rheumatism, strains, swelling of joints, and upon the chest. Burgundy pitch, Canada turpentine, crude turpentine, Venice turpentine, Bordeaux turpentine and Frankincense have much the same action and uses as oil of turpentine internally. They are administered in the same manner and in nearly identical doses, but are less commonly employed than the latter.

\section{Resina. Resin. (U. S. \& B. P.)}

The residue left after distilling off the volatile oil from turpentine.

Synonym.-Colophony, rosin, E. ; colophonum, P.G.; colophane, Fr.; kolophonium, geigenharz, G.

Properties.-A transparent, amber-colored substance, hard, brittle, pulverizable ; fracture glossy and shallow-conchoidal; odor and taste faintly terebinthinate. Spec. gr. 1.070-1.080. Soluble in alcohol, ether, and fixed or volatile oils; also in solution of potassium or sodium hydrate.

Constituents.-Chiefly abietic acid anhydride $\left(\mathrm{C}_{44} \mathrm{H}_{62} \mathrm{O}_{47}\right)$, 80-90 per cent.

\section{PREPARATIONS.}

Ceratum Resinc. Resin Cerate. (U. S. P.)

Synonym.-Basilicon ointment.

Resin, 350 ; yellow wax, 150 ; lard, 500 .

Emplastrum Resinc. Resin Plaster. (U. S. \& B. P.)

Synonym.-Adhesive plaster.

Resin, 140 ; lead plaster, 800 ; yellow wax, 60.

\section{ACTION AND USES OF RESIN.}

Resin is a local stimulant and antiseptic externally. The cerate is an excellent preparation for burns, wounds, ulcers, and abraded surfaces. The fumes arising from burning resin (on a hot shovel) are said to be of value when inhaled in chronic or subacute bronchitis. 
Pix Liquida. Tar. (U. S. \& B. P.)

An empyrenmatic oleoresin obtained by the destructive distillation of the wood of Pinus palustris Miller, and of other species of Pinus (nat. ord. Coniferæ).

Synonym.-Resina empyreumatica liquida - goudron, goudron végétal, Fr.; theer, G.

Habitat.-United States.

Properties.-Thick, viscid, semi-fluid, blackish-brown; heavier than water, transparent in thin layers, becoming granular and opaque with age ; odor empyreumatic, terebinthinate; taste sharp, empyreumatic.

Tar is slightly soluble in water; soluble in alcohol, fixed or volatile oils, and solutions of potassium or sodium hydrate.

Constituents.-Mainly-1, oil of tar ; 2, methylic alcohol; 3 , creosote ; 4 , guaiacol ; 5 , phenol ; 6 , pyrocatechin ; 7 , toluol ; 8 , xyol ; 9 , acetic acid ; 10 , acetone; 11 , resins.

Dose.-H. \& C., 3 ss.-i. (15.-30.); Sh. \& Sw., 3 i.-ii. (4.-8.); D., $m \times x .-3$ i. $(1 .-4$.).

PREPARATTON.

Unguentum Picis Liquidce. Tar Ointment. (U. S. \& B. P.)

Tar, 500 ; yellow wax, 125 ; lard, 375.

\section{Oleum Picis Liquide. Oil of Tar. (U. S. P.)}

A volatile oil distilled from tar.

Properties.-An almost colorless liquid when freshly distilled, but soon acquiring a dull, reddish-brown color, and having a strong tarry odor and taste. Spec. gr. about 0.970 . Soluble in alcohol.

\section{PIx Nigra. Pitch. (Non-official.)}

A solid, shining, black, bituminous substance. Soluble in ether, oils, and aqueous alkaline solutions. It contains an altered resin, and a crystalline principle, Retine $\left(\mathrm{C}_{18} \mathrm{H}_{18}\right)$. 


\section{Olemu Cadinum. Oil of Cade. (U. S. P.)}

A product of the dry distillation of wood of Juniperus Oxycedrus Linné (nat. ord. Coniferæ).

Synonym.-Oleum juniperi empyreumaticum, E. ; huile de cade, Fr. ; cadöl, G.

Habitat.-North Africa, Spain, France, and Portugal, on the borders of the Mediterranean, in waste places and stony hill-sides.

Properties.-A brownish or dark brown, clear, thick liquid ; having a tarry odor, and an empyremmatic, burning, somewhat bitter taste. Spec. gr. about 0.990 . It is almost insoluble in water, but imparts to it an acid reaction. Partially soluble in alcohol; completely soluble in ether, chloroform, or carbon disulphide.

Constituents. - The composition is similar to that of tar. Dose.-Same as that of tar.

ACTION AND USES OF TAR, OIL OF CADE AND PITCH.

Externally, tar produces hyperæmia, and, when rubbed continually into the skin, sometimes papules and pustules. It is a stimulant, rubefacient, antiseptic and parasiticide externally. Poisoning may follow the extensive application of large quantities of tar over a denuded surface, or if it be licked off the skin. Tar is mainly employed in veterinary medicine on the skin, and is a valuable remedy to relieve itching and as a local stimulant in chronic eczema ("grease"), sometimes in moist eczema and erythema, psoriasis, pityriasis, pruritus, and lichen. It also destroys the parasites of mange and ringworm. It is applied with fat, vaseline, soap or alcohol, in the proportion of 1-2 to 10. The official ointment (1-2), is generally appropriate in the above-mentioned skin diseases, but may require dilution. The following preparation is serviceable on patches of psoriasis :

Tar, soft or green soap, each two ounces; alcohol, two ounces. Pure tar painted over the surface with a brush, is often most efficient in obstinate cases of eruptive disorders. 
A lotion of oil of tar, sulphurated potassa, or Pernvian balsam, is more cleanly and suitable for house dogs with eczema. (See diet for eczerna in dogs, section on foods and feeding, p. 685.) Tar is of the greatest utility in stimulating the growth of hom, and is the principal ingredient of hoof ointments for horses. It may be mixed with an equal part of lard for this purpose, or the following mixture is well spoken of: tar, yellow wax and honey, $\frac{1}{4}$ lb. each; lard, $1 \frac{1}{2}$ lbs.; glycerin, 3 ounces. Melt and mix the lard and wax together; add the other ingredients; stir while cooling. Oakum, soaked with tar, is frequently packed under leather, beneath the shoes on horses' feet, to soften and stimulate the horn and to cure thrush and canker. Tar is also a beneficial application for foot rot of slieep. Tar is nsually kept on hand for farming purposes, and is therefore a popular antiseptic and protective in the treatment of wonnds and broken horns in cattle.

Internally. - Tar-on account of its constituents, phenol and creosote-in large quantities causes toxic effects, with symptoms resembling carbolic acid poisoning: e.g., abdominal pain, vertigo, signs of gastro-intestinal irritation, and the passage of dark-colored urine. It is not exceedingly poisonous, however, as recovery has been reported in man after the ingestion of an amount varying from one to two pints. Tar is eliminated by the kidneys, with the production of irritation and diuresis; also by the mucous membrane, and affects more especially that lining the bronchial tubes, where it acts as a local stimulant and antiseptic. For this reason the drug is an excellent expectorant in subacute or chronic bronchitis when given internally or by inhalation. The latter process may be conducted by pouring tar on a heated shovel, or, better, by dissolving tar in boiling sodium carbonate solution and steaming the patient with the vapor. Tar is occasionally exhibited in chronic gastro-intestinal catarrh and obstinate diarrhoea, with good results.

Tar is of valne internally in influencing those skin disorders which are benefited by its external application. 
Inhalations of tar are sometimes serviceable in pharyngitis and laryngitis, as well as in bronchitis. Oil of tar may be applied, dissolved in alcohol (1-8), as a lotion, and is an agreeable substitute for tar in chronic eczema or psoriasis of dogs. It is used in the pure state as a parasiticide for mange, scab, ringworm or favus. Oil of cade represents oil of tar and tar in their actions and uses, but its odor is pleasanter. It is prescribed externally in chronic eczema and pruritis, as follows: Oil of cade, 1 ; soft soap and alcohol, 4 parts each; or in equal parts with wax. Oil of cade can also be used in any proportion or manner in which tar is applicable. Pitch likewise possesses the same action as tar, and is sumetimes employed for making hoof ointments and plasters.

\section{Balsamum Perutianum. Balsam of Peru.}

\section{(U. S. \& B. P.)}

A balsam obtained from Toluifera Pereiræ (Royal) Baillon (nat. ord. Leguminosæ).

Synonym.-Balsamum peruvianum nigrum, balsam indicum, baume de Péron, baume des Indes, Fr.; Peru balsam, G.

Habitat.-Central America.

Properties.-A liquid having a syrupy consistence, free from stringiness or stickiness; of a brownish-black color in bulk, reddish-brown or transparent in thin layers; of an agreeable, vanilla-like, somewhat smoky odor, and a bitter taste, leaving a persistent aftertaste. On exposure to air it does not become hard. Spec. gr. 1.135 to 1.150. Miscible in all proportions, with absolute alcohol, chloroform, or glacial acetic acid. Completely soluble in 5 parts of alcohol.

Constituents. - 1 , a volatile oil, cinnamein, $\mathrm{C}_{9} \mathrm{H}_{7}\left(\mathrm{C}_{7} \mathrm{H}_{7}\right) \mathrm{O}_{2}$, about 60 per cent. ; 2, cinnamic acid, $\mathrm{C}_{19} \mathrm{H}_{8} \mathrm{O}_{2} ; 3$, a resin $(32$ per cent.), yielding benzoic acid, $\mathrm{HC}_{7} \mathrm{H}_{5} \mathrm{O}_{2}$, on dry distillation; 4 , styrol, $\mathrm{C}_{8} \mathrm{H}_{8} ; 5$, stilbene, $\mathrm{C}_{14} \mathrm{H}_{12} ; 6$, a volatile oil, benzylic benzoate, $\mathrm{C}_{7} \mathrm{H}_{5}\left(\mathrm{C}_{7} \mathrm{H}_{7}\right) \mathrm{O}_{2} ; 7$, benzylic alcohol, $\mathrm{C}_{7} \mathrm{H}_{8} \mathrm{O}$. 
Duse.-H. \& C., 亏 i.-ii. (30.-60.) ; Sh. \& Sw., 3 i.-ii. (4.-8.); D., Mx.-xxx. (.6-2.).

Balsamum Tolutanum. Balsam of Tolu. (U. S. \& B. P.)

A balsam obtained from Toluifera Balsamum Linné (nat. ord. Leguminosæ).

Synonym.-Baume de Tolu, banme de Cathagène, Fr.; Tolubalsam, G.

Habitat.-New Grenada and Venezuela.

Properties.-A yellowish-brown, semi-fluid, or nearly solid mass, becoming more brittle when exposed to the cold; transparent in thin layers ; having an agreeable odor, recalling that of vanilla, but distinct from it, and a mild aromatic taste. Soluble in alcohol, chloroform and solutions of fixed alkalies; almost insoluble in water.

Constituents. -1 , a volatile oil, tolene, $\mathrm{C}_{10} \mathrm{H}_{16}, 1$ per cent.; 2 , a volatile oil, benzylic benzoate, $\mathrm{C}_{7} \mathrm{H}_{5}\left(\mathrm{C}_{7} \mathrm{H}_{7}\right) \mathrm{O}_{2} ; 3$, benzoic acid, $\mathrm{HC}_{7} \mathrm{H}_{5} \mathrm{O}_{2} ; 4$, cinnamic acid, $\mathrm{C}_{9} \mathrm{H}_{8} \mathrm{O}_{2} ; 5$, benzylic cinnamate, $\mathrm{C}_{9} \mathrm{H}_{3}\left(\mathrm{C}_{7} \mathrm{H}_{7}\right) \mathrm{O}_{2} ; 6$, resins.

Dose._Same as balsam of Peru.

\section{PREPARATION.}

Syrupus Tolutanus. Syrup of Tolu. (U. S. \& B. P.)

Balsam of Tolu, 10 ; precipitated calcium phosphate, 50 ; sugar, 850 ; alcohol, 50 ; water to make 1000 . (U. S. P.)

Dose.-D., 3 i.-iv. (4.-15.).

\section{ACTION AND USES OF BALSAMS OF PERU AND TOLU.}

Externally, the balsams are stimulant, antiseptic and parasiticide. Balsam of Peru is a useful remedy in alcoholic solution (1-8) for chronic eczema of dogs. It may also be serviceable in ointment (1-8) for sore teats in cows, or as an application to kill lice and the parasites of (sarcoptic) mange and ringworm. It is undiluted a most excellent stimulaut and antiseptic dressing upon wounds and ulcers.

Internally, the balsams are stomachic and carminative, and are eliminated by the skin, mucous membranes and 
urinary organs, stimulating these parts. They are therefore occasionally prescribed in chronic bronchitis, pyelitis and cystitis. The syrup of Tolu is an exceedingly mild preparation, but forms an agreeable vehicle for congh mixtures in canine practice. The balsams may be administered in emulsion rubbed up with either glycerin, mucilage, or white of egg and water.

\section{Benzoinum. Benzoin. (U. S. \& B. P.)}

Synonym.--Resina benzoe, asa dnlcis, gum benjamin, E.; benzoin, Fr.; benzoe, G.

A balsamic resin obtained from Styrax Benzoin Dryander (nat. ord. Styraceæ).

Hubitut.-Sian, Sumatra, Java and Borneo.

Properties.-In lumps consisting of agglutinated, yellowish-brown tears, which are internally milk-white; or in the form of a reddish-brown mass, more or less mottled from whitish tears imbedded in it. It is almost wholly soluble in 5 parts of moderately warm alcohol, and in solutions of the fixed alkalies. When beated it gives off fumes of benzoic acid. It has an agreeable, balsamic odor, and a slight aromatic taste.

Constituents.-1, benzoic acid, 12 to 20 per cent.; 2, cinnamic acid, sometimes; 3 , several resins ; 4 , a volatile oil.

PREPARATIONS.

Adeps Benzoinatus. Benzoinated Lard. (U. S. \& B. P.)

Made by melting lard, 1000, with benzoin, 20 ; and straining. (U. S. P.)

Tinctura Benzoini. Tincture of Benzoin. (U. S. P.)

Made by maceration of benzoin, 200, in alcohol ; filtration, and addition of alcohol to make 1000 .

Dose.-H. \& C., 3 i. (:30.); D., 3 ss.-i. (2.-4.).

Tinctura Benzoini Composita. Compound Tincture of Benzoin. (U. S. \& B. P.)

Synonym.-Friar's balsan.

Benzoin, 120 ; purified aloes, 20 ; storax, 80 ; balsam of Tolu, 40 ; alcohol to make 1000. Marle by digestion and filtration. (U. S. P.) 
Acidum Benzoicum. Benzoic Acid. $\mathrm{HC}_{7} \mathrm{H}_{5} \mathrm{O}_{2}$.

(U. S. \& B. P.)

Synonym. - Acide benzoique, fleurs de benjoin, Fr.; benzoesäure, benzoeblumen, $\mathrm{G}$.

Derivation.-Obtained from benzoin by sublimation.

Properties. - White, or yellowish-white, lustrous scales or friable needles; odorless, or having a slight characteristic odor resembling that of benzoin, and of a warm, acid taste. Somewhat volatile at a moderately warm temperature, and rendered darker by exposure to light. Soluble, when pure, in about 500 parts of water, and in 2 parts of alcohol. Also soluble in 3 parts of ether, 7 parts of chloroform, and readily soluble in carbon disulphide, benzol, fixed and volatile oils, but sparingly soluble in benzin.

Incompatibles.-Alkalies, ammonium carbonate.

Dose.-H. \& C., 3 ii.-iv. (8.-15.) ; D., gr.v.•xv. (.3-1.).

\section{Ammonit Benzons. Ammonium Benzoate. $\mathrm{NH}_{4} \mathrm{C}_{7} \mathrm{H}_{6} \mathrm{O}_{2}$.}

$$
\text { (U. S. \& B. P.) }
$$

Made by the action of benzoic acid and ammonia water. In white crystals. Soluble in 5 parts of water; in 28 parts of alcohol.

Dose._Same as benzoic acid.

Sodir Benzoas. Sodium Benzoate. $\mathrm{Na}_{7} \mathrm{H}_{5} \mathrm{O}_{2}$. (U. S. P.)

Made by the action of a hot solution of sodium carbonate on benzoic acid. Occurs in a white powder. Soluble in 1.8 parts of water ; in 45 parts of alcohol.

Dose.-Same as benzoic acid.

ACTION OF BENZOIN, BENZOIC ACID AND BENZOATES.

These substances may be represented by benzoic acid. Action External.-Benzoic acid is an irritant applied externally, also when its vapor is inhaled. It is an efficient 
antiseptic; a solution (1-1000) will inhibit the growth of many forms of bacteria.

Action Internal.-Medicinal doses of benzoic acid exert only an antiseptic action in the alimentary canal. Large doses occasion increased bronchial and cutaneous secretion, with accelerated pulse. Euormous doses cause gastro-intestinal irritation, disturbance of the pulse and respiration, a species of intoxication and paraplegia. Benzoic acid is absorbed into the blood, acts as an antipyretic in fever, and is converted (probably in the kidneys) into hippuric acid and eliminated in the urine as such. It thus renders an alkaline urine acid, and stimulates and exercises an antiseptic influence upon the urinary mucous membrane. The change into hippuric acid is due to combination with a nitrogenous body-glycocoll-but the source of glycocoll is uncertain.

Benzoic acid $\mathrm{HC}_{7} \mathrm{H}_{5} \mathrm{O}_{2}+$ glycocoll $\mathrm{C}_{2} \mathrm{H}_{5} \mathrm{NO}_{2}=$ hippuric acid $\mathrm{C}_{9} \mathrm{H}_{9} \mathrm{NO}_{3}+$ water $\mathrm{H}_{2} \mathrm{O}$.

Benzoic acid is somewhat diuretic, but does not alter the composition of the urine in any constant manner, although metabolism is said to be increased. It is eliminated by the bronchial mucous membrane, augmenting secretion, and acting as an antiseptic in the bronchial tubes. When the vapor of any of the substances under consideration is inhaled in proper dilution, a similar expectorant action is attained. Benzoic acid is likewise excreted by the skin and salivary glands, exciting their functional activity.

Uses External.-The compound tincture of benzoin is a valuable stimulant and antiseptic application for wounds, sores and ulcers. It is often applied to the part on gauze or lint, followed by bandaging.

Uses Internal. - Benzoic acid is sometimes given in powder, pill, or ball, as an intestinal antiseptic, and as a remedy for rheumatism. It is inferior to salicylic acid in the latter disorder. The benzoates are said to be equally efficient as antiseptics and have been highly recommended in diarrhœea and dysentery. 
Benzoin, in the form of the tincture, and the benzoates, are serviceable in laryngitis, tracheitis, and bronchitis, to promote secretion and antisepsis. An inhalation of the tincture ( 3 ii.-Oi. hot water) is also very serviceable in these diseases for the same purposes.

The benzoates are of value in pyelitis and cystitis, particularly in carnivora with a normal acid urine, to acidify and disinfect the decomposing urine and stimulate the tract.

\section{Sinapis Alba. White Mustard.}

Synonym.-Sinapis albæ semina, B.P.; semen erucæ, yellow mustard seed, E. ; moutarde blanche, Fr.; weisser senf, G.

The seed of Brassica alba (Linné) Hooker filius et Thompson (nat. ord. Cruciferæ).

Habitat.-Southern Europe and Asia; cultivated in temperate climates.

Description.-About $2 \mathrm{Mm}$. in diameter; almost globular, with a circular hilum; testa yellowish, finely pitted, hard; embryo oily, with a curved radicle and two cotyledons, one folded over the other; free from starch; inodorous; taste pungent and acrid.

Constituents. - 1, a glucoside, sinalbin $\left(\mathrm{C}_{30} \mathrm{H}_{41} \mathrm{~N}_{2} \mathrm{~S}_{2} \mathrm{O}_{16}\right)$, and a ferment, myrosin, $10-15$ per cent. The latter converts the former, in the presence of water, into the active principle of the drug, acrinyl sulphocyanide $\left(\mathrm{C}_{7} \mathrm{H}_{7} \mathrm{ONCS}\right)$, a very acrid, volatile body, sinapine sulphate $\left(\mathrm{C}_{16} \mathrm{H}_{23} \mathrm{NO}_{5} \mathrm{H}_{2} \mathrm{SO}_{4}\right)$, and glucose ; 2, a bland, fixed oil, 25 per cent.; 3, gum, 20 per cent.

Dose.-H., 3 ss. (15.); C., इ ss.-i. (15.-30.); Sh. \& Sw., 3 i.-ii. (4.-8.) ; D., gr.x.-xv. (.6-1.).

\section{Sinapis Nigra. Black Mustard.}

Synonym.-Sinapis nigræ semina, B.P.; semen sinapis, P.G.; moutarde noire (Grise), Fr.; schwarzer senf, G.

The seed of Brassica nigra (Linné) Koch (nat. ord. Cruciferæ). 
Habitat.-Sonthern Europe and Asia; cultivated in temperate climates.

Description.-Abont $1 \mathrm{Mm}$. in diameter, almost globular, with a circular hilum; testa blackish-brown or grayishbrown, finely pitted, hard; embryo oily, with a curved radicle and two cotyledons, one folded over the other; free from starch; inodorous when dry, but when triturated with water, of a pungent, penetrating, irritating odor; taste pungent and acrid.

Constituents. - 1, a glucoside, sinigrin (or potassinm myronate), aud a ferment, myrosin. In the presence of water the latter converts the former into the acrid, volatile, official oil of mustard (allyl sulphocyanide, $\mathrm{C}_{3} \mathrm{H}_{5} \mathrm{CNS}$ ), acid potassium sulphate, and glucose; 2, a fixed, bland oil, similar to that in white mustard; 3 , gum.

Dose.-Same as white mustard.

Commercial form of mustard is a mixture of black and white mustard.

\section{PREPARATION.}

\section{Oleum Sinapis Volatile. Volatile Oil of Mustard.}

Synonym.-Oleum sinapis, B.P.; allyl sulphocyanide $\left(\mathrm{C}_{3} \mathrm{H}_{5} \mathrm{CNS}\right)$, oleum sinapis æthereum, E.; essence de moutarde, Fr.; ætherisches senföl, G.

Derivation. - A volatile oil obtained from black mustard by maceration with water and subsequent distillation.

Properties. - A colorless or pale yellow, limpid and strongly refractive liquid, having a very pungent and acrid odor and taste. Freely soluble in alcohol, ether, or carbon disulphide, the solutions being neutral to litmus paper. Spec. gr. 1.018 to 1.029 .

Action External.-Mustard quickly dilates the vessels of the skin and causes lyyperæmia. If its application is frequently repeated, there is so much vascular irritation that transudation of serum occurs under the epidermis, and blisters or even pustules are formed. Mustard induces a sensation of burning in man, but is not so irritating as oil 
of turpentine to horses, and the primary irritation is followed by partial anesthesia. It is one of the most useful counterirritants, and by this action contracts ressels in the underlying parts, relieres pain and congestion.

In rapidly stimulating the skin, mustard reflexly excites the nerrous system, respiratory functions and heart. Mustard is therefore a rubefacient, resicant, and counter-irritant exterually.

Action Internal.-Mustard stimulates gastric vascularity, secretion and motion, and promotes the appetite in small doses. Large doses occasion romiting in animals capable of the act. Intestinal peristalsis and secretion are probably likewise angmented by mustard. It is thus a stomachic, carminative and emetic internally, but is rarely administered save as an emetic because of its pungency and the difficnlty attending its exhibition. Mustard is absorbed to some extent, but we are ignorant concerning its ultimate fate or remote action. It is said to be a dinretic.

Uses External. - Mustard is an extremely valuable counter-irritant for relieving pain or congestion in almost any internal part. It is more commonly employed in the acute respiratory disorder's of the domestic animals, as in laryngitis, bronchitis, congestion of the lungs, plenrisy, pneumonia, to stop incessant cough, and after exposure to severe cold ; and the flour of mustard is used in a very thin paste made with warm water and applied with friction to the skin. Boiling water should not be mixed with mustard, nor vinegar, nor alcohol, as they interfere with its action. The volatile oil of mustard is a cleanly and convenient substitute for the crude drug. A small quantity, diluted with olive oil, or cottonseed oil (1-15), may be rubbed into either side of the chest in bronchitis, pleurisy, and other chest disorders, as a counter-irritant.

After the application of mustard, the part may be bandaged, or hot blankets may be placed over the body and the treatment reinforced by the use of mustard on the limbs and bandaging. If mustard is employed continuously 
to keep up constant counter-irritation, the drug should be washed off in 20 or 30 minutes, and the process repeated once in 2 hours. It is unwise to induce much vesication over an extensive surface with mustard, as the result is painful and resolution is slow. Mustard is also serviceable in painful abdominal diseases, as colic, tympanites, enteritis and peritonitis, but oil of turpentine appears here to be more efficient.

A sinapism (mustard application) over the loins is useful in acute nephritis, and will not lead to irritation of the kidneys from absorption which may ensue after cantharidal blistering. Cantharides has, however, a more active, permanent and revulsant effect in most local inflammatory conditions, and is more potent in acute laryngitis. The action of mustard is rapid and fleeting, unless applied continually in considerable strength. For this reason it is indicated to impress the nervous system instantaneously, in opium, alcohol and other narcotic poisoning; in respiratory failure, in collapse and extreme depression in the course of acute diseases (pneumonia and parturient apoplexy), applied all over the body, or over the cardiac region in syncope. Mustard is of utility in muscular or articular rheumatism, and is employed on swollen glands (strangles), inflamed joints and tendons; but is usually less efficacious than a good cantharidal blister in these three latter conditions.

Uses Internal.-Mustard is an efficient emetic for dogs, in tablespoonful doses, given in a cup of tepid water. It is usually at hand, and not only empties the stomach in poisoning, but reflexly stimulates the heart and respiration. If administered for its carminative or stomachic effect, mustard must be given in pill or ball.

\section{Eucalyptus. Eucalyptus. (U. S. P.)}

Synonym. - Feuilles d'eucalyptus, Fr.; eucalyptusblätter, G. 
The leaves of Eucalyptus globulus Labillardière (nat. ord. Myrtaceæ), collected from the older parts of the tree.

Description.-Petiolate, lanceolately scythe-shaped; from 15 to $30 \mathrm{~cm}$. long; rounded below, tapering above; entire, leathery, grayish-green, glandular, feather-veined between the midrib and marginal veins; odor strongly camphoraceous; taste pungently aromatic and somewhat cooling, bitter and astringent.

Constituents.-1, a volatile oil (see below); 2, a crystallizable resin; 3 , a crystallizable, fatty acid; 4, cerylic alcohol. Dose.-H. \& C., 3 ii.-iii. (60.-90.); D., 3 ss.-ii (2.-8.).

\section{PREPARATION.}

Extractum Eucalypti Fluidum. Fluid Extract of Eucalyptus. (U. S. P.)

Made by maceration, percolation and evaporation, so that $\mathrm{L} \mathrm{Cc.}=$ $1 \mathrm{Gm}$. of the crude drug.

Dose.-Same as for eucalyptus.

Oleum Eucalypti. Oil of Eucalyptus. (U. S. \& B. P.)

A volatile oil distilled from the fresh leaves of Eucalyptus globulus Labillardière, Eucalyptus oleosa F. V. Mueller, and some other species of Eucalyptus (nat. ord. Myrtaceæ).

Properties. - A colorless or faintly-yellowish liquid, having a characteristic, aromatic, somewhat camphoraceous odor, and a pungent, spicy, and cooling taste. Spec. gr. 0.915 to 0.925 . Soluble in all proportions in alcohol, carbon disulphide, or glacial acetic acid.

Constituents. - 1, a volatile oil, encalyptol or cineol $\left(\mathrm{C}_{10} \mathrm{H}_{18} \mathrm{O}\right), 70$ per cent.; 2, eucalyptene $\left(\mathrm{C}_{10} \mathrm{H}_{16}\right)$; 3 , cymene $\left(\mathrm{C}_{10} \mathrm{H}_{14}\right)$.

Incompatibles.-Alkalies, mineral acids, and metallic salts. Dose.-H., 3 i.-ii. (4.-8.); D., Mii.-x. (.12-.6).

Eucalyptol. Eucalyptol. $\mathrm{C}_{10} \mathrm{H}_{18} \mathrm{O}$. (U. S. P.)

A neutral body obtained from the volatile oil of Eucalyp- 
tus globulus Labillardière, and of some other species of eucalyptus (nat. ord. Myrtaceæ).

Derivation.-Crude eucaly ptol distills over from eucalyptus leaves at a temperature varying from $338^{\circ}$ to $352^{\circ} \mathbf{F}$., and is purified by redistillation from potassium hydrate or calcium chloride.

Properties.-A colorless liquid, haring a characteristic, aromatic and distinctly camphoraceous odor, and a pungent, spicy and cooling taste. Spec. gr. 0.930. Soluble in all proportions, in alcoliol.

Dose.-Same as oil of encalyptus.

ACTION OF EUCALYPTUS, OIL OF EUCALYPTUS AND EUCALYPTOL.

External.-The oil is a powerful antiseptic and disinfectant, and is even said to be three times more efficient in this respect than carbolic acid. It is but slightly irritating to the skin, unless its vapors are confined by bandaging, when it may cause vesicles and pustules. Some local anæsthesia follows primary skin irritation.

Internal.-Digestive Tract.-Oil of eucalyptus excites gastric and salivary secretion, and acts, both locally and during elimination, as a stimulant to the mucous membrane of the alimentary canal. Large doses occasion diarrhœea, and the fæcal discharges are impregnated with the odor of the oil. It is a stomachic, carminative, antiseptic and anodyne in the digestive tract.

Circulation.-Oil of eucalyptus arrests the amceboid movements of the white blood corpuscles, and diapedesis, in inflammatory areas; inhibits the growth of the plasmodia malariæ; is an antipyretic and antiperiodic, and generally comports itself like quinine; but is nevertheless distinctly inferior to it. Small doses reflexly stimulate the heart and cause an increase in blood pressure; while toxic doses depress the heart's action and lower vascular tension.

Respiration. - Small doses accelerate the respiratory movements. Large doses make the respiration slower and weaker, and death ensues through respiratory failure. 
Nervous System. - Poisonous quantities depress the brain, medullat and spinal cord. Reflex activity is lost. Animals stagger, suffer great loss of muscular power and sensation in their limbs, and fall ; the breatling is slow and irregular, the pulse weak, and there are occasional convulsions. The breathing stops before the cardiac pulsations.

Elimination.-Oil of eucalyptus is excreted by the skin, kidneys, and mucons membrane of the bronchial tubes and bowels, and therefore stimulates and disinfects these parts during its elimination. Hence the drug is a diarphoretic, diuretic, and genito-urinary stimulant, stimulating expectorant, and carminative.

Administration. - The oil, or encaly ptol, are administered in emulsion with gum; dissolved in alcohol; or in capsules.

\section{USES OF EUCALYPTUS, OIL OF EUCALYPTUS AND EUCALYPTOL.}

External.-Encalyptol is probably more generally useful than either eucalyptus or the oil. It is employed as an antiseptic with vaseline (1-8), on sores, wounds, and ulcers. and in lubricating instruments for use in the cavities of the body. It partially disguises the odor of iodoform, and is frequently combined with the latter in ointment. Encalyptol is serviceable as a stimulating, antiseptic and deodorant inhalation in catarrhal diseases of the respiratory tract with putrid discharges, and in pulmonary gangrene. The ordinary doses (by the mouth) are placed in hot water for this purpose. Eucalyptol, with sweet oil (1-5), forms an efficient stimulating and anodyne liniment.

Internal.-In chronic bronchitis, encalyptol is often valuable in stimulating and disinfecting the bronchial mucous membrane during its elimination. It is also efficacious in chronic pyelitis and cystitis, for the same reason. The oil has been given with asserted success in various bacterial diseases, as septicæmia, canine distemper, influenza, etc., for its antiseptic action. In human medicine, eucalyptus and its derivatives are mainly of worth as substitutes for quinine in malaria, when the latter drug is inadmissible. Eucalyptol 
may be given as a stomachic and carminative in digestive disorders with foul-smelling fæcal evacuations.

\section{Arnic瓜 Flones. Arnica Flowers.}

Synonym.-Fleurs d'arnique, Fr.; wollverleihblüthen, arnikablüthen, $\mathrm{G}$.

The flower heads of Arnica montana Linné (nat. ord. Compositæ).

Habitat.-Mountainous regions of Europe and Northern Asia.

Description.-Heads about $3 \mathrm{Cm}$. broad; depressedroundish, consisting of a scaly involucre in two rows, and a small, nearly flat, hairy receptacle, bearing about sixteen yellow, strap-shaped, ten-nerved, ray-florets, and numerous yellow, fire-toothed, tubular disk-florets having slender, spindle-shaped achenes crowned by a hairy pappus. Odor feeble, aromatic; taste bitter and acrid.

Constituents. -1, an ethereal oil ; 2 , an amorphous, yellow, acrid, bitter principle, arnicin; 3 , resins; 4, tannic acid; 5, caprylic and capronic acids.

Dose.—H \& C., 3 ss.-i. (15.-30.); D., gr.x.-xx. (.6-1.3).

\section{PREPARATION.}

Tinctura Arnice Florum. Tincture of Arnica Flowers. (U. S. P.)

Made by percolation of arnica flowers, 200 , with diluted alcohol to make 1000 .

Dose.-H. \& C., 亏i. (30.) ; D., M.xv.-xl. (1.-2.6).

ArNice Radix. Arnica Root.

Synonym.-Arnicæ rhizoma, B.P.; racine d'arnique, Fr.; arnikawurzel, G.

The rhizome and roots of Arnica montana Linné (nat. ord. Compositæ).

Description.-Rhizome about $5 \mathrm{Cm}$. long, and 3 or $4 \mathrm{Mm}$. thick; externally brown, rough from leaf scars; internally whitish, with a rather thick bark containing a circle of resin 
cells, surrounding the short, yellowish wood wedges, and large, spongy pith. The roots numerous, thin, fragile; grayish-brown, with a thick bark containing a circle of resin cells. Odor somewhat aromatic; taste pungently aromatic and bitter.

Constituents.-Identical with those of flowers.

Dose.-Same as flowers.

PREPARATIONS.

These are unnecessarily numerous considering the insignificance of the drug.

Extractum Arnica Radicis. (U. S. P.)

Extractum Arnicce Radicis Fluidum. (U.S. P.)

Dose.-Same as root.

Tinctura Arnice Radicis. (U. S. P.)

Dose.-Half that of the tincture of the flowers.

Tinctura Arnicce. (B. P.)

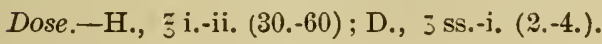

Action and Uses.-Externally, arnica, especially the tinc-' ture of the flowers, increases the vascularity of the integument, and may cause various grades of superficial inflammation on sensitive skins if not properly diluted. Internally, the drug stimulates the mucous membrane of the digestive tract, and is stomachic and carminative. Arnica is eliminated by the kidneys and mucous membranes and stimulates these parts during its excretion. Arnica slows and stimulates the heart and increases vascular tension in medicinal doses, while large doses augment the frequency of the pulse. This action follows, first, stimulation, and later, depression of the inhibitory apparatus. Toxic quantities occasion gastrointestinal irritation and nerrous symptoms, as giddiness, stupor, delirium, insensibility, and convulsions; with rapid, feeble pulse and sometimes syncope.

The only rational use for arnica is in stimulating the skin in sprains and bruises. The tincture of the flowers is generally diluted with water (1-12) for this purpose. It is doubtful if it is more serviceable than alcohol in these con- 
ditions. There is no sufficient evidence to show that arnica possesses any therapentic value when given internally, althongh it has been used and is recommended for dysentery, chronic bronchitis, rhenmatism, nervous disorders, etc.

\section{Mrrena. Myrrh. (U. S. \& B. P.)}

Synonym.-Myrrhe, F.

A gum-resin obtained from Commiphora Myrrha (Nees) Engler (nat. ord. Burseracer).

Habitat. - Eastern Africa and Southwestern Arabia, along the borders of the Red Sea.

Description.-In roundish or irregular tears or masses; dusty, brownish-yellow or reddish-brown; fracture waxy, somewhat splintery; translucent on the edges, somewhat marked with whitish veins; odor balsamic; taste aromatic, bitter and acrid. When triturated with water, myrrh yields a brownish-yellow emulsion; it is soluble in alcohol.

Constituents.-1, an active resinous principle, myrrhin $\left(\mathrm{C}_{48} \mathrm{H}_{32} \mathrm{O}_{10}\right), 30$ to 40 per cent. ; 2 , myrrhol $\left(\mathrm{C}_{10} \mathrm{H}_{14} \mathrm{O}_{1}\right)$, a volatile oil, 28 per cent.; 3 , arabin, a gum, 50 to 60 per cent. ; 4, a bitter substance.

Dose.-H. \& C., 3 ii.-iv. (8.-15.); Sh. \& Sw., 3 ss.-i. (2.-4.) ; D., gr.v.--xxx. (.3-2.).

\section{PREPARATIONS.}

Tinctura Myrrhoe. Tincture of Myrrh. (U. S. \& B. P.)

Made by maceration of myrrh, 200, with alcohol, and filtration to make 1000. (U. S. P.)

Dose.-H. \& C., 3 i.-ii. (30.-60.) ; Sh. \& Sw., 3 iii.-vi. (12 -24.) ; D., 3 ss -i. (2.-4.).

Tinctura Aloes et Myrrhoe. Tincture of Aloes and Myrrh.

(U. S. \& B. P.)

Made by maceration and percolation of myrrh, 100; purified aloes, 100; liquorice root; with alcohol and water to make 1000 . (U. S. P.)

Dose.-H. \& C., $\frac{\pi}{3}$ ii.-iv. $(60 .-120$.) ; Sh. \& Sw., 3 ss.-i. (15.-30.) ; D., 3 i.-ii. (4.-8.).

Action and Uses.-Myrrh, externally, is a mild stimulant 
and antiseptic by virtue of its resin and rolatile oil. It is a stomachic and carminative interually, exciting the appetite and increasing the secretion, motion and blood supply of the stomach and bowels. Myrrh is eliminated by the mucous membranes of the bronchial and genito-urinary tracts, aud stimulates and disinfects these parts during its excretion. The drug is occasionilly prescribed as a stimulating expectorant in chronic bronchitis; as a stimulant and antiseptic in chronic cystitis ; also as a uterine stimulant and emmenagogue in ammenorrhœen, and in chronic lencorrhœa.

Myrrh is thought to prove beneficial in anæmia, when combined with iron. It assists the action of purgatives, and myrrh may be exhibited as a laxative in the form of the tincture of aloes and myrrh. The tincture forms a serviceable mouth-wash in aqueous emulsion (1-16), and is sometimes employed as a stimulant and antiseptic on wounds, sores and ulcers, diluted with 4 to 8 parts of water. Myrrh is administered in tincture, ball or pill.

\section{Class 2.-Used Mainly for their Stomachic and Carminative Action Upon the Digestive Tract.}

Capsicum. Capsicum. (U.S. P.)

Synonym.-Capsici fructus, B. P.; cayenne pepper, African or pod pepper, E.; capsiqua, piment des jardins, piment rouge, poivre de cayenne, Fr. ; spanischer pfeffer, G. Called commonly "red pepper," when dried and powdered.

The fruit of Capsicum fastigiatum Blume (nat. ord. Solanaceæ).

Habitat. - Tropical America; cultivated also in other tropical countries.

Description.-Oblong-conical, from 10 to $20 \mathrm{MIm}$. long; supported by a flattish, cup-shaped, five-toothed calyx with a red, shining, membranous and trauslucent pericarp enclosing two cells and containing flat, reniform, yellowish seeds, attached to a thick, central placenta. It has a peculiar odor, and an intensely hot taste. 
Constituents. - 1 , capsaicin $\left(\mathrm{C}_{9} \mathrm{H}_{14} \mathrm{NO}_{2}\right)$, a crystallizable, acrid body; 2 , capsicin, a volatile alkaloid; 3 , a fixed oil ; 4 , fatty matter; 5 , resin.

Dose. - H., gr.xx.-3i. (1.3-4.); C., 3 i.-ii. (4.-8.); D., gr.i.-viii. (.06-.5).

\section{PREPARATIONS.}

Extractum Capsici Fluidum. Fluid Extract of Capsicum. (U. S. P.)

Made by maceration and percolation with alcohol, and evaporation, so that $1 \mathrm{Cc} .=1 \mathrm{Gm}$. of the crude drug. $(.06-.5)$.

Dose.-H., $\Pi \times x \times .-3$ i. $(1.3-4$.$) ; C., 3$ i.-ii. (4.-8.); D., Mi.-viii.

Tinctura Capsici. Tincture of Capsicum. (U. S. \& B. P.)

Made by percolation of capsicum, 50, with alcohol and water to make 1000 . (U. S. P.)

Dose.-H., 3 ii.-iv. (8.-15.); C., 3 ss.-i. (15.-30.); D., mv.- 3 i. (.3-4.).

Oleoresina Capsici. Oleoresin of Capsicum. (U.S. P.) residue.

Made by percolation with ether, distillation, and evaporation of the

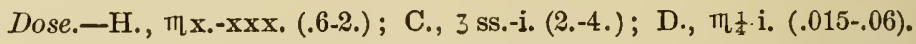

Administration.-Capsicum and the oleoresin are given in ball or pill. The fluid extract should be freely diluted with water.

Action and Uses.-Capsicum generally resembles the volatile oils in its action. Externally, it is rubefacient and counter-irritant, producing about the same degree of irritation as mustard, but causing considerably more pain, while its fumes are unbearable. Capsicum is used mainly as a stomachic and a carminative in angmenting the appetite, gastric vascularity, secretion and motion, and intestinal peristalsis. Capsicum is employed on the skin in local paralysis-as of the lip-in horses, with mustard in paste; or as the fluid extract painted on plaster splints to prevent dogs from gnawing them off.

Internally, capsicum is of greater value than black or white pepper, and is indicated in atonic indigestion and flatulent colic in horses (see ammonium carbonate, p. 141). 
It may be combined advantageously with bitters, as nux vomici. Capsicum is a favorite stimulant and tonic remedy - to the digestion-with bird fanciers. It is also said to increase the laying of eggs when given to hens.

\section{Zingiber. Ginger. (U. S. \& B. P.)}

Synonym.-Ingwer, G.; gingember, Fr.

The rhizome of Zingiber officinale Roscoe (nat. ord. Scitamineæ).

Habitat.-East and West Indies and India ; cultivated in tropical climates.

Description.-About 5 to $10 \mathrm{Cm}$. long, 10 to $15 \mathrm{MIm}$. broad, and 4 to $8 \mathrm{Mm}$. thick; flattish on one side; lobed or clavately branched; deprived of the corky layer; pale, buffcolored, striate; breaking with a mealy, rather fibrous fracture, showing numerous small, scattered resin cells and fibro-vascular bundles, the latter enclosed by a nucleus sheath ; agreeably aromatic and of a pungent and warm taste. Constituents. -1 , a volatile oil ; 2 , a resin ; 3 , gingerol, said to supply pungent taste, while the oil gives flavor.

Dose. -H., 3 ii.- $\tilde{3}$ i. (8.-30.); C., żi.-iv. (30.-120.) ; Sh. \& Sw., 3 i.-ii. (4.-8.) ; D., gr.v.-xv. (.3-1.).

\section{PREPARATION.}

Extractum Zingiberis Fluidum. Fluid Extract of Ginger.

(U. S. P.)

Made by maceration and percolation with alcohol, and evaporation, so that $1 \mathrm{Cc}$. $=1 \mathrm{Gm}$. of the crude drug.

Dose.-Same as that of ginger.

Action and Uses.-Ginger is chiefly administered in powder as a stomachic and carminative in atonic indigestion of horses and ruminants. It is frequently combined with sodium bicarbonate and bitters. Ginger also aids the action of purgatives and prevents griping. The powder or fluid extract should be added to magnesium sulphate when it is given in full purgative doses to cattle or sheep. (See magnesium and sodium sulphate, pp. 136 and 163). 
Mentha Pipenita. Peppermint. (U. S. P.)

Synomym.-Folia (herba) menthæ piperitæ, P.G.; menthe poivrée, Fr.; pfefferminze, G.

The leaves and tops of Mentha piperita Smith (nat. ord. Labiatre).

Habitut.-Indigenous in North America,Europe and Asia.

Description.-Leaves abont $5 \mathrm{Cm}$. long, petiolate, ovatelanceolate, acute, sharply serrate, glandular, nearly smooth; the few hairs containing crystals of menthol in one or more thin cells; branches quadrangular, often purplish ; flowers in terminal, conical spikes, with a tubular, five-toothed, often purplish calyx, a purplish four-lobed corolla, and four short. stamens; odor aromatic; taste pungent and cooling.

Constituents. - 1, a volatile oil : 2 , menthol ; 3 , menthene $\left(\mathrm{C}_{10} \mathrm{H}_{18}\right)$.

\section{Oleum Menthe Piferite. Oil of Peppermint.}

(U. S. \& B. P.)

Synonym. - Essence de menthe poivrée, Fr.; pfefferminzöl, G.

A volatile oil distilled from peppermint.

Pioperties.-A colorless, or yellowish, or greenish-yellow liquid, becoming darker and thicker by age and exposure to. the air ; having the characteristic strong odor of peppermint, and a strongly aromatic, pungent taste, followed by a sensation of cold when air is drawn into the mouth. Spec. gr. 0.9 to 0.920 .

It forms a clear solution with an equal volume of alcohol, but becomes turbid when somewhat further diluted.

Constituents.-1, menthol; 2, menthene, a liquid terpene obtained by distillation.

Dose.-H. \& C., Mlxv.-xxx. (1.-2.); D., Mi-v. (.06-.3).

PREPARATIONS.

Spiritus Menthce Piperitce. Spirit of Peppermint. (U. S. \& B. P.)

Syuouym-Essence de menthe poivrée, Fr.; Englisch pfeffermin? senz, G.; spiritus menthæe piperitæ anclicus, P.G. 
Oil of peppermint, 100 ; peppermint, 10 ; alcohol to make 1000 . Made by maceration and filtration. (U. S. P.)

Dose.-H. \& C., 3 ii.-iv. (8.-15.); D. $\pi \times \mathrm{xv} .-\mathrm{xxx}$ (1.-2.).

Aqua Menthce Piperitce. Peppermint Water. (U. S. \& B. P.)

Oil of peppermint, 2 ; precipitated calcium phosphate, 4 ; water to make 1000. Made by trituration and filtration. (U. S. P.)

Dose.-Used as vehicle in canine practice.

\section{Menthol. Menthol. $\mathrm{C}_{10} \mathrm{H}_{19} \mathrm{OH}$. (U. S. P.)}

Synonym.-Mint or peppermint-camphor:

A stearopten (having the character of a secondary alcohol) obtained from the official oil of peppermint (from Mentha piperita Smith), or from Japanese or Chinese oil of peppermint (from Mentha avensis Linné, var. piperascens Holmes, and Mentha Canadensis Linné, var. glabrata Holmes ; nat. ord. Labiatre).

Derivation.-Made from the oil of peppermint by fractional distillation ; freezing of the ligher boiling point product, and crystallization.

Properties.-Colorless, acicular or prismatic crystals, having a strong and pure odor of peppermint, and a warm, aromatic taste, followed by a sensation of cold when air is drawn in the montl. Slightly soluble in water; freely soluble in alcohol, ether, chloroform, carbon disulphide or glacial acetic acid. When it is triturated with about an equal weight of camphor, thymol, or chloral hydrate, the mixture becomes liquid. Menthol may be dissolved by heat in oleic acid, fats or liquid vaseline.

Dose.-H., gr.vii.-xv. (.5-1.); D., gr.ss.-ii. (.03-.12).

ACTION AND USES OF PEPPERMINT AND MENTHOL.

Peppermint and oil of peppermint owe their medicinal virtues chiefly to the menthol they contain. They resemble the other volatile oils in most respects, but are more anæsthetic and antiseptic than some. Menthol is used mostly externally, and is extremely valuable in relieving itching and neuralgic pain. It may be employed with alcohol or 
chloroform in solution ( 3 ss. to $\overline{3}$ i.), in urticaria or pruritus. An ointment is also serviceable, or a solution by heat in oleic acid (1 to 24 ). For burns, the following will be found beneficial: Sweet oil and lime water, each one ounce; menthol, one drachm. The cooling sensation produced by menthol is due to a specific effect upon the nerves of temperature. The anrsthetic and antiseptic action of menthol has been taken advantage of in the treatment of boils and superficial abscesses. A 10 to 50 per cent. solution in ether is said to abort these lesions when painted frequently on the inflamed parts. A menthol and camphor solution, obtained by dissolving fifteen grains of each in an ounce of liquid petrolatum, is one of the best preparations to use in an atomizer or dropper for acute or chronic nasal catarrh of dogs.

Internally, the essence of peppermint or oil are of worth in cases of mild colic and flatulence on account of their anæsthetic, carminative and antiseptic action. Menthol may be given to dogs to relieve vomiting. Peppermint water assuages thirst in fever, and this preparation is also used as. a pleasant vehicle in the administration of disagreeable drugs to dogs. The oil is prescribed in pill or ball to prevent the griping of cathartics.

Administration.-The essence is the preparation in most common use and is given in water. The oil is dissolved in spirit, or exhibited to dogs on sugar. Menthol may be administered in alcohol and syrup, equal parts ; or in pill or capsule to dogs.

Mentha viridis (spearmint) is official together with oleum menthæ viridis (oil of spearmint), spiritus menthæ viridis (spirit of spearmint), and aqua menthæ viridis. The actions, uses and doses are the same as those of peppermint and its preparations, but the latter are more popular and pleasant.

Anisum. Anise.

Synonym.-Anisi fructus, B.P.; anis, Fr.; anis, G. 
The fruit of Pimpinella Anisum Linné (nat. ord. Umbelliferæ).

Habitat.-Sontheastern Europe, Egypt, Western Asia; also cultivated.

Description.-About 4 or $5 \mathrm{Mm}$. long, ovate, compressed at the sides; grayish, finely hairy, and consisting of two mericarps, each with a flat face and five light brownish, filiform ridges, and about fifteen thin oil tubes, which can be I seen in a transverse section by the microscope. It has an agreeable, aromatic odor, and a sweet, spicy taste. It may be distinguished from conium fruit by the odor and taste, while the latter has usually single mericarps which are smooth, grooved upon the face, and have crenate ridges with wrinkles between them, and no oil tubes.

Constituents.-Oil of anise.

Dose.-H. \& C., ₹ i.-ii. (30.-60.); Sh. \& Sw., 3 ii.-iii. (8.-12.) ; D., gr.x.-xxx. (.6-2.).

\section{Illicium. Illicium. (U. S. P.)}

Synonym.-Anisi stellati fructus, B.P.; star arise.

The fruit of illicium verum Hooker filius (nat. ord. Magnoliaceæ).

Habitat.-North Anam.

Description.--The fruit is pedunculate and consists of eight stellately arranged carpels, which are boat-shaped, about $10 \mathrm{Mm}$. long, rather woody, wrinkled, straight-beaked, brown, dehiscent on the upper suture, internally reddishbrown, glossy, and containing a single, flattish, oval, glossy, brownish-yellow seed; odor anise-like, taste of the carpels sweet and aromatic, and of the seeds oily. Similar to the i poisonous fruit of Illicium anisatum Linné (Illicium religiosum Siebold), the capsules of which are more woody, shrivelled, and have a thin, mostly curved beak; a faint, clove-like odor, and an unpleasant taste.

Constituents.-Oil of anise.

Dose.-Same as that for anise. 


\section{Oleum Anisi. Oil of Anise. (U. S. \& B. P.)}

A volatile oil distilled from anise.

Properties.-A colorless or pale yellow, thin and strongly refractive liquid, having the characteristic odor of anise, and a sweetish, mildly aromatic taste. Spec. gr. about 0.980 to 0.990. Soluble in an equal volume of alcohol.

$$
\begin{aligned}
& \text { Dose.-H., Mxx.-xxx. (1.3-2.); D., Mi.-v. (.06-.3). } \\
& \text { PREPARATIONS. } \\
& \text { Aqua Anisi. Anise Water. (U.S. \& B. P.) }
\end{aligned}
$$

Oil of anise, 2 ; precipitated calcium phosphate, 4 ; water to make. 1000. (U. S. P.) Used as vehicle.

Spiritus Anisi. Spirit of Anise. (U. S. \& B. P.)

Oil of anise, 100 ; deodorized alcohol, 900. (U. S. P.)

Dose.-D., 3 i.-ii. (4.-8.).

ACTIONS AND USES OF ANISE AND ILLICIUM.

Oil of anise resembles in action the other volatile oils. It is employed with olive oil or alcohol (1-10) to kill fleas or lice on dogs, rubbed over the skin; and one drop of the pure oil may be placed on the feathers of fowl to cause destruction of lice. The oil is sometimes prescribed to disguise the taste or odor of drugs (see potassa sulphurata), and is ordered in cough mixtures for its expectorant properties.

Anise fruit is given to horses and ruminants on their food-frequently with sodium bicarbonate and ginger-to relieve mild forms of indigestion and flatulence through its. stomachic and carminative action.

\section{Cardamonum. Cardamom.}

Synonym.-Cardamomi semina, B.P.; fructus vel semen cardamomi minoris, P.G.; cardamomes, Fr. ; cardamomen, kleine kardamomen, G.

The fruit of Elettaris repens (Sonnerat) Baillon (nat. ord. Scitamineæ).

Habitat.-Malabar. 
Description.-Fruit ovoid or oblong, from 10 to $15 \mathrm{Mm}$. long; of a pale buff color, with a thin, tasteless pericarp. Seeds $4 \mathrm{Mm}$. long, reddish-brown, and have an agreeable odor aud a pungent, aromatic taste. The seeds are active; the pericarp las no medicinal virtue.

Constituents. -1 , a volatile oil, which is a terpene $\left(\mathrm{C}_{10} \mathrm{H}_{16}\right)$; 2, a fixed oil.

Dose.-Same as that of anise.

A tinctura and tinctura carlamomi compositre are official. They serve as coloring (red) and flavoring agents, and may be employed as vehicles in doses of one to two drachms, in canine practice. The dose of the fluid extract is the same as that of the drug.

\section{Coriandrum. Coriander.}

Synonym.-Coriandri fructus, B.P. ; coriander fruit, E. ; coriandre, Fr.; koriander, G.; fructus coriandri, P.G.

The fruit of Coriandrum sativum Linné (nat. ord. Umbelliferæ).

Habitat.-Southern Europe or Central Asia.

Description. - Globular, about $4 \mathrm{Mm}$. in diameter, brownish-yellow; odor and taste agreeably aromatic.

Constituents. - 1, the volatile oil, oleum coriandri, a colorless, or slightly yellow liquid, having the characteristic odor of coriander, and a warm, spicy taste.

Dose of coriander and its oil, same as for anise and its oil.

\section{Fæniculum. Fennel. (U. S. P.)}

Synonym. - Fœniculi fructus, B.P.; semen fœniculi, fennel fruit or seeds, E. ; semences de fenouil, Fr.; fenchelsamen, G.

The fruit of Fœniculum capillaceum Gilbert (nat. ord. Umbelliferæ).

Habitat.-Southern Europe and Levant. 
Description.-Oblong, nearly cylindrical, from 4 to $8 \mathrm{Mm}$. long; brownish or greenish-brown; odor and taste aromatic, anise-like.

Constituents. - A volatile oil of almost similar action and composition to oil of anise, oleum foniculi. A colorless, or pale yellowish liquid, having the characteristic aromatic odor of fennel, and a sweetish, mild and spicy taste. Soluble in alcohol.

Dose of fennel and its oil, same as that for anise and its oil.

FGnugreer. (Non-official.)

The seeds of Trigonella Fœnum Græcum, cultivated in France and Germany. They are oblong, cylindrical, somewhat compressed, obliquely truncated at each end; 1 to 2 lines long; of a brownish-yellow color, and have a strong, peculiar odor, and oily, bitterish taste. Fœnugreek contains both a volatile and fixed oil.

Dose.-Same as for anise.

ACTIONS AND USES OF CARDAMOM, CORIANDER, FENNEL AND FENUGREEK.

These drugs resemble anise in actions, uses, and doses. They enter into the composition of many popular tonic or. "condition" powders and drinks, and, by their stomachic and carminative properties, aid digestion. Ginger is perhaps. in more frequent demand than other agents of this class, by the profession.

Class 3.-Used Mainly for Their Antispasmodic Action in Stimulating the Nervous System.

VAleriana. Valerian.

Synonym.-Valerianæ rhizoma, B.P.; valeriane, Fr.; baldrianwurzel, G.

The rhizome and roots of Valeriana officinalis Linné (nat. ord. Valerianeæ). 
Habitat.-Europe and Northern Asia. Naturalized in New England.

Description.-Rhizome from 2 to $4 \mathrm{Cm}$. long, and 1 to 2 Cm. thick; upright, subglobular, or obconical; truncate at both ends; brown or yellowish-brown, internally whitish or pale brownish, with a narrow circle of white wood under the thin bark. Roots numerous, slender, brittle, brown, with a thick bark, and slender, ligneous cord. Odor peculiar, becoming stronger and unpleasant on keeping; taste camphoraceous and somewhat bitter.

Constituents.-1, a volatile oil ; 2 , valerianic acid $\left(\mathrm{C}_{5} \mathrm{H}_{10} \mathrm{O}_{2}\right)$, a colorless, oily acid, with burning taste aud odor of valerian. Soluble in alcohol and ether, and in 30 parts of water. Valerianic acid is also made artificially by a complicated process from the distillation of chromic acid and amylic alcohol. 3 , tannic acid ; 4 , resin ; 5 , malic, formic and acetic acids.

Dose.-H. \& C., 3 i.-ii. (30.-60.); D., gr.x.- zi. (6.-4.).

PREPARATIONS.

Extractum Valeriance Fluidum. Fluid Extract of Valerian.

(U. S. P.)

Made by maceration and percolation with water, and evaporation, so that $1 \mathrm{Cc} .=1 \mathrm{Gm}$. of the crude drug.

Dose.-H. \& C., इ i.-ii. (30.-60.); D., $\pi x_{.}-z$ i. (.6-4.).

A tinctura valerianæ $(1-\overline{5})$ and a tinctura valerianæ ammoniata (1-5), prepared with aromatic spirit of ammonia, are also official. The dose of either is 3 ss.-ii. (2.-8.), for dogs.

Ammoni Valerianas. Ammonium. Valerianate.

$$
\mathrm{NH}_{4} \mathrm{C}_{5} \mathrm{H}_{9} \mathrm{O}_{2} \cdot \quad \text { (U. S. P.) }
$$

Made by the action of ammonia gas upon valerianic acid, and crystallization.

Piroperties.-Minute, colorless, cubical crystals, or a white granular powder; without odor when colorless, but emitting a slight odor of iodine when colored, and having a sharp, saline taste. Very hygroscopic. Soluble in 1 part of water, and in 9 parts of alcohol.

Dose.-D., gr.ii.-v. (.12-.3) 
Ferri Valerianas. Ferric Valerianate. (U. S. P.)

Made by precipitating a solution of ferric sulphate with a solution of sodium valerianate, and washing and drying the precipitate.

Properties.-A dark, brick-red, amorphous powder of somewhat rarying chemical composition ; having the odor of valerianic acid and a mildly styptic taste; permanent in dry air. Insoluble in cold water, but readily soluble in alcohol. Dose.-D., gr.i.-iii. (.06-.18).

Zinci Valerianas. Zinc Valerianate. $\mathrm{Zn}\left(\mathrm{C}_{5} \mathrm{H}_{9} \mathrm{O}_{2}\right)_{2}+2 \mathrm{H}_{2} \mathrm{O}$.

$$
\text { (U. S. \& B. P.) }
$$

Made by crystallization from a mixture of hot solutions of zinc sulphate and sodium valerianate.

Properties.-White, pearly scales, having the odor of valerianic acid, and a sweetish, astringent and metallic taste. On exposure to the air it slowly loses valerianic acid. Solnble in 100 parts of water, and in 40 parts of alcohol.

Incompatibility.-Incompatible with acids, metallic salts and soluble carbonates; also vegetable astringents.

Dose.-D., gr.i.-iii. (.06-.18).

Administration.-Valerian should be given in the form of the fluid extract to horses, and this preparation or the tinctures may be exhibited to dogs in dilution. Valerianic acid is not used in medicine except to make valerianates. Of the salts, the zinc valerianate is the most popular, and is administered in pills in canine practice.

ACTION AND USES OF VALERIAN AND VALERIANATES.

The physiological action of valerianic acid and the valerianates is an unknown quantity, but clinical evidence supports their value. The volatile oil in valerian has much the same properties as other volatile oils in stimulating secretion, motion, vascularity and appetite, in relation to the digestive organs; and, in its elimination, the oil excites the 
mucons membranes of the bronchial tubes and genito-uriuary tract. The oil also stimulates the circulation reflexly. Toxic doses of the oil paralyze the brain and cord and depress the circulation; while lethal guantities of ammonium valerianate are said to first excite the spinal motor tract and cause convulsions, and to finally occasion spinal depression and paralysis. Valerian and the valerianates are called antispasmodics in stimulating and strengthening an enfeebled nervous system and thus combating disorders which are created by an increased susceptibility to impulses originating within the brain, or ontside of the body. Valerian is both recommended and used in the treatment of polyuria and diabetes insipidus of the horse; in chorea of dogs resulting from distemper, and occasionally in hysteria, epilepsy, convalescence from acute diseases, and nervous restlessness. Although the drug is of secondary importance, it finds a much larger field of usefulness in human medicine.

Zinc valerianate is more commonly employed in cauine practice for chorea. Ferric valerianate is supposed to combine the tonic and antispasmodic action of the two constituents in one preparation. The oil of valerian is a useful remedy (in emulsion) as a carminative in flatulence. It may be given to horses in doses of 3 ss.-i.; and to dogs in quantities of ๆ ii.-v.

\section{Asafetida. Asafetida. (U. S. \& B. P.)}

Synonym.-Gummi-resina asafotida, ase fétide, asafotida, Fr.; stinkasant, teufelsdreck, G.

A gum-resin obtained from the root of Ferula foetida (Bunge) Regel (nat. ord. Umbelliferæ).

Habitat.-Persia, Afghanistan and Turkestan.

Properties.-In roundish tears, from 2 to $6 \mathrm{Mm}$. or more in diameter; externally pale yellowish-brown, internally milk-white; brittle when cold, and breaking with a flat, conchoidal, and waxy fracture ; or the tears are superficially united into irregular masses without any intervening dark- 
colored substance. It has a peculiar odor, and a bitter, acrid, nauseous taste. When triturated it readily yields a milk-white emulsion.

Constituents. - 1, a volatile oil (3-9 per cent.), containing as its most important ingredient oil of garlic, which gives asafœetida its disagreeable odor ; 2 , gnm, about 25 per cent.; 3 , resin, 50 to 60 per cent., containing ferulaic acid $\left(\mathrm{C}_{10} \mathrm{H}_{10} \mathrm{O}_{4}\right)$.

Dose.-H. \& C., 3 ss.-i. (15.-30.); Sh. \& Sw., 3 i.-ii. (4.-8.); D., gr.iii.-xii. (.18-.8).

Administration.-Asafoetida is given in ball to the larger animals or in an extemporaneous emulsion which is readily made-owing to the gum in the drug-by trituration with water. Asafetida is administered to dogs in pill. The drug may also be injected in aqueous mixture per rectum.

Preparations._Pilulæ asafotida (gr.iii. each); dose-D., 1-4. Tinctura asafoetidie (1-5); dose-H., 3 ii.-iv. (60.-120.); D., Јss.-i (2.-4.). Emulsum (mistura) asafœticlæ, milk of asafetida (1-25); dose-D., 3 ss.-i. (15.-30.).

Action and Uses.-Asafetida is of value by reason of its volatile oil, and therefore possesses much the same action as cther agents of this class. In experiments on man asafetida caused "stomachache," activity of the bowels, increased pulse rate and respiratory movements, headache, dizziness, and sexual desire.

Asafeticla is chiefly used as a carmiuative, stimulating expectorant, and nerve stimulant or antispasmodic. Liquid preparations may canse nausea aud vomiting in dogs owing to the nauseous taste. The drug is of most service in flatulent colic of horses, when it is combined with ammonium carbonate in ball, or is given in this form simultaneously with linseed oil and oil of turpentine.

In atonic constipation of liorses, asafetida is prescribed with aloes in ball. Asafetidi is occasionally employed as a stimulating expectorant in chronic bronchitis, and in the later stages of brocchial catarrh, but it is probably inferior to anmoniacum for this purpose. As an antispasmodic agent, asafetida is useful in functional spasmodic affections, 
including hysteria, chorea and convulsions. The emulsion may be given in enema to dogs, in the two latter disorders.

Finally, tincture of asafetida is recommended to be added to alcoholic liquors in veterinary practice to prevent. their " misappropriation" by stable attendants.

Ammoniacum. Ammoniac. (U.S. \& B. P.)

Synonym.-Gummi-resina ammoniacum, E. ; ammoniaque, gommresine ammoniaque, Fr.; ammoniakgummi, G.

A gum resin obtained from Dorema Ammoniacum Don (nat. ord. Umbelliferæ).

Habitat.-Eastern Persia and Turkestan.

Properties.-In roundish tears, from 2 to $6 \mathrm{Mm}$. or more in diameter; externally pale yellowish-brown, internally milk-white; brittle when cold, and breaking with a flat, conchoidal and waxy fracture; or the tears are superficially united into irregular masses without any intervening darkcolored substance. It has a peculiar odor and a bitter, acrid and nauseous taste. When triturated with water it readily yields a milk-white emulsion.

Constituents.-1, a volatile oil, $1 \frac{1}{2}-4$ per cent. ; 2, a resin, 70 per cent.; 3, a gum, 20-28 per cent.

Dose.-H. \& C., इi.-ii. (30.-60.); Sh. \& Sw., 3 ii.-iv. (8.-15.) ; D., gr.v.-xxx. (.3-2.)

\section{PREPARATIONS.}

Emulsum Ammoniaci. Emulsion (or mixture) of Ammoniac (1-25). (U. S. \& B. P.)

Dose.—D., ऊ̄ ss.-i. (15.-30.)

Emplastrum Ammoniaci cum Hydrargyro. Ammoniac Plaster with Mercury. (U. S. \& B. P.)

Administration.-In emulsion, ball or pill. ACTION AND USES.

Ammoniac is a drug of minor importance, resembling asafoetida and containing a small quantity of a volatile oil having the same action as other oils of this class. Am- 
moniac is cccasionally employed externally as a mild counterirritant, in plasters. Since its rolatile oil is eliminated by the bronchial mucons membrane, ammoniac is given internally as a stimulating and slightly disinfecting expectorant in chronic bronchitis with or withont excessive secretion.

\section{Class 4.-Used Mainly for their Stimulant and Diuretic Actions on the Kidneys and Genito- Urinary Tract.}

Buchu. Buchu. (U. S.P.)

Synonym.-Buchu folia, B.P.; feuilles de bucco, Fr.; buckublätter, buccoblätter, $G$.

The leaves of Barosma betulina (Thunberg) Bartling et Wendland and Barosma Crenulata.(Linné) Hooker (nat. ord. Rutaceæ).

Habitat.-South Africa.

Description.-About $15 \mathrm{Mm}$. long, roundish obovate, with at rather wedge-shaped base, or varying between oval and obovate, obtuse, crenate or serrate, with a gland at the base of each tooth; dull yellowish-green; thickish, pellucidpunctate; odor and taste strongly aromatic, somewhat mintlike, pungent and bitterish.

Constituents. - 1, a volatile oil having an odor somewhat like peppermint, $1_{2}^{1}$ per cent.; 2 , a stearopten (Buchu camphor or diosphenol, $\mathrm{C}_{10} \mathrm{H}_{16} \mathrm{O}_{2}$ ), possessing an odor like peppermint and in solution in a liquid hydrocarbon, but crystallizing on exposure to the air; 3 , barosmin, a glucoside, soluble in ether, volatile oils, diluted acids and alkalies ; 4, gum ; 5 , rutin, a bitter substance.

Dose.-H. \& C., 亏 i.-ii. (30.-60.); D., gr. xv.-xxx. (1.-2.).

\section{PREPARATIONS.}

Extractum Buchu Fluidum. Fluid Extract of Buchu. (U. S. P.)

Made by maceration, percolation and evaporation, so that $1 \mathrm{Cc}$. $1 \mathrm{Gm}$. of Buchu.

Dose. -H. \& C., 亏 i.-ii. (30.-60.); D., $\Pi \times \times v_{0}-\mathbf{x x x} \cdot(1 .-2$. 
An infusion (1-20) by steeping leares in boiling water for half an hour in a closed ressel, is sometimes preferred, and will be taken voluntarily by the larger animals in linseed tea.

Tinctura Buchu. Tincture- of Buchu. (B. P.)

Dose.-Twice that of the fluid extract.

\section{ACTION AND CSES.}

The volatile oil and bitter principle act upon the digestive organs as an aromatic bitter, promoting appetite and digestion in small doses, while large doses cause nausea and vomiting in dogs. The volatile oil is absorbed and eliminated by the mucous membranes, particularly of the bronchial tubes and genito-urinary tract. It thus stimnlates and disinfects the mucous membranes, slightly increases the secretion of urine, and imparts its peculiar odor to the latter.

The drug is of considerable value in the treatment of chronic or subacute pyelitis, cystitis and urethritis. It is stimulating, but only slightly irritating. Buchu has been recommended in chronic nephritis, and is useful iu irritation. of the urinary bladder, with frequent micturition, combined with spirit of nitrous ether. Buchu is occasionally prescribed in the later stages of bronchitis or in the chronic form of this disease, and is employed in its native country as a remedy for chronic diarrhoea and dysentery.

\section{Oleum Juniperi. Oil of Juniper. (U. S. \& B. P.)}

Synonym.-Oleum fructus (Vel Baccæ) juniperi, oil of juniper berries, E.; essence de genièvre, Fr.; wachholderbeeröl, G.

A volatile oil distilled from the fruit of Juniperus Communis Linné (nat. ord. Coniferæ).

Habitat.-Canada and United States; Rocky Mountains, south to New Mexico.

Properties. - A colorless, or faintly greenish-yellow liquid, becoming darker and thicker by age and exposure to air; having the characteristic odor of juniper, and a warm, 
aromatic, somewhat terebinthinate and bitterish taste. Spec. gr. 0.850 to 0.890 . Soluble in abont four times its volume of alcohol.

Composition.-Oil of juniper is a terpene $\left(\mathrm{C}_{10} \mathrm{H}_{16}\right)$, and is isomeric with oil of turpentine.

Dose.-H. \& C., 3 i.-ii. (4.-8.); D., Mii.-x. (.12-.6).

PREPARATIONS.

Spiritus Juniperi. Spirit of Juniper. (U. S. \& B. P.)

Oil of Juniper, 50 ; alcohol, 950. (U. S. P.)

Dose.-H. \& C., 亏3i.-ii. (30.-60.); D., 3 ss.-i. (2.-4.).

Spiritus Juniperi Compositus. Compound Spirit of Juniper.

Oil of juniper, 8 ; oil of caraway, 1 ; oil of fennel, 1 ; alcohol, 1400 ; water to make 2000 .

Dose.-H. \& C., 亏3 ii.-iv. (60.-120.) ; D., 3 i.-iv. (4.-15.).

\section{ACTIONS AND USES.}

Oil of juniper resembles oil of turpentine physiologically as well as chemically. It is a stomachic and carminative, particularly when combined with alcohol and other aromatic oils (Sp'r. Juniper. Co.), but is used in medicine chiefly for its stimulant and diuretic action upon the kidneys and genitourinary tract during its elimination. Oil of juniper is capable of irritating the kidneys in large doses, and causing congestion, strangury, and even suppression of urine. It is less likely, however, to disturb digestion than oil of turpentine, and does not so readily occasion hæmaturia and albuminuria. Oil of juniper is indicated in chronic nephritis, pyelitis and cystitis ; also in dropsy of cardiac, renal, or hep. atic origin. It is efficient in assisting absorption of effusions into serous cavities, through its diuretic properties. The compound spirit of juniper approximates gin in composition, although it is not the official name for that liquor. This preparation is useful in the convalescent period of acute bronchitis and influenza, stimulating the bronchial mucous membrane by virtue of the volatile oil, and acting as a circulatory stimulant and diuretic. The oil of juniper is an 
efficient renal stimulant in passive congestion of the kidneys, aud following the active stage of acute nephritis.

Juniper berries are sometimes given to the larger

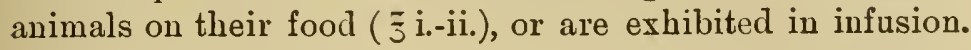

\section{Class 5.-Used Mainly for its Emmenagogue Action on the Female Generative Organs.}

\section{Sabina. Savine.}

Synonym. - Sabinæ cacumina, B.P.; savin tops, E.; sabine, Fr.; sadebaumspitzen, sevenkraut, G.; summitates (herba) sabinæ, P.G.

The flowering tops of Juniperus Sabina Linné (nat. ord. Coniferæ).

Habitat.-Canada, Northern United States, Europe and Siberia.

Description.-Short, thin, sub-quadrangular branchlets; leaves rather dark green, in four rows, opposite, scale-like, ovate-lanceolate, more or less acute, appressed, imbricated on the back with a shallow groove containing an oblong or roundish gland; odor peculiar, terebinthinate; taste nansenus, resinous and bitter. The chief constituent is the volatile oil, about 2 per cent.

Dose.-H., 亏̈i.-ii. (30.-60.) ; D., gr.v.-xv. (.3-1.).

\section{PREPARATION.}

Extractum Sabince Fluidum. Fluid Extract of Savine. (U. S. P.)

Made by maceration and percolation with alcohol, and evaporation, so that $1 \mathrm{Cc} .=1 \mathrm{Gm}$. of the crude drug.

Dose.-H. \& C., ₹ i.-ii. (30.-60.) ; D., mv.-xv. (.3-1.).

\section{Oleum Sabine. Oil of Savine. (U. S. \& B. P.)}

Synonym.-Essence de sabine, Fr.; sadebaumöl, G.

A volatile oil distilled from savine.

Properties.-A colorless, yellowish liquid, having a peculiar terebinthinate odor, and a pungent, bitterish and 
camphoraceons taste. It becomes darker and thicker by age and exposure to the air. Spec. gr. 0.910-0.940. Soluble in an equal volume of alcohol and glacial acetic acid. It is composed of several terpenes.

Dose. -H. \& C., 3 ii.-iv. (8.-15.); D., Mi.-v. (.06-.3).

Administration.-The oil is given in capsules or pills to small animals; in emulsion with gum, or in bland oil, to the larger animals.

Action External.-The oil is a powerful irritant to the skin, producing redness, vesication and even pustulation.

Action Internal. - The oil resembles oil of turpentine, but is more irritating. Full doses canse gastric stimulation, reflex circulatory excitement, and frequent micturition. Toxic quantities occasion gastro-enteritis with vomiting (in dogs), purging, colic, painful micturition, and the passage of bloody, albuminous urine. There are also unconsciousness, stertor, rapid breathing and pulse, convulsions and collapse. Lesions of gastro-enteritis are observable after death, except. in rare cases, when only congestion of the brain and lungs ocenr. The oil is eliminated by the skin and bronchial mucous membrane, but chiefly by the kidneys, with consecquent stimulation of the genito-urinary organs. The uterus. and ovaries are irritated and congestion of them follows with acceleration of ovulation. The oil also excites uterine contractions in the pregnant state. The drug is therefore an emmenagogne and ecbolic.

Uses.-The Unguentum (B.P.) may be applied externally as a counter-irritant. The oil is occasionally employed as an anthelmintic, but is inferior to other agents for this. purpose. It may be given in atonic ammenorrhoa, or in metrorrhagia dne to uterine relaxation, with benefit, but it shonld be need cautionsly. The oil is not to be used us an abortifacient, since sufficient doses to cause abortion will usually endanger the life of the mother or fœtus, both. 


\section{Tallianine. (Non-official.)}

Derivation.-Tallianine is said to be the result of the action of ozone upon a terpin-bearing volatile oil, the action being arrested at a point when the latter has absorbed a quantity equal to four volumes of ozone. It is put on the market in sealed glass tubes containing each 10 c.c.

Properties.-A clear, colorless, slightly syrupy liquid, having a strong odor of terpene and a taste both terebinthinate and saline.

Dose.-(Intravenously) H. \& C., 3 iis.-v. (10-20 c.c.); Sh. \& Sw., $M 75-3$ iiss. (5-10 c.c.); D., $M 330-75$ (2-5 c.c.); Cats, m15-30 (1-2 c.c.).

Actions and Uses.-External.-It has empirically given the most favorable clinical results, according to recent reports, in the moist and pustular forms of eczema when applied once daily upon the skin in the pure state in conjunction with the intravenous administration of 2 c.c. also once daily, in the treatment of dogs. The skin shonld first be well cleansed of dirt and sebaceous matter, with soap, warm water and a scrubbing brush. There is no sort of local reaction or disturbance when the drug is injected intravenously.

Internal.-Tallianine appears to be non-toxic in any reasonable amounts; 300 to 400 c.c. produce no untoward effects when given intravenously to horses. The most pronounced action of tallianine is exerted apparently upon the blood. In ordinary doses it produces in the horse a leucocytosis amounting to two or three times the normal and within the space of two or three hours. The effect is the same in large and small animals, although more rapid in the latter. This action is maintained for from five to twenty hours. The action of the heart seems to be moderately stimulated, and also that of the kidneys, with accompanying diuresis. The physiological details of these latter actious 
have not been worked out satisfactorily. The appetite is also reported as being wonderfully improved under the influence of the drug-whether directly or indirectly has not been made clear. Tallianine is a French proprietary preparation, and as such its chemistry and mode of manufacture have been withheld from the profession. Neither has a thoroughly scientific study been made to determine accurately its exact physiological action. The clinical reports in this country and abroad have been so exceptionally favorable that it was deemed proper to include the drug in this work, notwithstanding our disinclination to include proprietary preparations. Whether tallianine acts by virtue of its ozonizing properties or by stimulating the phagocytic action of the white blood cells in combating the micro-organisms of disease or their toxins, or whether it is purely due to altered metabolic activity, has yet to be determined; but clinically it has proven of great service in the following diseases when given intravenously once (or in very severe cases twice) daily, notably in pneumonia of all types, but also in pleurisy, pulmonary congestion, bronchitis, influenza, purpura hemorrhagica, heat apoplexy and prostration, anasarca, tetanus and diphtheria. General improvement with corresponding betterment in the local pathological conditions have been generally noted. Other medication should be withheld. The types of disease benefited by tallianine are so diverse that it is impossible to formulate a logical theory as to its mode of action. We have only the empirical, clinical results to base our remarks upon at present, but these thus far give promise that we have in tallianine a substantial addition to our pharmacopeia. 


\section{So-called Solid Volatile Oils or Stearoptens.}

Camphora. Camphor. $\mathrm{C}_{10} \mathrm{H}_{16} \mathrm{O}$. (U. S. \& B. P.)

Synonym.-Gum camphor, laurel camphor, E.; camphre, Fr.; kampfer, G.

A stearopten (having the nature of a ketone) obtained from Cinnamomum Camphora (Linné) Nees et Ebermaier (uat. ord. Laurineæ), and purified by sublimation.

Habitat. - China, Japan, Cochin China and Sunda Islands.

Properties.-White, translucent masses, of a tough consistence and a crystalline structure, readily pulverizable in the presence of a little alcohol, ether, or chloroform ; having a penetrating, characteristic odor, and a pungently aromatic taste. Spec. gr. 0.995. Very sparingly soluble in water, but readily soluble in alcohol, ether, chloroform, carbon disulphide, benzin, and in fixed and volatile oils, and milk. When camphor is triturated, in about molecular proportions, with menthol, thymol, phenol, or chloral hydrate, liquefaction ensues. On exposure to the air it evaporates, and when moderately heated, it sublimes without leaving a residue.

Composition.- "A stearopten is a solid crystalline substance separated from any volatile oil on long standing or at low temperature." Camphor is a stearopten and is chemically an oxidation product of a terpene,- the principal constituent of all volatile oils. A terpene is a hydrocarbon containing 10 atoms of carbon, and tlie terpene $\left(\mathrm{C}_{10} \mathrm{H}_{16}\right)$ from which camphor is derived is isomeric with that of oil of turpentine and many other volatile oils.

Dose.-H., 3 i.-iii. (4.-12.) ; C., 3 ii.-iv. (8.-15.); Sh. \& Sw., gr.xv.-3 i. (1.-4.) ; D., gr.iii.-xx. (.18-1.3).

\section{PREPARATIONS.}

Aqua Camphorce. Camphor Water. (U. S. \& B. P.)

Triturate camphor, 8 , with alcohol, 5 , and precipitated calcium phosphate, 5 ; then with water to make 1000 . Filter. (U. S. P.)

Dose.-Ad, lib. 
Spiritus Camphorce. Spirit of Camphor. (U. S. \& B. P.)

Dissolve camphor, 100, in alcohol, 800 ; filter, and add alcohol to make 1000 . (U. S.)

Dose.-H. \& C., § i.-ii. (30.-60.) ; D., 3 ss.-i. (2.-4.).

Linimentum Camphorce. Camphor Liniment. (U. S. \& B. P.)

Synonym.-Camphorated oil.

Camphor, 200 ; cottonseed oil, 800 . (U. S. P.)

Ceratum Camphorce. Camphor Cerate. (U. S. P.)

Camphor liniment, 100 ; white wax, 300 ; lard, 600.

Camphora Monobromata. Monobromated Camphor. $\mathrm{C}_{10} \mathrm{H}_{15} \mathrm{BrO}$.

(U. S. P.)

Derivation.-Made by heating camphor and bromine together at a temperature of $172^{\circ} \mathrm{F}$. $\left(77.7^{\circ} \mathrm{C}\right.$.) and solution in benzin. $\mathrm{C}_{10} \mathrm{H}_{16} \mathrm{O}+2$ $\mathrm{Br}=\mathrm{C}_{10} \mathrm{H}_{15} \mathrm{Br} \mathrm{O}+\mathrm{H} \mathrm{Br}$. Recrystallized from hot alcohol.

Properties.-Colorless, prismatic needles or scales, having a mild, camphoraceous odor and taste; permanent in the air, unaffected by light, and neutral to litmus paper. Almost insoluble in water; freely soluble in alcohol, ether, and chloroform, hot benzin and fixed and volatile oils; slightly soluble in gly cerin.

Dose.-D., gr.ii.-x. (.12-.6).

Action External.-Camphor resembles the volatile oils chemically and physiologically. It is a slight antiseptic externally, and parasiticide. The vapor of camphor kills moths, fleas, bugs, etc. Camphor is a mild irritant, producing a rubefacient action followed by partial anæsthesia. It is eliminated in part by the skin and occasions some diarphoresis.

Action Internal.-Alimentary Canal.-Camphor stimulates the stomach, increasing the secretion, motion and vascularity of the organ. In the bowels camphor is supposed to overcome pain, spasm, and check secretion in diarrhoea, but has little effect in normal conditions and in therapentic doses.

Circulation.-The heart is stimulated by camphor, and the pulse is increased in force and frequency by medicinal doses. The drug acts in part directly and in part reflexly 
from irritation of the stomach. Poisonous quantities of camphor depress the heart and the pulse becomes feeble and rapid. Leucocytosis is favored by camphor.

Respiration.-Camphor, like volatile oils, stimulates the bronchial mucons nembranes in its elimination by the lungs, and increases the blook supply and secretion of these parts. The characteristic odor is imparted to the breath after the ingestion of camphor. The drug is believed to relieve spasm and congh in bronchitis.

Nervous System.-Camphor is aften classed as an antispasmodic. It stimulates the nerve centres in the brain, medulla, and spinal cord, and thus orercomes spasm due to nervous weakness and incoördination. Poisonous doses depress and paralyze the higher nervous centres.

Kidneys and Sexual Organs.-Camphor is broken up in the body and eliminated in the breath and sweat, but mainly in the urine, as campho-glycuric acid. The drug influences the sexual organs, in some cases, but in most instances does not affect them. Full medicinal doses sometimes stimulate the sexual functions (aphrodisiac action). Very large doses are said to depress sexual desire (anaphrodisiac action), but these quantities may irritate the genito-urinary tract and produce erotic excitement.

Temperature.-Camphor is a slight antipyretic.

Toxicology.-Two to four ounces of camphor given to horses or cattle induce convulsions, with rapid pulse and breathing, but usually recovery ensues. Two to four Trachms cause, in dogs, vomiting, unsteady movements, asphyxia, coma and death.

Administration.-Camphor is exhibited internally in the form of the spirit, in pill or ball; and in solution in oil or milk.

Uses External.-Camphor is applied in powder as a stimulant and antiseptic on indolent sores; mixed with chalk or zinc oxide, as a dusting powder, in ehafing or erythema, for its anæsthetic properties. It is employed in liniments (Lin..Saponis, Lin. Camphoræ), in strains, bruises, 
rheumatism and myalgia, as a rubefacient and local anodyne.

Uses Internal.-Camphor is a valuable nerve and circulatory stimulant in collapse, heart failure, and poisoning by alcohol, opium, belladonna, etc. The Germans praise it lighly for this purpose, butit is not so frequently employed by English-speaking practitioners. It should be given subcutaneously in solution in ether ( 1 to 5 or 10$)$, or in olive oil ( 1 to 5 or 10), in doses of 2 to 3 grains for dogs; 15 to 30 grains for horses, hourly.

B

Camphoræ........................

Etheris........................ 3 ss.

Ol. Olivæ...................... 3 ii.

M.

S. Inject whole, for a horse ; 15 to 30 drops for a dog.

Camphor is of benefit in exhausting acute diseases (influenza and canine distemper), for the same reason and because it possesses diarphoretic and antipyretic properties. It may be combined with alcohol, spirit of nitrous ether, and ammonia compounds, in these affections.

Respiratory disorders are improved by camphor, since it is an expectorant, diarphoretic, stimulant and antiseptic. It is prescribed in spasmodic congh, bronchitis and pharyngitis. For the latter, in electuary with belladonna.

Camphor is a valuable drug in diarrhœa, particularly in the serons variety and in that form following exposure to cold. It is not useful in inflammatory conditions, but checks secretion and pain. Camphor is prescribed alone in diarrhœa, or with brandy and laudanum.

Camphor is sometimes given as an antispasmodic in hysteria and "thumps" (spasm of diaphragm) of horses; and in nervons palpitation of the heart, and chorea (monobromated camphor) of dogs.

Spirit of camplor and nitrous ether are efficient in relieving irritation of the genito-urinary tract. 
Thyмог. Thymol. $\mathrm{C}_{10} \mathrm{H}_{14}$ O. (U. S. \& B. P.)

A phenol (or stearopten, B.P.) occurring in the volatile oils of Thymus Vulgaris Linné, Monarda punctata Limné (nat. ord. Labiatæ), and Carum Ajowan (Roxburgh) Bentham et Hooker (nat. ord. Umbelliferæ).

Habitat. - Thymus vulgaris, Southern Europe, cultivated. Monarda punctata, United States, west to Colorado and Texas. Carum Ajowan, India, Egypt and Persia.

Derivation.-Thymol is made from the terpenes of the three volatile oils mentioned above, by fractional distillation, by saponifying the result with caustic soda to remove more terpenes, aud by cooling. The resulting soap, or soda-thymol compound, is decomposed with hydrochloric acid, and thymol is crystallized from an alcoholic solntion.

Properties.-Large, colorless, translucent crystals of the hexagonal system, having an aromatic, thyme-like odor, and a pungent, aromatic taste, with a very slight caustic effect upon the lips. Its specific gravity, as a solid, is 1.069, but when liquefied by fusion it is lighter than water. It melts at $50^{\circ}$ to $51^{\circ} \mathrm{C}$. $\left(122^{\circ}\right.$ to $123.8^{\circ} \mathrm{F}$.), remaining liquid at considerably lower temperatures. When triturated with about equal quantities of camphor, menthol, or chloral, it liquefies.

Soluble in about 1200 parts of water, and in less than its own weight of alcohol, ether or chloroform; also readily soluble in carbon disulphide, glacial acetic acid, and in fixed or volatile oils.

Dose.-H., 3 ss.-ii, (2.-8.) ; D., gr.i.-xv. (.06-1.).

Action and Uses.-Thymol resembles carbolic acid chemically and physiologically. It is less poisonons and irritant, more costly, and possesses greater antiseptic powers. It is much less valuable, however, medicinally, on account of its expense, and odor which strongly attracts flies.

Poisoning is not produced readily, as absorption from: the digestive tract is slow; but after considerable doses by the mouth, or when injected into the blood, toxic symptoms occur. One drachm given intravenously to a dog caused: 
prostration, coma and respiratory failure. Recovery ensued after the use of artificial respiration. Often no lesions are discoverable after death. At other times there is hyperæmia of the lungs and kidneys cansed by elimination of the drug. The urine is colored greenish or yellowish-brown by trausmitted light.

Thymol is used externally for general autiseptic purposes, for application to ulcers, and as an injection in cystitis in aqueous saturated solution. It is employed in ointment with vaseline (1-15) to destroy ringworm and to relieve itching in pruritus, eczema, lichen, psoriasis, etc. It may be applied as follows for the same purposes:

$\mathrm{P} x$

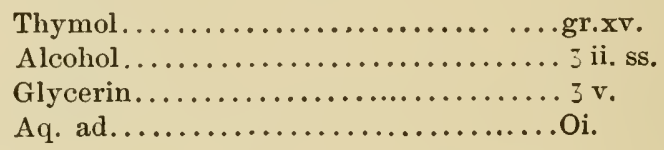

M.

An efficient antiseptic mouth wash consists of borax, gr. 40 ; thymol, gr. 20 ; water, 3 iv. It is inclicated in stomatitis. Thymol is of little worth for jnterual use. It is recommended as an intestinal antiseptic; as a remedy for tape worm, and as a urinary antiseptic in cystitis. It is given in diluted alcoholic solutions; better in oil or capsules.

\section{SECTION X.-VEGETABLE BITTERS.}

\section{Gentiana. Gentian. (U. S. P.)}

Synonym.-Gentianæ radix, B.P. ; radix gentianæ rubræ (vel lutæ vel majoris), gentian root, E.; radix gentianæ, P.G.; racine de gentiane (de gentiane janne), Fr.; enzianwurzel, bitterwurzel, rother (gelber) enzian, $G$.

The root of Gentiana lutea Linné (nat. ord. Gentianeæ).

Habitat.-The yellow gentian is indigenous in the Alps and mountains of southern and central Europe. 
Description.-In nearly cylindrical pieces or longitudinal slices, abont $25 \mathrm{Mm}$. thick; the upper portion closely anmulate; the lower portion longitudinally wrinklecl ; externally deep yellowish-brown; internally lighter; somewhat flexible and tough when damp; rather brittle when dry; fracture nneven; the bark rather thick, separated from the somewhat spongy meditnllium by a black cambium line; odor peculiar, faint, more prominent when moistened; taste sweetish and persistently bitter.

Constituents.-The chief one is gentiopicrin, a bitter crystalline.glucoside, soluble in alcohol and water. There is also gentisic acid $\left(\mathrm{C}_{14} \mathrm{H}_{10} \mathrm{O}_{5}\right)$, combined with gentiopicrin, sugar, gum, and a trace of volatile oil. Contains no tannin.

Incompatibles.-Iron in solution forms a black compound with the coloring matter in gentian. Silver nitrate and lead salts are incompatible with gentian.

Dose.-H., 3 ss.-i. (15.-30.); C., 3 i.-ii. (30.-60.); Sh. \& Sw., 3 i.-ii. (4.-8.) ; D., gr.v.-xxx. (.3-2.).

\section{PREPARATIONS.}

Extractum Gentiance. Extract of Gentian. (U. S. \& B. P.)

Made by maceration and percolation with water, and evaporation to a pilular consistence.

Dose.-A bout one-third that of gentian.

Extractum Gentiance Fluidum. Fluid Extract of Gentian. (U. S. P.)

Made by maceration and percolation with diluted alcohol, and evaporation, so that $1 \mathrm{Cc}$. $=1 \mathrm{Gm}$. of the crude drug.

Dose.-Same as gentian.

Tinctura Gentiance Composita. Compound Tincture of Gentian.

(U. S. \& B. P.)

Gentian, 100 ; bitter orange peel, 40 ; cardamon, 10 ; made by maceration and percolation with alcohol and water, to 1000. (U.S. P.)

Dose.-H. \& C., 亏 3 i.-iv. (30.-120.); D., 3 i.-iv. (4.-15.).

Administration.-Gentian is usually given to horses, cattle and sheep in powder, or to the former in the form of the compound tincture. The extract is suitable for dogs 
when exhibited in pills. Gentian is often employed as an excipient in the preparation of balls.

Action.-The simple bitters, as gentian, act as stomachics and bitter tonics. They are stomachics in promoting gastric digestion by stimulation of the gustatory nerves, thus improving the appetite and reflexly cuusing dilatation of the blood vessels in the stomach and increasing salivary and gastric secretions. Furthermore, the bitters excite gastric and intestinal peristalsis to a slight extent. The bitters only act as tonics by their local effect in facilitating the digestion and assimilation, and by increasing the appetite. Externally the bitters are mildly antiseptic; while internally they are inimical to intestinal parasites.

Uses.-Gentian is serviceable in simple loss of appetite. It is especially indicated in feeble gastric digestion caused by acute disease, overwork, insufficient and poor food, and in that form associated with general debility and anæmia. In the latter state, characterized by a pasty tongue, anorexia, rough coat and pallid mucous membranes, which may often be co-existent with the presence of intestinal worms, powdered gentian is most efficient when given to horses on the food three times daily with dried ferrous sulplate.

Again, loss of appetite, general weakness, and feeble digestion occurring in horses during convalescence from acute diseases, as influenza and pneumonia, is favorably met by a combination of compound tincture of gentian and whisky ( 1 ounce each), or by diluted hydrochloric acid and the compound tincture. The drug is useful in atonic indigestion, or mild chronic gastric or intestinal catarrh of young animals, when conjoined with sodium bicarbonate, which acts as a sedative and solvent of mucus.

The simple bitters, including gentian, are contra-indicated in any acute inflammation of the digestive tract, since they are mild irritants. Gentian is a valuable bitter for cattle and sheep, but quinine is more commonly given to dogs. 


\section{QUASSIA. Quassia.}

Synonym.-Quassiæ lignum, B.P. ; quassia wood, bitter wood, bitter ash, E. ; quassie, bois amer, Fr.; quaissienholz, G.

The wood of Picrœena Excelsa (Swartz) Lindley (nat. ord. Simarubeæ).

Habitat.-Jamaica and West Indies.

Description.-In billets of various sizes, dense, tough, of medium hardness, porous, with a minute pith and narrow medullary rays ; inodorous and intensely bitter. In the shops it is usually met with in the form of chips or raspings of a yellowish-white color.

Constituents.-Chiefly, quassiin $\left(\mathrm{C}_{10} \mathrm{H}_{12} \mathrm{O}_{5}\right)$, a bitter, neutral principle occurring in crystalline rectangular plates. There is also a volatile oil, but no tannin.

Dose.-Quassiin, D., gr. $\frac{1}{8}-\frac{1}{3}$ (.008-.02).

\section{PREPARATIONS.}

Extractum Quassire. Extract of Quassia. (U. S. \& B. P.)

Made by percolation with water, boiling and evaporation to pilular consistence.

Dose.-H., zi.-ii. (4.-8.) ; D., gr.ss.-iii. (.03-.18).

Extractum Quassice Fluidum. Fluid Extract of Quassia. (U. S. P.)

Made by maceration and percolation with alcohol and water, and evaporation, so that $1 \mathrm{Cc} .=1 \mathrm{Gm}$. of quassia.

Dose.-H. \& C., 亏i.-ii. (30.-60.); Sh. \& Sw., 3 ii.-iv. (8.-15.); D., $\eta \times x v,-3$ i. (1.4.).

Tinctura Quassice. Tincture of Quassia. (U. S. \& B. P.)

Made by maceration and percolation of quassia, 100 ; with alcohol and water to make 1000 . (U.S. P.)

Dose.-Twice that of fluid extract

Administration.-Quassia may be given to horses in the official preparations, - preferably the fluid extract,-or in infusion (1-80, in cold water for half an hour, B.P.). The, dose of the infusion is $\bar{j} \mathrm{iv}$. for horses; 3 ii.-iv. for dogs.

Actions.-Quassia is the most active and bitter stomachic we possess. Large doses irritate the digestive tract. The. 
drug is poisonous to the lower forms of animal life. One grain will kill a frog with the production of convulsions and respiratory and heart failure. A sweetened infusion is often employed to destroy flies. Considerable doses of quassia increase the secretion of bile and urine, and stimulate peristaltic action and eontraction of the minary bladder. It is an antiseptic and prevents fermentation in the digestive canal. Quassia acts generally in the same manner as gentian, by sharpening tho appetite, and increasing salivary and gastric secretions, together with vascularity and peristalsis of the stomach. The volatile oil assists the stomachic action.

Uses.-Quassia, like gentiau, is very serviceable in promoting appetite and digestion in atonic dyspepsia. It has this advantage, however, that it may be combined with liquid preparations of iron without incompatibility. Quassia is the most efficient vermicide in our possession for the destruction of Oxyuris curvula, horse; and O. vermicularis, dog, in the lower bowel. An infusion is employed for this purpose, made by soaking quassia chips in cold water (3 ii.-Oi.) for half an hour. The rectum should be first thoroughly washed out with soap and water, and one-half pint of this infusion is given in enema to dogs; two quarts to horses.

\section{Cascarilla. Cascarilla. (U. S. P.)}

Synonym.-Cascarillæ cortex, B.P.; cascarille, Fr.; cascarilla, kascarillrinde, G.

The bark of Croton Eluteria Bennett (nat. ord. Euphorbiaceæ).

Habitat.-Bahama Islands.

Description.-In quills or curved pieces about $2 \mathrm{Mm}$. thick, having a grayish, somewhat fissured, easily detached, corky layer, more or less coated with a white lichen, the uncoated surface being dull brown, and the inner surface smooth. It breaks with a short fracture, having a resinous and radially striate appearance. When burned, it emits a 
strong, aromatic, somewhat musk-like odor; its taste is war'm and very bitter.

Constituents.-1, cascarillin, a neutral, bitter, crystalline body; 2, two resins, 15 per cent.; 3 , a volatile oil, 1.5 per cent.; 4 , tannic acid ; 5 , gum.

Incompatibles.-Metallic salts, mineral acids, and lime water.

Dose.-H. \& C., ङ ss.-i. (15.-30.) ; Sh. \& Sw., 3 i.-ii. (4.-8.); D., gr.x.-xxx. (.6-2.).

Administration.-Cascarilla may be given in powder to the larger animals on their food, or in infusion (1-10, B.P.), or tincture $(1-8$, B.P.), 3 iv.-vi. of either for horses ; 3 ss.-i. of the tincture for dogs. The infusion does not keep.

Action and Uses.-Cascarilla is called an aromatic bitter, since it combines the action of a volatile oil in stimulating gastro-intestinal secretion, motion, and vascularity, with that of the bitters in exciting the appetite, gastric and salivary secretions, together with vascularity and peristalsis of the stomach. The drug is suitable for the same cases as gentian, but is particularly indicated in the treatment of atonic gastro-intestinal indigestion with flatulence (on account of its volatile oil). Mineral acids precipitate the resins in tinctures, so that the infusion may be combined to better advantage with acids.

\section{Calumba. Calumba. (U. S. P.)}

Synonym.-Calumbæ radix, B.P.; columbo, E.; columbo, Fr.; kolumbowurzel, G.

The root of Jateorhiza palmata (Lamarck) Miers (nat. ord. Menispermaceæ).

Habitat.-Mozambique, East Africa. Cultivated in the East Indies.

Description.-In nearly circular disks, 3 to $6 \mathrm{Cm}$. in diameter, externally greenish-brown and wrinkled, internally yellowish or grayish-yellow; depressed in the centre, with a few interrupted circles of projecting wood bundles, dis- 
tinctly radiate in the outer portion; fracture short, mealy; odor slight; taste mucilaginous; slightly aromatic, very bitter.

Constituents. -1 , calumbin $\left(\mathrm{C}_{21} \mathrm{H}_{22} \mathrm{O}_{7}\right)$, a neutral, bitter, crystalline substance; 2 , an alkaloid, berberine $\left(\mathrm{C}_{20} \mathrm{H}_{17} \mathrm{NO}_{4}\right)$, found in berberis, hydrastis, etc. ; 3 , calumbic acid $\left(\mathrm{C}_{22} \mathrm{H}_{22} \mathrm{O}_{6}\right)$; 4, starch, 33 per cent.

Dose.-H. \& C., 3 ss.-i. (30.-60.) ; Sh. \& Sw., 3 i.-ii. (4.-8.); D., gr.v.-xxx. (.3-2.).

PREPARATIONS.

Extractum Calumber Fluidum. Fluid Extract of Calumba. (U. S. P.)

Made by maceration and percolation with alcohol and water, and evaporation, so that $1 \mathrm{Cc} .=1 \mathrm{Gm}$. of the crude drug. (U. S. P.)

Dose.-Same as Calumba.

Tinctura Calumbce. Tincture of Calumba. (U. S. \& B. P.)

Made by maceration and percolation of calumba, 100 ; in alcohol, - and water to make 1000 . (U. S. P.)

Dose.-H. \& C., z 3 ii.-iv. (60.-120.); D., 3 i.-iv. (4.-15.). Dose of tincture (B. P.) half that of U. S. P. tincture.

Administration.-Calumba is given in powder on the food, or in the official preparations to the larger animals. The infusion (1-16, B.P.) may be used in the same doses as that of cascarilla. The tincture, and extract (gr.ii.-x., B.P.) are the best preparations for dogs.

Actions and Uses.-Calumba is a mild but pure bitter. Berberine, calumbin and calumbic acid are all bitter, but none of them possess any powerful physiological action. Calumba is indicated in the same cases as gentian, but, being free from tannin, may be combined with iron preparations withont producing an unsightly, inky mixture. It is less irritating than other bitters, and may be prescribed in more irritable conditions of the stomach. Calumba is frequently

- used during convalescence from the acute diseases and diarrhœa. 


\section{Taraxacum. Taraxacum. (U. S. P.)}

Synonym.-Taraxici radix, B.P.; dandelion, E.; pissenlit, dent de lion, Fr.; löwenzahn, G.

The root of Taraxacum officinale Weber (nat. ord. Compositæ), gathered in autumn.

Habitat.-Naturalized in the United States and growing commonly in waste places. Indigenous in Europe.

Description.-Slightly conical, about $30 \mathrm{Cm}$. long, and 1 or $2 \mathrm{Cm}$. thick above, crowned with several short, thickish heads, somewhat brancled, dark brown, - lougitudinally wrinkled, when dry breaking with a short fracture, showing a yellowish, porous central axis, surrounded by a thick, white bark, containing numerous milk vessels arranged in concentric circles; inodorous; bitter. It should be free from the root of Cichorium Intybus Linné (nat. ord. Compositæ), which closely resembles it, but is usually paler, and has the milk-vessels in radiating lines.

Constituents.-1, taraxacin, a bitter, soluble, crystalline substance ; 2 , inulin ; 3 , taraxacerin $\left(\mathrm{C}_{8} \mathrm{H}_{16} \mathrm{O}\right)$; 4 , resin, causing the milky juice.

Dose.-H., 亏 i.-ii. (30.-60.); Sh. \& Sw., 3 ii.-iv. (8.-15.); D., 3 i.-ii. (4.-8.).

\section{PREPARATIONS.}

Extractum Taraxaci. Extract of Taraxacum. (U. S. \& B. P.)

Made by bruising and expressing the juice from the fresh roots gathered in autumn. The juice is strained and evaporated to a pilular consistence.

Dose.-H. \& C., 3 i.-iv. (4.-15.); D., gr.v.-xx. (.3-1.3.).

Extractum Taraxaci Fluidum. Fiuid Extract of Taraxacum.

(U. S. P.)

Made by maceration and percolation with diluted alcohol, and evaporation so that $1 \mathrm{Cc} .=1 \mathrm{Gm}$. of taraxacum.

Dose.-Same as taraxacum.

Administration.-The fresh juice squeezed from the root (succus, B.P.) may be given to horses; or the official preparations may be used. 
Action and Uses.-Taraxacum is a simple stomachic and bitter asd may be employed in place of gentian or calumba. It has been generally taught that taraxacum is an hepatic stimuiant and increases the secretion of bile. This has been proved fallacious. The extract is often used as an excipient in preparing masses.

\section{Hydrastis. Hydrastis. (U. S. P.)}

Synonym.-Golden seal, yellow root, yellow puccoon, orange root, Indian dye, Indian tumeric, E.; racine d'hydrastis de Canada, Fr.; Canadische gelbwurzel, G.

- The rhizome and roots of Hydrastis canadensis Linné (nat. ord. Ranunculaceæ).

Halitat.-North America in woods, west to Missouri and Arkansas.

Descripion.-Rhizome abont $4 \mathrm{Cm}$. long and $6 \mathrm{Mm}$. thick; oblique, with short branches, somewhat annulate and longitudinally wrinkled; externally brownish-gray; fracture short, waxy, bright reddish-yellow, with a thickish bark, abont ten narrow wood-wedges, broad, medullary rays and large pith. Roots thin, brittle, with a thick yellow bark and subquadrangular, woody centre. Odor slight; taste bitter.

Constituents. - 1 , berberine $\left(\mathrm{C}_{20} \mathrm{H}_{17} \mathrm{NO}_{4}\right)$, an alkaloid occurring in yellow crystals and found in many plants of the families Berberaceæ, Ranunculaceæ, and Menispermaceæ ; 2, hydrastine $\left(\mathrm{C}_{21} \mathrm{H}_{21} \mathrm{NO}_{6}\right)$, a colorless, crystalline alkaloid, soluble in alcohol and ether ; 3 , canadine $\left(\mathrm{C}_{21} \mathrm{H}_{21} \mathrm{NO}_{4}\right)$, occurring in white, acicular crystals.

Dose.-H. \& C., 3 ii.- 3 i. (8.-30.); Sh. \& Sw., 3 i.-ii. (4.-8.); D., gr.v.- 3 i. (.3-4.).

\section{PREPARATIONS.}

Extractum Hydrastis Fluidum. Fluid Extract of Hydrastis.

(U. S. P.)

Made by maceration and percolation with alcohol, glycerin and water, and evaporation, so that $1 \mathrm{Cc} .=1 \mathrm{Gm}$. of hydrastis. 
Dose.-H. \& C., 3 ii.- $\frac{z}{3}$ i. (8.-30.); Sh. \& Sw., 3 i.-ii. (4.-S.); D., आlv.- 3 i. $(.3-4$.$) .$

Tinctura Hydrastis. Tincture of Hydrastis. (U. S. P.)

Made by maceration and percolation of hydrastis, 200 ; with diluted alcohol, to 1000 .

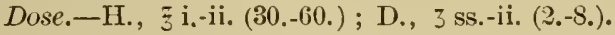

Glyceritum Hydrastis. Glycerite of Hydrastis. (U. S. P.)

Made by maceration and percolation of hydrastis, 1000 ; add water to the percolate and evaporate. Add water to the residue, set aside 24 hours and filter ; add enough water to the filtrate to make 500 ; then add glycerin, 500 .

Dose.-Same as fluid extract.

Hydrastininæ Hydraschloras. Hydrastinine Hydrochlorate. $\mathrm{C}_{11} \mathrm{H}_{11} \mathrm{NO}_{2} \mathrm{H}$ Cl. (U. S. P.)

The hydrochlorate of an artificial alkaloid derived from hydrastine by the action of oxidizing agents.

Properties.-Light, yellow, amorphous granules, or a pale yellow crystalline powder; odorless, and having a bitter, saline taste; deliquescent on exposure to damp air. Soluble in 0.3 part of water, and in 3 parts of alcohol.

Dose.-H., gr.i.-ii. (.06-.12); D., gr. $\frac{1}{12}-\frac{1}{6}(.005-.01)$.

\section{Hydrastin. (Non-official).}

The commercial name for a mixture of variable composition, consisting chiefly of berberine, together with hydrastine, and a resin. A greenish-yellow powder, having a bitter taste. Wrongly termed hydrastine.

Dose.-H., gr.xv.-xxx. (1.-2.); D., gr.iii.-v. (.18-.3).

Actions.-Hydrastis and its alkaloids, berberine and hydrastine, act as simple bitters and stomachics, in small doses, by improving the appetite and stimulating the secretion, motion and vascularity of the stomach. Hydrastis causes contraction of the nou-pregnant uterus, and may induce abortion in pregnant animals. It also increases the flow of bile and urine. The drug is a mild anti-periodic, but 
is decidedly inferior to quinine in this respect. Hydrastine and berberine resemble each other in actions, uses and doses. Berberine sulphate and hydrastine liydrochlorate are to be found in the market, and are used in the same doses as the pure alkaloids. Poisonous doses of hydrastine and berberine are followed by convulsions and paralysis; the former is more convulsant. Hydrastine is said primarily to markedly increase vascular tension. It is uncertain whether this action is due to vascular contraction or cardiac stimulation. In poisoning by either alkaloid there is great cardiac and vasomotor depression.

Uses.-Hydrastis, berberine, and hydrastine are employed in anorexia and atonic indigestion. The fluid extract of hydrastis and hydrastine (which is, however, expensive) are especially efficient for horses in combination with other bitters and iron, as follows:

$\mathrm{B}$

Extr. Capsici Fl.................. 3 ii.

Extr. Hydrastis Fl.

Extr. Nucis Vomicæ FI............. āā

M. (Furnish 3 ii. bottle)

Sig. Small bottleful tid. on tongue.

or: :-

$\mathrm{P}$

Hydrastinæ..........................

Pulv. Gentianæ

Pulv. Nucis Vomice

Ferri Sulph. Exsicc.............. āā ž ii.

M. et div. in ch't, no. xii.

Sig. One powder on food tid.

Hydrastis is exhibited empirically (probably as a local stimulant and antiseptic) in atonic and inflammatory conditions of the digestive organs, with great benefit, as in chronic gastro-intestinal catarrh or catarrhal jaundice. Hydrastis is used most frequently in liuman medicine to stop uterine hæmorrhage of all descriptions, and is often conjoined with the fluid extract of ergot for this purpose. Hydrastinine hydrochlorate has been employed with great success as 
a hæmostatic in metrorrhagia. Hydrastine is given to horses as a bitter tonic in doses of gr.iii.-v.; and to dogs in quantities of gr. $\frac{1}{4}-\frac{1}{2}$. Externally, the fluid extract of hydrastis (1-8 to 1-2), or liydrastine (gr.v.- $\bar{j}$ i), in aqueous solution, are most serviceable as local stimulants in the treatment of the subacute stages in inflammatory diseases of mucous membranes, and in relaxed or atonic conditions of these tissues. The solutions are applied as injections, or lotions, in lencorrhœa, endometritis, balanitis, otorrhœa, stomatitis, etc., and upon indolent ulcers.

Calamus. Calamus. (U. S. P.)

Synonym.-Sweet flag, radix acori, E.; rhizoma calami, P.G.; acore vrai, acore odorant, Fr.; kalmuswurzel, G.

The rhizome of Acorus Calamus Linné (nat. ord. Aroideæ.)

Description.-In sections of various lengths, unpeeled, about $2 \mathrm{Cm}$. broad, subcylindrical, longitudinally wrinkled; on the upper surface marked with leaf scars forming triangles, and on the lower surface with the circular scars of the rootlets in wavy lines; externally reddish-brown, somewhat annulate from remnants of leaf-sheaths; internally whitish, of a spongy texture, breaking with a short, corky fracture, showing numerous oil cells and scattered wood-bundles; the latter crowded within the subcircular endoderm. It has an aromatic odor, and a strongly bitter taste.

Constituents.-1, acorin $\left(\mathrm{C}_{36} \mathrm{H}_{60} \mathrm{O}_{6}\right)$, a liquid, yellow glucoside having a bitter taste ; 2 , a volatile oil, 1-2 per cent.; 3 , calamine ; 4 , choline.

Dose.-H. \& C., 亏 i.-ii. (30.-60.); Sh. \& Sw., 3 i.-iii. (4.-12.); D., gr.xv.-3 i. (1.-4.).

\section{PREPARATION.}

Extractum Calami Fluidum. Fluid Extract of Calamus. (U. S. P.)

Made by maceration, percolation and evaporation, so that $1 \mathrm{Cc} .=$ $1 \mathrm{Gm}$. of the crude drug. 
Dose.-H. \& C., इi.-ii. (30.-60.); Sh. \& Sw., zi.-iii. (4.-12.); D., $\pi \times \mathrm{xv} .-\mathrm{j}$ i. (1.-4.).

The powdered root may be given on the food to the larger animals; the fluid extract, or an infusion (1-16), may be exhibited to any patients.

Action and Uses.-Calamus is a mild aromatic bitter, and is therefore useful in anorexia and indigestion associated with mild forms of flatulence. The powdered root is employed as an excipient in powders, balls and electuaries. It is innocuous, and the dose is therefore unimportant.

\section{SECTION XI.-VEGETABLE CATHARTICS.}

\section{Class 1.-Simple Purgatives.}

Aloe Barbadensis. Barbadoes Aloes. (U. S. \& B. P.)

Synonym.-Curacoa aloes, E.; aloés des Barbades, Fr.; Barbados-aloe, G.

The inspissated juice of the leaves of Aloe vera (Linné) Webb (nat. ord. Liliaceæ).

Habitat.-The island of Barbadoes.

Properties.-In hard masses, orange-brown, opaque, translucent on the edges ; fracture waxy or resinous, somewhat conchoidal; odor saffron-like; taste strongly bitter. Almost entirely soluble in alcohol.

\section{Aloe Socotrina. Socotrine Aloes. (U. S. P.)}

Synonym.-Aloe succotrina, aloés sucotrin, s. socotrin, Fr.; socotora s. socotrinische aloe, G.

The inspissated juice of the leaves of Aloe Perryi Baker (nat. ord. Liliaceæ).

Habitat.-Eastern Africa.

Properties.-In hard masses, occasionally soft in the interior; opaque, yellowish-brown, orange-brown, or dark ruby-red, not greenish, translucent on the edges; fracture resinous, somewhat conchoidal. When breathed upon, it. 
emits a fragrant saffron-like odor; taste pecnliar, strongly bitter. Almost entirely soluble in alcoliol and in 4 parts of boiling water. The aqueous solution becomes turbid on cooling and yields a deposit.

The color of socotrine aloes is lighter, and it is less opaque than Barbadoes aloes. The powdered socotrine aloes is brighter and redder, and the odor less disagreeable than that of Barbadoes aloes.

\section{Aloe Capensis. Cape Aloes. (Non-official.)}

Syno:rym.-Aloés der Cap, Fr.

Habitat.-Africa.

Properties.-Occurs in dark-brown or olive-green resinous masses; fracture conchoidal; odor strong, sour and disagreeable. Yields a gamoge-yellow powder. Solubility same as socotrine aloes. Product of several varieties of aloes obtained from Cape Town and Natal.

Dose of Aloes.-H., 3 ss.-i. (15.-30.); C., 3 i.-ii. (30.-60.); Sh., $\tilde{3}$ ss.-i. (15.-30.); Sw., 3 ii.-iv. (8.-15.); D., gr.xx.- 3 i. (1.3-4.).

Constituents. -1 , aloin; 2 , a resin ; 3 , a volatile oil ; 4 , a trace of gallic acid.

Alornum. Aloin. (U. S. \& B. P.)

Synonym.-Alöine, Fr.

A neutral principle obtained from several varieties of aloes, chiefly Barbadoes aloes (yielding Barbaloin), and Socotra or Zanzibar aloes (yielding Socaloin), differing more or less in chemical composition and physical properties according to the source from which it is derived.

Derivation.-Obtained by pulverizing and macerating Barbadoes aloes in cold water, and evaporating the resulting solution in vacuo. Aloin crystallizes out and is dried between folds of bibulous paper. It is purified by repeated solution in hot water, filtration, recrystallization, and finally 
by solution in hot alcohol and crystallization. Nataloin is derived from Cape aloes.

Properties.-Minute, acicular crystals, or a micro-crystalline powder, varying in color from yellow to yellowishbrown; odorless, or possessing a slight odor of aloes; of a characteristic bitter taste, and permanent in the air. Barbaloin and socaloin are soluble in about 60 parts of cold water. Barbaloin is soluble in 20 parts of alcohol. Socaloin in 30 parts of absolute alcohol.

Dose.-H. \& C., 3 ii.-iii. (8.-12.); D., gr.ii.-xx. (.12-1.3), in combination with other purgatives.

\section{PREPARATIONS OF ALOES.}

The official preparations are numerous, but are not applicable to veterinary practice.

Tinctura Aloës et Myrrhœ. Tincture of Aloes and Myrrh.

(U. S. P.)

Synonym._- Elixis pro," elixir proprietas Paracelsi, E.

Made by maceration and percolation of purified aloes, 100 ; myrrh, 100 ; and liquorice root, 100; with alcohol and water to make 1000.

Action External.-Aloes is a slight stimulant to raw surfaces. It is absorbed from the denuded skin and thus may occasion purging.

Action Internal.-Alimentary Canal.-Aloes is first of all a purgative. In addition to this it is a bitter, and therefore small doses excite salivary and gastric secretion, together with the movements and vascularity of the stomach, and appetite. Experiments on dogs show that large doses of aloes increase the secretion of bile by stimulation of the liver. Aloes is absorbed from the digestive tract and is eliminated by the bowels, kidneys and mammary glands. It may be excreted in sufficient quantity in the milk to create looseness of the bowels in nursing animals.

Aloes stimulates peristalsis of the large intestines, but does not notably increase the secretions in the bowels. Moreover, its action is very slow (12-24 hours). This probably happens because the drug does not act till it reaches 
the large intestines, locally, or through elimination. Aloes is preëminently the best purgative for horses, but does not operate so well on the other domestic animals. Epsom salts, glauber salts or linseed oil are preferable for cattle; linseed oil or carron oil for foals and calves; and castor oil or calomel for dogs. A full dose of aloes often creates some general disturbances in horses, including nausea, slight colic, diuresis, elevation of temperature $\left(1^{\circ}-2^{\circ} \mathrm{F}\right.$.) and pulse, with purging lasting from 2 or 3 to 24 hours. Aloes also possesses anthelmintic properties because of its bitter qualities and purgative action. Socotrine aloes is the basis of the official preparations, but Barbadoes aloes finds most favor in veterinary medicine, and is probably the stronger of the two. Cape aloes is a little inferior to the other varieties and is more apt to produce dinresis. Aloes and aloin lead to catharsis, whether injected under the skin, into the blood, or applied on raw surfaces. Administration by the mouth is more effective. Aloin appears to contain the active principles of aloes, and is usually as operative, but some manufactures are ineffective.

Kidneys and Sexual Organs.-Aloes canses reflex, or sympathetic irritation of the female pelvic organs in its operation on the lower bowel; is an emmenagogue, and may prove abortifacient. The drug sometimes excites diuresis.

Administration.-Aloes is given to horses in semi-solution after being rubbed up with hot $\left(115^{\circ}-120^{\circ} \mathrm{F}\right.$.) water; or in ball. The patient should, if possible, be previously prepared by a diet of bran mashes and salt only, for 2 or 3 feedings before exhibition of the purge. The aloes ball in use for many years in the Harvard Veterinary Hospital is made by melting and mixing Barbadoes aloes ( $1 \mathrm{lb}$.) with glycerin and molasses (each $\overline{3}$ ii.), and powdered ginger root $(\xi i$.), on a water bath. When the mass is properly mixerl it is removed from the fire and alcohol $(\overline{\mathrm{v}}$. $)$ is added. The mass is poured on a layer of flaxseed meal to cool, and then is weighed into portions of $310 \frac{1}{2}$ each. These are rolled into balls, covered with tissue paper, and preserved in tight 
tin or glass vessels. Horses should not be worked after receiving aloes balls, but should be given a little walking exercise 12 hours after the administration of the dose. Colic and superpurgation may follow if the dose is repeated within 48 loours, or if large quantities of cold water are allowed during the action of the catliartic. If aloes does not operate satisfactorily, it is safer to give linseed oil by the mouth and rectal injections, than to administer a second dose of aloes.

Uses External.-The tincture of aloes and myrrh is sometimes applied as a stimulant to wounds, and powdered aloes is mixed with plaster of Paris in making splints for dogs, to prevent these animals from biting and tearing them off.

Uses Internal.-Aloes is employed in the treatment of the horse, whenever an active purge is desirable, with the following exceptions: It must not be used in acute diseases of the respiratory tract lest metastasis occur, and the inflammation attack the bowels. Neither in acute inflammation of the alimentary canal nor of the kidneys is it desirable; nor in intestinal obstruction or impaction of the colon. In the first two named conditions, aloes is too irritating to the organs implicated; in the two last, the drug may aggravate the trouble by the production of impotent peristaltic movements. Pregnancy contra-indicates the use of aloes, lest abortion ensue. The therapeutic scope of aloes being large, it is impossible to enumerate all the diseases in which it is useful. Perhaps this cathartic is more commonly serviceable in indigestion and spasmodic or flatulent colic.

In acute inflammatory diseases of the brain and cord aloes is often combined with calomel ( $3 \mathrm{i}$.) in ball to enhance the effect. The administration of an aloes ball is followed by that of small doses of Epsom salts ( $\bar{z}$ iv.) in the drinking water in the treatment of hæmoglobinæmia of horses, or in conditions when we wish to assist the depleting action of aloes. Turpentine is followed by aloes, or aloes is given prior to a course of iron sulphate and gentian, for the 
destruction of round-worms in horses. A laxative ball may be composed of aloes ( 3 ii.-iv.), ginger and powdered nux vomica (each 3 ii.), mixed with glycerin or molasses.

It is often tanght that alnes is coutraindicated in hæmorrhoids, but this teaching does not obtain unless the piles are inflamed. In piles, associated with an atonic condition, aloes may be beneficial by improving the tone of the bowels. Aloes may be useful in jaundice due to constipation, but in general it is inferior to salines, calomel and podophyllin in the treatment of this disorder. According to Brunton, the presence of bile in the intestines is essential for the cholagogue action of aloes. Therefore the absence of bile in the bowels would forbid the use of aloes as a cholagogue. Laxative doses of aloes are valuable in amenorrhoea, about the time that "heat" should occur, in combination with iron.

Oleum Lini. Linseed Oil. (U. S. \& B. P.)

Synonym.-Oil of flaxseed, E.; huile de lin, Fr.; leinöl, leinsamenöl, G. heat.

A fixed oil expressed from linseed without the use of

Properties.-A yellowish or yellow, oily liquid, having a slight, peculiar odor and bland taste. When exposed to the air it gradually thickens and acquires a strong odor and taste ; and if spread in a thin layer on a glass plate and allowed to stand in a warm place, it is gradually converted into a hard, transparent, resin-like mass (absence of non-drying oils).

Spec. gr. 0.930 to 0.940 at $59^{\circ}$ F. Soluble in about 10 parts of absolute alcohol, and in all proportions in ether, chloroform, benzin, carbon disulphide, or oil of turpentine.

Constituents.-1, linolein; 2, myristin; 3 , palmitin ; 4, albumin, which gives the oil its drying qualities.

Dose.-H., Oss.-i. (250.-500.). Mild laxative, on bran mash. C., Oi.-ii. (500.-1000.); Sh. \& Sw., 3 vi.-xii. (180.-360.); D. \& C., $\overline{3}$ ss.-ii. (15.-60.). 
Action and Uses.-Linseed oil exerts a laxative, or mild. purgative effect by its mechanical action in lubricating the bowels and their contents. It is suitable for horses when a derivative or depleting action is not desirable, as in frecal impaction or overloaded bowels in weak animals, and in those suffering from inflammatory diseases of the respiratory tract or digestive organs; diarrhoea, dysentery, and in pregnancy. Aloes, on the other hand, wonld be contraindicated in these conditions. Carron oil (linseed oil and lime water, equal parts), is particularly appropriate as a remedy for "heaves" in horses ( $\overline{3}$ ii.-iv.), and is one of the best eatharties for foals, lambs and calves ( $\Xi$ ii.-iv.). The laxative and antacid properties of this preparation tend to combat intestinal fermentation which is so common in young avimals with digestive disorders and diarrhœa. The same qualities of carron oil prevent flatulence and interference with the already impeded breathing in "heaves" of horses.

Linseed oil is frequently given to ruminants, although Epsom salts is geverally the best purge for them. It is indicated for these animals when a milder operation than that obtained by a fnll dose of salts is required, and for its demulcent action in irritable states of the digestire organs. By combining linseed oil with croton oil we procure a potent purge for cattle. Castor oil or sweet oil are usually preferable to linseed oil in the treatment of dogs. Soap suds enemata are made more effective by the addition of 1 or 2 pints of linseed oil (for horses), and 1 or 2 ounces (for dogs). The oil may be given in its pure state, but more uncommonly is prescribed with gruel, glycerin, mucilage, or molasses. One ounce each of linseed oil and molasses may be given to the larger animals; or one drachm of either to the smaller animals, as an expectorant in bronchitis. The mixture is often a most serviceable one and probably acts by improving the nutrition of the bronchial mucous membrane. Linseed oil and sweet oil, resemble cod liver oil in this respect, and while both are probably inferior to the latter, as expectorants, they are more palatable and cheaper. 
Oleum Ricini. Castor Oil. (U. S. \& B. P.)

Synonym.-Oleum palmæ christi, huile de ricin, Fr.; ricinusül, G.

A fixed oil expressed from the seed of Ricinus communis Linné (nat. ord. Euphorbiaceæ).

Habitat.-India. Cultivated in many countries.

Properties.-A pale, yellowish and almost odorless, transparent, viscid fluid, liaving a bland, afterwards slightly acrid, and generally offensive taste. Spec. gr. 0.950-0.970 at $59^{\circ} \mathrm{F}$. Soluble in equal volumes of alcohol, and in all proportions in absolute alcohol, or in glacial acetic acid; also soluble in three times its volnme of a mixture of 19 volumes of alcohol and 1 volume of water (absence of more than about 5 per cent. of most other fixed oils).

Constituents.-1, ricinolein, or ricinoleic acid glyceride, $\mathrm{C}_{3} \mathrm{H}_{5}\left(\mathrm{C}_{18} \mathrm{H}_{34} \mathrm{O}_{3}\right)_{3} ; 2$, an acrid principle; 3 , palmitin, stearị and myristin.

Dose.-H. \& C., Oi. (500.); Sh. \& Sw., 亏ii.-iv. (60.-120.); D. \& Cats, 亏 i.-ii. (30.-60.); ; poultry, ji. (4.).

Castor Oil Seeds.-These are not official. The name Ricinus, is applied to the plant becanse of the resemblance of the seed to a ricinus, or tick. The seeds are of a shing gray color, marked with brownish spots and streaks. They are about the size of small beans ( $17 \mathrm{Mm} . \times 8 \mathrm{Mm}$.), ovoid, flattened, and white inside. They contain 50 per cent, of oil, and an acrid, poisonons substance. Three seeds have cansed death in man, and they are ten times more purgative than the oil.

Action and Uses.-Castor oil probably contains some unknown purgative principle which exists in larger amount in the seeds. It is often taught that ricinoleic acid, derived from the decomposition of ricinolein in the bowels, prodnces purgation. This is apparently not the case. Castor oil is mild, but has a more decided purgative action than linseed oil and often occasions griping. It acts in 4 or 5 hours and will purge when absorbed from the skin or rectum. Castor 
oil is a galactagogue and is said to stimulate the secretion of milk when the castor leaves are applied to the mammary glands. Castor oil is specially applicable in canine practice, to unload the bowels, and in irritated conditions of the digestive tract. It is useful in constipation only as an occasional remedy, since it is followed by greater tendency in this direction; also in overloaded bowels, indigestion, diarrhea, and pregnancy; after the ingestion of foreign or putrid matters; and to assist the action of anthelmintics. Castor oil is inferior to linseed oil for horses, as a simple laxative, because it is more prone to cause colicky pains, and because it is more expensive. Castor oil is thought to be notably useful in irritation and inflammation of the intestines in these animals, however, as in diarrhoea, dysentery, and enteritis; and can be combined with anodynes and antispasmodics to prevent griping. Two or three ounces of castor oil are suitable for calves or foals with gastro-intestinal disorders. One or two teaspooufuls are suitable for poultry. Administration.-Castor oil is given to dogs with syrupus rhamni cathartici in the proportion of 1 ounce of the former to 1 drachm of the latter; or with glycerin (equal parts) and a few drops of oil of wintergreen. It is administered to puppies ( 3 i.-ii.) with an equal volume of sweet oil. Castor oil may be exhibited to horses with oil of peppermint (Mxx.); or in digestive irritation, in warm cooked flour gruel with laudanum ( $3 \mathrm{ss}$.) and fluid extract belladonna ( 3 i.); to foals and calves with mucilage or gruel and 5 drops of oil of peppermint.

Rhamnus Purshiana. (U. S. P.) Cascara Sagrada. (B. P.)

Synonym.-California buckthorn, sacred bark, chittem bark.

The bark of Rhamnus Purshiana de Candolle (nat. ord. Rhamnaceæ).

Habitat.-United States from northern Idaho west to Pacific Ocean. 
Description.-In quills or curved pieces about 3 to 10 $\mathrm{Cm}$. long and about $2 \mathrm{M}$.m. thick; outer surface brownishgray aud whitish; the young bark having numerous rather broad, pale-colored warts; inner surface yellowish to light brownish, becoming dark brown by age; smooth or finely striate; fracture short, yellowish; in the inner layer of thick bark somewhat fibrous; inodorous ; taste bitter.

Constituents.-1, three resins; 2 , a neutral body; 3 , a volatile oil; 4 , malic and tannic acids.

Dose.-D., gr.v.-xxx. (.3-2.).

PREPARATIONS.

Extractum Rhamni Purshiance Fluidum. Fluid Extract of Rhamnus Purshiana.

Made by maceration and percolation with diluted alcohol, and evaporation, so that $1 \mathrm{Cc} .=1 \mathrm{Gm}$. of the crude drug.

Dose.-D., m $\nabla_{.}-\mathrm{xxx}$. (.3-2.).

Extractum Cascarce Sagradie Liquidum. (B. P.)

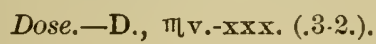

Frangula. Frangula. (U. S. P.)

Synonym.-Rhamni frangula cortex, B.P.; buckthorn, alder buckthorn, black alder, E.; bourdaine, bourgène, Fr.; faulbaumrinde, G.; cortex frangulæ, P.G.

The bark of Rhamnus Frangula Linné (nat. ord. Rhamneæ), collected at least one year before being used.

Habitat.-Europe and northern Asia.

Description.-Quilled, about $1 \mathrm{Mm}$. thick; onter surface grayish-brown, or blackish-brown, with numerous small, whitish, transversely-elongated lenticles; inner surface smooth, pale brownish-yellow; fracture in the outer layer short, of a purplish tint; in the inner layer fibrous and pale yellow; when masticated, coloring the saliva yellow; nearly inodorous; taste sweetish and bitter.

Constituents. - 1 , a glucoside, frangulin $\left(\mathrm{C}_{20} \mathrm{H}_{20} \mathrm{O}_{10}\right)$, converted in time into (2) emodin $\left(\mathrm{C}_{15} \mathrm{H}_{10} \mathrm{O}_{5}\right)$, a glncoside, to which the drug owes its purgative action. Frangulin yields. 
emodin, a constituent of rhubarb as well, and rhamnose $\left(\mathrm{C}_{6} \mathrm{H}_{12} \mathrm{O}_{5}\right)$, by hydrolysis.

$$
\text { Dose.-D., } 3 \text { ss.-i. (2.-4.). }
$$

\section{PREPARATIONS.}

Extractum Frangule Fluidum. Fluid Extract of Frangula.

Made by maceration and percolation with alcohol and water, and evaporation, so that $1 \mathrm{Cc}$, of the preparation $=1 \mathrm{Gm}$. of the crude drug. Dose.-D., 3 ss.-i. (2.-4.).

Extractum Rhamnce Frangule Liquidum. (B. P.) Dose.-D., 3 i.-iv. (4.-15.).

\section{Rhamnus Catharticus. (Non-official.)}

The berries of $R$. catharticus, from which an acrid, nauseous, bitter juice is obtained. A syrup-syrupus rhamni cathartici-is prepared from the juice (B.P.) with sugar, ginger and pimento.

Dose of Syrup.-D., $\overline{3}$ i.-ii. (30.-60.); Cats, $\overline{3}$ ss.-i. (15.-30.). Action and Uses.-The buckthorns are laxatires in the doses employed in medicine. The fresh bark of $\mathrm{R}$. frangula produces violent gastro-enteritis (frangulin), and therefore must be kept a year before using. The same remark applies with less force to $\mathbf{R}$. Purshiana. Cascara sagrada is probably the best mild purgative remedy for dogs with chronic constipation, and the dose does not require to be increased on repetition. In fact, the tone of the bowels is improved by the drug. It is more reliable and active than frangula. Cascara may cause some griping. It is very bitter and therefore may promote gastric digestion and appetite. The aromatic fluid extract made by a well-known firm is a most serviceable preparation in preventing colic and in disguising the taste. The average dose for the dog is $M x x .-\mathrm{xxx}$. each night, or $m_{v .-x}$. thrice daily. The syrup of buckthorn is commonly prescribed to dogs with castor oil, of each one ounce; or one drachm of the former with an onnce of the latter, for an occasional purgative. 
Rheum. Rlinbarb. (U.S. P.)

Synonym.-Rhei radix, B.P.; rhubarbe, Fr.; rhubarber, $\mathrm{G}$.

The root of Rheum officinale Baillon (nat. ord. Polygonaceæ).

Description.-In cylindrical, conical or flattish segments, deprived of the dark brown, corky layer, smoothish or somewhat wrinkled; externally covered with a bright yellowishbrown powder, marked with white, elongated meshes, containing a white, rather spongy tissue and a number of short, reddish-brown or brownish-yellow striæ; compact, hard; fracture uneven; internally white, with numerous red, irregularly-curved and interrupted medullary rays, which are radially parallel only near the cambium line; odor somewhat peculiar, aromatic; taste bitter, somewhat astringent. When chewed, rhubarb feels gritty between the teeth, and imparts a yellow color to the saliva.

Constituents. - 1 , a glucoside, chrysarobin $\left(\mathrm{C}_{27} \mathrm{H}_{30} \mathrm{O}_{14}\right)$, which yields 2-3 per cent. of chrysophanic acid $\left(\mathrm{C}_{15} \mathrm{H}_{10} \mathrm{O}_{4}\right)$, also called rhein or chrysophan ; 2 , rheotannic acid $\left(\mathrm{C}_{26} \mathrm{H}_{26} \mathrm{O}_{4}\right)$, which gives astringency to rhubarb; 3 , calcium oxalate (35 per cent.), causing grittiness ; 4 , resinous bodies : phroretin, emodin, aporetin and erythroretin. Chrysophanic acid and the resins are somewhat purgative, but the exact purgative principle has yet to be discovered.

Dose.-Stomachic-H. \& C., 3 i.-ii. (30.-60.); Sh., 3 i. (4); D. \& Cats, gr.v.-x. (.3-.6).

Mild Purgative-Foals and Calves, 3 i.-ii. (4.-8.); D., 3 i.-ii. (4.-8.).

\section{PREPARATIONS.}

Extractum Rhei Fluidum. Fluid Extract of Rhubarb.

$$
\text { (U.S. P.) }
$$

Made by maceration and percolation with alcohol and water, and evaporation, so that $1 \mathrm{Cc} .=1 \mathrm{Gm}$. of the crude drug.

Dose.-Same as that of rhubarb. 
Pulvis Rhei Compositus. Compound Powder of Rhubarb.

(U. S. \& B. P.)

Synonym. - Gregory's powder. Rhubarb, 25; magnesia, 65; ginger, 10.

Dose._Foals and Calves, 3 ss.-i. (15.-30.).

Extractum Rhei. Extract of Rhubarb. (U. S. \& B. P.)

Dose.-D., gr.v.-x. (.3-.6).

There are many other official preparations, but they possess no value in reterinary medicine.

Action Internal.-Alimentary Canal.-Rhubarb is a bitter, and therefore in small doses improves digestion in all animals by increasing the flow of salivary and gastric juices, and by stimulating the appetite, vascularity, and movements of the stomach. It is called a stomachic and bitter tonic. Larger doses canse mild purgation in the case of dogs and cats, but horses and cattle are but slightly affected in this way. Rhubarb augments the secretion of bile. It is commonly described as an agent which stimulates peristaltic action, but it is not certainly known how purging is brought about. Secondary constipation is more apt to follow the use of rhubarb than other drugs, because of rheotannic acid. This substance may be absorbed and eliminated into the bowels after the occurrence of purgation. Rhubarb, by virtue of chrysophanic acid, stains the fæces, urine, milk and sweat yellow in its excretion.

Uses.-Rhubarb is an efficient laxative remedy for the treatment of indigestion in young animals associated with diarrhœa. In this condition it sweeps ont the source of irritation and then exerts an astringent effect. The drug often acts most favorably with an antacid in the disorders noted. Gregory's powder is useful in the care of foals, calves and lambs with diarrhoea. The fluid extract may be given to dogs, but rhubarb is not so generally useful a purgative for these animals as castor oil, calomel, or cascara sagrada. Rhubarb has been recommended when a laxative is desirable, in cases of hæmorrhoids, to improve local tone, and also as a purgative in diarrhœa of young animals due to worms. 
One or two drops of the tincture of rhubarb in the drinking water form a serviceahle laxative for small birds.

\section{Chrisarobinum. Chrysarobin. (U. S. \& B. P.)}

Synomym.-Goa powder, araroba powder.

A nentral principle, in its commercial, more or less impure form, extracted from Goa Powder, a substance fonnd deposited in the wood of Andira Araroba Aginiar (nat. ord. Legnminosæ).

Habitat.-Brazil.

Properties. - A pale orange-yellow, microcrystalline powder, odorless and taisteless; turuing brownish-yellow on exposure to the air. Very slightly soluble in cold water or alcohol. Solnble in solutious of alkalies.

Constituents. - Chiefly ehrysarobin $\left(\mathrm{C}_{27} \mathrm{H}_{30} \mathrm{O}_{14}\right)$ - also called rhein and chrysophan,-an orange-yellow, crystalline glucoside, somewhat soluble in alcohol and ether; freely soluble in chloroform and benzol. It is oxidized into chrysophanic acid $\left(\mathrm{C}_{15} \mathrm{H}_{10} \mathrm{O}_{4}\right)$, and glucose.

\section{PREPARATION.}

Unguentum Chrysarobini. Chrysarobin Ointment. (U. S. \& B. P.) Chrysarobin, 5 ; benzoinated lard, 95 . (U. S.)

Action and Uses.-Chrysarobin is a powerful irritant to the skin and destroys parasites. It stains the skin and other materials dark brown. This may be removed, nnless fixed. by an alkali, with a weak solution of chlorinated lime or canstic soda. Chrysarobin is also a strong irritant in the gastro-intestinal tract, cansing vomiting and purging in carnivora. It is elimiuated by the kidneys, coloring the nrine yellow.

Chrysarobin is used as a parasiticide in the treatment of ringworm, and as a stimulant to the skin in chronic cutaneous disorders, as eczema (with mach itching and scaling), pityriasis, and particularly psoriasis The official 5 per cent. ointment should be diluterl $2 \% 3$ times for delicate skins. 
It should be applied over a large area with care, but is one of the most efficient remedies in obstinate diseases of the skin.

\section{Senna. Senna.}

Synonym.-Senna Alexandrina, senna Indica, B.P.; senna leaves, folia sennæ, E.; feuilles de séné, Fr.; sennesblätter, G.

The leaflets of Cassia acutifolia Delile (Alexandria Senna), and of Cassia augustifolia Vahl (India Senna); (nat. ord. Leguminosæ).

Description.-Alexandria Senna.-It consists of leaflets about $25 \mathrm{Mm}$. long and $10 \mathrm{Mm}$. broad, lanceolate or lanceoval, subcoriaceous, brittle, rather pointed, unequally oblique at the base, entire, grayish-green, somewhat pubescent, of a peculiar odor and a nauseous, bitter taste.

Impurities.-Argel leaves (Solenostemma Argel Hayne, nat. ord. Asclepiadx), are frequently present. They are thicker, one-veined, wrinkled, glancous, and even at the base.

India Senna.-It consists of leaflets 3 to $5 \mathrm{Cm}$. long, and 10 to $15 \mathrm{Mm}$. broad; lanceolate, acute, unequally oblique at the base; entire, thin, yellowish-green, nearly smooth; odor peculiar, somewhat tea-like; taste mucilaginous, bitter and nauseous. It should be free from stalks, discolored leares and other admixtures.

Habitat.-Alexandria Senna - Upper Egypt, Nubia, and Central Africa.

Indian Senna, or Tinnivelly Senna-Eastern Africa to India.

Constituents. - 1 , the purgative properties are chiefly due to magnesium and calcium cathartates; salts of cathartic acid $\left(\mathrm{C}_{180} \mathrm{H}_{96} \mathrm{~N}_{2} \mathrm{SO}_{82}\right)$, a black, amorphous glucoside; 2, two glucosides, sennacrol and sennapicrin, insoluble in water; 3 , chrysophanic acid; 4 , cathartomannit $\left(\mathrm{C}_{21} \mathrm{H}_{44} \mathrm{O}_{19}\right)$, an unfermentable sugar.

Dose.-H. \& C., 亏̈iv.-v. (120.-150.); Sh. \& Sw., ₹i.-ii. (30.-60.); D. \& C., 3 i.-iv. (4.-15.). 
PREPARATIONS.

Extractum Sennce Fluidum. Fluid Extract of Senna. (U. S. P.)

Made by maceration and percolation with diluted alcohol, and evaporation, so that $1 \mathrm{Cc} .=1 \mathrm{Gm}$. of the crude drug.

Dose.-Same as senna.

Pulvis Glycyrrhizce Compositus. Compound Powder of Glycyrrhiza. (U. S. \& B. P.)

Synonym.-Compound liquorice powder.

Senna, 180 ; glycyrrhiza, 236 ; washed sulphur, 80 ; oil of fennel, 4; sugar, 500.

Dose.-D., 3 ss.-ii. (2.-8.).

Action Internal.-Senna stimulates and increases the vascularity of the intestinal mucons membrane, and causes increased peristalsis of the large intestines, particularly of the colon. It produces copious pale-yellow and watery evacuations. The drug has a nauseous taste and purging is accompanied by some griping and flatulence. Senna acts more satisfactorily when combined with other purgative agents. It is absorbed and will occasion catharsis in sucklings after administration to their mothers, and after intravenous injection. The urine may be colored red or yellow by its elimination. It is extremely doubtful if senna exerts any influence on biliary secretion.

Uses.-Senna is but rarely employed in veterinary medicine. It may be used where a simple, vigorous cathartic is indicated in constipation, or in cases of slight fæcal accumulation.

The drug acts more effectively when given in conjunction with salts. The fluid extract, or an infusion made by pouring boiling water over the leaves and allowing them to macerate until the water becomes cold, are added to solutions of magnesium sulphate. This combination is more suitable for cattle or sheep. Compound liquorice powder may be given to dogs as a simple purgative in occasional or habitual constipation. 


\section{Class 2.-Drastic Purgatives.}

\section{Oleum Tiglif. Croton Oil. (U. S. P.)}

Synonym.-Olenm crotonis, B.P.; huile de croton, huile de graines de tilly, Fr.; crotonöl, G.

A fixed oil expressed from the seed of Croton Tiglium Linné (nat. ord. Euphorbiacex).

Habitat.-India, Indian Archipelago, and Philippine Islands. Also cultivated.

Properties.-A pale yellow or browuish-yellow, somewhat viscid, and somewhat fluorescent liquid, having a slight fatty odor, and a mild, oily, afterwards burning and acrid taste (great cantion is necessary in tasting). Spec. gr. 0.940 to 0.960 at $59^{\circ} \mathrm{F}$. When fresh it is soluble in about 60 parts of alcohol, the solubility increasing by age. It is freely soluble in ether, chloroform, carbon disnlphide, and in fixed and volatile oils.

Constituents. - 1, crotonol $\left(\mathrm{C}_{18} \mathrm{H}_{24} \mathrm{O}_{4}\right)$, a non-purgative body causing irritation of the skin; 2 , tiglinic acid $\left(\mathrm{C}_{5} \mathrm{H}_{8} \mathrm{O}_{2}\right)$, and many volatile acids existing as glycerides and accounting for the odor of croton oil; 3 , free and combined fatty acids. The purgative principle is undiscovered.

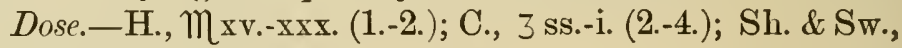

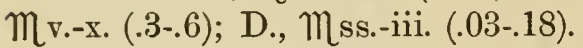

Croton seeds resemble castor seeds in size, but are not mottled or shiny. They are $13 \mathrm{Mm}$. long by $8 \mathrm{Mm}$. wide; oval in shape; white within, and possessing a mild, mucilaginous taste at first, but soon becoming hot and sharp. They contain from 50 to 60 per cent. of croton oil.

Action External.-Croton oil is a most powerful irritant, cansing pain, redness and swelling of the skin, soon followed by vesicles and pustules. Permanent destruction of the hair follicles succeeds with loss of hair and cicatrices. When applied to the skin, injected into the blood or under the skin, it is eliminated to some extent by the bowels, producing. 
purging; and sometimes by the kidneys, creating diuresis, irritation of these organs and strangury.

Action Internal.-Croton oil is also an intense internal irritant. It increases the vascularity of the stomach and bowels and in large doses creates gastro-enteritis. Medicinal doses notably augment the intestinal secretions-but not that of bile-and to a degree peristalsis. The drug may act in half an hour, but usually within a few hours, and purgation is attended witl colicky pain and griping. The movements are very fluid and sometimes contain blood. Croton oil is therefore a drastic hydragogue cathartic. The purgative action is probably due in part to direct irritation of the intestinal mucous membrane; in part to absorption and elimination of the oil by the bowels.

Toxicology.-Ten drops of croton oil will kill a dog unless vomiting occurs. Thirty drops prove fatal to a horse, intravenously. The treatment of poisoning includes the use of emetics or stomach tube, demulcents and opium.

Administration.-Croton oil may be placed on the tongue of an unconscious animal, in a small quantity of linseed oil, olive oil, or lard. The oil can also be given in enema with a pint of linseed oil. It may be exhibited to dogs in pill, castor oil, or rubbed up with a little butter and smeared on the back of the tongue. Croton oil (in a pint of linseed oil) is valuable in assisting the action of salts in obstinate constipation of cattle. It may be administered to horses $(M x$.$) , when a powerful derivative and purgative action is$ indicated, as in acute inflammation of the brain and cord, with calomel and aloes in ball.

Uses External.-Croton oil is ordinarily superseded by milder counter-irritants, as turpentine, mustard, or stimulating liniments; but it may be employed so as to secure any degree of irritation according to its strength. It is occasionally used for horses in acute diseases of the brain, applied around the poll and on the back of the neck; 1 part (20-30m), with 30 parts each of oil of turpentine and lin- 
seed oil ; in acute disorders of the chest $(10-15 \mathrm{~m}$ on either side) and abdomen (40 $\mathrm{m}$ ), similarly diluted.

Absorption, purging and revulsant action may be secured by the exterual application of croton oil. Croton oil is more often employed in cattle (1-6 or 10, with cod liver oil) for its counter-irritant effect when rubbed into the skin, as in laryngitis, glandular enlargement, and rheumatic joints. It is less likely to cause severe inflammation and blemishing than in horses. For swine, croton oil is diluted with 2 volumes of linseed or cod liver oil.

Uses Internal.-Croton oil is more suitable as a drastic cathartic for cattle, than for horses. It is prescribed in obstinate constipation (not of organic origin) when other remedies fail. Also in unconscious conditions where its small bulk will allow of its exhibition. Again, for its derivative and rapid effect in cerebral congestion, parturient apoplexy, etc. Croton oil may be given to dogs and pigs in similar conditions. The oil is too irritant for horses to justify its use save in exceptional cases.

Scammonium. Scammony. (U. S. \& B. P.)

Synonym.-Scammonée, Fr.; scammonium, G.

A resinous exudation from the living root of Convolvulus Scammonia Linné (nat. ord. Convolvulaceæ).

Habitat.-Syria.

Description.-In irregnlar, angular pieces or circular cakes, greenish-gray or blackish; internally porous, and breaking with an angular fracture; of a resinous lustre; odor peculiar, somewhat cheese-like; taste slightly acrid; powder gray or greenish-gray. When triturated with water, scammony yields a greenish emulsion; ether dissolves at least 75 per cent. of it.

Constituents. - 1, a resin (80-95 per cent.); 2, gum; 3, starch.

Dose.-D., 3 i.-ii. (4.-8.); Cats, 3 ss.-i. (2.-4.); Sw., 3 ii.-iv. (8.-15.). 
PREPARATION.

Resina Scammonii. Resin of Scammony. (U. S. \& B. P.) with water.

Derivation.-Made by solution in boiling alcohol, and precipitation

Properties. - Yellowish-brown or brownish-yellow masses or fragments, breaking with a glossy, resinous fracture; translucent at the edges; or a yellowish-white or grayish-white powder, having a faint, peculiar odor, and a slight, peculiar taste. Soluble in alcohol, ether, and oil of turpentine. jalapin.

Constituents. - Mainly scammonin $\left(\mathrm{C}_{88} \mathrm{H}_{156} \mathrm{O}_{42}\right)$, identical with

Dose.-One-half that of scammony.

JALAPA. Jalap. (U. S. \& B. P.)

Synonym.-Tuber jalapæ, P.G.; jalap, radix jalapæ, Fr.; jalape, jalapenknollen, $\mathrm{G}$.

The tuberous root of Ipomœa Jalapa Nuttall (nat. ord. Convolvulaceæ).

Habitat.-Mexico.

Description.-Napiform, pyriform or oblong, varying in size; the large roots incised, more or less wrinkled; dark brown, with lighter-colored spots, and short transverse ridges; hard, compact, internally pale grayish-brown, with numerous concentric circles composed of small resin-cells; fracture resinous, not fibrous; odor slight, but peculiar, smoky and sweetish; taste sweetish and acrid. Contains 12 per cent. of resin of jalap obtained by solution of jalap in alcohol and precipitation with water.

Constituents.-1, a hard resin, chiefly the glucoside jalapurgin ; 2, a soft resin.

Dose.-D., 3 i.-ii. (4.-8.); Cats, 3 ss.-i. (2.-4.); Sw., 3 ii.-iv. (8.-15.).

\section{PREPARATION,}

Resina Jalapce. Resin of Jalap. (U. S. \& B. P.)

Derivation.-Made by maceration and percolation with alcohol, partial distillation; precipitation with water; washing and drying.

Properties. - Yellowish-brown or brown masses or fragments, breaking with a resinous, glossy fracture, translucent at the edges, or 
a yellowish-gray or yellowisl-brown powder, having a slight, peculiar odor, and a somewhat acrid taste. Permanent in the air. Soluble in alcohol; insoluble in carbon disulphide, benzol, and fixed and volatile oils. Not more than 10 per cent. of it is soluble in ether.

Constituents. - 1, jalapurgin $\left(\mathrm{C}_{62} \mathrm{H}_{1{ }_{0}} \mathrm{O}_{32}\right)$, the most active principle; insoluble in ether ; 2 , convolvulin or jalapin, identical with scammonin; 3 , starch or gum.

Dose.-One-quarter that of jalap.

\section{ACTION OF SCAMMONY AND JALAP.}

Scammony and jalap are powerful liydragogue cathartics in their action on swine, dogs and cats. Horses and cattle are but slightly affected by them in ordinary doses. Their resins are dissolved by the bile in the duodenum, and a purgative substance is formed which chiefly stimulates the intestinal glands and causes a copious outpouring of secretion.

Both drugs excite peristaltic action and increase the vascularity of the intestinal mucous membrane, particularly scammony, so that griping may occur. They produce gastrointestinal irritation, with vomiting and purging, in animals capable of the act, after large doses. While jalap and scammony are active purgatives, they are not always certain, and are therefore more frequently employed in combination with other cathartics. They are indirectly cholagoguelike calomel-in sweeping out bile from the small intestines and preventing its reabsorption, and are said to be anthelmintics as well. The active principles of both drugs are absorbed, as death has taken place in an infant after exhibition of scammony to its nurse, and purging has followed the rubbing of jalap into the shaven skin of dogs.

Uses.-Jalap is in more common use than scammony because it is somewhat more of a hydragogue and less prone to cause griping. It is particularly indicated, in dropsy or ascites of dogs, made into pills with from 3 to 5 grains of calomel. Jalap may also be given to expel round or thread worms; in torpidity of the liver; and in obstinate constipation in dogs. The latter is treated more satisfactorily by 
massage, rectal enemata, manual evacuation and repeated doses of sweet oil.

\section{Cambogra. Gamboge. (U. S. \& B. P.)}

Synonym.-Gutti, P.G.; gummi resina guttæ s. gutti, gutta gamba, cambodia, gutte, gomme-gutti, Fr.; gummigutt, gutti, G.

A gum-resin obtained from Garcinia Hanburii Hooker filius (nat. ord. Guttiferæ).

Habitat.-Siam, Anam, and Camboja.

Properties.-In cylindrical pieces, sometimes hollow in the centre, 2 to $5 \mathrm{Cm}$. in diameter, longitudinally striate on the surface; fracture flattish, conchoidal, of a waxy lustre, orange-red; in powder bright-yellow ; inodorous ; taste very acrid; the powder sternutatory. Gamboge is partially soluble in alcohol and ether. When triturated with water it yields a yellow emulsion, and forms with solution of potassium or sodium lyydrate an orange-red solution from which, on the addition of hydrochloric acid, a yellow resin is precipitated.

Constituents. - 1, gambogic acid, a bright yellow or orange-red resin, to the extent of about 75 per cent. of the crude drug; it is not so active as the crude drug; 2 , a soluble gum.

Dose.-H., 3 ss.-i. (15.-30.); C., 3 i.-i.ss. (30.-45.); Sh. \& Sw., gr.xx.- 3 i. (1.3-4.); D., gr.v.-x. (.3-.6).

Action and Uses.-Gamboge is a drastic, hydragogue purgative, and slightly diuretic. Its action is uncertain and often violent, with production of griping pains. Large doses cause vomiting in carnivora and omnivora, and gastro-enteritis in all animals.

Gamboge is dissolved by the bile and alkaline intestinal juices and some of it is absorbed, since it colors the urine yellow in its elimination and occasions diuresis. Attempts to create catharsis by injection of gamboge into the blood, have proved futile. Gamboge should never be prescribed 
alone and is rarely used in veterinary medicine. It has been recommended in obstinate constipation, indigestion, impaction of the third stomach, and cerebral diseases of cattle, combined with salts. The smaller of the foregoing doses are to be employed, as the amount of the active principle is variable. The gum forms an emulsion when the drug is triturated with water.

\section{Colocynthis. Colocynth. (U. S. P.)}

Synonym. - Colocynthidis pulpa, B.P.; coloquintida, bitter apple, $\mathrm{E}$; coloquinte, Fr.; koloquinten, G.

The fruit of Citrullus Colocynthis Schrader (nat. ord. Cncurbitacer), deprived of its rind.

Habitat.-A vine growing in North and Sonth Africa, South and West Asia, and Japah, etc.

Description.-From 5 to $10 \mathrm{Cm}$. in diameter; globular, white or yellowish-white; light, spongy, readily breaking into three wedge-shaped pieces, each containing, near the rounded surface, many flat, ovate, brown seeds; inodorous; taste intensely bitter. The pulp only should be used; the seeds separated and rejected.

Constituents.-1, the chief purgative principle is colocynthin $\left(\mathrm{C}_{56} \mathrm{H}_{84} \mathrm{O}_{23}\right), 1-2$ per cent. An amorphous or crystalline bitter glucoside. Soluble in water and alcohol. There is also (2) an insoluble, resinous body called colocynthitin, or citrullin.

Dose. - Colocynthin - H., 3 ss.-i. $\quad$ (2.-4.); D., gr. $\frac{1}{4}-$ i. (.015-.06).

Colocynth-D., gr.ii.-iii. (.12-.18).

Elaterinum. Elaterin. $\mathrm{C}_{20} \mathrm{H}_{28} \mathrm{O}_{5}$. (U. S. \& B. P.)

Synonym.-Cucumis asininus, cucumis agrestis, wild or squirting cucumber, E.; concombre sauvage (purgatif d'ane), Fr.; springgurke essels-kürbis, spritzgurke, G.

A neutral principle obtained from elaterium, a substance. 
deposited by the juice of the fruit of Ecballium Elaterium (Linnè) A. Richard (nat. ord. Cucurbitaceæ).

Habitat.-Elaterium grows in North Africa, West Asia. and Southern Enrope. It is also cultivated.

Derivation.-Elaterium is exhausted with chloroform. Ether is added and elaterin is precipitated and is purified by redissolving in chloroform and crystallizing.

Properties.-Minute, white, hexagonal scales, or prismatic crystals, without odor, and having a slight, acrid, bitter taste. Soluble in 4250 parts of water, and in 337 parts of alcohol; also soluble in 543 parts of ether, or in 2.4 parts of chloroform. Permanent in the air.

Dose.-D., gr. $\frac{1}{20}-\frac{1}{1} \frac{1}{2}(.003-.005)$.

ACTIONS AND USES OF COLOCYNTH AND ELATERIN.

The action of colocynth and elaterin is similar in man, but the latter is more powerfnl. Both greatly increase secretions and, slightly, the flow of bile; while they stimulate peristalsis in some degree. Large doses cause painful griping, gastro-intestinal inflammation, excessive watery purging, and collapse. Elaterin frequently fails to purge horses and dogs, although death may follow large doses. Colocynth acts more certainly in the lower animals and is recommended by P. Cagny in dropsy and cerebral disease. Neither drug, however, is of any importance in veterinary medicine. Colocynth is contained in the compound cathartic pill given to dogs in doses of from one to three pills.

\section{Class 3.-Cholagogue Purgatives.}

Podophyllum. Podophyllum.

Synonym.-Podophylli rhizoma, B.P.; May apple, mandrake root, E., podophylle, Fr.; fussblatwurzel, G.

The rhizome and roots of Podophyllurn pellatum Linné (nat. ord. Berberideæ).

Habitat.-North America. 
Description.-Of horizontal growth, consisting of joints about 5 Mm. thick, but somewhat enlarged at the end, which has a circular scar on the upper side, a tuft of about 10, nearly simple, fragile ronts on the lower side, and is sometimes branched laterally; smooth or somewhat wrinkled, orange-brown, internally white and mealy, with a circle of small wood bundles; pith large; nearly inodorous; taste sweetish, somewhat bitter and acrid.

Constituents.-1, a resin, podophyllin (4-5 per cent.); 2, a coloring matter, podophyllinic acid.

\section{PREPARATION.}

Resina Podophylli. Resin of Podophyllum. (U. S. \& B. P.)

Synonym.-Podophyllin.

Derivation.-Made by maceration and percolation of podophyllum with alcohol; partial evaporation, and precipitation of the resin with diluted hydrochloric acid; washing with water, drying.

Properties.-An amorphous powder, varying in color from grayishwhite to pale greenish-yellow or yellowish-green; having a slight, peculiar odor, and a peculiar, faintly bitter taste. Permanent in the air. Soluble in alcohol and in solutions of potassium and sodium hydrate.

Constituents.-Chiefly podophyllotoxin $\left(\mathrm{C}_{23} \mathrm{H}_{24} \mathrm{O}_{9}+2 \mathrm{H}_{2} \mathrm{O}\right)$, stated to be a mixture of a purgative principle, picropodophyllin, and an inert body, podophyllinic acid, associated with a coloring matter, podophylloquercitin, and other resins.

Dose.-H. \& C., 3 i.-ii. (4.-8.); D., gr.i.-ii. (.06-.12).

Action.-Podophyllin is essentially a cholagogue cathartic of slow and uncertain action. Large doses cause purging and vomiting in animals, and lethal quantities occasion gastro-enteritis, colic, super-purgation, with bloody frcal evacuations, convulsions and death. It acts in the same way whether it is applied externally, injected into the blood, or given internally. Podophyllin must therefore exert its effect after absorption, and about 10 hours are required to produce purgation. The action is exerted mainly on the duodenum, which is intensely inflamed and even ulcerated in poisoning. Podophyllin directly increases the secretion of bile in small doses, while prrgative quanti- 
ties hasten its excretion by stimulation of the muscular coat of the gall bladder (except in the horse) and small intestines. It is probable that the intestinal secretions are somewhat angmented. The frecal movernents, after medicinal doses of podophyllin, are liquid, often stained with bile, and may be accompanied by some nausea and griping. Since podophyllin is an uncertain purgative, affecting different patients unequally, it should be combined with other agents when a purgative action is desired; preferably calomel and aloes. The time required for the action of these drugs is nearly the same as that necessary for podophyllin. Aloes increases biliary secretion, while calomel is an indirect cholagogue in sweeping bile ont of the small intestines and preventing its reabsorption.

Administration.-Podiophyllin should be given to dogs in pills; to horses in ball with calomel and aloes, if purgation is desired; or dissolved in liquor potassa and diluted with water.

Uses.-Podophyilin is particularly indicated as a choiagogue purgative in constipation associated with jaundice and hepatic disorders. The result of its action is said to be more favorable when the fæcal discharges are dark colored, whereas calomel is more successful if the evacuations are of a light hue. Diarrlıœa, with frequent watery discharges, and vomiting in dogs, due to atonic dyspepsia and a torpid liver, may be relieved by podophylliv.

\title{
SECTION XII.-TANNIC ACID AND DRUGS CONTAINING IT.
}

\author{
GaLLA. Nutgall. (U. S. \& B. P.)
}

Synonym.-Galls, E.; gallæ, P.G.; noix de galle, galle de chêne, Fr.; galläpfel, G.

An excresence on Quercus lusitanica Lamarck (nat. ord. Cupuliferæ), caused by the punctures and deposited ova of 
Cynips Gallæ tinctoriæ Olivier (class Insecta ; order Hymenoptera).

\section{Habitat.-Levant.}

Description.-Subglobular, 1 or $2 \mathrm{Cm}$. in diameter, more or less tuberculated above, otherwise smooth, heavy, hard; often with a circular hole near the middle communicating with the central cavity; blackish olive-green or blackishgray; fracture granular, grayish ; in the centre a cavity containing either the partly developed insect, or pulverulent remains left by it; nearly inodorous; taste strongly astringent. Light, spongy, and whitish-colored nutgall should be rejected.

Constituents.-1, (gallo) tannic acid, 60 per cent.; 2, gallic acid, 2.3 per cent.; 3 , sugar ; 4 , resin.

PREPARATIONS,

Unguentum Gallce. Nutgall Ointment. (U. S. \& B. P.)

Nutgall. 20; benzoinated lard, Ł0. (U.S. P.)

Unguentum Galle Cum Opio. (B. P.)

Acidum Tannicum. Tamnic Acid. $\mathrm{HC}_{14} \mathrm{H}_{9} \mathrm{O}_{9}$.

(U. S. \& B. P.)

Synonym.-Acidum gallo-tannicum, tanninum, tannin, digallic acid, E.; acid tannique, tannin, Fr.; gerbsäure, tannin, G.

An organic acid obtained from nutgall.

Derivation.-Powdered nutgall is exposed to damp air for 48 hours. It is then treated with water and ether. The water dissolves tannic acid; the ether removes gallic acid, coloring matters and impurities. The mixture is filtered and allowed to stand, when the lower aqueous layer yields tannic acid on evaporation.

Properties. - A light yellowish, amorphous powder, usually in the form of glistening scales or spongy masses; odorless, or having a faint, characteristic odor, and a strongly astringent taste; gradually turning darker when exposed to air and light. Soluble in about 1 part of water, and in 0.6 
part of alcohol ; also in 1 part of glycerin, with the intervention of moderate heat; freely soluble in diluted alcohol; sparingly in absolute alcohol; almost insoluble in absolute ether, chloroform, benzol or benzin.

Incompatibles.-Alkaloids, alkalies, mineral acids, silver, ferric, lead and antimony salts, gelatin and emulsions.

Dose.-H. \& C., 3 ss.- 3 ss. (2.-15.); Sh. \& Sw., 3 ss.-i. (2.-4.); D., gr.i-xv. (.06-1.).

PREPARATIONS.

Collodium Stypticum. Styptic Collodion. (U. S. P.)

Tannic acid, 20; alcohol, 5; ether, 25; collodion, to 100 . Made by solution.

Unguentum Acidi Tannici. Ointment of Tannic Acid. (U.S. P.)

Tannic acid, 20; benzoinated lard, 80 .

Glyceritum Acidi Tannici. Glycerite of Tannic Acid.

(U. S. \& B. P.)

Tannic acid, 20; glycerin, 80 . (U.S. P.)

Administration.-Tannic acid is given in solution in aromatic waters, alcohol, syrup, wine, glycerin, and water; also it is exhibited in powder. The drug is used externally in powder, ointment, glycerite, and lotion. Suppositories containing tannic acid are sometimes introdnced into the rectum.

Action External.-Tannic acid coagulates albumin, gelatin, and fibrin. It has little effect upon the unbroken skin, but when applied to a raw surface or mucous membrane, it coagulates and dries secretion, and so fills up the mouths of glands, making the tissues harder, denser, and drier. Tannic acid causes a species of "tanning" compatible with life, by occasioning coagulation of the interstitial fluid in the tissues, abstraction of moisture, and contraction of the cells of the part. It is the most important astringent principle contained in vegetable drugs. Tannic acid is, moreover, a local styptic or hæmostatic in arresting hæmorrhage by contraction of the smooth muscles of the vessel walls, by coagulation of the blood, and constriction of the tissues surrounding the blood 
vessels. The production of vascular contraction is denied by some experimenters, but this statement is not substantiated. Tamic acid, although a slight local irritant to raw surfaces, exerts a depressing action upon the sensory nerve endings, and is essentially a sedative in inflammatory conditions by causing ischrmia. There are several kinds of tannic acid, possessing slightly different chemical and physiological properties. The official taunic acid - gallotannic acid-is contained in nutgall and oak bark, while another variety-catechutannic acid-is found in kino, catechu, etc.

Action Internal.-Tannic acid dries the mouth by closure of glandular (mucous) ducts with coagulated secretion, and by constriction of the surrounding parts. It lessens the flow of mucus and of the digestive juices in the stomach and intestines by the same process. The drug therefore interferes with digestion for this reason, and also because it precipitates pepsin; so that tannic acid should not be given after eating. Moreover, the astringent action is arrested in the stomach by combination with albuminous and gelatinous material. Large doses irritate the alimentary caual and may create vomiting and diarrhoea. Tannic acid is converted into gallic acid in the bowels and is absorbed and eliminated in the urine as gallic and pyrogallic acids. Gallic acid does not coagulate albumin or gelatin and has a very feeble astringent action, so that tannic acid should always be used for a local effect. The remote astringent influence of tannic acid (in the form of gallic acid) is slight, but gallic acid is preferable when an astringent action on the tissues of the body is desirable, since it is less irritating to the mucous membrane of the digestive organs. Catechu and kino are often chosen in place of tannic acid in the treatment of diarrhoea, becanse they are less solnble (than tannic acid) and the contained catechutannic acid comes in contact with the intestinal mucons membrane for a louger time. The salts of tannic acid (tannates) are not astringent.

Uses External.-Tannic acid is a valnable astringent in a great variety of local inflammatory lesions. In the form of 
the glycerite, tannic acid may be applied advantageously to the skin in moist eczema, and as a remedy for frost bites. It is a useful application for sore and cracked teats. The mouth is painted with the glycerite for the cure of ulcerative or apthous stomatitis. The same preparation is injected into. the ear in otorrhoea of dogs, and into the vagina to arrest vaginitis and lencorrhoea. Pure tannic acid is an excellent agent when dusted upon raw surfaces, ulcers, and sores; and to stop bleeding in slight wounds. An aqueous solution is useful in lencorrhœa (2-5 per cent.), in eczema (5-10 per cent.), as a high enema in dysentery (1 per cent.), and to kill ascarides (1-2 per cent.) in the rectum. A one per cent. aqueous solution is sometimes ntilized as an inhalation in subacute laryngitis, tracheitis, and bronchitis. Powdered opium and nutgall ointment (1-14), or glycerite of tannin, are serviceable in hæmorrhoids aud rectal fissures, ulcers, or prolapse of the rectum.

Uses Internal.-Tannic acid is exhibited in powder or solution to arrest bleeding in the stomach. It is a good astringent in diarrhœa and hæmostatic in intestinal hæmorrhage, given in ball or pill, and often with opium. Tannic acid is an antidote to alkaloids, metallic salts, and tartar emetic, forming comparatively insoluble tannates, which should be removed if possible by evacuation of the stomach.

Acidum Galicicum. Gallic Acid. $\mathrm{HC}_{7} \mathrm{H}_{5} \mathrm{O}_{5}+\mathrm{H}_{2} \mathrm{O}$.

(U. S. \& B. P.)

Synonym.-Acide gallique, Fr.; gallussäure, G.

An organic acid, usually prepared from tannic acid.

Derivation.-Made by the exposure of paste of nutgall and water to the air for a month, when tannic acid undergoes hydration (tannic acid) $\mathrm{HC}_{14} \mathrm{H}_{9} \mathrm{O}_{9}+\mathrm{H}_{2} \mathrm{O}=2 \mathrm{HC}_{7} \mathrm{H}_{5} \mathrm{O}_{5}$ (gallic acid). The liquicl is then expressed from the paste and the residue is boiled with distilled water and filtered, when hot, throngh animal charcoal. Gallic acid crystallizes ont from the filtrate. 
Properties.-White, or pale fawn-colored, silky, interlaced needles or triclinic prisms; odorless; having an astringent or slightly acidulous taste; permanent in the air. Soluble in 100 parts of water, and in 5 parts of alcohol; in 40 parts of ether, and in 12 parts of glycerin. Very slightly soluble in chloroform, benzol, or benzin.

Incompatibles.-Metallic salts and spirit of nitrous ether. Dose.-H. \& C., 3 ii.- 3 ss. (8.-15.); Sh. \& Sw., 3 ss.-j. (2.-4.); D., gr.v.-xx. (.3-1.3).

Action and Uses.--Since tannic acid is absorbed as gallic acid, and since the latter drug is less irritating, it follows that gallic acid is preferable when it is proposed to exert an astringent effect through the circulation. Gallic acid is a feeble local astringent, and therefore tannic acid is always a better agent for topical use. The remote astringent action of gallic acid is questionable, but successful results are alleged to have followed its administration in the treatment of hæmorrhage from the lungs, uterus and kidneys, and in polyuria, albuminuria, bronchorrhœea, leucorrhœe, and excessive sweating.

Ergot has been generally considered superior to gallic acid for the purpose of producing general constriction of the smaller vessels, and arrest of internal hæmorrhage.

\section{Prrogallol. Pyrogallol. $\quad \mathrm{C}_{6} \mathrm{H}_{3}(\mathrm{OH})_{3}$. (U. S. P.)}

Synonym.-Pyrogallic acid.

Derivation.-A triatomic phenol obtained chiefly by the dry distillation of gallic acid, $\mathrm{HC}_{7} \mathrm{H}_{5} \mathrm{O}_{5}=\mathrm{C}_{6} \mathrm{H}_{3}(\mathrm{OH})_{3}+\mathrm{CO}_{2}$.

Properties. -Light, white, shining laminæ, or fine needles; odorless, and having a bitter taste; acquiring a gray or darker tint on exposure to the air and light. Soluble in 1.7 parts of water, and in 1 part of alcohol; also soluble in 1.2 parts of ether. 
Actions and Uses.-Pyrogallol is an excellent agent for the treatment of chronic psoriasis and for ringworm. An ointment coutaining an amount of pyrogallol, varying from gr.x.- 3 i. to the ounce of lard, is commonly employed. Toxic symptoms may follow its extensive application.

\section{Quercus Alba. White Oak. (U. S. P.)}

Synonym.-Quercus cortex, B.P.; cortex quercus, écorce de chêne, Fr.; eichenrinde, G.

The bark of Quercus alba Linné (nat. ord. Cupuliferæ).

Habitat.-North America, in woods.

Description.-In nearly flat pieces, deprived of the corky layer, about $5 \mathrm{Mm}$. thick; pale brown; inner surface with short, sharp longitudinal ridges; tough ; of a coarse, fibrous fracture; a faint, tan-like odor, and a strongly astringent taste. As met with in the shops, it is usually in irregularly coarse, fibrous powder, which does not tinge the saliva yellow.

Constituents. - 1, quercitannic acid, 6 to 11 per cent; 2, a bitter principle, quercin; 3 , resin ; 4 , a sugar, quercite.

Dose.-H., $\overline{3}$ ss.-i. (15.-30.); C., $亏$ i.-ii. (30.-60.); Sh. \& Sw., 3 i.-ii. (4.-8.); D., gr.x.--xxx. (.6-2.).

Action and Uses. - White oak bark is identical in action with tannic acid, but the latter is preferable for internal use. Oak bark is a cheap substitute for tannic acid applied externally in poultices, infusions, and decoctions, as an astringent. It is administered internally in infusion, or decoction (1-8), in diarrhœa and dysentery. The infusion may be given in gruel and combined with ginger, opium and alcohol in the treatment of "scouring" in foals and calves.

\section{Catechu. Catechu. (U. S. \& B. P.)}

Synonym.-Catechu nigrum, terra japonica, cutch, E.; cachou, Fr.; katechu, pegucatechu, G. 
An extract prepared from the wood of Acacia Catechu (Linné filius) Willdenow (nat. ord. Leguminosæ).

Habitat.-India and Africa.

Properties.-In irregular masses, containing fragments of leaves, dark brown, brittle, somewhat porous and glossy when freshly broken. It is nearly inodorous, and has a strongly sweetish and astringent taste.

Constituents.-1, catechutannic acid (35-40 per cent.) is the active principle; it is converted into the isomeric inactive catechuic acid, or catechin $\left(\mathrm{C}_{21} \mathrm{H}_{20} \mathrm{O}_{9}+5 \mathrm{H}_{2} \mathrm{O}\right)$, by the saliva and by boiling, a red color being developed. There is also (2) catechu-red.

Incompatibles.-Metallic salts, alkalies, and gelatin.

Dose.-H., 3 ss.-i. (15.-30.); C., 3 i.-ii. (30.-60.); Sh. \& Sw., 3 i.-ii. (4.-8.); D., gi.v.- $\mathrm{xxx}$ (.3-2.).

\section{PREPARATIONS.}

Tinctura Catechu Composita. Compound Tincture of Catechu. (U. S. P.)

Made by maceration of catechu, 100; cassia cinnamon, 50; with: diluted alcohol to make 1000 .

Dose.-H. \& C., 3 i.-ii. (30.-60.); Foals, Calves and Sheep, 3 ss.-i. $(15 .-30)$; D., 3 ss.-ii. (2.-8).

\section{Tinctura Catechu. (B. P.)}

Same dose as compound tincture.

Pulvis C'atechu Compositus. (Kino, Rhatany and Catechu. B. P.)

Dose.-Same as catechu.

Administration.-The compound tincture, or an infusion. (made by pouring boiling water over catechu, digesting for an hour, and straining), and the powder, are employed internally. The powder is given in flour gruel. The powder, or an infusion of any strength may be applied externally.

Catechu is represented in the B.P. by catechu pallidum, an extract of the leaves and young shoots of Uncaria Gambier, Eastern Archipelago. It occurs in brown cubes, about an inch square, and possesses a bitter, astringent taste. It is 
employed in the same doses and for the same purposes as catechu.

Action and Uses.-The action of catechu is exactly like that of tannic acid. The latter is preferable for external use on account of its greater solubility and astringency. Catechu acts more slowly and persistently in the digestive tract, by virtue of its tardy solubility, and is a useful remedy in diarrhœa, particularly in that of a watery or serous nature. It is frequently prescribed in this disorder with other synergistic agents, as opium, ginger and chalk. Finlay Dun recommends the following combination: Catechu, prepared chalk, and ginger, each three ounces; powdered opium, six drachms. Divide into eight balls, for horses; into six doses suspended in starch gruel for cattle; and into eight or ten doses (given in gruel) for calves or sheep. The compound tincture of catechu with landanum is an equally suitable combination for all animals with diarrhœa, given in drench. If there is much mucus in the fæcal discharges, showing a catarrhal state of the intestinal mucous membrane, it is advisable to order oil, salts, or calomel before locking up the bowels with an astringent. Catechu has been given internally in dysentery, and to stop uterine and other hæmorrhages.

\section{Kino. Kino. (U. S. \& B. P.)}

The inspissated juice of Pterocarpus Marsupium Roxburgh (nat. ord. Leguminosæ).

Habitat.-East Indies. Kino, indigenous in the West Indies, is occasionally imported here.

Properties.-Small, angular, dark brownish-red, shining pieces; brittle, in thin layers, ruby-red and transparent; inodorous, very astringent and sweetish, tinging the saliva deep red. Soluble in alcohol; nearly insoluble in ether, and only slightly soluble in cold water.

Constituents. - The most important is (1) kinotannic acid $\left(\mathrm{C}_{18} \mathrm{H}_{18} \mathrm{O}_{8}, 75\right.$ per cent.), resembling catechu-tannic acid, but 
not identical with it. There are also: 2 , kinoin, a crystalline, neutral substance; 3 , pyrocatechin, $\mathrm{C}_{6} \mathrm{H}_{4}(\mathrm{OH})_{2} ; 4$, gum ; 5 , pectin ; 6 , kino-red, formed by oxidation from kinotaunic acid.

Incompatibles. - Mineral acids, metallic salts, strong solutions of alkaloids, alkalies, and gelatin.

Dose.-H., 亏 ss.-i. (15.-30.); C., ₹ i.-ii. (30.-60.); Sh. \& Sw., 3 i.-ii. (4.-8.); D., gr.v.-xxx. (.3-2.).

\section{PREPARATIONS.}

Tinctura Kino. Tincture of Kino. (U. S. \& B. P.)

Prepared by maceration and filtration of kino, 100, with glycerin, 150; and water, 200; and the addition of alcohol to make 1000. (U. S. P.)

Dose.-H. \& C., żi.-ii. (30.60.); Foals, Calves and Sheep, zss.-i. (15.-30.); D., 3 ss.-ii. (2.-8.).

Pulvis Kino Compositus. Compound Powder of Kino. (B. P.)

(Pulv. cinnamon, 4 grs.; kino, 15 grs.)

Dose.-Dog, 1 powder; Foals and Calves, 4 powders.

Administration.-Kino may be given in ball, powder, infusion (1-32), or tincture.

Action and Uses.-The physiological actions and therapeutics of kino are nearly similar to those of catechu. The gum and pectin contained in kino renders its effect milder and more sootling to mucons membranes. Aqueons solutions gelatinize on standing, on acconnt of the gum in them. The drug is prescribed chiefly in serous diarrhoa, and also. is occasionally exhibited in dysentery and internal hæmorrhages.

Krameria. Krameria.

Synonym. - Kramerix radix, B.P.; radix rhatanhiæ, rhatany root, E.; radix ratanhæ, P.G.; ratanhia, Fr.; ratanhawurzel, G.

The root of Krameria triandra Ruiz et Pavon, and of Krameria Ixina Linné (nat. ord. Polygaleæ).

Habitat.-Peru and Bolivia.

Description.-From 1 to $3 \mathrm{Cm}$. thick, knotty and several 
headed above, branched below, the branches long; bark smooth or in thinner pieces; scaly, deep-rust browu; 1 to 2 Mm. thick; very astringent; inodorous; wood pale brownish-red, tough, with fine medullary rays nearly tasteless. The root of Krameria Ixina is less knotty and more slender, and has a dark purplish brown bark abont $3 \mathrm{Mm}$. thick.

Constituents.-The chief principle is (1) kramero-tannic acid, $\mathrm{C}_{54} \mathrm{H}_{21} \mathrm{O}_{21}$, about 20 per cent. There are also : 2, rhatanin ; 3, rhatanic-red $\left(\mathrm{C}_{26} \mathrm{H}_{22} \mathrm{O}_{11}\right)$, the coloring matter.

Incompatibles.-Metallic salts, alkalies, gelatin, and lime water.

Dose.-H., 3 ss.-i. (15.-30.); C., 3 i.-ii. (30.-60.); Sh. \& Sw., 3 i.-ii. (4.-8.); D., gr.v.-xxx. (.3-2.).

PREPARATIONS.

Extractum Kramerice Fluidum. Fluid Extract of Krameria.

(U. S. P.)

Made by maceration and percolation of krameria with glycerin and diluted alcohol, and evaporation, so that $1 \mathrm{Cc} .=1 \mathrm{Gm}$. of the crude drug.

Dose.-Same as krameria.

Extractum Kramerice. Extract of Krameria. (U. S. \& B. P.)

Made by filtration, and evaporation of a cold, aqueous infusion to dryness.

Dose.-H. \& C., 3 ii.-iii. (8.-12.); Sh. \& Sw., gr.xxx.-xl. (2.-2.6); D., gr..$-\mathrm{x} .(.3-.6)$.

Tinctura Kramerice. Tincture of Krameria. (U.S. \& B. P.)

Made by maceration and percolation of krameria, 200 ; with diluted alcohol to 1000 . (U. S. P.)

Dose-H. \& C., ₹ i.-ii. (30.-60.) Foals, Calves and Sheep, 3 ss.-i. (15.-30.); D., 3 ss.-ii. (2.-8.).

Action and Uses.-Krameria and its preparations nearly resemble catechu and kino in all respects as astringents. The fluid extract is a serviceable preparation in. watery diarrhœa, and in arresting hæmorrhage from the stomach and bowels. An infusion (1-20, B.P.) is an efficient remedy for leucorrhœa when injected into the vagina. The powdered extract is blown into the uostrils, or applied to the rectum to stop bleeding in these parts. 
HæMatoxylon. Hæunatoxylón.

Synonym. - Hæmatoxyli lignum, B.P; logwood, E. ; lignum campechianum, P.G.; lignum cœruleum-bois de campêche, bois d'inde, bois de sang, Fr.; blanholz, campecheholz, G.

The heart wood of Hromatoxylon Campechianum Linné (nat. ord. Leguminosæ).

Habitat.-Central America; naturalized in the West Indies.

Description.-Heavy, hard, externally purplish-black, internally brownish-red, and marked with irregular, concentric circles, splitting irregularly; odor faint, agreeable ; taste sweetish, astringent; when chewed it colors the saliva dark pink. Logwood is generally met with in the form of small chips or coarse powder of a dark brownish-red color; often with a greenish lustre.

Constituents. - The most important is (1) tannic acid. There are also: 2, hrmatoxylin, $\mathrm{C}_{16} \mathrm{H}_{14} \mathrm{O}_{6}$ (12 per cent.), a coloring matter, but in nearly colorless crystals when pure. It turns red on exposure to light, and solutions are used to stain pathological specimens; 3 , hæmatein, $\mathrm{C}_{16} \mathrm{H}_{12} \mathrm{O}_{6}$, formed from hæmatoxylin by oxidation, and possessing a green, metallic lustre.

Incompatibles.-Lime water, and tartar emetic, with metallic salts, forms a blue compound.

PREPARATION.

Extractum Homatoxyli. Extract of Hæmatoxylon. (U. S. \& B. P.)

Made by boiling in water, straining, and evaporating to dryness.

Dose.-H. \& C., 3 ss.-iv. (2.-15.); Sh. \& Sw., 3 ss.-i. (2.-4.); D., gr.v.-xv. (.3-1.).

A non-official fluid extract is often found in commerce.

Dose.-Three times that of extract.

Action and Uses.--Hæmatoxylon is a mild astringent, coloring the freces and urine red during its elimination. The extract is given in diarrhœe of young animals, and may be combined for this purpose with aromatic sulphuric acid, 
ginger, chalk and opinm. It is also employed internally in dysentery, atonic indigestion, and in leucorrhoa. The decoction (1-16, B.P.) may be exhibited in Oss.-i. doses to the larger animals; in 3 i.-ii. doses to smaller patients.

\section{Hamamelis. Hamamelis. (U. S. P.)}

Synonym. - Witch-hazel.

The leaves of Hamamelis virginiana Linné (nat. ord. Hamamelaceæ), collected in autumn.

Description. - Short, petiolate, about $10 \mathrm{Cm}$. long, obovate or oval, slightly heart-shaped and oblique at the base, sinuate-toothed, thickish, nearly smooth; inodorous; taste astringent and bitter.

Constituents.-The most important principle is (1) tannic acid, 8 per cent; there are also: (2) a bitter substance, and (3) a resin.

\section{PREPARATIONS}

Extractum Hamamelidis Fluidum. Fluid Extract of Hamamelis. (U. S. P.)

Extractum Hamamelidis Liquidum. (B. P.)

Made by maceration and percolation with alcohol, glycerin and water, and evaporation, so that 1 Cc. $=1 \mathrm{Gm}$. of the crude drug. (U.S. P.)

Dose.-H. \& C., 亏3 i.-ii. (30.-60.); D., 3 ss.-ii. (2.-8.).

Action and Uses.-Hamamelis is apparently physiologically inert, as shown by experiments on healthy animals. It nevertheless possesses considerable medicinal virtue as an astringent and styptic. Witch-hazel is a valuable agent, applied externally, to stop venous oozing in wounds, and to reduce swelling and pain of bruises and sores. The fluid extract may be diluted with 8 , or less, parts of water, for these purposes; or the B.P. ointment (1-10) may be employed. Hamamelis is useful in diarrhœe and mucous discharges. It arrests hæmorrhage from the uterus, kidneys, lungs and digestive tract; sometimes in a surprising manner. The flnid extract is a successful hæmostatic in bleeding from the 
bladder or rectum (piles) when injected (1-8) into these parts. It lessens soreness and swelling of blind piles-with an equal part of glycerin and a little starch-and the same preparation is beneficial in eczema, pruritus, and cutaneous irritations. The clear, colorless, proprietary extracts (as Pond's) are often more efficient externally and internally (in the same doses) than the official extract.

\section{SECTION XIII.-VEGETABLE DEMULCENTS.}

Oleum Olfve. Olive Oil. (U. S. \& B. P.)

Synonym.-Sweet oil, E. ; oleum olivarum, P.G.; huile d'olive, Fr.; vilvenöl, G.

A fixed oil expressed from the ripe fruit of Olea Europoa Linné (nat. ord. Oleaceæ).

Habitat.-Southern Europe and Asia.

Properties. - A pale yellow, or light greenish-yellow, oily liquid, having a slight peculiar odor, and a nutty oleaginous taste, with a faintly acrid after-taste. Spec. gr. 0.9150.918. Very sparingly soluble in alcohol, but readily soluble in ether, chloroform, or carbon disulphide. Very frequently adulterated with cotton seed, or other seed oils, which probably are of equal medicinal value, however.

Constituents.-1, olein, $\mathrm{C}_{3} \mathrm{H}_{5}\left(\mathrm{C}_{18} \mathrm{H}_{33} \mathrm{O}_{2}\right)_{3}, 72$ per cent., a fluid oil, a combination of oleic acid $\left(\mathrm{HC}_{18} \mathrm{H}_{33} \mathrm{O}_{2}\right)$ and glyceryl; 2, palmitin, $\mathrm{C}_{3} \mathrm{H}_{5}\left(\mathrm{C}_{16} \mathrm{H}_{31} \mathrm{O}_{2}\right)_{3}$, about 28 per cent., a combination of palmitic acid $\left(\mathrm{HC}_{16} \mathrm{H}_{3} \mathrm{O}_{2}\right)$ and glyceryl; and (3) cholesterin $\left(\mathrm{C}_{26} \mathrm{H}_{44} \mathrm{O}\right)$.

Dose._Laxative-H. \& C., Oi.-ii. (500.-1000.); D., 3 ii.-iv. (60.-120.).

Oleum Gossypil Seminis. Cotton Seed Oil. (U. S. P.)

A fixed oil expressed from the seed of Gossypium herbaceum Iinné and of other species of Gossypium (nat. ord. Malvaceæ), and subsequently purified. 
Habitat.-S. United States and other semi-tropical countries ; cultivated.

Properties. - A pale yellow, oily liquid, withont odor, and having a bland, nut-like taste. Spec. gr. 0.920-0.950. Very sparingly soluble in alcohol, but readily soluble in ether, chloroform, or carbon disulphide.

Constituents.-1, olein; 2, palmitin; 3 , coloring matter.

Dose.-Same as that of olive oil.

Action and Uses.-Olive oil is in common use as an emollient in burns and skin irritation. It assists in the performance of massage for sprains and bruises. Cotton seed oil has superseded it in liniments, as a matter of economy. Administered internally, sweet oil (with an equal part of castor oil) is a useful laxative for dogs. Linseed oil is more frequently given to the larger animals. An enema of $\frac{1}{2}$ pint, or more, of olive oil is serviceable in softening hard freal masses in dogs, and should be followed by the use of warm soap suds.

Sweet oil is a food, but is rarely used as such. Like other bland oils, it improves the nutrition of the bronchial mucous membrane in subacute or chronic bronchitis, and is of considerable benefit in these disorders, but inferior to cod liver oil or linseed oil. Olive oil is an efficient demulcent in. inflamed conditions of the alimentary tract, and in poisoning by irritants. Large quantities form soap-like masses,-with the alkaline intestinal juices,-which have been mistaken for gall stones. Cotton seed oil is of equal therapentic. value with sweet oil.

\section{Sapo. Soap.}

Synonym.-Sapo duris, B.P.; hard soap, white castile soap, E.; savon, Fr.; seife, G.

Derivation.-Soap is made by boiling olive oil with a solution of canstic soda, $\mathrm{C}_{3} \mathrm{H}_{5}\left(\mathrm{C}_{18} \mathrm{H}_{33} \mathrm{O}_{2}\right)_{3}$ (olein) $+3 \mathrm{NaOH}=$ $3 \mathrm{NaC}_{18} \mathrm{H}_{33} \mathrm{O}_{2}$ (sodium oleate or soap) $+\mathrm{C}_{3} \mathrm{H}_{5}(\mathrm{OH})_{3}$ (glycerin).

Properties.-A white, or whitish solid, hard, yet easily cut when fresh; having a faint, peculiar odor free from 
rancidity; a disagreeable alkaline taste, and an alkaline reaction. Soluble in water and in alcohol; more readily with the aid of heat.

\section{PREPARATIONS.}

Linimentum Saponis. Soap Liniment. (U. S. \& B. P.)

Synonym.-Opodeldoc. Lin. sapo. campl.

Soap, 70; camphor, 45 ; oil of rosenary, 10 ; alcohol, 750 ; water to make 1000. Made by solution, agitation and filtration. (U.S. P.)

Emplastrum Saponis. Soap Plaster. (U. S. P.) ation.

Soap, 100; lead plaster, 900; made by solution in water and evapor-

Sapo Molurs. Soft Soap. (U. S. \& B. P.)

Synonym.-Potassium oleate, sapo viridis, green soap.

A soap prepared from potassa and a fixed oil.

Derivation.-Heat linseed oil, 400 , to $140^{\circ} \mathrm{F}$. Dissolve potassa, 90, in water, 450 ; add alcohol, 40 ; and stir the mixture into the oil at the same temperature until it is soluble in boiling water without the separation of oily drops.

Properties.-A soft, unctuous mass, of a yellowish-brown or brownish-yellow color. Soluble in about 5 parts of hot water; also in 2 parts of alcohol without leaving more than 3 per cent. of insoluble residue.

PREPARATION.

Linimentum Saponis Mollis. Liniment of Soft Soap. (U. S. P.)

Synonym.-Tinctura saponis viridis.

Soft soap, 650 ; oil of lavender flowers, 20 ; alcohol, 300 ; water to make 1000. Made by solution and filtration.

Castile soap is the best example of a pure soap. Mottled castile soap contains iron as the coloring matter. The household "soft soap" is not sapo mollis, but is made of all kinds of rancid fats and is generally unfit for medicinal use. Yellow laundry soap owes its color to resin. Super-fatted soaps are of neutral reaction and unirritating. They are used as a basis for medicinal soaps containing tar, carbolic acid, etc. 
Action and Uses.-Most soaps are alkaline. Soap is a detergent or cleansing agent. The lather mechanically removes dirt, while the alkalinity assists in the removal of grease, dead epidermis, and sebaceous matter from the skin. The canstic alkali contained in soap relieves itching and is stimulating to the skin; so much so, that cheap soaps are harmful in normal conditions of the integnment.

Liniment of soft soap is frequently employed in chronic eczema and psoriasis, to remore scales and crusts; to stimulate the parts; and to quiet itching. It should be rubbed smartly into the skin, washed off, and followed by the application of a suitable ointment. Gauze saturated with soap suds (soap suds poultice) is an excellent agency to cause the exfoliation of the epidermis in patches of old scaly eczema and psoriasis, when applied for several hours. Soft soap, oil of cade, and alcohol, equal parts, are recommended as a useful preparation for the treatment of chronic eczema and pruritus. The application of soap and water is a necessary preliminary to the employment of a vesicating ointment, or parasiticide, since it cleanses the skin, and, by removing. epidermis, exposes the burrows of acari in mange and scab. Soap liniment is a favorite remedy for sprains and bruises. If a more stimulating action is desirable, it is advisable to combine oil of turpentine or water of ammonia with it. If an anodyne effect is indicated, tincture of aconite or opium are added.

Chafing of the skin produced by harness, should be treated by washing the skin with soap and water, and then by dusting with zinc oxide and starch, equal parts. Sapo mollis, together with an equal amount of flour of mustard, forms a most satisfactory cleansing and disinfectant mixture for the hands of the operating surgeon when employed in the same manner as ordinary soap. Soap may also be used as a lubricating agent for the hands or instruments in making examinations. Soap is a useful excipient for balls, pills, and plasters, and it is a constituent of liniiments. 
Soap is employed both as a qualitative and quantitive test for hard water. This contains salts of the alkaline eartl metals, as sulphates and carbonates of magnesium and calcium. Soap is decomposed by these salts, and insoluble soaps, i.e., calcium and magnesium stearate, are precipitated. The free alkali of the soap is then converted into insoluble sulphates and carbonates. These reactions produce a milky precipitate when a solution of soap is added to hard water.

Internally soap is an antacid and somewhat irritating, and may occasion vomiting and stimulation of intestinal peristalsis. These actions are taken advantage of in emergencies, when it may be given to dogs as an emetic, or to all animals in poisoning by acids. A piece of soap, when shaped by the hands into a conical form, dipped an instant into water, and introduced into the rectum, is one of the best agents for moving the bowels in the case of puppies and all young animals. Enemata of soap suds are in every day use. A mixture of sapo mollis, molasses, and water, in varying proportions, is a more efficient preparation. Oil of turpentine may be added in flatulence.

\section{Glycerinum. Glycerin. $\mathrm{C}_{3} \mathrm{H}_{5}(\mathrm{OH})_{3}$. (U. S. \& B. P.)}

Synonym. - Glycerine, E. ; glycerine, Fr.; glycerin, œlsüss, G.; glycerinum, P.G.

Derivation.-A liquid obtained by the decomposition of vegetable or animal fats or fixed oils, and containing not less than 95 per cent. of absolute glycerin. It occurs as a byeproduct in the manufacture of soap, but is made chiefly from palm oil by the action of superheated steam at a temperature of about $600^{\circ} \mathrm{F}$.

Properties.-A clear, colorless liquid, of a thick, syrupy consistence, oily to the touch, odorless, very sweet and slightly warm to the taste. Spec. gr. not less than 1.250. Soluble in all proportions in water or alcohol; also soluble in a mixture of 3 parts of alcoliol and 1 part of ether, but 
insoluble in ether, chloroform, carbon disulphide, benzin, benzol, and fixed and volatile oils. Reaction nentral. Glycerin is a solvent for alkaloids, digestive ferments, fixed alkalies, bromine, iodine, tannin, extracts, salicin, borax, boric acid, carbolic acid, etc.

$$
\text { Dose.-H. \& C., } 3 \text { i. (30.); D., } 3 \text { ss.-i. (2.-4.). }
$$

PREPARATIONS.

Glyceritum Amyli. Glycerite of Starch. (U. S. \& B. P.)

Starch, 10; water, 10; glycerin, 80. Made by solution with heat. There are also official glycerites of carbolic acid (1-4), of tannic acid (1-4), of boroglycerin (31 per cent.), of hydrastis, and of yolk of egg (glyceritum vitelli or glyconin, 45 per cent).

Suppositoria Glycerini. Suppositories of Glycerin. (U. S. P.)

Glycerin, $60 \mathrm{Gm}$. ; sodium carbonate, $3 \mathrm{Gm}$.; stearic acid, $5 \mathrm{Gm}$. Made by solution with heat and moulded into ten suppositories containing $6 \mathrm{Gm}$. or 3 i.ss. each.

Action External.-Glycerin is hydroscopic, emollient, sometimes parasiticidal, and antiseptic. It does not evaporate or become rancid. The clief medicinal value of glycerin depends upon its affinity for water, so that (in solution) it keeps moist the surface to which it is applied. Pure glycerin is, however, slightly irritant to the skin and may canse some inflammation of raw surfaces and mucous membranes on account of withdrawal of water from the tissues. It should therefore be diluted with water for most therapeutic purposes.

Action Internat. - Glycerin is absorbed but is only slightly oxidized in the body, and is of little value as a nutritive. It may give rise to a substance in the urine which reduces cupric oxide and renders the sugar test positive. It is somewhat antiseptic in the digestive tract, and appears to inhibit the formation of glycogen in the liver in some cases of glycosuria. Large doses are slightly purgative. Enormous quantities cause poisoning in animals, with the production of lææroglobinuria, muscular weakness, dryness of the mucous membranes, collapse, and death. 
Large amounts injected into the blood have occasioned convulsions.

Uses External. - These are mauifold. It is largely employed in lotions, ointments, and as a vehicle for the substances of which it is a solvent. Glycerite of starch is a successful remedy for rough, dry skin, and scaly eczema. Glycerite of carbolic acid is an appropriate application for fetid sores and nlcers, and, diluted with an equal part of glycerin, will destroy the acari of mange and scab. It should be used with caution to prevent poisoning. Glycerite of boroglycerin is an excellent preparation for the treatment of apthous stomatitis and thrush. In dryness of the meatus, and in canker of the ear (otorrhœa) in dogs, a mixture of tincture of iodine, 1 part, and glycerin, 4 parts, is recommended. Scratches and cracked heels of horses, fissured and excoriated surfaces, and erythema, are successfully treated with the following prescription:

B

Tinc. Opii.......................... 3 i.

Liq. Plumbi Subacetat.................

Glycerini........................... 3 ii.

M.

Aquæ.............................. 3 viii.

S. Apply externally.

Uses Internal._Glycerin is employed as an excipient for balls and pills, and as a vehicle for nauseous and irritating drugs. It is not a valuable remedy for internal use, but is sometimes given with the food to prevent intestinal fermentation and relieve flatulence. It may prove curative in cases of glycosuria; and in trichinosis when given by the month, and in high rectal injections after active purgation. Glycerin is a useful addition to congh mixtures in moistening and soothing the throat, and in not interfering with digestion. When injected into the rectum in quantities of $\bar{\xi}$ iv.-vi. for horses, or $\bar{z}$ ss.-i for dogs, it often causes prompt evacuation of the lower bowel. The suppositories may be employed in canine practice. 


\section{Glycyrrhiza. Glycyrrhiza.}

Synonym.-Glycyrrhize radix, B.P.; liquorice or licorice root, E.; réglisse, bois de réglisse, boix doux, racine douce, Fr.; spanisches süssholz, spanische süssholz-wurzel, G.

The root of Glycyrrhiza glabra Linné and of the variety glandulifera (Waldstein et Kittaibel) Regel et Herder (nat. ord. Legumiuoræ).

Halitat.-S. Europe and W. Asia; cultivated.

Description.-In long, cylindrical pieces, from 5 to 25 Mm. thick; longitudinally wrinkled,. externally grayishbrown, warty; internally tawny-yellow; pliable, tough; fracture coarsely fibrous; bark rather thick; wood porous but dense, in narrow wedges; medullary rays linear; taste sweet, somewhat acrid. The drug derived from the variety glandulifera (so-called Russian liquorice) consists usually of roots or root-branches 1 to $4 \mathrm{Cm}$. thick, 15 to $30 \mathrm{Cm}$. long, frequently deprived of the corky layer, the wood rather soft, and usually more or less cleft.

Constituents.-1, a yellow, sweet, amorphous glucoside, glycyrrhizin $\left(\mathrm{C}_{24} \mathrm{H}_{35} \mathrm{O}_{9}\right)$, abont 6 per cent.; 2, glycyramin; 3 , asparagin, about 3 per cent.; 4 , an acrid resin; 5 , starch; 6 , glucose.

\section{PREPARATIONS.}

Extractum Glycyrhizøe Fluidum. Fluid Extract of Glycyrrhiza.

Made by maceration and percolation with water of ammonia, alcohol and water, and evaporation, so that $1 \mathrm{Cc} .=1 \mathrm{Gm}$. of the crude drug.

Dose of the root or fluid extract is unimportant.

Extractum Glycyrrhizce Liquidum. (B. P.)

Dose.-Unimportant

Action and Uses.-Liquorice is demulcent and slightly laxative. The powderel ront is employed as an excipient in making electuaries, since it is soothing to the throat. It is also used as an excipient in the preparation of balls, and more or less successfully conceals, in the form of the fluid 
extract, the taste of aloes, cascara sagrada, ammonium chloride, turpentine, hyoscyamus and quinine sulphate.

\section{Linum. Linseed.}

Synonym.-Lini semina, B.P.; flaxseed, E.; semence de liu, Fr.; leinsamen, flachssamen, G.; semen lini, P.G.

The seed of Linum usitatissimum Linné (nat. ord. Lineæ).

Habitat. -Most temperate climates.

Description.-Abont. 4 or $5 \mathrm{Mm}$. long, oblong-ovate, flattened, obliquely pointed at one end; brown, glossy, covered with a transparent, mueilaginous epithelium, which swells considerably in water; the embryo whitish or pale greenish, with two large oily, planoconvex cotyledons and a thin perisperm; inodorous; taste mucilaginous, oily and bitter.

Constituents.-1, linseed oil, 30 to 35 per cent. in the nucleus; 2, gum, 15 per cent. in the epidermis ; 3 , proteids, 25 per cent.; 4 , a trace of amygdalin.

Action and Uses.-Linseed is a food. Oil cake or linseed cake from which the oil has been expressed, is exceedingly rich in protein (25-30 per cent.), and is also richer in fat (10 per cent.) than most foods. Cottonseed meal, which contains considerably more protein and fat, is more frequently employed in the United States. Gruel made from crushed linseed meal cake, or linseed meal, is useful as a restorative in all animals recovering from acute and debilitating diseases. The cake $(1 \mathrm{lb}$.), or a pint of cottonseed meal per diem, is a good addition to the ordinary fodder for horses suffering from malnutrition, with rough staring coats and dry skin, and for those affected with "broken wind." Gruel of linseed meal or cake is also serviceable for calves or lambs when reared on skimmed milk or other poor food. Linseed tea, made by steeping 1 part of whole linseed in 20 parts, by weight, of boiling water, for 1-4 hours, followed by straining, is a valuable demulcent preparation in pharyngitis, 
bronchitis, gastro-enteritis, and is possibly useful in acute cystitis and nephritis. It may be given in any amount. which an animal will take voluntarily. The mucilage contained in linseed tea cannot be carried throngh the blood. and eliminated by the kidneys, so that it must act by virtue of the water contained in it and perhaps by some intrinsic diuretic property.

The addition of a few drachms of gum arabic to the quart of linseed tea will improve the demulcent action. Linseed, linseed meal or farina lini is the best substance to use in the preparation of poultices. It should be mixed with an equal quantity of bran, when the poultice is applied directly to the part. If the ponltice is enclosed in a bag, the ontside should be oiled to prevent its sticking to the skin. Linseed meal, mixed with an equal amount of molasses, forms a common excipient for ball masses. Linseed tea, made thicker than usual, is a good local application in irritation of the rectum, or vagina.

\section{ACACIA. Acacia.}

Synonym.-Acaciæ gummi, B.P.; gum arabic, E.; gomme . arabique, Fr.; arabisches gummi, $\mathrm{G}$.

A gummy exudation from Acacia Senegal Willdenow (nat. ord. Leguminosæ).

Habitat.-N., E., and W. Africa.

Properties.-In roundish tears of various sizes, or broken into angular fragments with a glass-like, sometimes iridescent fracture; opaque from numerous fissures, but transparent and nearly colorless in thin pieces ; nearly inodorous; taste insipid, mucilaginous ; insoluble in alcohol, but soluble in 2 parts of water, forming a thick, mucilaginous liquid.

Constituents.-Arabiu or arabic acid $\left(\mathrm{C}_{12} \mathrm{H}_{22} \mathrm{O}_{11}\right)$, in combination with about 3 per cent. of magnesium, potassium and calcium.

Incompatibles. - Alcohol, ferric salts, lead subacetate, borax, and sulphuric acid. 
Preparations.-Mucilago acaciæ, U. S. \& B. P. (34 per cent., U. S.), and syrupus acaciæ, U. S. P. (25 per cent.)

Dose.-Ad lib.

Action and Uses.-Gum arabic is but slightly nutritious. It is a useful demulcent in covering and protecting inflamed mucous membranes of the upper respiratory and digestive tracts. It may be given freely in water, but large quantities may undergo fermentation and cause indigestion and diarrhoea. A 10 per cent. aqueous solution is sometimes injected into the bladder, vagina or rectum in inflammation of these parts. Acacia is chiefly of value in medicine for the preparation of mixtures, emulsions, pills, balls and electuaries. About 3 iii. of mucilago acaciæ are required to suspend $\tilde{3}$. of oil or resinous tincture. Acacia is sometimes prescribed in genito-urinary irritation. Animals will voluntarily drink aqueous solutions.

Tragacantha. Tragacanth. (U. S. \& B. P.)

Synonym.-Gomme adragante, Fr.

A gummy exudation from Astragalus gummifer Labillardière, and from other species of Astragalus (nat. ord. Leguminosæ).

Habitat.-Asia Minor.

Properties.-In narrow or broad bands, more or less curved or contorted, marked by parallel lines or ridges; white or faintly yellowish, translucent, horn-like, tough, and rendered more easily pulverizable by a heat of $122^{\circ} \mathrm{F}$. Very sparingly soluble in water, but swells into a gelatinous. mass which is tinged blue with iodine.

Constituents. - 1, arabin, 53.3 per cent., not identical with arabin of acacia, however; 2, basisorin $\left(\mathrm{C}_{6} \mathrm{H}_{10} \mathrm{O}_{5}\right), 33.1$ per cent., a gum, swells up with water but does not dissolve ; 3 . starch ; 4, ash.

\section{PREPARATION.}

Mucilago Tragacanthce. Mucilage of Tragacanth. (U. S. \& B. P.)

Tragacanth, 6; glycerin, 18; water to make 100 . (U. S.)

Dose.-Ad. lib. 
Action and Uses.-Tragacanth is a demulcent, but is chiefly used in the preparation of mixtures and emulsions to suspend oils, resins and insoluble powders.

\section{AlthæA. Althæa. (U. S. P.)}

Synonym.-Marshmallow root, E.; racine de guimauve, Fr. ; altheewurzel, eibischwurzel, G.; radix althæae, P.G.

The root of Althæa officinalis Linné (nat. ord. Malvaceæ).

Habitat.-N. and W. Asia and Europe. Cultivated in Europe, aud naturalized in E. United States and Australia, growing in salt marshes.

Constituents. - 1, bassorin, 35 per cent.; 2, pectin, 10 per cent.; 3 , asparagin, 1 per cent.; 4 , sugar.

\section{PREPARATION.}

Syrupus Althaece. Syrup of Althaea. (U. S. P.) 1000.

Althæa, 50 ; alcohol, 30 ; glycerin, 100 ; sugar, 700 ; water to make

Dose.-Ad lib.

Action and Uses.-Althæa is occasionally employed as a demulcent in irritable conditions of the digestive canal, and as a vehicle in the form of syrup.

\section{Saccharum. Sugar. $\mathrm{C}_{12} \mathrm{H}_{22} \mathrm{O}_{11}$. (U. S. P.)}

Synonym.-Saccharum purificatum, B.P.; 'refined sugar, cane sugar, E.; sucre, sucre de canne, Fr.; zucker, rohrzucker, G.

The refined sugar obtained from Saccharum officinarum Linné, and from various species or varieties of sorghum (nat. ord. Gramineæ); also from one or more varieties of Beta vulgaris Linné (nat. ord. Chenopodiaceæ).

Habitat.-Indigenous in S. Asia, but cultivated in many tropical and sub-tropical countries.

Properties. - White, hard, dry, distinctly crystalline granules, odorless, and having a purely sweet taste. Permanent in the air. Soluble in 0.5 part of water; in 0.2 part 
of boiling water, and in 175 parts of alcohol. Insoluble in ether, chloroform, or carbon disulphide.

PREPARATION.

Syrupus. Syrup. (U. S. P.)

Synonym.-Simple syrup, E.; sirop de sucre, Fr.; weisser syrup, G.; syrupus simplex, P.G.

Made by solution of sugar, 850 ; with heat in distilled water, straining, and addition of distilled water to make 1000. (U. S. P.)

\section{Molasses. (Non-official.)}

Synonym.-Theriaca, B.P.; sacchari faex, syrupus fuscus, treacle, E.; mélasse, Fr.; melasse, G.

The brown, uncrystallizable syrup that drains away from the crystals of raw sugar in the refining process.

Action and Uses.-Sugar, syrup and molasses are demulcents, and are sometimes employed in medicated syrup or electuary, for their soothing action on the throat in catarrh of the upper air passages. They are liable to ferment in the alimentary canal if given contmuously, with the production of acidity and indigestion, so that they are not suitable for general use às demulcents. Sugar, syrup and molasses are mainly useful as vehicles, corrigents, preservatives, and excipients in pharmacy. Sugar is utilized as a constituent. of powders, and syrup and molasses are excipients in the preparation of balls and electuaries. Sugar increases the solubility of calcinm salts (see Syrupus Calcis, p. 156) and protects ferrons compounds from oxidation (see Ferri Carb. Sacch., p. 194).

Sugar is an antiseptic, and, in syrup, prevents the fermentation of active medicinal substances. Brown sugar and molasses are laxative, in large doses, and are prescribed in veterinary practice, with ginger, to aid the action of salts on cattle (Oss.-i.) and sheep ( 3 ii.-vi.). (See Epsom salts, p. 163.); 


\section{SECTION XIT.-VEGETABLE DRUGS KILLING PARASITES.}

\section{Class 1.-Used to Destroy Tape-Worms.}

\section{Aspidium. Aspidium. (U. S. P.)}

Synonym.-Felix mas, B.P.; radix filicis maris, male fern, male shield fern, E.; rhizome (racine) de fougère mâle, Fr.; wurmfarnwurzel, waldfarnwurzel, johanniswurzel, G. ; rhizoma filicis, P.G.

The rhizome of Dryopteris Felix-mas Schott, and of Dryopteris Marginalis Asa Gray (nat. ord. Filices).

Habitat.-D. filix-mas, Europe ; D. marginalis, U. S.

Description. - From 5 to $15 \mathrm{Cm}$. long, 10 to $25 \mathrm{Mm}$. in thickness, and, together with the closely imbricated, darkbrown, roundish, and slightly curved stipe-remnants, 50 to $75 \mathrm{Mm}$. in diameter; densely curved, with brown, glossy, transparent and soft, chaffy scales; internally pale-green; rather spongy; vascular bundles about ten (Dryopteris filixmas) or six (Dryopteris marginalis) in number, arranged in an interrnpted circle; odor slight, but disagreeable; taste sweetish, acrid, somewhat bitter, astringent and nanseous.

Constituents. - The active principle is (1) filicic acid $\left(\mathrm{C}_{35} \mathrm{H}_{42} \mathrm{O}_{13}\right)$, a white, amorphous crystalline substance; there are also : 2 , a fixed oil, 6 per cent.; 3 , resin, 4 per cent.; 4 , filicin $\left(\mathrm{C}_{35} \mathrm{H}_{40} \mathrm{O}_{12}\right)$, a crystalline principle soluble in chloroform, benzol, fixed and volatile oils ; 5 , filix-red, a coloring matter ; 6 , a small quantity of a volatile oil.

Dose.-H. \& C., 亏 v.-vi. (150.-180.); Sh., 亏 ii.-iv. (60.-120.); Lambs, 3 i.-ii. (4.-8.); D. \& C., 3 ss.-i. (15.-30.).

\section{PREPARATION.}

Oleoresina Aspidii. Oleoresin of Aspidium. (U. s. P.)

Made by percolation with ether, distillation and evaporation of the ether.

Dose (also of the extractum filicis liquidum, B. P.)-H. \& C., 3 iii.-vi .(12.-24.); Sh. \& Sw., 3 i.-ii. (4.-8.); D. \& C., $\pi \times \nabla .-3$ i. (1.-4.) 
Action and Uses.-Aspidium is chiefly of value in veterinary medicine as a treniacide or agent destroying tape-worms, particularly those inhabiting dogs. Large quantities of the drug cause hæmorrhagic gastro-enteritis, tremors, weakness, stupor, coma, acute nephritis and cystitis. Six drachms of the oleoresin have proved fatal in man and sheep; five drachms in a medium-sized dog; and three ounces in the case of a cow. Aspidium must never be given with oil, which aids its absorption. Dogs should be fasted 24 hours or fed on a little milk; tlien the oleoresin should be administered, and the dose repeated in 3 hours. After the expiration of 12 hours from the administration of the first dose, a purgative quantity of castor oil is to be exhibited. An injection of salt and water assists the expulsion of segments of trnia from the rectum. If the head of the tænia is not expelled the treatment may be repeated in three days or a week. The oleoresin may be flavored with a few drops of oil of peppermint, and is often combined with a small dose of areca nut (gr.i. to the lb. live weight) in emulsion with mucilage of tragacanth, or with fluid extract of kousso, $3 \mathrm{i}$. to $3 \mathrm{ii}$. The oleoresin may also be exhibited in pills or capsules. It is on the whole the best agent against the tapeworms of dogs, including Tænia serrata, T. marginata, T. cœnurus and T. echinococcus.

\section{AreCA. (Non-official.)}

Synonym.-Areca-nut, betal-nut, E. ; noix d'aréque, Fr.; areca-nuss, G.

The seed of Areca Catechu (nat. ord. Palmacex).

Habital.-India, Coromandel and Malabar coasts: also in warm parts of Asia.

Description.-The seeds resemble nutmeg in size, shape and color. They yield a brown powder, partially soluble in water and alcohol. The taste is astringent.

Constituents. - 1 , the active principle is the liquid alkaloid, arecoline $\left(\mathrm{C}_{8} \mathrm{H}_{13} \mathrm{NO}_{2}\right)$; arecoline hydrobromate is the 
commercial salt, ocenrring in white crystals, soluble in alcohol and water; dose-H. \& C., gr. $\frac{1}{3}-\mathrm{l}$ (.02-.06), subcut.; 2 , an inert alkaloid ; 3 , red tannic acid; 4 , an oil.

Dose.-Areca nut-H., 3 ss.-i. (15.-30.); Lamb, 3 i. (4.); D., gr.ii. for each lb. of live weight, or gr.xv.- 3 ii. (1.-8.); Fowl (against A. gibbosa), gr.x. (.6) in pill.

Action and Uses.-Areca nut is an anthelmintic more commonly classed as a tæniacide, but capable of killing round-worms satisfactorily. It acts more successfully as a vermicide in dogs than in the case of the larger animals. Areca nut is an astringent in small doses, but large amonnts induce catharsis. When the drug is used as an anthelmintic the animal should be deprived of food for 24 hours previous to its administration. The powder is given to dogs in milk, frequently with oleoresin of male fern in small quantity. If purgation does not follow the use of areca nut within a short time, a dose of castor oil is indicated. The fluid extract is a more convenient preparation.

Arecoline hydrobromate $\left(\mathrm{C}_{8} \mathrm{H}_{13} \mathrm{NO}_{2} \mathrm{H} \mathrm{Br}\right)$ has been recently employed subcutaneously-H., gr. $\frac{1}{3}-\mathrm{i} .(.02-.06)$; D., gr. $\frac{1}{60}-\frac{1}{12}(.001-.005)$-as a rapidly-acting cathartic, exciting peristalsis, in colic and indigestion of horses, and in gastritis ('Fardel bound") of ruminants. Experiments have been made I. M Muir* with arecoline to prove its efficacy as a rapidly acting cathartic. He finds that while the drug produces salivation, it does not markedly increase intestinal secretion, but acts chiefly by stimulating peristalsis; that it frequently causes severe colic and nausea; that there is also incontinence of urine; that the pulse becomes slower and softer, and that sweating and reduction of temperature occur during its action; that arecoline hydrobromate may be safely given in the dose of $\frac{1}{3}$ grain (0.02) intravenously or subcutaneously, and repeated in an hour if necessary. Finally, arecoline is less desirable than eserine on account of the distressing symptoms accompanying its purgative

* Journal of Comp. Med. and Vet. Archives, Jan. and Feb., 1899. 
action, and is still less so than barium chloride, which causes no unpleasant effects at all in most cases and appears to be the most satisfactory of the rapidly acting cathartics which may be given under the skin. In 1 per cent. solution the alkaloid is used in the eye as a myotic.

\section{Kamala. Kamala. (U. S. \& B. P.)}

Synonym.-Rottlera.

The glands and hairs from the capsules of Mallotus philippinensis (Lamarck) Mueller Arg. (nat. ord. Euphorbiacer).

Hirbitat.-India, China and the Philippine Islands.

Properties. - A granular, mobile, brick-red or brownishred powder, inodorous and nearly tasteless; imparting a deep red color to alkaline liquids, alcohol, ether or chloroform, and a pale yellow tinge to boiling water. Under the microscope it is seen to consist of stellately arranged, colorless hairs, mixed with depressed-globular glands, containing numerous red, club-shaped vesicles.

Constituents. - The chief principle is (1) rottlerin $\left(\mathrm{C}_{22} \mathrm{H}_{20} \mathrm{O}_{6}\right)$, occurring in yellow acicular crystals, soluble in hot alcohol, ether, benzol, and carbon disulphide. There are also (2) resins, 80 per cent.

Dose.-D., 3 ss.-ii. (2.-8.); H. \& C., 3 i. (30.).

Action and Uses.-Kamala is an antlelmintic. It is employed more frequently as a tæniacide, but will also kill ascarides and oxyurides. Large doses may give rise to nausea and vomiting in dogs and cats. Kamala is also a purgative, so that it is rarely necessary to employ one after its administration. It should be given in syrup to the fasting animal, and repeated in eight hours if the first dose is not operative by that time. 


\section{Cusso. Kousso. (U. S. \& B. P.)}

Synonym.-Brayera, kooso, kusso, E.; cousso, kousso, Fr.; kosso, cusso, kusso, G.; flores kosso, P.G.

The female inflorescence of Hagenia abyssinica (Bruce) Gmelin (nat. ord. Rodaceæ).

Habitat.-Abyssinia.

Description.-In bundles, rolls, or compressed clusters consisting of pannicles about $25 \mathrm{Cm}$. long, with a sheathing. bract at the base of eacly branch; the two roundish bracts at the base of each flower, and the four or five obovate, outer sepals are of a reddish color, membranous and veiny; calyx top-shaped, hairy, euclosing two carpels or nutlets; odor slight, fragrant and tea-like; taste bitter, acrid and nauseous.

Constituents. - 1, the active principle is kosin or koussin, a yellow, tasteless, crystalline glucoside, soluble in alcohol, chloroform, benzol and ether, but insoluble in water ; dosedogs, gr.x.-xl. (.6-2.6); 2 , a volatile oil ; 3 , gum; 4, tannic acid; 5 , two resins. (8.-15;)

Dose.-Small dogs, 3 ss.-i. (2.-4.); large dogs, 3 ii.-iv.

\section{PREPARATION.}

Extractum Cusso Fluidum. Fluid Extract of Kousso. (U. S. P.)

Synonym.-Exiractum brayeræ fluidum.

Made by maceration and percolation of kousso with alcohol, and evaporation, so that $1 \mathrm{Cc} .=1 \mathrm{Gm}$. of the crude drug.

Dose.-Same as kousso.

Action and Uses.-Konsso is an effective tæniacide in dogs and cats. Large doses cause nansea, colicky pains and some catharsis. Kousso is administered in milk, or as an infusion flavored with peppermint; also in the form of the fluid extract, or glucoside in capsules, to the fasting animal. It should be repeated 3 times, at hour intervals, and followed by a small dose of castor oil if the bowels are not sufficiently relaxed. There is little danger of poisoning eren by great. quantities of the drug. 


\section{Granatum. Pomegranate.}

Synonym.-Granati radicis cortex, B.P.; écorce de la racine de grenadier (de balaustier), Fr.; granat-wurzelrinde, G.; cortex radicis granati, P.G.

The bark of the stem and root of Punica Granatum Linné (nat. ord. Lythrarier).

Habitat.-India and S. W. Asia. Also cultivated and naturalized in sub-tropical countries.

Description.-In thin quills or fragments, from 5 to 10 $\mathrm{Cm}$. long, and from 1 to $3 \mathrm{Mm}$. thick; outer surface yellowishgray, somewhat warty, or longitudinally and reticulately rigid; the stem-bark often partly covered with blackish lichens; the thicker pieces of the root-bark more or less scaly externally; inner surface smooth, finely-striate; grayish-yellow ; indistinctly radiate ; inodorous ; taste astringently, very slightly bitter.

Constituents.-The active principle is (1) pelletierine $\left(\mathrm{C}_{8} \mathrm{H}_{13} \mathrm{NO}\right), \frac{1}{2}$ per cent., a colorless, oily, aromatic alkaloid, soluble in alcohol, chloroform and ether; four salts occur in commerce: the tannate, sulphate, hydrobromate and hydrochlorate; the first is more frequently used; doseD., gr.ii.-v. (.12-.3); 2, punico-tannic acid, 22 per cent.; 3, methyl, pseudo, and iso-pelletierine; the latter is a tæniacide.

Dose.-Dogs, 3 ss.-i.ss. (2.-6.).

There is a (non-official) fluid extract which may be given in same dose.

Action and Uses.-Pomegranate is inferior to the foregoing drugs as an anthelmintic, since it is disagreeable and prone to produce vomiting. Pomegranate is an astringent, but, in large doses, acts as an emetic and purgative and has occasioned weakness, colic, dizziness and convulsions. In sufficient amount pelletierine is said to paralyze motor nerves, like curare. Granatum is an anthelmintic, chiefly against tape-worm. The decoction (1-8, B.P.) may be employed (with $\frac{1}{4}$ part syrup of ginger) in 3 doses, at 
hour intervals, for dogs. The patient should be previously fasted for 24 bours, and castor oil is indicated if purging is not produced by pomegranate. Tamnate of pelletierine is a yellowish, astringent-tasting powder, soluble in 80 parts of alcohol and 700 parts of water. It is invariably used in human medicine in preference to the crude drug, and should be followed in 2 hours by a dose of castor oil. The use of pelletierine is undesirable in young animals.

\section{Class 2.-Used to Destroy Round-worms}

Santonica. Santonica. (U.S. \& B. P.)

Synonym.-Levant worm seed, ssnıp.. dicæ s. "ontra. s. sanctum, s. santonici, E; barbsúue, semencine, Fr.; wurmsamen, zitwersamen, G.; flores cinæ, P.G.

The nnexpanded flewer-heads of Artemisia pauciflora Weber (nat. ord. Cumpesitæ).

Habitat.-Northern middle Europe and Asia.

Description. - From 2 to $4 \mathrm{Mm}$. long; oblong-ovoid, obtuse, smooth, somewhat glossy, grayish-green, consisting of an involucre of abont 12 to 18 closely imbricated, glandnlar scales with a broad midrib, enclosing four or five rudimentary florets; odor strong, peculiar, somewhat camphoraceons; taste aromatic and bitter.

Constituents. - The active principle is (1) santonin. There is also (2) a volatile oil.

Santoninum. Santonin. $\mathrm{C}_{15} \mathrm{H}_{18} \mathrm{O}_{3}$. (U. S. \& B. P.)

Synonym.-Santonine, Fr.; santonin, G.

A neutral priuciple obtained from santonica.

Derivation.-Made from a mixture of lime and santonica by exhausting with alcohol, evaporation of the latter, and by the addition of acetic acid to the residue. Santonin is obtained by treating an alcoholic solution of the residue with animal charcoal and erystallization. 
Properties.-Colorless, shining, flattened, prismatic crystals; odorless and nearly tasteless when first put in the mouth, but afterwards developing a bitter taste; not altered by exposure to air, but turning yellow on exposure to light. Nearly insoluble in cold water; soluble in 40 parts of alcohol, in 140 parts of ether, in 4 parts of chloroform, and in solutions of caustic alkalies.

Dose.-Puppies, gr. $\frac{1}{4}-\frac{1}{2}(.015-.03)$; D., gr.i.-iii. (.06-.18); H., $3 \frac{1}{4}$-iv. (1.-15.).

Action and Uses.-Santonin is preferable to santonica. Santonin is chiefly valuable as a parasiticide against roundworms; viz. : A. lumbricoides of cattle and swine, A. mystax and marginata of cats and dogs, and A. megalocephala of horses. It has no influence on tape-worms, nor probably on oxyurides.

Large doses cause poisoning, with the occurrence, in dogs, of nausea and vomiting, weakness, giddiness, muscular trembling, salivation, slow pulse, rapid respiration, dilated pupils, jaundice, unconsciousness, convulsions and deatl. While 5 to 6 grains induce symptoms of poisoning in dogs, $\frac{1}{2}$ to 1 drachm has often failed to produce a fatal result. Santonin is eliminated by the kidneys, increasing their secretion, coloring an acid urine yellow, and an alkaline urine purplish-red, or bloody hue. The humors of the eye are stained yellow, occasioning yellow sight in man,xanthopsy. There is congestion of the heart, lungs and nervous centres observed after death, but no gastro-enteritis. Santonin is very slowly converted in the intestines into sodium santoninate, and absorbed in this form. The best treatment of poisoning is undetermined, but inhalations of ether and enemata of chloral, together with laxatives, when consciousness returns, are said to be useful. The administration of santonin should be followed or accompanied by that of a cathartic.

The drug may be given to fasting dogs as follows : 
R Hydrarg. Chlorid. Mitis.

Santonini..................... āà gr.v.

Sacchari............................. 3 i.

M. et div. in chart. no. $x$.

Sig. One powder tid. (for round-worms).

or :-

Pe

Santonini..........................

Ol. Ricini....................... ${ }_{3}$ ii.

M.

Sig. Give one-third every third day (for round-worms).

Santouin may be given in pill to dogs combined with oleoresina filicis and areca nut. Santonin is not so commonly or successfully used in the treatment of round-worms in horses, as turpentine and aloes, creolin, etc. A ball containing $3 \mathrm{ii}$. of santonin and $3 \mathrm{i}$. of calomel, or a combination of 3 iv. santonin and 1 pint of castor oil, are suitable prescriptions for the horse. Santonin is often remedial in incontinence of urine in young animals, when belladonna fails, and is frequently beneficial in the treatment of amaurosis.

\section{Class 3.-Used to Destroy Lice.}

Staphisagria, Staphisagria.

Synonym. - Staphisagriæ semina, B.P.; stavesacre, semina staphidis agrice s. pedicularis, E.; staphisaigre, Fr.; stephauskörner, lansekörner, G.

The seeds of Delphinium Staphisagria Linné (nat. ord. Ranunculaceæ).

Habitat.-Shores of Mediterranean ; cultivated.

Description.-About $5 \mathrm{Mm}$. long, 3 or $4 \mathrm{Mm}$. broad, flattish-tetrahedral, one side convex, brown or brownishgray, with reticulate ridges, containing a whitish, oily, albumen and a straight embryo; nearly inodorous, taste bitter and acrid.

Constituents.-1, the important principle is delphinine $\left(\mathrm{C}_{22} \mathrm{H}_{35} \mathrm{NO}_{6}\right)$, a white, poisonous, crystalline alkaloid resem- 
bling veratrine and aconite; solnble in alcohol, chloroform, and ether; 2, delphisine $\left(\mathrm{C}_{27} \mathrm{H}_{46} \mathrm{~N}_{2} \mathrm{O}_{4}\right) ; 3$, delphinoidine $\left(\mathrm{C}_{42} \mathrm{H}_{68} \mathrm{~N}_{2} \mathrm{O}_{7}\right) ; 4$, staphisagrine $\left(\mathrm{C}_{22} \mathrm{H}_{22} \mathrm{NO}_{5}\right)$; 5, a fixed oil.

Action and Uses.-Powdered staphisagria is employed solely to kill lice (pediculi) in ointment (1-2) with benzoinated lard or vaseline. Creolin solutions (3-6 per cent.), tobacco infusious (5-10 per cent.), and oil of anise with sweet oil (1-10 per cent.), are also nsed for the same purpose. The latter mixture is an elegint preparation for pet dogs.

The tincture of larkspur(Delphinium consolida), another species of the same genus, is also a very efficient parasiticide against pedicnli. It contains 1 part of larkspur seeds to 16 of alcohol.

\section{Class 4.-Used to Destroy Fleas.}

Pyrethrum. Pyrethrum. (Non-official.)

Synonym. - Persian, Caucasian or Dalınatian insect. powder.

The flowers of Pyrethrum roseum and carneum.

Habitat.-Caucasian Mountains; cultivated in California, U. S.

Description.-A coarse, greenish-yellow, pungent powder. Pyrethrum is the best parasiticide for fleas (pulicidæ). It is used more frequently to kill these parasites on cats and dogs. Pyrethrum is simply dusted over the whole body or is applied in the form of a tincture (1-4), diluted with 10 parts of water.

The application of Dalmatian insect powder to kittens and puppies, or to dogs and cats in enfeebled condition, may be attended with danger unless the powder is brushed off within 10 or 15 minutes after its use. Deaths have occurred in these animals following its free and careless employment. 
SECTION XV.-VEGE'TABLE DRUGS STIMULATING UNSTRIATED MUSCLE, PARTICULARLY THAT OF THE UTERUS.

\section{Ergota. Ergot. (U. S. \& B. P.)}

Synonym.-Ergot of rye, spurred rye, E.; ergot, ergot de seigle, ergot de blé, blé cornu, Fr.; mutterkorn, kornmutter, zapfenkoru, G.

The sclerotium of Claviceps purpurea (Fries) Tulasne (Class Fungi), replacing the grain of rye, Secale cereale Linné (nat. ord. Gramineæ).

Habitat.-Ergot is obtained mainly from Spain and Russia.

Description.-Somewhat fusiform, obtusely triangular, usually curved, about 2 or $3 \mathrm{Cm}$. long and $3 \mathrm{Mm}$. thick; three-furrowed, obtuse at both ends, purplish-black, internally whitish with some purplish strie, breaking with a short fracture; odor peculiar, heavy; taste olly and disagreeable.

Constituents.-It is doubtful whether the principles so far discovered wholly represent the activity of ergot. The following are the more important constituents : 1 , coruntine, an alkaloid which, with (2) sphacelinic or sphacelic acid (a nou-nitrogenous, unstable substance, insoluble in water but soluble in alkalies), constitute, according to many, the more important active principles; they both cause contraction of the uterus and blood vessels ; 3 , three alkaloids: ergotine $\left(\mathrm{C}_{50} \mathrm{H}_{52} \mathrm{~N}_{2} \mathrm{O}_{3}\right)$, soluble in water and alcohol; ecboline, amorplions, soluble in water and alcohol; ergotinine $\left(\mathrm{C}_{35} \mathrm{H}_{40} \mathrm{~N}_{4} \mathrm{O}_{6}\right)$, crystalline; 4 , two acids : ergotic or ergotinic acid and sclerotinic acid (1-4 per cent.); both are soluble in witer and alkalies and are said to possess ecbolic actions; 5 , a fixed oil, about 35 per cent.; 6 , tannin; 7 , phosphoric acid. Alcohol extracts cornutine; water, the salts of the acids. Therefore ergotin is believed to represent the activity of the drug. Ammonia is considered the best solvent by the Brit. 
Pharm. Couference, which recommends a tinc. ergotre ammoniata consisting of ergot, 1 part, aromatic spirit of ammonia, 2 parts. The dose is the same as that of the fluid extract.

Dose.-H. \& C., żss.-i. (15.-30.); Sh. \& S., 3 i.-ii. (4.-8.); D., 3 ss.-i. (2.-4.).

\section{PREPARATIONS.}

Extractum Ergoto. Fluidum. Fluid Extract of Ergot. (U. S. P.)

Made by maceration and percolation with diluted alcohol, and evaporation, so that $1 \mathrm{Cc} .=1 \mathrm{Gm}$. of ergot.

Dose.-H. \& C., 3 ss.-i. (15.-30.); Sh. \& Sw., 3i.-ii. (4.-8.); D., 3 ss.-i. (2.-4.).

Extractum Ergotce. Extract of Ergot. (U. S. P.)

Synonym.-Ergotin.

Made by evaporation of the fluid extract to a pilular consistence.

Dose.-H. \& C., gr.xx.-3 i. (1.3-4.) ; D., gr.ii.-x. (.12-.6). (By mouth or subcutaneously.)

\section{Extractum Ergotce Liquidum. (B. P.)}

( $\leftleftarrows$ i. $\in$ rgot $=\Xi$ i. of preparation.) (1.-2.).

Dose.-H. \& C., 3iv. (15.); Sh. \& Sw., 3 i. (4.); D., आxv.-xxx.

Tinctura Ergotoe. (B. P.)

$$
\text { (109 gr. to } \xi \text { i.) }
$$

Dose.-H. \& C., 3 ss.-ii. (15.-60.); Sh. \& Sw., 3 i.-iv. (4.-15.); D., 3 ss.ii. (2.-8.).

\section{Ergotinum. Ergotin. (B. P.)}

Dose.-H. \& C., gr.xx.- 3 i. (1.3-4.); D., gr.ii.-x. (.12-.6).

Injectio Ergotini Hypodermica. (B. P.)

Doзe.-H., 3 3/4-11/2 (3.-6.); D., miii.-x. (.2-.6).

Ergot deteriorates rapidly with age. Both it and its preparations should be fresh and the drug should be discarded when over a year old.

Action Internal. - Digestive Organs.-Ergot stimulates the involnutary muscle of the stomach and intestines, but rarely sufficiently to cause any ontward sigus. The blood vessels in the walls of the digestive tract are contracted 
directly by the drug. and also because of the constriction of the muscular walls themselves.

Circulation.-Ergot is absorbed into the blood but does not influence that fluid. The essential action consists in constriction of the arterioles and veins throughout the body, with rise of blood pressure. This is due to stimulation of the spinal vasomotor centres. The pulse rate is also somewhat reduced, owing, probably, to stimulation of the peripheral cardiac vagi. When ergot is thrown directly into a vein, vascular tension is much lowered. This phenomenon follows depression of the heart muscle and does not occur after ordinary therapeutic administration of ergot by the mouth or under the skin. Toxic doses paralyze both the vasomotor centres and heart muscle.

Nervous System.-The nervons system is not affected by medicinal doses of ergot, nor by large single doses of the drug. Certain changes occur in poisoning, but these are not understood.

Uterus.-Ergot causes contraction of the pregnant womb. It does not invariably induce abortion, but does always act on the parturient organ. Small doses increase the duration and force of the uterine contractions, but full medicinal doses produce a tonic, continuous spasm of the womb without the usual intermissions. Ergot possesses little influence on the normal unimpregnated uterus. The action on the womb is probably to be ascribed to stimulation of the uterine unstriated muscle, and the spinal lumbar centres controlling this organ. Ergot is the oxytocic in most common use. The secretion of urine, sweat, saliva and milk is lessened by ergot, owing to general vascular contraction.

Administration.-The fluid extract is generally given by the mouth. Some proprietary preparations are made for subcutaneous ase. Bonjean's ergotin, or the official extract, are employed hypodermatically.

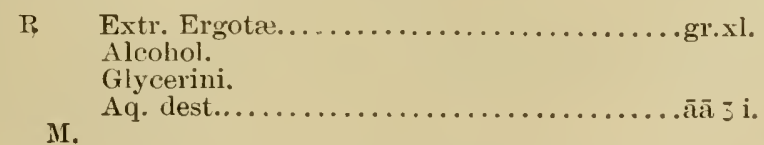

Sig. Give one-half subcutoneously to a horse; 10 to $15 \mathrm{M}$. to dogs. 
Injections should be made deeply into the muscular tissue to aroid abscess. Ergot should be repeated frequently to arrest hæmorrhage.

Toxicology. - Enormous single doses are required to poison animals or man. When as much as two drachms of ergot to the pound, live weight, are given to dogs, death is not constant. Three ounces, however, have proved fatal to small dogs. Acute poisoning is characterized by vomiting (in $\operatorname{dogs}$ ), profuse salivation, dilation of the pupils, rapid breathing, and frequent pulse. The animal cries out, has convulsive twitchings, staggering gait, paraplegia, intense thirst, and coma, terminating in death. Horses, cattle, and sheep are unaffected by any ordinary quantity of the drug.

Chronic poisoning or ergotism rarely occurs in animals owing to continuous ingestion of ergotized grains. It is characterized by gastro-intestinal indigestion, with nausea, vomiting, colic, diarrhœa or constipation, and abortion ensues in pregnant animals. In addition to gastro-intestinal irritation the symptoms naturally assume two forms: 1, the gangrenous form; 2, the spasmodic form. In the first variety of ergotism there are coldness and anæsthesia of the extremities, including the feet, ears, and tail of quadrupeds; the comb, tongue and beak of birds, - followed by the appearance of passive congestiou, blebs, and dry gangrene in the vicinity of these parts. The hoofs and beaks often drop off. Death ensues from general exhaustion. In the spasmodic form are seen tonic contraction of the flexor tendons of the limbs and anæsthesia of the extremities; muscular trembling and general tetanic spasm, with opisthotonos, convulsions and delirium. Death also occurs from asthenia.

Uses. - Two therapeutic indications for the use of ergot can be directly dednced from its physiological actions: 1, to cause uterine contraction; 2 , to produce general vascular contraction.

1. Ergot is occasionally of service in simple nterine inertia when there is no malposition of the fœetus, or mechan- 
ical obstruction (pelvic deformity, rigid os nteri) to its passage. Very small doses must be given for this purpose in order to intensify the force of the uterine contractions without inducing spasm of the nterus. The more common causes of dystocia are remedied most advantageously by manual interference. Ergot is of chiefest value in obstetric practice to prevent or arrest post-partum hæmorrliage which sometimes occurs in cows and ewes. If administered before delivery of the placenta, ergot may give rise to tonic contraction of the womb and retention of the afterbirth.

Ergot is of benefit in some disorders of the unimpregnated and non-parturient uterus. Thus to aid the expulsion of cysts, and to contract the uterus and its blood vessels in hypertrophy, subinvolution, chronic metritis and fibroid tumors.

In paralysis of the bladder, ergot is occasionally useful by creating contraction of the muscular coat of its walls. This condition is most satisfactorily treated by the injection of an ordinary dose of ergotin into the empty urinary bladder.

2. Ergot is commonly recommended for combating internal hæmorrhage, when surgical measu res are impossible. Under this head may be included bleeding from the nose, mouth, stomach, intestines, lungs, uterus and kidneys. The drug should usually be given under the skin (ergotin) in these emergencies. It is, however, extremely doubtful whether the increase of blood pressure caused by ergot does not more than offset its beneficial action in contracting vessels when used to stop internal hæmorrhage, and the best clinicians to-day condemn its use and resort to ice and opium in this condition. Ergot may be beneficial in causing constriction of the blood vessels in the early stages of some hyperæmias and inflammations,-notably pulmonary, cerebral and spinal congestion, parturient apoplexy, cerebritis, cerebro-spinal meningitis, spinal meningitis and myelitis; and in vasomotor palsy following surgical shock. In most of these states (except the latter, where ergotin is indicated, under the skin) ergot must be exhibited in very large doses three times daily. 
For the same physiological reason that ergot is employed as a hæmostatic, it has been prescribed with alleged advantage in the treatment of chronic diarrhoa, dysentery, and bleeding piles. Ergotin is injected between the skin and vessel walls for the cure of aneurism and varicocele. This treatment is of doubtful utility.

\section{Gossypil Radicis CoRTEx. Cotton Root Bark.}

$$
\text { (U. S. P.) }
$$

Synonym.-Ecorce de racine de cottonnier, Fr.; baumwollen-wurzelrinde, $\mathrm{G}$.

The bark of the root of Gossypium herbaceum Linné, and of other species of Gossypium (nat. ord. Malvacex).

Habitat.--Sub-tropical Africa and Asia; also cultivated in the United States.

Description.-In thin, flexible bands or quilled pieces; outer surface brownish-yellow, with slight, longitudinal ridges or meslies, small, black, circular dots, or short, transverse lines, and dull brownish-yellow patches, from the abrasion of the thin cork; inner surface whitish, of a silky lustre, finely striate; bast-fibres long, tough and separable into papery layers; inodorous ; taste very slightly acrid and faintly astringent.

Constituents. - 1, a fixed oil ; 2 , a resin; 3 , tannic acid.

$$
\text { PREPARATION. }
$$

Extractum Gossypii Radicis Fluidum. Fluid Extract of Cotton

Root Bark. . (U. S. P).

Made by maceration and percolation witl glycerin and alcohol, and eraporation, so that $1 \mathrm{Cc} .=1 \mathrm{Gm}$. of the crude drug.

Dose.-H. \& C., ₹ ss.-i. (15.-30.); Sh. \& Sw., z ss.-i. (2.-4.).

Action and Uses. - Cotton root bark resembles ergot in its actions and uses, but the drug has not been scientifically studied in full detail. It is an oxytoxic, abortifacient, and emmenagogue.

Cotton root bark is employed during parturition in 
uterine inertia, to prevent post-partum hemorrhage, to induce abortion, and to arrest metrorrhagia. A decoction ( $\xi \mathrm{iv}$. to $\mathrm{qt}$. boiled down to Oi.) is said to be more active than the fluid extract. Dose.-H. \& C., Oi. (500.); D., ₹ i.-ii. (30.-60.).

Ergot is generally to be preferred as a more thoroughly understood and reliable drug.

\section{SECTION XVI.-COLCHICUM.}

\section{Colchici Radix. Colchicum Root. (U.S. P.)}

Synonym.-Colchici cormus, B.P.; meadow saffron root, colchicum corm, bulbus seu tuber colchici, E; bulbe de colchique, de safran bâtard, Fr.; zeitlosenknollen, G.

The corm of colchicum autumnale Linné (nat. ord. Liliaceæ).

Habitat.-England and continental Europe.

Description.-About $25 \mathrm{MIm}$. long, ovoid, flattish and with a groove on one side; externally brownish and wrinkled; internally white and solid; often in transverse slices, reniform in shape, and breaking with a short, mealy fracture; inodorous; taste sweetish, bitter and somewhat acrid.

Constituents. - 1 , the alkaloid colchicine $\left(\mathrm{C}_{22} \mathrm{H}_{25} \mathrm{NO}_{6}\right)$, is the active principle; it exists to the extent of 0.5 per cent.; is amorphous or crystalline, and soluble in water and alcohol; 2, colchiceine $\left(\mathrm{C}_{21} \mathrm{H}_{23} \mathrm{NO}_{6}\right)$, occurs naturally and is produced artificially by the action of acids on colchicine; slightly soluble in water, soluble in alcohol; 3 , sugar; 4, starch; 5 , gum. iodine.

Incompatibles.-Astringents, tincture of guaiacum and

Dose.-H. \& C., 3 ss.-ii. (2.-8.); Sh., gr.x.-xx. (.6-1.3); Sw. \& D., gr.ii.-viii. (.12-.5) in powder.

Dose.-Colchicine-H. \& C., gr. $\frac{1}{6}-\frac{1}{2}$ (.01-.03); D., gr. $\frac{1}{100}{ }^{-}$ $\frac{1}{50}(.0006-.0012)$, by mouth or subcutaneously. 
PREPARATIONS.

Extractum Colchici Radicis Fluidum. Fluid Extract of Colchicum Root. (U. S. P.)

Made by maceration and percolation with alcohol and water, and evaporation, so that $1 \mathrm{Cc} .=1 \mathrm{Gm}$. of colchicum root.

Dose.-H. \& C., 3 ss.-ii. (2.-8.); Sh., $\Pi x .-x x .(.6-1.3)$; Sw. \& D., Mii.-viii. (.12-.5).

Vinum Colchici Radicis. Wine of Colchicum Root. (U. S. P.)

Made by percolation of colchicum root, 400, with alcohol, 150; and white wine to make 1000 .

Dose.-Twice that of colchicum root.

Dose.-Twice that of the root.

Vinum Colchici. (B. P.)

\section{Colchici Semen. Colchicum Seed. (U. S. P.)}

Synonym.-Colchici semina, B.P.; semences de colchique, Fr.; zeitlosensamen, G.; semen colchici, P.G.

The seed of Colchicum autumnale Linné (nat. ord. Liliaceæ).

Description. - Subglobular, about $2 \mathrm{Mm}$. thick, very slightly pointed at the hilum; reddish-brown, finely pitted, internally whitish ; very hard and tough ; inodorous; taste bitter and somewhat acrid.

Constituents.-1, colchicine, 3 per cent.; 2, colchiceine; 3 , a fixed oil, 6-8 per cent.; 4, starch ; 5 , sugar ; 6 , gum.

Dose.-Same as root, or a little larger.

PREPARATIONS.

Extractum Colchici Seminis Fluidum. Fluid Extract of Colchicum

Seed. (U. S. P.)

Made by maceration and percolation with alcohol and water, and evaporation, so that $1 \mathrm{Cc}$. $=1 \mathrm{Gm}$. of colchicum seeds.

Dose.-Same as fluid extract of root.

Vinum Colchici Seminis. Wine of Colchicum Seed. (U. S. P.)

Made by maceration of colchicum seed, 150; alcohol, 150; and white wine to make 1000 .

Dose.-H. \& C., 3 iii.- 3 i. (12.-30.); D., Txx.-xxx. (.6-2.). 
Tinctura Colchici Seminis. Tincture of Colchicum Seed.

(U. S. \& B. P.)

Made by maceration and percolation of colchicum seed, 150; with alcohol and water to make 1000 . (U. S. P.)

Dose.-H. \& C., 3 iii.- $\overline{3}$ i. (12.-30.); D., $\Pi x x_{0}-\mathrm{xxx}$. (.6-2.).

Action External:-Colchicum is an irritant to the skin and mucous membranes.

Action Internal. - Digestive Organs. - Colchicum and colchicine are identical in action withont regard to their method of introduction into the system. Colchicum is essentially a gastro-intestinal irritant in large doses. Small therapeutic quantities are cholagogue and increase the flow of bile. Full medicinal doses occasion anorexia, uansea, celicky pains, loud intestinal rumblings (borborygmi), and purging. Colchiceine appears to closely resemble colchicine physiologically. Neither the circulation, nervous system, respiration, nor temperature are affected by colchicum save in poisonous doses.

Circulation.-Toxic doses reflexly influence the circulation, but colchicum does not appear to affect it directly nutil late in poisoning, when the vagus endings become depressed and paralyzed. Full medicinal doses lower the force and frequency of the pulse.

Nervous System.-Toxic quantities depress and paralyze the motor cells of the inferior cornua in the spinal cord, and also depress the sensory nerves. The motor nerves and muscles are unaffected.

Kidneys and Elimination.-It is doubtful whether colchicum exerts any decided or uniform action on the kidneys. Experiments relating to this matter are perpiexingly conflicting. It is stated by many authors that the excretion of both the organic and inorganic solids in the urine is increased; that of urea more than uric acid.

Toxicology.-Colchicum is a very poisonous drug. Accidental lethal poisoning occasionally occurs among herbivora from eating meadow saffron at pasture, or in hay. In such 
cases it is naturally impossible to estimate the quantity , the plant ingested. Two and one-half drachms of the wine of the root, and one-half grain of the alkaloid, have proved fatal to man. Two drachms of the corm have killed a dog, and one-tenth of a grain of colchicine has destroyed a cat. The symptoms of poisoning comprise: anorexia, nausea, dulness, salivation, violent vomiting (in carnivora), purging, at first watery, then mucous and often bloody, and accompanied by great tenesmus, tympanites, and colic. There are often such nervous symptoms as tremors, stupor, coma, and paralysis. The animal becomes very weak, the respiration is slow and feeble, the pulse rapid-and imperceptible, the skin is cold and covered with a clammy sweat, and death occurs in collapse following severe gastro-enteritis. After the injection of large doses of colchicine, increasing the amount does not aggravate the symptoms.

Post-Mortem Appearances.-The mucous membrane of the stomach and intestines is swollen, congested, and eroded. Sometimes free blood is found within their lumen. There is also acute hyperæmia of the kidneys.

Treatment.-This consists in the use of the stomach pump, emetics, and cathartics, unless there has been free purging and vomiting. Tunnic acid is the best chemical antidote, but is not wholly satisfactory. It should be used, however. Demulcents (oil and egg albumin) and opium relieve the local irritation, pain and purging. Stimulants, as atropine, strychnine, and alcolool, together with external heat, combat collapse.

Administration.-The crude drug is not suitable for use. The wine of the root is the best preparation.

Uses.--It is difficnlt to estimate the therapentic value of colchicum. It is called an alterative by many anthorities, for want of a better term to describe a drug whose physiological actions do not explain its medicinal virtues. Colchicum is the most successful single remedy for gout in human medicine, but does not possess so great a value in veterinary practice. It is sometimes used with considerable advantage 
in the treatment of subacute and chronic rheumatism, and in that form complicating influenza in horses.

Potassium iodide is generally prescribed with colchicum in these disorders, and also in pericarditis and pleurisy of rheumatic origin. Colchicum is commonly believed to be a serviceable diuretic in various diseases (although this hypothesis is not physiologically substantiated). It is therefore recommended in hæmaglobinæmia and swelling of the legs in horses; in cerebral congestion ; ascites ; pleural and pericardial effusions, and dropsies, as an eliminative. Colchicine, subcutaneously, is especially lauded in rheumatism.

\section{SECTION XVII.-VEGETABLE DRUGS ACTING MECHANICALLY.}

Amylum. Starch. $\mathrm{C}_{6} \mathrm{H}_{10} \mathrm{O}_{5^{\circ}} \quad$ (U. S. \& B. P.)

Synonym.-Corn starch, E.; fecule (amidon) de froment, de blé, Fr.; kraftmehl, weizenstärke, G.

The fecula of the seed of Zea Mays Linné (nat. ord. Gramineæ).

Habitat.-Tropical Asia and Africa, but cultivated in tropical, sub-tropical and temperate countries.

Description.-In irregular, angular masses, which are easily reduced to a fine powder ; white, inodorous and tasteless; insoluble in ether, alcohol or cold water. Under the microscope appearing as granules, nearly uniform in size, more or less angular in outline, with indistinct strix and with a distinct hilum near the centre.

Constituents.-1, starch-granulose ; 2 , starch-cellulose.

PREPARATION.

Glyceritum Amyli. (See p. 581).

Action and Uses.-Starch is a mechanical protective externally, used as a dusting powder, alone or with zinc oxide (1-4), in chafing, erythema, and moist eczema. The. 
glycerite of starch is a serviceable demulcent. Boiled starch paste, mixed with glue, is used to stiffen bandages by painting the mixture on in layers with a brush. Boiled starch gruel (2 tablespoons of starch to a pint of water) is a suitable diet for diarrhoea, and is frequently injected into the rectum as a demulcent in diarrhoea and dysentery, and as a vehicle for enemata. Starch is an antidote to iodine. It is ntilized in pharmacy as a vehicle to suspend insoluble powders or oils, and in mucilage $(1-40$, B.P.), as a basis for ointments. Zinc oxide, one part; starch and vaseline, each 3 parts, form a very satisfactory preparation for acute eczema in dogs, which does not rub off so readily as zinc ointment.

\section{Oleum Theobromatis. Oil of Theobroma.}

$$
\text { (U. S. \& B. P.) }
$$

Synonym.-Butter of cacao, E.; beurre de cacao, Fr.; cacaobutter, G.; oleum (butyrum) cacao, P.G.

A fixed oil expressed from the seed of Theobroma Cacao Linné (nat. ord. Sterculiaceæ).

Properties. - A yellowish-white solid, having a faint, agreeable odor, and a bland, chocolate-like taste. Readily soluble in ether or chloroform.

Constituents. -1 , olein ; 2 , stearin ; 3 , laurin ; 4, arachin ; 5 , glycerides of formic, butyric, and acetic acids.

Uses. - Cacao butter melts at the temperature of the body, and is chiefly used as an excipient for suppositories and electuaries. It also has a demulcent action and may be employed on raw surfaces or in inflammation of the throat and digestive tract.

\section{Gossypium Purificatum. Purified Cotton. (U. S. P.)}

Synonym.-Absorbent cotton, E.; bombyx, lana gossypii, - coton, Fr.; baumwolle, G.

The hairs of the seed of Gossypium herbaceum Linné, and of other species of Gossypium (nat. ord. Malvaceæ), . 
freed from adhering impurities and deprived of fatty matter.

Habitat.-Tropical Asia aud Africa; cultivated in subtropical and tropical countries, mostly in the Sonthern United States.

Description.-White, soft, fine filaments, appearing under the microscope as hollow, flattened and twisted bands, spirally striate and slightly thickened at the edges; inodorous and tasteless; insoluble in ordinary solvents, but soluble in copper ammonium sulphate solution.

Uses.-Absorbent cotton is used as a cheap, convenient and cleanly substitute for ordinary sponges; to make poultices by soaking it in antiseptic solutions (as creolin 1-2 per cent.) and placing it between layers of gauze; and for surgical dressings.

Oakum, consisting of the fibres of old rope, is often employed as a cheap absorbent material, saturated with tar, in packing horses feet.

Tow, - the coarser unbleached fibres of flax; and lint,the scrapings of soft, loosely woven linen,-_are also utilized as absorbent substances for surgical purposes.

Pyroxylinum. Pyrosylin. (U. S. \& B. P.)

Synonym.-Gun cotton, soluble gun cotton, colloxylin.

Derivation.-Marle by maceration of purified cotton, 100 ; in a mixture of nitric acid, 1400 ; and sulphuric acid, 2200 ; at a temperature of $32^{\circ} \mathrm{C}$. $\left(90^{\circ} \mathrm{F}\right.$.), until a sample is soluble in a mixture of 1 volume of alcohol and 3 volumes of ether; washing with cold and boiling water, draining, and drying in small pellets.

\section{PREPARATIONS.}

Collodium. Collodion. (U. S. \& B. P.)

Pyroxylin, 30; ether, 750; alcohol, 250. Made by solution, agitation, and decantation of clear portion.

Collodium Cantharidatum. ('antharidal Collodion. (U. S. P.)

Synonym.-Blistering collodion.

Made by percolation of cantharides, 60 ; with chloroform, distilla- 
tion of the chloroform and evaporation of the residue until it weirhs 15 (Gm.), and solution in flexible collodion, 85.

Collodium Flexile. Flexible Collodion. (U. S. \& B. P.)

Mix collodion, 920; Canada turpentine, 50; and castor oil, 30. (U. S. P.)

Collodium Stypticum. Styptic Collodion. (U. S. P.)

Mix tannic acid, 20; alcohol, 5; ether, 25; add collodion to make 100.

Action and Uses.-Collodion, when painted on dry skin, rapidly dries and leaves a thin, protective coating. It is a useful agent to seal and secure coaptation of small wounds and to keep them aseptic. Also to protect abraded surfaces, as fissures of teats. Flexible collodion is less apt to crack. Collodion contracts the superficial tissues and will often abort boils when applied directly over them. Collodion is employed as a vehicle for the application of many other agents, as corrosive sublimate, carbolic acid, iodoform, etc.

Euphorbium. Euphorbium. (Non-official.)

The dried juice of a cactus-like plant, Euphorbium resinifera, growing in Morocco and regions contiguous to the Atlas Mountains. Obtained by incising the stems aud branches.

Description.-In dull yellowish tears, of the size of peas; odorless; taste acrid; powder of a grayish color; insoluble in water, but soluble in alcohol, ether and oil of turpentine.

Constituents. -1 , an acrid resin $\left(\mathrm{C}_{20} \mathrm{H}_{32} \mathrm{O}_{4}\right), 38$ per cent., the active principle; 2 , euphorbon.

Action and Uses.-Euphorbium is an intense irritant, both externally and internally. It is sometimes employed in veterinary medicine as a constituent of resicating preparations to enhance their effect, but if applied alone it is liable to cause extensive irritation, sloughing, and destruction of tissue. Euphorbium may, however, be safely applied in tincture (1-16), or in ointment witl cantharides, as a vesicant for horses. The following combination is recommended as a powerful blister for the latter animals. Euphorbium and 
cautlarides, each two parts; corrosive sublimate, one part; vaseline, eight parts; cerate, twelve parts. There is no danger of absorption and genito-urinary inflammation from the use of euphorbium, as with cantharides.

\section{SECTION XVIII.-MEDICINAL AGENTS OF ANIMAL ORIGIN.}

\section{Adrenalin.}

This is an active principle of the suprarenal glands, and was discovered by Dr. Jokichi Takamine* and made public in 1901. It is a light, white, microcrystalline substance, slightly soluble in cold water, more so in hot. It has a somewhat bitter taste and produces a benumbing of the tongue. Adrenalin is permanent in powder, but changes color and is oxidized in aqueous solution. It is said to be the active principle of the suprarenals, but non-toxic, which can not be said of the glands or their extracts (and probably not of adrenalin). Adrenalin is from 825 to 1,000 times more powerful than the glands in its physiological effect. Solutions may be boiled withont impairing their therapeutic activity.

Action.-The most important action is exerted upon the circulatory apparatus, which is comparable to that of digitalis but much more transient, lasting not more than ten minutes when given intravenously. (The drug acts very uncertainly when given by the mouth-except locally on the digestive tract-because of poor absorption.) The force of the heart is increased but the rate decreased (by excitation of the vagi) and blood pressure is more markedly heightened than by any other known drug, owing to central and peripheral vasomotor stimnlation or to excitation of

\footnotetext{
* Therapeutic Gazette, April 15, 1901.
} 
the muscular coat of the vessels. Respiration is also strengthened and both voluntary and involuntary muscles are stimulated by adrenalin. Large doses of the adrenal grand cause poisoning with failure of the heart and respiration and paralysis of the vagi. Recent experiments with mrenalin intravenonsly have resulted not infrequently in sudden death from paralysis of the heart, so that the drug must be used in this manner with caution until its action lias been more thoroughly studied.

Reichert, on the other hand, in experiments on dogs, found that $.00025 \mathrm{gm}$. of adrenalin per kilo of live weight ( $\mathrm{gr} \cdot \frac{1}{2+0}$ for each $2 \mathrm{lbs}$. 3oz.) stimulated the circulation, respiration and metabolic gravity. In poisoning by large doses of the adrenal gland, there have been observed restlessness and loss of muscular strength from action on the spinal cord or muscles, or both. Glycosuria has also been induced by the subcutaneous injection of the suprarenal extract and stimulation of the secretions of the buccal, œesophageal and bronchial mucous membranes. The predominant and valuable effect of adrenalin and extracts of the adrenals consists in the complete blanching of mucous membranes and raw surfaces after the local application of these substances. Adrenalin is undoubtedly the most powerful astringent and hemostatic known.

Subcutaneous injection of a 1 to 1000 solution causes blanching over an area about two inches in diameter within a minute and lasts. for six to twelve hours. Solutions containing adrenalin to the amount of 1 to 5000,1 to 10,000 , or even 1 to 20,000, will produce an ischemia after their hypodermic use within a few minutes and lasting for three to six hours. Neither ecchymoses nor sloughing occur after the injection of tho drug. Secondary hemorrhage does not. ordinarily appear after the nse of adrenalin, but it will prevent the secondary relaxation, congestion and pain sometimes seen following the use of cocaine.

Uses.-External._Adrenalin chloride is commonly sold 
in a 1 to 1000 aqueous solution in the shops. It has proven of most value when combined with cocaine for subcutaueous use in operative work. One part of the 1-1000 solution should be added to uiue parts of normal salt solution (one heaping teaspoonful of sodium chloride to the quart of sterile water), to which is added 1 per cent. of cocaine, or more if desired. A stronger solution of adreualin chloride than this ( 1 to 10,000 ) is not usually desirable in operative work, because it is irritating, and in some instances when strouger solntions were used so much ischemia was produced that the blood vessels could not be found and ligated and secondary hemorrhage ensued. This would apply only to major operations, however.

The combination of cocaine with adrenalin is coming into general favor and marks a distinct progress in local anæsthesia. Four advantages are claimed for this combination: 1, that the operation is made comparatively bloodless; 2 , that the cocaine is retained in the operative field a longer time, owing to the contracted state of the vessels, and does not escape into the general circulation; this is a double advantage-in prolonging anæsthesia and in preventing systemic effect of cocaine; 3 , that adrenalin is a circulatory stimulant and will offset the toxic action of cocaine; this is somewhat problematical and theoretical; 4 , that adrenalin comnteracts the vascular relaxation seen sometimes after the nse of cocaine.

Adrenalin has also proven of great service in arresting hemorrhages from wounds, mucous membranes and cavities of the body. A solution equivalent to a 1 to 5000 of adrenalin in sterile salt solution may be applied for this purpose directly to the bleeding surface, or gauze saturated with it may be packed into wounds and cavities, as the uterus and vagina. Adrenalin chloride is useful also in many inflammatory diseases of the eye and nose in the same strength. Catarrhal conjunctivitis, keratitis, episcleritis and iritis yield to its iufluence, particularly when it is combined with other astringents on account of its transient 
action; with atropine in iritis. Adrenalin chloride in a 1 to 5000 solution containing 2 per cent. of boric acid forms a useful preparation for general application in inflammations of mucous membranes.

Internal.-Adrenalin is employed internally to arrest bleeding from the stomach, intestines, lungs and uterus. Its local application in hemorrhage from the bladder, rectum, vagina and uterus is, however, much more effective. The drug is said to have yielded good results in human practice in the treatment of diabetes insipidus, purpura lemorrhagica, acute and chronic bronchitis, pulmonary congestion and oedema of glottis and lungs. Reichert, as the result of his experiments on morphinized dogs, believes that adrenalin is a valuable and rapidly acting stimulant to the heart, vasomotor system and respiration in poisoning by opium and anæsthetics. It has also been used in emergencies for the same purpose and with reported good results. In opium poisoning, metabolism is checked and vital activity lowered, together with inhibition of the normal secretion of the adrenals, which Reichert thinks is relieved by adrenalin. Martin* has recently given 3 i. (4.0) of adrenalin solution ( 1 to 1000 ) in a pint of normal salt solution subcutaneously in conditions of shock and circulatory failure in human patients, with some satisfactory results and no toxic effects.

In emergencies, adrenalin should be injected intravenously in solutions not stronger than 1 to 5000 ; but, in view of some deaths reported from the experimental injection of the drug, this mode of treatment must be regarded with some suspicion.

Administration.-Adrenalin is preferable to the so-called suprarenal extracts, the dried and powdered suprarenal capsules of sheep and oxen. When the drug is given by the mouth or rectum, its action on the system at large is slow and uncertain owing to the tardiness of alssorption, presumably due to the vascular constriction it occasions and

* American Med., Nov, 21, 1903. 
to its rapid decomposition. Intravenous injection is most effectire, ilthough attencied with some danger in large doses. A stronger solution than 1 to 10,000 may cause an abscess if the drug be given lispodermically. The doses of adrenalin chloride in 1 to 1000 solntion are: Dogs, m 10.-60. (0.6-4.0); horses, 32-4 (S.-15.). Adrenalin should be repeated once in two hours when given internally as an hemostatic. Of the suprarenal extract, the dose would be about half that advised for the adrenalin solution.

\section{Cantharis. Cantharides. (U. S. \& B. P.)}

Synonym.-Spanish flies, blister beetles, muscæ hispanicæ, E.; cantharides, Fr.; spanische fliegen, canthariden, G.; cantharides, P.G.

Cantharis is the beetle, Cantharis vesicatoria De Geer (class Insecta ; order Coleoptera).

Habitat. - Southern Europe, Germany and Russia; living chiefly on Oleaceæ and Caprifoliaceæ.

Description.-Abont $25 \mathrm{Mm}$. long and $6 \mathrm{Mm}$. broad; flattish cylindrical, with filiform antennæ, black in the upper part, and with long wing-cases, and ample, membranous, transparent, brownish wings, elsewhere of a shining, copperygreen color. The powder is grayish-brown, and contains green shining particles. Odor strong, and disagreeable; taste slight, afterwards acrid.

Constituents. - 1 , the active principle is cantharidin, $\mathrm{C}_{10} \mathrm{H}_{12} \mathrm{O}_{4}$ (2 per cent.), in colorless scales, insoluble in water, soluble in alcohol, ether, chloroform, oils, acetic acid and acetic ether; it is found chiefly in the generative organs, eggs, and blood of the beetles ; 2 , a volatile oil ; 3 , a bland, green oil; 4, acetic and uric acids, extractives and salts; cantharides deteriorates with age and should be kept unpowdered in tightly stoppered bottles.

Dose.-H. \& C., gr.v.-xx. (.3-1.3); Sh. \& Sw., gr.iv.-viii. (.24-.5); D., gr.i.-ii. (.06-.12). 
PREPARATION.

Tinctura Cantharidis. Tincture of Cantharides. (U.S. P.)

Made by percolation of cantharides, 50; with alcohol to mare 1000. (U. S. P.)

Dose.-H. \& C., 3 ii.-iv. (8.-15.); D., mii.-xv. (.12-1.).

Action External.-Cantharides, by virtue of cantharidin, is an intense irritant. When applied to the skin in ointment it produces no effect for several hours, but after that time causes dilatation of the cutaneous vessels, hyperæmia, and blister's, which appear in from 3 to 12 hours. The blisters soon break, discharge their serous contents, and then dry and crust the surface. If the action of cantharides is maintained continuonsly; if the application is repeated, or covered with a bandage; or if the skin was previously inflamed, then inflammation of the deeper-seated parts ensues, followed by suppuration, sloughing, loss of tissue, destruction of hair follicles, and scars. The drug is therapeutically a rubefacient and vesicant, and counter-irritant, in occasioning dilatation of the superficial vessels, and reflexly, contraction of those in the remote underlying parts. Cantharides acts more powerfully on the skin of horses and dogs, than on that of cattle and swine. If applied over an extensive surface, absorption and poisoning may occur.

Action Internal.-Cantharides affects mainly the digestive and genito-urinary tracts. It is a violent gastro-intestinal irritant. Toxic doses cause vomiting, in animals capable of the act, at first bilions (and containing greenish specks of the wings aud wing cases), then mucous, and finally bloody. There is purging in all, associated with great pain and straining, of a mucous, fibrinous, and often hæmorrhagic character. There are salivation, swelling and pain in the salivary glands. The gastro-enteritis is accompanied by general prostration, and feeble, rapid pulse. A few hours after the occurrence of the preceding symptoms there is enough absorption of cantharidin to induce lumbar paiv, followed by frequent, scanty and painful micturition (strangury). The urine is albuminous and often bloody. Can- 
tharides is eliminated chiefly by the kidneys, but also to some extent by the other excretory organs, including the skin. Sexual excitement may be present in poisoning. It is more common with small than large toxic doses. There are erections and great heat in the penis, and even inflammation and sloughing of the organ. Abortion is precipitated in the pregnant, and "heat" is hastened in the nonpregnant female. Stupor, coma, and collapse close the scene after lethal doses. Twenty grains of cantharides have killed a man ; forty, a dog; and one drachm has destroyed a horse.

The treatment includes the use of emetics or the stomach pump; opium, to relieve pain and strangury; albuminous, mucilaginous drinks; and, in collapse, external heat, alcoholic stimulants, strychnine and atropine under the skin. Oils and fats dissolve cantharidin and must not be giveu in poisoning as demulcents.

Post-Mortem Appearances.-Swelling, congestion, ecchymoses, and erosion of the gastro-intestinal mucous membrane are observed after death by cantharides. There are, moreover, lesions of acnte nephritis and cystitis, with inflammation of the whole genito-urinary mucous membrane. Hyperæmia of the brain and spinal cord have also been reported.

Uses External.-Cantharides is employed more frequently than any other counter-irritant, to cause blistering, in veterinary practice. The reader is referred to the section on counter-irritants (p. 696) for an account of their actions. The action of cantharides is too tardy and irritating, and there is too much danger of absorption and poisoning to recommend it for the production of counter-irritation over an extensive area (chest and abdomen) in acute pulmonary congestion, pneumonia, bronchitis, colic, enteritis and peritonitis. Mustard, turpentine, and external heat are generally preferable in these disorders.

A blister of cantharides is serviceable in pericarditis and pleuritis with effusion, and, applied over the throat in severe, acute laryngitis, may obviate the necessity of tracheo- 
tomy. Again, blisters are useful on the poll in inflammation of the brain and its membranes; and over the spine in myelitis and meningitis; over the lumbar region in paraplegia.

A cantharidal application is often efficacions in muscular or joint rheumatism when placed over the affected area, or near by, in acute conditions. Furthermore, a cantharides. blister is of advantage in acute diseases of the ear, when rubbed in above and behind this organ; and will relieve pain in the stomach, and vomiting when applied to the epigastrium.

Cantharides is the remedy usually employed in the treatment of diseases of the bones, joints, bursæ, ligaments, and tendons. In exostoses, as spavin and ringbone, the ointment is used most effectively after the actnal cautery, to secure absorption and resolution, or anchylosis. Cantharidal ointment is often sufficient, together with complete rest, in the treatment of synovitis, and strains of tendons and ligaments. A cantharides blister is, sometimes, beneficial in hastening the formation of abscess, ("strangles"); or to aid their resolntion after paracentesis ; also to stimulate indolent. ulcers or wounds; and to assist absorption of tranmatic indurations, when applied around these lesions. The actual cautery, followed by a cantharides blister, will cause sweiling and close the opening in the abdominal parietes of small umbilical hernias of foals and calves.

Likewise, blisters are valnable in closing and sealing. punctured wounds into joints and synovial cavities. Cantharides is commonly employed in ointment ( 1 to $4-8$ ) made by melting and mixing the excipients in a double boiler; i.e., over a water bath, and stirring in thoroughly the powdered drug. The following is a good preparation :

$\mathrm{P}$

Pulv. Cantharidis.

Ceræ flav.......................... āa $\bar{z}$ ii.

M.

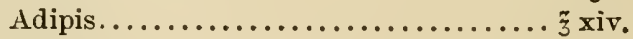

S. External use. 
More powerful ointments are made with powdered euphor. bium and cantharides, each 2 parts; corresive sublimate, 1 part; vaseline, 8 parts ; cerate, 12 parts ; or,

Tar and resin, each 4 parts ; yellow wax, 3 parts ; cottonseed oil, 10 parts ; powdered euphorbium, 2 parts ; cantharides, 6 parts.

The technique of blistering consists in cutting the hair and washing the part to be blistered, and rubbing the blister long and thoroughly in to the skin. The animal should be controlled by tying up the head, or using a cradle, or side-bar attached to the halter and surcingle, to prevent horses from biting the blistered area. The tail should be tied up if the blister is applierl within its reach. Dogs should be muzzled, but are apt to rub the, sore spot. The serum discharged from the blister must be continually sponged off with soap suds and water, to prevent excoriation of the subadjacent skin, or the latter may be covered with a solution of resin in alcohol, by means of a brusl.

The blister is washed off in 36 or 48 hours after its application, and vaseline shonld thereafter be kept on the part. The use of cantharidal blisters is contraindicated in weak or young animals ; on the flexures of joints; or delicate skin on the inner aspect of the upper part of the limbs; on acutely inflamed areas; and in renal disease.

Uses Internal. - Cantharides is rarely administered internally. It is sometimes suceessful in stopping incontinence of urine, when due to relaxation of the neck of the bladder, and it may be used as a stimulant in chronic cystitis and pyelitis. Cantharides is recommended to increase sexual desire in cows and mares, but it has usually to be given in toxic doses to produce an aphrodisiac action. The tincture should be employed when the drug is exhibited internally. 
Adeis. Limil. (U. S. P.)

Synonym.-Aieps preparatus, B.P.; axmngia, axmmin porci s.porcina, prepared lard, hog's lard, E.; axonge, grain: : de porc, Fr.; schweineschmalz, G.; adeps suillus, P.G.

The prepared internal fat of the abilomen of Sus Scrofa Linné (class Mammalia; order Pachydermata), purified by washing with water, meltiug, aud straining.

Properties.-A soft, white, unctuous solid, having a faint odor free from rancidity, and a bland taste; insoluble in water; slightly soluble in alcohol ; readily soluble in ether, chloroform, carbon disnlphide, or benzin. Spec. gr. about 0.932 .

\section{PREPARATIONS.}

Ceratum. Cerate. (U. S. P.)

Synonym.-Cerat simple, Fr.; einfaches cerat, wachssalbe, G. White wax, 300; lard, 700.

Unguentum. Ointment. (U. S. P.)

Synonym.-Unguentum simplex, B.P.; simple ointment, E.; pommade simple, Fr.; wachssalbe, G. Lard, 800; yellow wax, 200. (U. S. P.)

Ceratum Resina. (See p. 506.)

Adeps Benzoinatus. (See p. 512.)

\section{Sevum. Suet.}

Synonym.-Sevum præparatum, B.P.; mutton suet, E.; suif, Fr.; talg, hammeltalg, G.; sebum, P.G.

The internal fat of the abdomen of Ovis aries Linné (class Mammalia; order Ruminantia), purified by melting and straining.

Properties.-A white, solid fat, nearly inodorous, and having a bland taste when fresh, but becoming rancid on prolonged exposure to the air. Insoluble in water or cold alcohol; soluble in about 60 parts of ether, and slowly in 2 parts of benzin.

Constituents. -1, olein ; 2, stearin ; 3, palmitin ; 4, hircin. 
Adeps Lane Hydrosus. Hydrous Wool Fat. (U. S. P.)

Synonym.-Lanolin, œеsypum.

The purified fat of the wool of sheep (Ovis aries, Linné ; class Mammalia; order Ruminantia), mixed with not more than 30 per cent. of water.

Properties.-A yellowish-white, or nearly white ointmentlike mass, having a faint, peculiar odor. Insoluble in water, but miscible with twice its weight of the latter, without losing its ointment-like character.

Constituents.-1, cholesterin, $\mathrm{C}_{26} \mathrm{H}_{43}(\mathrm{OH}) ; 2$, ethers of oleic, stearic, palmitic and other acids.

ACTION AND USE OF LARD, SUET, AND HYDROUS WOOL FAT.

Lard is used mainly as a basis of ointments and cerates. Benzoin is commonly added to it to prevent or retard rancidity. Lard is inferior to petrolatum as a lubricant. It is rarely given internally as an antidote to caustic alkalies, and as a demulcent.

Suet is contained in certain ointments and plasters. It is harder than lard and becomes rancid on prolonged exposure. Lanolin is not subject to rancidity, but possesses no particular medicinal action. It is indicated where absorption of some drug is desired (mercury, potassium iodide) by inunction, as it is believed to be more readily absorbed from the skin than any other fat. Lanolin is used as a basis of ointments.

Cera Flata. Yellow Wax. (U. S. \& B. P.)

Synonym.-Cera citrina, beeswax, B.P.; cire jaune, Fr.; gelbes wachs, G.

A peculiar, concrete substance prepared by Apis mellifica Linné (class Insecta ; order Hymenoptera).

Properties. - A yellowish to brownish-yellow solid, having an agreeable, honey-like odor, and faint, balsamic 
taste. Spec. gr. 0.955-0.967. It is brittle when cold; by the heat of the hand it becomes plastic. Insoluble in alcolool; sparingly soluble in cold alcohol, but completely soluble in ether, chloroform, fixed and volatile oils.

Constituents. - 1 , myricin or myrical palmitate $\left(\mathrm{C}_{30} \mathrm{H}_{61}\right.$, $\mathrm{C}_{16} \mathrm{H}_{31} \mathrm{O}_{2}$ ), a spermaceti-like substance; 2 , cerin or cerotic acid $\left(\mathrm{C}_{27} \mathrm{H}_{54} \mathrm{O}_{2}\right)$, an imperfectly saponifiable waxy body; 3 , hydrocarbons $\left(\mathrm{C}_{27} \mathrm{H}_{56}\right.$ and $\left.\mathrm{C}_{34} \mathrm{H}_{64}\right)$; 4, an alcohol $\left(\mathrm{C}_{25} \mathrm{H}_{62} \mathrm{O}\right) ; 5$, ceryl alcohol $\left(\mathrm{C}_{27} \mathrm{H}_{56} \mathrm{O}\right)$.

\section{Cera Alba. White Wax. (U. S. \& B. P.)}

Synonym.-Cire blanche, Fr.; weisses wachs, G. moisture.

Yellow wax, bleached by exposure to air, light and

Properties.-A yellowish-white solid, somewhat translucent in thin layers, having a slightly rancid odor, and an insipid taste. Spec. gr. 0.965-0.975. Solubility and composition the same as those of yellow wax.

Uses.-Yellow and white wax are used as bases for plasters, ointments and cerates, since they do not decompose nor melt at the temperature of the body.

\section{Cetaceum. Spermaceti. (U. S. \& B. P.)}

A peculiar, concrete, fatty substance, obtained from (the sperm whale) Physeter macrocephalus Linné (class Mam-. malia ; order Cetacea).

Habitat.-Indian and Pacific Oceans.

Properties. - White, somewhat translucent, slightly unctuous masses of a scaly-crystalline fracture and a pearly lustre; odorless, and having a bland, mild taste. It becomes yellow and rancid by exposure to the air. Spec. gr. about 0.945. Insoluble in water, nearly so in cold alcohol; soluble in ether, chlornform, carbon disulphide. fixed and volatile. oils. 
Constituents. - 1, chiefly a fat, cetin or cetyl palmitate $\left(\mathrm{C}_{16} \mathrm{H}_{33} \mathrm{C}_{16} \mathrm{H}_{31} \mathrm{O}_{2}\right)$, composed of cetylic alcohol $\left(\mathrm{C}_{16} \mathrm{H}_{33} \mathrm{OH}\right)$ and. palmitic acid $\left(\mathrm{HC}_{16} \mathrm{H}_{31} \mathrm{O}_{2}\right) ; 2$, sperm oil, a small quantity.

\section{PREPARATION.}

Ceratum Cetacei. Spermaceti Cerate. (U. S. P.)

Spermaceti, 100; white wax, 350 ; olive oil, 550 . Made by melting and mixing.

\section{Unguentum Cetacei. (B. P.)}

Action and Uses.-Spermaceti resembles wax. It is used as an emollient and as a basis for plasters, ointments, and cerates. It is rarely used alone.

\section{MeL. Honey. (U. S. \& B. P.)}

Synonym.-Miel, Fr.; honig, G.

A saccharine secretion deposited in the honeycomb by Apis mellifica Linné (class Insecta ; order Hymenoptera).

Properties.-A syrupy liquid of a bright yellowish to a pale-yellowish-brown color; translucent when fresh, but gradually becoming opaque and crystalline; having a characteristic, aromatic odor, and a sweet, faintly acrid taste. Nearly soluble in water.

Constituents.-1, grape sugar (dextrose); 2, fruit sugar (glucose); 3 , a volatile oil ; 4, wax; 5 , formic acid, a trace. Fresh honey contains sucrose or cane sugar, which is changed into grape and fruit sugars.

\section{PREPARATION.}

Niel Despumatum. Clarified Honey. (U. S. P.)

Synonym.-Mel depuratum, B.P.; P.G.; miel despumé, mellite simple, Fr.; gereinigter honig, G.

Derivation.-Melt honey with two per cent. of its weight of paperpulp in water bath ; skim, strain, and add five per cent. of its weight of glycerin.

Dose.-Ad lib.

Honey is employed as an excipient in electuaries and confections. It is a demulcent and mild laxative for young 
animals. Oxymel (clarified honey, 8 parts; water and acetic acid, each 1 part) is a soothing preparation for the throat.

Saccharum Lactis. Sugar of Milk. $\left(\mathrm{C}_{12} \mathrm{H}_{22} \mathrm{O}_{11}+\mathrm{H}_{2} \mathrm{O}\right.$. (U. S. \& B. P.)

Synonym.-Lactose, lactin, milk sugar, E.; sucre de lait, Fr.; milchzucker, G.

A peculiar, crystalline sugar obtained from the whey of cows' milk, hy evaporation, and purified by recrystallization.

Properties. - White, hard, crystalline masses, yielding a white powder, feeling gritty on the tongue; odorless, and having a faintly sweet taste. Permanent in the air. Soluble in about 6 parts of water; insoluble in alcohol, ether, or chloroform.

Dose.-Ad lib.

Uses.-Cugar of milk is less soluble and therefore less sweet than cane sugar. It is harder, and thus assists in the subdivision of drugs, and serves as a vehicle in the making of powders and triturates. It also forms the basis of homœopathic preparations.

Sugar of milk is a considerable diuretic and may be given to dogs in 2 to $4 \mathrm{drachm}$ doses daily, in solution in the drinking water, for dropsy of renal or cardiac origin.

Pepsindm. Pepsin. (U. S. \& B. P.)

A proteolytic ferment or enzyme obtained from the glandular layer of fresh stomachs from healthy pigs; and capable of digesting not less than 3000 times its own weight of freshly coagulated and disintegrated egg albumin.

Derivation.-The chopped mucous membrane of a pig's stomach is macerated for several days in a weak, aqueous solution of hydrochloric acid, with frequent stirring. The pepsin is precipitated from this solution by the addition of sodium chloride and rises to the surface. The floating mix- 
ture is skimmed off, drained, pressed and dried. Sometimes the surface of the clean mucous membranes of the stomach of pigs, calves, or sheep is simply scraped off and dried.

Properties.-A fine, white, or Jellowish-white, amorphous powder, or thin, pale yellow or yellowish, transparent or translucent grains or scales, free from any offensive odor, and having a mildly acidulous or slightly saline taste, usually followed by a suggestion of bitterness. It slowly attracts moisture when exposed to the air. Soluble, or for the most part soluble, in abont 100 parts of water, with more or less opalescence; more soluble in water acidulated with hydrochloric acid; insoluble in alcohol, ether or chloroform.

Dose.-D., Calves and Foals, gr:x.- 3 i. (.6-4.).

PREPARATION.

Pepsinum saccharatum (pepsin, 1; sugar of milk, 9) is a weak preparation of little value.

Action and Uses.-Pepsin is of some value in the treatment of dogs and young animals. It assists the digestion of proteids in the stomach, but has no action on fat or carbohydrates of the food. The drug should usually be given along with hydrochloric acid, which converts any pepsinogen, in the gastric tubules, into pepsin. Pepsin contains the unorganized digestive ferment of the gastric juice, but is not by any means the pure ferment, which has never been isolated.

Much of the commercial pepsin is inert, or is composed largely of mucus, albumin and peptone, which later gives the preparation a musty odor and causes it to absorb moisture when exposed to the air, and to become sticky.

Pepsin is serviceable in gastric indigestion of young animals, which is sometimes accompanied by diarrhœa, and in dyspepsia and feeble digestion cansed by acute illness. Its use must not be long persisted in, as the normal functions of the stomach will fail from lack of use. Pepsin is administered in pill, or solution with hydrochloric acid. 


\section{Pancreatinum. Pancreatin. (U. S. P.)}

Synonym.-Zymine, B.P.

A mixture of the enzymes naturally existing in the pancreas of warm-blooded animals, usually obtained from the fresh pancreas of the hog (Sus Scrofa Linné; class Mammalia; order Pachydermata).

Derivation.-Chopped hog's pancreas is macerated in a dilnte aqueous solution of hydrochloric acid for 48 hours, and pancreatin, which is separated by adding a saturated solution of sodium chloride, rises to the surface and is skimmed off, drained, washed, and when nearly dry, is diluted with sugar of milk until 10 grains will exactly emulsify 2 drachms of cod-liver oil.

Properties. - A yellowish, yellowish-white or grayish, amorphous powder; odorless, or haring a faint, peculiar, not unpleasant odor, and a somewhat meat-like taste. Slowly and almost completely soluble in water ; insoluble in alcohol.

Dose.-D., gr.v.-xv. (.3-1.).

Action and Uses.-Pancreatin fulfils a fourfold function by virtne of the four ferments contained in it. It digests proteids by means of the ferment, trypsin; it decomposes and emulsifies fat owing to the ferment, steapsin; it converts starch into sugar by reason of the ferment, amylopsin; it coagulates milk through the action of a milk-curdling ferment. Pancreatin is thus more useful than pepsin on account of its more extended actions.

It does not digest food in an acid medium, but may aid digestion in the stomach, before much gastric juice has been secreted, during the first half hour after the ingestion of food. Pancreatin acts more efficiently in intestinal indigestion because of the presence of an alkaline secretion. For this reason pancreatin is commonly prescribed with sodium bicarbonate, and, if given for intestinal indigestion, it is administered in pill or tablet to dogs one or two hours after feeding. It is indicated in diarrhoea, when the freal move- 
ments contain particles of undigested food, and in other furms of deficient digestion due to general disease. Pancreatin is more especially valuable to digest food previous to its administration by the mouth or rectum (see Artificial Feeding, p. 694:) For this purpose a good preparation can be made extemporaneously by washing and cntting up a fresh pig's pancreas, soaking it in absolute alcolol for 24 hours, pressing out the alcohol, macerating it in ten times its weight of glycerin for 48 hours, and filtering. The filtered glycerin extract is added in the proportion of $3 \mathrm{i}$. to the pint of warm milk, with a little sodium bicarbonate, to artificially digest it.

\section{Fel Bovis. Oxgall. (U. S. P.)}

Synonym.-Fel tauri, inspissated oxgall, E.

The fresh bile of Bos Taurus Linné (class Mammalia; order Ruminantia).

Properties.-A brownish-green or dark green, somewhat viscid liquid, laving a peculiar, unpleasant odor, and a disagreeable, bitter taste. Spec. gr. 1.018 to 1.028. It is neutral, or has a slightly alkaline reaction.

Dose.-D., gr.v.-xv. (.3-1.).

PREPARATION.

Fel Bovis Purificatum. Purified Oxgall. (U. S. P.)

Synonym.-Fel bovinum purificatum, B.P.; fiel de boeuf purifie, Fr.; gereinigte ochsengalle (rindsgalle), G.

Evaporate oxgall, 300, to 100; add alcohol, 100. The alcohol is distilled off from the decanted and filtered solution, and the remainder evaporated to pilular consistence.

Properties.-A yellowish-green, soft solid, having a peculiar odor and a partly sweet and partly bitter taste. Very soluble in water and alcohol.

Dose.-Same as for oxgall.

Action and Uses.-Purified oxgall contains all the active elements of bile,- - biliary acids, coloring matter and cholesterin. One part of oxgall represents about fifteen parts of bile. Oxgall is a laxative, and substitute for deficiency in 
the normal biliary secretion. It is also an intestinal antiseptic. Oxgall is indicated in constipation with pale-colored freces. It interferes with gastric digestion and shonld be given to dogs in pills some lours after meals. Twenty grains dissolved in an onnce or two of water may be given with advantage to logs (in enema) for chronic constipation witl impacted frees.

\section{Papain. (Non-official.)}

Synonym.-Papayotine, papaya, papayine, papoid.

A digestive ferment obtained from the juice of the unripe fruit of Carica papaya (Papaw), an herbaceous tree growing in the East and West Indies. Papain or papayotine are often used to describe the dried juice itself, which exists in the form of a powder similar to that of gnm arabic. The ferment is precipitated with alcolıol, from the juice, and albuminoid matters are removed by basic learl acetate. Papain occurs in the form of a white, or grayish-white, nearly tasteless powder, soluble in glycerin and water. Papain is said to digest both proteids and carbohydrates, in either an acid or alkaline medium, and is recommended in gastric or intestinal indigestion in pill or powder. It has also been used to destroy pyogenic membrane of fistulæ and abscess, in 5 per cent. solution; or tumors and malignant growths, injected into the tissues in 10 per cent. solution. This latter use is accompanied by pain and febrile temperature, although the substance is said to merely dissolve diseased tissues without canstic effect. Papain may be given to dogs, foals, or calves in doses of gr.ii.-x. (.12-.6). Its exact therapeutic value has yet to be determined.

\section{Oleum Morrhuæ. Cod Liver Oil. (U. S. \& B. P.)}

Synonym.-Oleum jecoris ascelli, P.G.; oleum hepatis morrhnæ, cod oil, E.; linile de morne, huile de foie de morue, Fr.; leberthran, stockfischleberthran, G. 
A fixed oil obtained from fresh livers of Gadus Morrhuæ Linné and other species of Gadus (class Pisces; order Teleostei ; family Gadidæ).

Habitat.-North Atlantic Ocean.

Properties.-A pale-yellow, thin, oily liquid, having a peculiar, slightly fishy, but not rancid odor, and a bland, slightly fishy taste. Spec. gr. 0.920-0.925. Scarcely soluble in alcohol, but readily soluble in ether, chloroform or carbon disulphicle; also in 2.5 parts of acetic ether. Brown oils are not desirable therapentically.

Constituents.-1, glycerin oleate, 70 per cent.; 2, palmitiu and stearin, 25 per cent.; 3, oleic, margaric, palmitic, stearic, butyric and acetic acids, in small quantities; 4 , biliary matter, as cholic, fellinic and bilifellinic acids; 5 , gaduin $\left(\mathrm{C}_{35} \mathrm{H}_{46} \mathrm{O}_{9}\right)$; 6, morrhuol, a crystalline substance containing iodiue, phosphorns and bromine; 7, traces of iron, lime, and magnesia; 8, decomposition prodnets or cadaveric alkaloids, in brown oils.

Dose.-H. \& C., z̈ii.-iv. (60.-120.); Sh. \& Sw., żss.-i. (15.-30); D. \& C., 3 i..iii. (4.-12.).

Action Internal.-Cod liver oil resembles other oils in aiding untrition, the accumulation of fat, and the maintenance of bodily heat, but surpasses them in three particulars: 1 , cod liver oil is more easily absorbed; this has been proven comparatively by injecting varions oils into separate ligated portions of the living animal bowel ; 2 , cod liver oil is more readily oxidized after absorption; this is shown by ins lact that it reluces and therefore changes the color of potassinm permanganate solutions more quickly than other oils; 3 , cod liver oil increases the number of red blood cormmscles in anæmia; this has been demonstrated by bloodcom u. bnt mot ly comparison with the effect of other oils. Th "asise of aborptom is thonglit by some to be due to his. I prin inies which ail the diffirsion of substances $t$ ol a mmens membrame (usmosis) when the latter is

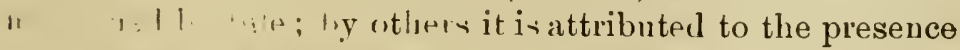
I 1 w onl whin.ls wonld tend to saponify and 
emulsify the drug. The medicinal superiority of cod liver oil has led many writers to classify it as an alterative, and special properties have been referred to the minute traces of iodine, phosphorus, and bromine in the oil. These agents exist in too infinitesimal an amount to exert much therapeutic action.

It is probable that no one constituent, or group of constituents, yet separated from the oil truly represent its medicinal effect. Cod liver oil is inferior to other oils in one respect, however, and this consists in its liability to cause nausea, incligestion, diarrhœea and vomiting, in large doses and in some patients.

Administration.-Cod liver oil may be given pure, or if this does not agree, it may be exhibited in various ways : 1 , with an equal quantity of lime water and a little syrup; 2 , with ether ( $m x$.$) , small animals; 3 \mathrm{i}$. to large animals; or with whiskey; 3, shaken with white of an egg, or mucilage of tragacanth, and a few drops of oil of peppermint; 4 , to dogs, in one of the proprietary emulsions, or with malt extract. The oil should be given after feeding and administration begun with the smaller doses as recommended above.

Uses.-Cod liver oil is indicated generally in conditions of malnutrition occurring primarily; in the course of chronic diseases; or following acute diseases. It is especially indicated in diseases of the respiratory tract, when it improves the nutrition of the mucous membranes, as well as the general nutrition. Thus cod liver oil is one of the best remedies in tardy convalescence from canine distemper; from influenza, bronchitis, pneumonia and strangles in horses; also in "heaves," empliysema, or broken wind in horses. Carron oil is cheaper and very efficient in the latter disorder, given on the food.

Cod liver oil is valuable in the treatment of chronic bronchitis, chronic eczema, and chorea of dogs; and in rickets, anæmia, weakness, and emaciation in all young animals. It often proves curative in various forms of muscular and chronic articular rheumatism, and facilitates the absorption 
of chronically enlarged glands. The use of cod liver oil is contra-indicated in hot weather, and in animals suffering from indigestion or acute diarrhœa. It is often beneficial, however, in chronic diarrhœa.

Linseed oil, oil cake, and cottonseed meal may often be conveniently and properly substituted for cod liver oil, in the case of the larger patients; while morrhuol (gr.iii. $=1$ teaspoonful cod liver oil?) given in pills, or lipanin (oleic acid, 6 ; olive oil, 100) may be exhibited in drachm doses to dogs, when cod liver oil does not agree.

\section{Icthyouus. Icthyol. (Non-official.)}

Synonym.-Ammonium icthyol sulphonate.

Derivation.-A bituminous quartz occurring in the Tyrol Mountains, containing the fossil remains of fish, is distilled with strong sulphuric acid, and sulphurous acids are removed from the distillate by sodium chloride, while sulphonic acid separates out. The latter is usually saturated with ammonia, forming icthyol; but similar preparations are made by the combination of sulphonic acid with sodium, lithium and zinc.

Properties.-A thick, dark, reddish-brown liquid, of a tarry consistency, and possessing a peculiar, disagreeable odor, and hot, bituminous taste. It is soluble in water, glycerin, alcohol, benzol, fats and fixed oils.

Constituents.-Icthyol contains about 10 per cent. of sulphur; also an inseparable volatile oil, to which its disagreeable odor is due.

Action and Uses.-Icthyol is one of the most widely useful drugs recently introduced into medicine. It is particularly valuable in the treatment of acute and chronic diseases of the skin and subadjacent tissues, accompanied with inflammation, pain, swelling, and induration; also in epidermal proliferation. Icthyol is supposed to readily permeate the skin, and there act to relieve inflammation and pain, and aid resolution. It is one of the most successfnl remedies in 
chronic eczema, psoriasis, and urticaria; in erysipelas muscular and articular rheumatic disorders; and in bruised and strained muscles, tendons, and ligaments.

The drug has proved of great worth in the treatment of frost bites, burus, and in causing absorption of lymphatic enlargements. Icthyol is recommended as a cure for sarcoptic mange and scab. It is donbtful whether the drug is an antiseptic, but is stimulant, anodyne, and resolvent, locally. Ictlyol is most satisfactorily applied to the urbroken skin. in ointment, with lauolin or lard, 25 to 50 per cent. Solutions in water, glycerin, oils or alcohol, are sometimes employed of various strengths. Icthyol is rarely given internally for chronic rheumatism.

Thiol is a substitute for icthyol, lacking the unpleasant odor of the latter medicament. Thiol is derived from browncolored paraffin or gas-oils, by a complicated process, and consists of a mixture of sulphuretted hydrocarbons. The drug exists in two forms: 1, thiolum liquidum, a thin, brownish-black liquid, soluble in water and glycerin; 2, thiolum siccum, occurring in lustrous scales. Thiol is. cheaper than icthyol and is said to be as efficacious as the latter. This remains to be proved. Liquid thiol is employed. in 10 per cent. aqueous solution or in ointment; and thiolum. siccum in powder, dusted on inflamed parts, as in acute. moist eczema. 


\section{DOSES OF DRUGS.}

In the following table three doses are usually given for each drug. The first dose is for horses and cattle, in botlr the apothecaries' and metric systems of weights and measures. The second dose is for sheep and swine, in both the apothecaries' and metric systems of weights and measures. The third dose is for dogs (and also cats) in both the apothecaries' and metric systems of weights and measures.

Letters are used to signify the name of the animal for which the dose is intended. Thus : H., Horses; C., Cattle; Sh., Sheep; Sw., Swine; D., Dogs (which also includes cats in most cases).

The following abbreviations are also employed: $l b$, pound ; pt., pint; oz., ounce ; dr., drachm ; m., minim ; gr., grain; gm., gram; cc., cubic centimeter. These doses are suitable for animals of average weight.

\section{Dose Table.}

ACetanilid.-H. \& C., dr. 1-2 (gm. 4.-8.). Sh. \& Sw., dr. $\frac{1}{3}-1$ (gm. 2.-4.). D., gr. 3-7 (gm. .2-.5).

ACETUM OPII.-H., oz. 1-2 (cc. 30.-60.). C., oz. 2-3 (cc. 60.-90.) Sh. \& Sw., dr. 2-6 (cc. 8.-24.). D., m. 3-20 (cc. .2-1.3).

ACID, ARsenous.-H. \& C., gr. 2-3 (gm. .12-.2); single dose, gr. 5-10 (gm..3-.6). Sh. \& Sw., gr. 1-2 (gm. .06..12). D., gr. $\frac{1}{30}-\frac{1}{10}$ (gm. $.002-.006)$.

ACID, Benzorc.-H., dr. 2-4 (gm. 8.-15.). D., gr. 5.-15 (gm. .3-1.).

ACID, BORIC.-H., dr. 2-4. (gm. 8.15.). D., gr. 5-15 (gm. .3-1.).

ACId, Carbolic.-H. \& C., gr. 15-30 (gm. 1.-2.). Sh. \& Sw., gr. 5-10 (gm. .3-.6). D., gr. $\frac{1}{2}-1$ (gm. .03-.06).

ACID, Citric.-H., dr. 2-4 (gm. 8.-15.). D., gr. 10-20 (gm. .6-1.3).

ACL, GALLIC.-H. \& C., dr. 2-4. (gm. 8.-15.). Sh. \& Sw., dr. $\frac{1}{2}-1$ (gm. 2.-4.). D., gr. 5-20 (gm. .3-1.3).

ACID, HYDRIODIC (syrup of).-D., dr. 1 (cc. 4.). 
ACID, HYDROCHLORIC (dilute).-H., dr. 1-2 (cc. 4.-8.). C.. dr. 2-4 (cc.8.15.). Sh. \& Sw., m. 15-20 (cc. 1-1.3). Sw. \& D., m. 5-20 (cc. .3-1.3). Acid, Hydrocyanic (dilute).-H. \& C., m. 20-40 (cc. 1.3-2.6). Sh., m. 10-15 (cc. .6-1.). Sw., m. 2-5 (cc. .12-.3). D., m. 1-3 (cc. .06-.2). ACID, LaCTIC.-H. \& C., dr. $2-4$ (cc. 8.-15.). D., dr. $\frac{1}{2}-1$ (cc. 2.-4.).

ACID, Nitric (dilute).-H., dr.1-2 (cc.4.-8.). C., dr. 2-4 (8.-15.). Sh., dr. $\frac{1}{2}-1$ (cc. 2.-4.). Sw. \& D., m. 5-30 (cc. .3-2.)。

ACID, NitrohydROCHLORIC.-H., m. 20-40 (cc. 1.3-2.6). D., m. 3-5 (cc. .2-.3).

ACID, Nitrohydrochloric (dilute).-H., dr. 1-2 (cc. 4.-8.). C., dr. 2-4 (cc. 8.-15.). Sh., dr. $\frac{1}{2}-1$ (cc. 2.-4.). Sw. \& D., m. 5-30 (.3-2.).

Acid, Phosphoric (dilute).-H., dr. 1-2 (cc. 4.-8.). C., dr. 2-4 (cc. 8.15.). Sh. \& Sw., dr. $\frac{1}{2}-1$ (cc. 2.-4.). D., m. 5-30 (.3-2.).

ACid, Salicylic.-H. \& C., dr. 2-8 (gm. 8-30). Sh., dr. 1-4 (gm. 4.-15.). Sw., dr. $\frac{1}{2}-1$ (gm. 2.-4.). D., gr. 5-30 (gm. .3-2.).

ACID, SUlPhuric (dilute).-H., dr. 1-2 (cc. 4.-8.). C., dr. 2-4 (cc. 8.15.). Sh., dr. $\frac{1}{2}-1$ (cc. 2.-4.). Sw. \& D., m. 1 u-30 (cc. .6-2.).

ACid, Sulphuric, Aromatic.-H., dr. $\frac{1}{2}-1$ (cc. 2.-4.). C., dr. 2.4. (cc. 8.-15.). Sh., m. 15-30 (cc. 1.-2.). Sw. \& D., m. 5-15 (cc. .3-1.).

AcID, Sulphurous.-H. \& C., oz. 1-2 (cc. 30.-60.). Sh. \& Sw., dr. 1-2 (cc. 4.-8.). D., dr. $\frac{1}{2}-2$ (cc. 2.-8.).

Acid, Tannic.-H. \& C., dr. $\frac{1}{2}-4$ (gm. 2.-15.). Sh. \& Sw., dr. $\frac{1}{2}-1$ (gm. 2.-

4.). D., gr. 1-15 (gm. .06-1.).

ACid, Tartaric.-H. \& C., dr. 2-4 (gm. 8.-15.) D., gr. 10-30 (gm. .6-2.). Aconite.-H., gr. 3-20 (gm. .2-1.3). D., gr. $\frac{1}{1}-2$ (gm. .006-.12).

Aconite, Extract of Leaves.-H., gr. 3-6 (gn? .2-.4). D., gr. $\frac{1}{2}-1$ (gm. .03-.06).

ACONITE, Extract of.-H., gr. 1-3 (gm. .06-.2) · D., gr. ${ }_{10}^{\frac{\pi}{4}-\frac{1}{4}}$ (gm. .006$.015)$.

ACONite, Fluid Extract of.-H., m. 3-20 (cc. .2-1.3). D., m. $\frac{1}{10}-2$. (cc. .006-.12).

Aconite, Tincture of. -H., m. 5-40 (cc. .3-3.6). C., dr. $\frac{1}{2}-1$ (cc. 2.-4.). Sh. \& Sw., m. 5-10 (cc. .3-.6). D., m. $\frac{1}{2} \cdot 5$ (cc. .03-.3). (See p. 459.)

Aconitine Nitrate (Squibb).-H., gr. $\frac{1}{30}$ (gm. .002). D., gr. $\frac{1}{20 \overline{0}}-\frac{1}{100}$ (gm. .0003-.0006).

Adrenalin Solution.-(By mouth), H., dr. 2-4 (cc. 8.-15.). D., m10-60 (cc. 0.6-4.).

AloES.-H., oz. $\frac{1}{2}-1$ (gm. 15.-30.). C., oz. 1-2 (gm. 30.-60.). Sh., oz. $\frac{1}{2}-1$ (gm. 15.-30.). Sw., dr. 2-4 (gm. 8.-15). D., gr. 20-60 (gm. 1.3-4.). 
Alorn.-II. \& C., dr. 2-3 (gn. 8.-12.). D., gr. 2-20 (gm. .12-1.3).

Alum.-H. \& C., dr. 2-4 (gm. 8.-15.). Sh. \& Sw., gr. 20.-60. (gm. 1.3-4.). D., gr. 5-10 (gm. .3-.6). Emetic, dr. 1 (gm. 4).

Ammonia, Aromatic Spirit of.-H. \& C., oz. 1-2 (cc. 30.-60.). Sh. \& Sw., dr. 2-4 (cc. 8.-15.). D., dr. $\frac{1}{2}-1$ (cc. 2.-4.)

Ammonia, Spirit of. -H. \& C., oz. $\frac{1}{2}-1$ (cc. 15.30.). Sh. \& Sw., dr. 1-2 (cc. 4.-8.). D., m. 10-20 (cc. .6-1.3).

Ammonia, Water of.-H. \& C., oz. $\frac{1}{2}-1$ (cc. 15.-30.). Sh. \& Sw., dr. 1-2 (cc. 4.-8.). D., m. 10-20 (cc. .6-1.3).

Ammonia, Stronger Water of.-H. \& C., dr. .2-6. (cc. 8.-24.). Sh. \& Sw., dr. 1 (cc. 4.). D., m. 5-10 (cc. .3-.6).

Ammoniacum.-H. \& C., oz. 1-2 (gm. 30.-60.). Sh. \& Sw., dr. $2-4$ (gm. 8.-15.). D., gr. $5-30$ (gm. .3-2.)

Amoniaccm, Emulsion of.-D., oz. $\frac{1}{2}-1$ (cc. 15.-30.).

Ammonium, Solution of Acetate.-H. \& C., oz. 2-4 (cc. 60.-120.). D., dr. 2.8 (cc. 8.-37.).

Aunonium Benzoate.-H., dr. 2-4 (gm. 8.-15.). D., gr. 5-15 (gm. .3-1.)。

Ammonium Carbonate.-H., dr. 2 (gm. 8.). C., dr. 3-6 (gm. 12.-24). Sh. \& Sw., gr. 15.-40. (gm. 1.-2.6). D., gr. 3-10 (gm. .2-.6). Emetic, D., gr. 15 (gm. 1).

Amionium Chloride.-H., dr. 2 (gm. 8.). C., dr. 3-6 (gm. 12.-24.) Slı. \& Sw., gr. 15-40 (gm. 1.-2.6). D., gr. 3-10 (gm. .2-.6).

Amionium Valerianate. -D., gr. 2-5 (gm. .12-.3).

AMYl NitRite.-H., dr. $\frac{1}{2}-1$ (cc. 2.-4.). D., m. 2-5 (cc. .12-.3), by. inhalation.

Axise.-H. \& C., oz. 1-2 (gm. 30.-60.). Sh. \& Sw., dr. 2.3 (gm. 8.-12.). D., gr. $10-30$ (gm. .6-2.).

ANISE, Oil of.-H., m. 20-30 (cc. 1.3-2.). D., m. 1-5 (cc. .06-.3).

AnIse, Spirit of.-D., dr. 1-2 (cc. 4.-8.).

Antimony and Potassium Tartrate.-H., dr. $2-4$ (gm. 8.-15.). Sw.; emetic, gr. 4-10 (gm. .24-.6). D., gr. $\frac{1}{10}-\frac{1}{2}$ (gm. .006-.03). Emetic, D., gr. 1-2 (gm. .06-.12).

Antmiony, Wine of.-D., m. 5-60 (cc. .3-4.)

ANTIPYRIN.-H. \& C., dr. 3-4 (gm. 12.-15.). Sh. \& Sw., dr. 1 (gm. 4). D. . gr. 5-20 (gm. .3-1.3).

ANTISTREPTOCOCCUS SERUM.-H., 20-50 cc.

Antitoxins, Tetanus.-H., 20 cc. (See p. 742.)

APOMORPHINE HYDROCHLORATE. -D., emetic subcutaneously, gr. $\frac{1}{30}-\frac{1}{10}$ (gm. .002-.006); by mouth, gr. $\frac{1}{8}-\frac{1}{5}$ (gm. .008-.012). D., expectorant, gr. $\frac{1}{40}-\frac{1}{25}$ (gm. .0015-.0024). 
AreCA Nut.-H., oz. $\frac{1}{2}-1$ (gm. 15.-30.). Lamb, dr. 1 (gm. 4.). D., gr. 15-60 (gm. 1.-4.). Fowl, gr. 10 (gm. .6).

AreCA, Fluid Extract of.-Dose same as nut.

ARECOLINE Hydrobromate.-H. \& C., gr. $\frac{2}{3}-1 \frac{1}{2}$ (gm. .04-.1), subcutanoously.

Arnica Flowers.-H. \& C., oz. $\frac{1}{2}-1$ (gm. 15.-30.). D., gr. $10-20$ (gm. .61.3).

ARnica Flowers, Tincture of.-H. \& C., oz. 1 (cc. 30.). D., m. 15-40 (cc. 1-2.6).

ARNicA Root.-H. \& C., oz. $\frac{1}{2}-1$ (gm. 15.-30.). D., gr. 10-20 (gm. .6-1.3).

ARnica Root, Fluid Extract of.-H. \& C., (oz. $\frac{1}{2}-1$ (cc. 15.-30.) D., m. 10-20 (cc. .6-1.3).

ARNica Root, Tincture of.-H. \& C., dr. 4 (cc. 15.). D., m. 7-20 (cc. .51.3).

ARNica Root, Tincture of (B. P.) -H. \& C., oz. 1-2 (cc. 30.-60.). D., dr. $\frac{1}{2}-1$ (cc. 2.-4.).

Arsenic.-See Acid, Arsenous.

ARSENIC, Fowler's Solution of.-H. \& C., dr. 2-8 (cc. 8.-30.). Sh. \& Sw., dr. 1-2 (cc. 4.-8.). D., m. 2-10 (cc. .12-.6).

ASAFETIDA.-H. \& C., oz. $\frac{1}{2}-1$ (gm. 15.-30.). Sh. \& Sw., dr. 1-2 (gm. 4.8.). D., gr. $3-10$ (gm. .2-.6).

ASAFETIDA, Emulsion of.-D., oz. $\frac{1}{2}-1$ (cc. 15.-30.)

Asafetida, Pilî.-D., (1.-4.).

ASAFETIDA, Tincture of.-H. \& C., oz. $2-4$ (cc. 60.-120.). D., dr. $\frac{1}{2}-1$ (cc. 2.-4.).

ASPIDIUM, Fluid Extract of (B. P.).-H. \& C., dr. 3-6 (cc. 12.-24.). Sh. \& Sw., dr. 1-2 (cc. 4.-8.). D., m. 15-60 (cc. 1.-4.).

ASPIDIUM, Oleoresin of.-H. \& C., dr. 3-6 (cc. 12.-24.). Sh. \& Sw., dr. 1.2 (cc. 4.-8.). D., m. 15-60 (cc. 1.-4.).

Atropine Sulphate.-H., gr. 1-1 $\frac{1}{2}$ (gm. .06-.09). C., gr. 1-2 (gm. .06-.12). Sh. \& Sw., gr. $\frac{1}{16}-\frac{1}{12}$ (gm. .004-.005). D., gr. $\frac{1}{20}-\frac{1}{30}$ (gm. .0005-.002). A verage dose, D., gr. $\frac{1}{100}$ (gm. .0006).

Balsam of Peru.-H. \& C., oz. 1-2 (cc. 30.-60.). Sh. \& Sw., dr. 1-2 (cc. 4.-8.). D., m. 10-30 (cc. .6-2.)

BARIUM ChLORIDE.-H., dr. 1-2 (gm. 4.-8.). Intertracheally, gr. 7-15 (gm. .5-1.).

Belladonin, Alcoholic Extract of Leaves.-H. \& C., gr. 10-20 (gm. .6-

1.3). Sh. \& Sw., gr. 2-4 (gm. .12-.24). D., gr. $\frac{1}{8}-\frac{1}{2}$ (gm. .008-.08). 
Belladonna, Tincture of Leaves.-D., m. 15-30 (cc. 1.-2.).

Belladonna Root, Fluid Extract of.-H., dr. 1.2 (cc. 4.-8.). C., dr. 2-3 (cc. 8.-12.). Sh. \& Sw., m. 10-15 (cc. .6-1.). D., m. 1-3 (cc. $.06-.2)$.

Benzoin, Tincture of.-H., oz. 1 (cc. 30.). D., dr. $\frac{1}{2}-1$ (cc. 2.-4.)

Bismuth Salictlate.-D., gr. 5-10 (gm. .3-.6).

Bismcth Subcarbonate. - H., dr. 2-4 (gm. 8.-15.). D., gr. 10-30 (gm. $.6 \cdot 2)$.

Bismuth Subnitrate.-Dose same as subcarbonate.

Brandy.-H. \& C., oz. 2-4 (cc. 60.-120.). Sh. \& Sw., oz. 1-2 (cc. 30.-60.), D., dr. 1-4 (cc. 4.-15.).

BuCHU, Fluid Extract of.-H., oz.1-2 (cc. 30.-60.). D., m.5-30 (cc. .3-2.).

BuckTHORN (Rhamnus Catharticus), Syrup of.-D., oz. 1-2 (cc. 30.-60.). Cats, oz. $\frac{1}{2}-1$ (cc. 15.-30.).

Caffeine.-H., gr. 7-15 (gm. .5-1.). D., gr. $\frac{1}{2}-3$ (gm. .03-.2).

Caffeine, Citrate of.-H., gr. 15-30 (gm. 1.-2.). D., gr. 1-6 (gm. .06-.36).

Calamus.-H. \& C., oz. 1-2 (gm. 30.60.). Sh. \& Sw., dr. 2.3 (gm. 8.12.). D., gr. 15-60 (gm. 1.-4.).

Calames, Fluid Extract of.-Dose same as Calamus.

Calcium, Precipitated Carbonate of.-H., oz. 1-2 (gm. 30.-60.). C., oz. 2-4 (gm. 60.-120). Sh. \& Sw., dr. 2-4 (gm. 8.-15.). D., gr. 10-60 (gm. 6-4).

Calciem Úhloride.-H. \& C., Oz. $\frac{1}{2}-1$ (gm. 15-30). D., gr. 5-20 (gm. .3-1.3).

Calcium, Lactophosphate, Syrup of. - Foals anc Calves, oz. $\frac{1}{2}-1$ (cc. 15.-30.). D., dr. 1-4 (cc. 4.-15.)

Calcium, Precipitated Phosphate of.-H., dr. 2-4 (gm. 8.-15.). C., oz. $\frac{1}{2} 1$ (gm. 15.-30.). Sh. \& Sw., dr. 1-2 (gm. 4.-8.). D., gr. 5-20 (gm. .3-1.3).

CALOMEL.-See Mercury.

Calumba.-H. \& C., oz. $\frac{1}{2}-1$ (gm. 15.-30.). Sh. \& Sw., dr. 1-2 (gm. 4.-8.). D., gr. 5-30 (gm. .3-2.).

Calumba, Fluid Extract of.-Dose same as Calumba.

Calumba, Tincture of.-H. \& C., oz. 2.4 (cc. 60.-120.). I’., dr. 1-4 (cc. 4.-15.).

CAMPhOR.-H., dr. 1-3 (gm. 4.-12.). C., dr. 2-4 (gm. 8.-15.). Sh. \& Sw., gr. 15-60 (gm. 1.-4.). D., gr. 3-20 (gm. .2-1.3).

CAMPHoR, Monobromated.-D., gr. 2-10 (gm. .12-.6).

CAMPhOR, Spirit of.-H., oz. 1-2 (cc. 30.-60.). D., dr. $\frac{1}{2}-1$ (cc. 2.-4.). 
Cannabis Indica, Extract of.-H., dr. 1-2 (gm. 4.-8.). D., gr. $\frac{1}{6}-1$ (gm. $.015-.06)$.

Cannabis Indica, Fluid Extract of.-H., dr. 4-6 (cc. 15.-24.). D., m. 3-10 (cc. .2-.6).

Cannabis Indica, Tincture of.-D., m. 15-30 (cc. 1.-2.).

Cantharides.-H. \& C., gr. 5-20 (gm. .3-1.3). Sh. \& Sw., gr. $4-8$ (gm. .24-.5). D., gr. 1-2 (gm. .06-.12).

Cantharides, Tincture of.-H., dr. 2-4 (cc. 8.-15.). D., m. 2-15 (cc. .12-1.).

CAPsicum.-H., gr. 20.60 (gm. 1.3-4.). C., dr. 1-2 (gm. 4.-8.). D., gr. 1-8 (gm. .06-.48).

CaPsicum, Fluid Extract of.-Dose same as Capsicum.

Capsicum, Oleoresin of.-H., m. 10-30 (cc. .6-2.). C., dr. $\frac{1}{2}-1$ (cc. 2.-4.). D., m. $\frac{1}{4}-1$ (cc. .015-.06).

Capsicum, Tincture of. - H., dr. $2-4$ (cc. 8.-15.). C., oz. $\frac{1}{2}-1$ (cc. 15.-30.). D., m. 5-60 (cc. .3-4.).

Carbon Bisulphide.-H., dr. 2-4 (cc. 8.-15.).

Cardamon.-H. \& C., oz. 1-2 (gm. 30.-60.). Sh. \& Sw., dr. 2-3 (gm. 8.12.). D., gr. 10-30 (gm. .6-2.).

Cardamon, Fluid Extract of.-Dose same. (Non-official.)

Cardamon, Compound Tincture of.-D., dr. 1-2 (cc. 4.-8.).

Cardamon, Tincture of.-D., dr. 1-2 (cc. 4.-8.).

Cascara Sagrada.-D., gr. 5-30 (gm. .3-2.).

Cascara Sagrada, Fluid Extract of.-D., m. 5-30 (cc. .3-2.).

Cascara SAgrada (Solid) Extract of.-D., gr. $2-8$ (gm. .12-.5).

Cascarilla.-H. \& C., oz. $\frac{1}{2}-1$ (gm. 15.-30.). Sh. \& Sw., dr. 1-2 (gm. 4.8.). D., gr. 10-30 (gm. .6-2.).

Castor Orl.-H. \& C , pt. 1 (cc. 500.). Sh. \& Sw., oz. 2-4 (cc. 60.-120.). D., dr. 1-2 (cc. 4.-8.). Fowl, dr. 1 (cc. 4.).

Catechu.-H., oz. $\frac{1}{2}-1$ (gm. 15.-30.). C., oz. 1-2 (gm. 30.-60.). Sh. \& Sw., dr. 1-2 (gm. 4.-8.). D., gr. 5-30 (gm. .3-2.).

Catechu, Fluid Extract of.-(Non-official.)-Dose same as Catechu.

Catechu, Compound Tincture of.-H. \& C., oz. 1-2 (cc. 30.-60.). Foals,

Calves and Sheep, oz. $\frac{1}{2}-1$ (cc. 15.-30.). D., dr. $\frac{1}{2}-2$ (cc. 2.-8.).

Cerium Oxalate.-D., gr. 3-5 (gm. .2-.3).

Chalk, Compound Powder of.-D., gr. 10-60 (gm. .6-4.). 
Chalk Mixture.-D., oz. 1-2 (cc. 30.-60.).

Chalk, Prepared.-H., oz. 1-2 (gm. 30.-60.). C., oz. 2-4 (gm. 60.120.). Sh. \& Sw., dr. 2-4 (gm. 8.-15.). D., gr. 10-60 (gm. .6-4.).

Charcoal (Wood, and animal, purified).-H. \& C., oz. 1-2 (gm. 30.60.). Sh. \& Sw., dr. 2-4 (gm. 8.-15.). D., gr. 20-60 (gm. 1.3-4.).

Chloral.-H. \& C., oz. 1-2 (gm. 30.-60.). Sh. \& Sw., dr. 1-2 (gm. 4.-8.). D.. gr. 5-20 (gm, .3-1.3).

Chloroform.-H. \& C., dr. 1-2 (cc. 4.-8.). Sh. \& Sw., m. 20-30 (cc. 1.3-2.). D., m. 2-20 (cc. .12-1.3).

Chloroform, Spirit of.-H. \& C., oz. 1-2 (30.-60.). Sh. \& Sw., dr. 2-4 (cc. 8.-15.). D., dr. $\frac{1}{2}-1$ (cc. 2.-4.)

Cinchona Bark.-H., dr. 2-4 (gm. 8.-15.). C., oz. 1-2 (gm. 30.-60.). Sh. \& Sw., dr. 1-4 (gm. 4.-15.). D., gr. 10-60 (gm. .6-4.).

Crychoxa, Compound Tincture of.-H., oz. 2-4 (cc. 60.-120.). D., dr. $\frac{1}{2}-4$ (cc. 2.-15.).

Cinchona, Extract of.-H., dr. 1-2 (gm. 4.-8.). D., gr. 5-30 (gm. .3-2.).

Cischosa, Fluid Extract of.-H., dr. 2-4 (cc. 8.-15.). C., oz. 1-2 (cc. 30.-60.). Sh. \& Sw., dr. 1-4 (cc. 4.-15.). D., gr. 10-60 (cc. .6-4.).

Cinchonine Sulphate, Tonic Dose.-H., gr. 20-60 (gm. 1.3-4.). C.,

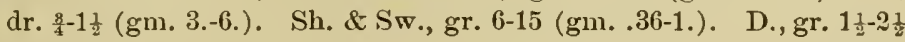
$(.1-.15)$.

Cinchonine Sulphate, Antipyretic Dose.-H., dr. $2 \frac{1}{2}-5$ (gm. 10.-20.). Sh. \& Sw., gr. 40-50 (gm. 2.6-3.3). D., gr. i-15 (gm. .5-1.).

Cixchonidine Sulphate.-Dose same as Cinchonine Sulphate.

Cocaine Hrdrochlorate.-H., gr. 5-10 (gm. .3-.6). D., gr $\frac{1}{8}-\frac{8}{4}$ (gm. $.008-.045)$.

Cod Liver Oil.-H., oz. 2-4 (cc. 60.-120.). Sh. \& Sw., oz. $\frac{1}{2}-1$ (cc. 15.-30.). D., dr. 1-3 (cc. 4.-12.).

CODEINE.-D., gr. $\frac{1}{4}-2$ (gm. .015-.12).

Colchicine.-H. \& C., gr. $\frac{1}{6}-\frac{1}{2}$ (gm. .01-.03). D., gr. $\frac{1}{100^{-\frac{1}{5}}}$ (gm. .0006$.0012)$.

Colchicur Root.-H. \& C., dr. $\frac{1}{2}-2$ (gm. 2.-8.). Sh., gr. 10-20 (gm. .61.3). Sw. \& D., gr. 2.-8 (gm. .12-.5).

Colchicum Root, Fluid Extract of.-Dose same as Colchicum.

Colchicum Root, Tincture of.-H. \& C., dr. 3-8 (cc. 12.-30.). D., m. $11)-30$ (cc. .6-2.)

Colchicum Root, Wine of.-H. \& C., dr. 3-8 (cc. 12.-30.). D., m. 10-30 (cc. .6-2.).

Collargol (Colloidal Silver).-See p. 180. 
Colocynth.-D., gr. 3-8 (gm. .2-.5).

Colocynthin.-H., dr. $\frac{1}{2}-1$ (gm. 2.-4.). D., gr. $\frac{1}{4}-1$ (gm. .015-.06).

Conine Hrdrobromate.-H \& C., gr. $\frac{8}{4}-1 \frac{1}{2}$ (gm. .045-.1). Sh. \& Sw., gr. $\frac{1}{5}-\frac{2}{5}(\mathrm{gm} . .012-.024)$. D., gr. $\frac{1}{60}-\frac{1}{30}(\mathrm{gm} . .001-.002)$.

Conium.-H. \& C., dr. 1-2 (gm.-4.-8.). Sh. \& Sw., gr. 10-20 (gm. .6-1.3). D., gr. 2-5 (gm. .12-.3).

Conium, Fluid Extract of.-Dose same as Conium.

Conium, Extract of.-H. \& C., gr. 12-24 (gm. .72-1.5). Sh. \& Sw., gr. 2-4 (gm. .12-.24). D., gr. $\frac{1}{4}-1$ (gm. .015-.06).

Convallaria, Fluid Extract of.-H. \& C., dr. 1-2 (cc. 4.-8.). D., m. 5-10 (cc. .3-.6).

Copper Sulphate.-H. \& C., dr. 2-4 (gm. 8.-15.). Sh. \& Sw., gr. 20-40 (gm. 1.3-2.6). D., gr. 1-2 (gm. .06-.12). Emetic-D., gr. 6-20 (gm. .36-1.3).

Corrosive Sublimate.-(See Mercury.)

CotTon Root Bark.-(See Gossypium.)

Creasote.-H., m. 15-30 (cc. 1.-2.). C., dr. $\frac{1}{2}-1$ (cc. 2.-4.). Sh. \& Sw., m. 5.-15 (cc. .3-1.). D., m. $\frac{1}{2}-2$ (cc. .03-.12).

Creolin.-H. \& C., dr. 1-2 (cc. 4.-8.). D., m. 1-5 (cc. .06-.3). Single dose as anthelmintic. H., oz. $\frac{1}{2}-1$ (cc. 15.-30.).

Croton Oil.-H., m. 15-30 (cc. 1.-2.). C., dr. $\frac{1}{2}-1$ (cc. 2.-4.). Sh \& Sw., m. 5-10 (cc. .3-.6). D., m. $\frac{1}{2}-3$ (cc. .03-.2).

Digitalein.-H., gr. $\frac{1}{8}-\frac{1}{4}$ (gm. .008-.015). D., gr. ${ }_{100}^{\frac{1}{00}}$ (gm. .0006).

Digitalin.-H., gr. $\frac{1}{4}-\frac{1}{2}$ (gm. .015-.03). D., gr. $\frac{1}{60}-\frac{1}{30}$ (gm. .001-.002).

Digitalis.-H., gr. 10-60 (gm. .6-4.). C., dr. $\frac{1}{2}-1 \frac{1}{2}$ (gm. 2.-6.). Sh. \& Sw., gr. 5-15 (gm. .3-1.). D., gr. $\frac{1}{2}-3$ (gm. .03-.2).

Digitalis, Fluid Extract of. -Dose same as Digitalis.

Digitalis, Extract of.-H., gr. 5-10 (gm. .3-.6). D., gr. $\frac{1}{8}-\frac{1}{2}$ (gm. .008$.03)$.

Digitalis, Infusion of.-H. \& C., oz. 2-6 (cc. 60.-180.). Sh. \& Sw., oz. $\frac{1}{2}-1$ (cc. 15.-30.). D., dr. 1-4 (cc. 4.-15.).

Digitalis, Tincture of.-H. \& C., dr. 2-4 (cc. 8.-15.). Sh. \& Sw., dr. $\frac{1}{2}$ $1 \frac{1}{2}$ (cc. 2.-6.). D., m. 5-30 (cc. .3-2.).

DOVER's POWDER.-H., oz. $\frac{1}{2}-1$ (gm. 15.-30.). D., gr. 5-10 (gm. .3-.6).

Dover's POWder, Liquid.-H., oz. $\frac{1}{2}-1$ (cc. 15.-30.). D., m. 5-10 (cc. .3-.6).

ELATERIN.-D., gr. $\frac{1}{80}-\frac{1}{12}(\mathrm{gm} . .003-.005)$. 
Ergot.-H. \& C., oz. $\frac{1}{2}-1$ (gm. 15.-30.). Sh. \& Sw., dr. 1-2 (gm. 4.-8.). D., dr. $\frac{1}{2}-1$ (gm. 2.-4.).

ERGot, Fluid Extract of.-Dose same as Ergot.

ERgot, Extract of (Ergotin).-H. \& C., gr. 20-60 (gm. 1.3-4.). D., gr. 2-10 (gm. .12-.6).

ERgot, Tincture of. - H. \& C., oz. $\frac{1}{2}-2$ (cc. 15.-60.). Sh. \& Sw., dr. 1-4 (cc. 4.-15.). D., dr. $\frac{1}{2}-2$ (cc. 2.-8.).

EsERINe.-(See Physostigmine.)

ETHER.-H. \& C., oz. 1-2 (cc. 30.-60.). Sh. \& Sw., dr. 2-4 (cc. 8.-15.). D., m. 10-60 (cc. .6-4.).

ETHER, Spirit of, and Compound Spirit of.-H. \& C., oz. 1-2 (cc. 30.-60.). Sh. \& Sw., dr. 2-4 (cc. 8.-15.). D., m. 10-60 (cc. .6-4.).

Ether, Nitrous, Spirit of (Sweet Spirit of Nitre).-H. \& C., oz. 1-4 (cc. 30.-120.). Sh. \& Sw., dr. 2-4 (cc. 8.-15.). D., m. 10-60 (cc. .6-4.)

Eucalyptus, Fluid Extract of.-H., oz. 2-3 (cc. 60.-(40.). D., dr. $\frac{1}{2}-2$ (cc. 2.-8.).

EUCalyptus, Oil of.-H., dr. 2-4 (cc. 8.-15.). D., m. 5-20 (cc. .3-1.3).

EUCALYPTOL.-Dose same as Oil of Eucalyptus.

Fennel.-H. \& C., oz. 1-2 (gm. 30.-60.). Sh. \&Sw., dr. 2-3 (gm. 8.-12.). D., gr. 10-20 (gm. .6-1.3).

FeL Bovis.-(See Oxgall.)

Fendareek.-H. \& C., oz. 1-2 (gm. 30.-60.). Sh. \& Sw., dr. 2-3 (gm. 8.12.). D., gr. 10-30 (gm. .6-2.).

Frangula, Fluid Extract of. - D., dr. $\frac{1}{2}-1$ (cc. 2.-4.).

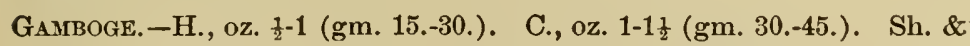
Sw., gr. 20-60 (gm. 1.3-4.). D., gr. 5-10 (gm. .3-.6).

Gaultheria, Oil of.-H. \& C., dr. 2 oz.1 (cc. 8.-30.). Sh. \& Sw., dr. $\frac{1}{2}$ 2 (cc. 2.-8.). D., m. 5-15 (cc. .3-1.).

Gelsemine.-H., gr. $\frac{1}{4} \frac{1}{2}$ (gm. .015-.03). D., gr. $\frac{1}{60}-\frac{1}{20}$ (gm. .001-.003).

GELSEMIUM.-H., dr. 1-2 (gm. 4.-8.). D., gr. 5-10 (gm. .3-.6).

GELseminu, Fluid Extract of.-Dose same as Gelsemium.

Gelsemium, Tincture of. - H., oz. $\frac{1}{2} 2$ (cc. 15.-60.). D., m. 15-60. (cc. 1.-4.).

Gentian.-H., oz. $\frac{1}{2}-1$ (gm. 15.-30.). C., oz. 1-2 (gm. 30.-60.). Sh. \& Sw., dr. 1-2 (gm. 4.-8.). D., gr. 5-30 (gm. .3-2.).

Gentian, Fluid Extract of.-Dose same as Gentian. 
Gentlan, Compound Tincture of.-H. \& C., oz. 1-4 (cc. 30.-120.). D., dr. 1-4 (cc. 4.-15.).

GINGER.-H., dr. 2-oz. 1 (gm. 8.-30.). C., oz. 1-4 (gm. 30.-120.). Sh. \& Sw., dr. 1-2 (gm. 4-8). D., gr. 5-15 (gm. .3-1.).

GINGER, Fluid Extract of.-Dose same as Ginger.

GLYCERIN.-H. \& C., oz. 1 (cc. 30.). D., dr. $\frac{1}{2}-1$ (cc. 2.-4.).

GLYCYRRHIZA.—(See Liquorice.)

Gossypium Root, Fluid Extract of Bark of.-H. \& C., oz. $\frac{1}{2}-1$ (cc. 15.30.). Sh. \& Sw., dr. 1-2 (cc. 4.-8.). D., dr. $\frac{1}{2}-1$ (cc. 2.-4.).

Granatum (Pomegranate).-D., dr. $\frac{1}{2}-1 \frac{1}{2}$ (gm. 2.-6.).

Granati Radicis Cortex, Fluid Extract of.-D., dr. $\frac{1}{2}-2$ (cc. 2.-8.).

HжMatoxylon, Extract of.-H. \& C., dr. $\frac{1}{2}-4$ (gm. 2.-15.). Sh. \& Sw., dr. $\frac{1}{2}-1$ (gm. 2.-4.). D., gr. 5-15 (gm. .3-1.).

HÆMATOxYLoN, Fluid Extract of.-H. \& C., oz. $\frac{1}{2}-1 \frac{1}{2}$ (cc. 15.-45.) Sh. \&

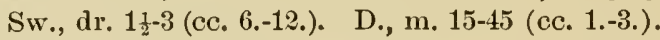

HaMamelis, Fluid Extract of.-H. \& C., oz. 1-2 (cc. 30.-60.). D., dr. $\frac{1}{2}-2$ (cc. 2.-8.).

Hrdrastin.-H., gr. 15-30 (gm. 1.-2.). D., gr. 3-5 (gm. .2-.3).

Hydrastine Hydrochlorate.-H., gr. 1-2 (gm. .06-12). D., gr. $\frac{1}{12}-\frac{1}{6}$ gm. .005-.01).

Hrdrastis, Fluid Extract of.-H. \& C., dr. 2-oz. 1 (cc. 8.-30.). Sh. \& Sw., dr. 1-2 (cc. 4.-8.). D., m. 5-60 (cc. .3-4.).

HrdRAstis, Glycerite of.-Dose same as Fluid Extract.

Hrdrastis, Tincture of.-H., oz. 1-2 (cc. 30.-60.). D., dr. $\frac{1}{2}-2$ (cc. 2.-8.).

Hroscine Hydrobronate, -H., gr. $\frac{1}{6}-\frac{1}{4}$ (gm. .01-.015). D., gr. $\frac{1}{150-10} \frac{1}{100}$ (gm. .0004-.0006).

Hyoscyamine Hydrobromate and SulPhate.-H., gr. 1-2 (gm. .06-.12).

D., gr. $\frac{1}{60}-\frac{1}{30}(\mathrm{gm}$. .001-.002).

Hroscrasius.-H. \& C., oz. $\frac{1}{2}-1$ (gm. 15.-30.). D., gr. 5-15 (gnı. .3-1.).

Hroscyayus, Fluid Extract of.-Dose same as Hyoscyamus.

Hroscyanus, Extract of.-H. \& C., gr. 20-60 (gm. 1.3-4.). D., gr. $\frac{1}{2}-2$ (gm. .03-.12).

Hyoscramus, Tincture of.-D., dr. 1-2 (cc. 4.-8.).

Hroscyanus, Succus of.-D., dr. 1-2 (cc. 4.-8.).

Hrpophosphites, Compound Syrup of.-D., dr. 1 (cc. 4.).

IODINE.-H. \& C., dr. $\frac{1}{2}-1$ (gm. 2.-4.). Sh. \& Sw., gr. 10-20 (gm. .6-1.3). D., gr. 2-5 (gm. .12-.3). 
Iobrve, Compound Solution of. - H., dr. 2-4 (cc. 8.-15.). D., m. 2-10 (cc. .12-.6).

IODINE, Tincture of. - H., dr. 2-4 (cc. 8.-15.). D., m. 2-10 (cc. .12-.6).

IPECAC. -H., dr. 1-2 (gm. 4.-8.). C., dr. 2-1 (gm. .8-15.). Sh., dr. $\frac{1}{2}-1$ (gm. 2.-4.). D., gr. $\frac{1}{2}-2$ (gm. .03-12). Emetic-D. \& Sw., gr. 15-30 (gm. 1.-2.). Cats, gr. 5-12 (gm. .13-.72).

IPECAC, Fluid Extract of.-Dose same as Ipecac.

IPECAC, Syrup of.-D., Expectorant, m. 15-60 (cc. 1.-4.).

IPECAC, Wine of.-D., Expectorant, m. 15-60 (cc. 1.-4.).

Iron AND Ammonium Citrate. - D., gr. 5-10 (gm. .3-.6).

Iron, Carbonate, Saccharated.-H., dr. 1-2 (gm. 4.-8.). C., dr. 2-4 (gm. 8.-15.). Sh. \& Sw., (gr. 20-30 (gm. 1.3-2.). D., gr. 1-5 (gm. .06-.3).

Iron, Chloride, Solution of.-H. \& C., dr. 2-4 (cc. 8.-15.). Sh. \& Sw., m. 10-20 (cc. .6-1.3). D., m. 2-10 (.12-.6).

Irox, Chloride, Tincture of.-H. \& C., oz. 1-2 (cc. 30.-60.). Sh. \& Sw., m. 20-30 (cc. 1.3-2.). D., m. 5-60 (cc. .3-4.).

Iron, Iodide, Syrup of.-D., m. 5-10 (cc. .3-6.).

IRox, (and) Quinine Citrate.-DD., gr. 5-10 (gm. .3-.6).

Iron, Reduced.-H., dr. 1-2 (gm. 4.-8.). C., dr. 2-4 (gm. 8-15). Sh. \& Sw., gr. 20-30 (gm. 1.3-2.). D., gr. 1-5 (gm. .06-.3).

Irox, (and) Strychnine Citrate.-D., gr. 1-2 (gm. .06-.12).

Iros, Sulphate and Dried Sulphate.-H. \& C., dr. 1-2 (gm. 4.-8.). Sh. \& Sw., gr. 20-30 (gm. 1.3-2.). D., gr. 1-5 (gm. .06-.3).

JABORANDI.-H. \& C., dr. 2-4 (gm. 8.-15.). Sh. \& Sw., dr. $\frac{1}{2}-1$ (gm. 2.-4.). D., gr. $5-60$ (gm. .3-4.).

JABORANDI, Fluid Extract of.-Dose same as Jaborandi.

JALAP.-Sw., dr. 2-1 (gm. 8.-15.). D., dr. 1-2 (gm. 4.-8.). Cats, dr. $\frac{1}{2}-1$ (gm. 2.-4.).

JALAP, Fluid Extract of.-Dose same as Jalap.

JALAP, Resin of.-Sw., dr. $\frac{1}{2}-1$ (gm. 2.-4.). D., gr. 15-30 (gm. 1.-2。). Cats, gr. $7-15$ (gm. .5-1.).

Juniper, Compound Spirit of.-H. \& C., oz. 2-4 (cc. 60.-120.). D., dr. 1-4 (cc. 4.-15.).

JuNiper, Oil of.-H. \& C., dr. 1-2 (cc. 4.-8.). D., m. 2-10 (cc. .12-.6).

KAMALA.-H., oz. 1 (gm. 30.). D., dr. $\frac{1}{2}-2$ (gm. 2.-8.).

Kino.-H., oz. $\frac{1}{2}-1$ (gm. 15.-30.). C., oz. 1-2 (gm. 30.-60.). Sh. \& Sw., dr. 1-2 (gm. 4.-8.). D., gr. 5-30 (gm. .3-2.). 
KIno, Fluid Extract of.-Dose same as Kino.

Kino, Tincture of.-H. \& C., oz. 1-2 (cc. 30.-60.). Foals, Calves and Sheep, oz. $\frac{1}{2}-1$ (cc. 15.-30.). D., dr. $\frac{1}{2}-2$ (cc. 2.-8.).

Koussin.-D., gr. 5-40 (gm. .3-2.6).

Kousso.-Small dogs, dr. $\frac{1}{2}-1$ (gm. 2.-4). Large dogs, dr. 2-4 (gm. 8.-15.)

Kousso, Fluid Extract of.-Dose same as Kousso.

Krameria.-H., oz. $\frac{1}{2}-1$ (gm. 15.-30.). C., oz. 1-2 (gm. 30.-60.). Sh. \& Sw., dr. 1-2 (gm. 4.-8.). D., gr. 5-30 (gm. .3-2.).

Krameria, Fluid Extract of.-Dose same as Krameria.

Krameria, Extract of.-H. \& C., dr. 2-3 (gm. 8.-12.). Sh. \& Sw., gr. 20-40 (gm. 1.3-2.6). D., gr. 5-10 (gm. .3-.6).

Krameria, Tincture of.-H. \& C., oz. 1-2 (cc. 30.-60.). Foals, Calves and Sheep, oz. $\frac{1}{2}-1$ (cc. 15.-30.). D.. dr. $\frac{1}{2}-2$ (cc. 2.-8.).

Lead ACETATE.-H. \& C., dr. 1 (gm. 4.). Sh. \& Sw., gr. 15-20 (gm. 1.1.3). D., gr. 1-2 (gm. .06-.12).

Lime, Solution of (Lime Water).-H. \& C., oz. 4-6 (cc. 120.-180.). Calves and Foals, oz. 2 (cc. 60.). D., dr. 1-8 (cc. 4.-30.).

Linseed Oil.-H., pt. $\frac{1}{2}-1$ (cc. 250.-500). C., pt. 1-2 (cc. 500.-1000.). Sh. \& Sw., oz. 6-12 (cc. 180.-360.). Dogs and Cats, oz. $\frac{1}{2}-2$ (cc. 15.-60.).

LIQUORICE. - Ad lib.

LIQUORICE, Fluid Extract of.-Ad lib.

LIQUORICE, Extract of.-Ad lib.

Liquorice, Compound Powder of.-D., dr. 1-2 (gm. 4.-8.).

Lithium Carbonate.-D., gr. 3-10 (gm. .2-.6).

Lithium Citrate. -D., gr. 5-20 (gm. .3-1.3).

Magnesia.—Foals and Calves, dr. 1-2 (gm. 4.-8.). D., gr. 5-60 (gm. .3-4.).

Magnesium Carbonate.-Dose same as Magnesia.

Magnesium Sulphate.-H. (laxative), oz. 2-4 (gm. 60.-120.). C. (purgative), lb. 1-2 (gm. 500.-1000.); (laxative), oz. 3-4 (gm. 90.-120.). Calves, dr. 2-3 (gm. 8.-12.). Sh., oz. 4-6 (gm. 120.-180.). D., dr. 1-4 (gm. 4.-15.).

MALE Fern, Oleoresin and Fluid Extract of.-H. \& C., dr. 3-6 (cc. 12.24.). Sh. \& Sw., dr. 1-2 (cc. 4.-8.). D., m. 15-60 (cc. 1.-4.).

Menthol.-D., gr. 2 (gm. .12).

Mercury with Chalk.-Foals and Calves, gr. 10-15 (gm. .6-1.). D., gr.. $1-10(\mathrm{gm} . .06-.6)$. 
Mercury, Corrosive Chloride of.-H. \& C., gr. 5-8 (gm. .3-.5). Sh. \& Sw., gr. 2 (gm. .12). D., gr. $\frac{1}{3} 0^{-\frac{1}{8}}$ (gm. .002-.008).

Mercury, Iodide of (red).-Dose same as Corrosive Chloride.

Mercury, Mass of (Blue Pill).-D., gr. 1-10 (gm. .06-.6).

Mercury, Mild Chloride of.-H., dr. $\frac{1}{2}-1$ (gm. 2.-4.). C., dr. 5-6 (gm. 20.-24.). D., gr. ${ }_{10}^{\frac{1}{2}-\frac{1}{2}}$ (gm. .006-.03), in divided doses; gr. $3-5$ (gm. $.2-3)$, in single doses.

Methyl Salicylate.-H., dr. 2-8 (cc. 8.-30). D., m. 5-15 (cc. .3-1.).

Morphine ANd ITS SAlts.-H. \& C., gr. 3-10 (gm. .2-.6). Sh., gr. $\frac{1}{2}-2$ (gm. .03-.12). Sw., gr. $\frac{1}{10-\frac{1}{2}}(\mathrm{gm} .006-.03)$. D., gr. $\frac{1}{8}-\frac{1}{2}$ (gm. .008$.03)$. Subcutaneously-H., gr. $3-4$ (gm. .2-.24). D., gr. $\frac{1}{8}-\frac{1}{3}$ (gm. $.008-.02)$.

MorrhuOL.-D., gr. 1-5 (gm. .06-.3).

MustaRd.-H. \& C., oz. $\frac{1}{2}-1$ (gm. 15.-30.). Sh. \& Sw., dr. $\frac{1}{2}-2$ (gm. 2.-8.). D., gr. 10-15 (gm. .6-1.). Emetic-D., oz. $\frac{1}{2}$ (gm. 15.).

Naphthalin.-H., dr. 2-4 (gm. 8.-15.). D., gr. 1-20 (gm. .06-1.3).

NAPHTOL.-H., dr. 2-3 (gm. 8.-12.). D., gr. 1-10 (gm. .06-.6).

NiCotiNe.-H. \& C., gr. $\frac{1}{60}-\frac{1}{20}$ (gm. .001-.003).

Nitroglycerin (1 per cent. solution).-H. \& C., dr. $\frac{1}{2}-1$ (cc. 2.-4.). D., m. 1-2 (cc. .06-.12).

Nux VonicA.-H. \& C., dr. 1-2 (gm. 4.-8.). Sh., gr. 20-40 (gm. 1.3-2.6). Sw., gr. 10-20 (gm. .6-1.3). D., gr. 1-2 (gm. .06-.12).

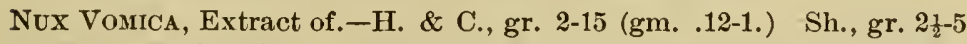
(gm. .15-.3). Sw., gr. 1-2 (gm. .06-.12). D., gr. $\frac{1}{8}-\frac{1}{4}$ (gm. .008-.015).

Nux Vomica, Fluid Extract of.-Dose same as Nux Vomica.

Nux Vomica, Tincture of.-D., m. 5-10 (cc- .3-.6).

Olive Oil. -H. \& C., pt. 1-2 (cc. 500.-1000.). D., oz. 2-4 (cc. 60.-120.)'.

OPIUM, Camphorated Tincture of (Paregoric).-D., dr. 1-4 (cc. 4.-15.).

OPIUM, Extract of.-H., dr. $\frac{1}{2}-1$ (gm. 2.-4.). C., dr. 1-2 (gm. 4.-8.). Sh., gr. 5-10 (gm. .3-.6). Sw., gr. 2-10 (gm. .12-.6). D., gr. $\frac{1}{6}-\frac{1}{2}$ (gm. $.01-.03)$.

Opium, Powder.-H., dr. 1-2 (gm. 4.-8.). C., dr. 2-4 (gm. 8.-15.). Sh., gr. 10-20 (gm. .6-1.3). Sw., gr. 5-20 (gm. .3-1.3). D., gr. $\frac{1}{2}-3$ (gm. .03-.2).

OPIUM, Tincture of.-HI., oz. 1-2 (cc. 30.-60.). C., oz. $2-3$ (cc. 60.-90.). Sh. \& Sw., dr. $2-6$ (cc. 8.-24.). D., m. 330 (cc. .2-2.).

OpIUM, Wine of.-Dose same as Tincture. 
OXGALL. -D., gr. 5-15 (gm. .3-1.).

PANCREATIN,一D., gr. 5-15 (gm. .3-1.).

Papain. -Foals, Calves and Dogs, gr. 2-10 (gm. .12-.6).

Pelletierine Tannate.-D., gr. 2-5 (gm. .12-.3).

Peppermint, Oil of.-H. \& C., m. 15-30 (cc. 1.-2.). D., m. 1-5 (cc. $.06-.3)$.

Peppermint, Spirit of.-H. \& C., dr. 2-4 (cc. 8.-15.). D., m. 15-30 (cc. 1.-2.).

PePsin.-Dogs, Foals and Calves, gr. 10-60 (gm. .6-4.).

Phenacetin.-H., dr. 2-3 (gm. 8.-12.). D., gr. 5-10 (gm. .3-.6).

Phosphorated Oil.-H., dr. 2-3 (cc. 8.-12.). D., m. 1-5 (cc. .06-.3).

Phosphorus.-H., gr. 1-2 (gm. .06-.12). C., gr. 2-3 (gm. .12-.2). Sh., Sw. \& D., gr. $\frac{1}{100}-\frac{1}{20}(\mathrm{gm} . .0006-.003)$.

Phosphorus, Spirit of,-D., m. $7-20$ (cc. .5-1.3).

Physostigma. -H., gr. 15-30 (gm. 1.-2.). D., gr. $\frac{1}{4}-1$ (gm. .015-.06).

Physostiglia, Fluid Extract of.-Dose same as Physostigma.

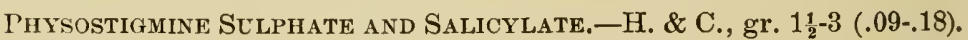
D., gr. $\frac{1}{100}-\frac{1}{30}$ (gm. .0006-.002), subcutaneously and intravenously.

Pilocarpine and its Salts.-H. (purgative), gr. $2-5$ (gm. .12-.3). C. (purgative), gr. 5-10 (gm. .3-.6). H. (diarphoretic; dangerous), gr. 6-12 (gm. .36-.72)。 Sh., gr. 1 (gm. .06). D., gr. $\frac{1}{10-\frac{1}{3}}$ (gm. .006$.02)$.

Pilocarpus.-H. \& C., dr. 2-4 (gm. 8.-15.). Sh. \& Sw., dr. $\frac{7}{2}-1$ (gm. 2.-4.). D, gr. 5-60 (gm. .3-4.).

Pilocarpus, Fluid Extract of.-Dose same as Pilocarpus.

Podophyllin.-H. \& C., dr. 1-2 (gm. 4.-8.). D., gr. 1-2 (gm. .06-.12).

Pomegranate.-See Granatum.

Potassa, Solution of.-H. \& C., oz. $\frac{1}{2}-1$ (cc. 15.-30.). Sh. \& Sw., dr. $\frac{1}{2}-1$ (cc. 2.4.). D., (m. 5-20 (cc. .3-1.3).

Potassium Acetate.-H. \& C., oz. $\frac{1}{2}-1$ (gm. 15.-30.). Sh. \& Sw., dr. $\frac{1}{2}-$ 1 (gm. 2.-4.) D., gr. 5-20 (gm. .3-1.3).

Potassicm Bicarbonate.-Dose same as Acetate.

Potassium Bitartrate.-H. \& C., oz. $\frac{1}{2}-1$ (gm. 15.-30.). Sh. \& Sw., Oz. $\frac{1}{2}$ (gm. 15.). D., dr. $\frac{1}{2}-1$ (gm. 2.-4.).

Potassium Bromide.-H. \& C., oz. 1-2 (gm. 30.-60.). Sh. \& Sw., dr. $2-$ 4 (gm. 8.-15.). D., gr. 5-60 (gm. .3-4.). 
Potassium Carbonate.-H. \& C., oz. $\frac{1}{2}-1$ (gm. 15.-30.). Sh. \& Sw., dr. $\frac{1}{2}-1$ (gm. 2.-4.). D., gr. 5-20 (gm. .3-1.3).

Potassium Chlorate.-Dose same as Carbonate.

Potassium Citrate.-Dose same as Carbonate.

Potassiun Nitrate.-Dose same as Carbonate.

QUASSIA, Extract of.-H., dr. 1-2 (gm. 4.-8.). D., gr. $\frac{1}{2}-3$ (gm. .03-.2).

QUassia, Fluid Extract of.-H. \& C., oz. 1-2 (cc. 30.-60.). Sh. \& Sw., dr. 2-4 (cc. 8.-15.). D., m. 15-60 (cc. 1.-4.).

Quassia, Tincture of.- oz. 2-4 (cc. 60.-120.). D., dr. $\frac{1}{2}-2$ (cc. 2.-8.). QUASSIIN.—D., gr. $\frac{1}{8}-\frac{1}{3}$ (gm. .008-.02).

Quercus Alba.-H., oz. $\frac{1}{2}-1$ (gm. 15.-30.). C., oz. 1-2 (gm. 30.-60.). Sh. and Sw., dr. 1-2 (gm. 4.-8.). D., gr. 10-30 (gm. .6-2.).

Quercus Alba, Fluid Extract of.-Dose same as Quercus Alba.

Quinidine.-Tonic dose-H. (gr. $20-60$ (gm. 1.3-4.). C., dr. $\frac{8}{4}-1 \frac{1}{2}$ (gm.

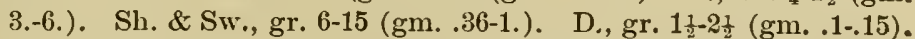

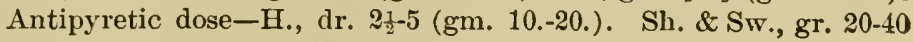
(gm. 1.3-2.6). D., gr. 7-15 (gm. .5-1.).

Quinine And its Salts.-Tonic dose-H., gr. 15-60 (gm. 1.-4.) C., dr. $\frac{1}{2}-1 \frac{1}{2}$ (gm. 2.-6.). Sh. \& Sw., gr. 5-10 (gm. .3-.6). Dogs and Cats, gr. 1-2 (gm. .06-.12). Antipyretic dose-H. \& C., dr. 2-4 (gm. 8.15.) Sh. \& Sw., gr. 20-40 (gm. 1.3-2.6). Dogs and Cats, gr. 5-10 (gm. .3-.6).

QUinoldin.-Dose three or four times that of Quinine.

Resorcin.-H., dr. 1-2 (gm. 4.-8.). Foals and Calves, dr. $\frac{1}{2}-1$ (gm. 2.-4.). D., gr. $2-5$ (gm. .12-.3).

Rhamnus Catharticus, Syrup of.-D., oz. 1-2 (cc. 30.-60.). Cats, oz. $\frac{1}{2}-1$ (cc. 15.-30.).

Rhamnus Catharticus, Fluid Extract of.-D., dr. $\frac{1}{2}-1$ (cc. 2.-4.)

Rhubarb.-Stomachic-H. \& C., oz. 1-2 (gm. 30.-60.). Sh. \& Sw., dr. 1 (gm. 4.). D., gr. 5-10 (gm. .3-.6). Purgative-Foals, Calves and Dogs, dr. 1-2 (gm. 4.-8.).

Rhubarb, Fluid Extract of.-Dose same as Rhubarb.

Rhubarb, Compound Powder of.-Foals and Calves, oz. $\frac{1}{2}-1$ (gm. 15.-30.).

Sabina, Fluid Extract of.-H., oz. 1-2 (cc. 30.-60.). D., m. 5-15 (cc. .3-1.).

Sabina, Oil of.-H. \& C., dr. 2-4 (cc. 8.-15.). D., w. 1-5 (cc. .06-.3). 
Salicin,-H. \& C., dr. 2-8 (gm. 8.-30.). Sh., dr. 1-4 (gm. 4.-15.). Sw., dr. $\frac{1}{2}-1$ (gm. 2.-4.). D., gr. 5-30 (gm. .3-2.).

SALOL.-H., dr. $\frac{1}{4}-4$ (gm. 1.-15.). D., gr. 1-3 (gm. .06-.2). Puppies, gr. $\frac{1}{4}-\frac{1}{2}(\mathrm{gm} . .015-.03)$.

Savin.-See Sabina.

SCAMMONY.-D., dr. 1-2 (gm. 4.-8.). Cats, dr. $\frac{1}{2}-1$ (gm. 2.-4.).

SCAMMONy, Resin of.-D., dr. $\frac{1}{2}-1$ (gm. 2.-4.). Cats, gr. 15-30 (gm. 1.-2.).

Senna.-H. \& C., oz. 4-5 (gm. 120.-150.). Sh. \& Sw, oz. 1-2 (gm. 30.60 ). Ir., dr. 1-4 (gm. 4.-15.).

Senna, Fluid Extract of.-Dose same as Senna.

Serum, Antistreptococcus.-See p. 663.

Silver Nitrate.-H. \& C., gr. 5-10 (gm. .3-.6). Sh. \& Sw., gr. 1-2. (gm. .06-.12). D., gr. $\frac{1}{8}-\frac{1}{2}(\mathrm{gm} . .008-.03)$.

SODA, Solution of.-H. \& C., oz. $\frac{1}{2}-1$ (cc. 15.-30.). Sh. \& Sw., dr. $\frac{1}{2}-1$ (cc. 2.-4.). D., m. 5-20 (cc. .3-1.3).

Sodium Bicarbonate.-H. \& C., oz. $\frac{1}{2}-2$ (gm. 15.-60.). Sh. \& Sw., dr. $\frac{1}{2}-1$ (gm. 2.-4.). D., gr. 5-30 (gm. .3-2.).

Sodium Carbonate.-H. \& C., dr. 2-6 (gm. 8.-24.). Sh. \& Sw., gr. 2040 (gm. 1.3-2.6). D., gr. 5-20 (gm. .3-1.3).

Sodium ChloRide.-Purgative-Cattle, lb. $\frac{1}{2}-1$ (gm. 250.-500.). Sh., oz. 1-2 (gm. 30.-60.).

Sodium Bronide.-H. \& C., oz. 1-2 (gm. 30.-60.). Sh. \& Sw., dr. 2-4 (gm. 8.-15.). D., gr. 5-60 (gm. .3-4.).

SOdIUM IODIDE,-H., dr. $2-4$ (gm. 8.-15.). C., dr. 6 (gm. 24.). Sh. \& Sw., gr. 15-30 (gm. 1.-2.). D., gr. 2-10 (gm..12-.6).

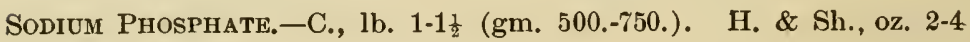
(gm. 60.-120.). D., dr. 1-2 (gm. 4.-8.).

Sodium Salicylate.-H. \& C., dr. $2-8$ (gm. 8.-30.). Sh., dr. 1-4 (4.-15.). Sw., dr. $\frac{1}{2}-1$ (gm. 2.-4.). D., gr. 5-30 (gm. .3-2.).

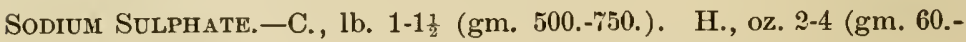
120.). Sh., oz. 2-4 (g. 60.-120.). D., dr. 1-4 (gm. 4.-15.).

Sodium Sulphite, Bisulphite and Hyposulphite.--H. \& C., oz. 1 (gm. 30.). Sh. \& Sw., dr. $\frac{1}{2}-1$ (gm. 2.-4.). D., gr. 5-30 (gm. .3-2.).

SQUILL.-H., dr. 1-2 (gm. 4.-8.). C., dr. 2-4 (gm. 8.-15.). Sh., gr. $15-$ 30 (gm. 1.-2.). D., gr. 1-5 (gm. .06-.3).

SQuill, Fluid Extract of.-Dose same as Squill.

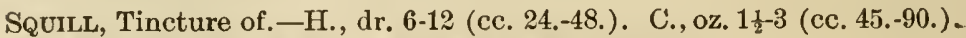

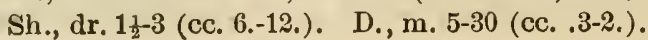


SQuill, Syrup of.-H., oz. $\frac{1}{2}$ (cc. 15.). D., dr. $\frac{1}{2}-1$ (cc. 2.-4.).

SQuill, Compound Syrup of.-D., m. 5-30 (cc. .3-2.):

Strophanthus, Tincture of.-H. \& C., dr. 1-4 (cc. 4.-15.). D., m. 2-10 (cc. .12-.6).

Strophanthin.-H., gr. $\frac{1}{5}-\frac{1}{2}(\mathrm{gm} . .012-.03)$. D., gr. 10 $^{\frac{1}{0}}-\frac{1}{60}(\mathrm{gm} . .0006-$ $.001)$.

Strichnine ANd ITS SAlts.-H., gr. $\frac{1}{2}-2$ (gm. .03-.12). C., gr. 2-3 (gm. $.12-.2)$. Sh., gr. $\frac{1}{4}-1$ (gm. .015-.06). D., gr. $\frac{1}{120}-\frac{1}{60}$ (gm. .0005-.001).

Stlphur.-H. \& C., oz. 2-4 (gm. 60.-120.). Sh. \& Sw., oz. 1-2 (gm. 30.60.). Do, dr. $\frac{1}{2}-4$ (gm. 2.-15.).

Tallianine (intravenously).-H. \& C.. dr. $2 \frac{1}{2}-\tilde{5}$ (cc. 10.-20.). Sh. \& Sw., m. 25-dr. $2 \frac{1}{2}$ (cc. 5.-10.). D., m. 30-75 (cc. 2.-5.). Cats, m. 15-30 (cc. 1.-2.).

Taraxacui. - H. \& C., oz. 1-2 (gm. 30.-60.). Sh. \& Sw., dr. $2-4$ (gm. 8.-15.). D., dr. 1-2 (gm. 4.-8.).

Taraxacum, Fluid Extract of.-Dose same as Taraxacum.

Taraxacum, Extract of.-H. \& C., dr. 1-4 (gm. 4.-15.). D., gr. 5-30 (gm. .3-2.).

Terebene.-H. \& C., dr. 2-6 (cc. 8.-24.). D., m. 5-15 (cc. .3.1.).

Terpin HYdrate.-H., dr. $\frac{1}{2}-2$ (gm. 2.-8.). D., gr. 5-20 (gm. .3-1.3).

Tetanes Antitoxin.-See p. 663.

Thymol.-H., dr. $\frac{1}{2}-2$ (gm. 2-8.). D., gr. 1-15 (gm. .06-1.).

TurPentine, Oil of.-Carminative-H. \& C., oz. 1-2 (cc. 30.-60.). Sh. and Sw., dr. 1-4 (cc. 4.-15.). D., m. 10-30 (cc. .6-2.). Anthelmintic -H. \& C., oz. 2-4 (cc. 60.-120.). D., dr. $\frac{1}{2}-4$ (cc. 2.-15.). DiureticH. \& C., dr. 2-6 (cc. 8.-24.).

VAlerian.-H. \& C., oz. 1-2 (gm. 30.-60.). D., gr. 10-60 (gm. .6-4.).

VAlerian, Fluid Extract of.-Dose same as Valerian.

VAlerian, Tincture of.-D., dr. $\frac{1}{2}-2$ (cc. 2.-8.).

VAlerian, Ammoniated Tincture of.-D., dr. $\frac{1}{2}-2$ (cc. 2.-8.).

VAlERIAN, Oil of.-H., dr. $\frac{1}{2}-1$ (cc. 2.-4.). D., m. 2-5 (cc. .12-.3).

VAlerianate of Amronium.-D., gr. 2-5 (gm. .12-.3).

VALERIANATE OF IRON.-D., gr. 1-3 (gm. .06-.2).

VAlerianate OF Zinc.-D., gr. 1-3 (gm. .06-.2).

Veratrine.-H., gr. $\frac{1}{2}-2$ (gm. .03-.12). C., gr. 2-5 (gm. .12-.3). D., gr. $\frac{1}{5}-\frac{1}{10}(\mathrm{gm} . .0012-.006)$.

Veratrum Viride.-H. \& C., dr. $\frac{1}{2}-1$ (gm. 2.-4.). Sh. \& Sw., gr. 2030 (gm, 1.3-2.). D., gr. $x_{10}-1$ (gm. .006-.06). 
Veratrum Tiride, Fluid Extract of.-Dose same as Veratrum Viride.

Veratrum Viride, Tincture of.-H. \& C., dr. 1-2 (cc. 4.-8.). Sh. \& Sw. m. 40-60 (cc. 2.6-4.). D., m. $\frac{1}{5}-2$ (cc. .012-.12). (See p. 453.)

WHISKEY.-H. \& C., oz. 2-4 (cc. 60.-120.). Sh. \& Sw., oz. 1-2 (cc. 30.60.). D., dr. 1-4 (cc. 4.-15.).

WiLd Cherry. Syrup) of. -D.. dr. 1-4 (cc. 4.-15.).

Wintergreen, Oil of.-See Gaultheria.

Zisc ACETATe.-H. \& C., dr. 1-2 (gm. 4.-8.). Sh. \& Sw., gr. 10-20 (gm. .6-1.3). D., gr. 2-3 (gm. .12-.2).

Zinc Oxide.-H. \& C., dr. 1-2 (gm. 4.-8.). D., gr. 5-10 (gm. .3-.6).

Zinc Phosphide.-D., gr. $\frac{1}{10}(\mathrm{gm} . .006)$.

Zinc Sulphate.-H. \& C., dr. 1-2 (gm. 4.-8.). Sh. \& Sw., gr. 10-20 (gm. .6-1.3). D., gr. 2-3 (gm. .12-.2). Emetic-D., gr. 10-15 (gm. $.6-1$.$) .$ 


\section{GENERAL THERAPEUTIC MEASURES.}

\section{Food and Feeding.}

In order to comprehend the rationale of feeding in disease it is essential to kuow something of the principles of feeding in health. A food has been defined as "that which, being innocnous in relation to the tissues, is a digestible, absorbable substance that can be oxidized in the body and decomposed in such a way as to gire up to the body the forces it contains."

A complete food is composed of organic and inorganic constituents. The inorganic matters, with the exeeption of common salt, and rarely phosplate of lime and sodium, are usually present in sufficient quantity in ordinary food. The organic components of regetable food stuffs are divided into nitrogenons and non-nitrogenous classes. These are analogons to the constituent parts of the animal body into which they are transformed. The greater portion of animal tissue is made up of nitrogenons elements, while the larger part of plants is composed of non-nitrogenons material. Among the nitrogenons elements the most important are the proteids. G! uten of flour is an example of a vegetable proteid ; while white of egg, casein of milk, and fibrin of blood represent animal proteids. Fat exists as such in both plants and anmals. A single, chemical compound, as protein, is known as a nutrient in relation to feeding. The nutrients of importance are proteids, fat, and carbohydrates. The first two are common to animal and plant structure; the latter to plants alone. A complete food contains the three nutrients just mentioned and inorganic substances. Carbohydrates include such bodies as sugar, starch, and cellulose, or woody matter of plants. $6 \% 9$ 
Proteids consist of carbon, hydrogen, oxygen, nitrogen, and sulphur, united in different proportions. Carbohydrates are composed of carbon, hydrogen and oxygen. Fat is similar in composition to carbolydrates, but in its combustion ontside the body yields approximately two and a half times as much heat as that produced by an equal weight of carbohydrate. Fat as a rutrient, is therefore empirically regarded as equivilent to two and a half times the same weight of carbohydrate material. The percentage of digestibility of the common food stuffs has been determined experimentally and is pretty uniform in all healthy animals. In $100 \mathrm{lbs}$. of hay there are approximately:

$\begin{array}{lrcc} & \text { Lbs. } & \begin{array}{c}\text { Per cent. } \\ \text { digested. }\end{array} & \begin{array}{c}\text { Lbs. } \\ \text { digested. }\end{array} \\ \text { Protein......... } & 9.7 & .56 & 5.4 \\ \text { Carbohydrates...... } & 67.3 & .60 & 40.4 \\ \text { Fat........... } & 2.5 & .48 & 1.2\end{array}$

If we multiply the 1.2 lbs. of digestible fat by 2.5 to reduce it to the same terms as carbohydrates according to the empirical premises stated above, we have 3 lbs. of carbohydrates. The total amounts of carbohydrates digestible in $100 \mathrm{lbs}$. of hay is therefore $40.4 \mathrm{lbs} .+3 \mathrm{lbs} .=43.4 \mathrm{lbs}$. of non-nitrogenous matter. The nitrogenous nutrient consists of $5.4 \mathrm{lbs}$. of digestible protein, and the proportion of the latter to the digestible non-nitrogenons material is as 1 to 8 . This proportion in any given fodder is called the nutritive ratio, or the relation of the nitrogenons to the non-nitrogenous nutrients. The mutritive ratio is of the greatest importance, as it enables us to compose a proper ration with almost any kinds of fodder at hand, by so adjusting them that we secure the right proportion of nutrients. The proper untritive ratio is indeed that proportion of nutrients which has been found to be most economically and physiologically valuable in any given class of animals and for any given purpose. The uutritive ratio varies accordingly for different animals and for the same animals under different conditions. Such ratios 
have been formulated by means of physiological experiments on healthy animals as employed for economic purposes.

It is self-evident that nutritive ratios camnot be laid down for diseased animals, and we must content ourselves with observiug how disease alters, in a general way, the requirements of the unrmal animal. The following table of nutritive ratios suitable for the animals and purposes named, is taken from Armsby's "Cattle Feeding." The mutritire ratio is estimated for 1000 lbs. live weight per diem.

\begin{tabular}{|c|c|c|}
\hline & $\begin{array}{l}\text { Nutritive } \\
\text { Ratio. }\end{array}$ & $\begin{array}{l}\text { Total lbs. of } \\
\text { Nutrients. }\end{array}$ \\
\hline Horses at work .......... & $1: 7$ & 13.00 \\
\hline Horses heavily worked.... & $1: 5.5$ & 17.00 \\
\hline Milch cows ............ & $1: 5.4$ & 15.40 \\
\hline Oxen at rest.............. & $1: 12$ & 8.85 \\
\hline Sheep for wool............ & $1: 9$ & 11.70 \\
\hline
\end{tabular}

For example, the nutritive ratio, or the proportion of lbs. of proteids to lbs. of carbohydrates and fat for horses at work, should be as one to seven; and the total number of lbs. of pure fat (transformed into terms of carbohydrates), carbohydrates, and protein requirerl to support 1000 lbs. of live weight of horses at work, per diem, is thirteen.

Horses doing hard work, and all animals supplying nitrogenous products, as wool and milk, demand more nitrogen in their food. The proteids, as we have seen, constitute a large proportion of the solids and fluids of the body. A greater part of the assimilated protein circulating in the blood and fluids of the body is termed circulating protein, and is mainly instrumental in supplying the inmediate needs required in the performance of the vital functions. A smaller part of the proteids furnished the economy is fixed as a constituent part of the tissues, and is called organic, or tissue protein. If the amount of circulatory protein is deficient, then the organized protein is called upon, the tissues are robbed of their substance, and the boly emaciates. When a larger amount of protein is contained in the bluod, we have a proportionately larger elimination of nitrogenous 
matter in the urine, as equilibrinm is soon established in the adult animal of constant weight, so that the amount of nitrogen eliminated equals that ingested. In young and growing animals a portion of the nitrogen does not reappear in the urine, but is ntilized in tissue formation. An excess of circulating protein, besicles being wasteful economically, is harmful in causing plethora and various disordered couditions, resulting in the formation of uric acid and other products of imperfect oxidation. Nevertheless, a certain surplns of protein appears to be essential for the well-being of animals; more than is necessary to make good the loss of protein through tissue waste as determined by estimation of the nitrogenous elimination in the mine of fasting animals.

The vegetable proteids are transformed into bodies of simpler chemical composition in the stomach and are there converted in part by the gastric juice, but chiefly by pancreatic (trypsin), biliary and intestinal ferments in the intestines, into peptone, the only form in which protein can be absorbed by the digestive tract.

The epithelial cells of the intestines possess the power not only to absorb the peptone but to transmute it into different isomeric compounds, as serum albumin, serum globulin and fibrinogen; special cells being employed in the formation of particular compounds.

Any peptone not so converted by the intestinal epithelinm becomes a poison when absorbed into the entero-hepatic circulation, but its toxicity is destroyed by the liver cells. The protein elements (as serum albumin) in the blood consist of those absorbed unchanged from the intestines and those different isomeric forms which have been used in the body, and all are eliminated and oxidized by the excretory glands with the production of heat and escape of the products of tissue waste, as urea, uric acid and water from the kidneys; bilirubin, taurocholic acid, etc., from the liver. Heat is evolved in this oxidation of proteid substances in the glands equivalent to 1,812 kilogram meters for each gramme of protein consumed. 
This heat formation is of extreme importance in stimulating and sustaining nervons action.

Among proteids in food are the nucleoalbumins, so-called because they exist in both animal and vegetable cells. The nucleoalbumins are extremely valuable, since they contain iron and phosphorus. They are absorbed by special epithelial cells of the intestinal walls and are converted in part into animal nucleoalbumin, as found in the various cells of the body and in milk and eggs; while a portion is oxidized and split up in the liver, forming lirmoglobin, the iron compound of the blood, and lecithin, the phosphorus compound essential to nerrous tissue. It has even been held that iron can only be supplied the blood as a nucleoalbumin and that when iron is given as medicine it acts indirectly by protecting the iron in the nucleoalbumins of the food from destruction. This is, however, probably too sweeping a statement.

Protein is first of all a tissue builder, and is also a source of energy by its decomposition. A dog can live on lean meat (pure protein) for months and gain in weight.

Carbolyydrates in the food, as sugar and starch, are digested in part by the salivary ferment (ptyalin), but chiefly by the pancreatic ferment (trypsin) in the intestines, and are converted by them into glucose. The glucose is then absorbed by the small intestines, passes thence as dextrose into the enterohepatic circulation and is oxidized within muscles and liver cells and elsewhere in the form of dextrose (glucose) and glycogen, with the production of heat equivalent to 1,657 kilogram meters for each gramme of carbohydrate consumed, while carbou dioxide and water are formed as end-products. The heat production, as in the case of the combustion of proteids, is of great importance in sustaining nerve action.

Animal fat in the food is emulsified by the bile and by the pancreatic and intestinal secretions and is absorbed by special cells of the intestinal mucous membrane and passes into the lymphatics; thence into the thoracic duct, and is also oxidized into carbonic dioxide and water with production of heat and energy. The fat consumed in the body is thought to be oxidized in part in the pulmonary alveoli. 
Carbodydrates do not directly furnish tissue elements, but do so indirectly in preventing decomposition (to some extent) of protein in the body, and in lessening its consumption. In this way the comparatively inexpensive carbohydrates will compeusate for an insufficient ration of costly proteids.

It is asserted that this action of carbohydrates is explained by the fact that these nutrients have a greater affinity for oxygen than proteids and so are the first to undergo combustion.

The term albuminoid was formerly used as synonymous with proteid, lut is now employed to include nitrogenons bodies (gelatin) derived from protein in the body but not convertible into proteids. These bodies do not take the place of proteids, but appear to fulfil the functions of circulating protein, and, like carbohydrates, conserve protein consumption, but are uot tissne builders.

Carbohydrates aid directiy in the production of fat. Fat in the food may be directly assimilated as such if there is a sufficient carbohydrate and proteid ration to protect it from decomposition. The role carbohydrates play includes:

1. The formation of tissue indirectly by protecting the proteid elements from combustion with an insufficient nitrogemous diet.

2. The generation of heat, energy, or mechanical work.

3. The production of fat.

Carbohyclrates cannot be relied npon as sole articles of food. The fat of the body is derived from the decomposition of proteids and carbohydrates, and directly from fat contained in the food. Fat lnbricates the tissues, and a reasonable accumulation serves as a store of potential energy to be called upon in time of need, when its action is similar to that of carbohydrates. The main source of muscular energy, movement, or mechanical work, is thought to ::esicle in the carbohydrates, because with excessive muscular work there is increased evolution of heat, carbonic dioxide and water. In so far as muscular energy and power depend upon a good 
machine, in so far do the proteids aid the work of the machine by keeping it in good repair and working order. From this point of view the proteids represent the mechanism by which the work is done; the carbohydrates the fuel necessary for its performance. The exact relative value of carbohydrates and proteids in the production of the vital forces is still sub judice. Nervous activity is inseparable from muscular activity; both seem to require a highly nitrogenous regimen, and such a regimen is conducive to both.

The classes of food stuffs for the larger animals embrace both green and dry fodder, consisting of the whole plant minus the roots; parts from which the more valuable portions have been removed, as straw; tubers and roots. containing a large percentage of water; the seeds or grains, constituting the most concentrated food; and the seed coverings or chaff.

Refuse and bye-products of manufacture are extensively used, as bran, cottonseed meal, linseed meal, and brewers' grains. In addition, animal matter, as flesh meal, bone meal, and dried blood, are sometimes of value. Pasture grass may be taken as a standard of comparison for green fodders. In $100 \mathrm{lbs}$. of grass there are approximately digestible :

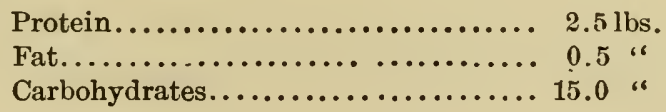

Green clover contains considerably more, green rye. slightly more, and green oats somewhat less protein; while in green corn fodder there is only about half as much protein. Roots, as compared with green pasture grass, possess only one-third the quantity of protein and solids. Potatoes, however, have double the nutritive value of roots. Comparison is made in reference to protein, as that is by far the most valuable nutrient, and the amount of carbohydrates and fat do not vary much in similar kinds of fodder.

Meadow hay, including such common varieties as timothy and red top, may be taken as a standard of comparison 
for dry fodder. In $100 \mathrm{lbs}$. of hay there are approximately digestible :

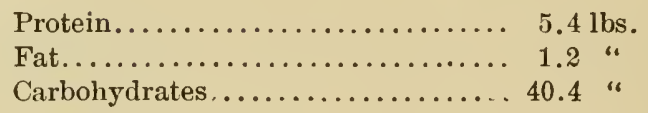

Clover hay has double the amount, and rowen or aftermath about one-third more protein than is contained in ordinary meadow hay. Oats may be selected as a standard of comparison for grains. $100 \mathrm{lbs}$. of oats contain of digestible nutrients as follows:

Protein...................... 10.0 lbs.

Fat......................... 4.5 '

Carbohydrates................ 45.0 “

Corn yields slightly less protein and considerably more carbolyydrates and fat, while cottonseed meal contains nearly three times as much protein, and four times as much fat as are found in oats. Bran (or shorts) has approximately the same chemical composition as oats. The straw of wheat, barley, rye, oats, and corn have a somewhat similar value. In 100 lbs. of wheat straw there are digestible :

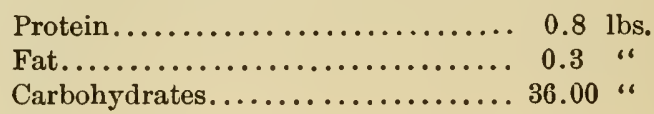

In order to compare the composition of food with that of the tissues into which it is converted, we submit the following :

PERCENTAGE COMPOSITION OF LIVE ANIMAL.

IN 100 LBS, OF LIVE OX THERE ARE :

Protein........................ 8.7 lbs.

Fat.......................... 19.2 "

Ash....................... 5.9 “

Water..........................66 66 "

Certain of the fodders are especially adapted or otherwise for the various domestic animals. Green clover is prone to fermentation and the formation of flatulency if given in large quantities to any animal. A sudden change 
from dry to luxuriant green food is always undesirable for a similar reaion. Green grass cut short by a lawn mower should not be gireu horses, as it is swallowed in an unmasticated condition and leads to indigestion and colic. Potatoes and roots are more snitable for ruminants than for horses; yet upon the latter they act as natural and agreeable laxatives, and form a palatable addition to dry fodder, particuliarly carrots. The best variety of hay for liorses consists of good bright timothy or herds grass with a slight admixture of red top and clover: Carrots shonld be sliced longitudinally to prevent choking when given to horses. Clover hay is dusty and apt to provoke "heaves" in horses, and that, together with rowen, is more appropriate for ruminants.

Bran is but poorly digested by horses, yet acts favorably as a laxative when given once or twice a week mixed with boiling water and plenty of salt, constituting a "bran mash." Bran takes the place of oats as a nitrogenons food for ruminants, and is less expeusive. Cottonseed meal, being extremely rich in nitrogen, and usually in oil or fat, is not easily digested by any animal, but may be given in quantities of a quart or two to ruminants, and from half a pint to a pint to horses, daily. Cottonseed meal is supplied cattle to compensate for a deficiency of nitrogen in the food, while in horses it acts as a slight laxative and may improve the general condition. Straw and corn fodder are not readily digested by horses unless cut and steamed, but are suitable for ruminants and are often preferable to a poor quality of hay. The demands of the system for food vary in relation to tissue change, which is diminished by rest, increased by work, and either accelerated or decreased by disease. The requirements for nutriment are greater during the growing period and for the formation of the various natural products, as milk or wool. The state of the digestive organs and assimilative powers guide us in selecting the kind and quantity of food desirable. In acute disease it is advisable to feed little and often, the food being prepared in the most digestible and palatable form, and in as great a variety as 
attainable. We may restrict the diet as a whole or in part. Starving diminishes circulatory protein, increases tissue waste, weakens an animal, and lessens the natural resistance agrinst disease. Restricting the diet is useful in controlling unmanageable animals, in diminishing sexnal excitement, and in the treatment of plethora when combined with proper exercise. In most acnte inflammatory diseases, as in acute laminitis, a light laxative diet is desirable, as steamed oats with bran and salt, roots and green fodder. In the milder forms of acute gastro-enteritis we must restrict the diet to small quantities of easily digested food, as cracked or steamed oats, chopped hay and gruels, with the addition of a little green fodder or roots for horses; while carnivora are given unilk and lime water. In chronic digestive disorders the food must be readily digestible and assimilable, and of a. nitrogenous character, since anæmia and malnutrition follow the defective digestion and absorption. In chronic indigestion or gastro-enteritis of horses, Zuill recommends oats (boiled, scalded or steamed, and allowed to stand 12 hours), 2 parts; bran, 1 part; and malted barley, 1 part. The addition of salt and a little green fodder to this ration is palatable and desirable. The dietary for constipation in horses should consist of bran mashes twice a week with plenty of salt; roots and green fodder at frequent intervals, combined with suitable exercise and appropriate drug treatment. Dogs suffering from constipation may be given raw liver twice a week, or may be put on an occasional or exclusive diet of one of the commercial dog breads or biscuits. These are laxative and are invaluable in eczema of dogs, commonly resulting from lack of exercise and over-indulgence in food. If constipation is very obstinate, total abstinence from all food, water excepted, for a time, followed by the use of lean meat with salt and beef tea, are indicated till the bowels are emptied manually or by enemata. The ration for diarrhœa embraces the partial restriction of water, which increases the bulk and fluidity of the intestinal contents aud so stimulates the movements of the bowels. If the diarrhœa. 
is so severe as to endanger life, an abundance of pure or boiled water should be allowed in order to compensate for the loss of fluid from the blood.

Theoretically, an albuminous diet is indicated in diarrhoea because of the loss from the blood and tissues, and because intestinal digestion is disordered and starchy food would be undigested and cause fermentation, etc. Practically, a certain amount of starchy food seems to be serviceable in the treatment of diarrhoea. Horses and cattle should be given cooked flour or barley gruel and roasted oatmeal and cracked oats. Coarse foods, as bran and straw and green fodder, are not allowable. Swine should be supplied with gruels of barley, flour or oatmeal (strained).

Fowl with diarrhœa may be fed on boiled rice and given a few drops of laudanum two or three times daily. Dogs and cats should have boiled milk, strained rice gruel, cooked lean meat and crackers. Broths and beef tea are not desirable, but beef juice and white of egg in water are of value. These dietaries should be employed in conjunction with other measures, as the preliminary use of a laxative, rest, quiet, and external heat and drug treatment. Young suckling animals, as foals and calves, may be fed on cooked and strained oatmeal or barley gruel made with milk, if the mother's milk does not agree. In severe attacks of gastroenteritis, or in gastric or intestinal ulceration with hæmorrhage from the stomach or bowels, the food should be bland and fluid, as soaked bread, oatmeal, barley or flour gruels, linseed tea (made by boiling linseed in a muslin bag immersed in water), and small quantities of green fodder for the larger animals; while milk and lime water, white of egg and water, broths and beef juice are indicated for carnivora. In the latter animals we may have to resort to predigested food given by the mouth, or, if vomiting is persistent, by the rectum.

The diet in cases of catarrhal jaundice should be easily digestible, bland, and such as will not require much bile for its digestion. The larger patients should be given gruels, 
steamed cracked oats, young and tender green food, cooked potatoes, together with alkalies and other appropriate remedies. Dogs are allowed milk and lime water, crackers, bread and cooked lean meat. Feeding in hæmoglobinæmia or "black water" of horses must be restricted to the use of gruels, green fodder and a little hay in the early stages of the disorder. Food is usually withheld 12 hours before surgical operations, and this, in addition to the administration of a cathartic, will prevent injury in casting the larger animals, which might follow were the digestive tract overfull. It will also lessen the danger of intestinal fermentation and absorption of toxins from the bowels, which may occur after operation owing to an enfeebled digestive action. If dogs are starved before surgical operation, vomiting is prevented during or after etherization. Water alone may be restricted to advantage in obesity, cardiac disease with adema, or in the treatment of chronic exudations, as in hydrothorax. Water may be allowed in these conditions only once daily, or eren every other day; and this treatment may be combined with the use of saline cathartics in strong animals. The specific gravity and density of the blood and the tendency to absorption from the tissues and cavities is increased. By the same process the quantity of blood is diminished and the load put npon the heart is lessened, both of which may prove beneficial in cardiac diseases.

A full, or restorative diet shonld be especially rich in protein. Generons feeding is distinctly in order in the treatment of general debility, malnutrition, anæmia, weakness of the digestive organs, convalescence from acute diseases and in animals particnlarly sensitive to cold, or in those which sweat easily. A full diet is also useful in overworked animals and in those subject to losses from increased secretion, excretion, or exuclation, as in chronic suppuration, .diarrhœa, albuminnia, ascites and oodema. A restorative diet for herbivora includes grain, as corn, bran, outs aud cottonseed meal; hay and grass, with occasionally milk and eggs. For omnivora, corn, potatoes, blood, milk and soups. 
For carnivora, meat extracts, milk, eggs, broths and meat' juice. In most wasting diseases, fat, protein and water are the food elements especially needful. An abundance of water stimulates the appetite, secretions, excretions, tissue changes and vital processes generally. Salt should be given freely as an aid to digestion in increasing the formation of hydrochloric acid, and indirectly that of pepsin. Alcohol, being a nutritive and capable of easy absorption, assimilation and decomposition in the body, forms a most valuable adjunct to a restorative diet. A deficiency of lime in the food is occasioually the cause of rickets in the young, and fragilitas ossium in the old, but more frequently these diseases are due to defective digestion, assimilation, or excessive lactation. Bone meal may be fed to advantage in such affections. It contains both lime and phosphoric acid and should be given in small quantities ( 1 tablespoonful to large animals ; 1 teaspoonful to small patients) on the food in connection with the administration of hydrochloric acid and bitters.

In fever a restricted diet is often secessary in the more acute stages, with loss of appetite, diminished secretions, and morements of the stomach, but as soon as convalescence sets in the increased tissne waste prodnces an excessive demand for food and the digestive orgaus may become overtaxed. The initial dietetic treatment of fevers consists in the use of oats, bran mashes, and gruels, with the addition of a small quantity of grass or roots for horses. The change from this cliet should be very gradual to a dry, coarse fodder, in order to avoid digestive disorders. The bitters, alcohol, hydrochloric acid, and salt, together with a copious supply of water, will furthermore aid convalescence. Caruivora, with fever, should be fed milk, beef juice, broth, bread, oatmeal and a small quantity of cooked lean meat.

Obesity is treated most advantageously by proper feeding. A certain amount of fat is essential in the body in Inbricating the tissnes, in acting as a protection against cold, in serving as an enveloping and shielding cushion to the un- 
derlying tissues, and finally in supplying a store of nutrition. Animals living in cold climates are covered symetrically by fat, but those indigenous in hot countries have accumulations of fat in masses to avoid over-heating the body. This is seen in the hump of the camel, zebu and Brahmin bull. When the camel is severely taxed, the fat is consumed and the hump becomes loose and flabby. House dogs overfed and insufficiently exercised, horses, and other animals kept for breedingpurposes, are those most commonly afflicted with obesity. Fat in the body may be formed from fat, albuminoids, and carbohydrates of the food. Carbohydrates, if in excess of the needs of the economy, protect the fat in the food from decomposition and so enable it to be stored in the body. Protein may also protect the consumption of fat of the food, for it is broken up into nitrogenous and non-nitrogenous. elements, and the latter may be transformed into body fat. The accumulation of fat proceeds most readily when there is. an abundance of fat in the food, in addition to the other nutrients; less so when the ration consists of fat and albuminoids, or of carbohydrates and albuminoids; and least of all with a diet consisting of pure protein. The latter, then, is the food to be approached as near its purity as advisable. If protein ration is followed too closely, digestive disorders, loss of strength and nervous disturbances are likely to occur. The fat resulting from the decomposition of a pure protein fodder is usually not sufficient to supply the needs of the body, and the organized fat is gradually called upon to supply the deficiency in the food.

Exercise, cathartics, diuretics and diarphoretics, together with venesection, are synergistic measures. As emaciation proceeds, we must add more and more nonnitrogenous material to our ration. Fut is said to accumulate most readily in the vicinity of vascular areas where the flow of blond is returded, and therefore oxidation, combustion, and molecular activity diminished. Exercise, on the other hand, stimulates the circulation, while deprivation of water makes the blood-current more rapid by decreasing the 
amount of blood. Both therefore favor the destruction of fat. Vogel has reported good results in reducing obesity by the use of the following rations. From 19 to 26 weeks are required for a cure.

DAILY RATION FOR THE HORSE.

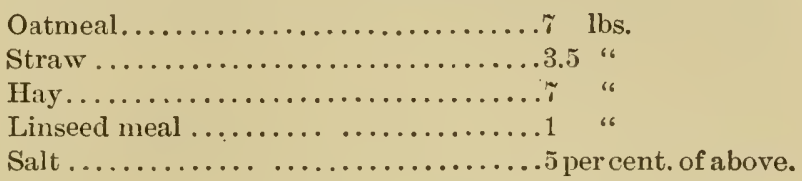

DAILY RATION FOR DOG WEIGHING 115 LBS.

Cooked oatmeal.................. lbs.

Fat.................... "

SAME LATER.

Oatmeal........................ $1 \mathrm{~b}$.

Flesh meal ................... 1/2 "

Fat..................... "

Salt ........................ teaspoonful.

DAILY RATION FOR FAT SETTER OR POINTER.

Lean meat..................... $1 \frac{1}{2} \mathrm{lbs}$.

Bread.........................11/2 "

Fat ............................

Animals suffering from fatty degenerative changes do not stand such a rigid diet as the foregoing. These animals are affected with a weak heart, due to fatty degeneration of the myocardinm, with atrophy of its muscular fibres. They have dyspnœe on exertion. The cardiac insufficiency leads, in its turn, to secondary troubles, as catarrh of the digestive and respiratory organs, and disorder of the liver and kidneys, following general passive congestion. The treatment should be directed in such cases to lessening the amount of blood and the work put upon the heart, by limiting the ingestion of water, and by strengthening the heart with appropriate stimulants. The activity of the skin should be excited by frequent grooming and the use of diarphoretics, while the activity of the kidneys should be enhanced by the employment of diuretics. We are prevented 
from feeding an exclusively nitrogenous diet in these cases, as the oxidizing power of the blood is not sufficient to decompose it, neither is it safe or advisable to quickly remove the fat, as cardiac failure might ensue. Small quantities of water are allowed, given two hours after feeding. The general ration shonld be rich in proteids and also contain a moderate amount of fat and carbohydrates.

\section{ARTIFICIAL FEEDING.}

Artificial feeding consists in the introduction of food into the body other than in the ordinary way by the mouth. Rectal feeding is the only procedure of much value. The agents employed must be bland, and capable of easy absorption and assimilation. If the food is at all irritating, tenesmus and ejection quickly occur. From two to four ounces of liquid nourishment may be given to small or mediumsized dogs ; from four to eight ounces to large dogs. One quart may be employed for horses. The nutrient enema should be introduced into the bowel through a flexible rubber tube carried up into the colon. An hour before the enema is given the bowel is to be washed out thoroughly with cold water. The nutrient injection should not be given oftener than once in six hours, and if the bowel is irritable, not oftener than once in twelve hours. The absorptive power of the rectum is slight, but that of the colon is considerably greater. Predigested food is most valuable. Leube's Beef Peptone may be used. A bullock's pancreas is finely chopped and rubbed up with eight ounces of glycerin. This extract will keep fresh several weeks in a cold place. To one-third of the extract are added five ounces of finely chopped beef, and the mixture is ready for immediate use. The peptonizing powders of Fairchild Brothers \& Foster are most convenient in preparing digested food. Each powder consists of five grains of pancreatic extract and fifteen graius of sodium bicarbonate.

A useful nutrient enema for a large dog may be made of two eggs and six ounces of milk. Four to six eggs may be 
added to a quart of milk for use as an enema for a horse. The mixture is then to be peptonized and introduced into the rectum at the temperature of the body. In nsing the peptonizing powders, one is placed in a quart glass jar together with a teacripful of cold water. Then a pint of the mixture to be peptonized is poured into the jar, and the latter placed in a vessel containing water as hot as the hand will easily bear. The jar is kept in the hot water for twenty minutes and put on ice. When the mixture is used it shonld be heated to $100^{\circ} \mathrm{F}$. If predigested food is to be given by the mouth, it is well not to keep the glass jar immersed in hot water more than five minutes, as otherwise the taste will be bitter and disagreeable. A small dose of landanum is always useful to prevent the expulsion of enemata. Braudy may be added in the proportion of one ounce to the pint of milk after peptonizing. The addition of salt to egg-albumin greatly facilitates absorption. Gruels of all kinds, and broths, may be peptonized, as well as milk. It is not essential, however, to peptonize milk and other fluids, although absorption is rendered somewhat more complete. The digestive powers of the large intestines are but slight. Sugar is absorbed unaltered; undigested proteils (with certain exceptions) and fat are not absorbed. Peptones, soluble proteids, as milk, meat juice, egg albnmin, and emulsified fat are absorbed. Nevertheless, absorption from the lower bowel is trivial compared with that from the stomach ancl in. testines generally, and as the extent of surface with which rectal injections come in contact is swall, and their sojourn short, it follows that rectal feeding cannot take the place of normal alimentation.

In tetauns, paralysis of muscles of deglutition, fracture of the jaw in horses, persistent vowiting and convulsions in dogs, and in all animals refusing food, rectal feeding is indicated. It is possible in horses to feed through a stomach tube. 


\section{Counter-Irritants.}

A counter-irritant is an irritant which acts counter, or against an existing irritation, result of irritation, or pain. In applying a "twitch" to a horse, we are inflicting an irritation to relieve some other somrce of irritation elsewhere. It is taken for granted that the damage and pain caused by the artificial irritant are not so severe as those already existing. The amount of injury produced by an irritant depends upon the nature of the material, its strength, the duration of its action, the mode of application, and the part to which it is applied. We may consider the effects occasioned by a mild and increasing action following the continued use of a single agent, or representing the action of materials of different degrees of potency. There first appears redness of the skin, accompanied by some burning or pain (rubefacient action), and if the irritation progresses there is a serons exudate poured ont into the mucons layer of the skin. This leads to swelling and œdema. Greater irritation canses nore exndation of serum and an elevation of the epidermis in a circumscribed area or areas, and the formation of blebs or blisters (vesication). If the irritation ceases at this point, the blisters break open and their coutents dry on the surface, covering the parts with a thick scab. Synchronons with vesication we observe a similar process attacking the hair follicles. The hairs are loosened and fall, but as the papillæ are usually unaffected, the growth of hair is soon renewed. The recovery of hair is facilitated by the application of grease to the part. Certain agents canse circumscribed inflammation of the skin with the formation of pustules (pustulants), as croton oil, and these create necrosis of the hair papilla and, therefore, permanent loss of hair. If the irritant is severe, suppuration follows vesication; or, if an ordinary irritant is applied with violent friction, is covered with a bandage, or placed over an already inflamed part, the same result 
happens. The terms referring to the degree of action inherent in ageuts are as follows :

1. A rubefacient, causing hyperæmia.

2. A vesicant, or epispastic, inducing blistering.

3. A pustulant, creating pustules.

4. An escharotic, or caustic, occasioning death of the tissues.

The same agent, as has already been pointed out, may produce one or more of these actious according to circumstances. The inner aspect of limbs and the flexures of joints are peculiarly sensitire, owing to the thinness of the skin over these areas. If a counter-irritant is rubbed properly into the skin it may penetrate into the mucons layer.

Reference has been made to the local influence of irritants. We will now direct attention to their remote effect. It is certainly known that irritation of the surface decidedly affects distant organs. Brown-Séquard noted contractiou of vessels in one arm when the other was immersed in cold water. Severe burns of the surface are followed by duodenal ulcers. The preceding and succeeding remarks enable us to partially account for the remote influence of counterirritants, but although we know their practical value, it is not within our present knowledge to offer theories wholly explaining their effect.

The influence of counter-irritants may be summed up in reflex action; $i$. e., the production and conduction of an impulse from the periphery to nerve centres, thereby modifying the nerve functions and blood supply in distant parts.

The skin is commonly the point of application. It normally is an organ of protection, respiration, secretion and special sense, and, through its medium, a regulator of temperature, responding to such natural stimuli as heat, cold, moisture and dryness. Such an unnatural and considerable stimulation as is produced by counter-irritants consequently creates very sensible alterations in the bodily functions. 
Extensive counter-irritation causes the breathing to become slower and deeper by reflex stimulation of the vagi, and also by making the respiratory movements more painful, if the application be made to the chest wall. The circulation is likewise affected, and both the force of the heart and blood pressure are increased, unless the irritation is very widespread and severe, when the reverse happens. In accordance with the foregoing remarks, the use of considerable heat, together with mustard or turpentine, is of great value in conditions of vital depression, surgical shock, collapse and coma. Moreover, the effect on local blood supply is still greater and full of importance, since it may explain the beneficial action obtained in the ordinary use of counterirritants. In experiments conducted upon animals, it has been observed that when sinapisms are placed over the head, the blood vessels in the pia mater are first dilated, but soon contract and remain in that condition for some time. Likewise there was seen, following energetic connter-irritation of the chest, anæmia of the underlying parts, including the muscles, pleura, and even the pulmonary tissue. It is essential to bear in mind, then, that while comnter-irritants induce local congestion in their immediate vicinity, they also cause reflexly vascular contraction in more remote areas. In accordance with this demonstration, the importance of these agents in inflammation lies not so much in their bringing blood to the surface, as in forcing it ont of distant parts. This fact is not generally appreciated. The use of the word "drawing" signifies the common idea of a counter-irritant, and implies the first proposition.

Temperature is not materially affected by the therapentic use of counter-irritants, and they are not necessarily contraindicated in fevers. Experiments, however, appear to show that mild counter-irritation may lead to a slight elevation of body-heat, owing to stimulation of the calorifacient centres, while extensive and prolonged action lowers temperature by diarphoresis and depression of the heart and heat centres. 
Counter-irritants notably relieve pain. This result is not snly due to overcoming congestion, but occurs when pain is purely neuralgic. The phenomenon is not altogether explicable. The subduing influence of a twitch in the case of pain inflicted upon a horse is an analogous example. Wechsberg, in some late experiments, notes, as a most striking effect of counter-irritants, cedematous infiltration of the skin, subcutaneous tissue and muscle in subadjacent parts, with compression of blood vessels in the deeper-lying structures. He attributes the relief of pain afforded by counter-irritants to anæmia and rapid compression brought to bear on the nerves in these underlying parts; a result similar to the anodyne iufluence observed following the injection of normal salt solution over a nerve-truuk, which is the priuciple of Schleich's method of local anæsthesia by tissue infiltration.

Still this explanation does not interpret the relief of pain sometimes seen in parts remote from the point of application of counter-irritants.

Pain is usually referable to the peripheral ends of an affected nerve. It is good practice to apply counter-irritation directly over a deep-seated inflammation or seat of pain, but in assuaging superficial pain it is found, that where the treatment can be made over the root of the painful nerve, better results are obtained. In pain in the chest wall a blister should be placed next the spine over the root of the spinal nerve involved; in pain in the head in man, counterirritation is applied over the back of the neck. Counterirritation should be done behind the ear to relieve pain and inflammation in the eye and ear.

In the treatment of enlarged glands and in acute inflammations, as abscess, boils and carbuncles, by counterirritants, the application should be about the lesions rather than directly upon them.

Among other actions accomplished by counter-irritants are: possible stimulation of trophic nerves and nutrition of a part; augmentation of tissue change, locally and geuer- 
ally, and dilatation of vessels (when applied after the subsidence of acute inflammation or in chronically inflamed parts), with renewed activity of the circulation and consequent absorption of inflammatory exudations. Furtlermore, counter-irritants reflexly overcome spasm and pain occurring in colic, by stimulating and replacing normal peristaltic action in place of abnormal localized contractions. In like manner they excite uterine contractions by stimulation of the involuntary muscular fibres of the womb.

\section{INDICATIONS FOR COUNTER-IRRITANTS.}

1. To overcome congestion and inflammation in remote parts.

2. To promote absorption of inflammatory products locally.

3. To relieve pain.

4. To stimulate the heart, respiration and nervous functions.

Rubefacients.-In this class are included the volatile oils-turpentine, oil of wintergreen, etc.-alcohol, chloroform, balsams, resins, iodine, tincture of camphor, tincture of cantharides, mustard, and lieat. These agents are nsed when it is desirable to stimulate the nervons system rapidly, and to relieve pain and congestion. To attain this end, we employ comparatively mild agents in order that we may apply them over an extensive surface withont causing serious or permanent results. Mustard is rubbed with warm water into a thin paste (a simapism), and rubbed over the chest of lorses in congestion of the lungs, in acute bronchitis, or in the first stage of plemitis, to obtund pain and lessen congestion. Sinapisms also relieve obstinate congh, revive failing respiration, and stimulate reflexly the vital functions in collapse, shock and narcotic coma. Applied over the cardiac region, they avert syncope. To assist the action of mustard, we often cover the application with hot blankets, and then with dry ones. Stimulating liniments are sometimes preferred. One volume of oil of mustard. 
may be combined with fifteen volumes of oil of turpentine: or ammonia water, thirty parts, and oil of turpentine, fifteen parts, are added to spirit of camphor and soap liniment, each fifty parts.

Turpentine is more valuable in abdominal disorders in horses. It is sprinkleci on hot blankets, and applied as a stupe to stop pain, spasm, and stimulate normal peristalsis. in colic; and to relieve pain and congestion in enteritis, peritonitis, diarrhœa and other difficulties. The beneficial result accruing from the use of external comnter-irritants in bowel troubles is often facilitated by the injection of hot $\left(115^{\circ}\right.$ F.) rectal enemata.

Stimulating liniments are serviceable in aiding resolution of swelling following the acute stage of cellulitis, lymphangitis, neuritis, mammitis, rheumatism, strains and bruises. They are often employed in laryngitis. In chronic skin diseases, as eczema, mild counter-irritants (tar, oil of cade, Peruvian balsam, etc.) substitute an active reparative process, tend to aid absorption of exudation and induration, and relieve pain and itching. The tincture of iodine may abort incipient inflammatory lesions, as boils and abscess, by means of its counter-irritant and antiseptic properties.

\section{VESICANTS AND THE ACTUAL CAUTERY}

Cantharides, red iodide of mercury, and croton oil, are more commonly used in veterinary medicine to cause blistering. Reference will be had, hereafter, to cantharidal blisters. Blisters and the cautery are especially indicated. to canse resolution of inflammatory products and modification of inflammatory processes; to secure fixation and rest of parts, and to relieve pain. It is impossible to enumerate all the conditions in which they are useful. In the treatment of severe sprains, as curb and "breakdown;" and in exostoses, as ringbone and spavin, the actual cautery (firing) is used before and in conjunction with blistering to exaggerate the connter-irritant effect. Absorption is attained in the foregoing conditions by the production of an acute inflam- 
mation, with increase of vascularity, tissue change and fatty degeneration. In "breakdown," the formation of scar tissue is thought (without reason) to assist in supporting the limb. Sometimes, on the other hand, osseous deposit is unabsorbed, but anchylosis and freedom from pain in a diseased joint is secured by the enforced maintenance of rest and fixation of the joint, together with the production of new bone.

In exndative diseases of serons membranes, as pleuritis, pericarditis, peritonitis, meningitis, arthritis, and synovitis, blisters facilitate absorption and recovery after the acnte stage is over. This favorable result is not due to loss of sernm, but to modification of the inflammatory process. In the first three diseases named above, blisters-flying blisters-may be applied.in spots every few days in different places over the affected area.

Blisters reflexly stimulate the nerve centres in meningitis, in addition to their action on the inflammatory lesion. They should be applied over the poll or spine according to the location of the trouble. Absorption in chronically enlarged glands is assisted by blisters. They also hasten "ripening" of suppurating glands or abscess when this process is slow, and hasten their resolution after evacuation of pus. The blister should be rubbed on abont the inflamed area in these lesions.

Vesicants are also valuable in pharyngitis and laryngitis for serere cases, when stimnlating liniments are ineffectual; and, in lessening pain, exudation and swelling of the throat, may avert the necessity of tracheotomy. A blister applied abont the coronet in diseases of the feet is serviceable in stimulating the growth of the hoof and promotes repair in navicular disease, laminitis, and cartilaginous quittor, after the acute stage is passed. In arthritis and synovitis, the whole diseased area, with the exception of the flexure of the joint, is covered with a blister. Since it is often impossible to immobilize a part, in veterinary practice, by splints, blisters are sometimes employed for this end after reduction of dislocations. 
The hair shonld be clipped from an area to be blistered, the skin washed with soap and water, and the animal tied up or restrained in some way from biting the part. It is the custom to cover immediately the surrounding parts with grease, but protection from the acrid discharge can be secured more effectively by frequent sponging with soap suds and water; or painting the skin, under the blistered surface, with a solution of resin in alcohol. Grease is not so good a protective, since it is a solvent for cantharides. Vaseline should be applied following the active stage.

Caustics or Escharotics are agents which destroy tissue. They comprise such substances as the caustic alkalies, mineral acids, silver nitrate; iron, zinc and copper sulphates; ferric, zinc and mercuric chlorides; carbolic acid, arsenic, together with the actual cantery. They are indicated in the treatment of exuberant granulations, morbid growths, septic, slonghing and necrotic parts, pyogenic membranes, fistulons tracts, and for the destruction of poisons, as in rabid dog and snake bite. Escharotics stimulate and modify untrition in unhealthy wounds and ulcers. Many form chemical compounds with the tissue elements. Heat oxidizes animal tissues, and also coagulates albumin and abstracts water from them.

The alkalies produce greasy compounds with the proteids, saponify the fats and withdraw water from the tissues. They are the most widely destructive agents. Silver nitrate forms an insoluble albuminate with the tissues to which it is applied, and protects the underlying structures from further action. It is most superficial in its action, but possesses specific properties in altering the condition of unhealthy wounds for the better. Extreme heat, exemplified by the white-liot iron, acts as a counter-irritant in stimulating reflexly the mervous system; in increasing the rascularity ci surrounding parts, and in favoring revulsion of blood; in aiding absorption and resolution, and in reliering pain apart from its direct destructive effect. Caustics may be applied in either the solid or liquid state, and in various forms, as 
the stick, powder, paste and ointment. Canstics are sometimes employed to stop hæmorrhage. (See Styptics, p. 63). The reader is referred to the articles on special agents for further details).

Suppurants.-Any of the more active connter-irritants, as croton oil, may induce suppuration. Under this head may be mentioned setons and issues of rowel. A seton is a piece of tape or other material, introduced through an incision in the skin, and thence nnder the skin in the connective tissne, and finally ont through the skin at a short distance from the point of entrance. The loose ends are then knotted together, and the whole loop is drawn through the wound once daily to keep up constant irritation and suppuration. An issue of rowel is a piece of ganze or tow, which is passed into an incision, where the substance remains, and causes continual irritation and suppuration. To intensify their irritant action, both setons and issues of rowel may be first saturated with cantharides ointment or oil of turpentine. They have been employed in acute diseases of the eye, meningitis, "strangles," and in joint and shoulder lameness, near the seat of trouble. Setons and issues of rowel are barbarous and dirty, and fortunately are becoming obsolete.

\section{Cold and Heat.}

Cold.-Cold and heat are only relative terms. As used here, they refer, respectively, to a thermal intensity below or above that of the body. Cold is usually applied by meaus of water in some form. In veterinary practice we are limited in the employment of cold air, as a medium, to the use of free ventilation and protection of animals from the solar heat. Cool air is especially desirable in the treatment of most febrile affections by lowering temperature and serving as a stimulus to the respiratory, circulatory and nervous functions generally. Locally, cold causes contraction of the peripheral vessels and muscles of the skin, forcing ont fluids from the part and reducing local temperature. 'This is more 
distinctly noticeable in congested areas. If the application is very severe or long continued, the vessels lose their tone, become paralyzed, and we have passive congestion, iuflummation, and finally death.

Ordinurily, reaction sets in after the use of cold, more particulitrly if followed by heat, when an active hyperæmia is substituted for the ischæmia. This is brought about both by reflex stimulation of the heart by the cold, and local d.lation of the vessels. Therefore, when we wish to constringe parts, we use moderate cold continuously; but, by alternating cold and heat we may accelerate the blood supply, and by first forcing out, and then bringing back the fluids of the tissues, we can maintain such an activity of the circulation that even solid exudations are absorbed. Cold, locally, lessens nervous irritability and pain directly, and, also, by contracting the afferent vessels, it diminishes the impact of the blood on sensitive parts. Moderate heat is said, nevertheless, to produce much the same result by relaxing the capillaries of the collateral circulation, thus draining off the blood and relieving tension in the inflamed part. Tissue change is diminished, locally, by the action of cold. Suppuration and sloughing proceed but slowly under its retarding influence. Acute abscess is converted into wiat might be logically termed a "cold abscess" in the most literal sense of the word. Remotely, moderate cold applierl to the body for a short period actually increases general temperature by stimulating reflexly the heat centres, increasing oxidation and lessening radiation from the contracted superficial vessels. If cold is in contact with the whole body for a longer time, the temperature gradually sinks and the reduction continues for some hours, when, in healthy animals, the temperature rises above normal. Such effects are much more pronounced in pyrexia. The action upon the nervous system is exceedingly important therapeutically. The most powerful stimulating action is exerted upon the centres of inuervation controlling the circulatory, respiratory, digestive and excretory organs. In fever, this treatment 
invigorates the failing digestive functions, relieves the nervous irritability by cooling the blood going to the great nerve centres in the medulla, and strengthens and stimulates the entire vervous organization. Cold applications and drinks stimulate the circulation reflexly, increase blood tension, and, therefore, the secretion of urine in fever, which is very important for the elimination of toxins. Locally, heat and cold are often used interchangeably in medicine. In human practice it is a rule to be guided in the choice of ove or the other by the desire of and effect mpon the patient, and this should apply, as far as possible, in veterinary practice. Cold is employed, locally, in congestions of superficial parts, and tends to abort inflammation and relieve pain.

In meniugitis, rubber ice bags or continual irrigation of the head and spine are used. Laryngitis may be successfully treated by ice ponltices (cracked ice aud sawdust in linen bags) or by thin rubber ice bags surrounding the throat. In the same way are treated sprained tendons, capped hocks, broken knees, recent curbs, and lymphangitis. In fact, most superficial inflammatory surgical affections are benefited by cold applications. Ice and ice water are useful in checking venous or capillary hæmorrhage, although hot water is often more serviceable. Cold water enemata are valuable in atonic constipation, diarrhœea, and to reduce temperature in fever. In the uterus, ice in small lumps will arrest metrorrhagia, and, in the rectum, aid in reducing prolapse. Ice bags, placed aloug the spine over the sympathetic ganglia, will cause dilatation of arterioles in regions corresponding to the point of application.

\section{MODES OF EMPLOYING COLD WATER.*}

Ablution is the simplest method of applying water to the surface of the body. It is merely bathing. Water at the temperature of $50^{\circ}$ to $60^{\circ} \mathrm{F}$. is applied by a rough, coarse

* The writer is much indebted to the writings of Simon Baruch in connection with uses of water. 
cloth. The water is thrown on in considerable quantities, beginning with the head and going rapidly over the neck, trunk and limbs successively, rubbing the skin briskly all the while with the cloth. Two attendants are required for the application of an ablution to the larger animals. The patient is finally chied and warmily blanketed. The process nay be. repeated each half hour in fever, or twice daily as a tonic measure in chronic diseases. The rationale consists in the stimulus afforded by the cold to the nervous system, accompanied by contraction and subsequent dilatation of the peripheral blood vessels, with consequent cooling of the blood and increased radiation of heat from the surface. In most hydriatic methods for reducing temperature in fever, friction of the skin should be the sine qua non, as otherwise the physical cooling of the body is confined entirely to the periphery, the contraction of the surface vessels driving the blood inward to the vital organs. The superficial muscles then act as non-conductors, and heat production being stimulated reflexly by the cold, an actual increase of internal temperature may obtain.

It is only by securing dilatation of the superficial vessels by friction that the result first described can be prevented, for after the first shock the peripheral vessels dilate, an increased supply of blood is bronght to the surface, is rapidly cooled and courses inward, only to be replaced by more over-heated blood. The internal temperature is thus lowered, and instead of an internal congestion being brought about, as may happen when the skin is simply exposed to cold, we have a constant withdrawal of heated blood from the interior. In this perpetual interchange not only does cooling of the blood and body occur, but the circulation is equalized and congestions are overcome.

The Sheet Bath. - Whole baths are impracticable for our larger patients, and the sheet bath may be substituted to advantage for antipyretic and other purposes to which the cold bath is adapted. A cloth soaked in cold water, or ice poultice, is placed on the poll to prevent determination of 
blood to the head, and a linen sheet, wet in water, of from $50^{\circ}$ to $80^{\circ} \mathrm{F}$., is placed over the animal, the surface being rubbed, while dashing on the sheet water at the temperature of $50^{\circ}$ to $60^{\circ} \mathrm{F}$. This process is continued for fifteen or twenty minutes, unless rigor is induced. The method is valuable in the treatment of fever and insolation. The general rationale is the same as in the case of ablutions, but the antipyretic effect is more marked and permanent. The sheet may be covered, while wet, by blankets, and conrerted into a wet pack.

The Wet Pack is applicable for general or local use, and for varions purposes. A linen sheet is dipped in water at a. temperature of $50^{\circ}$ to $70^{\circ} \mathrm{F}$., and wrung out very thoroughly. A cold application is put on the head and the sheet applied and covered with dry woolen blankets. The duration of application is from one quarter to three hours, according to. the object in view. If it is used as a strictly antipyretic measure, it should be changed frequently. The wet pack differs materially from other hydriatic procedures in that reaction occurs slowly, for there is no artificial stimulus in the shape of friction to accelerate it. The primary contraction of the vessels is succeeded by partial dilatation of them, when the blood from the interior of the over-heated body is. cooled on the surface by contact with the sheet and by vaporization of the water. Vascular contraction again occurs owing to the cooling, forcing the chilled blood inward. So there is continual interchange of cooled and heated blood, until the sheet has become thoroughly warmed. After the wet pack is removed, the skin should be dried and the patient well blanketed.

The interchange of blood is useful in relieving congestion of the internal organs, in aiding nutrition by bringing to the periphery nutriment absorbed from the gastro-intestinal tract, and for its tonic effect on the nervous system. In fever, it abates cerebral hyperæmia, delirium and excitement, and promotes rest and quiet.

The Priessnitz Poultice is similar to the wet pack, but a 
waterproof protective is interposed between the wet sheet, sponges or cloths on the inside and the outside woolen coverings. Evaporation is thus prevented, but not vaporization. Such an application may be used with safety in febrile diseisses, as plemitis and premmonia. If it is not renewed frequently, hyperæmia of the surface occurs, and this may be beneficial iu aborting or relieving internal congestion. It then acts as an ordinary ponltice, and is of value in various local inflammations resulting from strains, blows and bruises; also, in laryugitis. Besicles relieving internal congestion, the Priessnitz poultice stimulates absorption, removes induration and hastens suppuration, locally. The wet pack and Priessnitz poultice are more appropriate for practical use than the other methods in veterinary practice, since the evaporation from the hair of animals contraindicates methods suitable in human medicine.

Cold Baths are only practicable for the smaller animals. Dogs may be immersed in water at $90^{\circ} \mathrm{F}$., which is rapidly cocled down to $60^{\circ}$. The bath should last about fifteen minutes, the surface of the body being rubbed constantly. Ice water should be frequently poured over the head. After removal from the water, the patient must be thoroughly dried, wrapped in warm blankets, and a stimulant given if necessary. Such treatment may be used as an antipyretic measure if the temperature is over $103^{\circ} \mathrm{F}$. in the rectum.

Douches. - A douche is a forcible impact of water against the surface of the body. It is not used for its antipyretic effect, but acts as a stimulant to the nervons system at large, whereby the respiratory action is deepened and strengthened, and the circulation invigorated. The douche is given advantageously in the treatment of coma, of alcohol, chloroform, ether or opinm, applied to the lead. The water may be dashed from a pail or applied by means of a garden lose or from a tap. Theumatic lameness and peripheral paralysis are suitable cases for the donche treatment, followed by vigorous rubbing and dry bandaging. Syncope may often be quickly relieved by douching of the head and chest. 
Local Baths are good, especially in inflammatory conditions of the feet in horses. Tubs may be employed for the animals to stand in, the water being changed frequently or being kept cool by ice. It is not advisable to allow anịmals to stand in large bodies of water on acconnt of the danger they incur of "catching cold" from surface evaporation. The value of the local use of water in acute laminitis is worth mentioning here. After the shoes have been removed the extreme pain may be alleviated by standing the horse in quite hot water, sufficient to reach up to or above the foot-locks. Good results are obtained by changing to ice water after the first day and continuing this for several days, in this way causing a contraction of the arteries, lessening the amount of blood supplied to the part and the danger of chronic laminitis and dropped sole. If convenient to a running stream, about the same results may be obtained by standing the animal in it.

Irrigation with cold water is done in inflammatory diseases of the joints, tendons and feet. Running water may be permitted to flow continnously through perforated rubber tubes, connected with a tap, or used as siphons and closed at their distal extremities. The holes may be made in the tube with red-hot needles. The rubber tubes should be wound about the limb or part and held in position by bandages. Leiter's expensive block tin tubes are easily bent and rendered useless by the movements of our patients. The treatment of lacerated wounds by means of a stream of water from a convenient hydrant, causes the part to granulate quickly and greatly facilitates the healing process, but care should be taken not to allow the water to run over the wound more than three or four hours at a time each day, otherwise the part will become "waterlogged" and tend to break down and slough rather than to fill in with healthy granulation tissue.

Cold Drinks are both refreshing and antipyretic in action. Cool water should be placed where the patient can take it as he desires. In stomatitis, tetanus and angina, cool water 
is distinctly grateful and comforting. In the latter two diseases, it should be arranged so that the animal can reach it withont bending the neck. The mouth can be rinsed out continually, removing decomposing food and mucus, the thirst be slaked and heat and inflammation relieved.

Cold Enemata are valuable antipyretic agencies. From five to fifteen quarts of cold water may be thrown up through a flexible rubber tube, six feet long, far into the bowel of the horse.

Evaporating Solutions.-Methylene bichloride or ether spray may be applied for a short time by means of an atomizer, to induce local anæsthesia of a part, through the powerful refrigeration produced in their evaporation, and is most satisfactorily empioyed in conjunction with cocaine injections. One turn of a cotton or linen bandage, or a single thickness of similar stuff, put about a part and wet continuously with cold water, forms a good evaporating medium in allaying superficial inflammation and pain. A mixture of clay, and equal parts of water, vinegar and diluted solution of lead acetate, make a cheap and efficient cooling application for external use in the treatment of bruises and sprains. The paste should be removed as quickly as it dries.

Refrigerants. - Certain medicines either produce a subjective feeling of coolness or actually cause it, applied externally or given internally. The subjective sensation is due to some inexplicable action on the local nerve supply. Soma are stimulants and astringents, and diminish the circulation in the part. Externally, acetate of lead, chloride of ammonium, nitrate of potash, and vinegar, are used most frequently as refrigerants.

The mineral acids and salts of potassium and sodium, especially potassium nitrate, are administered more commonly, internally, both for their cooling effect and to allay thirst. 
HEAT

Water at a moderate degree of heat-what is termed lukewarm-i. e., $86^{\circ}$ to $95^{\circ} \mathrm{F}$, applied to the body, stimulates the action of the skin, relaxes peripheral vessels and diminishes nervons excitability, pain and spasm in neighboring parts. Water at a temperature of $112^{\circ}$ to $120^{\circ} \mathrm{F}$. contracts blood vessels of underlying parts, relieving congestion and pain. Such a degree of heat resembles cold in its effect, and they may often be used interchangeably. Still stronger heat has much the same effect as excessive cold, only acting more quickly, causing dilatation of the vessels, pain, inflammation and destruction of tissue. The action of powerful heat on the whole body, or upon single organs, is similar to that of counter-irritants. Mild, moist heat is beneficial in the treatment of wounds in poorly rascular parts where there is a tendency to indolent granulation, as about the feet in horses. Again, in low grades of inflammation with induration, as in strains of tendons, where moderate heat tends to stimulate the circulation and hasten absorption. In the treatment of abscess and burns, with destruction of tissue and suppuration, moist heat applied locally macerates the dead tissue, hastens sloughing and relieves pain, and in softening parts prevents the burrowing of pus and the formation of deep-seated pockets and sinus's.

A modern view of poulticing is that it aids the migration of lencocytes, and therefore is productive of good in assisting their phagocytic action.* The abscess can thus be more speedily formed and more quickly defined. Herein heat differs from cold. In irritable and spasmodic troubles of muscular origin in various organs, heat is distinctly remedial, as in pelvic and abdominal pain and colic, when

* Hot applications increase exulation, congestion ancl proliferation of cells and local chemical activity. All these results are inimical to bacterial growth. 
employed in the form of rectal injections. Heat may, in many conditions, be used interchangeably with, or in the place of, cold, according to the preference of the practitioner, or the effect upon the patient. As, for instance, in the case of pneumouia, pleuritis, angina, and in checking hæmorrhage. Heat may be utilized in simply preventing the natural radiation of it from the body. Thus, simple, warm, dry blankets, applied all over the surface of the body, may abort catarrhal or rheumatic conditions by merely causing retention of the body heat, dilatation of the peripheral vessels and equalization of the circulation. Covering a portion of the skin with such dense preparations as tar, pitch or collodion, in mild superficial inflammatory lesions, is said to produce farorable results by restraining radiation and increasing heat and blood supply in the part. Even thickened tendons and indurated glands may be benefited thereby. The Priessnitz poultice continuously applied has a similar action. In vasomotor paralysis, seen in collapse, following loss of blood or poisoning, and in shock due to traumatism or surgical operation, heat is eminently a life-saving means. In such conditions the loss of vascular tone and dilatation of the vessels leads to dangerous, and even fatal, cooling of the body. Heated dry blaukets, or those wrung out in hot water, should be applied to the larger animals, together with hot rectal injections; while the smaller auimals may be placed in baths at the temperature of $105^{\circ} \mathrm{F}$., till the temperature becomes normal. Such treatment should be combined with the use of vascular and cardiac stimulants, atropine, digitalis and strychuine, and saliue infusions.

MODES OF APPLYING HEAT.

Poultices or Cataplasms. - Cataplasms are compositions for the local application of heat and moisture. They are made, commonly, of flaxseed meal, bran, oatmeal, bread, potatoes and carrots. One or other of these is stirred up in boiling water until a thick, pasty consistency is reached. This mass may then be applied, while very hot, directly to 
the part when we wish to produce a softening of the tissues, as in abscess or tender feet in horses, and the whole is covered by a cloth. In poulticing horses' feet, the materialusually bran and flaxseed meal, equal parts-is mixed in a pail, with boiling water, and spread on a piece of bagging (double thickness, and about two feet square), in sufficient quantity to surround and cover the entire foot. The bagging is then folded and tied about the pasterns, and over around the front and sole of the foot. The whole should occasionally be immersed in water to prevent drying of the poultice. When a poultice is used merely for its continued heat, in relieving heat and congestion, the material should be enclosed in a flannel bag, in order the longer to retain and radiate its warmth.

A very hot poultice acts as a counter-irritant in contracting blood vessels in more remote parts, besides its effect in abating pain. A warm, moist poultice causes a mild local hyperæmia, softens broken down and dead tissues, and aids suppuration and sloughing. Poultices are not employed very much, except in the treatment of horses' feet, as they are clumsy, laborious contrivances, and dificnlt to keep in place. If long-continued, they cause tissnes to become swollen, sodden and macerated, destroying their vitality. Antiseptic ponltices are made by soaking sheet cotton, ganze, or other absolhent material, in lot antiseptic solutions, as corrosive sublimate, 1-1000; creolin or sulpho-naphtol, 1-100. The material is very lightly wrung out, wrapped about with dry ganze, covered with oil paper, silk or rubber protective, and applied to the part with a bandage. Antiseptic ponltices are useful in the treatment of septic injuries, and when there is much pain, destruction of tissue, slonghing and suppuration. Otherwise, poultices are deciderlly contraindicated in the case of wounds, as dry antiseptic or aseptic absorbent dressings are far preferible in securing one of the cardinal requirements in the process of healing, i.e., dryness. As substitutes for ordinary poultices, we have spongio-pilene, counter-irritants, stupes and fomentations. 
Spongio-pilene occurs in sheets, about an inch in thickness, made of a mixture of sponge and felt, backed with i flexible covering of gutta-percha. Its main objection is the expense. It forms, when soaked in water, a cleanly and easily applied poultice for non-suppurating parts.

Counter-irritation, as has been pointed out, is produced by hot poulticing as well as by drugs. A combination of the two is obtained in stupes.

Stupes, Stupa.-A stupe consists of a fliunel or other cloth, wrung ont in plain or medicated hot witer, and applied to the skin. These are often covered by waterproof protection, the better to retain heat. In the veterinary art, hot blankets are often applied orer the whole chest or abdomen to relieve internal congestion and pain in pleuritis, pneumonia and colic. Turpentine stupes are more in favor with abdominal troubles. These are made by simply sprinkling oil of turpentine over the hot blankets, or by saturating flannel cloths in turpentiue and wringing them out in very hot water. To get a very active counter-irritant effect, a mustard paste may be rubbed over the chest, and then hot blankets applied.

Hot Water Bags, made of rubber, and enclosing water at a temperature of $120^{\circ} \mathrm{F}$., may be placed along the spine, and by stimulating the cord and sympathetic ganglia, cause stinulation of the vaso-constrictors in regions corresponding to the controlling areas over which the heat is applied. In this manner inflammatory conditions of the throat, chest, and abdomen' are said to have been aborted, and internal hæmorrhage effectually arrested. Conversely, cold may be used over the spine to dilate distal arterioles.

Fomentations are simply local baths. As technically employed, the word refers to bathing parts with plain or medicated hot water, by means of sponge or cloths. They may be used to cleanse wounds or parts of dried discharges; they act as counter-irritants if very hot, or as mild, stimulating, soothing and softening applications if warm. In order to produce much effect, besides a mere detergent one, they 
should be applied for a considerable length of time-one half hour at least-and be followed by drying and bandaging. Fomentations reduce swelling and pain, and hasten repair in bruises, strains and local inflammatory lesions.

Injections of hot water are employed to cleanse wounds, stop bleeding and relieve pain and spasm. Injections may be thrown into the rectum or vagina at a temperature of $115^{\circ}$ to $120^{\circ} \mathrm{F}$. In the vagina, hot water may stop postpartum hæmorrhage, pain and congestion in the pelvis, by producing uterine contractions, and atonic constriction of vessels in neighboring parts, which lasts for several hours following its use. Hot rectal injections $\left(115^{\circ} \mathrm{F}\right.$.) subdue abdominal and pelvic pain or spasm, as intestinal or renal colic and spasm of the neck of the bladder. The heat per se in such injections may be invaluable in shock and collapse as noted above.

The water may either be led off through a rubber tube, from a stop-cock in a pail or reservoir, placed a few feet above the patient, or else siphonage may be done off-hand through a bit of small-sized hose. Having hung a pail filled with water three or four feet above the patient, the hose is filled with water, and, closing the ends to keep it full, the spper part is put in the pail, while the lower, smooth and greased, is passed into the rectum or vagina; or water may be poured through a large tin funnel into the upper end of the tube. The ordinary fountain syringe is the best apparatus for smaller animals.

Dry Heat may be applied by means of hot blankets, hot water bags, hot salt or sand in cloth bags, a flatiron or hot, wet cloths between waterproof coverings. Dry heat is often preferable to moist heat for simply relieving pain and congestion, as animals are less apt to become chilled by drafts and evaporation from the surface afterwards. It is generally more difficult to obtain, however.

Inhalations of plain or medicated steam are given for their local effect on the mucous membranes of the upper air passages. The moist heat has a soothing action on the 
nerves, and tends to loosen dry exudations. Agents may be: incorporated in the inhalation having a sedative, stimulating or antiseptic action. (Vid. "Agents Acting on the Respiratory Organs," pp. 47,48 .)

The technique consists in placing a bucket containing a boiling mash under the horse's nose, or in pouring cool. water over a heated brick or iron in the bottom of a pail. The practice of tying a bag over a horse's head, and steaming him therein, is bad, if the animal is suffering from. respiratory troubles, as insufficient pure air is obtainable. A dog may be placed on the seat of a cane bottomed chair, and covered loosely with a sheet over the whole, the steam: being generated in a vessel beneath.

Hot Baths at a temperature of $98^{\circ}$ to $110^{\circ} \mathrm{F}$. are impracticable for larger animals. They can be given to the smaller animals in collapse, shock, rheumatism, and to abort cold. after exposure. Glowing heat is applied by means of heated metal, and is treated under the section on counter-irritation.

\section{ACTION OF HEAT CONTRASTED WITH THAT OF COLD.}

The action of intense heat or cold on animal tissue is very similar in effect, producing vasomotor paralysis, congestion, inflammation, destruction of tissue and death. Even the sensations to which they give rise resemble each other so closely, that the coolies on first handling ice said they could not hold it because it burued their fingers. Strong heat $\left(115^{\circ}\right.$ to $120^{\circ} \mathrm{F}$.) contracts blood vessels in underlying parts. and overcomes pain and congestion. Heat of this degree. approaches cold in similarity of action. A moderate degree of heat dilates vessels, while cold of like intensity contracts them. Moderate heat relieves pain by relaxing. tissue, diminishes vascular tension by dilating efferent vessels of the collateral circulation, and draining off blood from. the congested areas. Moderate cold, on the other hand, accomplishes a similar result in benumbing nervous sensation and lessening the impact of blood in the painful region 
by constringing the afferent vessels. Tissue change is increased by moderate heat, but decreased by cold applied locally, or generally in fever. Swelling of tissue is reduced by cold directly; only indirectly by heat, which may, indeed, increase it. Softening and sloughing of parts, suppuration and "ripening" of abscesses and "cleaning off" of wounds, are facilitated by moderate heat, but hindered by cold.

\section{Disinfectants, Antiseptics and Deodorants.}

Disinfectants, or germicides, are agents which destroy the micro-organisms, causing infectious and contagious diseases, fermentation and putrefaction. Antiseptics are agents which prevent the growth and development of the microorganisms, occasioning fermentation, putrefaction and disease; more especially the micrococci producing suppuration.

Deodorizers, or deodorants, are agents which destroy or counteract a foul odor. They are not necessarily antiseptic or disinfectants. Considerable confusion exists in relation to the terms disinfectant and antiseptic, because the latter is often described as an agent which inhibits the growth, or destroys the life of the micro-organisms of fermentation, putrefaction, and disease. This definition makes antiseptics synonymous with disinfectants. The distinction exists, however, according to common usage, that while disinfectants may, in dilution, act as antiseptics, antiseptics are not often disinfectants, and in the nature of things are not strong enough to kill germs, although they may hinder their growth. Antiseptics may then be regarded as a subdivision of disinfectants. The two terms are unnecessary and misleading, as either might embrace both interference with the growth and destruction of micro-orgauisms. Disinfection may fall short of sterilization; $i$. e., death of all germs. Boiling a fluid containing micro-organisms wholly kills them; but, while disinfectants may destroy the germs of disease, they often fail to kill more resistant and harmless organisms, as the spores of $\mathrm{B}$. subtilis. The scope of anti- 
septics has been extended by some authors (Hare) to include agents which destroy the toxie products of bacteria (iodoform) and prevent their absorption. A discrimination between disinfectants and antiseptics may be made in relation to their connection with the body. Those agents employed to kill germs, in matter distinct from the living body, are disinfectants; while those agents applied on the surface, or introduced within the body, may be classed as antiseptics, since they can rarely be used in such strength as to kill all micro-organisms without injuring or killing their host.

\section{DISINFECTANTS AND DISINFECTION.}

Air, sunlight, heat and water are naturally the best disinfectants. Air scatters and dilutes micro-organisms, making them pathologically inactive. There is no more effective way to disinfect a stable, in which animals are living, than by free ventilation with pure air. It is well known that animals are less liable to contract infections diseases in the comparatively pure air of the conntry than in closely crowded and ill-ventilated city buildings. Liliewise, the contagious diseases of children mostly occur in winter, when they are herded together in schools and in poorly ventilated dwellings.

To attempt to disinfect the air surrounding a patient is the height of absurdity. The generation of chlorine and sulphurous acid gases for this purpose, although recommended in text books, is futile, and by irritating the respiratory mucons membrane, accomplishes more harm than good, since a congested surface offers a more suitable field for bacterial growth. Air, on the other hand, may be a medium of infection when contaminated with dust containing pathogenic bacteria (B. tuberculosis). Sunlight is prejudicial to the vitality of bacteria. Whereas, the bacilli of tuberculosis will live almost indefinitely in dark, damp places, they quickly succumb to sunlight and dry air. Sunlight and pure air are, then, imperative for both the immediate and preventive treatment of germ diseases. Heat is the most 
powerful agency for disinfection at our command. Dry heat, to be efficacious, must be applied at a temperature of $140^{\circ}$ C. $\left(284^{\circ} \mathrm{F}\right.$.) for three hours to kill all bacteria and spores; but this degree of heat scorches most fabrics and destroys many materials. Boiling water quickly kills all non-spore-bearing pathogenic bacteria, and these iuclude most of the organisms causing the common contagious and infectious diseases (tuberculosis and anthrax excepted). Two hours of continuous boiling will not destroy the most resistant of micro-organisms-the spores of the hay bacillus-but moist or saturated steam, at $230^{\circ} \mathrm{F}$., will infallibly kill any spores whatsoever within a few minutes. Fire is the most complete disinfectant, because it not only destroys germs, but their food and products. Water, like air, dilutes germs and aids oxidation and destruction of organic matter; but, again like air, driuking water may be the source of infection when sufficiently contaminated.

\section{MECHANICAL MEANS OF PROCURING ASEPSIS.}

The placing of sole reliance upon chemical agents for surgical antisepsis, in relation to the body, is a connmon error. 'Th'sse agents may damage denuded tissue, and do mot rach 1 he micro-organism burirl in the tissues. Therefore, it is imposwible to render iufected, living tissue absolutely aseptic, or sterile, by merely bathing it with chemical solutions. Far more efficacious is mechanical cleansing of the skin with soap, water and the scrubbing brush, and even of infected raw surfaces with gauze and corrosive sublimate solution, ${ }^{*}$ supplemented, if necessary, by the knife, caustic and drainage, to remove necrosed tissue and septic material.

\section{CHEMICAL AGENTS.}

Mercuric bichloride, carbolic acid, quicklime, chlorinated lime, sulphurous acil, and chlorine, are more frequently employed as disinfectants. Corrosive sublimate.

* Harrington's solution, see p. 216. 
solutions are decomposed by keeping, and by contact with albumin and ammonia. Acids, or common salt, added to bichloride solutions prevent, in i measure, this decomposition; but, nevertheless, mercuric bichloride is rendered nufit for the disinfection of masses of decomposing albuminons matter, as manure. One of the best solutions, employed by the Paris Disinfection Service, is composed of corrosire sublimate, 2 grammes; tartaric acid, 4 grammes; and water, 1 litre (1-500), colored with 5 drops of a 5 per cent. solution of indigo carminate. An English solution, in common use, consists of corrosice sublimate, 1 ounce; hydrochloric acid, 2 ounces, and water to make 3 gallons (1-768). The usual strength of corrosive sublimate solutions, for disinfection, varies from 1-500 to 1-1000. These solutions are suitable for articles made wholly, or in part, of leather, rubber and fur; for blankets, cotton and woolen fabrics, and for floors, walls, and wood work of stables. Surgical instruments, and other metallic implements and fixtures, are injured by corrosive sublimate solutions. Carbolic acid is more expensive than corrosive sublimate, and less efficient in cases where the latter is applicable. Carbolic acid can, however, be used to disinfect albuminons material and metallic substances. It is employed on animal excreta in 5 per cent. aqueons. solution (abont 8 ounces to the gallon of hot water). This solution will cause the hands to dry, crack and fissure if: they are immersed in it for any length of time.

Chlorinated lime and quicklime are good disinfectant agents to mix with animal evacuations. In fact, bleaching powder is probably the best and cheapest disinfectant we possess for use in privies, drains, sinks, cesspools, and sewers, and for the destruction of micro-organisms on floors, and in freces and urine.

A few pounds of this preparation may be thrown into privies or cesspools once a week, and the pure compound, or a satmraterl solution, may be scattered over floors or mixed with mannre. A 1 per cent. solution is used to disinfect harness, which shonld be washed and greased directly 
afterwards. Stagnant and putrid water may be rendered drinkable, after some hours, by the addition of 1 to 2 ounces to each 65 gallons of water. Chlorinated lime is a powerful deodorant as well as disinfectant, but is of no value in either capacity unless the compound contains so much chlorine gas that the face cannot be held near it without the production of great irritation to the eyes. Bleaching powder should be placed upon decomposing animal bodies, and sheets wet with a saturated solution should be wrapped abont the carcasses of animals dead from contagions diseases, to prevent infection during transportation. Disinfection by sulphurous acid and chlorine gas is done to destroy germs which caunot be reached by other metheds. Three pounds of sulphur and two ounces of turpentine or alcohol (to afford moisture and aid combustion) are needful for every 1000 cubic feet of air space. Sulplur is generally burned in an iron vessel placed on sand, or floating in a tub of water. If the building is sufficiently tight to insure proper disinfection, it is difficult to secure combustion of the proper amount of sulphur. To obviate this, the sulplur may be saturated with turpentine, ignited and placed in an iron kettle on a tripod over an alcohol lamp. Chlorine is disengaged from chlorinated lime, to which is added crude muriatic acid, one pound of former $t$ ( ) three of latter for every 5,000 cubic feet of air space. Buildings must be tightly sealed and made completely irrespirable for animals during the space of three hours. Sulphurous acid disinfection is not of much value, and has been discarded by most health anthorities both here and abroad. It certainly will not kill the spores of anthrax and tuberculosis, and should never be allowed to replace thorough mechanical cleansing and disinfection with other chemical agents, but may be utilized as an additional safeguard. Chlorine gas is more reliable. Formaldehyde is now being employed by most boards of health for geveral disinfectant purposes, and it appears to be the best means of gaseous disinfection. (See p. 338.) 
ANTISEPTICS.

It is perhaps well to consider here the sources of infection and the natural defenses or immunity possessed by animals against parasitic invasion. Pathogenic microorgauisms are commonly brought in contact with the body through the agency of the air, drinking water and food, and insects (flies and mosquitoes), and gain entrance by means of the air passages, digestive canal and blood; but even in the two former cases, the micro-organisms are in a certain sense outside of the boly, since it is not easy for them to penetrate the intact and healthy ciliated mucous membrane of the respiratory tract. But when the mucous membrane is damaged by inflammation, and the cilixe becomes paralyzed, and abnormal secretions are formed, then a furorable opportunity is offered for their growth and entrance into the circulation. In the digestive tract the hydrochloric acid of the gastric juice and bile* act as natural antiseptics, while the liver is thought to destroy toxins resulting from bacterial life.

When digestion is in a normal condition, putrefaction and fermentation do not occur; but when its activity is diminished, and the secretion of the digestive juices is lessened, and the hepatic functions are depressed, then a chance is offered for bacterial growth, fermentation and absorption of toxins, or even actual transmigration of micro-organisms through the intestinal walls. To these natural agencies of defense, which may be likened to outlying pickets shielding the animal from bacterial invasion, we must add the intrinsic pcwer of resistance vested in the tissues, blood serum and leucocytes in combating microorganisms; and the production of antitoxins in the body, antagonizing the toxius formed by bacterial action. Microorganisms are always to be found on the surface of the body and within its natural cavities open to the air, but patho-

* Recent experiments in human patients show that many forms of pathogenic bacteria may live in bile. It is but moderately bactericidal. 
genic bacteria are less likely to do harm if the animal is in a healthy condition. A limited number of bacteria (micrococci) may even exist within the blood in health, and this fact accounts for suppuration occurring when the tissues are severely injured, withont solution of continuity. The internal use of antiseptics is of comparatively little value, except when these agents come in direct contact with germs in the digestive tract. This follows becanse it is impossible to administer antiseptics in sufficient amonut to serionsly interfere with bacterial growth in the tissnes, withont injuring or even killing the patient.

There is known but a single instance (malaria) where the extibition of an antiseptic will inhibit the development of micro-orgauisms of a general infections disease, and so absolutely arrest it. It is very possible that mercury as a specific remedy in syphilis, and salicylates in rhenmatism, act therapentically as internal antiseptics. Antiseptics are of benefit in rendering the contents of the digestive tube more or less aseptic, and (after absorption) they exert some antiseptic action on the mucous membrane of the respiratory tract (volatile oils), and also on the urinary tract, during their elimination. The principal agents nsed as antiseptics for surgical purposes are : corrosive sublimate, carbolic acid, creolin, hydrogen dioxide, potassinm permanganate, zinc chloride, iodoform, salicylic acid, aristol, iodol, and boric acid. Those employed internally include naphtol, salol, creolin, carbolic acid, bismuth salicylate and subuitrate, quivine, salicylic acid, and many others. For a more detailed description the reader is referred to special articles on these agents in the preceding pages.

\section{DEODORIZERS OR DEODORANTS.}

Deodorants are not of any practical value in simply exchanging one odor for another, but, as in the case of chlorine, they sometimes actually destroy compounds which give rise to the stench. Sewer and other malodorous gases, resulting from foul decomposing matter and excreta, may be 
freer from bacteria than ordinary air, and are not usually the carriers of micro-organisms, nor the cause of specific infectious diseases. These gases do, however, occasion indefinite symptoms of ill-health. Deodorizers, which are also disinfectants, are of service in destroying noxions emanations and their source; but, to accomplish this, it is necessary that they come into direct contact with putrefying material, and should not be placed about the habitations of man or animals, with the ridiculous idea that they are achieving more than the production of a vile odor.

\section{PRACTICAL DISINFECTION.}

The premises occupied by animals suffering from contagious diseases, together with all articles contained therein, such as harness, blaukets, stable implements, and evacuations, must be disinfected after the removal of all animals and isolation of the sick. The excreta shonld be mixed with milk of lime ( 1 part of freshly slacked quicklime, with 2 parts, by volume, of water), or with pure chlorinated lime. The floors and walls must be scraped and washed. Boiling water should then be poured over every available part of the premises, and these brushed with a saturated solntion of chlormated lime. Clothing may'be treated by boiling in water, or by soaking in a solution of corrosive sublimate (1-500), or carbolic acid (1-20), for twelve hours. Haruess is disinfected by washing with soap and water, and then with a 2 per cent. crude carbolic acid or creolin, or 1-1000 corrosive solution. Valueless articles are given to the flames. Stable and metallic instrmments and fixtures are to be freed from dirt, scrubbed with soap and hot water, drenched with boiling water, and then with : 2 per cent. crude carbolic acid or creolin solution.

Gaseous disinfection is now in orter to kill microorganisms in remote and inaccessible places. Live steam is the most efficient means at our disposal for this jurpose, when a suitable apparatus for its application to 
woodwork, haymows, etc., is obtainable. In place of this we may resort to formaldehyde, chlorine or sulphurous acid gas. The walls are finally painted or covered with whitewash containing 2 per cent. of crude carbolic acid. Healthy animals, which have not been exposed to infection, may now be allowed to return to their disinfected quarters.

\section{SURGICAL ANTISEPSIS AND ASEPSIS.}

It may be fitting, and not out of place, to briefly outline here the use of antiseptics and asepsis in veterinary surgery. Since the days when Lister introduced antisepsis, surgery has advanced in a manner which appears, however, like retrogression. It is now conceded that asepsis can be attained more satisfactorily and safely without the general use of antiseptics. For antiseptics, as has been noted, inflict a certain amount of damage mpon denuded surfaces, and, in so far, lessen the resistance of the body to the inroads of bacteria. Modern surgery attempts to secure a comparative asepsis by mechanical cleanliness, which is more efficient, simpler, and harmless to the body. Antiseptics are indicated to assist asepsis in the toilet of the unbroken skin, and when sepsis has already occurred, or is unavoidable. The gross neglect of aseptic precautions, often seen in the operations of veterinary surgery, would be considered criminal practice in liuman surgery.

Asepsis is, nevertheless, very difficult to secure in the lower animals living among filthy surroundings and lying on frecal discharges. In addition to these disadvantages, the trouble of controlling animal-movements during operation, and of keeping dressings in place, make the attainment of perfect asepsis embarrassing and frequently impossible. The more common administration of anæsthetics would facilitate asepsis by preventing movements of the patient and enntact of the operative field with dirt.

But there are all degrees of infection, and while, with the best methods of securing cleanliness at onr command, it is impossible to completely sterilize normal skin and 
tissues, yet the surgical result may be perfect. Therefore, in surgical operations, we should endeavor to procure as small an amount of infection, or dosage of micro-organisms, as possible, consistent with existing environment and conditions.

The following aseptic technique is especially applicable in the case of any surgical operation undertaken upon a noninfected part. If it is possible to carry out all the details, and the result is successful, healing will take place withont suppuration.

Operations upon suppurating and infected areas should be conducted with cleanliness, and antiseptics are more desirable, particularly hydrogen dioxide in full strength, after thorough cleansing with normal salt solution.

To prepare the surface of the body for operations, the hair is first clipped and shaved, the skin is thoronghly scrubbed with a brush, green soap and water for five minutes, and then with 70 per cent. alcohol. After the skin is incised there is no further necessity for antiseptics unless the wound is already infected, or becomes so by exposure to impure air or contact with dirt. The hands of the operator, including the finger nails, should be brushed until clean with green soap and water, and then with 70 per cent. alcohol. It is well for all participating in an operation to wear thin rubber surgical gloves after thorongh hand-disinfection-in pus cases, to prevent contamination of the hands; in clean cases, to avoid infection of the wound from the hands. If gloves are not worn in operating upon clean cases, they are all the more useful in dressing or operating upon pus cases to avoid contamination of the hands which later might give rise to wound infection when the naked hands come in contact with a clean wound. Instruments are thoroughly scrubbed with soap and water, and boiled for ten minutes in an aqueous solution of sodium bicarbonate ( 1 teaspoonful to the quart), and then placed in a solution of carbolic acid (1-40), or removed to a sterile towel. New snonges only should be employed, which have been previ- 
ously cleansed, and then soaked in carbolic acid (1-40) solution, or pieces of sterile gauze may be used.

If irrigation is desirable, normal salt solution (1 heaping teaspoonful to the quart of sterile water) is appropriate. Nothing else but this is allowable within the non-infected abdominal cavity.* Sutures of silk, and needles, are prepared by boiling in water for thirty minutes. The area ahont the operative field is to be surrounded with cloths, or towels, which have been boiled or baked, and instruments and sponges may be laid on these.

Dressings may cousist of gauze which has been exposed for three hours to dry heat at $140^{\circ} \mathrm{C}$. $\left(284^{\circ} \mathrm{F}\right.$.), or placed in an oven of an ordinary cooking stove, in closerl tin cans, until it becomes scorched and slightly brown. The same ganze may be used for sponges. Unsterilized articles are not to be suffered to come in contact with the operator, or wound, during the operation.

Wound infection from exposure to the air and other media, is prevented by immediate dressing and baudaging, or by collodion applications.

\section{Venesection.}

Venesection, or blood-letting, formerly abused, has, for that reason, fallen into almost complete disuse. This is unfortunate, since blood-letting is a valuable and often lifesaring measure. The indications for venesection are chiefly limited to conditions associated with a general high arterial pressure and local engorgement of some organ.

In such cases venesection very rapidly reduces general blood-tension to a point lower than that existing in the engorged region, so that congestion is relieved. A full, incompressible pulse is said to indicate the desirability of

* It may be noted here that the peritoneum of the horse is so extremely delicate and liable to infection, as compared to that of man and dogs, that most abdominal operations are practically contraindicated in the case of this animal. 
venesection in severe acute disorders-in accordance with the above-but this is not by any means invariably the fact. as will be shown.

Venesection leads to a reduction of temperature, anr vascular tension is lowered for from 3 to 48 hours, accorting to the quantity of blood withdrawn, but the blood vessels quickly aljust themselves to the smaller mass of blood, and the original quintity of this vital fluid is soon restored (24 to 48 homrs) throngh absorption from the tissnes and alimentary canal. The heart beats more rapidly, owing to the lessened resistance in the vessels, and venesection is accompanied by nansea and prostration.

The blood is less dense and more fluid after bloodletting, and for this reason, if inflammatory processes follow, exudation is more apt to ensue. The fibrin is first regained, then the normal number of white, and finally that of red corpuscles, in from one to five weeks. Circulatory depressants-as veratrum viride-accomplish much the same results as blood-letting, by causing general reduction of rascular tension and relief from local congestion, thus "bleeding an animal into its own veins" withont loss of blood, it is true, but with less rapid and certain effect.

Catharties, diureties and diarphoretics also lower blood pressure by abstraction of fluid from the vessels, but their action is slow. The following disorders are those most suitable for treatment by venesection when they exist in an alarming form in robust animals :

Cerebral congestion. In insolation and tympanitis.

Apoplexy, particularly parturient apoplexy of cows.

Encephalitis.

Acute cerebral meningitis.

Active pulmonary congestion and apoplexy.

Passive pulmonary congestion in cardiac disease.
Sthenic pneumonia.

Sthenic pleuritis.

Urticaria.

Lymphangitis.

Toxamia $\left\{\begin{array}{l}\text { Bacterial, } \\ \text { Mineral, } \\ \text { Vegetable. }\end{array}\right.$

(Followed by saline infusion.)

Venesection from the jugular in cerebral congestion is, 
in fact, a species of local blood-letting by directly draining blood away from the brain; and it preserves life by preventing pressure on, and paralysis of, the great vital medullary centres controlling the respiration and heart. Moderatn blood-letting is sometimes advisable in the early stages of severe inflammatory attacks of the brain or its membranes.

In cerebral congestion, and dyspnoa due to gastric tympany and pressure on the diaphragm, bleeding may give relief. Blood-letting is particularly applicable in the treatment of parturient apoplexy of cows, and, when the disease has once occurred, it may be employed as a prophylactic measure in plethoric animals immediately before parturition. Venesection alleviates dangerous pulmonary congestion, removes the venous load on the right heart, and relieves dyspnoea and cyanosis by making it possible for the heart to force a smaller quantity of blood through the less obstructed lungs.

A feeble and easily compressible pulse does not necessarily contraindicate venesection in engorgement of the lungs, for this condition leads to stasis in the pulmonary circulation, prevents the proper flow of blood into the left ventricle, and thus canses arterial anæmia. Therefore, so far from contraindicating blood-letting, this condition urgently demands it. Alarming dyspnœa, great cyanosis, together with a general plethoric state, should guide us in blood-letting in sthenic pneumonia and pulmonary congestion, rather than the state of the pulse. Venesection is serviceable in advanced cases of cardiac disease in dogswith failing compensation, venons engorgement of the lungs, and dyspnoa-by relieving the obstruction to the right heart. Moderate blood-letting is occasionally useful in severe cases of acute pleuritis, laminitis, lymphangitis and urticaria in plethoric horses.

Finally, in various toxæmias, blood-letting drains away both the blood and its contained poisoll. The mass of blood removed may be advantageously replaced by injection 
of normal salt solution into a vein or under the skin. This method is not in prevalent use in veterinary medicine, but is applied wtth notable success in human practice. Every veterinary practitioner should be competent to bleed an animal. An amount greater than $\frac{1}{5}$ of the total quantity of blood should not be withdrawn. The total quantity of blood is equal to about 13.5 per cent. of the body weight in horses; to 2.2 per cent. of the body weight of fat swine; to 6.6 per cent. of the body weight in dogs, and to 7.7 per cent. of the body weight in man. Large horses or cattle may be bled to the extent of from 4 to $6 \mathrm{qts}$; smaller subjects, 2 to $4 \mathrm{qts}$; sheep, $\frac{1}{2}$ to 1 pt. ; dogs, 4 oz. t's 1 pt.

Blood-letting is generally done to animals in the upright position by shaving the hair and cleansing the skin over the jugular vein in the upper part of the neck. The vein is made prominent by pressure below the site of operation, and a fleam, or knife carefully guarded, is plunged into the vein, making a good clean incision. The blood should be quickly withdrawn and carefully measured and the effect on the pulse noted, and the blood-letting maintained until there is noticeable reduction in the vascular tension and other symptoms, for the relief of which venesection is employed. The bleeding is arrested by suturing the lips of the wound and by pressure with a bandage.

Local Blood-letting, or Scarification, is often useful in relieving tension and pain in locally congested or inflamed tissues, and may even arert death of the part. Furthermore, stasis is removed and exudation from the engorger vessels may be prevented, while a fresh supply of arterial blood flows in to reinstate the vital processes.

Scarification is practiced by making mumerous small, parallel incisions into the skin, fascia or other tissues in the long axis of a limb or part. In inflammation of the periosteum it is necessary to puncture this membrane. Bleeding is facilitated by warm poulticing, and is arrested by packing the incisions with sterile gauze. 
INDICATIONS.

Lampas.

Glossitis.

Periostitis.

Cellulitis.

Conjunctivitis.

Mastitis.

Laminitis.

(To secure blood for microscopic examination.)

Sometimes the veins leading from an inflamed area are opened, thus securing local abstraction of blood; e. g., the digital veins in laminitis; the milk veins in mammitis.

Scarification, or puncture, is indicated in the abovementioned conditions whenever there is great swelling, pain and tension in the affected parts, and not otherwise.

\section{Transfusion.}

Transfusion is the transfer, directly or indirectly, of blood from one living animal to another. In this process the blood must be obtained from an animal of the same species as the patient, but even then disintegration of the blood corpuscles follows, resulting in nephritis from the extra work put upon the kidneys in their effort to eliminate the destruction-products of the transfused blood. Embolism and sepsis are added dangers even when the blood is defibrinated, after removal from the body of the host, and only the sernm is injected into the patient.

The injection of warm, normal salt solution (.6 of 1 per cent.) has been found to fill all the indications for transfusion of blood, and yet is free from the dangers and difficulties besetting the latter.

\section{Saline Infusion.}

Saline infusions are intended to replace the normal blood plasma, and, therefore, should contain approximately the amount of sodium chloride-. 6 of 1 per cent.-contaiued in this fluid. The solutions should be filtered and boiled previous to their use, when this is possible, and are made 
by adding a heaping teaspoonful of sodium chloride to the quart of sterile water, which is used at a temperature of usually $103^{\circ}$ to $115^{\circ} \mathrm{F}$., according to the mode of introduction and circumstances.

Simple distilled and ordinary water are noxious to the tissues, while salt solution is entirely innocuous unless it contains three times the quantity of sodium chloride normally present in the biood.

Mode of Introduction.- Saline infusions are introduced within the body (1) by intravenous injection; (2) by injection into mnscular tissue (hypodermoclysis); and (3) by rectal injection (enteroclysis).

Intravenous injection is the most rapid and certain method, but not so simple and practicable as hypodermoclysis.

Any superficial vein which can be readily seen and isolated, may be utilized; preferably the jugular or internal saphena vein in animals; the median basilic, or cephalic, at the bend of the elbow in man.

The apparatus consists of a glass funnel or rubber bag connected by four or more feet of rubber tubing, with a canula or curved piece of glass trubing 4 inches long and $\frac{1}{4}$ inch in diameter for horses; $\frac{1}{8}$ inch in diameter for dogs. The apparatus should be boiled immediately before using. The vein is made prominent by manual pressure exerted by

* The true proportion of sodium chloride in blood plasma of mammals is .8 per cent., or 123 grains to the quart. 0.6 of 1 per cent. of sodium chloride is not really "normal " for mammals, but was deduced from that found in the plasma of frogs. The injection of a too dilute saline solution will cause the red blool cells to swell and part with their hemoglobin and will lead to great sweating and diuresis in the effort of nature to restore the plasma to its proper composition. A more exact solution for saline infusion consists of : Sodium chloride, .8 per cent.; potassium chloricle, . 3 per cent.; calcium chloride, .02 per cent.; water, 100 . In emergencies, ordinary table salt (which contains a slight amount of calcium chloride, causing its deliquescence), in the proportion of 123 grains to the quart or a level teaspoonful to pint of sterile water, may be employed for intravenous infusion or hypodermoclysis. 
an assistant, or by a bandage, applied proximally to the seat of operation. The hair is shaved from the part, which is cleansed, and an incision $1 \frac{1}{2}$ to $2 \frac{1}{2}$ inches long is made by lifting a fold of the skin directly over the vein and snipping off the top of the fold with scissors parallel to its long axis. The sheath of the vein is exposed, raised by dissecting forceps, and divided. The vein is tien lifted from its bed with an aneurism needle, and two silk or catgut ligatures are drawn under it about an inch apart. The vein is now incised longitudinally, and, as the blood begins to spurt ont, the distal ligature is tied about the vessel. The canula is next passed into the incision in the vein toward the heart and the proximal ligature is tied, with the first part of a surgeon's knot, about the rein and canula, holding the latter in place and preventing leaking of the salt solution from the vessel. When the injection is completed, the tube is withdrawn and the proximal suture is tied on the heart side of the incision, and thus the vessel is occluded on either side of the seat of operation. The apparatus is filled with salt solution-iucluding the funuel, tubing, and canula-at a temperature of $103^{\circ}$ to $110^{\circ} \mathrm{F}$. before its introduction into the vein, and the funnel should be kept full during its use to prevent the entrance of air into the ressel. Any pressure, previously employed between the incision and the lieart, should of course be remored before beginning the injection. A little clean absorbent cotton may be placed at the bottom of the funnel before the salt solution is poured into it, if the solution has not been previonsly filtered. In using the apparatus the funnel is raised abont two feet above the vein. The quantity of salt solution to be injected will vary from a few ounces to two pints in the case of dogs; from one to many quarts for horses. Enormous quantities of normal salt solution may be introlnced into the blood without harm, even an amount equal to fonr times that of the blood, providing the inflow is not too rapid; $i$. e., exceeding one fluid drachm to the pound of live weight in fifteen minutes. When this amount is exceeded the heart and kidneys cannot 
take care of the great quantity of fluid in the vessels and tissues. A return to the normal volume, force, and rate of the pulse, and of color to the mucous membranes, will lead us to stop the saline infusion. The use of intravenous saline injections is frequently followed by a reaction within half an hour, characterized by a severe rigor, succeeded by sweating, labored breathing, a stroug pulse and increased urinary secretion.

\section{Hypodermoclysis.}

Injection of warm $\left(103^{\circ}\right.$ to $105^{\circ} \mathrm{F}$.) normal salt solution into the muscular tissue of the neck, abdomen or flank, is done aseptically with the same apparatus employed for intravenous saline infusions, using a large hollow needle to thrust under the skin directly into the muscular tissue, instead of the glass tube for intravenous injection; or a fountain syringe filled with saline solution and attached to a sterile aspirating needle may be used; or a reversed aspirator apparatus may be utilized: i.e., by filling the jar with salt solution and forcing the air into the jar, thus displacing the fluid. The fountain syringe is the best apparatus. Hypodermoclysis may be employed in the same cases as intravenous infusion, and is a better method on account of its simplicity. We are guided as to the quantity of solution desirable by the same indications noted above as referring to intravenons saline injections. Hypodermoclysis may be done in several places, and absorption is assisted by massage. Salt solutions are injected under the udder in females, and are occasionally thrown into the peritoneal cavity, particularly after operations in this region, before closing the abdominal walls. 


\section{Enteroclysis.}

Enteroclysis applies to the rectal injection of normal salt solution $\left(105^{\circ}\right.$ to $120^{\circ} \mathrm{F}$.) to secure absorption. This method may be applied in cases not so urgent as to demand intravenous saline infusion or hypodermoclysis, more especially moderate degrees of hrmorrhage, shock, collapse, and circulatory depression, when the intrinsic heat of the injection is valuable in restoring the normal bodily temperature.

\section{USES.}

INDICATIONS FOR SALINE INFUSIONS.

Grave hæmorrhage.

Shock, traumatic, operative, and electric.

Suppression of urine.

Severe diarrhoea.

Eclampsia.

Purpura hemorrhagica.
Toxanria $\left\{\begin{array}{l}\text { Bacterial. } \\ \text { Mineral. } \\ \text { Vegetable. }\end{array}\right.$

In threatened death from any accidental cause.

In any disease with feeble heart and low vascular tension.

Hypodermoclysis, or the intravenous injection of saline infusions, find their greatest usefulness as life-saving measures in severe hæmorrhage. While these methods are not in vogne in veterinary practice, they lave become recognized procedures of great practical value in human medicine. The indications, following hæmorrhage, are to fill up the vessels and to restore vascular tension, since danger is imminent, not from loss of blood corpuscles, but from lack of a circulating medium. There is a sufficient number of red corpuscles to carry on the respiratory and oxygen-bearing functions even after the greatest loss of blood possible from ordinary canses. In fact, respiration is but slightly inpaired in human subjects suffering from pernicious anæmia, when there is a 90 per cent. reduction in the normal number of red corpuscles, and two-thirds of the blood may be withdrawn from animals and replaced with normal salt solutious without serious damage resulting. In shock there is general vasomotor paralysis, so that most of the blood collects in. 
the abdominal veins, while the ventricles and arteries are emptied. In this condition saline infusions $\left(105^{\circ}\right.$ to $110^{\circ} \mathrm{F}$.) are of infinite value, becanse absorption of drugs from the digestive canal and subentaneons tissue is impaired. Saline infusions greatly dilute the blood-and, therefore, poisons in the blood-in toxamia, while they increase the activity of the kidners and elimination of toxins. The intrinsic heat of the injections is thought to stimnlate antitoxin formation, and the restoration of rascular tension is believed to assist the natural bodily resistance of the patient.

A great viriety of disorders have been treated successfully in hnman medicine with saline infusions, on this hasis, including: septicæmia, pnenmonia, uræmia, diabetic coma, purpura hæmorrhagica, tetanus, nlcerative endocarlitis, pyelitis; acnte alcohol, ether, chloroform, carbonic monoxicie, arsenic and mushroom poisoning; and toxæmias resulting from acute infectious disorders. The same treatment might be applied to hæmoglobinæmia and other toxæmias peculiar to the domestic animals. Venesection for the purpose of removing the poisoned blood should, in most cases, be resorted to prior to practising saline injection in the toxæmias. Exchding shock and hremorrhage, where heat is invaluable, saline infusions are generally given at the temperature of $103^{\circ} \mathrm{F}$. by the rectum, nuder the skin, or into a vein.

\section{Kunsel's Treatment for Milk Fever in Cows.}

This special form of treatment merits the attention of the veterinary profession becanse of the remarkably successful results which have been almost universally secured in the case of milk fever, which is not only a very common disease, but one which has hitherto baffled the best therapentic attempts of the veterinarian. Following the Schmidt treatment with his intramammary injections of potassium iodide-which was productive of great diminution of the mortality of milk fever, but was often followed by local 
injury to the udder-M. Kunsel, of Lncerne, in March, 1903, made his first report of the method under discussion. This consists in the following: A tauk of compressed oxygen; which can be had of any of the wholesale drug houses, is connected by rubber tubing six feet in length to a milking tube and firmly wired to the nozzle on the tank and to the tube, which should be boiled previons to use. The ndder of the cow affected with milk fever should be stripped of milk and thoronghly washed with warm water and soap and the teats cleansed with 70 per cent. alcohol or some other effective antiseptic. The milking tube is then introduced into one of the upper teats and the oxygen gas is allowed to flow slowly into the teat until the corresponding quarter of the udder is tense and well distended. While pinching the teat to prevent the escape of gas, the tube is withdrawn and a strip of bandage or tape is bound about the lower part of the teat to retain the oxygen. The same procedure is repeated in each of the remaining teats. The ligatures on the teats may be permitted to remain in place for an hour and a half, when they shonld be removed. The inflation of the udder may be repeated in six hours, if necessary, owing to non-improvement of the patient. As synergistic measures, the subcutaneous injection of one-half grain of strychnine nitrate, the use of enemata to empty the bowels, and catheterizution are important in aiding recovery.

The animal should also be comfortably propped up with bags of hay.

If oxygen can not be readily obtained, the use of a bicycle pump connected with a milking tube may be employed with much success; some veterinarians claining that the results are as good as with the use of oxygen, providing the air is pure which is pumped into the udder.* This purity of the air may be attained by blowing the air through

* d ery convenient arrangements are now commonly sold at a small price for inflating the cow's udder with air. These consist of a rubber bulb and tubing, a chamber containing sterile cotton (through which the air is filtered) and a milking tube for introduction into the teat. They may be used by the laity, and their employment has been as satisfactory as when oxygen was injected. 
a wash bottle containing 2 per cent. carbolic acid solution. Light massage of the udder shonld follow the inflation. The results of the Kunsel treatment are wonderful. Kunsel reported a series of twenty-two cases of milk fever, without a death, following the use of his method. Similar results have been secured in this country. The rationale of the treatment has yet to be elucidated. Various hypotheses have been advanced, such as the effect of the oxygen on a hypothetical anærobic bacillus in the udder ; the action of the oxygen on the blood and general metabolism in destroying toxic products in the economy; the action of air-compression in the udder in overcoming conjestion in this part; and a possible stimulation of the secretory function of the mammary gland with elimination of toxins. The fact that injection of milk in to the mammary gland has produced a condition simulating milk fever appears to augur a local cause of the disease.

\section{Lavage.}

Lavage is a term applied to washing out the stomach with the stomach tube. This process, while an every-day occurrence in human medicine, has been too long neglected in Veterinary practice. Fortunately, new interest has been awakened in this useful procedure by Phillips, of St. Louis, who has perfected a tube and demonstrated the practicability of its use.*

The passage of the tube is chiefly of value in acute indigestion of the horse, with gastric flatulence and distention, where pain and danger of rupture of the organ are averted by permitting escape of gas. By further washing ont the stomach in such conditions, and in gastritis and engorgement, toxic, fermenting ingesta are immediately removed and the evil results, as tympanites and local inflammation of the stomach and of the intestines, are prevented. In choking, as by oats, the passage of the tube may afford relief, while in poisoning the washing out of the stomach is the one essential treatment. Gastric indiges-

*Amer. Vet. Review, Мay, 1904. 
tion and flatulence are shown by colic, distention in the region of the stomach, difficulty in thoracic breathing and eructations of gas by the mouth, or attempts at retching and romiting.

To pass the tube, the hor'se may be backed into a stall. The operator stands to the animal's left and an assistant, holding up the horse's head and the distal end of the tube, to the patient's right.

The tubo is placed in warm water and the surface is dusted with powdered slippery elm or smeared with vaseline. The left nostril of the liorse is also lubricated in the same way.

The operator pushes the tube gently along the floor of the left nasil fossi with the left hand, while guiding its direction with the right hand.

The first obstruction is likely to be met, when the tube has been entered about a foot, by its contact with the turbinates. The point of the tube should then be held downwards, by the pressure of the right forefinger pushed as far as possible into the nostril, while the outer part of the tube is lifted upward to force the point down into the pharynx. When the tube enters the pharynx attempts at swallowing are likely to occur and these are just what are needed to close the epiglottis over the larynx and to force the tube into the gullet. If swallowing is not evident it may be brought on by pushing the end of the tube gently backward and forward into the pharynx, and, when an attempt at deglutition occurs, the tube should be thrust forward. If the tube goes into the trachea instead of the cesophagus, it will meet with little resistance and expired air may be felt coming from it, while coughing often results. If it is in the gullet, the tube will be held more firuly by its walls and only fetid gas may escape with stomach contents. It shonld by these means be definitely established then that the tube is in the gullet before introdncing it farther.

The tube should be made with white marks on the rubber to show when it may be expected to have reached the gullet and again the stomach. 
During the course of passing the tube it must be well Iubricated.

When the stomach is reached the gas may have already escaped and fluid contents may be siphoned off by filling the tube with warm water from a funnel or syringe, holding the distal end tightly closed aud lowering it to the ground so as to permit of the escape of stomach contents by siphonage. If the contents are largely solid, the stomach must be repeatedly filled with 2 to 4 quarts of warm water and allowed to escape again by lowering the onter end of the tube to the ground. If the flow stops, owing to choking of the tube, it may be started again by injection of water into the tube with a syringe or pump.

The latter must not be used to suck out the contents of the stomach except so far, if necessary, as to start the siphonage. The stomach should thus be repeatedly washed until the water comes away clear. If water is injected with a syringe, care must be taken to avoid forcing air into the stomach.

When passage of the tube becomes impossible through one nostril, the other one may be tried. Phillips reports failure to pass the tube in the liorse in only 5 per cent. of trials. The tube is best made of red Para rubber and long enough to reach from the stomach to the ground when in place.

\section{Serum Therapy.*}

Antitoxic Serum. - The microscopic parasites which cause infections diseases-of which the bacteria are the most common-do so chiefly through the production of cer-

* In the use of serum therapy the strictest aseptic precautions are to be used. The animal is prepared by shaving off the hair from the part and the skin is washed with soap and water and then with alcohol and water, 2 parts of the former and 1 part of the latter, or with 5 per cent. carbolic acid watery solution, which is harder on the hands. The syringe and needle must be boiled for five minutes before using. When many animals are injected at one time, it may be sufficient to wipe off the needle after each injection with the alcohol solution. The puncture may be covered with collodion to advantage. Glass syringes, with asbestos packing on plunger, are most suitable for boiling, the needle connected by rubber tubing. 
tuin complex, poisonons, proteid bodies called toxins, which combine with the cells of the vital organisin and destroy their vital functions. Indeed, all the clissic symptoms of some of the infectious diseases may be caused by injection of the toxins of the bacteria which cause these diseases; such is the case with tetanus and diphtheria. Under favorable circumstances, however, when the dose of toxins is not too large, the animal organism reacts against these toxins through the possession of antitoxic principles in the fluids of the boly or by means of the secretion of antitoxius by the cells of the animal tissues.

The antitoxins set free from the cells then combine with the toxins in the blood and so prevent the toxins from uniting with the cells of the body and thus destroying them. The subject is a very intricate one and is as yet in a somewhat nebulous state and we here present but the briefest and crudest outline of the present and generally accepted hypothesis.

To illustrate the method of artificially manufacturing antitoxins, we will take as an example the production of tetanus antitoxin.

Tetanus Antitoxin.-Tetanus bacilli are grown in bouillon for two or three weeks in an incubator, when the culture is filtered free of bacilli and furnishes tetanus toxins. A horse is injected with one-half a cubic centimeter of toxin and an equal amount of Lugol's solution to lessen its virulence. The injections are repeated in increasing quantities till the seventy-second day, when as much as $150 \mathrm{cc}$. are injected. The horse is very susceptible and reacts with local inflammation at the site of each injection and generally by the production of antitoxius in its blood. A few days after the last injection the horse's immunity is at its maximum-that is, its blood is highest in antitoxic strength. The horse's blood is then withdrawn under strictest aseptic precautions and the serum is decanted after a day or two. The serum is placed in aseptic bottles holding 10 cc., which are sealed. 
They are put in an incubator for several days to prove their sterility; if the serum turns cloudy it is rejected. Most serums will keep a year if preservecl with a slight amount of carbolic acid or if treated by repeated sterilization below $100^{\circ} \mathrm{C}$. Cloudiness in a serum indicates that it is unfit for use.

The activity of a serum is estimated in two ways. First, the amount of antitoxic serum required to nentralize a given volume of toxin of known strength. Second, the strength is stated in units. Thus a test toxin is prepared, $\frac{1}{100}$ cc. of which constitutes the smallest fatal dose of a guinea pig. This amount of toxin is neutralized by $\frac{1}{1000}$ of a unit of antitoxiu. Or, to put it auother way, 1 unit of antitoxin will protect 1,000 guinea pigs against the smallest fatal dose of toxin.

The dosage is, then, reckoned in units of antitoxin, which is the most accurate method. While this mode of standardization is applied to the use of diphtheria antitoxin, unfortunately it is not employed in the case of the other serums where the dose is given in cc. This is inadvisable, as different manufactures possess different antitoxic strengths.

The toxin of tetanus is developed by the bacteria of this disease, which gain entrance through wounds of the tissues of the body. The bacilli of tetanus are not distributed by the blood, but remain at the site of infection, and the toxins they produce are taken up by the peripheral nerve endings in the vicinity and carried along the axis cylinders of the motor nerves to the central nervous system. The same thing happens in rabies. Wheu the toxins reach the spinal cord the symptoms of tetanus appear and finaliy death occurs, when the important centers of the mednilit become intoxicated. Tetanus bacilli live in the soil, ami wounds which are contaminated with dirt or foreign bodies, and those which do not have free access to air-as bruised or punctured wounds-are chiefly liable to the development of tetanus. It will thus be seen that tetanus does nut 
become apparent until some time after the involvement of the nervous system, and for this reason the use of tetanus antitoxin is not so actively preventive at this period, when the toxins have combined with proteid elements of the nervons system and are therefore unable to combine with and be neutraiized by the antitoxin. Nevertheless, tetauns antitoxin is of some value as a curative remedy in tetamns if used within thirty hours of the beginning of the attack.

As a preventive agent when employed before infection, or immediately after it, tetanus antitoxin is almost certain. Nocard injected 2,727 horses with tetanus antitoxin in a certain district, and while none of these developed tetanus, there were 259 cases in the same region in unprotecter horses. The immunity produced by tetanus antitoxin is thought to last from fifteen to thirty days. So-called idiopathic tetanus is in reality traumatic, resulting from small unseen wounds of the mucous membranes or integument. Tetanus antitoxin is then. indicated for use in the horse when the case is seen early, or as a preventive when tetanus is prevalent, or following wounds the character of which (see above) suggests the possibility of the development of the disease. The remedy may be employed without fear of doing any damage if properly administered. There are three ways of giving tetanus antitoxin; as a preventive it way be given under the skin or intravenously, and, as a cure for the disease, it may be used in these ways or be injected within the brain. The latter method has been practiced consiclerably in human medicine and with somewhat uncertain results as compared with the intravenous injection, althougl it is generally considered superior. Roux saved 35 out of 45 guinea pigs by intracerebral injection, whereas by subcutaneous injection of antitoxin he had but two recoveries in seventeen cases of tetanus. The injection is made with a blunt needle into the substance of the brain through a small hole in the skull made with a drill at a point midway between the onter angle of the orbit and the centre of a line 
drawn orer the top of the head from one auditory meatus to the other.

In most cases in veterinary practice the subcutaneous, or, better, intravenous, administration will be found. most practicable. The dose is 5 to $20 \mathrm{cc}$; the smaller dose as a prophylactic and when injected into the brain. $20 \mathrm{cc}$. is the usual curative dose for the horse, although the dose may vary according to the manufacturer.

Tetanus bacilli and spores may live in the tissues for weeks, and thus it is safer to repeat the dose-when given for immunizing purposes-at the end of the first and third week to antagonize any later intoxication cansed by a new crop of bacilli.

Anti-infectious Serum.-Antistreptococcus serum is included under this head becanse it appears to be especially antagonistic to streptococci themselves and to possess less antitoxic power. In the preparation of this serum (after M[armorek) streptococci are grown in human serum or serous exudate mixed with two parts of peptonized bouillon, and their virulence is greatly increased by repeatedly injecting them from rabbit to rabbit. This virulence becomes in this process so great that an amount of culture equivalent to one single streptococcus will certaiuly kill a rabbit. Small doses of a culture of living virnlent streptococei from the rabbit are injected into the lıorse from time to time, this animal reacting vigorously to the injections. It is not until the horse has undergone this treatment for a ye:tr and has recovered from each injection that a serum is obtained of a sufficient strength to combat living streptococei and their toxins. The blood of the horse being withdrawn furnishes anti-streptococcic serum. It is now thought by many anthorities that the serum to be most effective should be polyvalent-that is, should be active against the many varieties of streptococci by the cultivation of large numbers of streptococci to represent their different toxins. 
Marmorek holds, however, that the streptococci causing erysipelas, cellulitis, abscess, sore throat, parturient infections, scarlet fever, etc., are identical; that all produce the same toxins, and that all are antagonized by an antiinfections or antitoxic serum made from any one or all of them.

The therapeutic results of antistreptococcic serum are not so certain as those following the use of some other serums-notably anti-diphtheritic serum in man-because so-called streptococcic infection is often a mixed infection, by which is meant that produced in part by other bacteria, and because serums produced by the cultivation and inoculation of apparently the same varieties of streptococci seem to differ considerably in their protective value. As in the case of other serums, the therapeutic result is much more favorable when antistreptococcic serum is used as a prophylactic or in the early stages of the infection.

The injection of antistreptococcic serum is practically devoid of danger.

With the qualifications above noted, antistreptococcic serum has proven serviceable in the following morbid conditions: Medical and surgical septicemia, pyemia, parturient infections, traumatic infections, peritonitis, empyema, cellulitis, erysipelas, broncho- and contagions pneumonia of horses, and cerebro-spinal meningitis. As some of the above diseases are often caused by infections other than streptococcus, the employment of antistreptococcic serum may be unavailing in them unless the etiology is known to be streptococcic invasion.

Dosage.-In the larger animals from 20 to 50 ce. are injected at all ages at a single operation, and the dose should be repeated every twelve or twenty-four hours until the symptoms are abated. 
A fresh specimen of autistreptococcic serum is always desirable, as its power to destroy streptococci is soon lost.

\section{Toxins.}

Tuberculin.-Koch's tuberculin is prepared by growing tubercle bacilli in flasks, containing peptonized bouillon and glycerin, in an incubator at $37^{\circ} \mathrm{C}$. for six weeks. The cultures are boiled and filtered through porcelain to remove the dead bacilli, and the toxic substance is concentrated to one-tenth of its bulk by boiling. The result is a solution of the toxins of the tubercle bacilli in glycerin, and to this is added a $\frac{1}{2}$ per cent. aqueous solution of carbolic acid for injection. Tuberculin is used in veterinary medicine solely as a diagnostic test for tuberculosis in animals, chiefly cattle. It may be used with almost entire certainty for this purpose, Koch claiming 99 per cent. of correct results from its injection. Injections in tuberculons animals cause a rise of temperature of from one to three or more degrees F. in about twelve hours from the time of injection. If there is any focus of tuberculosis, as in the joints or bones, open to inspection, there will be a notable reaction observed in this locality, with heat, redness and decrease of function of the part. Erlich explains the fever following the injection of tuberculin to be due to a reaction of a zone of cells about the tuberculous focus which have been made unusually susceptible by the toxins of the disease so that they become inflamed by the sudden extra amount of toxin injected. In a tuberculous guinea pig, which has been killed by the injection of an overdose of tuberculin, zones of hyperemia may be seen surrounding each of the grey nodules characteristic of the disease. The injection of an ordinary dose of tuberculin is practically harmless and does not even render the milk of a cow unfit for food. In an advanced stage of tuberculosis the animal may not react to tuberculin, and this may be explained by the fact that the tissues are perhaps habituated to the toxins. 
The Test.-It is best to take the temperature of the animal from 6 A.M. every two hours until the tuberculin is injected on the evening of the same day between 8 and 10 P.Mr. The test is unreliable in animals whose temperature reaches $103^{\circ} \mathrm{F}$. during this period prior to the injection, and sometimes in those in advanced stages of the disease. The injection is made aseptically into the subcutaneous tissue in the side of the neck with a syringe and needle previously boiled. The animals should be kept in the stable during the time required for the test, and should not be allowed to drink large quantities of cold water to reduce their temperature while the test is being made. The temperature of the animal should be taken at 6 A.M. on the morning following the injection and from that time every two hours till 8 P.M.

A rise of two degrees $\mathrm{F}$. is necessary for a positive reaction; that is, a rise of two degrees over the maximum temperature of the animal in the fourteen to sixteen hours before the injection. Those animals in which the temperature does not rise over $103^{\circ} \mathrm{F}$. within fifteen or at most twenty hours after injection may be considered nontuberculous; when the temperature is between $103^{\circ}$ and $104^{\circ} \mathrm{F}$. the test is doubtful, and the animals should be reexamined after a month; when the temperature rises gradually to $104^{\circ} \mathrm{F}$. or over within fifteen hours after the injection, the animals may be classed positively tuberculous, provided the temperature constitutes a rise of two degrees over the maximum temperature recorded prior to the injection.

The average dose of tuberculin as prepared and diluted for immediate use by the U.S. Government is $2 \mathrm{cc}$ if tuberculin is to be kept for any period, it is better to procure the concentrated toxin and dilute it with a $\frac{1}{2}$ per cent. carbolic acid solution prior to injection. Tuberculin 
should be kept in a cool, dark place and should be rejected if it becomes cloudy.

Mallein.-This toxin is prepared in a manner almost precisely similar to that described under tuberculin. The sterilized cultures, from which the dead bodies of the Bacilli mallei have been filtered, and containing their toxins, are employed to test horses and mules for the existence of glander's and farcy. A rise of two degrees F. in an animal of normal temperature (not exceeding $101^{\circ} \mathrm{F}$.) within fifteen honrs of injection, together with a tender swelling five to ten inches in diameter at the site of injection, which is at its height in forty-eight hours, constitute positive proof of glanders. If the fever occurs without the swelling, or the swelling without the ferer, the animal should be re-tested in a week. In the normal animal a swelling occurs at the site of injection, without rise of temperature, but the swelling is much smaller and has almost disappeared by the encl of twenty-four hours, whereas in the glandered animal the swelling persists until the third or fourth day after the jujection. The temperature prior to the injection should not be over $102^{\circ} \mathrm{F}$., but if it is, the occurrence of a large, persistent swelling at the site of injection renders a diagnosis of glanders probable. The performance of the test is similar to that with the use of tuberculin, including the preparatory temperature-taking and that following the injection. The injection is made aseptically with a sterile syringe under the skin on the side of the neck, and a temperature of $104^{\circ}$ F. occurring within fifteen hours after the injection, together with a large and slowly disappearing swelling, is certain cvidence of glanders.

The usual dose for a diaguostic test of mallein in the horse is 1 cc., but the dose varies with different brands of manufacture, the proper dose of each being stated on the bottle. If several injections of mallein are given to a glandered horse, the reaction may disappear, and in this way glandered horses may be fraudulently prepared for sale is free from the disease. 
Recently claim has been made by a reputable veterin: riau that glinders may be cured by one or more injections (f mallein, and he bases his opinion on the failure of such inimals to react a second or third time to the mallein test. The fact above related that glandered animals become ordinarily insusceptible to the repeated test, together with. the danger of accepting such a statement until fully proved, should make one very cautious to act on such a belief in practice.

Semner* has, however, found that horses may be protected against infection with glanders by injections of mallein, and this lends some encouragement to the view of its possessing curative properties.

Mouilleron examined (at one time) four glandered liorses after death, following three or four negative tests with mallein, and found no evidence of the disease by microscopy, cultures and inoculation. Nocard has also shown the curability of glanders. The exact status of the matter is as yet undetermined, and until it is we may more safely refuse to accept the general curability of glanders by mallein.

\section{Vaccines.}

Varcination consists in introducing, within the animal body, bacteria (or their products) of a disease-with the intention of protecting the animal against the disease. The theory consists in the fact that, by modifying the virulence or action of the bacteria in some way, there are produced in the inoculated animal antitoxins. These not only prevent the occurrence of the inoculated disease, but even the development of the disease when introduced by natural channels. The activity of bacteria is lessened in various ways. Thus in preparing the vaccines against anthrax and blackleg the bacteria are exposed to heat. Then by inoculating the bacteria in a way, different from that in which they naturally

* Central l. f. Bacter., Bd. xvii, Nos. 9 and 10. 
gain entrance to the borly, their activity may be diminished; e.g., the bacillus of blackleg is introduced under the skin or intravenonsly, and the tail is sometimes chosen as a site, the blood supply being poor. The vaccines do not prove curative if employed after the development of the disease, against which they are preventives, and often they are ineffectual if used after the exposure of the animal to the infection of the disease. This is not the case in antirabic inoculation, however, as the treatment proves successful after the patient has been bitten and infected by a rabid animal. So in smallpox in the human, vaccination will modify and possibly prevent smallpox if done within four days after exposure to infection. The duration of the period of immunity conferred by vaccination differs in the case of the different vaccines.

Blackleg or Quarter Evil.-WThis disease is cansed by B. chan roi and is distinct from anthrax. Calves shonld be vaccinated when six months old and again within a year. The best time is that before the nsual occurrence of the disease, and no surgery, as branding, castration, marking, dehorning or spaying, should be done before, or within two weeks after, vaccination. Either one or (better) two vaccines are used, the first being the weakest and prepared by heating to $103^{\circ} \mathrm{C}$, and the second being leated to $93^{\circ} \mathrm{C}$. and used eight days later. Vaccination is done with a powder obtained by drying and triturating a piece of affected muscle. The directions for using the same are elaborate and furnished in detail by reliable makers. The use of this vaccine is very successful as a prophylactic against blackleg unless the animal has already been exposed to the disease or actually has it. Such must hare been the case when blackleg appears in a few days after vaccination.

Anthrax.-Anthrax is the most fatal disease attacking horses, sheep, goats, mules and cattle. It occurs in the United States, more often in Mississippi and Louisiana. Vaccination was done by Pasteur in 1880 and was the first 
attempt made in establishing artificial immunity in practice. Great crowds gathered to see the result of his treatmerit. He subjecter 24 sheep, 1 goat and 5 cattle to vaccination, and then 60 animals (including the vaccinated and unvaccinated) were inoculated with anthrax. Forty-eight hon's later the sight presented to the public beggars description. In the padilock were seen dead and dying all the mnvaccinated animals, while the raccinated ones appeared in perfect health. A small number of animals-especially sheep and goats-die from the treatment. In countries where anthrax is endemic, vaccination has reduced the mortality from 10 to $\frac{1}{2}$ of 1 per cent. Statistics also sliow that less than 1 per cent. of vaccinated animals die of the disease. When unvaccinated herds are attacked usually 80 per cent. die. The raccination should be practiced nsually in summer or fall, as these are the favorite seasons for development in infected regions. The protection lasts from six to twelve months. Two vaccines are nsed. Nnmber one, the weaker, is malle by growing bacilli in a current of air at $109^{\circ} \mathrm{F}$. for twenty-four days; number two is prepared in the same manner during twelve days. The weak number one is injected and followed in ten days by number two. Sick animals may infect a pasture for ten years, but vaccination will practically permit of pasturing on the infected land. The method of inoculation requires minute directions, which may be obtained from manufacturers. 


\section{INDEX OF DISEASES AND REMEDIAL MEASURES}

Abortion, accidental and epizoötic in cows and ewes.

Isolate and disinfect, 216.

Disinfect premises, 725 .

Empty uterus.

Antiseptic vaginal injections.

Lysol, 335.

Creolin, 334.

Carbolic acid, 329.

Corrosive sublimate, 216.

Ergot, 629.

ABscess.

Iodine, 246 .

Iodoform, 252.

Poultices, 714.

Rhigolene, 350.

Cocaine, 434.

Potassium permanganate, $20 \tilde{5}$.

Paracentesis.

Cantharides, 646, 702.

Acari or Mites. See Mange and Scab.

Parasiticides, 69.

ACtinomycosis.

Excise.

Potassium iodide, 24!.

Iodine, 244.

Carbolic acid, 331.

Good food and tonics.

ACNE.

Cleanse with soap and water.

Prevent chafing.

Sulphur, 258.

Arsenic, 266.

Phosphorus, 289.
AFt'TER-PAINS.

Remove elots or placental remains.

Opium, 368.

Morphine, 368.

Aged Animals, lestruction of.

Chloroform, $30 \%$.

Bullet, 307 .

Amaurosis.

Blister about orbit.

Strychnine, 409.

A MNenorrheaA. Sterility.

Full or restricted diet.

Tonies and emmenagogues, 58.

Oil of turpentine, 504 .

Myrrh, 525.

Oil of savine, 545 .

Cantharides, 647 .

Aloes, 569.

ANEMIA.

Full diet, 690 .

Fresh air.

Exercise.

Remove prinary cause.

Iron, 201.

Arsenic, 226.

Quinine, 488.

Gentian, 554.

Strychnine, 410.

Copper sulphate, 188.

Calcium phosphate, 158.

Cod liver oil, 657.

Myrrh, 525.

Clip horses with thick coats.

Angina. See Pharyngitis, Laryngitis, 
Anorexia. See Appetite, loss of.

Anthrax. Charbon.

Anthrax vaceine, 751 .

Corrosive sublimate, 215 .

Ipecac, 476.

Destroy or isolate animals.

Disinfeet premises, discharges and dear bodies, 725.

A Poplexy, cerebral, parturient.

Kunsel's treatment, 737.

Enemata, 737.

Strychnine, $73 \%$.

Ice to poll.

Ergot, 629.

Venesection, 730.

Bandage legs.

Eserine, 419.

Croton oil, 582.

Colocynth, 587.

Veratrum viride, $46 \%$.

Oil of turpentine, 504.

Change patient's position every few hours.

Avoid drenches if animal unconseious.

Empty bladder.

Stimulants, if collapse.

A PPETITE, loss of.

Gentian, 5.54.

Cinchona, 488.

Quinine, 488.

Quassia, 550.

Cusearilla, $55 \%$.

Cahumba, 558.

Taraxacnm, 560 .

Hydrastis, 562 .

Hydrastine, 562.

Hychastin, 562.

Calamus, 564 .

Capsicum, 526.

Aртиа. See Stomatitis.

\section{ARtiritis.}

Irr:gation, 710.

Iodine, 246 .
A rtwhritis (cont inued)

Oil of turpentine, 50\%.

Tartar emetic, 231.

Burgundy pitch, 506 .

Calomel, 219.

Corrosive sublimate, 217.

Red mereurie iodide, 219.

Cintharides, r02.

Rest.

Ascarides. See Worms.

Anthelmintics, 68.

Ascires. See Dropsies.

Astima in Dogs.

Bromides, 243.

Nitrites, 313.

Chloral, 318.

Belladonna, 386.

Arsenic, 226.

Eserine, 419.

Astuma in Horses. See Broken Wind.

Azoruria. See Ilamoglobinamia.

BaLANITIS.

Cleanse.

Urethral injection, 1 per cent. solution of zinc sulphate and lead acetate, equal parts.

IIytrastis, 503.

Barrenness, Sterility. See $A m$ menorrtoea.

$A_{\text {phrodisiacs and emmenagogues, }}$ $57,58$.

Brา Es.

Of rabid dogs, nitrie aeid, 272 .

of insects, : immonia, 147.

Of snakes, ammonia, 147 ; potassa, 123.

BLADDER, irritable.

Bèlladonna, 386.

Hyoscyamus, 390.

Potassium citrate, 127.

Potassinm aeetate, $12 \%$.

Spirit of nitrous ether, 313. 
BLADDER, irritable (continued).

Camphor, 550.

Buchu, 541.

Salol, 495 .

Urotropin, 341.

Rest.

Hot enemata.

Remove smegma, calculi and other sources of reflex irritation.

\section{Blepharospasm.}

Atropine, 384.

BleEdixg. See Hemorrhage.

Bog Spavin, See Arthritis.

Irrigation, 710 .

Rest.

High-heeled shoe.

Cantharides, 646.

\section{BoILs.}

Iodine, 252.

Collodion, $63 \%$.

Icthyol, 659 .

Carbolic acid, 330.

Menthol, 530.

Iodoform, 25:.

Glutol, 341.

Phosphorus, 236.

Ponltices, 714.

Paracentesis.

Bots. Larvae of Oestrus Equi.

Carbon disulphide, 278.

Chloroform, 68.

Anthelmintics, 68.

Green fodder.

Cathartics.

Break Down.

Cautery, 701.

Brokex Knees.

Cold, 706.

BrokeN WiNd.

Carron oil. 156, $5 \% 0$.

Arsenic, 226.

Strychnine, 408.
Brokex Wixt (continued).

Veratrine, $4 i 1$.

Restrict water, 118.

Concentrated diet.

Linseed meal, 610.

Cod liver oil, 658.

Bronchitis, aente and chronic.

Inhalations, $4 \%$.

Mustard, 517, 700.

Solution of ammonium acetate, 151.

Spirit of nitrons ether, 313.

Nitrites, 313.

Belladonna, 385.

Strychnine, 408.

Digitalis, 451.

Strophanthus, 455.

Squill, 458.

Dover's powder, 475.

Opium, 3r0.

Codeine, 366.

Heroin, 357.

Sodium bicarbonate, 136 .

Potassium bicarbonate and citrate, 127.

Ammoninm chloride, 150 .

Anmonimm carbonate, 149 .

Aconite, 463.

Quinine, 489 .

Potassium iodide, 249.

Arsenic, 226.

Cod liver oil, 658.

Linseed tea, 611.

Linseed oil. 570.

Olive oil, 60:3.

Tar, 509.

Benzoin, 515.

Eucalyptol, 521.

Ipecac, 475 .

Uil of turpentinc, 50)3.

Resin, 506.

Balsam of Peru, 512.

Balsam of 'Tohı, 512.

Myrrh, 525.

Asafetida, 538. 
Bronchitis, acute and chronic (continued).

Ammoniacuın, 540.

Buchu, 541.

Compound spirit of juniper, 542 .

Camphor, 550.

Sulphur, 258.

Sulphurous anhydride, 259.

Alum, 169.

Tannic acid, 593.

Eserine, 419.

Cantharides, 645.

Bruises.

Fomentations, 715.

Priessnitz poultice, 708.

Refrigerant mixtures, 711 .

Lead acetate, 174 .

Laudanum, 174.

Alcohol, 289.

Arnica, 523.

Camphor liniment, 549.

Hamamelis, 601.

Icthyol, 659.

Soap liniment, 605.

Stimulating liniments, 701.

Olive oil, 603 .

Burys.

Orthofor'm, 25:.

Aristol, 156.

Carron oil, 156.

Boroglyceride, $2 \div 5$.

Carbolic acid, 331.

Iodoform, 252.

Salicylic acid, 494 .

Sodium bicarbonate, 135 .

Chalk, 154.

Menthol, 530.

Oil of turpentine, 502.

Chlorinated lime, 240.

Chlorinated soda, 240 .

Moist heat, 712 .

Stimulants.

Good food.

Bursitis.

Ice, 706 .
Bursitis (continued).

Irrigations, 710.

Injections of iodine, 246 .

Injections of carbolic acid, 329.

Red mercuric iodide, 246 .

Cantharides, 645 .

Calculi, biliary. See Colic, Gall Stones.

Calculi, intestinal.

Enemata.

Rectal manipulation.

Anodynes. See Colic.

Eserine, 419.

Calculi, remal, vesical.

Hot enemata for pain, 716.

Enemata, 30.

Morphine, $36 \%$.

Potassium acetate and citrate, 127.

Lithium salts, 152.

Ammonium and sodium benzoate, 513.

11 ydrochloric or sulphuric acid for horses.

Canker of Ear. See Otorrhœa.

Chapped Elbow, Hock, Knee. See Bursitis.

Catarril. See Coryza, Ozœna.

Cerebral Hamorrhage. See Apoplexy.

Cerebritis. See Encephalitis.

Cerebro-spinal Meningitis.

Ice to head, 706 .

Ergot, 385, 629.

Bellarlonna, 385.

Aloes, 568.

Catheterize.

Strychnine, 409.

Cantharides, 645.

Chafing. See Intertrigo. 
Chorea.

Iron, 203.

Arsenic, 226.

Cod liver oil, 658 .

Antipyreties, 323.

Bromides, 243.

Chloral, 318.

Hyoscyamus, 390.

Cannabis indiea, 394.

Anrestheties, 307.

Eserine, 419.

Gelsemium, 422.

Conium, 430.

Valerian, 537.

Zine valerianate, $53 \%$.

Camphor, 550.

Strychnine, 409.

Quinine, 488.

Asafetida, 538.

Anthelmintics if due to worms, 68 .

Full diet.

CoLd, exposure to.

Mustard, 700.

Alcohol, 291.

\section{Colsc.}

With gastric flatulence, use stomach tube in horse, 739.

Opium, $36 \%$.

Morphine, 367.

Atropine, 385.

Chloral, 318.

Chloroform, $30 \%$.

Ether, 295.

Camnabis indica, 394.

Hyoscyamus, 390.

Capsicum and ammonium earbonate, 526.

Oil of peppermint, 530 .

Oil of turpentine, 503,701 .

Aconite, 463.

Asafetida, 538.

'Tobaceo, 426.

Aloes, 568.

Barium ehloride, 159.
Colsc (continued).

Arecoline, 617.

Eserine, 418.

Enemata, 30, 138.

Mustard, 518.

Stupes, 715.

Hot injections, 713 .

\section{Coma.}

Ice to head, $r 06$.

Cold ilouche, 709 .

Mustard and bandaging on regs, 700.

Venesection, 729.

Purgatives, 28.

Stimulants, in collapse.

Conjestion of Lungs.

Mustard, 517, 700.

Nitrites, 308.

Veratrum viride, $46 \%$.

Venesection, 729.

Aeonite, 463.

Conjunctivitis.

Cold eompresses.

Zine sulphate, 18\%.

Borie acid, 275.

Coraine, $43 \%$.

Silver nitrate, 1\%\%.

Protargol, 179.

Argyrol, 180.

Alum, 168.

Copper sulphate. 18\%.

Merenric oxides, 214.

Citrine ointment, 220 .

Creolin, 334.

Lysol, 335.

Corrosive sublimate, $21 \%$.

Scarification, 731.

Constipation.

Diet, 688.

Enemata, 30, 31.

Mamual evacuation, 585.

Abdominal massage, 585.

Exercise. 
Constipation (continued).

Ilorse-

Aloes, 568.

Linseed oil, $5 \% 0$.

Calomel, 218 .

Epsom salts, 163.

Cattle-

Epsom salts, 163.

Linseed oil, 570 .

Calomel, 218.

Foals and Calves-

Carron oil, $5 \% 0$.

Gregory's powder, 5i6.

\section{Dogs-}

Castor oil, 572.

Olive oil, 602.

Compoumd cathartic pill, 210.

Componsd liquorice powder, 579

Cascara sagrata, $5 \% 2$.

Syrup of purging buckthoru, 5\%4

Calomel, ¿218.

\section{Puppies- \\ Suppositories- \\ Soap. \\ Glycerin, 607.}

Phillips' milk of magnesia, 165.

\section{Birds-}

Tincture of rhubarb, 5\%.

Buckthorn, 5\%2.

Rhubarb, $5 \% 6$.

Senna, 579.

Croton oil, 582.

Jalap, 584.

Gamboge, 586.

Colocynth, 587.

Elaterin, 58\%.

Podophyllin, 589.

Arecoline, $61 \%$.

Ox gall, 655 .

Fserine, 418.

Pilocarpine, 444.

Barium chloride, 159.

Veratrine, 4i1.
Constipation (continued).

Strychuine, 410.

Glycerin, 608.

Belladomna, 386, 410.

Hyoscyamus, 390.

Sulphur, 258.

Myrrh, 525.

Asafetida, 538.

Convalescence.

Diet, 690.

Alcohol, 289.

Bitters, 289.

Strychnine, 410.

Gentian, 554.

Quinine; 489.

Calumba, 558.

Quassia, 556.

Hydrastin, 562.

Valerian, 53\%.

Pepsin, 653.

Cod liver oil, 658.

Convulsions.

Anesthetics, $30 \%$.

Chloral, 318.

Bromicles, 243.

Antipyretics, 323.

Valerian, 537.

Copper sulphate, 186.

Laxatives.

Warm bath.

Remove worms or source of irritation in digestive canal.

Corneal Opacities and Ulcers. See Keratitis.

Calomel, $21 \%$.

Coryza.

Inhalations, 47.

Spirit of nitrous ether, 312.

Aconite, 433.

Dover's powder, 475.

Opunm, 370.

Cocaine, $43 \%$.

Menthol, 530.

Quinine, 590. 
Coryza (continued).

Arsenic, 225.

Bismuth, 192.

Linseed oil, $5 \% 0$.

Cougri.

Inhalations, 47.

Opium, 369.

Heroin, 357.

Codeine, 366.

Belladonna, 385.

Phenacetin, 323.

Chloroform, 298.

Chloral, 318.

Wild cherry, 348.

Prussic acid, 347.

Cannabis indica, 394.

Bromides, 243.

Gelsemium, 422.

Tar, 509.

Balsam of Tolu, 512.

Arsenic, 226.

Camphor, 550.

Minstard, 517, 700.

Ammoninm chloride, 150.

Cracks or Fissures. See Fissures.

Cramps.

Atropine, 386.

Belladonna, 386.

Croup. See Laryngitis.

Curb.

Cold, 706.

Cautery, 701.

Cystitis.

Spirit of nitrous ether, 313.

Belladonna, $38 \%$.

Hyoseyamus, 396.

Aconite, 313.

Potassinm citrate and acctate, 313 .

Urotropin, 341.

Buchu, 541.

Oil of juniper, 542 .

Linseed tea, 611 .

Acacia, 606.

Cantharides, 647.
Crstitis (continued).

Boric acid, 275.

Creolin, 334.

Iıysol, 335.

Balsam of 'Tolu, 512.

Balsam of Perm, 512.

Salol, 495.

Oil of turpentine, 504.

Benzoates, 515.

Eucalyptol, 521.

Thymol, 552.

Myrrh, 525.

Rest.

Debility.

Alcohol, 290.

I'on, 201.

Arsenic, 226.

Strychnine, 409.

Quinine, 488.

Calcium phos],hate, 158.

Cod lives oil, $65 \%$.

Linseed meal, 610.

Cotton-seed meal, 610 .

Phosphor'us, 236.

Gentian, 554.

Calumba, 558.

Cardamom, 534.

Coriander, 5:34.

Fennel, 534.

Fenugreek, 534.

Ginger, 534.

Decubitis.

Chlorinated lime, 240.

Aristol, 253.

DELIRIUN.

Hyoscyamus, 390 .

Hyoscine, 390.

DIABETES IxSIPIDUS.

Iodine, 247 .

Gallic acid, 594.

Valerian, 587.

$\Lambda$ void bad fodder.

Diabetes Melitus, Sec Glyeosuria, 
Diaphraguatic Spasm, Hiccougm. See "Thumps."

\section{Diarritea.}

Laxatives-

Linseed oil, $5 \%$.

Castor oil, 572.

Rhubarb, $5 \% 6$.

Gray powder, 214.

Calomel, 218.

Carron oil, $5 \% 0$.

Magnesimm sulphate, 163 .

Diet and restriction of water, 688,689 .

Saline infusions, 736.

Enematr, 30.

Stareh, 636.

Rest.

Opium, 36\%.

Astringents.

Chalk, 155 .

Aluminum hydroxide, 169.

Silver nituate, 178.

Acids, $2 \% 2$.

Lead acetate, 175 .

Bismuth, 192.

Tannic aeid, 593.

Krameria, 599.

Hsmatoxylon, 600.

Hamamelis, 601.

White oak, 595.

Catechu, 597.

Kino, 598.

Ergot, 630.

Antisepties-

Carbolic aejd, 332.

Creosote, 333 .

Boric acid, 275.

Char'coal, $27 \%$.

Naphtalene, 33\%.

Salol, 495.

Oil of turpentine, 503 .

Tar, 509.

Benzoates, $5 \% 4$.

Arsenic, 225.
Diarritea (continued).

I pecac, 476.

Syrup of lime, $15 \%$.

Buchu, 541.

Camphor, 550.

\section{DIPHTHERIA.}

Insolate and disinfect discharges. Antitoxin when due to KlebsLoeffler bacillus.

Irrigate throat with normal salt solution.

\section{Alcohol.}

Milk and eggs.

Disinfect premises. 725 .

\section{DISINFECTION.}

Disinfeetants, 718-723.

Practical disinfection, 725.

Chlorine, 238.

Sulphur, 258.

Carbolic acid, 332.

Creosote, 333.

Formaldehyde, 339, 340.

Fol'malin, 339, 340.

Dislocations.

Anxstheties, 307.

Blistering, 702.

Distemper, Canine.

Isolate.

Country air.

Good food.

Quinine, 489.

Iron, 203.

Strychnine, 409.

Coffee, 399.

Calfeine, 399.

Camphor, 550.

Encalyptol, 521.

Chloral, 318.

Phenacetin, 323.

Bellaronna, 385.

Disinfect jlemises, 725. 
Dropsy, Cardiac, Hepatic, Renal. Digitalis, 219, 45:.

Squill, 458.

Caffeine, 400.

Strophanthus, 455.

Potassium iodide, 249 .

Pilocarpine, 444.

Calomel, 458.

Jalap, 584.

Colocynth, 584 .

Colchicum, 635 .

Sugar of milk, 652 .

Diet, 690 .

\section{Dysentery.}

Isolate.

Calomel, 218.

Magnesium sulphate, 163.

Linseed oil, 570.

Castor oil, $5 \% 2$.

Rhubarb, 576.

Ipecac, 476.

Opium, 367.

Lead acetate, 175.

Benzoates, 514.

Silver nitrate, 178.

Creolin, 334.

Lysol, 335.

Naphtalin, $33 \%$.

Oil of turpentine, 503.

Buchn, 541.

White oak, 595.

Catechu, 597.

Kino, 593.

Starch, 636.

Tannic acid, 593.

Haxmatoxylon, 600.

Arsenic, 225.

Disinfect premises and discharges, 725.

Feeding, 668, 669 .

Drspepsia, See Indigestion.

Dyspyea. See Laryngitis, Diphtheria and Chest Dispases.

Nitringlycerin, 312.
DYSPAEA (continued).

Nitrites, 312.

Inhalations, 47 .

Counter-irritation.

Tracheotomy.

Eclampsia. See Convulsions.

ECZEMA

Tar, 508.

Oil of tar, 510.

Oil of cade, 510 .

Icthyol, 660 .

Zine ointment, 185.

Sulphurated potash, 261.

Sulphur, 261.

Yellow wash, $21 \%$.

Black wash, $21 \%$.

Indine, 246.

Boric acid, 275.

Chalk, 154.

Lead acetate, 174

Zinc sulphate, $\mathbf{1 8 5}$.

Bismuth subnitrate, 191.

White precipitate ointment, 220 .

(itrine ointment, 220.

Curbolic acis, 331.

Salicylic acid, 494.

Balsam of Pern, 511.

Oleate of inercury, 214.

Blue ointment, 214.

Thymol, 552.

Chrysarobin, 5 r\%.

Glycerite of tannin, 593.

Hamamelis, 602.

Glycerite of starch, 608 .

Pilocarpine, 444.

Phosphorous, 235.

Cod liver oil, 658.

Diet, 688 .

Fypuysema. See Broken Wind. Arsenic, 226.

Stryehnine, 409.

Digitalis, 451.

Strophanthus, 455. 


\section{ENCEPIIALITIS.}

Ice on heal, 706.

Venesection, 629 .

Ergot, 629.

Croton oil, 582.

Veratrum viride, 467 .

Eserine, 419.

Pilocarpine, 444.

Aloes, 568.

Stimulauts.

Cantharides, 646.

linuocarditis. See also Rhenmatism.

Aconite, 464 .

Digitalis, 452.

Strophint hus, 455.

Potassium lodıde, 249.

\section{ENTERITIS.}

Opium. 369.

Atropine, 385.

Aconite, 463.

Veratrum viride, 468.

Mustard, 518.

Castor oil, 572.

Oil of turpentine, 503.

EPILePSy. Sce Convulsions.

EPISTAXis.

Adrenalin, 639.

Pack nostril with ganze.

Ice water or ice bag on forchead. Opium, 369.

Firgot, 629.

Alnm, 168.

Krameria, 599.

Erysipelas.

Icthyol, 660 .

Iodine, 246.

Carbolic acid, 329.

Creosote, 333.

Quinine, 489.

Alcohol, 291.

Tincture of ferric chloride, 195.

Milk and eggs.

Isolate and disinfect premises, 725.

\section{Erytiema.}

Zinc oxide, 185.

Lead acetate, 174.

Calamine, 185, 563.

Chalk, 155.

Boric acid, 275.

Tar, 508.

Camphor, 549.

Vaseline, 350.

Hamamclis, 602.

EYE, foreign bodies in.

Cocaine, 437.

Exostoses. See Spavin, Ringbone, Splint, etc.

Fainting. See Syncope.

FARDEL Bound. Inspection of $3 r d$ Stomach, Gastritis of Ruminants.

Strychnine, 410.

Veratrine, 471.

Arecoline, $61 \%$.

Epsom salts, 163.

Linseed oil, 570.

FEVER.

Spirit of nitrous ether, 313.

Solution of ammonium acetate, 151.

Magnesium sulphate, 163.

Aconite, 463.

Acetanilid, 323.

Phenacetin, 323.

Antipyrin, 323.

Quinine, 489.

Alcohol, 290.

Salicylic acid, 495.

Diet, 691 .

Refrigerants, 711.

Cold applications, 706-712.

Cold drinks, 706.

Cold enemata, 711.

Cool air. 
Fissure.

Of Rectum-

Orthoform, 353.

Belladonna, 383-386.

Iodoform, 251.

Tannic acid, 593.

Of Teats-

Silver nitrate, 178.

Balsam of Peru, 511.

Glycerite of tanuin, 5.93.

Collodion, 637.

Fistula of Wituers, Pold, IaAteral Cartilages, etc.

Corrosive sublimate, 216 .

Iodine, 246.

Arsenic, 224.

Carbolic acid, 329.

Creosote, 333.

Flatulexce, Trmpanites. Sce Indigestion and Colic.

Stomach tube in horses with gastric flatulence.

Oil of turpentine, 503.

Sodium bicarbonate, 135.

'Terebene, 504.

Chloroform, 298.

Oil of peppermint, 592 .

Charcoal, $2 \%$.

Strychnine, 410.

Ammonium carbonatc, 526.

Capsicum, 526.

Eserine, 418.

Naphtalin, 337.

Bismuth, 192.

Resorcin, 338.

Valerian, 53\%.

Asafetida, 538.

Cascarilla, 557 .

Calamus, 564.

Glycerin, 608.

Fleas (Pulex irritans).

Oil of anise, 532.

Pyrethrum, 624.

Carbolic soap.

Tobacco, 426.
Foot Rot in Sneep.

Glycerite of carbolic acid, 331.

Oil of Turpentine, 50\%.

Tar, 509.

Founder. Sec Laminitis.

Fractures.

Anesthetics, 307.

Calcium phosphate, 158.

Starch bandages, 636 .

Fracture of JAW.

Rectal feeding, 695 .

Fragilitas Ossium. Sce Osteomalacia.

Frontal Sinus, inflammation of. Iotloform, 251.

Frost Bite, Dermatitis, CongelATIONS.

Iethyol, 659 .

Oil of turpentine, 502 .

Glycerite of tannin, 593.

"Galls." See Ulcers, Wounds.

Gall Stones. See Colic.

Calomel, 219.

Salines, 136.

Oil of turpentinc, 503.

Exercise.

Gangrene.

Remove dead tissue with knife.

Oil of turpentine, 502.

Chlorinated sorla, 238.

Alcohol.

Good food.

Garget, See Mammitis.

Gastritis.

Opium, 368.

Bismuth, 192.

Solution of lime, 157 .

Ipecac, 475.

Prussic acid, 347.

Diet, 688, 695.

Nutritive enemata, 695 .

Ice by mouth. 
Gastroenteritis. Acute, Chronje. See Indigestion.

Opium, 368.

Linseed tea, 610 .

Acacia, 612.

Althan, 613.

Diet, 688,689 .

Gastroduonenitis, See Janudice.

Glandular Swellingis.

Iodine, 246.

Red mercuric iodide, 219.

Mercury, 214.

Mercuric oxides, 214.

Icthyol, 659.

Cantharides, 702.

Poultices, 714 .

Cod liver oil, 658.

Glaucona.

Physostigmine, 418.

Granular Lidos.

Alum, 168.

Copper sulphate, 187.

GLossitis.

Scarification, 731.

Glycosuria.

Glycerin, 608.

Granulations. See Wounds.

Grease.

Tar, 508.

Oil of cade, 508.

Griping of Catinartics.

Ginger, 527.

Belladonna, 386.

Hyoscyamus, 390.

Oil of peppermint, 530 .

Growths. See Warts.

\section{H TMATEMESIS.}

Opium, 369.

Ergot, 629.

lce by mouth. 629 .

Ileat to spine, 715 .

Oil of turpentine, 504.
II ÆuATENESIS (continued).

Silver nitrate, 178.

Tannic acid, 593.

Hamamelis, 601.

Rest.

Dict, 689.

II AMOG LOBINAMIA.

Aloes, 568.

Magnesium sulphate, 568.

Sodium bicarbonate, 136.

Colchicum, 635.

Diet, 690.

Prophylaxis, exercise.

Light feeding.

H FMIOPTYSIS.

Opium, 369.

Ergot, 629.

Heat to spine, 715 .

Digitalis, 452.

Oil of turpentine, 504 .

Gallic acid, 593.

Hamamelis, 601.

Rest.

HaMORRHAGES.

Ice, 706.

Opium, 369.

Ergot, 629.

Saline infusion, 735 .

Adrenalin, 639.

Antipyrin, 323.

Cocaine, 437.

Ferric salts, 201.

Oil of turpentine, 504.

Acids, 272.

Kino, 598.

Alcohol, 291.

Hemorrhage, Post-Partum.

Remove afterbirth.

Repair tears.

Ergot, 629.

Hot vaginal injections, 716 .

Pack uterus with gauze.

Saline infusion, 735.

Ifmorrhoids, See Piles. 
Heart Failure. See Syncope.

Heart, Fatty.

Diet, 690-694.

Stry chnine, 408.

Digitalis, 451.

Heart, Hypertrophied.

Potassium iodide, 249.

Aconite, 464.

Digitalis, 452.

Heart, Palpitation of.

Aconite, 464.

Digitalis, 452.

Strophanthus, 455.

Bromides, 243.

Belladonna, 384.

Camphor, 550.

Heart, Valvular Disease of.

Digitalis, 451.

Strophanthus, 455.

Strychnine, 408.

Diet, 690 .

Heat Stroke, See Sun Strolie.

Heaves. See Broken Wind, Emphysema, Asthma.

Hernia.

Anæsthetics, $30 \%$.

Cantharides, 646.

HERPES.

Lead acetate, 174.

Hydrophobia. See Rabies.

Hypertrophy of Heart. See Heart.

\section{HYSTERIA.}

Bromides, 243.

Valerian, 537.

Asafetida, 538.

Camphor, 550.

Impaction of Colon.

Magnesium sulphate, 163.

Linseed oil, 569.

Castor oil, 571.
ImpaCrion of Colos (continued).

Atropine, 386.

Strychnine, 410.

Hyoseyamus, 390.

Barium chloride, 159.

Eserine, 418.

INCONTINENCE OF URINE.

Bellatonna, 387.

Hyoseyamus, 390 .

Strychnine, 410.

Cantharides, 647.

Indigestion， Dyspepsia， Cinkonic

Gastro-intestinal Catarrh.

1)iet, 688 .

Aloes, 568.

Linseed oil, 5\%0.

Carron oil, $15 \%$.

Castor oil, 572.

A recoline, $61 \%$.

Rhubarb, 576.

Eserine, 419.

Acids, $2 \approx 2$.

Sodium bicarbonate, 135.

Arsenic, 225.

Strychnine, 410.

Capsicum, 526.

Ginger, 527.

Gentian, 554.

Hydrastin, 562.

Quinine, 488.

Pepsin, 653.

Pancreatin, 654.

Papain, 656.

Hydrochloric acid, 272.

Sulphurous acid, 260.

Carbolic acid, 332.

Creosote, 333.

Salicylic acid and salol, 495.

'Tartar' emetic, 232.

Hydrogen dioxide, 120.

Chareoal, 277.

Ammonium chloricle, 150.

Anise, 534.

Cardamom, 534.

Coriander, 534. 
Indigestron, ete. (continued).

Fennel, 534.

Fenugreek, 534.

Oil of turpentine, 503 .

Benzoie aeid, 514.

Eucalyptol, 521.

Calumba, 558.

Quassia, 556.

Cascarilla, $55 \%$.

Hæmatoxylon, 600 .

\section{InduRATIONS.}

Priessnitz poultice, 708.

Cantharides, 646.

Inflamation, Chronic and Acute. See Fever.

Laxatives, 570 .

Ice, 706.

Venesection, 728.

Scarification, 731.

Irrigation, 710.

Counter-irritants, 701.

Poultices, 712.

Diet, 691.

Aeonite, 463.

Opium, 369.

Veratrum viride, 467.

Mereury, 215.

Tartar emetic, 23:.

Sodium bicarlonate, 135.

Iodine, 246.

\section{INFLUENZA.}

Inhalations, 47.

Solntion of ammonium acetate, 151.

Spirit of nitrous ether, 313.

Potassium nitrate, 129.

Aleohol, 289.

Compound spirit of juniper, gin, 281.

Quinine, 489.

Strychnine, 408.

Coffee, 399.

Caffeine, 399.

Belladonna, 385 .
INFLUENZA (continued).

Euealyptol, 521.

Camphor, 550.

Linseed oil, 570.

Disinfection, 725 .

Intertrigo. See Erythema.

INTESTINAL H EMORRIIAGE.

Opium, 369.

Ergot, 629.

Tannie acid, 689.

Hamamelis, 601.

Diet, 689 .

Intestinal Jndigestion AND Ca TARRI. See Indigestion.

Intestinal Orstruction. See $I n$ tussusception and Twist.

INTUSSUSCEPTION.

Opium, 369.

Enemata, 30 .

Position, 418.

Aludominal section.

Fiserine, 418.

IRI'TIS.

Atropine, 384.

Calomel, 219.

Eserine, 418.

Itronva. See Pruritus.

JAUNDICE.

Magnesium sulphate, 164.

Calomel, 218.

Sorlinm phosphate, 142.

Nitrohydrochloric acid, 218.

Acids, 272.

Aloes, 569.

IIydrastis, 562 .

Podophyllin, 589.

Enemata, 30.

I)iet, 689 .

Jorv's, Inflammation aind Swelling of. See Arthritis. 
Keratitis.

Atropine, 374.

Calomel, 217.

LAMENESS.

Cocaine, 435.

\section{LaMinitis.}

Aconite, 463.

Veratrum viride, $46 \%$.

Pilocarpine, 444.

Venesection, 731.

Poultices, 713.

Local bath, 709 .

Vesication, 702.

Feeding in, 688.

\section{LAARXNGITIS.}

Inhalations, 47.

Benzoin, 515.

Priessnitz poultice, 709.

Ice bag, 706.

Mustard, 517.

Cantharides, 645.

Red mercuric iodide, 219.

Stimulating linıment, 701.

Spirit of nitrous ether, 312 .

Aconite, 463.

Belladonna, 384 .

Dover's powder, 495.

Ipecac, 475.

Iodine, 246.

Potassium iodide, 249.

Sulphurous anhydride, 25\%.

Alum, 168.

Tannic acid, 593.

Tar, 509.

Silver nitrate, 178.

\section{LEUCORRHEA.}

Creolin, 334.

Lysol, 335.

Corrosive sublimate, 216 .

Alum, 168.

Zinc sulphate, 187.

Chlorinated soda, 238.

Myrrh, 525.

Potassium permanganate, 205.
LEUCORRHEA (continued).

Bolic acid, 275.

IIydrustis, 563.

Glycerite of tannin, 593.

Gallic acid, 594.

Krameria, 599.

Hxmatoxylon, 601.

Lice. Pediculi.

Parasiticicles, 69

Balsam of Peru, 511.

Oil of anise, 532 .

Carbolic acid, 331.

Creosote, 383.

Creolin, 334.

Lysol, 335.

Oil of turpentine, 502.

Corrosive sublimate, 215 .

Mercury ointment and oleate, 214.

Staphisagria, 624.

Licilex. See Eczema.

LimiInasis. See Calculi.

Lockjaw. See Tetanis.

Luxations. See Dislocations.

LyMPILANGITIS.

Aconite, 463.

Colchicum, 635.

Cold, 709.

Venesection, 729.

Manyitis.

Belladonna, 383.

Aconite, 463.

Stimnlating liniments, 701.

Venesection, 729.

Mange. Scabies. Acariasis.

Parasiticides, 69.

Tar, 508.

Oil of tar, 5018.

Oil of cade, 508 .

Balsam of Peru, 511.

Sulphur, 25\%.

Iodine, 246.

Tobacco, 426.

Carbolic acid, 331. 
M ANGE, ete. (continued).

Creosote, 333.

Naphtol, 336

Creolin, 334.

Lysol, 335.

Chlorinated lime, 240 .

Blue ointment, 214.

Disinfection, 257.

Mastitis. See Mammitis.

Malaria.

Quinine, 489.

Aloes, 489.

Calomel, 489.

Meningitis.

Ice, 706.

Ergot, 629 .

Opinm, 368.

Calomel, 219.

Corrosive sublimate, 217.

Pilocarpine, 444.

Venesection, 729.

Cantharides, 646-702.

Metritis. Pelnic Lymphangitis.

Pelvic Peritonitis.

Removal of clots, placental or membranons remains.

Hot antiseptic vaginal injections, as:

Creolin, 334.

Lysol, 335.

Quinine, 489.

MEtrorrhagia.

Ergot, 629.

Hydrastis, 562.

Cotton-root bark, 630 .

Ice, 706.

Hot injections, 716 .

Ferric chloride, 200 .

Hanamelis, 601.

Digitalis, 452.

Savine, 544.

Gallic acid, 594.

Catechu, 577.

Milk Fever. See Parturient Apoplexy.

Mrositis (Interstitial). Myalgia, Muscular Rheumatisir.

Chloroform, 298.

Oil of turpentine, 502.

Camphor liniment, 550.

Aconite, 463.

Missage.

Veratrine, 471.

Quinine, 489.

Salicylate acid, 4!55.

Salicylates, 495.

Cod liver oil, 658 .

Nasal Gleet. See Ozcun.

NePhRITIS.

Mustard, 491.

Digitalis, 452.

Strophanthus, 455.

Buchu, 541.

Oil of juniper, 542 .

Linseed tea, 611.

Nervous Exilaustion. NeurasTHENIA.

Phosphorus, 2:36.

Neuralgia.

Opinm, 368.

Morphine, 366.

Antipyrin, 323.

Belladonna, 384.

Gelsemium, 422.

Conium, 430.

Aconitine, 463.

Aconite, 463.

Menthol, 529.

OBEstTy.

Feeding for, 691-694.

Salt in, 141.

Pilocarpine, 444.

Exercise.

Estrus equi. See Bots, 
UPACITY OF ConNeA. See lieratitis.

OPEN JOINTS.

Asepsis.

\section{Oрнтнацmi. See Conjunctivitis.}

Osteomalacia, Mollites Ossiun, Fragilitas Ossiun.

Diet, 691.

Calcium phosphate, 158.

Phosphorus, 236.

\section{Otitis Media.}

Cantharides, 646.

\section{Otorrhea, Otitis Externa.}

Boric acid, 275.

Zine sulphate, 185.

Alum, 169.

Potassium pelmanganate, 205.

Iydrastis, 563.

Glycerite of taunin, 593 .

Tincture of iodine, 608 .

Oxyurides. See Worms.

\section{Ozgena.}

Iodine, 246.

Chlorinated soda, 240.

Bismuth subnitrate, 191.

Potassium permanganate, 201 .

Boric acid, 275.

Copper sulpliate, 187.

Arsenic, 225.

\section{PaIN.}

Opium, 367.

Morphine, 367.

Codeine, 366.

Heroin, $35 \%$.

Chloral, 318.

Cannabis indica, 394.

Acetanilid, 323.

Gelsemium, 422.

Antipyrin, 323.

Phellacetiı, 323.

Palpitatioñ. See Heart.
Paralysis. Paraplegia, Hemiple(i1).

Strychnine, 409.

Cantharides, 646.

Cold douches, 709.

P'ARALYSIS, Local.

strychnine. 409.

('apsicum, 526.

Parturient Apoplexy. See Apoplexy.

Pericarditis.

Cantharides, 646, 702.

Potassium iodide, 249.

Calomel, 219.

A conite, 464.

Digitalis, 452.

Sitrophantlous, 45.).

Colchicun 631.

P'Eriostitis.

Iodine, 246.

Incision, 731.

Red mercuric iodicle, 219 .

Cantharides, 646, r02.

Perritonitis.

Opium, 369.

Morphine, 369.

Atropine, 386.

Aconite, 463.

Veratrum viride, 467.

Oil of turpentine (stupes), 503, r01.

Mustard, $5 \% 8$.

Alcohol, 284.

Pinaryagitis.

Inhalations, $4 \%$.

Belladonna, 385.

Aconite, 463.

C'amphor, 550.

spirit of nitrous ether, 313.

Licorice, 609.

Linseed oil, 570 .

Acacia, 612.

Solution of ferric chloride, 200.

Silver nitrate, 178. 
Pharyigitis (continued).

Cantharides, 702.

Cold drinks, 710.

Рноторіовіа.

Atropine, 384.

Eserine, 418.

Pinexitis. See Cerebritis.

Piitheiriasis. See Lice.

Piles.

Magnesium sulphate, 168 .

Sulphur, $25 \%$.

Aloes, 569 .

Orthoform, 253.

Glycerite of tamnin, 593 .

Hlamamelis, 602 .

Ergot, 630.

Cocaine, 437.

Iodoform, 251.

Acids, 272.

Bellarlonna, 383.

Pityriasis. See Eczema,

Pletilora.

Diet, 688.

Pleuritis.

Opium, 370.

Calomel, 219.

Linseed oil, 570.

Wet pack, ros.

Venesection, 729.

Aconite, 463.

Mustard, 517, 700.

Oil of turpentine, 503 .

Iodine, 246.

Potassium iodicle, 249.

Piloearpine, 444.

Digitalis, 451.

Strophanthns, 455.

Caffeine, 399.

Gelsemium, 422.

Salt, 411.

Diet, 690.

Cantharides, 702.

\section{Pxeumonia.}

Venesection, 729.

Mustard, 517.

Wet pack, 708.

Nitrites, 313, 408.

Potassium nitrate, 129.

Potassium chlorate, 130.

Quinine, 489.

Antipyretics, 323.

Opium, 370.

Aconite, 463.

Veratrum viride, 467.

Gelseminm, 422.

Solution of ammonium acetate, 151.

Strychnine, 408.

Digitalis, 451.

Strophanthus, 455.

Phosphorus, 236.

A rsenic, 226.

Poisoning. For Antidotes, see Special Drugs.

Stomach tube, 739.

Tannic acid, 593.

Charcoal, $27 \%$.

Alum, 169.

'/ine sulplate, 186.

Mustard, 518.

A pomorphine, 372.

Nitroglycerin, 312.

Atropine, 385.

Stryehnine, 408.

Camphor, 550.

Olive oil, 603.

Soap, 606.

Venesection, 729.

Saline infusion, 735.

Poll Fvil. See Fistula.

Polyuria. See Diabetes Insipidus.

Post Partum II mmorrhage. See Metrorrhagia.

Pregnancy.

Linseed oil, $5 \% 0$.

Castor oil, 572. 
Prolapse of Rectuin.

Tannic acid, 593.

Powdered opium and nutgall ointment, 593.

Ice, 706.

Pruritus, general, and of anus and viulva.

Carbolic acid, 331.

Boric acid, 275.

Hamamelis, 602.

Chloral, 318.

Alcohol, 289.

Prussic acid, 347.

Corrosive sublimate, 216.

Tobacco, 426.

Cocaine, 437.

Salicylic acid, 494.

Potassium bicarbonate, 123.

Tar, 508.

Oil of tar, 508 .

Oil of cade, 510 .

Menthol, 529.

Lime water, $15 \%$.

Alum, 168.

Yellow wash, 217.

Black wash, 217.

Silver nitrate, 178.

Thymol, 552.

Psoriasis, Scaly Eczema, MalLENDERS.

Oil of tar, 508.

Resorcin, 338.

Tar, 508.

Oil of cade, 510.

Iodine, 246.

Sulphur, $25 \%$.

Thymol, 552.

Chrysarobin, 577.

Pyrogallol, 595.

Soft soap, 605 .

Icthyol, 660 .

Ptralisy.

Bellarlonna, 38\%).

Atropine, 385.

Alum, 168.
Puerperal Fever. Sec Parturient Apoplexy, Metritis.

Pulionary (Edema.

Nitrites, 313.

Atropine, 385.

Digitalis, 451 .

Strophanthus, 455 .

Purpura Hamorriagica.

Mineral acids, 273.

Quinine, 488.

Oil of turpentine, 504.

Copper sulphate, 189 .

Iron 201.

Pyenia.

Alcohol, 290 .

Precitis.

Urotropin, 341.

Oil of turpentine, 504.

Balsams, 512.

Benzoates, 515.

Fucalyptol, 521 .

Buchu, 541.

Oil of juniper, 542 .

('antharicles, $64 \%$.

Quittor. Sec Fistula.

RABIES.

Quarantine suspeets.

Destroy rabid animals.

Cauterize bites made by rabid patients with nitric acid, 272.

Employ Pasteur treatment on men bitten by rabid animals.

Disinfect premises, 725 .

Renal Colic. See Calculus.

Retention of URINe.

Pressure on bladder through rectum, horse.

Catheterize.

Opium and belladomna per rectum.

Hot enemata, 30. 
Rineunatis.s.

Salicylic acid, $49 \pi$.

Ganltheria, 495.

Sialol, 495.

Methyl salicylate, $49 \%$.

Chloroform, 298.

$\Lambda$ tipyretics, 323.

Opium, 368.

Belladonna, 384, 385.

Strychnine, 409.

Gelsemium, 422.

Pilocarpine, 444.

Digitalis, 452.

Strophanthus, 455.

Sulphur, 258.

Potassium iodıle, 249.

Aconite, 463.

Veratrum viride, 46 \%.

Ioline, 246.

Veratrine, 471.

Dover's powder, 475.

Lithium salts, 152.

Tartar emetic, 231.

Quinine, 489.

Burgundy pitch, 506.

Benzoic acid, 514.

Mustard, 518.

Camphor liniment, 550.

Icthyol, 660.

Stimulating liniment, 701.

Cod liver oil, 658.

Colchicum, 635.

('antharides, 646.

Dry heat, 713.

C'old douche, 709.

RICKETS.

Sodium phosphate, 124.

Phosphoris, 236.

Calcimm phosphate, 159.

Syrup of calcium, lactophospliate, 159.

Syrup of ferrous iodicle, 202.

C'od liver oil, 6is.

\section{RingBone.}

Cautery, 701.
Ringbone (continued).

('antharides, 645.

RINGWORM.

Parasiticides, 68.

Glycerite of carbolic acid, 331.

Tincture of iodine, 246.

Citrine ointment, 220.

White precipitate, 220.

Mercurial ointment, 214.

Balsam of Pern, 511.

Sulphur, $25 \%$.

('hlorinated lime, 240.

Sulphurous acid, 260.

Boric acid, 253.

(reosote, 333.

Bichloride of mercury, 216.

Naphtol, 336.

Oil of turpentine, 502.

Thymol, 5is.

Chrysarobin, $57 \%$.

Pyrogallol, 594.

RoARING. See Thick Wind.

Salivation. See Ptyalism.

SaPRemia and Septictama.

Quinine, 489.

Collargol, 180.

Eucalyptol, 521.

Sulphites, 143.

Alcohol, 290.

Scab in Sheep, Sarcoptes orts.

\section{See Mange.}

Carbolic acid, 331, 332.

Creolin, 334.

I yssol, 33i.

Tobacco, 426.

Tar, 508.

Oil of tar, 510.

Oil of cade, 510.

Scabies. See Mange.

Scirrius (ord. See Champignon.

Scratciles. See Erythema.

Serricama. See Sapremill. 
Sllock.

Opium, 370.

Alcohol, 290.

Atropine, 385.

Strychnine, 408.

Digitalis, 451.

Ergot, 629 .

Ileat, 713.

Mustard, 700.

Saline infusions, 735.

\section{Shoulder Layeness.}

Veratrine, 451.

Turpentine, 502.

Sick Animals, destruction of.

('hloroform, 307.

Bullet, 307.

Prussic acid, 346.

\section{SitFAST .}

Turpentine, 502.

Sore Thront. See Pharyngitis, Laryngitis.

Sores. See Wounds and Tleers.

Spasms. See Convulsions, Epilepsy, Chorea, Tetanus, Colie, Asthma, Thumps, etc.

Spavin.

Cantharides, 646.

Cantery, $\% 01$.

SPLINT.

Red mercuric iolide, 219.

Cantharicles, 646 .

Sprains and Strains.

Irrigation, 710.

Refrigerant mixtmres. 711.

Priessnitz poultice, 708.

Fomentations, 715.

Chloroform, 298.

Uil of turpentine, 502 .

Iodine, 246.

Arnica. 523.

Lead acelate, 174.
Sprains and STrains (continued).

Camphor liniment, 549.

Olive oil, 603.

Soap liniment, 605 .

Ictliyol, 660.

Burgundy pitch, 506 .

Blister and cantery, 701.

Sterility. See Ammenorrhoea.

S'terilization, Hands, Instruments, Operative Field.

Surgical asepsis and antisepsis, 726.

Soap and mustard, 605.

Corrosive sublimate, 216.

Carbolic acid, 328.

Creolin, 334.

Lysol, 335.

Stomach Staggers. See Indigestion.

Stonatitis.

Potassium chlorate, 130

Borax, 275.

Nitric acid, 27\%.

lime water, 157.

Alum, 168.

Chlorinated lime, 240.

Glycerite of carbolic acid, 331.

Thymol, 552.

IIyrlrastis, 563.

Glycerite of tamnin, 593.

Cold drinks, 710.

Strains. See Sprains.

S'trangles.

Mustard, 518.

Canthariles, 646.

Stimulants.

Fresh air.

Good laxative diet.

Poultice the throat, $\mathbf{7 1 2}$

Isolation.

Treat generally as for influenza.

Disinfection, 725. 
Sunstroke, Heat Stroke, InsolaTION.

Antipyretics, 323.

Cold applications, 706 .

Venesection, 729 .

Surfeit, Nettrlerasit. See Utr-

- icaria.

Sycope, Ileart Failure, Weak IIEART.

Ammonia, 147 .

Spirit of ammonia. 147.

Alcohol, 291.

Ether, 294.

Hoffmann's anorlyne, 293.

Strychnine, 409.

Digitalis, 451.

Strophanthus, 455.

Convallaria, 456.

Camphor, 550.

Caffeine, 399.

A tropine, 385.

()il of turpentine, 503 .

Mustard, $5 i 0$.

Synovitis.

Ilrigation, 710.

Ioline, 246.

Mustard, 578.

Carbolic acid, 329.

Alum, 168.

Mereurial ointment, 214.

Cantharides, 646, 701.

TaPE WorM. See Worms,

Teats, Sore. See Fissure.

\section{Tenosynovitis}

Iodine, 246.

Rel mercuric iodide, 219 .

Cantharides, 646 .

Tetanus.

Chloral, 318. .

Carbolic acid, 330.

Bacelli treatment, 330.

Opium and morphine, 368 .

\section{Tetanus (continued).}

Belladonna, 387.

Hyoscyainus, 390.

Cannabis indica, 394.

Fserine, 419.

Gclsemium, 422.

Tobaceo, 426.

Bromides, 243.

Antitoxin, most suceessful, 742 .

Cold water, 710 .

Rectal feeding, 695.

THick Wind, RoARING.

Red mercuric iodide, 219.

Potassium iodicle, 249.

Arsenic, 226.

Strychnine, 408.

Tracheitis.

Benzoin, 515.

'Tamnic acid, 593.

Tuorovan Pin. See Bursitis.

Turusir. See Stomatitis.

Thrush in Frog of Iorse.

Calomel, 217.

Tar, 509.

Cleanliness.

Thumps, Hiccough, Spasm of Diapilragi.

Ether, 295.

Morphine, 368.

Belladonna, 386.

Camphor, 550.

Ticks, IODIDE.

Creolin, 225.

Sulpho-naphthol, 225.

Arsenic. 225.

Tinea, See Ringurorm.

Trichiniasis, Trichinosis,

Glycerin, 602. 
Tuberculosis.

Iodoform, 251.

Trupanitis." See Flatulence.

Twist. See Volvulus.

UDDER, Inflammation of. See Mammitis.

Ulcrrs. See Wounds.

Urinary Calculi. See Calculi.

Urinary Preserfative.

Chloral, 318.

URINE, Concentrated.

Spirit of nitrous ether, 313.

Potassium citrate and acetate, $12 \%$.

Urticaria, Nettlerasi, Surfeit.

Sodium bicarbonate, 135.

Calomel, 217.

Arsenic, 226.

Acids, 272.

Pilocarpine, 444.

Menthol, 529.

Icthyol, 659.

Venesection, 729.

\section{URETHRITIS.}

Urotropin.

Protargol, 179.

Argyrol, 180.

Zine sulphate, lead acetate, 1 per cent. solution, equal parts.

Buchu, 541.

UTERUS, Hypertrophy of.

Ergot, 629.

UTERUS, Inertia of.

E.rgot, 629 .
UTERUs, Inflammation of. See Metritis.

UTERUs, Subinvolution of.

Ergot, 629 .

Viginitis. See Leucorrhan.

Verminous Bronchitis. See

Worms.

Volvulus, Twist.

Enemata.

Opinm.

Eserine, 418.

Abdominal section.

VoMITING.

I[ot water, 118

Lime water, $15 \%$.

Cerium oxalate, 170 .

Bismuth subnitrate, 192.

Mereury, 214.

Calomel, 218.

Carbolic acid, 332.

Creosote, 333.

Prussic acid, 347.

Morphine, 368.

I pecac, 475.

Menthol, 530 .

Rectal feeding, 695.

Warts and Growths.

Glacial acetic acid, 266 .

Nitric acid, 271.

Carbolic acid, 329.

Sulphuric acid, 271.

Zine ehloride, 182.

Arsenic, 224.

WEeD. See Lymphangitis.

Winstling. See Thick Wind, Roaring.

Wind GaLls, See Bursitis.

Wiriers, fistulous. See Fistula. 
Worms.

Anthelmintics, 68.

Ascarides. Round Worms. Lumbricoid Worms.

Areca nut, 617.

Santonin, 623.

Creolin, 335.

Naphtol, 336.

Ether, 295.

Solution of ferric chloride, 201.

Calomel, 218, 219.

Oil of turpentine, 503.

Arsenic, 226.

Tartar cmetic, 232.

Copper sulphate, 188.

Tannic acid, 593.

Kamala, 618.

Tobacco, 426 .

Ascarides, etc. (cont.).

$\Lambda$ loes, 568.

Castor oil, 572.

Rhubarb, 576.

Jalap, 584 .

Oxyurides, Maw Worms.

Tobacco, 426.

Ether, 295.

Salt, 141.

Kamala, 618.

Quassia, 556.

Stronguli. S. micrurus and filaria. Husk Hoose. Verminous Bronchitis.

Sulphurous inhalations, 259 .

Carbolic acid, 331.

Oil of turpentine, 504.

Creosote, 333.

Syngamus trachealis, Gapes.

Oil of turpentine on a feather, 504.
Wonss (continued).

Tuenia, Tape Worm.

Aspidium, 616.

Oil of turpentine, 503 .

Areca nut, 617.

Pelletierine, 620.

Pomegranate, 620.

líousso, 619.

Kamala, 618.

Naphtol, 336.

Ether, 295.

Wounds, Granulahtions aND

ULCERS.

Ilydrogen dioxide, 120.

Lime and charcoal, 157.

Alum, 168.

Silver nitrate, 177.

Potassium permanganate, 205.

Mercuric oxides, 214.

Corrosive sublimate, 216.

Resin, 506.

Naphtalin, 336.

Chloral, 318.

C'hlorinated sorla, 240.

Chlorinated lime, 240 .

Iorloform, 251.

Iodol, $25 \%$.

Aristol, 253.

Acetanilid, 238.

Bismuth, 253.

Salol, 253, 494.

Boric acid, 253, 275.

Sulphurous acid, 260.

Nitric acid, 272.

Charcoal, $27 \%$.

Carbolic acid, 328.

Creosote, 333.

Creolin, 333.

Lysol, 335.

Formalin, 338.

Glutol, 341.

Vaseline, 350.

Salicylic acid, 494.

Tar, 509. 


\section{INDEX OF DISEASES AND REMEDIAL MEASURES}

Wounds, etc. (continued). Balsain of Peru, 511. Benzoin, 514. Myirl, 525.

Eucalyptol, 521. Zine sulphate, 185. Conium, 430.

Laudanum, $36 \%$. Oil of Turpentine, 502 . Camphor, 549.
Wounds, etc. (continued).

Thymol, 552.

Hydrastis, 563.

Tincture of aloes and myrrh, 568.

Collodion, 637 .

Glycerite of tamnin, 593 .

Cantharides, $645,646$.

Antiseptic ponltices. rit. 


\section{GENERAL INDEX.*}

\begin{tabular}{|c|c|}
\hline PAGE & $\begin{array}{l}\text { PAGE } \\
\text { PAg }\end{array}$ \\
\hline Abbreviations ........... 86 & Acid, nitromuriatic........ 264 \\
\hline Ablution ................ 706 & pliosphoric........... . 265 \\
\hline Absorbent cotton ..........636 & diluted.......... 265 \\
\hline Absorption of drugs......... 3 & salicylic ............ 490 \\
\hline Absorption through skin..... 10 & sulphuric ............ 263 \\
\hline Aca'cia.................6 611 & diluted $\ldots \ldots \ldots \ldots 263$ \\
\hline Accelerating apparatus, drugs & aromatic ........ 263 \\
\hline 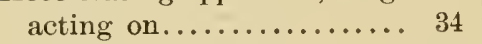 & sulpliurous............ 259 \\
\hline Ace'ta............... 78 & tartaric............. \\
\hline Acetanilid ................ 319 & Acids.................. 18 \\
\hline Acetanilidum ............ 319 & $\mathrm{~A}^{\prime}$ cidum ace'ticum......... 266 \\
\hline Acetic acid.............. 266 & dilu'tum........266 \\
\hline glacial ............. . 266 & gla'ciale.......... \\
\hline Acid, acetic.............. 266 & arseno'sum............ 220 \\
\hline diluted...........266 & benzo'icum ........... 513 \\
\hline glacial $\ldots \ldots \ldots \ldots 266$ & bo'ricum............ 273 \\
\hline arsenous..... ......... 220 & carbo'licum .......... \\
\hline solution of........ 221 & cru'dum .......... 324 \\
\hline benzoic............510-513 & liquefa'ctum...... 324 \\
\hline boracic ............. 273 & clırysopha'nicum ....... 575 \\
\hline boric ............... 273 & ci'tricum ............ $26 i$ \\
\hline carbolic................ 324 & ga'llicum ............. 593 \\
\hline crude............ 324 & liydrochlo' ricum ........ 262 \\
\hline citric .............. 267 & dilu'tum........ 262 \\
\hline syrup of $\ldots \ldots \ldots \ldots 268$ & hydrocya'nicum dilu'tum. 342 \\
\hline gallic............... 593 & la'cticum ...........268 \\
\hline hydriodic .............. 248 & méconicum ............ 352 \\
\hline syrup of $\ldots \ldots \ldots \ldots 248$ & ni'tricum ............ 264 \\
\hline hydrocyanic, diluted.... 342 & dilu'tum......... 264 \\
\hline hydrochloric.......... 262 & nitrolhydrochlo' ricum . . . . 264 \\
\hline diluted .......... 262 & dilu'tum......... 265 \\
\hline lactic.............. 268 & phosphor'icum........... 265 \\
\hline muriatic............ 262 & dilu'tum........ 265 \\
\hline nitric.............. 264 & salicy'licum ............ 490 \\
\hline diluted.......... 264 & sulphu'ricum.......... 263 \\
\hline nitrohydrochloric ....... 264 & dilu'tum......... 263 \\
\hline diluted .......... 264 & aroma'ticum ....... 263 \\
\hline
\end{tabular}

*The accented syllable in Latin words is indicated by the sign '. 778 


\begin{tabular}{|c|}
\hline PAGE \\
\hline A'cidum sulphuro'sum....... 259 \\
\hline ta'nnicum ........... 590 \\
\hline tarta'ricum $\ldots \ldots \ldots \ldots \ldots 267$ \\
\hline Aconine................ 459 \\
\hline Aconite ................ 458 \\
\hline Aconiti'na............. 459 \\
\hline ni'tras $\ldots \ldots \ldots \ldots .459$ \\
\hline
\end{tabular}

Aconitine............... 459

nitrate.............. 459

Aconi'tum................ 458

Acorin ................ 563

Acrinyl sulphocyanide....515, 516 Action of drugs-

cumulative..........4, 449

influenced by-

time given....... 13

habit............ 14

disease .......... 14

idiosyncrasy........ 14

mode of . ........... 2

on animals compared with

$\operatorname{man} . . . \ldots \ldots \ldots \ldots . . . .11$

on horses compared with

other auimals......... 11

on pigs and dogs....... 12

on ruminants.......... 12

heat contrasted with cold. 717

Actual cautery............ 701

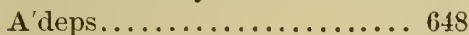

berizoina'tus ..........., 516

la'næ hydro'sus . . . . . . . 649

Adjuvant............... 89

Administration of drugs....6, 13

Adrenalin chloride......... 639

Ether................ 292

African pepper............ 525

A'l cohol.................. 278

absolute.............. 280

absolu'tum ............ 280

amylic............... 280

amy'licum .............. 280

deodora'tum............. 280

diluted................279

dilu'tum ............. 2 279

deodorized ............. 280
A'lcohol ethyl ........... PAGR.

ethy'licum............ 280

phenyl............... 324

Ale.................... 283

Alkaline earths ........... 153

metals.............. 120

Alkaloi'dea............... 70

Alkaloids ................ 70

Allyl sulphocyanide........ 516

Aloë.................... 564

barbade'nsis............ 564

cape'nsis ............ 565

socotrina............ 546

Aloin................... 565

Aloi'num ............... 565

Alterative............... 59

Althæ'a..............6 613

Alum.................. 166

ammonio ferric......... 64

burnt.................. 167

dried............. 167

potassium............ 166

Alu'men ................. 166

exsicca'tum............ 167

u'stum .............. 167

Alu'mina hydrated......... 167

Alu'mini hy'dras.......... 167

su'lphas.............. 166

Alu'minum............. 166

hydrate.............. 167

hydroxide.............. 167

sulphate............ 167

and potassium sulphate .. 166

American hellebore......... 464

Ammonia, aromatic spirit of.. 148

liniment ............ 14 .

spirit of ............. 145

aromatic......... 148

water................ 144

Ammoniac............... 539

emulsion............. 539

plaster with mercury.... 539

Ammoniacum............ 539

Ammoniated mercury....... 211

ointment ...........211 
Ammo'nii be'nzoas ......... 513

ca'rbonas .............. 147

chlo'riclum... .......... 149

valeria'nas........... 535

Ammonio ferric alum....... 64

Ammonium .............. 144

Ammo'nium acetate, sol'n of. . 1:0

Anmo'nium benzoate...... 513

carbonate............. 147

chloride............. 149

icthyol sulphonate......6 659

Amygdalin.............6 610

Amyl nitris ............. 308

Amyl nitrite.............. 308

Amylic alcohol............. 280

A'mylum ................. 609

Anæsthesia................ 299

dangers of ........... 301

practical............. 305

primary.............. 302

uses of.............. 307

Anæsthetics, general........ 40

local................ 43

Analgesics............... 319

Anaphrodisiacs............ 57

Anatomy, influencing action of drugs ............... 11

Anhidrotics ............... 66

Anise............... 530

oil................... 532

water................ 532

Ani'si fru'ctus............. 531

stella'ti fru'ctus....... 531

Ani'sum................ 530

Anodyne, Hoffman's ......... 293

Anodynes................. 39

local .............. 43

Antacids.............. 17

Anthrax vaccine.......... 751

Anthelmintics........... 67

Antidote, arsenic........... 196

Anti-emetics.............. 23

Antifebrin .............. 319

Antigalactagogues........ 59

Autimo'nii et pota'ssii tar'tras. 228
PAGE

Antimo'nii o'xidum ......... 228

su'lphidum........... 227

purifica'tum ...... 227

Antimo'nium.............. 227

sulphura'tum ......... 227

Antimony and potassium tar-

trate.............. 228

oxide................. 228

sulphide..............227

purified......... 227

Antimony, tartrated......... 228

trioxide .............. 228

trisulphide............227

wine of .............. 229

Antiparasitics............ 68

Antipyretics...........60, 319

Antipyrin........................... 320

Antipyri'num............ 323

Antiseptics... 19, 67, 319, 718, 723

Antisepsis, surgical ... . . . 726

Antisialagogues........... 15

Antispasmodics ........... 534

Antistreptococcic serum..... 745

Antitoxin, diphtheria ....... 760

tetanus.............. 742

Aphrodisiacs ............ 56

Apomorphi'næ hydrochlo'ras.. 370

Apomorphine hydrochlorate. . 370 .

Aporetin ............... 575.

$\mathrm{A}^{\prime}$ qua.................. 117

ammo'niæ.............. 144

fo'rtior........... 144

ani'si............... 532

ca'mphoræ.......... 547

chlo'ri ............... 237

chlorofo'rmi............ 296

destilla'ta............ 117

hydrogenii dioxide....... 119

me'nthæ piperi'tæ...... 529

me'nthæ vi'ridis........ 530

$A^{\prime}$ quæ................... 76

Arabin................524, 611

Arabic acid.............6611

Arachin .................6 637

Araroba powder.......... 577 


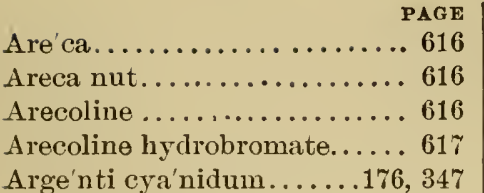

io'didum............ 176

ni'tras.............. 175

dilu'tus ......... 176

fu'sus............ 176

o'xidum............. 176

Arge'ntum............. 175

Argyrol................ 180

Aristol.................252

Aristo'lum............... 252

A'rnica ................. 522

A'rnice ra'dix ............ 522

flo' res.............. 522

root................ 522

Arnica flowers............ 522

Arnicin................ 522

Aromatics............... 16

bitters............... 16

Aromatic oils ..........71, 497 spirit of ammonia....... 148

Arseni trioxidum........... 220

Arsenic.................. 220

administration of........ 226

antidote..........196, 224

Fowler's solution of ...... 221

trioxide............... 220

white ............... 220

Arsenous acid.............. 220

solution of $\ldots \ldots \ldots 221$

A'rsenum............... 220

Artificial feeding .......... 694

Asafetida............... 537

Asafœ'tida ................ 537

Asepsis, means of procuring. . 720

surgical.............. 720

Aspi'dium.............. 615

Astringents............. 63

Atropi'na................. $3 \pi 4$

Atropi'næ su'lphas........ 375

Atropine .............. 374

Atropine sulphate ........ 375
PAGE

Bacelli's treatment......... 331

Baking soda............. 134

Balsam of Peru ........... 510

of Tolu ............. 511

of Fir ............... 505

Ba'lsama................ 72

Ba'Isamum Peruvia'num ..... 510

Toluta'num............ 511

Balls.................. 80

Barbadoes aloes............ 564

Barbaloin................ 565

Ba'rii chlo'riclum........... 159

Ba'rium ................ 160

Barium chloride .......... 160

Barosmin ................. 540

Basilicon ointment......... 506

Basis................. 89

Baths, cold... ........... r09

hot................. 717

sheet...............

Beer..................... 283

Bella'donna ............. 372

Bella'donnæe fo'lia.......... 372

ra'dix............... 373

Benzoic acid ...510,511, 512, 513

Benzoinated lard........... 512

Benzoi'num............... 512

Benzylic benzoate......... 511

cinnamate.............5 511

Berberine.............55s, 560

Beta naphtol.............. 335

Betal nut............... 616

Bhang................. 392

Bichloride of mercury ....... 209

Bile, drugs acting on....... 26

Bismuth ................. 189

salicylate ............. 190

subcarbonate .......... 189

subgallate........... 190

subnitrate............ 190

Bismu'tli salicy'las .... . . . 190

subca'rbonas........... 189

subga'llas............. 190

subni'tras............. 190 .

Bismu'tlum............ 189 
Bitters................ 171

Black antimony, purified..... 227 mustard............ 515 wash .............. 217

Black-leg vaccine .......... 751

Bleaching powder......... . 237

Bleeding. . . . . . . . . . . . . 729

local ................ 732

Blister beetles ............ 639

Blistering collodion.........6.638

Blood, drugs acting on ...... 32 vessels, drugs acting on .. 36

Blue mass. . . . . . . . . . . . . 207 pill ................. 207 stone............... 187 vitriol.............. 187

Bodily heat, drugs influencing. 60 Bo'li.................... 80

Boluses ................. 80

Bone black...............276

Boracic acid ............. 273

Borax................ 273

Boric acid ............. 273

Boroglyceride, solution of.... 273

Boroglycerin, glycerite of .... 273

Brain, drugs acting on....... 38

Brandy .................281

Bray'era.................619

Bromides................2 240

Bromine.................240

Bro'mum.................. 240

Bronchial tubes, drugs influencing the ............. 49

Bronchial spasm .......... 50

Brucine................ 401

Bucco .................. 540

Buchu................. 540

Buchu-camphor ........... 540

Buckthorn.........572, 573, 574

Burgundy pitch...........504 plaster.............. 505

Burnt alum.............. 167

Butter of cacao...........636

Cacao butter. 636
PAGE

Cade, oil of ............. 508.

Caffei na............... 395

Caffei'na citra'ta........... 396

Caffeine ................. 395

citrated.............. 396

Calabar bean. ............. 410

Calabarine............... 411

Calamine............ 185, 563

Ca'lamus................ 563

Ca'lcii ca'rbonas praecipi'ta'tus 154 pho'sphas praecipita'tus. . 157

Calcined magnesia . . . . . . . . 164

Ca'lcium................ 153 carbonate precipitated.... 154 chloride............. 159 hydrate, solution of..... 155 lactophosphate, syrup of . 158 phosphate precipitated ... 157

Calisaya bark............ 476

Calomel................210

Calu'mba ................ 557

Calumbic acid............ 558

Calumbin................ 558

Calx.................. 155

chlora'ta ............ 237

Cambo'gia................ 585

Camphor................ 547

Ca'mphora............... 547

Camphorated oil........... 548

Camphor gum............ 547

laurel............... 547

monobromated......... 548

Canadine ............... 560

Cannabin .............. 392

Cannabinine ............ 392

Cannabinon............... 392

Ca'nnabis i'ndica........... . 391

Ca'nnabis I'ndian ... . . . . . . . 391

Cantharides.............. 643

Cantharidin..............643

Ca'ntharis.............. 643

Cape aloes... . . . . . . . . . . . . 565

Capronic acid............ 523

Caprylic acid ............ 522

Capsaicin............... 526 
Ca'psici fru'ctus ........ 525

Capsicin................. 526

Ca'psicum.............. 525

Ca'rbo.................... 276

anima'lis.............. $2 \approx 6$

purifica'tus ....... 276

li'gni .............. 276

Carbolic acid............. 324

Carbon.................. 2i6

disulphide............. $2 \pi 8$

Carbo'nei bisu'lphidum...... 278

disu'Iphidum........... 278

Cardamom .............. 532

Cardamo'mum............ 532

Carminatives .............. 19

Carron oil................. 156

Casca'ra sagra'da .......... 572

Cascari'lla............... 556

Cascarillin................ $55 \pi$

Castile soap..............603

Castor oil............... 571

seeds................ 571

Cataplasms.............. 713

Catechin............... 596

Ca'techu................ 595

red ................... 596

tannic acid. ........... 596

Catechuic acid............. 596

Cathartic acid ............ 578 pills, compound........ 210

Cathartics................ 24

Cathartomannit. ........... 578

Caucasian insect powder..... 624

Caustic, lunar............. 176

mitigated............. 176

potash............... 122

soda .................. 132

Caustics..............63, 703

Cayenne pepper.......... 525

Ce'ra a'lba..............6.650

fla'va ...............6.649

Cera'ta...............79, 648

Cerates................79, 648

Cera'tum ...............6 648

Cera'tum ca'mphoræ
PAGE

Cera'tum ceta' cei ......... 651

resi'næ............... 506

Cerebral depressants......... 38

excitants............. 38

Ce'rii o'xalas.............. 170

Ce'rium.................. 17

Ce'rium oxalate............ 170

Cerous oxalate ............ 170

Ceta'ceum...............6.650

Cetin.................. 651

Cetylic alcohol...........651

Ceradine............... 46t

Chalk, drop............... 153

mixture............ 1.53

powder, compound...... 153

prepared.............. 153

troches.............. 153

Champagne.............. 283

Charcoal, animal.......... $2 \pi 6$

purified.............. 2i6

Chareoal, wood ........... $2 \pi 6$

Charges............... 79

Cha'rtæ................ 79

Cherry, wild .............. 348

Chinoidine.............. 482

Chittem bark............. 5\%2

Chloral .................... 314

hydrate............... 314

Chloralum hydratum.... ... 314

Chloride of lime........... 159

Chlorinated lime.......... 237

solution of........238

soda, solution of ........ 238

Chlorine.................23\%

water ............... 237

Chloroform ............. 295

liniment.............. 296

spirit ............... 296

water............... 296

Chlorofo'rmum ........... 295

purifica'tum. ........... 295

Chlo'rum ............... 237

Cholagogues.............. 26

Choline................392, 563

Cholesterin .............602 


\begin{tabular}{|c|c|}
\hline & \\
\hline Chrysophan. .........555,577 & Cold ene'mata.......... \\
\hline Chrysophanic acid....575, 5r7, 578 & Collargol .......... \\
\hline Chrysarobin ............. 5i7 & Colloidal silver ... . . . . . . 180 \\
\hline Chrysarobi num.......... 577 & Collidine................ 428 \\
\hline Churrus................. 392 & Collo'dia............... \\
\hline Cidler .................. 283 & Collodion............... 637 \\
\hline Cinclio'na ................ 476 & blistering $\ldots \ldots \ldots \ldots \ldots 637$ \\
\hline Cinchona red ............ $4 \pi 9$ & cantharidal... ........6 637 \\
\hline Cincho'na ru'bra.......... 479 & flexible............. 638 \\
\hline Cincho'næe co'rtex.......... 476 & styptic...........591, 638 \\
\hline Cinchonidine............. 48:3 & Collodions............79, 637 \\
\hline Cinchonidi'næ su'lphas... . . . 48:3 & Collo'dium cantharida'tum... 637 \\
\hline Cinchonidi'na............ 483 & flexi'le.............. 638 \\
\hline Cinchonidine ............ 447 & sty'pticum..........591, 637 \\
\hline sulphate............ 483 & Colloxylini.............. 687 \\
\hline Cinchoninæe sulphas........ 483 & Colly'ria.............. 86 \\
\hline Cinchonine............. 477 & Colocynth ............... 586 \\
\hline sulphate.............. 483 & Colocynthin ............ 581 \\
\hline Cinchonism............. 486 & Colocy'nthis............... 586 \\
\hline Cinchotamnic acid.......... 478 & Colocynthitin ........... 586 \\
\hline Cineol.................... 519 & Colophony ............... 506 \\
\hline Cinnamein............. 510 & Columbo............... 557 \\
\hline amic acid.........510, 511 & Common salt............. 13 \\
\hline Circulation, drugs acting on.. 32 & Compound cathartic pills..... 210 \\
\hline Citrated caffeine .......... 397 & liquorice powder....... 579 \\
\hline Citric acid............ 267 & powder, catechu........ 596 \\
\hline Citrine ointment...........211 & powder, kino........... 598 \\
\hline Citralin................. 586 & solution of iodine. ...... 244 \\
\hline Claret................. 282 & syrup of squill.......... 229 \\
\hline Classification............ 113 & Conféctiones ............ 79 \\
\hline Clysters.................9, 30 & Confections.............. 79 \\
\hline Co'ca................ 431 & Conhydrine.............. 427 \\
\hline Cocai'næ hydrochlo'ras....... 431 & Conine................ 427 \\
\hline Cocaine................. 431 & Coni' næ hydrobro'mas....... 427 \\
\hline Codei'na.................. 350 & Conium................ 427 \\
\hline Cod liver oil.............. 656 & Convallamarin............ 456 \\
\hline Coffee............396, 397, 400 & Convalla'ria.............. 455 \\
\hline Colchiceine ............. 632 & Convallarin............. 456 \\
\hline Colchicine............... 632 & Convolvulin .............. 584 \\
\hline Co'lchicum ............... 631 & Copper.................. \\
\hline root................6 631 & acetate................ \\
\hline seed................ 632 & sulphate............ 186 \\
\hline Cold as a remedy.......... r04 & Copperas... .... ....... 193 \\
\hline baths. .............. r09 & Corjander............... 533 \\
\hline drinks.............. 710 & Coria'ndri fructus. ......... 533 \\
\hline
\end{tabular}


Cornutine...............625

Corrosive mercuric chloride . . 208

Corrosire sublimate........ 208

Cosmoline................. 348

Cotton...................6837

absorbent............637

purified ..............6.637

root bark ............6630

seed oil .............. 602

soluble gun cotton....... 638

Counter-irritants.....62, 696, 715

Cox's hive syrup........... 457

Cream of tartar............ 131

Credés ointment............ 181

Creolin .................. 333

Creoli'num............... 333

Creosote.................... 333

Creoso'tum............... 333

Cre'ta præpara'ta.......... 154

Croton oil................. 580

seeds................ 580

Crotonol................ 580

Crude carbolic acid.......... 324

Cryptopin................. 352

Cu'ca...................4 431

Cumulative action.......... 4

Cupri acetas............... 188

Cu'pri su'lphas............. 186

Cupric sulphate............. 186

Cu'prum................ 186

Cusso................... 619

Cutch................... 595

Cymene................. 519

Dalmatian insect powder.... 624

Deadly nightshade ......... 3\%2

Deco'cta................ 76

Decoctions............... $\quad r 6$

Definitions.............. 1

of drugs .............. 2

Delphinine..............6 623

Delphinoidine ............. 624

Delphinisine.............. 624

Demulcents.............. 64
Deodorants........67, 724, 718

Deodorized alcohol......... 280

opium................ 354

Deodorizers.............. 67

Dermatol.................. 190

Diachylon plaster.......... 1r1

Diaphoretics............. 6.5

Digestion .............. 75

Digestive organs, drugs acting on.................... 15

Digestives............... 19

Digitalein.............. 445

Digitalin.............445, 416

Digita'lis............... 445

fo'lia................ 445

Digitonin............... 445

Digitoxin............... 445

Diosphenol............... 540

Diphtheritic antitoxin ...... 760

Disease, affecting action of

drugs................. 14

Disinfectants.......67, 718, 719

Disinfection, practical ...... 725

Displacement or percolation. . $\%$

Dissolution, law of,

$11,39,287,299,362$

Distilled oils............71, 497

water................. 11,

Diuretics............... ह

Domestic measures......... 92

Dosage................ 1n

Doses..................... 10

Dose table............... 661

Double muriate of quinine and

urea .................448

Douches................ 709

Dover's powder ............ 353

Drastics.................. 24

Draught............... 81

Drench .................. 81

Dried alum.............. 167

sodium carbonate....... 133

Drop chalk ............. 153

Drugs acting on the-

budily heat........... 
Drugs acting on the- PAGE

blood................ 32

vessels ........... 36

brain................. 38

circulation............ 32

digestive organs........ 15

ears................ 46

eуе................ 44

heart.............. 33

metabolism ........... 59

nerves............... 43

special sense ..... 44

nervous system ........ 38

respiratory organs....... 46

sexual organs......... 56

skin ............... 62

spinal cord........... 42

urinary organs......... 52

Drugs, absorption of ....... 3

classification of ......... 113

cumulative action of..... 4

definition of............ 2

destroying parasites.. $\ldots 6^{7}$

doses of .............661

elinination of $\ldots \ldots \ldots \ldots, 4$

excretion of ............ 5

general action of $\ldots . . .2 \quad 2$

influencing the composition of urine ......... 55

reaction of urine... 54

secretion of sweat. . 65

local action of ......... 2

mode of action of....... 2

mode of administration .. 6

primary action of....... 2

secondary action of ...... 2

selective action of. ..... 2

Dry heat ............... 716

Ears, drugs acting on ...... 46

Ecbolics................. 58

Ecboline................. 625

Egg, yolk of ............667

Elaterin ................ 586

Elateri'num............. 586
PAGE

Electua'ria............. 82

Electnaries ............... 82

Elimination............. 4

Elixirs................ 77

Eli'xir proprieta'tis parace'lsi

500,540

Elutriation.............. 73

Emetics................. 29

Emetine................ 472

Emmenagogues .......... 57

Emodin.............573, 575

Emollients.............. 61

Empirical therapeutics...... 1

Empla'stra ............... 79

Empla'strum............ 79

ammoni'aci cum hydra'r-

gyro............... 539

pi'cis Burgu'ndicæ...... 505

resi'næ.............. 506

sapo'nis .............6 604

Emu'lsa ............... 77

Emulsions.............. 77

Emu'lsum ammoni'aci....... 539

asafœe'tidæ............ 538

Enemas .................9, 30

Ene'mata...............9, 30

Enteroclysis ...........733, 736

Epispastics. .............6 697

Epsom salt.............. 163

Ergot ................6. 625

Ergot of rye............. 625

Ergo'ta................ 625

Ergotic acid.............6 625

Ergotin................626

Ergotine...............6. 625

Ergotinic acid............ 625

Ergotinine..............625

Ergoti'num .............. 626

Errhines................ 46

Erythroretin........... 575

Erythro'xylon............. 431

Escharotics............63, 697

Eseridine................ 411

Eserine..$\ldots \ldots \ldots \ldots \ldots \ldots .411$

salicylate...........4 411 
Eserine sulphate.......... 412

Essences................ 71

Esse'ntiæ .............. i1 $^{2}$

Essential oils........... 1,497

Ether................. 292

nitrous, spirit of ....... 308

spirit of ............. 292

spirit of, compound ...... 293

sulphuric ............ 292

Ethereal oils...........71, 49 \%

Ethyl alcohol............ 280

Ethyl nitrite. ........... 308

Ethyl oxide............. 292

Eucain hydrochlorate....... 428

Eucalyptene ............. 519

Eucalyptol ............. 519

Euca'lyptus............. 518

Eupho'rbium.............6638

Euphorbon..............6638

Evaporating solutions....... 711

Excipient............... 75

Excretion............... 5

Expectorants............ 49

depressing........... 49

stimulating........... 49

Extra'cta.............. 78

flu'ida................ is

Extracts............... 78

Extra'ctum aco'niti........ 459

a'rnicæ ra'dicis......... 523

bella'donnæ folio'rum alcoho'licum........... 373

ca'nnabis i'ndicæ....... 392

cincho'næ............478

coni'i ..............4 428

digita'lis..............446

ergo'tr.............66 626

gentia'næ........... 553

hæmato'xyli .........6 600

hyosc' yami ............ 388

jaborandi............ 440

krame'riæ ............ 599

nu'cis vo'micæ........ 400

o'pii............................. 353

opii liquidum......... 354
Extra'ctum qua'ssiæ....... 555

rhe'i................ 576

tara'xici............. 559

casca'ræ sagra dæ li'qui-

(lum ...............573

ergo'tæ li'quidum.......626

fi'licis li'quiclum........615

fra'ngulæ li' quidum...... 5\%4

glycyrrhi'zæ li'quidum... 609

hamame'lidis li'quidum... 601

o' pii li'quidtum..... . . . . 354

Eye, drugs acting on....... 44

lotions............. 86

Fats................ 79

Feeding, artificial ......... 694 rectal..............6.694

Fel bo'ris............... 655

purifica'tum......6.655

tau'ri..............6.695

Felix mas.............. 615

Fennel................ 533

Fern, male............ 615

Fer'ri ca'rbonas sacchara'tus. . 194 chlo'ridum............ 194

et ammo'nii cit'ras..... 197

et pota'ssii ta'rtras...... 196

et quini'næ ci'tras........ 197

solu'bilis......... 198

o'xidum hydra'tum cum

magne'sia ........196, 224

su'lphas............. 193

exsicca'tus........ 193

granula'tus ....... 194

valeria'nas........... 536

Ferric chloride.......... 19.

solution of ....... 195

tincture of ....... 195

hydrate with magnesia... 196

subsulphate, solution of.. 195

valerianate.......... 536

Ferrous carbonate, mass of ... 194

saccharated....... 194

iodide, syrup of ....... 194

sulphate.............. 193 
PAGE

Ferrous sulphate, dried...... 193

granulated ............ 193

Fe'rrum................ 192

redu'ctum........... 192

Ferulaic acid............. 538

Filicin................6 615

Fi'liz mas...............615

Filicic acid..............661j

Fixed oils..............

Flax seed...............6 610

oil of ............. 569

Fleming's tincture ......... 460

Flexible collodion..........638

Flies, Spanish............ 643

Flowers of sulphur......... 255

Fluid extracts............ 78

Fluidextractum aco'niti..... 459

bella'donnæ ra'dicis..... 373

bu'chu ............. 540

ca'lami .............. 563

calu'mbæ............ 558

ca'nnabis índica........ 392

ca'psici ............. 526

cincho'næ.............478

co'lchici ra'dicis se'minis . 632

coni'i ............... 428

convalla'riæ........... 456

cu'sso.................6 619

digita'lis . . ........... 446

ergo'tæ............. 626

eucaly'pti ............. 519

fra'ngula............ 514

gelse'mii............ 4:0

gentia'næ............ 553

glycyrrhi'zæ. .......... 609

gossy'pii ra'dicis ........630

hamame'lidis ........... 601

hydra'stis............ 560

hyoscy'ami ........... 388

ipecacua'nhæe ......... 472

krame'ria ............ 599

nu'cis vo'micre. . . . . . . 401

piloca'rpi. ........... 440

qua'ssia. ............. 5.55

rha'mni purshia'næ..... 573
PAGE

Fluidextractum rhe'i........ 575

sabi'næ............. 543

sci'llæ..............457

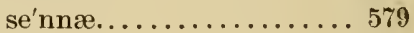

tara'xici ............. 559

valeria'næ ........... 535

vera'tri vi'ridis......... 465

zingibe'ris........... 527

Fœni'culi fru'ctus.......... 533

Foeni'culum .............. 533

Fo'nugreek............... 534

Fome'nta............... 715

Fomentations............ 715

Food and feeding ......... 679

artificial............ 694

in anæmia. . . . . . . . 690

in black water ......... 690

in clironic indigestion....6 688

in constipation......... 688

in convalesence......... 690

in debility............. 690

in diarrhoea . . . . . . . 688, 689

in fever............... 691

in gastroenteritis... . . 688, 689

in hæmorrhage from sto-

mach and bowels...... 689

in jaundice.......... 689

in laminitis . . . . . . . . . 688

in obesity............ 691

Formaldehyde............ 338

Formalin ................. 338

Formic acid .......... 535, 651

Formic aldeliyde ........... 338

Fox glove............... 445

Fowler's solution........... 221

Fra'ngula ............... 573

Frangulin.............. 575

Frial's balsam............. 512

Fusel oil............... 280

Gaduin..................657

Galactagogues ............ 58

Galls.................... . 589

Ga'lla.................. 589

Gallic acid.............. 593 


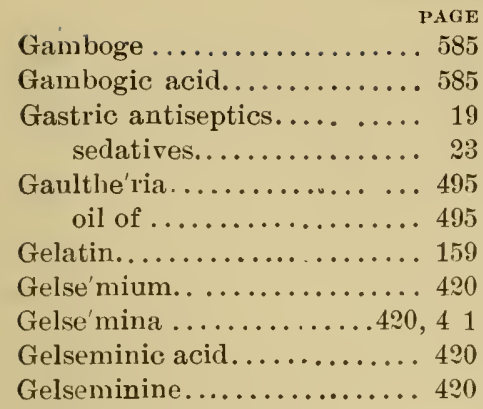

General action of drugs...... 2 therapeutics ........... 1

therapeutic measures.... 679

Gentian................ 552

Gentia'na............... 552

Gentisic acid............. 553

Gentiopicrin ............ 553

Germicides...........67, 718

Gin.................. 281

Ginger............... 527

Gingerol. .............. 527

Glacial acetic acid......... 266

Glauber's salt............. 136

Glonoin................. 309

spirit of . . . . . . . . 309

Glonoi'num .............. 309

Glucosi'dea.............. 70

Glucosides.............. $\tau_{0}$

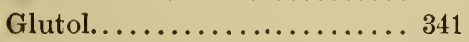

Glycerin ..............6606

suppositories of ........607

Glyceri'num .............6606

Glyceri'ta.............79, 607

Glycerites...........67, 607

Glyceri'tum a'cidi carbo'lici,

325,607

a'cidi ta'nnici.......591, 607

a'myli............636, 607

boroglyceri'ni......... 273

hydra'stis........... 561

vite'lli..............667 607

Glyceryl borate, glycerite of. . 273

trinitrate ............. 309

Glyconin..............6 607
PAGE

Glyryramin ............. 609

Glycyrrhetin............. 609

Glycyrrhiza.............6 603

Glycyrrhizin.............6 609

Goa powder.............. 5i氵

Golden seal............ 501

Golden sulphur........... 2:8

Gossy'pii ra'dicis co'rtex..... 690

Gossy'pium purifica'tum..... $6: 36$

Goulard's extract .......... 1;1

Grana'tum..............6 6:0

Granulated ferrous sulphate. . 194

Gray powder............. 20\%

Gregory's powder ......... $5 i 6$

Green hellebore........... 464

Green soap............... 604

Green vitriol. . . . . . . . . . . . 193

Guara'na................ 396

Guaranine............... 395

Gum arabic............. 611

Gum-resinæ............ $\tau_{2}$

Gum-resins ............. \%

Gu'mmi................ \%

Gun cotton, soluble.......667

Gunjah ................ 392

Gu'ttx............... 91

Habit, influencing action of drugs................ 14

Hæmatein .............. 600

Hrematinics. ............. 33

Hæmatoxylin............6 600

Hænatoxylon............6600

Hrmostatics............. 63

Hamame'lis.............. 601

Hard soap .............. 603

Hard petrola'tum .......... 349

Harrington's solution ....... 216

Haschisch................ 391

Hartshorn ............... 144

Hav'stus.............. 81

Heart, drugs acting on.....33, 415

Heat............... 12,704

Heat, bodily, drugs acting on . 60

Heavy magnesia. ........... 165 
Hellebore, American PAGE

Hemide'smus ............. 472

Hemlock ................ 464

Hemp, Indian. . . . . . . . . . . 391

Henbane................. 387

Hepatic stimulants......... 26

Heroin .................. 357

Heroin hydrochloride ....... 357

Hircin .................. 648

Hive syrup. . . ........... 229

Hock. .................... 282

Hoffman's anodyne........... 293

Holocain ................. 439

Homolle's digitalin......... 446

Honey.................. 651

clarified.............. 651

Honeys ................. 80

Hot bath................ 717

water bags............ 715

Hydragogues.............. 25

Hydra'rgyri chlo'ridum corro-

si'vum ................. 208

chlo'ridum mi'te........ 209

io'didum r'u'brum. . . . . . 209

o'xidum fla'vum........ 207

o'xidum ru'brum........ 207

subchlori'dum........... 209

Hydra'rgyrum............. 206

ammonia'tum.......... 210

cu'm cre'ta............206

Hydrastin ................. 561

Hydrastine ............ 560

Hydrasti'næ hydrochlo'ras.... 561

Hydra'stis .............. 560

Hydrated alu'nina......... 16i

Hydriodic acid, syrup of. .... 248

Hydrochloric acid.......... 262

solution of arsenic........221

Hydrocyanic acid, diluted.... 342

Hydrogen dioxide, solution of. 119 peroxide, solution of . . . 119

Hydrous wool fat ..........649

Hydroxide, potassiun....... 122 sodium.............. 132

Hyoscine ............... 389
PAGE

Hyosci'næ hydrobro'mas...... 389

Hyoscine hydrobromate...... 389

Hyoscyamine..........372, 388

Hyoscyamine hydrobromate. . 389

sulphate............... 388

Hyoscy'ami fo'lia........... 387

Hyoscy'amus .............. 387

Hypnotics............... 40

Hypodermatic injections...... 6

Hypodermoclysis.........733, 735

Hypophosphites.......... . 217

Ice bags ............... 706

Ice poultices.............. 706

Icthyol ............... 659

Icthy'olum . . . . . . . . . . . 659

Idiosyncrasy in action of drugs 14

Igasuric acid............ 400

Illi'cium............... 531

Incompatibility, clemical.... 83

physical............. 85

physiological........... 85

Index for diseases and reme-

dial measures ............. 753

Indian cannabis............ 391

Indian hemp . . . . . . . . . . . 391

Ineine..................4. 453

Infu'sa............... $r 6$

Infusions .............. 76

saline............... 732

Infu'sum ca'lami.......... 564

cascari'lla............. 557

cincho'næ........... 479

acidum .........4 479

digita'lis ............. 446

Inhalations..........6, $4 \pi, 716$

Inje'ctio apomorphi'næ hypo-

de'rmica .............. 3r

ergoti'ni hypode'rmica... 626

morphi'næ hypode'rmica.. 356

In jections, intratracheal..... \& 8

intravenous.........6, 733

rectal.............. 9

subcutaneous.......... 6

Intestinal antiseptics........ $20^{\circ}$ 
Intrar nous injection.......6, 73

In ${ }^{2}$ ratracheal injection........ 8

Inunctions.............. 6

Inu'in............... 559

Iodine. . . . . . . . . . . . . . . . 243

compound solution of..... 244

ointment............. 244

tincture of $\ldots \ldots \ldots \ldots \ldots .244$

Io'dinm.................. 241

Iodoform . . . . . . . . . . . . . . . 250

Iodofo jnum. . . . . . . . . . . . 250

Iodol. . . . . . . . . . . . . . . . . . 252

Iodo'lim. . . . . . . . . . . . 25 2

Ipecac. . . . ............ 4 4;

Ipecarma nha. . . . . . . . . . 4\%1

Ipecacuanhic acid..........4 4\%

Iron. . . . . . . . . . . . . . . 192

and ammonium citrate... 197 and potassium tartrate... 196 and quinine citrate...... $19 \%$

soluble........... 198

by hydrogen.......... 193

metallic............ 192

Querenne's.............192

roduced .............. 192

Irrigation ..............

I:ritants................ 62

Isupellctierine. . . . . . . . . . 6 620

Jabor:ndi . . . . . . . . . . . . . 439

Pernambuco........... 439

Rio Janeiro. . . . . . . . . . . 439

Jaborandine . . . . . . . . . . 440

Jalorine . . . . . . . . . . . . . . 439

Jalap.................... 583

Jala'pa. . . . . . . . . . . . . 58:3

Jalapin................ 583

Jalapurgin. . . . . . . . . . . . 583

Jasmine, yellow... . . . . . . . . 420

Jervine. . . . . . . . .4464, 465, 468

Kama'la.................618

Kermes mineral. .......... 227

Iinic a

5ino................. 597
Kinoin . . . . . . . . . . . . . . 598

Kinovic acid............. 478

Kinovin................448

Kino-tannic acid .......478, 597

Ko'la nut. . . . . . . . . . . . . 396

Kombé poison ............. . 453

Kombic acid............. 4.53

Kooso...................619

Kosin..................619

Koussin. . . .............. 619

Kousso...................9919

Krame'ria................ 598

Kramero-tannic acid........ 599

Kunsel's treatment, milk fever 737

Labarraque's solution ........238

Lac sulphurus. . . . . . . . . . . 256

Lactose ................ 65\%

Lanolin. . . . . . . . . . . . . . 649

Lard. . . . . . . ............. 648

benzoinated........... 648

Latin words and phrases...... 86

Laudanum. . . . . . . . . . . . 3 35

Laurel camphor........... 547

Laurin................6.636

Lavage ................. 739

Law of dissolution,

$11,39,286,299,362$

Laxatives................. 2t

Lead ................. 171

acetate............... 171

carbonate............ 1\%

iodide ............. 1\%

ointment............ 17\%

nitrate............. 1\%2

oxide ............... 1\%

plaster. .............. 171

poisoning ............ 1\%:3

subacetate, cerate of..... 1\%3

solution of ............ 1;1

sugar of............. 171

white.............. 17\%

water............... 171

Levant worm seed..........6 621 
Levigation.............. 73

Light magnesia . . . . . . . . . 164

Lily of valley............. 455

Lime................... 155

chlorinated............23i

chloride of ........... 159

liniment............ 156

phosphate, precipitated... 157

solution of............ 15i

syrup of . . . . . . . . 156

water............... 15.5

Linime'nta............. 79

Liniments.............. $\tau_{9}$

Linime'ntum ammo'niæ..... 137

bella'donnæ............ 3 st $^{4}$

Linime'ntum ca'lcis........ 156

ca'mphoræ............ 548

chlorofo'rmi........... 296

sapo'nis ..............6 604

mo'llis..........6 604

terebinthi'næ........... 499

Linseed................... 610

oil of.............. 569

Linolein ................ 569

Li'num..................610

Liquid petrolatum.......... 348

Li'quor a'cidi arseno'si....... 221

ammo'niæ............ 144

ammo'nii aceta'tis....... 150

ammo'niæ fo'rtior....... 144

arsenica'lis............ 206

arse'nici hydrochlo'ricus.. 221

ca'leis............... 155

chlora'tre.............238

fe'rri chlo'ridi.......... 195

perchlo'ridi........ 195

subsulpha'tis....... 196

hydroge'nii dio'xidi...... 119

io'di compo'situs. ....... 244

morphi'næ aceta'tis...... 356

morphi'næe hydrochlora'tis 356

plu'mbi subaceta'tis..... 171

dilu'tus......... 171

pota'ssæ............. 122

pota'ssi arseni'tis .......221
P.17F

L'iquor so'dx........... 182

so'dæ chlorina'tæ....... 2.

zi'nci chlo' ridi.......... 18.

Liquo'res.............. $\%$

Liquorice root............6 608

Liquorice powder, compound. 2.56

Litharge................ 170

Li'thii ca'rbonas. . . . . . . . . 1, 151

citras............... 15?

sali'cylas............. 112

Lithium............... 1,51

Liver, drugs acting on...... 23

Liver of sulphur........... 260

Lixiviation.............

Local action. ............ 2

anodyne............ 43

anæsthetics........... 43

blood letting.............

Logwood ..............600

Lotio flava............... 216

nigra .............. 217

Lugol's solution............ 244

Lunar caustic..........176, 1:3

Lysol . . . . . . . . . . . . . . 35

Lyso'lum............... 325

Maceration.............. $r_{5}$

Magne'sia............... 16t

calcined............... 104

heavy............... 164

light .............. 164

milk of, Phillip's......... 165

pondero'sa............. 164

Magne'sii ca'rbonas......... 164

su'lphas ............. 1C3

Magne'sium............... 163

carbonate............. 104

sulphate............... 163

Male fern ................ 615

Mallein ............... 749

Malic acid............. 5e.

Manganese .............. 203

Manga'num.............. 203

Marshmallow ............... C ${ }_{3}$

Mass, blue............. 203 
Mass of ferrous carbonate . . . 194

Ma'ssa fe'rri carbona'tis . . . . . 194 hydra'rgyri ...........206

Ma'ssæ.................. 79

Masses..................

Maté................... 396

Ma'teria me'dica............ 1

May apple................5 58 \%

Meadow saffron ............6 631

Measures, weights, tables of $90,91,92,93,94$

domestic.............. 92

Meconic acid............. 352

Meconin................ 352

Medicines, definition of...... 1

Mel...................6 651 despuma'tum..........651

Me'llita.................. 80

Menstruum ............... 75

Mentha piperi'ta .......... 528 vi'ridis. ............. 530

Menthene................528

Menthol..............528, 529

Mercurial ointment.........207

Mercuric ammonium chloride. 210 corrosive chloride....... 208 iodide, red............. 209 nitrate, ointment of..... 210 oxide, red............ $20 \%$ ointment of ......2 207 yellow...............207 ointment of.....2208

Mercurous chloride, mild..... 209

Mercury................. 206

ammoniated...........210 ointment.........207

bichloride............ 208

biniodide............. 209

chloride, corrosive......208 mild............ 209

iodide, red............ 209

mass of .............. 206

subchloride............ 209

with chalk............ 206

Metabolism, drugs influencing 59
Metacreosotic acid.......490,492

Methyl coniine...........4 427

pelletierine...........6 620

salḯcylas. ............ 496

salicylate............. 496

Metric system.............92-98

Milk, drugs influencing secre-

tion of............ 50

drugs eliminated in ..... 59

of asafetida........... 538

of sulphur............ 255

peptonized............694

sugar of. ............652:

Milk fever, Kunsel's treatment 737

Mindererus, spirit of. ...... 150

Mistu'ra cre'tæ............ 154

Mistu'ra................

Mixtures................ $\%$

Mitigated caustic........... 176

Molasses .................614

Monobromated camphor...... 548

Monsel's solution............ 196

Morphi'na...............351, 354

Morphinæ ace'tæ........... 355

hydrochlo'ras .......... . 355.

Morphi'næe su'lphas.......... 354:

Morphine ..............351, 354 acetate............... 354

hydrochlorate ........ 355 sulphate ............. 354

Morrhuol............... 657

Motor nerves, drugs acting on 43

Moulded silver nitrate....... 176

Mucilage of acacia ........612

Mucila'go aca'ciæ......... 612

tragaca'nthæ..........612

Muscarine.............. . . . 382

Muriatic acid..............262 diluted............... 262

Mustard................. 515

black............. 515

white.............. 51

Mutton suet. .............. 648

Mydriatics.............. 45

Myristin................ 569 


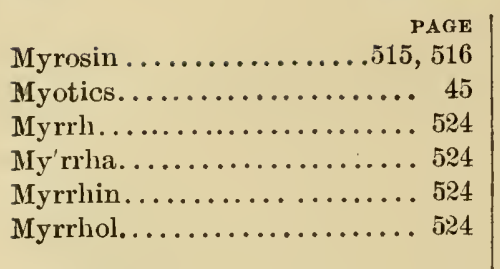

Naphtalene .............. 336

Naphtalin................ 336

Naphtali'num............. 336

Naphtol. . . . ............. 335

Narceine................ 351

Narcotics............... 40

Narcotine............... 351

Nataloin.............. 566

Nativelle's digitalin........ 445

Nerves, drugs acting on ...... 43

Nervous system, drugs acting on. ..................

Nerves of special sense, drugs acting on .......... 44

Nicotine.............423, 424

Nightshade, deadly ....... 372

Nitric acid. ............. 264 dilute................264

Nitrites................ 308

Nitre ................ 127 sweet spirit of......... 308

Nitroglycerin ............. 309 spirit of . . . . . . . . . . 309

Nitrohydrochloric acid...... 264 diluted ..............265

Nitrous ether, spirit of. ..... 308

Nutgall................ 580 ointment ............ 590

Nux vomica............. 490

Oak, white............. 595

Oakum................6 637

Official preparations......... 76

Oil of anise $\ldots \ldots \ldots \ldots \ldots . .531,532$

box berry............ 495

cade................. 508

carron.............. 156

castor .............. 5 5 1

checkerberry .........495
PAGE

Oil, cod liver............. 656

coriander ............ 533

cotton seed............6.602

croton .............. 580

ethereal ............. 71

eucalyptus. ........... 519

fennel.............. 534

flax seed. ............ 569

fusel................280

gaultheria............ 495

artificial ........4496

synthetic........ 496

juniper............. 541

linseed ............... 564

mustard, volatile........ 516

olive............... 602

peppermint...........5 528

phosphorated .......... 234

savine ............. 543

sweet.............6602

spearmint ............ 530

$\operatorname{tar} \ldots \ldots \ldots \ldots \ldots \ldots . \ldots 507$

theobroma............6 636

turpentine............ 498

Oil of turpentine, rectified.... 499

wintergreen.......... 495

artificial.........4 496

synthetic ....... 496

Oils...................71, 79

distilled ............ 71

essential............. 71

ethereal.............. 71

fixed.............. 71

volatile .............. $\tau_{1}$

O'lea.................. 71, 79

Olea'ta...............

Oleate of mercury.......... 209

veratrine............. 469

Oleates............... 79

Olein........602, 603, 637, 648

Oleoresina Fe'lix Mas....... 615

O'leoresi'næ........... 71,79

Oleoresins.............. 11,78

Oleum ani'si.........531, 532

ca'dinum............. 508 
Oleum capsici........... 526

coria'ndri............ 533

eucaly'pti. ........... 519

fœni' culi............. 534

fœnugreek............ 534

gaulthe'riæ........... 495

gossy'pii se'minis....... 602

juni'peri ............. 541

li'ni................ 569

me'nthæ piperi'tæ...... 528

vi'ridis.......... 530

mo'rrhuæ...........6 656

oli'væ.............6.602

phosphora'tum......... 234

pi'cis li'quidæ.........5 507

ri'cini.............. $55^{21}$

sabi'næ........... 543

si'napis rolati'le....... 516

terebi'nthinæe........ 498

rectifica'tum...... 499

theobro'matis..........6 636

ti'glii............... 580

Olive oil ............... 602

O'pii pu'lvis............... 352

O'pium ................... 351

denarcotizatum......... 354

deodoratum............ 354

Ordeal bean.............4 410

Organs, sexual, drugs acting on 56

Orthocreosotic acid......490,492

Orthoform............. 253

Orthoformum............253

Ox gall ..................655

purified.............6.655

Oxytocics............. 58

Pack, cold................6 67

Palmitin........6602, 603, 648

Pancreatin..............6.654

Pancreati'num...........6.654

Papain ................6.656

Papaverine................ 351

Papers.................

Papoid................656

Parasiticides............. 68
Paregoric PAGE

Pelletierine..................... 620

Pepper, cayenne.......... 525

red .................. 525

Peppermint............. 530

water............... 529

Pepsin................ 652

saccharated..........6.653

sacchara'tum..........6 653

Peptonized milk............ 694

Percolation .............. 73

Permanganate of potassi ..... 203

Persian insect powder....... 624

Peruvian balsam.......... 510

Petrola'tum............... 348

hard................ 349

liquid ............... 348

li'quidum............. 348

mo'lle............... 348

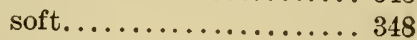

spi'ssum .............. 349

Petroleum ointment ........ 348

soft............. 348

hard........... 349

Phroretin .............. 575

Pharmaceutical processes..... 72

Pharmacognosy, definition of. 1

Pharmacology, definition of.. 1

Pharmacopoia, definition of

preparations of the.... $\quad 76$

Pharmaco-dynamics......... 1

Pharmacy, definition of..... 1

Phenacetin ................ 319

Phenaceti'num............. 319

Phenol.................. 324

Phillip's milk of magnesia... 16

Physical incompatibility....83, 84

Physiological incompatibility.

83,84

Phosphide of zinc.........236

Phosphorated oil.......... 233

Phosphoric acid........... 265

diluted............... 256

Phosphorus.............. 233

spirit of ............ 234 


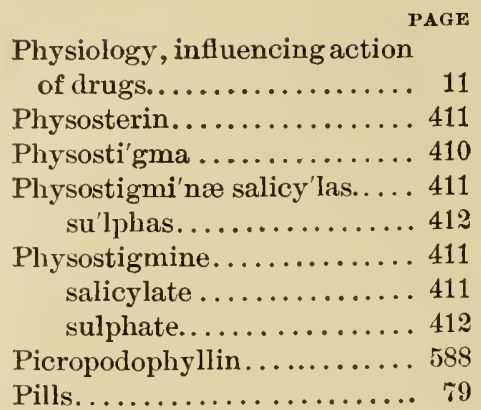

of asafetida...........538

blue ................ 207

cathartic, compound.... 210

Pilocarpi'na hydrochlo'ras ... 440 ni'tras.............. 441

Pilocarpine............. 439

lyydrochlorate......... 440

nitrate ...............441

Pilocarpidine............... 439

Piloca'rpus.............. 439

Pi'lula hydra'rgyri.......... 206

Pi'lulæ................. 79

Pinene................ 500

Pitch................. 507

Burgundy............ 504

plaster .............. 505

Pix Burgu'ndica........... 504

li'quida.............. 507

ni'gra.............. 5 ( 7

Plasma, blood, drugs acting on 32

Plasters................. 79

Plu'mbi ace'tas............. 171

ca'rbonas ............. 172

io'didum ............. 172

ni'tras .............. 172

Plu'mbi o'xidum.......... 170

Plumbum.............. 170

Pneumogastric, drugs acting

on................. 34

Podophyllin ............. 588

Podophyllinic acid......... 588

Podophy'llum............ 587

Podophylloquercitin........588

Podophyllotoxin.......... 588
PAGE

Pomegranate............6 620

Port...................... 282

Posology................... 10

table of.............661

Potash, caustic........... 122

Pota'ssa................. 122

cum ca'lce............. 123

solution of ............ 122

sulphura'ta ........... 260.

with lime............ 123

Pota'ssii ace'tas............ 125

bica'rbonas ............ 125

bita'rtras ............. 131

bro'midum ............ 240

ca'rbonas .............. 124

chlo'ras .............. 129

ci'tras ............... 125

cya'nidum............. 347

io'didum ............ 247

ni'tras............... 127

perma'nganas.......... 203

Potassium............... 121

acetate.............. 125

alum................ 166

arsenite, solution of..... 221

bicarbonate............ 125

bitartrate............ 131

bromide.............. 240

carbonate............. 124

chlorate .............. 129

citrate .............. 125

cyanide.............. 347

hydrate............. 122

solution of....... 122

hydroxide............ 122

iodide..... .. ....... 247

Potassium nitrate......... 127

permanganate.......... 203

tartrate, acid.......... 131

Powder, compound catechu.. 596 chalk .......... 153

liquorice......255, 579

Dover's............... 473

Gregory's ............ 576

ipecac and opium......473 


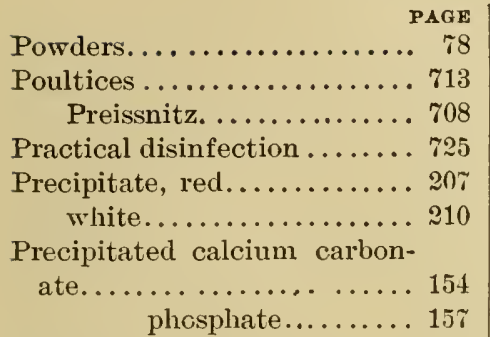

Preface................. iii

Preparations, pharmacopoial. 76

Prexpared chalk............ 153

Prescription, definition of.... 88 writing. ........... 86 words and phrases. used in......... 86

Prescriptions for balls....... 103 drenches............ 106 electuaries ............ 110 liniments ............. 112 mixtures............. 105 ointments............ 111 pills.................. 101 powders............. 107 suppositories......... 111

Primary action............ 2

Protargol................. 179

Protopine............... 352

Pru'nus virginia'na. ........ 318

Prussic acid.............. 342

Pseudoaconitine............ 459 aconine.............. 450 jervine.............. 464 Pelletierine..........6 620

Pu'lvis ca'techu conpo'situs .. 596 $\mathrm{Pu}^{\prime}$ lvis cre'tre compo'situs .... 153 Pu'lvis glycrrhi'za compo'situs...........255, 579 ipecachua'nhæe et o'pii. 353,473 kino co .............. 598 rhe'i compo'situs........ 5 5\%6

Pu'lveres................. 78

Punicotannic acid.........6 620

Pupil, drugs acting on....... 45

Purgatives............... 24
Purgatives, cho drastic............... 24 hydragogue ........... 25 laxative............. 24 saline................ 25 simple.............. 24 uses of.......... 28

Purified cotton............ 636 ox gall..............655 655

Pustulants..........662, 697 Pyre'thrum.............. 6 624 Pyridine................4 423

Pyrocatechin ............. 598

Pyrogallic acid............ 594

Pyrogallol................ 594

Pyroxylin.............6637

Pyroxyli'num........... 637

Quarter eril vaccine........ 751

Qua'ssia................. 555

Quassiin.................. 555

Quercin................ 595

Quercitannic acid......... 595

Quercite................. 595

Que'rcus a'lba........... 595

Querenne's digitalin........ 446

Quicksilver. .............206

Quinamine................ 477

Quinic acid .............. 4 48

Quinicine.............. 47

Quinidi'næ su'lphas......... 482

Quinidine............... 477

sulphate........... 483

Quini'na................. 47.

Quini'na bimuria'tis ca'rbamas...............482 bisu'lphas............. 480 hydrobro'mas .......... 481

Quini'næ hyrochlo'ras...... . 481 su'lphas............. 483 valeria'nas. ............ 482 Quinine...............4 47 bisulphate.......... 480 hydrobromate.........4 481 hydrochlorate.........4 481 
PAGE

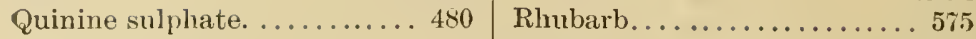

valerianate......... 482 Ricinolein ............ 571

Quinoidine..........44\%, 482 Rosin ...............506

Quinoidi'num........... 482 Rottle'ra..............618

Rational therapeutics....... 1 Rubefacients..........62, 00

Rectal injections.......... 9 Rubi jervine............. 464

Rectal feeding.......... 694 Rum................ 281

Rectified oil of turpentine... 499 Rutin................ 541

Rectification............ 75

Red corpuscles, drugs acting on................ 33

Red cinchona........... 479 mercuric oxicle........2 218 pepper................ 525 precipitate...........208 ointment.........208

wine................ 282

Reduced iron ............ 192

Refrigerants............6, 711

Remote action ............ 2

Resin cerate............. 506 plaster................ 506

Resi'na jala'pæ............ 583 podophy'lli............ 588 scammo'nii ........... 583

Resi'næ............... 71

Resins................ 71

Resorcin ................................. 337

Resorcinol............... 337

Rèsorci'num ............. 337

Respiratory organs, drugs acting on..........46, 471 mucous membrane, drugs acting on .......... 46

Rha'mnus cartha'rticus...... 574 purshia'na............ 572

Rhamnose................ 574

Rhatanic red............. 599

Rhatanin ............... 599

Rhatany ................ 598

Rheotannic acid........... 575

Rhe'um ................. 575

Rhein.............575, 577

Rhigolene............. 350

Sab'ina................ 543

Saccharated ferrous carbonate.............. 194

pepsin ..............6 653

Sacchari faex............ 614

Sa'ccharum.............. 613

la'ctis.............. 652

Sal ammoniac............ 149

soda................. 133

volatile............. 147

Salicin ... ............ 491

Salici'num............... 491

Salicylic acid............ 490

Salicylism .............. 492

Salicylous acid........... 492

Salicyluric acid........... 492

Saline purgatives........... 25

infusions ........... 732

Salivary glands, drugs acting on $\ldots \ldots \ldots \ldots \ldots \ldots, 15$

Salol.................. 491

Salt, common........... 137

Epson.............. 163

Glauber's ............ 136

Salts of tartar............ 124

Saltpetre............... 127

Santo'nica .............6.621

Santonin...............6. 621

Santoni'num.............6.61

$\mathrm{Sa}^{\prime}$ po ................6. 603

mo'llis. .............664 604

vi'ridis................ 604

Savine............... 543

Scaling................. 73

Scammonin............. 583 
PAGE

Scammo'nium. . . . . . . . . . 582

Scammony............... 582

Scarification..............

Scheele's prussic acid..... . . 342

Schmiedeberg's digitalin..... 446

Scilla . . . . . . . . . . . . . . 456

Scillin................ . $45 i$

Scillipicrin ............. 457

Scillitoxin.............. 457

Sclerotinic acid ......... 625

Secondary action.......... 2

Secretion of milk, drugs influencing............. 58

Sedatives, gastric.......... 23

Selective action........... 2

Se'nna................. 5\%8

Alexandria ........... 5\%8

Tinnivelly ........... 5\%8

Indian .............. 578

Sennacrol.............. 578

Sennapicrin............ 5\%8

Sensory nerves, drugs acting

on................4 431

Serum therapy............ 741

antistreptococcic....... 745

Se'vum................. 648

Sexual organs, drugs acting on 56

Sherry................. 283

Sheet bath............... 707

Sialagogues .............. 15

Silver.................. 175

cyanide..........176, 347

iodide.............. 176

nitrate ............. 175

diluted.......... 176

moulded......... 176

oxide............... 176

soluble............... 180

Simple purgatives.......... 24

Sinalbin................ 515

Sinapin sulphate......... 515

Si'napis................ 515

Si'napis a'lba ............ 515

ni'gra.............. 515

Sinapism............... 518

Sinigrin............... 516

Skin, drugs acting on....... 62

Soap.................603

composition of........6 603

green...............664 604

hard...............603

liniment............604

plaster..............604 604

soft..............664

white castile.........6 603

Socaloin ... ............ 5 565

Ścotrine aloes............ . 56t

Solla..................... 182

baking.............. 134

caustic.............. 132

solution of ........... 132

washing ............ 183

So'dii be'nzoas ............. 513

bica'rbonas............ 134

bisu'lphis ............ 142

bo'ras.............. 273

bro'midum............ 241

ca'rbonas ............ 133

exsicca'tus....... 133

chlo'ridun............ 139

hyposu'lphis ......... 143

io'didum............ 247

pho'sphas............ 141

salicylas.............491

su'lphas ............. 136

su'lphis............. 142

Sodium ................. 132

benzoate............ 513

bicarbonate.......... 134

bisulphite............ 142

borate.............. 273

bromide.............. 241

carbonate............ 133

dried............ 133

chloride............. 139

hydrate............. 132

solution of ....... 132

hydroxide............ 132

hyposulphite........... 139

iodide.............. 247 
Sodium phosphate......... 141

salicylate ........... 491

sulphate ............ 136

sulphite ............ 142

Soft petrola'tum.......... 348

petroleum ointment...... 348

soap...............664 604

liniment........664 604

Soluble silver ............ 180

Solution of acetate of ammon-

ium............... 150

ammonia............... 144

stronger ......... 144

arsenite of potassium.... 221

arsenous acid.......... 221

chloride of iron ......... 195

zinc.............. 182

chlorinated lime.......... 238

soda... ........ 238

hydrogen dioxide....... 119

morphine acetate....... 356

hydrochlorate...... 356

iodine, compound....... 244

line............... 155

potash .............. 123

soda................. 133

subacetate of lead....... 171

diluted........... 172

subsulphate of iron...... 196

Solutions ................ 74

Soporifics.................. 40

Spanish flies.............. 639

Spearmint............... 530

oil of............. 530

spirit of............. 530

water of............. 530

Spermace'ti.............650

Sphacelinic acid........... 625

Spinal cord, drugs acting on. . 423

Spirit of ammonia.......... 145

aromatic......... 148

camphor............. 548

chloroform...........296

anise.............. 582

ether................ 292
PAGE

Spirit of ether compound..... 293

glonoin............... $30 y$

juniper............. 542

compound.....281, 542

nitrous ether........... 308

peppermint.......... 528

pliosphorus............. 234

rectified,............, 278

Spearmint............. 530

Spirits .............. 77

Spi'ritus ...............

Spi'ritus $æ^{\prime}$ theris........... 29

compo'situs........2.:

nitro'si.......... 308

ani'si................ 583

ammo'niæ............. 145

Spi'ritus ammo'niæ aroma'ti-

cus................ 148

me'nthæ piperi'tæ...... 528

vi'ridis ......... 530

pho'sphori ............ 234

rectifica'tus............ 278

te'nuior.............. 278

vi'ni ga'llici........... 281

Squill................. $456^{6}$

compound syrup of ...... 457

Staphisa' gria ...........6.623

Staphisagrine............ 623

Stavesacre..............623

Stearin..............136, 648

Stearopten.............. 547

Sternuatories $\ldots \ldots \ldots \ldots \ldots$. 46

Stimulants, cerebral......... $3 \&$

hepatic .............. 26

Stomach, drugs acting on .... 16

Stomach tube, uses of........ 739

Stomachics.............. 16

Stout................. 283

Strophanthidin .......... 453

Strophanthin .........453, 454

Stropha'nthus........... 453

Strychni'na............401

Strychni'næ su'lphas........ . 402

Strychnine............... 401

sulphate............ 402

Stupes................ 715 


\section{PAGE}

Styptic collodion........591, 638

Styptics................ 63

Styrol.................. 510

Subcutaneous injections..... 6

Sublimate, corrosive ....... 208

Sublimed sulphur......... 255

Succus hyoscyami .......... 388

Sudorifics................ 65

Suet.................6648

mutton............6648

Sugar.................6 613

cane...............6 613

of milk............652

of lead.............. 171

Su'lphur............... 255

flowers of............ 255

liver of..............260

lo'tum.............. 255

milk of............. 256

ointment............. 256

precipitated........... 256

præcipita'tum ......... 256

sublima'tum ...........255

sublimed ............. 255

washed............... 255

Sulphuric acid ........... 263

aromatic............. 263

diluted................ 263

ether................ 292

Sulphurated pota'ssa ....... 260

Sulphurous acid........... 259

Su'pposito'ria............. 79

glyceri'ni...........6607

iodofo'rmi............ 252

morphi'næ............. 356

Suppositories............ 79

Suppurants.............. 704

Surgical antisepsis........ 726

Sweat, drugs influencing secre-

tion of ............. 65

Sweet flag.............. 563

Sweet spirits of nitre....... 308

oil.................. 602

Symbols (signs) ......... 90, 91

Synthetic oil of wintergreen.. 496
Syrup, simple............614

Sy'rupi................. 77

Syrups.............77, 614

Sy'rupus aca'ciæ..........612

a'cidi hydrio'dici ........ 248

althæ'æ............ 613

ca'lcii lactophospha'tis ... 158

ca'leis............... 156

fe'rri io'didi........... 194

fu'sci.............6 614

ipecacua'nha ......... 473

pru'ni virginia'næ....... 348

rha mui catha'rtici...... 574

sci'llæ............... 457

composi'tus.....229, 457

toluta'nus ........... 511

Taba'cum.............. 423

Tables of weights and meas-

ures................90-98

Tallianine............... 545

Tannic acid............. 590

Tannin.................. 590

Tar.................... 507

Taraxacin................ 559

Taraxacerin.............. 559

Tara'xacum............. 559

Tartrated antimony........ 228

Tartar emetic............. 228

Tartar, salts of........... 124

cream of............ 131

Tea ................396, 400

Terebene................ 500

Terebe'num............. 500

Terebi'nthina ............ 498

canade'nsis ........... 505

Terpenes................ 499

Terpin hydrate............ 500

Terpi'ni hy'dras........... 500

Tetanocannabine .......... 370

Tetanus antitoxin......... 742

Thebaine................ 351

Theine................ 39;

Theobro'ma, oil of ......... 626

Theobromine............ $89 \dot{0}$ 
Therapeutics............... 1

definition of........... 1

empirical ............. 1

general ............... 1

rational ................ 1

Theri'aca...............6 614

Thiol.................. 660

Thymol................ 5.51

Tiglinic acid............ 580

Tinctu'ra aco'niti ......... 459 alo'ës et my'rrhæ.....524, 566 a'rnica ............. 523

flo'rum .......... 522

ra'dicis .......... 523

asafoe'tidæ............ 538

bella'donnæe folio'rum.... 373

benzoi'ni.............. 512

compo'sita. .......512

bu'chu............... 541

calu'mbr........... 558

ca'nnabis i'ndicæ........ 392

cantha'ridis...........6 644

ca'psici ............. 526

cardamo'mi.......... 533

compo'sita ....... 533

cascari'llæ............ 557

ca'techu ............ 596

compo'sita....... 596

cincho'næ........... 479

compo'sita. ...... 479

co'lchici se'minis....... 632

digita'lis.............. 446

ergo'tæ $\ldots \ldots \ldots \ldots \ldots \ldots 626$

ammonia'tæ.....66 626

fe'rri chlóride ......... 195

perchlo'ridi....... 195

gelse'mii............ 420

gentia'næ compo'sita..... 553

hydra'stis............ 561

hyosey'ami............ 388

io'di ................. 244

ipecacua'nhæe et o'pii...353, 473

ki'no................. 598

krame'riæ............ 599

my'rrhæ............524
Tinetu'ra nu'cis vo'micæ...., 401 o'pii................ 353 camphora'tre...... 353 deodora'tæ........ 354

qua'ssiæ............ 555

sci'llæ.............. 457

stropha'nthi........... 453

valerianæ........... 535

ammoniatre..... 535

Tinctu'ræ..............

Tineture of larkspur......... 624

Tinctures............... $\%$

Tobacco.................. 423

Tolu.................... 511

Tonics ................. 60

Tow..................6 637

Toxicology, definition of ..... 1

Tragacanth...............6 612

Tragaca'ntha.............6 612

Transfusion.............. 732

Treacle.................. 614

Trituration................ $7_{2}$

Trituratio'nes............. 78

Triturations ............... 88

Tuberculin ............. 747

Turpentine .............. 498

Canada............... 505

liniment.............. 499

oil of .............. 499

Ungue'nta.............. 79

Ungue'ntum ............. 648

a'cidi carbo'lici.......... 324

ta'nnici ......... 591

aconiti'næ............ 463

bella'donnæ. ........... 373

ceta'cei.............6.651

ehrysarobi'ni .......... 5\%7

credé................ 181

ga'llæ............... 590

cum o'pio........ 590

hydra'rgyri ........... 207

ammonia'ti ....... 211

nitra'tis.......... 210

o'xidi fla'vi........ 208 


\section{PAGE}

Ungue'ntum hyra'rgyri ru'bri. 207

io'di.................. 244

iodofo'rmi............. 250

petro'lei.............. 348

pi'cis li'quide......... 507

pota'ssii io'didi......... 247

sabinæ.............. 544

su'lphuris............. 255

Ungue'ntum zi'nci o'xidi. .... 184

United States Pharmacopœia. 76

Urinary tract, drugs acting on 52 sedatires.............. 56

antiseptics ........... 56

Urine, drugs altering compo-

sition of ................

drugs increasing secretion

of $\ldots \ldots \ldots \ldots \ldots \ldots$.

acidifying....... 55

making alkaline.... 55

making aseptic..... 56

Urotropin............... 341

Uterus, drugs acting on..... 58

Uterine action, drugs restrain.ing.................. 58

Vaccines, black leg or quarter evil................751

anthrax..............751

Vagus, drugs acting on .....34, 35

Valerian................. 534

Valeria'na.............. 534

Valeria'næ rhizo'ma........ 534

Valerianic acid............ 535

Vaseline.................. 348

Vasomotor centre, drugs acting on................. 36

Vegetable bitters.......... 552

cathartics.............. 544

drugs................. 351

Venesection .............. 728

Veratralbine...............4468

Veratri'na...............4468

Veratrine...............4 468

Veratroidine...........464, 465

Vera'trum vi'ride.......... 464
Vera'trum al'bum . ..... PAGE 468

Vermicides................ 67

Vermifuges.............. 68

Vesicants.............62, 697

Vessels, drugs acting on ..... 36

Vienna paste............. 123

Vi'na................... 77

Vinegars................ 78

Vi'num a'lbum............. 282

Vi'num antimo'nii.......... 229

co'lehici ............6 632

colchici ra'dicis .........632

co'lchici se'minis........ 632

ipecacua'nlıæ.........4 473

o'pii................ 354

porte'nse............ 282

ru'brum................ 282

xe'ricum.............. 283

Vile'llus, glacerite of ....... 607

Vitriol, blue.............. 186

green................ 193

Volatile oils...........71, 497 oil of mustard......... 516

Warm baths............ 717

Wash, black .............. 217 yellow...............216

Washed sulphur .......... 235

Washing soda............. 133

Water.................. 117

Waters................. 77

Wax .................. 649

yellow................6 649

white...............650 650

Weights and measures .....92-98

Wet pack.............. r 08

Whiskey ................. 280

White arsenic............220

castile soap ...........6 603 corpuscles, drugs acting on................. 33

hellebore.............. 464

lead.................. 172

mustard .............. 515

oak................ 595 


\begin{tabular}{|c|c|}
\hline PAGE & PAGE \\
\hline White precipitate.......... 211 & Zinc............... \\
\hline ointment.........211 & acetate.............. 184 \\
\hline wax ................6 650 & carbonate, precipitated... 183 \\
\hline wine............... 282 & chloride............... 182 \\
\hline wild cherry............... 348 & oxide................ 184 \\
\hline Wine, white.............. 282 & Zinc oxide ointment......... 184 \\
\hline red................ 282 & phosphide............ \\
\hline Wines................. 77 & sulphate............. \\
\hline Wintergreen, oil of......... 495 & valerianate.......... \\
\hline artificial oil of $\ldots \ldots \ldots \ldots 496$ & Zi'nci ace'tas............ 184 \\
\hline synthetic oil of ........ 496 & ca'rbonas praecipita'tus .. 183 \\
\hline Witchhazel ............... 601 & chlo'ridum............. 182 \\
\hline Wool fat, hydrous ..........6 649 & o'xidum ......... \\
\hline Wormseed............... 621 & pho'sphidum............ 236 \\
\hline oil of $\ldots \ldots \ldots \ldots \ldots \ldots, 621$ & su'lphas.............. 183 \\
\hline Yellow jasmine........... 420 & 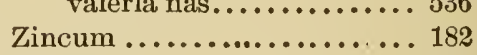 \\
\hline mercuric oxide.........207 & Zi'ngiber................. \\
\hline wash $\ldots \ldots \ldots \ldots \ldots \ldots 216$ & Zymine................ \\
\hline wax $\ldots \ldots \ldots \ldots \ldots \ldots, 649$ & \\
\hline Yolk of egg.............. 607 & \\
\hline
\end{tabular}





\section{CATAlocue of WILLIAM R. JENKINS'}

\section{Works Concerning \\ HORSES, CATTLE, SHEEP, SWINE, Etc. 1905}

(*) Single asterisk designates New Books.

(**) Double asterisk designates ${ }^{*}$ Recent Publications

ANDERSON. "Vice in the Horse" and other papers on Horses and Riding. By F. L. Anderson. Size, $6 \times 9$, cloth, illustrated.................. 75

ARMSTEAD. "The Artistic Anatomy of the Horse." A brief description of the various Anatomical Structures which may be distinguished during Life through the Skin. By Hugh W. Armstead, M.D., F.R.C.S. With illustrations from drawings by the author. Cloth oblong, $10 \times 12 \frac{1}{2} \ldots \ldots \ldots \ldots \ldots \ldots \ldots \ldots . \ldots \ldots$

$\boldsymbol{B A C H}$. "How to Judge a Horse." A concise treatise as to its Qualities and Soundness; Including Bits and Bitting, Saddles and Saddling, Stable Drainage, DrivIng One Horse, a Pair, Four-in-hand, or Tandem, etc. By Capt. F. W. Bach. Size, $5 \times 7 \frac{1}{2}$, clo., fully lllus. 100

(*)BANHAM. "6 Anatomical and Pliysiological Model of the Cow." Half life size. Composed of superposed plates, colored to nature, showing internal organs, muscles, skeleton, etc., mounted on strong boards, with explanatory text. Size of Model opened, $10 \mathrm{ft}$. $33 \mathrm{ft}$., closed $3 \mathrm{ft} . \times 1 \frac{1}{2} \mathrm{ft} \ldots \ldots \ldots \ldots \ldots \ldots 70$

- "Anatomical and Physiological Model of the Horse." Half llfe size. By George A. Banham, F.R.C.V.S. Size of Model 38 x 41 in................. 750 
BANHAM (continued)

- "Tables of Veterinary Posology and Therapentics," with weights, measures, etc. By Geo. A. Banham, F.R.C.V.S. New edition. Cloth, size 4 x 51-2, 192 pages................................ 00

BAUCHER. "Method of Horsemanship." Including the Breaking and Training of Horses. By F. Baucher........................... 00

(*)BELL. "The Veterinarian's Call Book (Perpetual)," By Roscoe R. Bell, D.V.S., editor of the American Veterinary Review. Revised every year.

A visiting list, that can be commenced at any time and used until full, containing much useful information for the student and the busy practitioner. Among contents are items concerning: Veterinary Drugs; Poisons; Solubility of Drugs; Composition of Milk, Bile, Blood, Gastric Juice, Urine, Saliva ; Respiration; Dentition; Temperature, etc., etc. Bound in flexible leather, with flap and pocket .......... 125

BITTING. "Caliot's Exercises in Equine Surgery." See "Cadiot."

BRADLEY. " "Outlines of Yeterinary Anatomy." By O. Charnock Bradley, Member of the Royal College of Veterinary Surgeons; Professor of Anatomy in the New Veterinary College, Edinburgh.

The author presents the most important facts of veterinary anatomy in as condensed a form as possible, consistent with lucidity. $12 \mathrm{mo}$.

Complete in three parts.

Part I.: The Limbs (cloth)................. 25

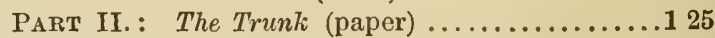

Part III.: The Head and Neck (paper)......... 25

The Set complete ...................... 25 
CADIOT. "Exercises in Equine Surgery." By P. J. Cadiot. Translated by Prof. A. W. Bltting, D.V.M. Edited by Prof. A. Liautard, M.D.V.M. Size, $6 \times 9 \frac{1}{4}$, cloth, illustrated.................. 250

- "Roaring in Horses." Its Pathology and Treatment. This work represents the latest development in operative methods for the alleviation of roaring. Each step is most clearly defined by excellent full-page illustrations. By P. J. Cadiot, Professor at the Veterinary School, Alfort. Translated by Thos. J. Watt Dollar, M.R.C.V.S., ete. Cloth, size 5 1-4 x 7 1-8, 77 pages, illustrated................... 75

- "Studies in Clinical Veterinary Medicine and Surgery." By P. J. Cadiot. Translated, edited, and supplemented with 49 new articles and 34 illustrations by Jno. A. W. Dollar, M.R.C.V.S. Cloth, size $7 \times 93.4,619$ pages, 94 black and white illustrations............. 25

(*)-" A Treatise on Surgical Therapeutics of the Domestic A nimils." By P. J. Cadiot and J. Alny. Translated by Prof. A. Liautard, M.D.,V.M.

Part I.-Restraint of horses, cattle, etc. ; general anxsthesia ; surgical antisepsis and asepsis ; hemostasis and cauterization; firing. 45 illus ......1 00

Part II.-Inflammations, gangrene, foreign bodies, traumatic lesions, chilblains, frost bites, congeliations, complications of traumatic lesions, etc...1 00 Part III.-Granulations, cicatrices, mycosis, virulent diseases, tumors, diseases of skin and cellular tissue, tendons, etc.................. 100 Part IV. (completing the volume) nearly ready.

CHAPMAN. "'Manual of the Pathological Treatment of Lameness in the Horse," treated solely by mechanical means. By George T. Chapman. Cloth, size $6 \times 9,124$ pages with portrait...........200

CHAUVEAU. "The Comparative Anatomy of the Domesticated Animals." By A. Chauveau. Revised by G. Fleming, F.R.C.V.S. 8vo, eloth, 585 illus..6 25 
CLARKE. "Chart of the Feet and Teeth of Fossil Horses." By W. H. Clarke. Card, size 9 1-2 x 12.. 25

-“Horses' Teeth." Fourth edition, re-revised, with second appendix. Cloth, size $51-4 \times 7$ 1-2, 322 pp., illus..2 50

CLEAVELAND. "Pronouncing Medical Lexicon." Pocket edition. By C. H. Cleveland, M.D. Cloth, size $31.4 \times 41-2,302$ pages............... 75

CLEMENT. "Veterinary Post Mortem Examinations." By A. W. Clement, V.S. The absence in the English language of any guide in making autopsies upon the lower animals, induced Dr. Clement to write this book, trusting that it would prove of practical value to the profession. Cloth, size $5 \times 71-2,64$ pages, illustrated.................... 75

(*) COURTENAY. "Manual of the Practice of Veterinary Medicine." By Edward Courtenay, V.S. Revised by Frederick T. G. Hobday, F.R.C.V.S. Second edition. Cloth, size $51-4 \times 71-2,573$ pages $\ldots \ldots \ldots \ldots .275$

COX. "Horses : In Accident and Disease." The sketches introduced embrace various attitudes which have been observed, such as in choking; the disorders and accidents occurring to the stomach and intestines; affection of the brain; and some special forms of lameness, etc. By J. Roalfe Cox, F.R.C.V.S. Cloth, size $6 \times 9,29$ full page illustrations.............. 50

CURTIS. "Hor'ses, Cattle, Sheep and Swine." By Geo. W. Curtis, II.S.A. Cloth, size 7 1-4 x 10, 343 pages, 117 illustrations..................250

("*) DALR YMIPLE. "Veterinary 0bstetries." A compendium for the use of advanced students and Practitioners. By W. H. Dalrymple, M. R. C. V. S., principal of the Department of Veterinary Science in the Louisiana State University and A. \& M. College; Veterinarian to the Louisiana State Bureau of Agriculture, and Agricultural Experiment Stations. Cloth, sire 6 × 9 1-4, 162 pages, 51 illustrations...2 50 
DALZIEL. " Brenking and Training Dogs." Part I, by Pathfinder. Part II, by Flugh Dalziel. Cloth, illustrated.......................... 50

- “The Collie." By Hugh Dalziel. Paper, illustrated.... 40

- "The Diseases of Dogs." Causes, symptoms and treatment. By Hugh Dalziel. Cloth, illustrated..........1 00

- “Diseases of Horses." Paper ................... 40

— “The Fox Terrier." By Hugh Dalziel. Paper, 40; clo.1 00

- "The Greylound." Cloth, illus................1 00

- "The St. Bernard." Cloth, illustrated............100

$D A N A$. "Tables in Comparative Physiology." By Prof. C. L. Dana, M.D. Chart, $17 \times 17 \ldots \ldots \ldots \ldots \ldots .25$

DANCE, "Veterinary Tablet." By A. A. Dance. Chart, $17 \times 24$, mounted on linen, folded in a cloth case for the pocket, size $33-4 \times 6$ 1-2. Shows at a glance the synopsis of the diseases of horses, cattle and dogs; with their cause, symptoms and cure.......... 75

(*)DE BRUIN. "Bovine 0bstetries." By M. G. De Bruin Instructor of Obstetrics at the State Veterinary School in Utrecht. Translated by W. E. A. Wyman, formerly Professor of Veterinary Science at Clemson A. \& M. College, and Veterinarian to the South Carolina Experiment Station. Cloth, size $6 \times 9,382$ pages, 77 illustrations...................5 00

Synopsis of the Essential Fentures of the Work

1. Authorized translation.

2. The only obstetrical work which is up date.

3. Written by Europe's leading authority on the subject.

4. Written by a man who has practiced the art a lifetime

5. Written by a man who, on account of his eminence as bovine practitioner and teacher of obstetrics, was selected by Prof. Dr. Frōhner and Prof. Dr. Bayer (Berlin and Vienna), to discuss bovine obstetrics both practically and scientifically.

b. The only work containing a thorough differential rias. nosis of ante and post partuı çiseases. 
DE BRUIN, “Bovine Obstetrics” (continued)

7. The only work doing justice to modern obstetrical surgery and therapeutics.

8. Written by a man whose practical suggestions revolutionized the teaching of veterinary obstetrics even in the great schools of Europe.

9. The only work dealing fully with the now no longer obscure contagious and infectious diseases of calves.

10. Absolutely original and no compilation.

11. The only work dealing fully with the difficult problem of teaching obstetrics in the colleges.

12. The only work where the practical part is not overshadowed by theory.

. A veterinarian, particularly if his location brings him in contact with obstetrical practice, who makes any pretence toward being scientific and in possession of modern knowledge upon this subject, will not be without this excellent work, as it is really a very valuable treatise.-Prof. Roscoe $R$. Bell, in the American Veterinary Review.

In translating into English Professor De Bruin's excellent textbook on Bovine Obstetrics, Dr. Wyman has laid British and American veterinary surgeons and students under a debt of gratitude. The wolks represents the happy medium between the booklets which are adapted for cramming purposes by the student, and the ponderous tomes which, although useful to the teacher, aro not exactly suited to the requirements of the everyday practitioner . We can strongly recommend the work to veterinary students and practitioner's.-The Journal of Comparative Pathology and Therapcuties.

(*)DOLLAR. "'Diseases of Cattle, Sheep, Goats and Swine." By G. Moussu and Jno. A. W. Dollar, M.R.C.V.S. Size 6 × 9 1-2, 785 pages, 329 illustrations in the text and 4 full page plates in colors.......8 75

(*) - "A Hand-book of Horse-Shoeing," with introductory chapters on the anatomy and physiology of the horse's foot. By Jno. A. W. Dollar, M.R.C.V.S., with the collaboration of Albert Wheatley, F.R.C.V.S. Cloth, size $6 \times 8$ 1-2, 433 pages, 406 illustrations ..4 75

- " Operative Techni(yue." Volume 1 of "The Practice of Veterinary Surgery." Cloth, size $63-4 \times 10,264$ pages, 272 illustrations........................ 375

_ "Geucral Surgery." Volume 2 of "The Practice of Veterinary Surgery." In preparation.

(")-"Regional Veterinary Surgery." Volume 3 of "The Practice of Veterinary Surgery." By Drs. Jno. A. W. Dollar and H. Möller. Cloth, size 6 1-2 x 10853 and xvi pages, 315 illustrations.............6 25 
DOLLAR-(continued)

- "Cadiot's Clinical Veterinary Medicine and Surgery." See "Cadiot."

- "Callot's Roaring in Horses."

See "Cadiot."

DUN. "Veterinary Medicines, their Actions and Uses." By Finlay Dun, V.S., late lecturer on Materia Medica and Dietetics at the Edinburgh Veterinary College, and Examiner in Chemistry to the Royal College of Veterinary Surgeons. Edited by James Macqueen, F.R.C.V.S. Tenth revised English edition.

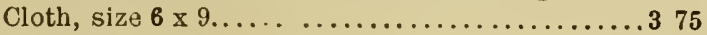

DWFER. "0n Seats and Saddles." Bits and Bitting, Draught and Harness and the Prevention and Cure of Restiveness in Horses. By Francis Dwyer. Cloth, size $5 \times 7,301$ pages, gilt, illustrated..........1 50

FLEMING. "Animal Plagnes." Their History, Nature, and Prevention. By Geo. Fleming, F.R.C.V.S., etc. First Series. Chronological History from B.C. 1490 to A.D. 1800. Cloth, size $6 \times 9,548$ pages......6 00 Second Series. Chronological History from A.D. 1800 to 1844 . Cloth, size $6 \times 9,539$ pages.......3 00

- "The Comparative Anatomy of the Domesticated Animals." By A. Chauveau. Translated by Dr. Fleming. See "Chauveau."

- "The Contagions Diseases of Animals." Their influence on the wealth and heaith of nations and how they are to be combated. Paper, size $5 \times 71-2,30$ pages ..... 25

- "Human and Animal Variolø." A Study in Comparative Pathology. Paper, size 5 1-2 $x 81$-2, 61 pages.... 25

- "Parasites and Parasitic Diseases of the Domesticated Animals." By L. G. Neumann. Translated by Dr. Fleming. 
FLEMING (continued)

- "Operative Veterinary Surgery." Vol. I, by Dr. Geo. Fleming, M.R.C.V.S. This valuable work, one of the most practical treatises yet issued on the subject in the English language, is devoted to the common operations of Veterinary Surgery ; and the concise descriptions and directions of the text are illustrated with numerous wood engravings. Cloth, size $6 \times 91-4,285$ and $x$ viii pages, 343 illustrations...........2 75

$\left({ }^{*}\right)$ Vol. II, edited and passed through the press by W. Owen Williams, F.R.C.V.S. Cloth, size $6 \times 91-4$, 430 and xxxvii pages, 344 illustrations.................. 25 Both volumes bound in one.............. 25

- "Roaring in Horses." By Dr. George Fleming, F.R.C.V.S. Its history, nature, causes, prevention and treatment. Cloth, size 5 1-2 $\times 83-4,160$ pages, 21 engravings, 1 colored plate................ 50

- "Tuberculosis." From a Sanitary and Pathological Point of View. By Geo. Fleming, F.R.C.V.S. Paper, size

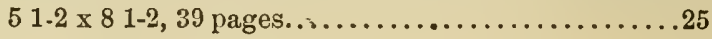

- "Veterinary Obstetrics." Including the Accidents and Diseases incident to Pregnancy, Parturltion, and the Early Age in Domesticated Animals. By Geo. Fleming, F.R.C.V.S. Cloth, size $6 \times 83-4,758$ pages, illus. 625

$\left.{ }^{*}\right)$ GOTTHIEL. "A Manual of General Histology." By Wm. S. Gottheil, M.D., Professor of Pathology in the American Veterinary College, New York; etc., etc.

Histology is the basis of the physician's art, as Anatomy is the foundation of the surgeon's science. Only by knowing the processes of life can we understand the changes of disease and the action of remedies; as the architect must know his building materlals, so must the practitioner of medicine know the intimate structure of the body. To present thls knowledge in an accessible and simple form has been the author's task. Second edition revised. Cloth, size 5 1-2 x 8, 152 pages, 68 illustrations...100 
GRESSWELL. "The Bovine Prescriber." For the use of Veterinarians and Veterinary Students. Second edition revised and enlarged, by James B. and Albert Gresswell, M.R.C.V.S. Cloth, size, $5 \times 7$ 1-2, 102 pages.......................... 75

- "The Equine Hospital Prescriber." For the use of Veterinary Practitioners and Students. Third edition revised and enlarged, by Drs. James B. and Albert Gresswell, M.R.C.V.S. Cloth, size $5 \times 71-2,165$ pages............................. 75

- "Diseases and Disorders of the Horse." A Treatise on Equine Medicine and Surgery, being a contribution to the science of comparative pathology. By Albert, Jas. B. and Geo. Gresswell. Cloth, size $53-4$ × 8 3-4, 227 pages, illustrated................. 175

- Manual of "The Theory and Practice of Equine Ifedicine." By James B. Gresswell, F.R.C.V.S., and Albert Gresswell, M.R.C.V.S. Second edition revised. Cloth, size 5 1-4 x 7 1-2, 539 pages............2 75

- "Veterinary Pharmacopaia and Manual of Comparative Therapy." By George and Charles Gresswell, with descriptions and physiological actions of medicines, by Albert Gresswell. Second edition revised and enlarged. Cloth, $6 \times 83-4,457$ pages.......... 50

HASSLOCH. "A Compend of Veterinary Materia Medica and Therapentics." By A. C. Hassloch, V.S., Lecturer on Materia Medica and Therapeutics, and Professor of Veterinary Dentistry at the New York College of Veterinary Surgeons and School of Comparative Medicine, N. Y. Cloth, size $51-4$ × $71-2,225$ pages................................ 50

HEATLEY. "The Stock 0wner's Guide." A handy Medical Treatise for every man who owns an ox or cow. By George S. Heatley, M.R.C.V.S. Cloth, size $51-4 \times 8,172$ pages..................... 25 
$\left({ }^{* *}\right)$ HILL. "The Diseases of the Cat." By J. Woodroffe Hill, F.R.C.V.S. Cloth, size $51.4 \times 7$ 1-2, 123 pagee, illustrated............................. 25

Vritten from the experience of many years' practice and close pathological research into the maladies to which our domesticated feline friends are liable-a subject which it must be admitted has not found the prominence in veterinary literature to which it is undoubtedly entitled.

- "The Management and Diseases of the Dog." By J. Woodroffe Hill, F.R.C.V.S. Cloth, size $5 \times 71-2$, extra fully illustrated..................200

HINEBAUCH. "Veterinary Dental Surgery." By T. D. Hinebauch, M.S.V.S. For the use of Students, Prac. titioners and Stockmen. Cloth, size $51-4 \times 8,256$ pages, illustrated........................... 00

HOARE. “A Manual of Veterinary Therapeuties and Plarmacology." By E. Wallis Hoare, F.R.C.V.s. Cloth, size $51-4 \times 71-4$, 560 pages.............2 75

$\left({ }^{*}\right)$ HOBDAY. "Canine and Feliue Surgery॰" By Frederick T. G. Hobday, F.R.C.V.S, Cloth, $53-4$ × $83-4,152$ pages, 76 illustrations.................200

$\left({ }^{*}\right)$ " The Castration of Cryptorchid Horses and the Ovariotomy of Troublesome Mares." By Frederick T. G. Hobday, F.R.C.V.S. Cloth, size 5 3-4 × 8 3-4, 106 pages, 34 illustrations......... 75

$\left.{ }^{* *}\right)$ HUNTING. The Art of Horse-shoeing. A manual for Horseshoers. By William Hunting, F.R.C.V.S., ex-President of the Royal College of Veterinary Surgeons. One of the most up-to-date, concise books of its kind in the English language. Cloth, size $6 \times 91-4$, 126 pages, 96 illustrations................ 00

$\left.{ }^{* *}\right)$ JENKINS. "Model of the Horse" and "Model of the Cow."

See "Banham." 
KEATING. "A New Unabridged Pronouncing Dictionary of Medicine." By John M. Keating, M.D., LL.D., Henry Hamilton and others. A voluminous and exhaustive hand-book of Medical and scientific terminology with Phonetic Pronunciation, Accentuation, Etymology, etc. With an appendix containing important tables of Bacilli, Micrococci, Leucomaines, Ptomaines; Drugs and Materials used in Antiseptic Surgery; Poisons and their antidotes; Weights and Measures; Themometer Scales; New Officinal and Unofficinal Drugs, etc., etc. Cloth, 818 pages ...5 00

("*)KOBERT. "I'ractical Toxicology for Physicians and Students." By Professur Dr. Rudolph Kobert, Medical Director of Dr. Brehmer's Sanitarium for Pulmonary Diseases at Goerbersderf in Silesia (Pr'ussia), late Director of the Pharmacological Institute, Dorpat, Kussia. Translated and edited by L. H. Friedburg, Ph.D. Authorized Edition. Practical knowledge by means of tables which occupy little space, but show at a glance similarities and differences between poisons of the same group. Also rules for the Spelling and Pronunciation of Chemical Terms, as adopted by the American Association for the Advancement of Science. Cloth, $61.2 \times 10,201$ pp..2 50

К ОСН. “Etiology of Tuberculosis." By Dr. R. Koch. Translated by T. Saure. Cloth, size $6 \times 9$ 1-4, 97 pages................................ 10

LAMBERT. "The Germ Theory of Disease." Bearing upon the health and welfare of man and the domesticated animals. By James Lambert, F.R.C.V.S. Paper, size 51.4 x $81.4,26$ pages, lllustrated.... 25

LAW. "Farmers' Veterinary Adviser." A Guide to the Prevention and Treatment of Disease in Domestic Animals. By Prof. James Law. Cloth, size $51-4$ × 7 1-2, illustrated...................3 00 
$\left.{ }^{(* *}\right) \boldsymbol{L E G G E . ~ " C a t t l e ~ T u b e r c u l o s i s . " ~ A ~ P r a c t i c a l ~ G u i d e ~}$ to the Farmer, Butcher and Meat Inspector, By T.M. Legge, M.A., M.D., D.P.H., and Harold Sessions, F.R.C.V.S. Cloth, size $51-2 \times 81-2,77$ pages....1 00

("*)LIAUTARD. "Animal Castration." A concise and practical Treatise on the Castration of the Domestic Animals. The onls work on the subject in the English language. By Alexander Liautard, M.D., V.S. Having a fine portrait of the author. Tenth edition revised and enlarged. Cloth, size $51-4$ × $71-2,165$ pages, 45 illustrations...................200

. . The most complete and comprehensive work on the subject in English veterinary literature. - American Agriculturist.

- "Cadiot's Exercises in Equine Surgery." Translated by Prof. Bitting and edited by Dr. Liautard.

\section{See "Cadiot."}

- "A Treatise on Surgical Therapentics of the Domestic Animals." By Prof. Dr. P. J. Cadiot and J. Almy. Translated by Prof. Liautard.

See "Cadiot."

- "How to Tell the Age of the Domestic Animal." By Dr. A. Liautard, M.D., V.S. Standard work upon this subject, concise, helpful and containing many illustrations. Cloth, size $5 \times 71-2,35$ pages, 42 illustrations........................... 60

- "Lameness of Horses and Diseases of the Locomotory Apparatus." By A. Liautard, M.D.,V.S. This work is the result of Dr. Liautard's many years of experience. Cloth, size $51-4 \times 71-2,314$ pages.......2 50

- "Manual of Operative Veterinary Surgery." By A. Liautard, M.D., V.M. Engaged for years in the work of teaching this special department of veterinary medicine, and having abundant opportunities of realizing the difficulties which the student who earnestly strives to perfect himself in his calling is obliged to encounter, the author formed the deter- 
LIAUTARD (continued).

mination to facilitate his acquisition of knowledge, and began the accumulation of material by the rompilation of data and arrangement of memorandum, with the recorded notes of his own experience, the fruit of a long and extended practice and a careful study of the various authorities who have illustrated and organized veterinary literature. Cloth, size $61-4$ × 9,786 pages, 563 illustrations........5 00

- "Pellerin's Median Neurotomy in the Treatment of Chronic Tendinitis and Periostosis of the Fetlock." Translated by Dr. A. Liautard.

See "Pellerin,"

- "Vade Mecum of Equine Anatomy." By A. Liautard, M.D.V.S. For the use of advanced stutents and veterinary surgeons. Third edition, Cloth, size $5 \times 71-2,230$ pages and 10 full page illustrations of the arteries. ......................... 00

- Zundel's "The Horse's Foot and Its Diseases."

See "Zundel."

LONG. "Book of the Pig." Its selection, Breeding, Feeding and Management. Cloth...........400

$\left.{ }^{* *}\right)$ LOWE. "6reeding Rachorses by the Figure Systell." Compiled by the late C. Bruce Lowe. Editerl by William Allison, "The Special Commissioner," London Sportsman, Hon. Secretary Sporting League, and Manager of the International Horse Ageney and Exchange. With numerous fine illustrations of celebrated horses. Cloth, size $8 \times 10,262$ pages............................. 50

LUDLOW. "Science in the Stable"; or How a Hor'se can be Kept in Perfect Health and be Used Without Shoes, in Harness or under the Saddle. With the Reason Why. Second Edition. By Jacob R. Ludlow, II.D. Late Staff Surgeon, U. S. Army. Paper, size $41-2 \times 53-4,166$ pages................... 50 
LUPTON. "Horses: Sound and Unsound," with Law relating to Sales and Warranty. By J. Irvlne Lupton, F.R.C.V S. Cloth, size $53-4 \times 71.2,217$ pages, 28 illustrations.................. 25

MAGNER. "Standarl Horse and stock Book." By D. Magner. Comprising over 1,000 pages, illustrated with 1756 engravings. Leather binding. .......6 00

$M C B R I D E$. "Anatomical Ontlines of the Horse." By J. A. McBride, M R C.V.S. Second edtion revised and enlarged. Cloth, size $51.4 \times 71-4$, illus ...2 50

(")M'FADYEAN. "Anatomy of the Horse." Second edition completely revised. A Dissection Gulde. By John M'Faryean, M.B., B.Sc., F.R.S.E. Cloth, size $6 \times 834,388$ pages, illustrated..........5 50

This book is intended for Veterinary students, and offers to them in its 48 full-page colored plates, 54 illustrations and excellent text, a valuable and practical aid in the study of Vetelinary Anatomy, especially in the dissecting room.

- "Comparative Anatomy of the Domesticated Animals." By J. M'Fadyean. Profusely illustrated, and to be issued in two parts.

Part I-Osteology, ready. Size 5 1-2 x 8 1-2, 166 pages, 132 illustrations. Paper, 2 50; cloth....2 75

(Part II in preparation.)

MILLS. "How to Keep a Dog in the City." By Wesley Mills, M.D., D.V.S. It tells how to choose, manage, house, feed, educate the pup, how to keep him clean and teach him cleanliness. Paper, size $5 \times 7$ 1-2, 40 pages........................... 25

(*)MÖLLER - DOLLAR. "Regional Veterinary Surgery." See "Dollar."

MOHLER. "I'andbook of Meat Inspection." By Robert Ostertig, M.D. Translated by Earley Vernon Wilcox, A.M., Ph.D. With an introduction by John R. Mohler, V.M.D., A M, See "Ostertag." 
MOSSELMAN-LIENAUT. "Manual of Veterinary Microbiology." By Professors Mosselman and Liénaux, Nat. Veterinary College, Cureghem, Belgium. Translated and edited by R. R. Dinwiddie, Professor of Veterinary Science, College of Agriculture, Arkansas State University. Cloth, size $51.2 \times 8,342$ pages, illustrated........................... 00

(*)MOUSSU. " "Diseases of Cattle, Sheep, Goats and Swille." By G. Moussu and Jno. A. W. Dollar, II.R.C.V.S.

See "Dollar."

NEUMANN. "A Treatise on Parasites and Parasitic Diseases of the Domesticated Animals." A work to which the students of human or veterinary medl. cine, the sanitarian, agriculturist or breeder or rearer of animals, may refer for full information regarding the external and internal Parasites-vegetable and animal-which attack various species of Domestle Animals. A Treatice by L. G. Nemmann. Professor at the National Veterinary School of Toulouse. Translated and edited by George Fleming, C. B., I.L. D..F.R.C.V.S. Cloth, size $63.4 \times 1$, 873 pages, 365 illustrations ........................ 50

NOCARD. "The Animal Tuberculoses, and their Relation to Human 'Tuberculosis." By Ed. Nocard, Prof. of the Alfort Veterinary College. Translated by H. Scurfield, M.D. Ed., Ph. Camb. Cloth, $5 \times 7$ 1-?, 143 pages... 100

Perhaps the chief interest to doctors of human medicine in Professor Nocard's book lies in the demonstration of the small part played by lieredity, and the great part played by contagion in the propagation of bovine tuberculosis. It seems not unreasonable to suppose that the sane is the case for human tuberculosis, and that, if the childrell of tuberculous parents were protected from infection by cohabitation or ingestion, the importance of heredity as a cause of the disease, or even of the predisposition to it, would dwindle away into insignificance. 
OSTERTAG. "Handbook of Meat Inspection." By Robert Ostertag, M.D. Authorized Translation by Earley Vernon Wilcox, A.M., Ph.D. With an introduction by John R. Mohler, V.M.D., A.M. The work is exhaustive and authorative aud has at once become the standard authority upon the subject Cloth, size $63-4$ × $93-4,920$ pages, 260 illustrations and 1 colored plate............................. 50

$P A L L I N$. "A Treatise on Epizoolic Lymphangitis." By Capt. W. A. Pallin, F.R.C.V.S. In this work the author has endearored to rombine his own experience with that of other writers and so attempts to give a clear and complete account of a subject about which there is little at present in English veterinary literature. Cloth, size $53-4 \times 81-2,90$ pages, with 17 fine full page illustrations................... 25

PEGLER. "The Book of the Goat." Thirr edition rewritten and enlarged. Cloth, 223 pages, illus...1 75

PELLERIN. "Median Neurotomy in the Treatment of Chronic Tendinitis and Periostosis of the Fetlock." By C. Pellerin, late repetitor of Clinic and Surgery to the Alfort Veterinary School. Translated, with Additional Facts Relating to It, by Prof. A. Liautard, M.D., V.M. Having rendered good results when performed by himself, the author believes the operation, which consists in dividing the cubito-plantar nerve and in excising a portion of the peripherical end, the means of improving the conditions, and consequently the values of many apparently doomed animals. Agriculture in particular will be benefited.

The work is divided into wo parts. The first covers the study of Median Neurotomy itself; the second, the exact relations of the facts as observed by the author. Boards, $6 \times 9$ 1-2, 61 pages, illustrated..100

PETERS. "A Tuberculons Herd-Test with Tuberculin." By Austin Peters, M. R. C. V.S., Chief Inspector of Cattle for the New York State Board of Health during the winter of 1892-93. Pamphlet... 25 
REYNOLDS. "An Essay on the Breeding and Management of Draught Horses." By R. S. Reynolds, M.R.C.V.S. Cloth, size $51-2 \times 83-4,104$ pages.. 140

ROBERGE. "The Foot of the Horse," or Lameness and all Diseases of the Feet traced to an Unbalanced Foot Bone, prevented or cured by balancing the foot. By David Roberge. Cloth, size 6 × 91 1-4, 308 pages, illustrated.............................. 00

SEWELL. "The Examination of Horses as to Sound. ness and Selection as to Purchase." By Edward Sewell, M.R.C.V.S. Paper, size 51-2 × 81.2 , 86 pages, lllustrated with 8 plates in color............ 50

.... It is a great advantage to the business man to know something of the elements of law, and nobody ought either to buy or own a horse who does not know something about the animal. That something this book gives, and gives in a thoroughly excellent way....

-Our Animal Friends.

\section{SMITH. "A Manual of Veterinary Physiology." By} Veterinary Captain F. Smith, M.R.C.V.S. Author of "A Manual of Veterinary Hygiene."

Throughout this manual the object has been to condense the information as much as possible. The broad facts of the sclences are stated so as to render them of use to the student and practitioner. In this second edition-rewritten-the whole of the Nervous System has been revised, a new chapter dealing with the Development of the Ovum has been added together with many additional facts and illustrations. About one hundred additional pages are given. Second edition, revlsed and enlarged. Cloth, slze 6 × $83-4$, 673 pages, 102 illustrations................ 75

- "Manual of Veterinary Hygiene." Second edition revlsed. Cloth, size 5 1-4 x 7 1-2, 477 pages, 93 illus......2 75

(")STRANGEWAY. " Veterinary Anatomy." Edited by I. Vaughan, F.L.S., M.R.C.V.S. New edition revised. Cloth, size 61 -4 × 9 1-2, 625 pages, 224 11lus.....5 00 
SUSSDORF. "Six Large Colored Wall Diagrans." By Prof. Sussdorf, M.D. (of Göttingen). Text translated by Prof. W. Owen Williams, of the New Veterinary College, Edinburgh. Size, 44 inches by 30 inches.
1.-Horse.
4. $-0 \mathrm{x}$.
2.-Mare.
5.-Boar and Sow.
3.-Cow.
6.-Dog and Bitch.

The above are printed in eight or nine colors.

Showing the position of the viscera in the large cavities of the body.

Prlce, unmounted...................175 each

" mounted on linen, with roller... ...3 50 "

(*)ТНОМРSON. "Elementary Lectures on Veterinary Science." For agrlcultural students, farmers and stock keepers. By Henry Thompson, M.R.C.V.S., lecturer on Veterinary Science at the Aspatria Agricultural College, England. It is complete yet concise and an up-to-date book. Cloth, 397 pp., 51 illus...3 75

VAN MATER. "A Text Book of Veterinary Ophthaimology." By George G. Van Mater, M.D., D.V.S., Professor of Ophthalmology in the American Veterinary College; Oculist and Aurist to St. Martha's Sanitarium and Dispensary; Consultlng Eye and Ear Surgeon to the Twenty-sixth Ward Dispensary; Eye and Ear Surgeon, Brooklyn Eastern District Dispensary, etc. Illustrated by one chromo lithograph plate and 71 engravings. Cloth, $6 \times 91-4,151$ pages...3 00 . . We intend to adopt this valuable work as a text book.-E. J. Creely, D.V.S., Dean of the San Francisco Veterinary College.

VETERINARY DIAGRAMS in Tabular Form. Size, $28 \frac{1}{2}$ in. $\times 22$ inches. Price per set of five...4 00 Mounted and folded in case.................7 00 Mounted on roller and varnisbed............10 00

No. 1. "The External Form and Elementary Anatomy of the Horse." Eight colored illustrations1. External regions; 2. Skeleton; 3. Muscles (Superior Layer); 4. Muscles (Deep Layer); 5. Respiratory Ap- 
VLTERINARY DIAGRAMS (continued).

paratus; 6. Digestive Apparatus; 7. Circulatory Apparatus ; 8. Nerve Apparatus ; with letter-press description ................................... 25

Mounted on roller and varnished............2 25

No. 2. "The Age of Domestic Animals." Forty-two figures illustrating the structure of the teeth, indicating the Age of the Horse, Ox, Sheep, and Dog, with full description ........................ 75 Mounted on roller and varnished............2 00

No. 3. "The Unsoundness and Defects of the Horse." Fifty figures illustrating-1. The Defects of Conformation; 2. Defects of Position; 3. Infirmities or Signs of Disease ; 4. Unsoundnesses; 5 . Defects of the Foot; with full description..................... 75 Mounted on roller and varnished............200

No.4. "The Shoeing of the Horse, Mule and 0x." Fifty figures descriptive of the Anatomy and Physiology of the Foot and of Horse-shoeing...........75 Mounted on roller and varnished............2 00

No. 5. "The Elementary Anatomy, Points, and Butcher's Joints of the 0x." Ten colored illustrations -1. Skeleton; 2. Nervous System: 3. Digestive System (Right Side); 4. Respiratory System; 5. Points of a Fat Ox; 6. Muscular System; 7. Vascular System; 8. Digestive System (Left Side); 9. Butcher's Sections of a Calf : 10. Butcher's Sections of an Ox; with full description ............................. 25 Mounted on roller and varnished...........2 25

WALLEY. "Four Bovine Scourges." (Pleuro-Pneumonla, Foot and Mouth Disease, Cattle Plague and Tubercle). By Thomas Walley, M.R.C.V.S. With an Appendix on the Inspection of Live Animals and Meat. Quarto, cloth....................6 40 
WALLEY (continued).

(")- "A Practical Guide to Meat Inspection." By Thomas Walley, M.R.C.V.S., late principal of the Edinburgh Royal (Dick) Veterinary College; Professor of Veterinary Medicine and Surgery, etc. Fourth Edition, thoroughly revised and enlarged by Stewart Stockman, M.R.C.V.S., Professor of Pathology, Lecturer on Hygiene and Meat Inspection at Dick Veterinary College, Edinburgh. Cloth, size 5 1-2 × 81 -4, with 45 colored illus., 295 pages....3 00

An experience of over 30 years in his profession and a long official connection (some sixteen years) with Edinburgh Abattolrs have enabled the author to gather a large store of information on the subject, which he has embodied in his book.

While Dr. Stockman is indeed indebted to the old for much useful information, this up-todate work will hardly be recognized as the old " Walley's Meat Inspection."

WILCOX. "Haulbook of Meat Inspection." By Robert Ostertag, M.D.

See "Ostertag."

WILLIAMS. "Principles and Practice of Veterinary Medicine." Author's edition, entirely revised and lllustrated with numerous plain and colored plates. By W. Williams, M.R.C.V.S. Cloth, size $53-4$ × $83-4$, 863 pages.......................... 50

- "Principles and Practice of Veterinary Surgery." Author's edition, entirely revised and illustrated with numerous plain and colored plates. By W. Williams, M.R.C.V.S. Cloth, size $61.2 \times 91-4,756$ pages ............................ 50 
THE MOST COMPLETE, PROGRESSIVE AND

SCIENTIFIC BOOK ON THE SUBJECT IN

THE ENGLISH LANGUAGE

(") WINSLOW. "Veterinary Materia Medica and Therapeutics." By Kenelm Winslow, B.A.S., M.D.V., M.D., (Harv.); formerly Assistant Professor of Therapeutics in the Veterinary School of Harvard University; Fellow of the Massachusetts Medical Society ; Surgeon to the Newton Hospital, etc.

Second Edition Revised.

Cloth, size $61.4 \times 91-4,7 € 1$ pages.. ........6 00

Your letter received and I am pleased to know that we are to have an American Materia Medica. -J. H. Wattles, Sr., M.D., D.V.S., The Wester'n Veterinary College, Kansas City, Mo.

- Am delighted with it. It is remarkably correct, complete and up-to-date and is bound to supersede any other work on the same subject heretofore before the profession.

No practitioner's library is complete without it and it will be indispensable for students, as it does away with the necessity of their having a number of collateral books on the subject.

It will be adopted as the text book in the Chicago Veterinary. College.-Dr.E. L. Quitman, Chicago Veterinary College.

. . The book is of admirable merit and full of valuable information from beginning to end, very explicit, rich and interesting, and should be in the hands of every student as well as practitioner of the art of Veterinary Medicine.-Thurston Miller, M.D., Professor of Materia Medica, Therapeutics and Chemistry, San Francisco Veterinary College.

I consider it the only work on materia medica and therapentics suitable to the American veterinary practitioner. It deserves a wide distribution among veterinarians. I have recommended it to my students.-Juhn J. Repp, V.M.D., Iowa State College, Ames, Iowa. 
(*)WYMAN. "Bovine Obstetrics," By M. G. De Bruin. Translated by W. E. A. Wyman, M.D.V.,V.S.

See also "De Bruin."

(") - "Catechism of the Principles of Veterinary Surgery." By W. E. A. Wyman, M.D.V., V.S. Cloth, size $6 \times 9$, 317 pages ............................. 50

Concerning this new work attention is called to the following points:

1.-It discusses the subject upon the basis of veterinar'y investigations.

2.-It does away with works on human pathology, histology, etc.

3.--It explains each question thoroughly both from a scientific as well as a practical point of view.

4.- It is writen by one knowing the needs of the student.

5.-It deals exhaustively with a chapter on tumors, heretofore utterly neglected in veterinary pathology.

6.-The only work in English specializing the subject.

7.-The only work thoroughly taking into consideration American as well as European investigations.

8.- Offering practical hints which have not appeared in print, the result of large city and country practice.

(**) " The Clinical Diagnosis of Lameness in the Horse." By W. E. A. Wyman, D.V.S., formerly Professor of Veterinary Science, Clemson A. \& M. College, and Veterinarian to the South Carolina Experiment Station. Cloth, size $6 \times 9$ 1-2, 182 pp., 32 illus....2 50

(") - "Tibio-peroneal Neurectomy for the Relief of Sparin Lameness." By W. E. A. Wyman, M.D.V., V.S.

Boards, size $6 \times 9,30$ pages, illustrated....... 50 Anyone wanting to perform this operation should procure this little treatise; he will find it of considerable help.-The Veterinary Journal.

ZUNDEL. "The Horse's Foot and Its Iiseases." By A. Zundel, Principal Veterinarlan of Alsace Lorraine. Translated by Dr. A. Liautard, V.S. Cloth, size $5 \times 73-4,248$ pages, lllustrated.............2 00

ZUILL. "Typloid Fever; or Contagious Influenza in the Horse." By Prof. W. L. Zulll, M.D.,D.V.S. Pamphlet, size $6 \times 9$ 1-4, 29 pages............ 25

Any book sent prepaid for the price WILLIATI R. JENKINS, 


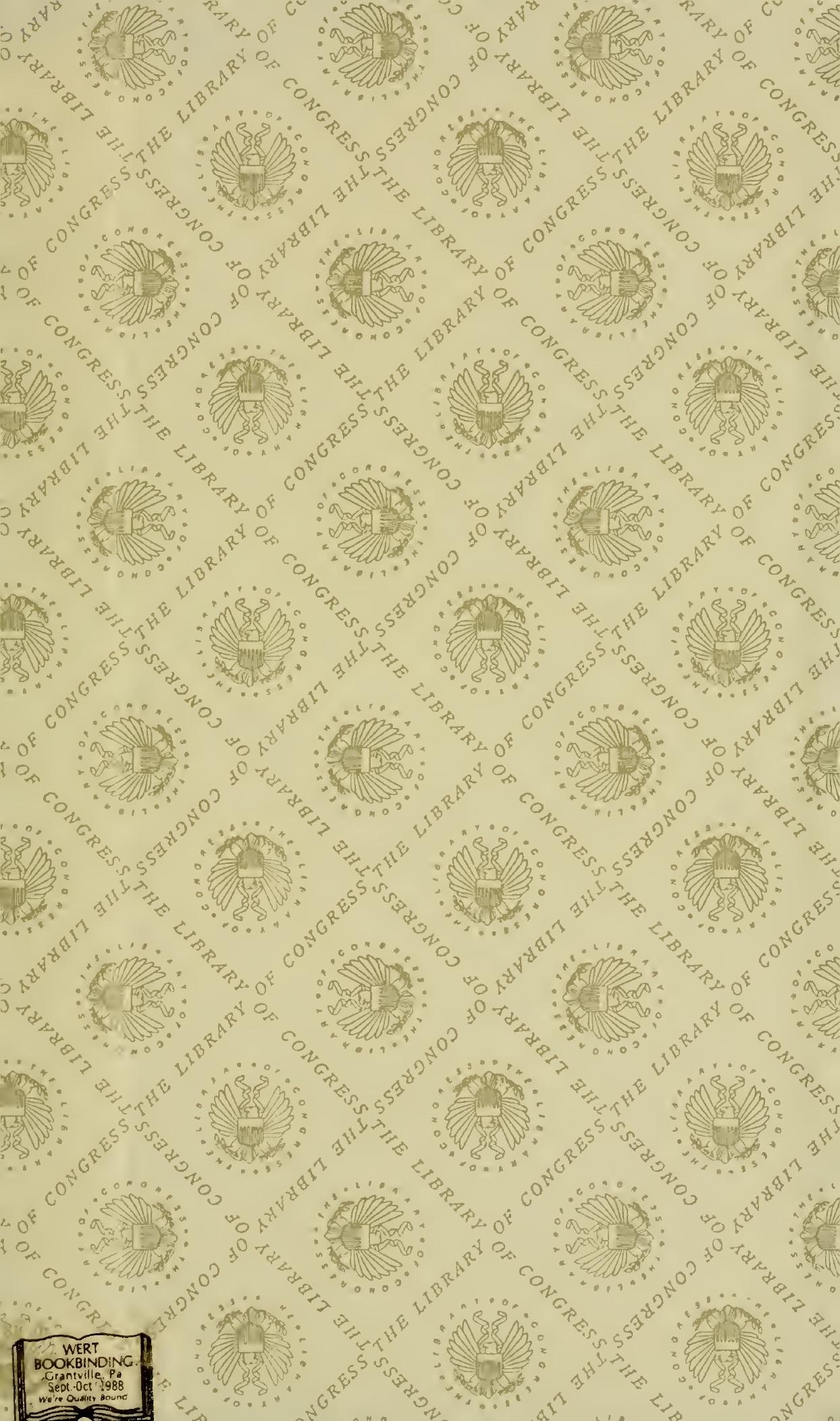
